\title{
WH1-77-64
}

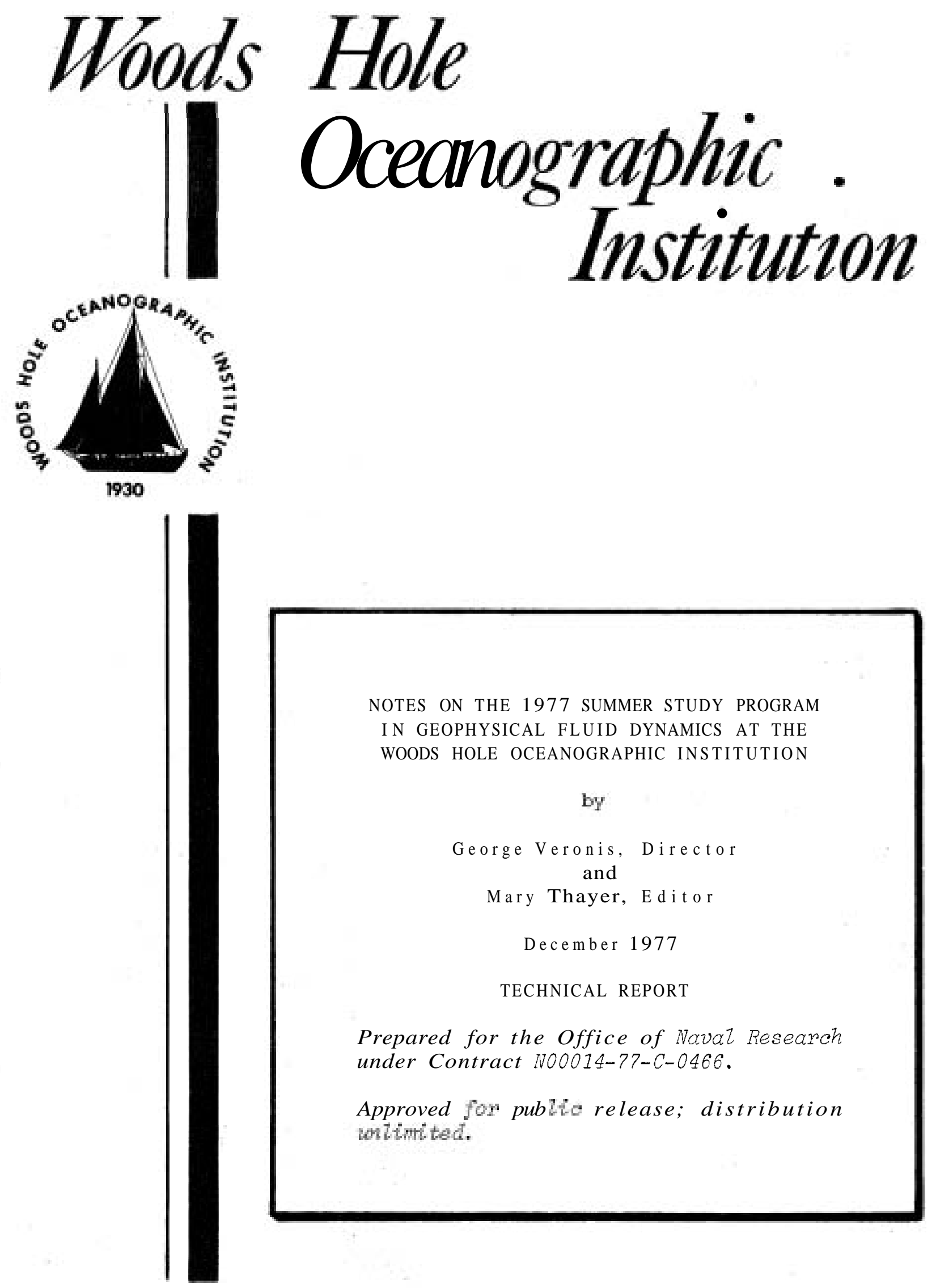

WOODS HOLE, MASSACHUSETTS $\mathbf{0 2 5 4 3}$ 
NOTES ON THE 1977 SUMMER STUDY PROGRAM IN GEOPHYSICAL FLUID DYNAMICS AT THE WOODS HOLE OCEANOGRAPHIC INSTITUTION

\author{
by \\ George Veronis, Director \\ and \\ Mary Thayer, Editor
}

WOODS HOLE OCEANOGRAPHIC INSTITUTION

Woods Hole, Massachusetts 02543

December 1977

TECHNICAL REPORT

Prepared for the Office of Naval Research under Contract No0014-77-C-0466.

Reproduction in whole or in part is permitted for any purpose of the United States Government. In citing this manuscript in a bibliography, the reference should be followed by the phrase: UNPUBLSHED MANUSCRIPT.

Approved for public retease; distribution unlimited.

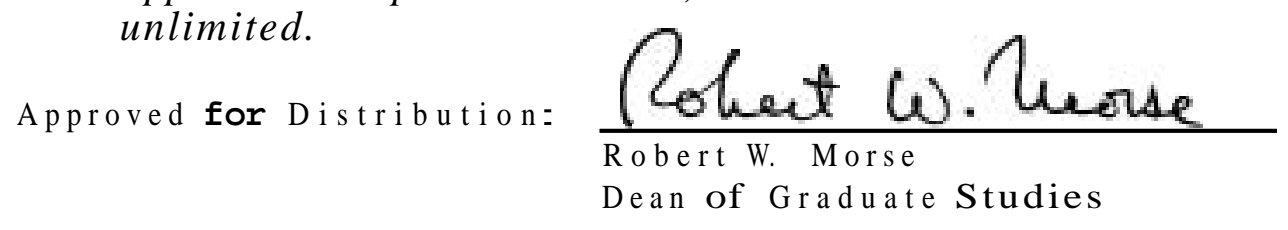




\title{
CONIENTS OF THE VOLUME
}

Course Lectures on Turbulence

\author{
Abstracts of Seminars \\ Microsymposium on Geostrophic Turbulence \\ (Abstracts)
}

Fellowship Lectures 


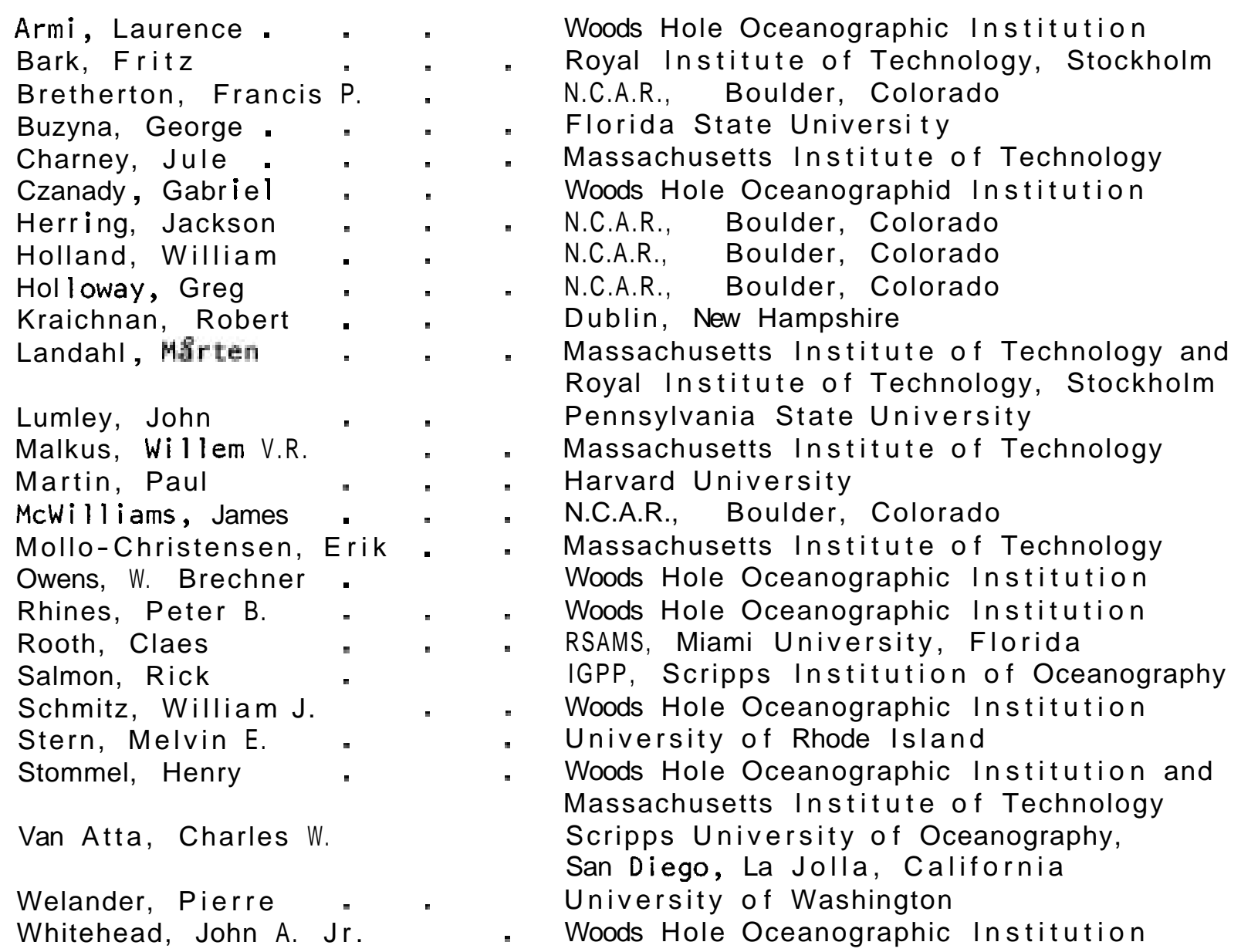

\section{Postdoctoral Participants}

Sarmiento, Jorge Louis Tsiang, Eugene $Y$
- . Lamont-Doherty Geological Observatory

- Goddard Institute of Space Studies

\section{Predoctoral Participants}

Gartrell, Gregory, Jr. Giuliani, John L. Jr. Gustavsson, Lars Hakan Hoffman, Ross $\mathrm{N}$ Holt, Rush D.

Lewalle, Jacques Loder, John Wayne. Masuda, Akira Maxey, Martin Richard Morris, John Stephen Poyet, Jean-Pierre. Stevenson, James W. .
- California Institute of Technology

- Yale University

- Royal Institute of Technology, Sweden

- Massachusetts Institute of Technology

- New York University

- Pennsylvania State University

- Dalhousie University, Hali fax, Nova Scotia

- Kyushu University, Japan

- University of Cambridge, England

- The Johns Hopkins University, Baltimore

- Columbia University

- Harvard University 


\section{Editors' Preface}

The lectures by Marten Landahl, recorded in the first part of this report, served as the introduction to the study of turbulence which was the principal theme of the nineteenth summer program in Geophysical Fluid Dynamics at the Woods Hole Oceanographic Institution. The reports of the lectures by the students were reworked by Professor Landahl so that they serve as a careful record of his presentation.

The abstracts of the seminars by invited speakers cover a broad range of topics, from astrophysics to experimental, laboratory fluid dynamics. The seminars served, to introduce the student fellows to the tremendous diversity of studies that fall within the scope of geophysical fluid dynamics. Included among the abstracts are the seminars presented during the one-week symposium on geostrophic turbulence. Peter Rhines organized the symposium and invited many of the principal contributors to this important, developing topic.

The twelve student lectures summarize the most creative product of the summer program. These reports are necessarily preliminary because the short time available to the students does not admit a carefullw worked, finished product. Some of the reports will appear later as published articles and will represent more effectively the product of the summer activity.

It is a pleasure to acknowledge the financial support that was arranged by Ralph Cooper of the Fluid Dynamics Division of the Office of Naval Research. In an emergency situation he contacted six agencies, three ONR, one NSF and two Air Force, and assembled the necessary funds for our operation. We are deeply indebted to him for his faith in the program and for his eagerness to help.

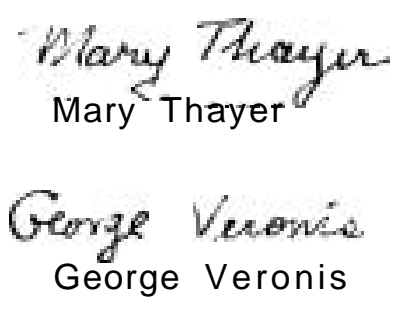




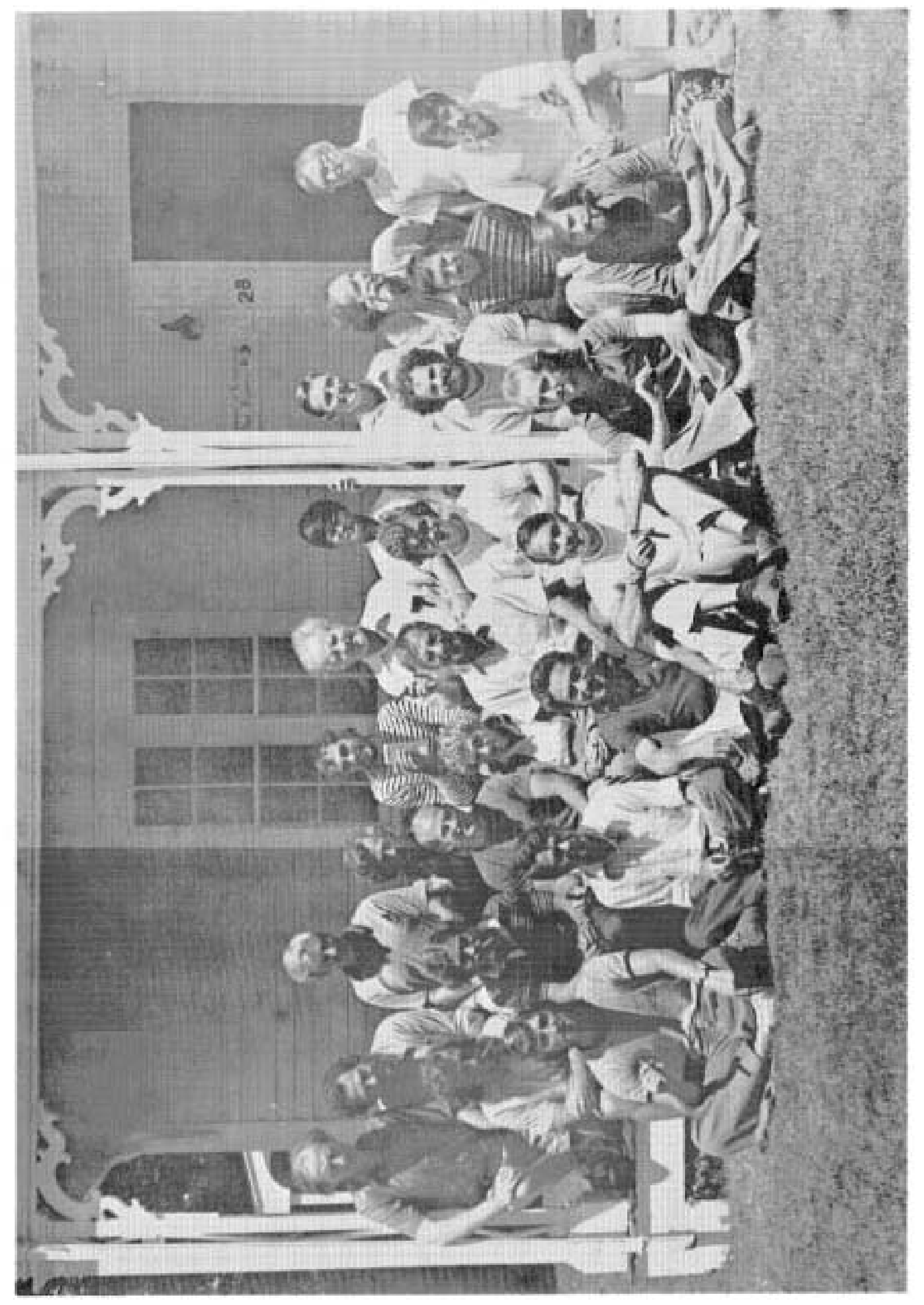

-

兵

x.

in

5 .

界

-

5

$8>$

$\approx-\overline{0}$

-

西

ô.

- $=$

-

등

政

$3=$

$8 \div$

ज证

.

등돈

$\leq$ 응

당

ํㅗㄹ 要

- w

昌的要

ㄴ.

的要

5․

중

悾

$\frac{7}{4}$

훈

$0^{2}$

o

+

u ㅜㅇ

일 문오 
Course Lectures on Turbulence

$$
\text { by }
$$

Mårten Landahl

Massachusetts Institute of Technology

and

Royal Institute of Technology, Stockholm, Sweden

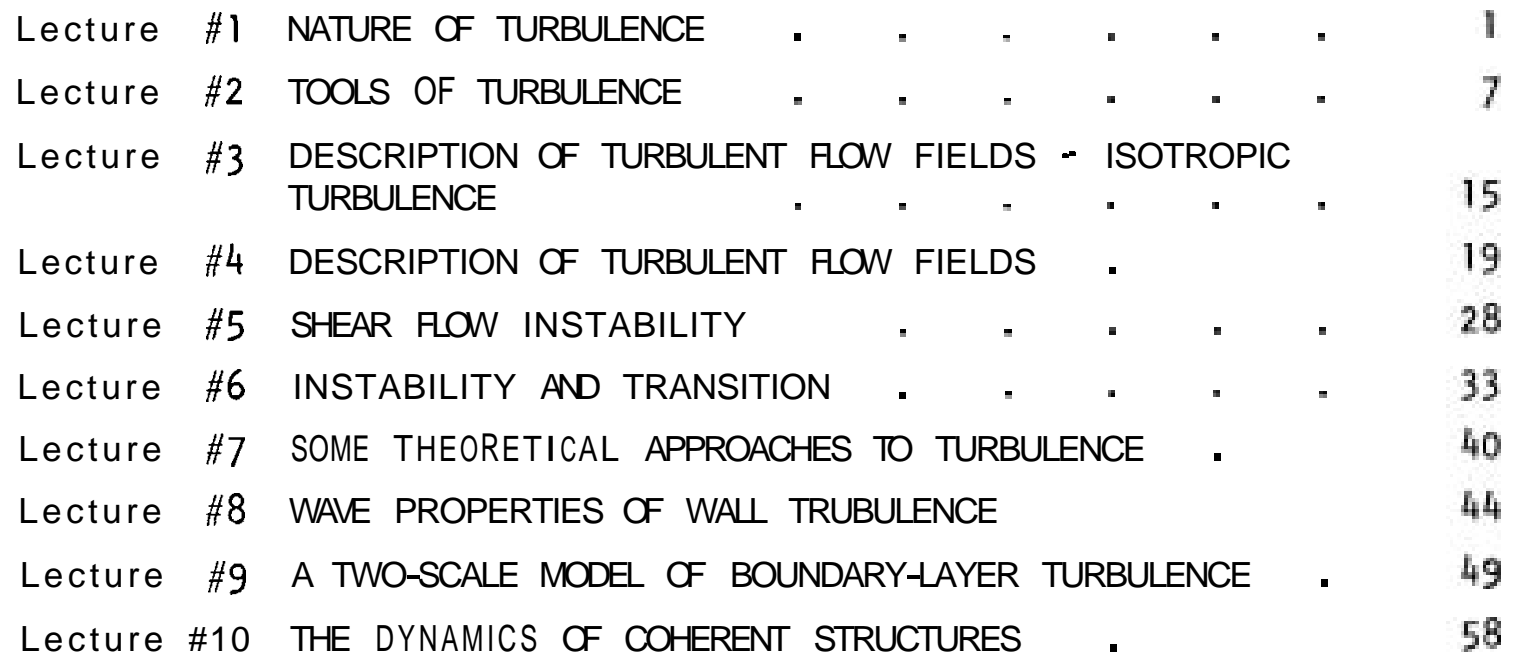

\section{Abstracts of Seminars}

Effects of Density Stratification on Turbulence $\quad 65$ Laurence Armi

Boundary Mixing and Lateral Advection: The Dynamic Duo Responsible for Vertical Mixing in the Deep Ocean 68 Laurence Armi

$\begin{array}{ll}\text { Spin-up of a Rapidly Rotating Gas } & 69\end{array}$ Fritz H. Bark

Turbulent Interface Layers . . . 70 Gabriel T. Csanady

The Return to Isotropy of Homogeneous Turbulence . 70 John L. Lumley

Turbulent Velocity Profiles from Stability Theory . $\quad 72$ Willem V.R. Malkus

Application of Phase Transition Methods to the Large Scale Spectral Properties of Stirred Fluids . Paul C. Martin

Gerstner's Edge Waves in a Rotating Stratified Fluid 75 Erik Mollo-Christensen

Wave Packets

Erik Mollo-Christensen 
Page No.

On Modelling the Superlayer

Stephen J. Morris

Photon Bubbles

Edward A. Spiegel

Extreme Discharge in Turbulent and Other Indeterminate Flows. Melvin E. Stern

The Beta Spiral and the Determination of the Absolute Velocity

Field from Hydrographic Station Data Henry Stommel

Coherent Temperature Structures in Shear Flow. Charles W. Van Atta

Model of World Ocean Circulation George Veronis

Building Blocks for Ocean Circulation Theory. Pierre Welander

Rayleigh-Bénard Convection - Laminar and Turbulent John A. Whitehead, Jr.

Hydromagnetic Turbulence Robert Kraichnan

1. An Axisymmetric Ocean Circulation Model

2. A Two-dimensional Model of the Thermocline Circulation Jule G. Charney

\section{Micro-Symposium on Geostrophic Turbulence} (Abstracts)

Two-dimensional Turbulence above Topography Francis P. Bretherton

The Transition to - and the Region of - Geostrophic Turbulence

in a Rotating, Differentially Heated Annulus George Buzyna

Low-frequency Fluctuations beneath the Gulf Stream at $55^{\circ} \mathrm{W}$ Ross Hendry

Statistical Theory of Stratified Flow above Topography . Jackson Herring

Statistical Accommodation among Quasigeostrophic Eddies (What can you learn from a box of red and blue marbles?) Greg Holloway

Approximate Models of Turbulence. Robert Kraichnan

The General Circulation in a B-Plane Channel James C. McWilliams

Simulated Dynamic Balance for Mid-Ocean Mesoscale Eddies

W. Brechner Owens

Eddy-induced Abyssal-Ocean Circulation

Peter B. Rhines and William R. Holland 


\section{Fellowship Lectures}

Wave Breakdown in a Stratified Shear Flow

Gregory Gartrell, Jr.

'Twin-Exhaust' Galaxy Models and Instabilities in Channels with

Flexible Walls . . . . . " John L. Giuliani

The Initial Value Problem for an Infinite Shear Layer with a Discontinuous Density Profile.

L. Hakan Gustavsson

Antarctic Water at the Equator Ross N. Hoffman

Steady Solutions for the Leighton Solar Dynamo Model . Rush D. Holt

On the Fractal Geometry of the Small-Scale Structure of High Reynolds Number Turbulence Jaques Lewalle

A Grid-Stirring Experiment with Buoyancy Input John W. Loder

Reflection and Transmission of Rossby Waves by Bottom Topography 191 Akira Masuda

Development of Disturbances to an Inviscid Shear Layer . Martin R. Maxey

A Model of the Upwelling Due to Topographically Guided Flow. Stephen J.S.Morris

Coupling between Pulsation and Convection in a Star Jean-Pierre Poyet

Coupling between a Surface Mixed Layer and Large Scale Steady

Interior Ocean Motions James W. Stevenson 
COURSE LECTURES

by

Mårten Landahl

Massachusetts lnstitute of Technology

and

The Royal Institute of Technology, Stockholm, Sweden

TURBULENCE

Lecture \#l.

1. NATURE OF TURBULENCE

1.1 Characteristics of turbulent flows

This lecture is an introduction into the subject of turbulence, a field that is very old but still far from being well understood. A 1514 drawing by da Vinci showing the discharge from a sewage outfall in Milan indicates both the age of practical interest in turbulent flows and some of the important features of turbulence. Among the latter are the sharp bound ary between the turbulent and nonturbulent regions, the presence of both large and small-scale eddies in the flow, and the strong mixing.

It is difficult to give a precise definition of turbulence; this is perhaps partly due to the incomplete understanding of the flow phenomena involved. Turbulent flows generally exhibit fluctuations which are random in space and time. An important characteristic of turbulence is the large range of scales of eddies present in the flow. This can be illustrated, for example, in photographs of jets with and without asbestos fibers suspended in the fluids. (Filipsson et $a l ., 1977)$. In the jet with fibers, small-scale eddies are inhibited, leaving only the large-scale eddies, which are seen to be fairly well organized. In the jet without fibers, the large scales are still there, but are obscured by the small-scale eddies.

The Reynolds number, $\boldsymbol{R}_{\boldsymbol{e}} \equiv \boldsymbol{\ell} \boldsymbol{V}_{\boldsymbol{0}} / \boldsymbol{\nu}$ gives a ratio of a typical viscous diffusion time in the fluid to a typical convection time. Here, Uo is a typical velocity in the fluid, $\boldsymbol{l}$ a reference length, and $\nu$ is the kinematic viscosity. While turbulent flows are usually characterized by high Reynolds number, indicating that viscous effects are small, it should be noted that a turbulent flow will always create a range of scales for which viscosity is important.

\subsection{Brief historical review}

We give here a brief review of some of the early important work in turbulence.

Reynolds (1883) carried out experiments with flow in a straight cylindrical pipe and found that there were basically three regimes of flow, namely, laminar and turbulent flows (termed by him "sinuous" flows), and a transition state between the two. The latter shows laminar and turbulent regions alternately. Reynolds was the first to point out that the dimensionless combination $\boldsymbol{\ell} \mathbf{V}_{0} / \mathcal{\nu}$ (later to be named the Reynolds number) is an important quantity in describing the flow regimes. For small values of $\operatorname{Re}$, the flow is laminar, at higher values, the flow may become turbulent. Turbulence cannot be maintained for Re $\sim 2000$. He also examined the Navier- 
Stokes equations and introduced the concept of turbulent stresses

$$
-\rho u_{i} u_{j}
$$

Boussinesq (1877) was the first to suggest the use of an eddy viscosity in turbulent flow problems which he assumed to be a constant. This assumption, however, was soon found to be inadequate for flow near a solid boundary .

Prandtl (1925) and Taylor (1915, 1922) introduced the mixing length hypothesis for boundary layer flows. The spectral theory of turbulence was initiated by Taylor $(1935,1938)$. Major advances in this field were made in the Russian school by Kolmogorov (1941), Obukhov (1941), and Millionshikov (1939, 1941). Other important contributions to the theory of homogeneous turbulence were those by Heisenberg (1948) and Batchelor (1951, 1953).

One problem area which is closely related to turbulence is that of flow instability and transition. Substantial advances have been made in this field over the years. Rayleigh (1880) found that a necessary condition for flow instability is an inflection in the velocity profile. Orr (1907) and Sommerfeld (1908) independently developed equations governing the stability of fluids. Some solutions of the eigenvalue problem were provided by Tietjens (1925), Tollmien (1933) and Schlichting (1935). Experimental verification of the Tollmien-Schlichting waves was provided by Schubauer and Skramstad (1947).

\subsection{Scaling considerations}

We now consider the scaling relations appropriate to some turbulent flows (see Fig.1). Large scales are often determined by the physical constraints of the flow. In pipe or channel flows, the large scales are set by the cross-sectional dimensions of the pipe or channel. In jets, or wakes, the large scales are determined by the width of the jet or wake, which is a function of downstream distance. Close to the jet source, or flow obstruction, the width of the turbulent flow region, as well as the large scale, are strongly dependent upon the size of the source, or obstruction. In a free shear layer, there is no externally imposed length scale, so the scale for the large structures is primarily set by the width of the turbulent region which depends on the downstream distance.

In order to estimate the size of the large scale velocity fluctuations for a free shear layer, we consider the following: the average time for the fluid to reach a location $x$ is given by

$$
t=x / U_{0} \text {, }
$$

where Uo is the mean velocity in the downstream direction. If the r.m.s. velocity fluctuation is $u^{\prime}$, then the sideways excursion and consequently the width $\delta$ of the turbulent layer at $x$ is of order:

i.e.,

$$
\begin{aligned}
& \delta u^{\prime} t \sim u^{\prime} x / U_{0}, \\
& \delta / x \sim u^{\prime} / u_{0}
\end{aligned}
$$

The smallest scales are those for which viscous dissipation will begin to quench the energy of the fluctuation. This will occur at scales for which the Reynolds number based on the eddy scale and fluctuating 


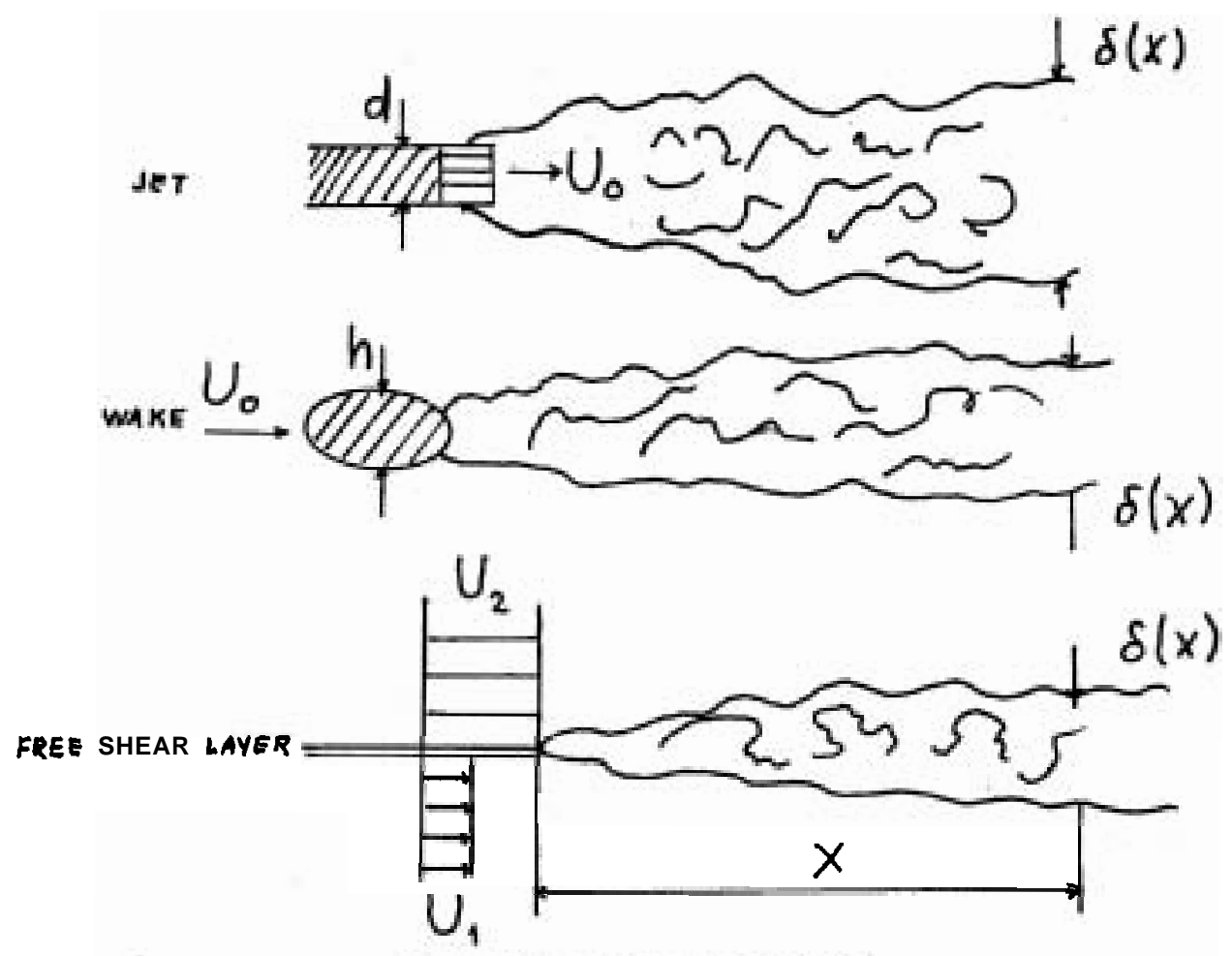

FREE TUREULENT SWEAR LAYER

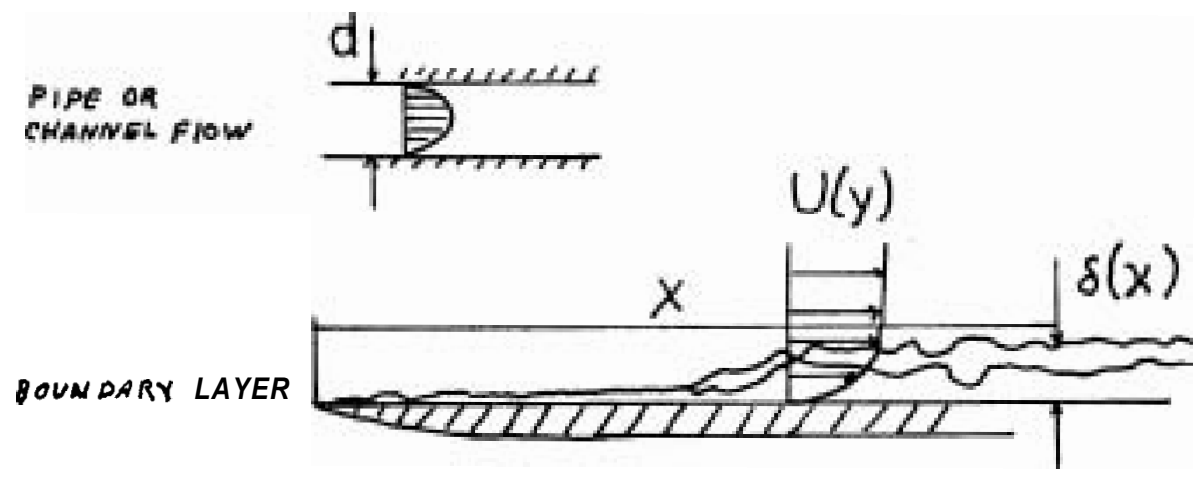

WALL-DOUWDEO MHFAR FLOWE

Fig. 1 
velocity is of order unity, hence we set $R e=\frac{u^{\prime} l}{V}=1$, where $l$ is the smallest scale. This gives

$$
\ell=\nu / u_{i}
$$

The rate of viscous dissipation $E$, per unit mass, is given by

$$
\epsilon \equiv \frac{\nu}{2}\left(\overline{\left.\frac{\partial u_{i}}{\partial x_{j}}+\frac{\partial u_{j}}{\partial x_{i}}\right)^{2}}\right.
$$

where the oberbar denotes an ensemble average. For an estimate of $\epsilon$ we use $\frac{\partial u_{i}}{\partial x_{j}} \sim u^{\prime} / \ell$ and find

$$
\epsilon \sim v\left(u_{i} / l\right)^{2}
$$

From (1.4) and (1.5) we obtain the Kolmogorov length scale,

$$
\ell_{K} \equiv\left(v^{3} / \epsilon\right)^{1 / 4}
$$

time scale,

$$
\tau_{k}=(v E)^{1 / 2}
$$

and velocity scale:

$$
v_{\mathrm{K}} \equiv(\nu \in)^{1 / 4}
$$

For the case of a boundary layer, a different set of scales may be derived on basis of conditions at the wall. From the shear stress at the wall, given by $\sigma_{w}=\mu\left(\frac{3 \bar{a}}{\partial y}\right)_{w a l l}$, one can define a friction velocity, $\mu_{k}=\left(\sigma_{w} / \rho\right)^{1 / 2}$.From this and the kinematic viscosity, one may form the wall length and time scales,

respectively.

$$
\begin{aligned}
& \ell^{+} \equiv \nu / u_{*} \\
& \tau^{+} \text {a } \nu / u_{*}
\end{aligned}
$$

\subsection{Equations of motion}

We assume that the density $\rho$ is constant, that the main flow is in the direction of the $x$-coordinate, and that the $y$-coordinate is normal to the boundary. An overbar $(-)$ denotes an ensemble average. For a fluid of constant density we have the equation of continuity,

$$
\frac{\partial V_{i}}{\partial x_{i}}=0
$$

(Double index implies a summation over the index in the usual fashion.) The momentum equations read

$$
\rho\left(\frac{\partial v_{i}}{\partial t}+V_{j} \frac{\partial v_{i}}{\partial x_{j}}\right)=-\frac{\partial P}{\partial x_{i}}+\mu \frac{\partial^{2} v_{i}}{\partial x_{j} \partial x_{j}}
$$

Now, we set $V_{i}=\vec{U}_{i}+u_{i}$, where $\vec{U}_{i}$ is the ensemble average of $U_{i}$ and $u_{i}$ is the fluctuating part of $\mathcal{U}_{i}$. By definition $u_{i}=0$. Similarly we set $P=P+P$. Taking the ensemble average of $(1.12)$, we obtain

$$
\rho\left(\frac{\partial V_{i}}{\partial t}+V_{j} \frac{\partial V_{i}}{\partial x_{j}}\right)=-\frac{\partial \bar{P}}{\partial x_{i}}+\frac{\partial}{\partial x_{j}}\left[\mu \frac{\partial \bar{U}_{i}}{\partial x_{j}}-\rho \overline{u_{i} u_{j}}\right]
$$


since $\quad u_{j} \frac{\partial u_{i}}{\partial x_{I}}=\frac{\partial\left(u_{i} u_{j}\right)}{\partial x_{j}}-u_{i} \frac{\partial u_{j}}{\partial x_{j}}=\frac{\partial\left(u_{i} u_{j}\right)}{\partial x_{j}}$

$\overline{a x_{j}}=-0$, by continuity. Subtracting Eq. (1.13) from Eq. (1.12), we get

$$
\left[\frac{\partial u_{i}}{\partial t}+\bar{v}_{j} \frac{\partial u_{i}}{\partial x_{j}}+u_{j} \frac{\partial \bar{v}_{i}}{\partial x_{j}}\right]=-\partial x_{i}^{n}+\frac{\partial}{\partial z_{j}}\left[\mu \frac{\partial u_{i}}{\partial u_{i}}-p\left(u_{i} u_{j}-\overline{u_{i} u_{j}}\right)\right]
$$

Here, $\rho\left(u_{i} u_{j}-\overline{u_{l} u_{j}}\right)$ may be considered as the "sluctuating Reynolds stresses". Multiplying (1.14) by $u_{i}$ and summing over the index $i$, we obtain

$$
u_{i} \frac{\partial u_{i}}{\partial t}-u_{j} U_{i} \frac{\partial u_{i}}{x_{i}}+u_{i} u \frac{\partial v_{i}}{\partial x_{i}}=-\frac{1}{\rho} u_{i} \frac{\partial p}{\partial x_{i}}+u_{i} \frac{\partial}{\partial x_{j}}\left[\nu \frac{\partial u_{i}}{\partial x_{j}}-\left(u_{i} u_{i}-u_{i} u_{j}\right)\right]
$$

Now, $g^{2} \equiv u_{i} u_{i}$, therefore $u_{i} \frac{\partial u_{i}}{\partial t}=\frac{1}{2} \frac{\partial}{\partial t} u_{i} u_{i}=\frac{1}{2} \frac{\partial q^{2}}{\partial t}$

Furthermore, $v_{j} u_{i} \frac{\partial u_{i}}{\partial x_{j}}=\frac{1}{2} V_{j} \frac{a}{\partial x_{j}}$ and $u_{i} \frac{\partial u_{i} u_{j}}{\partial x_{j}}=u_{i} u_{i} \frac{\partial u_{j}}{\partial x_{j}}+u_{i} u_{i} \frac{\partial u_{i}}{\partial x_{j}}=\frac{u_{i}}{2} \frac{\partial q_{i}^{2}}{\partial x_{j}}$, continuity again being used. The term $u_{i} \frac{\partial}{\partial x_{j}}\left(\frac{\partial \mu_{i}}{\partial x_{j}}\right)$ may be recast as follows:

Hence,

$$
\begin{aligned}
u_{i} \frac{\partial}{\partial x_{j}}\left(\frac{\partial u_{i}}{\partial x_{j}}\right)= & u_{i} \frac{\partial}{\partial x_{j}}\left(\frac{\partial u_{i}}{\partial x_{j}}+\frac{\partial u_{j}}{\partial x_{j}}\right)= \\
& \frac{a}{\partial x_{j}}\left[u_{i}\left(\frac{\partial u_{i}}{\partial x_{j}}+\frac{\partial u_{j}}{\partial x_{i}}\right)\right]-\frac{\partial u_{i}}{\partial x_{j}}\left[\frac{\partial u_{i}}{\partial x_{j}}+\frac{\partial u_{j}}{\partial x_{i}}\right]= \\
& \frac{\partial}{\partial x_{j}} u_{i}\left(\frac{\partial u_{i}}{\partial x_{j}}+\frac{\partial u_{i}}{\partial x_{i}}\right)-\frac{1}{2}\left(\frac{\partial u_{i}}{\partial x_{j}}+\frac{\partial u_{i}}{\partial x_{j}}\right) .
\end{aligned}
$$

$$
\begin{aligned}
\frac{1}{2}\left(\frac{\partial q^{2}}{\partial t}+V_{i} \frac{\partial q^{2}}{\partial x_{j}}\right)= & -u_{i} u_{j} \frac{\partial \bar{V}_{i}}{\partial x_{j}}-\frac{1}{\rho} \frac{\partial\left(p u_{i}\right)}{\partial x_{i}}+ \\
& +\nu \frac{\partial}{\partial x_{j}}\left[u_{i}\left(\frac{\partial u_{i}}{\partial x_{j}}+\frac{\partial u_{i}}{\partial x_{i}}\right)\right]-\frac{\nu}{2}\left(\frac{\partial u_{i}}{\partial x_{j}}+\frac{\partial u_{i}}{\partial x_{i}}\right)^{2}-\frac{1}{2} \frac{\partial}{\partial x_{j}}\left(u_{j} g^{2}\right)+u_{i} \frac{\partial}{\partial x_{j}}\left(\overline{u_{i}} u_{j}\right)
\end{aligned}
$$

The ensemble average of (1.16) gives the equation for the kinetic energy of the fluctuating flow.

$$
\begin{aligned}
\frac{1}{2}\left(\frac{\partial q^{2}}{\partial t}+v_{j} \frac{\partial q^{2}}{\partial x_{j}}\right)= & -\overline{u_{i} u_{j}} \frac{\partial \bar{U}_{i}}{\partial x_{j}}-\frac{1}{\rho} \frac{\partial \mu_{i}}{\partial x_{i}}+\nu \frac{\partial}{\partial x_{j}}\left[u_{i}\left(\frac{\partial u_{i}}{\partial x_{j}}-\frac{\partial u_{j}}{\partial x_{i}}\right)\right] \\
& -\frac{\nu}{2}\left(\frac{\partial u_{i}}{\partial x_{j}}+\frac{\partial u_{i}}{\partial x_{j}}\right)^{2}-\frac{1}{2} \frac{\partial}{\partial x_{j}} \overline{u_{j} q^{2}}
\end{aligned}
$$

or $\frac{\vec{D} \bar{e}}{D t}=\tilde{P}-\epsilon-\frac{\partial \bar{F}_{f}}{\partial x_{j}}$

where $\quad \frac{\bar{D}}{\bar{D} t} \equiv \frac{\partial}{\overline{a t}}+\bar{V}_{f} \frac{\partial}{\partial x_{i}}$

$\bar{e}=\frac{\dot{a}}{2} g^{2}=$ mean kinetic energy of fluctuations per unit mass.

$\widetilde{\mathrm{P}} \equiv-u_{i} \frac{\partial \overline{\bar{\nu}}}{\partial \bar{x}_{i}}=$ production term

$$
\begin{aligned}
\equiv-\left(\frac{\partial u_{i}}{\partial x_{i}}+\frac{\partial u_{j}}{\partial x_{k}}\right)^{2} & =\text { dissipation term } \\
\frac{\partial F_{f}}{\partial x_{j}}= & =\text { transport term, where }
\end{aligned}
$$




$$
\widetilde{F_{j}}=\frac{1}{p}\left(\overline{p u_{j}}\right)-\nu\left[u_{j}\left(\frac{\partial \overline{u_{j}}}{\partial \hat{x}_{j}}+\frac{\partial u_{i}}{\partial z_{i}}\right]+\frac{1}{2}\left(\overline{u_{j} q_{j}^{2}}\right)\right.
$$

is the flux of kinetic energy due to pressure work, to viscous stresses and

to turbulent diffusion, respectively.

\section{References}

Batchelor, G.K. (1951) Proc.Camb.Phil.Soc. 47: 359.

Batchelor, G.K. (1953) The theory of homogeneous turbulence. Camb.Univ.Press.

Boussinesq, J. (1877) Mém.prés.par div. savants à l'acad.sci.Paris 23: 46.

Fillipsson, L.G.R., T. Lagerstedt and F.H.Bark (1977) Hydrodynamic stability properties of fiber suspensions with application to drag reduction. (TO appear in J. Non-Newtonian Fluid Mechanics.)

Heisenberg, W. (1948) Proc.Roy.Soc.A, 195: 402.

Kolmogorov, A.N. (1941) C.R.Acad.Sci.U.S.S.R., 30: 301.

Millionschikov, M. (1939) C.R.Acad.Sci.,U.S.S.R., 22.231.

Millionschikov, M. (1941) C.R.Acad.Sci., U.S.S.R., 32 619.

Obukov, A.M. (1941) C.R.Acad.Sci., U.S.S.R., 32: 19.

Orr, W.M.F. (1907) Proc.R.Irish Acad. I7(A): 124.

Prandtl, L. (1925) ZAMM, 5. 136.

Rayleigh, Lord (1889) Proc.London Math.Soc., 11: 57 or Scientific Papers 1: 474 , Cambridge University Press.

Reynolds, 0. (1883) Phil.Trans. 174: 935 or Scientific Papers 2: 51, Cambridge University Press.

Schlichting, H. (1935) Nachr.Ges.Wiss.Gottingen, Math.Phys.Klasse, 1:47.

Schubauer, G. B. och H. K. Skrarnstad (1947) J.Aero.Sci., 14: 69.

Sommerfeld, A. (1909) Ein Beitrag zur hydrodynamischen Erklärung der turbulenten Flussigkeitsbewegung. Proc.4th Int.Congr.Math., Bome.

Taylor, G. I. (1915) Phi 1.Trans.Roy.Soc. London 215,A: 1.

"1 (1935) Proc.Roy.Soc.A 164: 15.

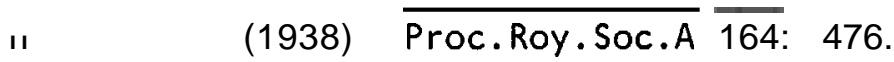

Tietjens, 0. (1925) Z.angew.Math.Mech. 5. 200.

Tol Irnien, W. (1935) Nachr,Ges,Wiss,Göttingen Math.Phys.Klasse,Fachgruppe $1: 79$.

Notes submitted by

c. Gregory Gartrell, Jr. 
Lecture \#2.

\section{TOOLS OF TURBULENCE}

\subsection{Experimental tools}

In experiments on turbulence, statistical quantities are usually sought. Although instruments have been designed to determine simple statistical quantities such as mean values directly, the recent tendency is to record the signal with the best resolution possible and then do the statistical analysis afterwards. We shall here review briefly the methods for measuring instantaneous velocities and pressures.

\section{Velocity measurements}

There are two principal instruments for measuring the fluctuating velocity. The first and most commonly used is the hot-wire anemometer. A thin $(1-10 \mu \mathrm{m})$ wire through which an electric current is passed is immersed into the fluid and the velocity is deduced from the heat transfer rate. (The latter is proportional to $\sqrt{|V|}$ ). It is important to use a very thin wire to minimize thermal inertia. There are two ways of operation. The first is to keep the current constant and measure the change in the temperature, which is related to the resistance in the wire. The second, and more recent approach, is to hold the temperature fixed by aid of an electronic circuit and observe changes in the resistance. The length of the wire is directly related to the problem of space resolution. If the wire is made too short, disturbances from the supports will dominate the flow, while if it is made too long, much of the small-scale phenomena will not be observed by the apparatus. A typical length is a few tenths of a $\mathrm{mm}$ with a resolution for wind tunnel experiments of $>10 \ell^{+}$. This is not quite sufficient for resolving a range of small scales, which, although they may contain little energy, could play an important role for the dynamics, for example, in the generation of vorticity.

The second instrument for measuring the instantaneous velocity field is the Laser-Doppler Velocity (LDV) meter. The most commonly employed principle of operation is to split the laser beam into two and have them cross in the fluid so as to create an interference fringe pattern

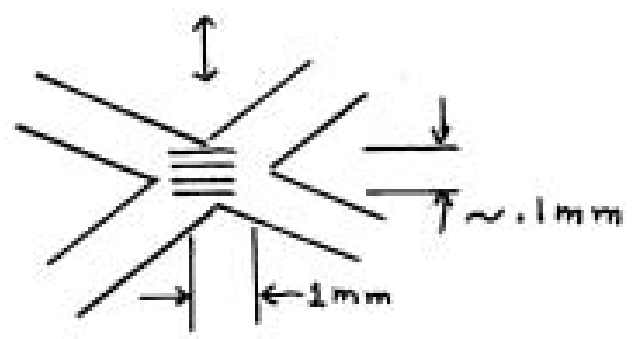

As a marked particle in the flow moves across the fringes an oscillatory signal is generated from which the velocity can be deduced. The advantage over the hot wire anemometers is that the technique of measurement does not interfere with the flow pattern. The disadvantage is that the interference fringes require a three-dimensional volume of non-negligible size to give a sufficient number of fringe crossings to determine the velocity accurately. This technique, however, does hold much promise for the future. 


\section{Surface pressure measurement}

Surface pressure measurements are commonly based upon the deflection of a membrane at the surface.

\section{microphone}

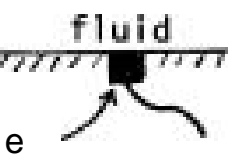

In a recent sophistocated application of this method Emmerling (1973) set up an array of membranes at a fluid surface and reflected light of them such that pressure disturbances would later alter the interference fringes.

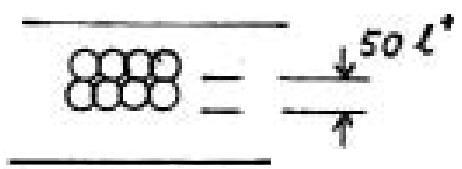

With this apparatus he found sizable fluctuations at $<50 \ell^{+}$, which is remarkable, since the pressure spectrum had been thought to decrease more rapidly according to previous experiments.

\section{Trace particle methods}

A final remark should be made on methods for observing streaklines. Besides the use of dye, which is only good for qualitatively following the flow, bubbles of hydrogen have been extensively used for quantitative measurements (see Kline, S.J., et al., 1967).

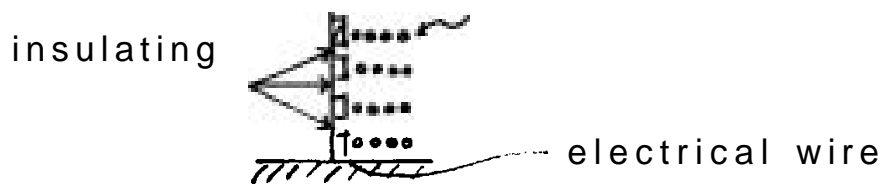

One problem with the method is the interpretation of the data since one does not observe an instantaneous Eulerian flow field but rather a Lagrangian field. Also, one runs into difficulties with resolution. The distance between the bubbles as well as the diameters of the bubbles themselves must be smaller than the scale of turbulence under investigation. Too small particles or bubbles (less than $1 \mu$ ) do not reflect visible light and are therefore of limited use for visualization purposes.

Notes submitted by John L. Guiliani, Jr. 
Lecture \#2. (continued)

\subsection{Statistical tools}

Generally, in studies of turbulent flows the details of the motion are not of interest and a statistical description provides one way of selecting the relevant properties. Moreover, the instantaneous flow at large Reynolds number is not necessarily independent of the exact initial conditions, whereas the average flow is. Although it is now accepted that simply averaging turbulent signals may obscure important processes within the flow (e.g. "bursting" in the viscous wall layer), as a prelude to the more sophisticated methods needed to study such processes (see 2.3) we first outline the concepts used in analyses fundamental of stochastic processes. A good condensed presentation of this subject may be found in Lin (1961).

With the macroscopic conditions of the flow held constant, the experiment is repeated a large number of times and measurements of some flow property $u(\vec{x} ; t)$ are made at a given point $(\vec{x} ; t)$. With this ensemble of experiments the following definitions may be made:

DEFINITION: The probability density of $(\vec{x} ; t), \rho_{u}(\alpha)$ is defined by $\theta_{u}(\alpha) d \alpha=$ fraction of experiments for which $u(\vec{x} ; t)$ falls between $\alpha$ and $\alpha+d \alpha$.

Thus $\int_{-\infty}^{\infty} d \propto P_{u}(\alpha)=1$.

DEFINITION: Mean value and central moments

(i) The mean value of the random function is $\bar{u}=\int d \propto x P_{u}(x)$

(ii) The $m^{\text {th }}$ central moment $\mu_{m} \equiv \int_{-\infty}^{\infty} d \alpha(\alpha-\vec{u})^{m} P_{u}(\alpha)$.

The first three are of most physical interest in turbulence:

$\mathrm{m}=2: \quad \mu_{z} \equiv \sigma^{2} \quad$ (variance)

The standard deviation $\sigma=\sqrt{\mu}$, measures the width of probability density function.

$\mathrm{m}=3$ : The skewness $S$ e $\mu_{3} / \sigma^{3}$ measures the asymmetry of the density function about its mean.

$m=4^{\prime \prime} \quad$ Kurtosis (flatness factor, $K=\mu_{4} / \sigma_{4}$ gives an estimate of the importance of the tails of the probability density function.
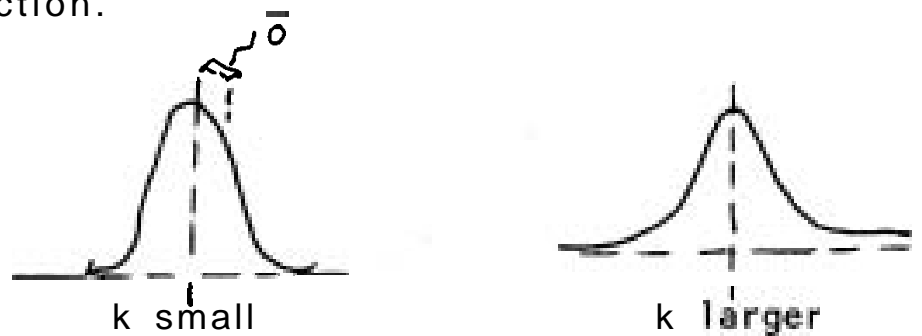

EXAMPLE 1: The random variable $u(t)$ is Gaussian (normal) if

$$
P_{u}(\alpha)=G(\alpha) \equiv \frac{1}{\sqrt{2 \pi \sigma}} e^{-\alpha^{2} / 2 \sigma^{2}}
$$

CENTRAL LIMIT THEOREM: The sum of independent, identically distributed random variables (each of which contributes roughly the same amount to the sum) is asymptotically Gaussian as the number of terms in the sum increases without bound. 
EXAMPLE 2: Gram-Charlier (probability density function often used to fit measured values).

$F_{u}(\alpha)=G(\alpha)\left\{1+\sum_{i}^{\infty} \lambda_{k} H_{k}(\alpha)\right\}$, where $\lambda_{k}(k=1, \ldots)$ are adjustable constants (related to the central moments of $\mathcal{P}_{k}(\alpha)$ ), and

$H_{k}(\alpha)=e^{\alpha / 2 \sigma^{2}}\left(\frac{\partial}{\partial \alpha}\right)^{\omega} G(\alpha)$, is the $k^{\text {th }}$ Hermite polynomial.

DEFINITION: Joint probability density. Given two signals $u(t), v(t)$, the joint probability density $\mathcal{F}_{\boldsymbol{u} v}(\alpha, \beta)$ is defined by

$\rho_{u v}(\alpha, \beta) d \alpha d \beta=$ fraction of experiments for which

$$
\alpha \leq u \leq \alpha+d \alpha \text { and } \beta \leq v \leq \beta+\alpha \beta
$$

Higher order densities (involving $n$ signals) are defined similarly.

DEFINITION: If the joint probability density of the signals $u(x, ; t)$,

$u x, u\left(x_{n} ; t\right)$ is independent of the origin chosen for the spatial coordinates for all $n$, then $u(x, t)$ is a homogeneous process; i.e. the joint probability density depends only on the configuration of the points $x_{1}, \ldots x_{n}$.

A similar condition applied to $t$ (only) defines stationarity.

Note: Weaker forms of homogeneity can be defined, e.g. only the autocorrelation $\overline{u\left(t_{1}\right) u\left(t_{2}\right)}$ may be required to be independent of the origin in time.

DEFINITION: If $P_{\underline{k v}}(\alpha, \beta)=\mathscr{P}_{\underline{u}}(\alpha) \cdot \mathscr{P}_{v}(\beta)$ then $u$ and $v$ are statistically independent; e.g. $u, v$ are jointly Gaussian if $\mathcal{P}_{u v}\left(\alpha_{3} \beta\right)=$ $=\frac{1}{2 \pi \sigma} \exp \left[-\left(\frac{\mu^{2}+v^{2}}{2 \sigma^{2}}\right)\right]$.

Two jointly Gaussian random variables are statistically independent.

DEFINITION: Correlation (covariance). If $u, v$ have zero mean, their correlation is

$$
Q_{u v} \equiv \overline{u v}=\iint_{-\infty}^{\infty} d \alpha d \beta \rho_{u v}(\alpha, \beta)
$$

Note: If $u$, v are statistically independent, then $Q_{u v}=0$; the converse need not be so.

Special cases:

(1) Replacing $v(t)$ by $u(t+\tau)$ gives the autocorrelation function,

$$
Q_{u u}(\tau)=u(t) u(t+\tau)
$$

(i i) Space-time correlation:

$$
Q_{u u}(\tau, x, \xi)=u(x, t) u(x+\xi, t+\tau) .
$$

(Stationarity has been assumed in the notation.) 
DEFINITION: The power spectrum of $u(t)$ is

$$
S_{u \psi}(\omega)=\frac{1}{2 \pi} \int_{-\infty}^{\infty} d \tau e^{i \omega \tau} Q_{u u}(\tau)
$$

The cross power spectrum of $u(x, t)$ is

$$
S_{\omega}(\omega, \xi)=\frac{1}{2 \pi} \int_{-\infty}^{\infty} \alpha \tau e^{i \omega \tau} Q_{\mu \omega}(\tau, z)
$$

THEOREM: If $u(t)$ is a stationary random function and $\tilde{u}(\omega)=\frac{1}{2 \pi} \int_{-\infty}^{\infty} d t e^{i \omega t} u(t)$, is its Fourier transform (interpreted as a generalized function) then $\tilde{u}^{*}\left(\omega_{1}\right) \tilde{u}(\omega)=S(\omega) \delta\left(\omega-\omega_{1}\right)$

PROOF: From the definition of $\tilde{u}(\omega)$,

$$
\overline{\tilde{u}^{*}\left(\omega_{1}\right) \bar{u}(\omega)}=\frac{1}{4 \pi^{2}} \int_{-\infty}^{\infty} d t_{2} \int_{-\infty}^{\infty} d t_{2} \mathrm{e}^{-i \omega_{1} t_{1}} e^{i \omega t_{2}} \overline{u\left(t_{1}\right) u\left(t_{2}\right)} .
$$

Letting $t_{2}=t_{1}+\tau$ and using the stationarity of $u(t)$ we find

$$
\begin{aligned}
\tilde{u}^{*}\left(\omega_{1}\right) \tilde{u}(\omega) & =\frac{1}{4 \pi^{2}} \int_{-\infty} d t_{1} \int_{-} d t e^{i\left(\omega-\omega_{1}\right) t_{1}+i \omega t} \cdot Q_{u u}(\tau), \\
& =\frac{1}{2 \pi} \int_{-\infty}^{\infty} d t_{1} e^{i\left(\omega-\omega_{1}\right) t_{1}} \cdot \frac{1}{2 \pi} \int_{-\infty}^{\infty} d \tau e^{i \omega t} Q_{u u}(\tau), \\
& =\delta\left(\omega-\omega_{1}\right) S(\omega),
\end{aligned}
$$

where $\delta\left(\omega-\omega_{1}\right)=\frac{1}{2 \tau} \int_{-\sigma} d t_{1} e^{i\left(\omega-\omega_{1}\right) t_{1}}$

DEFINITION: $E_{u u}(\tau, x, k)=\frac{1}{\tau} \pi \int_{\infty}^{\infty} d \xi e^{-[k \xi} Q_{u \mu}(\tau, x, \xi)$, is the wave number spectrum of $u$ and

$\widetilde{E}_{u u}(\omega, x, k)=\frac{1}{2 \pi} \int d \tau e^{i \omega \tau} E_{u u}(\xi, x, t)$ is the wave number frequency spectrum.

As above, it can be shown that if $u$ is homogeneous and stationary that-

$$
\text { , }(w, k) \cdot \delta\left(\omega-\omega_{1}\right) \delta(\&-\&,)=\overline{\hat{\omega}^{*}\left(\omega_{1} k_{1}\right) \hat{u}_{-},\left({ }^{*}\right)} \text {, }
$$

where

$$
\hat{u}(\omega, k)=\int_{-\infty}^{\infty} \int_{-\infty}^{\infty} d \xi d t e^{i(\omega t+k \xi)} u(x, t),
$$

is the space-time transform of $u(x, t)$.

Note: The definitions can easily be extended to vector functions of vector argument. 
EXAMPLE (1) Damped harmonic oscillator.

$m \ddot{u}+d \dot{u}+k u=q(t)$.

Fourier transforming gives $\tilde{u}(\omega)=(\omega) / Y(\omega)$, where

$Y(\omega)=($ F.T. of deflection $) /($ F.T. of forcing $)=$

$=\frac{1}{-m \omega^{2}-(\omega d+K},=\frac{-1}{m\left(\omega-\Omega^{(1)}\right)\left(\omega-\Omega^{(2)}\right)}$ is the

admittance of the system. Here

$$
\Omega_{d \rightarrow 0}^{(n)} \sim \pm \sqrt{\frac{k}{m}}-\frac{i d}{2 m}=\Omega_{R}^{(n)}+i \Omega_{I}^{(n)} .
$$

The power spectral density $S(\omega)$ can be found using the relationship between the Fourier transform and the power spectrum together with

$$
\tilde{u}(\omega)=\tilde{q}(\omega) Y(\omega) \text {. }
$$

For,

$$
\begin{aligned}
S_{u \mu}(\omega) \delta\left(\omega-\omega_{i}\right) & =\overline{\overline{u(\omega) \alpha^{*}\left(\omega_{1}\right)},} \\
& =\overline{q(\omega) \tilde{q}^{*}\left(\omega_{1}\right) \bar{Y}(\omega) \bar{Y}^{*}\left(\omega_{1}\right)}, \\
& =\overline{\tilde{q}(\omega) \tilde{q}^{*}\left(\omega_{1}\right)} \bar{Y}(\omega) \bar{Y}^{*}\left(\omega_{1}\right), \\
& =S_{q q(\omega) \delta\left(\omega-\omega_{1}\right) \cdot|\bar{Y}|^{2} .}
\end{aligned}
$$

i.e. $S_{u u}(\omega)=S_{q q}(\omega)|Y|^{2}$, and because the factor $|Y|^{2}$ appears on the right-hand side, resonances in the system will show up in $S_{u u}(\omega)$. Both the amplitude and width of the resonance peak are determined from $\Omega_{\Sigma}$, since

$$
\begin{aligned}
\mid I^{2} & =P P^{*} \\
& =\frac{1}{\left(\frac{\omega-\Omega_{R}^{(i)}}{\Omega_{I}^{(1)}}\right)^{2}+1}-\cdot \frac{1}{\left(\frac{\omega+\Omega_{R}^{(1)}}{\Omega_{I}^{(\omega)}}\right)^{2}+1} \cdot \frac{1}{\Omega_{I}^{4}}
\end{aligned}
$$

(Here the resonance peak occurs close to $\Omega_{R}^{[1]}$ because $\stackrel{\Omega_{L}}{\longrightarrow} \rightarrow 0$.)

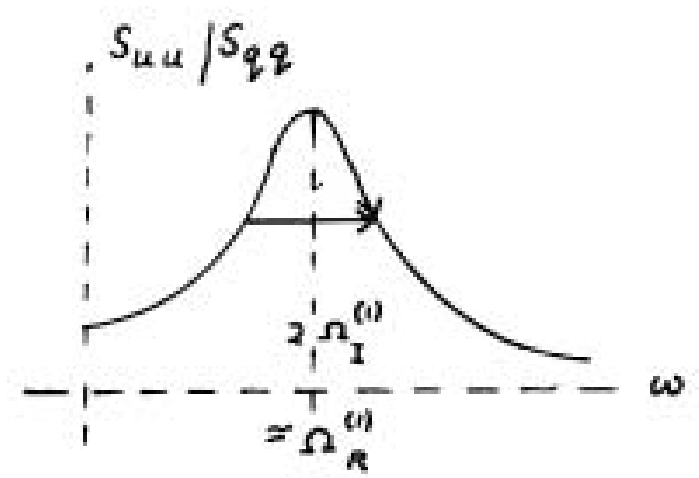


Note: If there were no damping, $\Omega_{I}^{[1]}=0$, and the resonance peak would be of infinite amplitude and nonintegratable, in this system damping must be present for statistical stationarity to hold.

\section{EXAMPLE (2). Shot noise. (space-time analogue of (1).)}

If an air-filled tube is struck at random, uniformly in space and time, waves propagate in the tube. The waves are governed by a linear wave equation with damping and a forcing term so that the system is a spacetime analogue of (1).

For each disturbance component of wavenumber $k$ the system has two resonance frequencies. Near each of these $Y$ is of the form

$$
P \sim \frac{1}{\omega-\Omega^{(n)}(K)} \quad n=1,2
$$

so that

Here

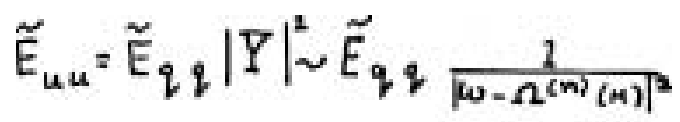

$$
\Omega^{(n)}=\Omega_{R}^{(n)}+i \Omega_{I}^{(\omega)} \text {. }
$$

For each wavenumber $k$ there is a resonance curve of the form:

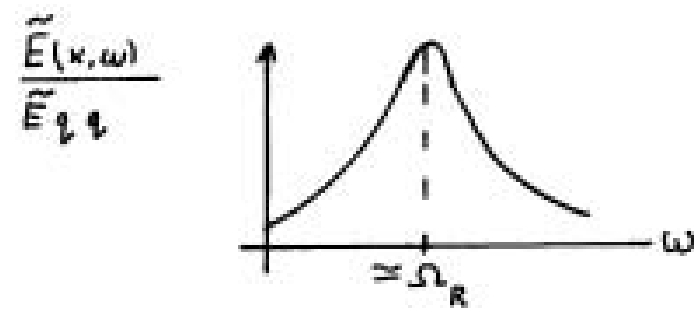

Note: The curve $\omega=\Omega_{x}(x)$ is the dispersion relation for waves in the tube, i.e. most of the energy is concentrated in slowly decaying waves.

Notes submitted by Stephen J.S.Morris

Lecture \#2 (continued).

\subsection{Conditional sampling methods}

Current experimental techniques allow one to record everything and then do the statistical analysis afterwards. But how does one know a priori what statistics are important? One approach is to average over only 'interesting' events hopefully isolating causal relationships. Much of this information may be obtained from the probability density function.

We denote the conditional mean of $u$, given that $u>u_{1}$ by $\bar{u}_{1}$. Then

$$
\bar{u}_{1} \hat{t}=\bar{u}_{1} \int_{u_{1}}^{\infty} P(u) d u=\int_{u_{1}}^{\infty} u(u) d u
$$

where $\hat{t}$ is the probability that (or time fraction that) $u>u_{\mathbf{l}}$.

The sorting of Reynolds stresses into the four quadrants may be obtained from the joint probability density function of $u$ and $v$. The total stress is 


$$
\bar{\sigma}=-\rho \overline{u v}=-\rho \int_{-\infty}^{\infty} u v P_{u v}(u, v) d u d v
$$

while the stress due to $(u, v)$ in quadrant 1 is

where $\quad \hat{t}=\int_{0}^{\infty} \int_{0}^{\infty} P_{u v} d u d v$ here.

$$
\sigma_{1}=-\rho u v_{1}=-\rho \int_{0}^{\infty} u v P(u, v) d u d v / \tilde{t}
$$

We may also find $P_{\sigma}(\sigma)$ from $P_{u v}(u, v)$. Since

$$
\begin{aligned}
\bar{\sigma} & =\int_{-\infty}^{\infty} \sigma p(\sigma) d \sigma=-\rho \int_{-\infty}^{\infty} u v P_{u v}(u, v) d u d v \\
& =\int_{-\infty}^{\infty} \int_{-\infty}^{\infty} P_{u, v}\left(u,-\frac{\sigma}{\rho u}\right) \frac{d u}{\rho u} d \sigma \text { with the change of variable } v=-\sigma / \rho u=
\end{aligned}
$$

So that

and

$$
P_{\sigma}(\sigma)=\int_{-\infty}^{\infty} P_{u v}\left(u_{1}-\frac{\sigma}{\rho u}\right) \frac{d u}{\rho_{u}}
$$

$$
P_{1 \sigma}(\sigma)=-\int_{0}^{\infty} P_{u v}\left(u_{1}-\frac{\sigma}{P_{u}}\right) \frac{d u}{\rho u} / \hat{t} \quad(\sigma<0)
$$

is the probability density function of $\sigma$ given $(u, v)$ is in quadrant 1.

Thus $\quad \bar{\sigma}_{1}=-\int_{0}^{\infty} \sigma P_{1 \sigma}(\sigma) d \sigma$

Thi's approach was recently used by Nakagawa and Nezu (1977).

Another set of sampling conditions might be

$$
\frac{\partial u}{a t} \geqslant 0 \text { and } \sigma \gtrless 0 \text {. }
$$

We must bear in mind that the sampling technique biases the results.

We note that for some approximations a normal Gaussian distribution may be useful (see Lin, 1961).

Blackwelder and Kaplan (1976) defined a variable integral time average (VITA) for any quantity $Q$ by

$$
\hat{Q}\left(x_{i}, t, T\right)=\frac{1}{2 T} \int_{t=T}^{t+T} Q\left(x_{i}, t^{\prime}\right) d t^{\prime}
$$

$T$ is chosen by trial and error so that the time integral is just large enough to capture events of interest. In their particular case $T$ was chosen to be 10 times $\nu / u^{2}$ (the viscous time scale), and an interesting event was considered to occur when $D(t)=1$ where $D(t)$ is an indicator function determined by

$$
D(t)=\left\{\begin{array}{l}
1 \text { if } \hat{u}^{2}-(a)^{2}>k u^{\prime 2} \\
0 \text { otherwise }
\end{array}\right.
$$

where $u^{\prime}$ is the RMS deviation velocity, and $k$ is an adjustable constant (typically 2 or 3 ). The results seem to be fairly insensitive to the choice of $k$. When tested on a pure white noise signal $D(t)$ was always zero as desired. The technique appears to be good for sampling intermittent events. 


\section{References}

Blackwelder, R.F. and R.E.Kaplan 1976 J.Fluid Mech. 76: 89.

Emmerling, R. Max-Planck Institut fur Stromungsforschung, Gottingen. Rep. No.9/1973.

KI ine, S. J., W.C. Reynolds, F.A Schraub and P.W. Runstadler 1967

J.Fluid Mech. 30: 741.

Lin, C.C. 1961 Statistical Theories of Turbulence, Princeton Aeronautical Paperback Series.

Nahagawa, H. and I. Nezu 1977 J.Fluid Mech. 80: 99.

Notes submitted by

Ross N. Hoffman

Lecture \#3.

\section{DESCRIPTION OF TURBULENT ROW FIELDS - ISOTROPIC TURBULENCE.}

We will first consider isotropic and homogeneous turbulence. In a typical wind-tunnel experiment the turbulence is ultimately produced by shear at a grid so the flow has greater variability in the $x$ direction until well down stream. Instead of measuring the spatial correlation $Q_{H}(\xi)=u_{i}\left(x_{1}, t\right) u_{1}\left(x_{1}+\xi_{1}, t\right)$ directly (which requires two probes of course) we may approximate this by the autocorrelation $u_{1}\left(x_{1}, t\right) u_{1}\left(x_{1}, t+\tau\right)$ if we assume Taylor's frozen flow field hypothesis holds. Taylor's hypothesis is that the turbulent structure does not change with time but is advected along with the mean flow, i.e. $\frac{a}{a t}=-\bar{u}_{i} \frac{d}{d x_{i}}$. Thus we take $T=-\xi_{1} / \bar{u}_{1}$. We must have $\left|\frac{u_{i}}{a_{i}}\right| \ll 1$ for this assumption to be valid. A more complete discussion of this hypothesis is given by Lumley (1965). Of course we cannot make this assumption for flows for which the turbulent fluctuations evolve rapidly with time such as shear flows.

In an isotropic and homogeneous turbulence, one needs to consider only two correlation functions. We first have the longitudinal one

$$
Q_{11}=u^{i z}(t) f\left(\xi_{1}\right)
$$

(where $t=x_{i} / \bar{u}_{l}$ ). $\mathrm{f}$ is called the normalized longitudinal correlation function. Similarly we have a transverse correlation function,

$$
Q_{12}=Q_{33}=u^{\prime 2} g\left(\xi_{1}\right)
$$

where $g\left(\xi_{l}\right)$ is the normalized transverse correlation. For all the others, when we have $\xi_{1} \neq 0, \xi_{2}=\xi_{3}=0$.

$$
Q_{i j}=0 \quad i \neq j \quad \text { because of isotropy. }
$$

It is left as an exercise to show for separation of the two points in an arbitrary direction

$$
\vec{r}=\left(\xi_{1}, \xi_{2}, \xi_{3}\right) \text { that }
$$




$$
Q_{i j}=u^{\prime 2}\left\{\frac{f(r)-g(r)}{r^{2}} \xi_{i} \xi_{j}+g(r) \delta_{i j}\right\}
$$

(see Hinze p.187, Fig.3-7, for examples of $Q$ ).

Finally if we apply the continuity equation

$$
\frac{\partial Q_{i j}}{2 \xi_{j}}=\overline{u_{i}\left(x_{k}, t\right) \frac{\partial}{\partial \xi_{j}} u_{j} \cdot\left(x_{k}+f_{k}, t\right)}=\overline{u_{i} \frac{\partial u_{j}}{\partial x_{j}}}=0
$$

The last two equations imply

$$
g(r)=\frac{1}{2 r} \frac{\partial}{\partial r}\left(r^{2} f\right)
$$

which is convenient to use for'checking the consistency of experimental results.

The Fourier transform of the autocorrelation function is equivalent, under Taylor's hypothesis to the 1-D wave number spectrum. Rather than use the spectrum tensor $\mathbf{E}_{\mathbf{i}} \mathbf{j}$ we are primarily interested in the sum of diagonal elements $E_{i j}$ because $E(k) d k=\left(\frac{1}{2} \oiint E_{i i} \alpha \sigma\right) d k$ is the kinetic energy in the interval $(k, k+d k)$ where the integration is over a sphere of radius $k$. $\left(k^{2}=k \cdot k=k_{j} k_{j}\right.$.)

Now for isotropic turbulence

$$
Q_{i i}=u^{\prime 2}[f(r)+2 g(r)]=u^{\prime 2} R(r)
$$

is a function of $\mathbf{r}$ alone if the flow is homogeneous. This suggests $\mathbf{E}_{\mathbf{i}} \mathbf{i}$ is only a function of $k$. Thus

$$
\begin{aligned}
E_{i i} & =\frac{u^{\prime 2}}{(2 \pi)^{3}} \iiint_{\int} e^{-i \vec{k} \cdot \vec{r}} R(r) d \xi_{1} d f_{2} d I_{3} \\
& =\frac{2 u^{\prime 2}}{(2 \pi)^{2}} \int_{0}^{\infty} \frac{\sin k r}{k r} R(r) r^{2} d r
\end{aligned}
$$

We have transformed to spherical coordinates with $\vec{k}$ the polar axis. Now since $E_{i i}=E_{i i}(k)$

$$
E(k)=2 \pi k^{2} E_{i i}(k)
$$

If we consider inverting back to correlations

$$
\begin{aligned}
Q_{i i} & =\iiint e^{i \vec{k} \cdot \vec{r}} E_{i i}(k) d k, d k_{2} d k_{3} \\
& =4 \pi \int_{0}^{\infty} k^{2} E_{i i} \frac{\text { sinkr }}{k r} d k=2 \int_{0}^{\infty} E(k) \frac{i m r}{k r} d k
\end{aligned}
$$

again transferring the polar coordinate but now choosing $\vec{r}$ as the polar axis. In the limit $\longrightarrow 0 \quad Q_{i i}=\overline{u_{i i}^{2}}=3 u^{\prime 2}$ so

$$
Q_{i i}=\int_{0} E(k) d k=\frac{3}{2} u^{12}=\overline{q^{2}} / 2
$$

and we see that $E(k)$ represents the kinetic energy of the turbulence. 
Dissipation may be described in a similar manner. Recall

$$
\epsilon=\frac{\gamma}{2} \overline{\left(\frac{\partial u_{i}}{\partial x_{i}}+\frac{\partial u_{i}}{\partial x_{i}}\right)^{2}}=\gamma \overline{\frac{\partial u_{i}}{\partial x_{j}}\left(\frac{\partial u_{i}}{\partial x_{j}}+\frac{\partial u_{i}}{\partial x_{i}}\right)}
$$

thus

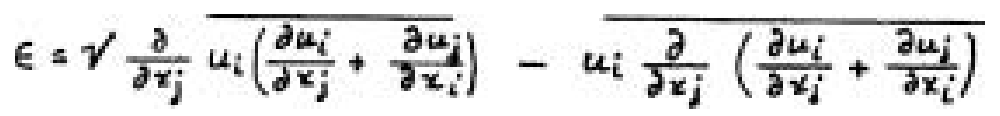

Now the first term vanishes when integrated over an appropriately chosen volume and applying continuity to the last term we have (for the volume)

$$
E=-\overline{u_{i} \frac{\partial^{2}}{\partial x_{j} \cdot \partial x_{j}} u_{i}}=-\frac{\partial^{2} Q_{l i}}{\partial \xi_{j} \partial \xi_{j}}
$$

The last step follows from the definition of $Q$. The wave number analog of $E$ is thus $2 \gamma \int k^{*} E(k) d k$ and we have

$$
\frac{\partial E(k)}{\partial t}=T-\partial \sqrt{ } h^{2} E(k)
$$

which states the time rate of change of energy at wave number $k$ is equal to the (nonlinear) transfer from other wave numbers $(T)$ less the dissipation $\bar{\varepsilon}=2 v k^{2} E(k)$.

Presentation of data

To present date in nondimensional form we use the Kolmogorov scales. The length scale

$$
l_{k}=\eta=\gamma^{3 / 4} \epsilon^{-1 / 4}=k_{k}^{-1}
$$

defines the reference wave number $\boldsymbol{k}_{k}^{-1}$ so that a normalized wave number is

$$
\tilde{k}=k / k_{k}=\left(\epsilon^{1 / 4} \gamma^{-3 / 4}\right)_{k}
$$

A normalized spectrum is

$$
\tilde{E}=E(k) h_{k} / v_{k}^{2}=\left(\varepsilon^{-k_{k}} \gamma^{-5 / 4}\right) E(h)
$$

where the Kolmogorov velocity is

$$
V_{k}=v^{k_{4}} \varepsilon^{1 / 4} \text {, }
$$

Normalized energy spectra are presented in this format by Von Atta and Chen (1969). They measured $E$ and $\frac{\partial E}{a t}$ (by moving downstream in a stationary flow)
and found $T$ as a residual.

The inertial subrange is the interval of $\mathrm{k}$ such that $k_{\mathrm{e}} \ll k \ll k_{\mathrm{k}}$ and $R_{e} \gg 1 . E\left(h_{e}\right)$ is the maximum value of $E(k)$. At the large scale end of the inertial subrange turbulent kinetic energy is produced; this energy cascades down to smaller and smaller scales where at the small scale end of the inertial subrange it is dissipated by viscosity. This concept was first advanced by Kolmogorov (1941). If one assumes that in this range $E$ depends only on $\mathrm{K}$ and $E$ dimensional analysis (Obukov, 1941) gives

$$
E(k, \epsilon)=\propto E^{2 / 3} k^{-5 / 3}
$$

Empirically $\approx \approx 1.5$. (See Gurvidi, Koprov, Tsvang $\varepsilon$ Yaglom (1967) and Pao (1965) for some experimental verification). This ' $-5 / 3$ law' seems to work better than it should in view of the limitations of the theory. 
Notes submitted by

Ross N. Hoffman

Addendum to Lecture 3.

Isotropic turbulence

It is also possible to obtain a measure of the length scales of isotropic turbulence from the spatial correlation functions, $f(\pi)$ and $g(n)$, of the velocity fluctuations.

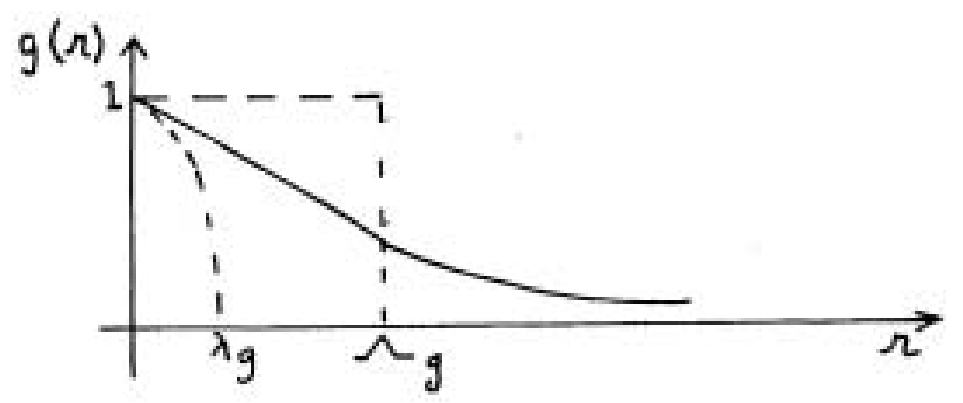

Taylor's microscale, $\lambda \mathrm{g}$, is defined by the curvature of the correlation function at $\mu=0:\left.\quad \frac{\partial^{2} g}{\partial r^{2}}\right|_{n=0} \leq-\frac{2}{\lambda g^{2}}$

A Taylor expansion of $g(n)$ about $\pi=0$ yields, for small $\Omega$,

$$
\begin{aligned}
g(r) & =1+r\left(\frac{\partial g}{\partial r}\right)_{n=0}+\frac{n^{2}}{2}\left(\frac{\partial^{2} g}{\partial r}\right)_{n=0}^{2}+\cdots \\
& \simeq 1-\left(\frac{r}{\lambda g}\right)^{2}
\end{aligned}
$$

with the first derivative taken to be zero at the origin on the assumption that for distances of the order of the Kolmogorov length scale or less, the velocity fluctuations should be perfectly well-correlated. The Taylor microscale can thus be taken as the intercept of the parabola that matches $g(\Omega)$ at the origin. Its physical meaning is somewhat less certain, since it appears to be too large a length scale to be used in the scaling of the dissipation rate for isotropic turbulence

$$
\varepsilon=15 \nu\left(\partial u_{1} / \partial x_{1}\right)^{2} \sim 15 \nu \overline{u_{1}^{2}} / \lambda^{2} \sim 15 \nu \frac{\mu^{2}}{\lambda^{2}}
$$

as originally intended by Taylor. It may, however, be used to obtain a Reynolds number for the flow from which the more commonly used Reynolds numbers (based on the Kolmogorov length scale, e.g.) can be calculated, and it is sometimes used as a rough estimate of the scales at which the energy starts to fall off due to inertial mixing of length scales. For large Reynolds number flows, the microscale lies in the inertial subrange. is defined as

The Taylor macroscale $\Lambda g$ (also commonly called the integral scale)

$$
\Lambda g=\int_{0}^{\infty} g(r) d \Omega
$$

and is taken to be a measure of the width of the correlation curve. It is considered to be the energy containing scale of the turbulent field, and is also used to calculate a Reynolds number for the flow. 
References

Blackwelder, R. and R. Kaplan 1976 J.Fluid Mech., 76: 89

Gurvidi, Koprov, Tsvang and Yaglom 1967 Atmospheric Turbulence and Radio Wave Propagation, 30

Kolmogorov, A. 1941 Compt.rend.acad.sc. U.R.S.S., 30: 301; 32: 16.

Lin, C. 1961 Statistical Theory of Turbulence

Lumley, J. 1965 Phys.Fluids, 8. 1056

Nakagawa, H. and I. Nezu 1977 J.Fluid Mech., 80: 99

Obukoff, A. 1941 Compt.rend.acad.sci. U.R.S.S. 32: 19

Pao, Y. 1965 J.Phys.Fluids, 8. 1063

Von Atta, C. and W. Chen 1969 J.Fluid Mech. 38: 743

Notes submitted by John W. Loder

Lecture \#4.

4. DESCRIPTION OF TURBULENT ROW FIELDS

\subsection{Shear flows}

Some generalizations can be made about the level of turbulence in the various types of shear flow. Jet flows have the highest turbulent intensities, with gross estimates for the ratio of the turbulent velocity fluctuations to the mean flow velocity $\left(u^{\prime} / \bar{U}\right)$ being in the range of .30-.60; consequently, jet flows are the most difficult to analyze. For boundary layers, $u^{\prime} / \bar{u} \approx .05-.10$ (see e.g. Hinze (1975), Fig.7-19), although the ratio can be up to 20 near the wall. Wake flows have lower turbulent intensities with $u^{\prime} / \bar{v} \approx .02-.05$.

Shear flows evolve to varying degrees in the direction of flow. Neglecting external influences, they commonly spread fairly slowly. In the following discussion, two-dimensional mean flows with slow downstream evolution will be assumed, so that the boundary-layer approximations can be used in the scaling of the equations of motion.

$$
\frac{\nabla}{\bar{u}} \ll 1 ; \frac{\partial}{\partial y} \gg \frac{\partial}{\partial x}
$$

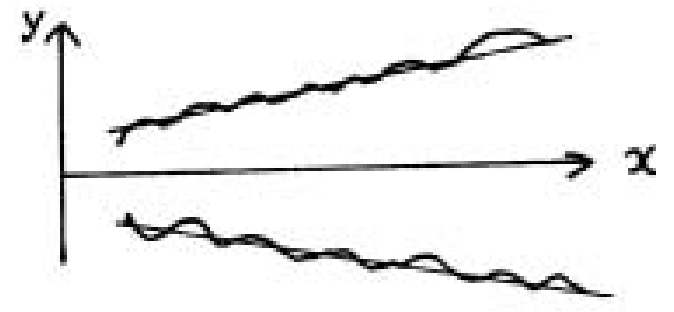


The dominant term in the energy equation associated with the production of kinetic energy will thus be

$$
-\overline{u v} \frac{\partial \bar{U}}{\partial y}
$$

while the most interesting stress term in the momentum equation will be $-p \overline{u v}$.

\section{D_Wake:}

We will now consider a simple situation to show that the flow field can be predicted with a minimum of experimental information. A cylinder of diameter $d$ lies with its axis in the $x-z$ plane and perpendicular to a constant flow U.

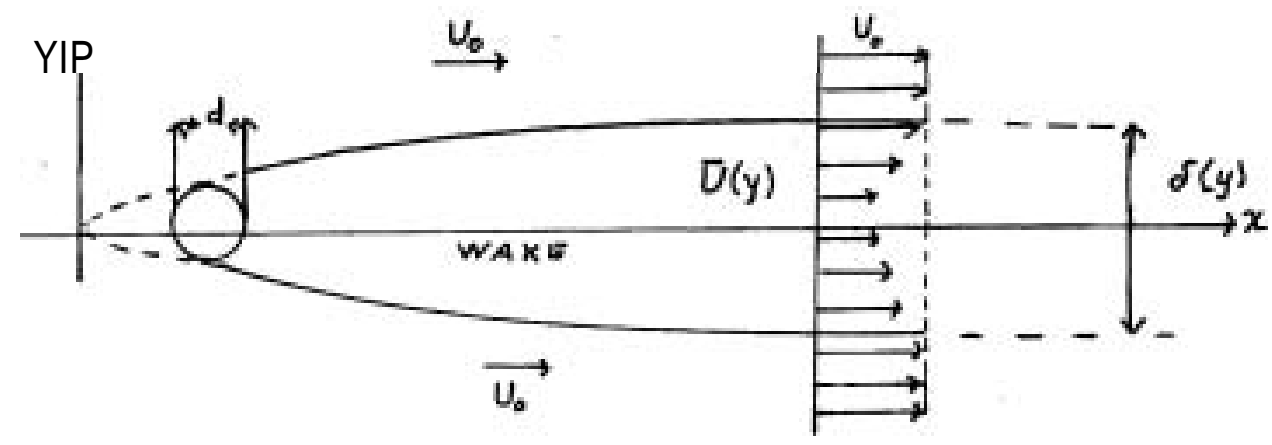

The resulting mean flow can be considered as two-dimensional (assuming homogeneity in the $z$ direction), but there will be three-dimensional turbulent velocity fluctuations in the wake downstream from the cylinder. We call the departure of mean velocity $\vec{U}(y)$ in the wake from that in the absence of the wake the velocity defect, $\mathbb{L}=U_{0}-\bar{U}$, and assume $\bar{u}$ to be a function of the nondimensional parameter $\eta=y / \delta(x)$ so that the profile of $\bar{a}$ looks the same downstream except for a change of scale (known as a selfsimilar or self-preserving flow field). We normalize $\bar{u}$ with respect to its value along the $x$-axis $u_{m}(x)$

$$
\bar{u}=\bar{u}_{m}(x) f(\eta)
$$

and consider the momentum balance across the wake for distances downstream such that $\frac{x}{d} \geq 50$ (so as to get away from the region where developing vortices are important). Assuming an inviscid fluid (i.e. large Reynolds number flow), we have the equations for steady mean flow

and

$$
\bar{U} \frac{\partial \hat{U}}{\partial x}+\bar{V} \frac{\partial \hat{U}}{\partial y}=-\frac{1}{\rho} \frac{\partial \bar{p}}{\partial x}-\frac{\partial}{\partial x}\left(\bar{u}^{2}\right)-\frac{\partial}{\partial y}(\overline{u v})
$$

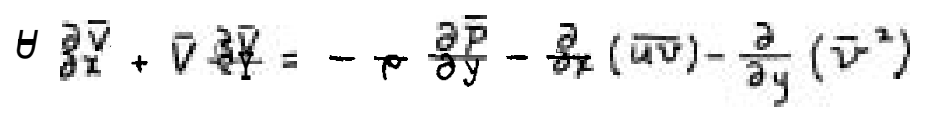

where derivatives with respect to $z$ of the stress terms $\bar{u} \boldsymbol{w}$ and $\bar{v}$ are zero due to our assumption of homogeneity in the $z$-direction. The length scales in the wake may be taken as $L$ in the $x$-direction, and a crossstream scale $\ell$ such that $\delta=O(\ell)$. At large distances downstream, $\ell \ll L$. $U_{a}$ may be taken as a velocity scale for the mean flow in the $x$-direction, and we take $U_{4}$ as a scale for the velocity defect, $\bar{U}=U_{0}-\bar{U}$. The Reynolds stresses may be scaled as $-\overline{u v}=O\left(\mu^{*}\right),-\bar{v}^{2}=O\left(\mu^{2}\right)$ and $-\bar{u}^{2}=O\left(\mu^{*}\right)$, and 
the mean equation of continuity may be used to scale the cross-stream component of mean velocity $\bar{\nabla}$ as $\nabla=O\left(U_{s} \ell / L\right)$. We replace $\bar{U}$ in the equations by $\bar{V}=U_{0}-\bar{u}$, and consider the terms in (4.2):

$$
\begin{aligned}
& -U_{0} \frac{\partial \bar{u}}{\partial x}: \frac{U_{0} U_{l}}{L}=\left(\frac{U_{0}}{\mu} \frac{U_{0}}{\mu} \frac{l}{L}\right) \frac{\mu^{2}}{l} \\
& \bar{u} \frac{\partial \bar{u}}{\partial x}: \frac{U_{0} U_{l}}{L}=\left(\left(\frac{U_{0}}{\mu}\right)^{2} \frac{l}{L}\right) \frac{\mu^{2}}{l} \\
& -\bar{v} \frac{\partial \bar{u}}{\partial y}: \frac{U_{0} l}{L} \frac{U_{l}}{l}=\left(\left(\frac{U_{l}}{\mu}\right) \frac{l}{L}\right) \frac{\mu^{2}}{l} \\
& -\frac{1}{\rho} \frac{\partial \bar{P}}{\partial x}: ? \\
& -\frac{\partial}{\partial x}\left(\bar{u}^{2}\right): \frac{\mu^{2}}{L}=\frac{l}{L} \frac{\mu^{2}}{l} \\
& -\frac{\partial}{\partial y}(\overline{u v}): \frac{\mu^{2}}{l}=1 \cdot \frac{\mu^{2}}{l}
\end{aligned}
$$

Since $\ell / L<1$ and assuming that $\frac{l}{L} \rightarrow 0$ faster than $\left(U_{s} / \mu\right)^{2}$ (see e.g. Tennekes and Lumley, 1972), we may neglect the terms $\bar{\mu} \frac{\partial \bar{z}}{\partial \bar{x}}=-\bar{\nabla} \frac{\partial \bar{u}}{\partial y}$ and $-\frac{a}{\partial x}\left(\bar{u}^{2}\right)$. We retain the pressure term for now, and also $-U_{0} \partial \bar{u} \partial y$ (since $U_{0}>U_{s}$ ) as necessary to balance the Reynolds stress term if the pressure term is found to be insufficient.

The terms in (4.3) are

$$
\begin{aligned}
U_{0} \frac{\partial \bar{V}}{\partial x}: \frac{U_{0}}{L} \frac{U_{A} l}{L}=\frac{U_{0}}{\mu} \frac{U_{s}}{\mu}\left(\frac{l}{L}\right) \frac{\mu^{2}}{l} \\
-\bar{u} \frac{\partial \bar{V}}{\partial x}: \frac{U_{1}}{L} \frac{U_{s} l}{L}=\left(\frac{U_{s}}{\mu}\right)^{2}\left(\frac{l}{L}\right)^{2} \frac{\mu^{2}}{l} \\
\bar{V} \frac{\partial \bar{V}}{\partial y}: \frac{U_{2}}{L} \frac{U_{1}}{l}\left(\frac{U_{t}}{\mu}\right)^{2} \frac{l}{L} \mu^{2} \\
-\frac{1}{\rho} \frac{\partial \bar{P}}{\partial y}: \quad ? \\
-\frac{\partial}{\partial x}(u v): \frac{\mu^{2}}{L}=\frac{l}{L} \frac{\mu^{2}}{l} \\
-\frac{\partial}{\partial y}\left(\bar{v}^{2}\right): \frac{\mu^{2}}{l}=1 \cdot \frac{\mu^{2}}{l}
\end{aligned}
$$

The terms $-\frac{\partial}{\partial x}(\overline{u v})$ and $\bar{v} \frac{\partial \bar{v}}{\partial y}$ may be neglected by previous arguments, while the factor $\left(\frac{\varepsilon}{L}\right)^{2}$ in $U_{0} \frac{\partial \vec{v}}{\partial \psi}$ and $-\bar{u} \frac{\partial \vec{v}}{\partial x}$ make these terms small, so that the only term of sufficient magnitude to balance the Reynolds stress term is the cross-stream pressure gradient term. Thus, we can re-write our momentum equations as

$$
-V_{0} \frac{\partial u}{\partial x} \approx-\frac{1}{\rho} \frac{\partial \hat{p}}{\partial x}-\frac{\partial}{\partial y}(\sqrt[U v]{ })
$$


and

$$
\frac{1}{\rho} \frac{\partial \vec{p}}{\partial y}+\frac{\partial}{\partial y}\left(\bar{v}^{2}\right) \approx 0
$$

integration of (4.5) yields

$$
\frac{\vec{p}}{\rho}+\vec{v}^{2}=\frac{P_{0}}{p}
$$

where $P_{a}$ is the pressure outside of the turbulent wake $(y \rightarrow \pm \infty)$. If we assume that there is no externally imposed downstream pressure gradient $\left(\frac{\partial P_{6}}{\partial x}=0\right)$, we obtain

$$
\frac{1}{\rho} \frac{\partial \bar{p}}{\partial x} \quad \frac{\partial\left(\bar{v}^{v}\right)}{\partial x}=0
$$

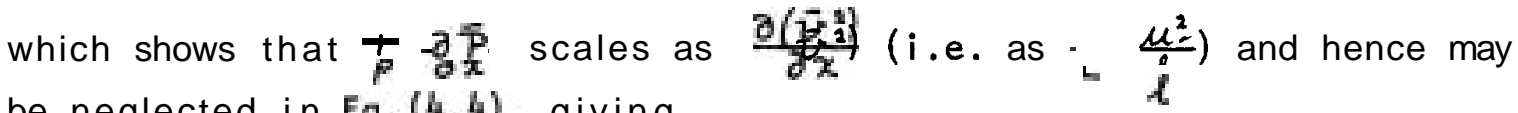
be neglected in Eq. (4.4), giving

$$
U_{0} \frac{\partial \bar{u}}{\partial x}-\frac{\partial}{\partial y}(\overline{u v})=0
$$

Integrating with respect to $y$, and knowing that the velocity fluctuations disappear as $y \rightarrow \pm \infty$, we obtain

$$
U_{0} \int_{-\infty}^{\infty} \frac{\partial \bar{u}}{\partial x} d y=\frac{\partial}{\partial x}\left\{U_{0} \int_{-\infty}^{\infty} \tilde{u} d y\right\}=0
$$

which may be integrated with respect to $x$ to give

$$
\begin{aligned}
& u_{0} \int_{-\infty} \bar{u} d y=A_{1} \\
& \text { or } \rho u_{0} \int_{-\infty} \bar{u} d y=A .
\end{aligned}
$$

This integral is known as the momentum integral, and states that the net flux of momentum defect per unit depth is constant across planes normal to the $x$-axis independent of distance downstream.

By substitution of (4.1) into (4.7), this may be re-expressed as

$$
\rho U_{0} \delta(x) \text { a, }(x)-f f(n) \alpha \eta=A
$$

and since $\int_{-\infty}^{\infty} f(n) d n=$ constant

$$
\delta(x) \vec{u}_{m}(x)=B \text {. }
$$

This may be considered as the result of momentum conservation in a selfsimilar flow field.

We now attempt to determine some description of the wake from the downstream momentum balance, Eq. (4.6). Differentiation of (4.1) yields

$$
\frac{\partial \bar{u}}{\partial x}=f(\eta) \bar{u}_{m}^{\prime}(x)-\frac{\bar{u}_{m}(x) \delta^{\prime}}{\delta(y)}(y) \eta f^{\prime}(\eta)
$$

(where primes indicate first derivatives) and assuming that the velocity fluctuations are proportional to the maximum velocity defect (this is part of the self-preservation hypothesis; see, e.g. Tennekes and Lurnley, 1972), i.e.,

$$
\overline{u v}=-\vec{u}_{m}^{2}(x) g(n)
$$

we may write (4.6) as 


$$
U_{0}\left[\bar{u}_{m}^{\prime}(x) f(\eta)-\frac{\vec{u}_{m}(x) \delta^{\prime}(x)}{\delta(x)} \eta f^{\prime}(\eta)\right]+\bar{u}_{m}^{2}(x) \frac{1}{\delta(x)} g^{\prime}(\eta)=0
$$

Multiplication by $\frac{\delta}{a_{m}^{2}}$ yields

$$
v_{0} \frac{\vec{u}_{m}^{\prime}}{u_{m}^{2}}(x) \delta(x) f(n)-\frac{v_{0} \delta^{\prime}(x)}{\vec{u}_{m}(x)} \eta f^{\prime}(\eta)=-g^{\prime}(n)
$$

The right-hand side of (4.9) is a function of $\eta$ only, and it can be easily shown that in order for the left-hand side to be a function of $\eta$ only then the coefficients

and

$$
C=v_{0} \frac{\bar{u}_{m}^{\prime}(x)}{\bar{u}_{m}^{2}} \delta(x)
$$

$$
D=\frac{v_{0} \delta^{\prime}(x)}{\vec{u}_{m}(x)}
$$

must be independent of $x$.

Equation (4.9) may be written (assuming $f(\eta) \neq 0$ )

$$
c(x)-D(x) \frac{\eta f^{\prime}(n)}{f(n)}=-\frac{g^{\prime}(\eta)}{f(n)}
$$

so that $C^{\prime}(x)-D(x) \phi(n)=0$, which can only be true for all $\eta$ if $C^{\prime}(x)=D^{\prime}(x)=0$.

The pair of equations, (4.10) and (4.11), together with the momentum integral as an additional constraint (see, e.g. Tennekes and Lumley, 1972) have solutions

$$
\begin{aligned}
\bar{u}_{m}(x) & =k_{1} x^{-u_{2}} \\
\alpha(x) & =k_{1} x^{1 / 2}
\end{aligned}
$$

which agree with experimental observations of plane turbulent wakes beyond about $x=80 \mathrm{~d}$ (Tennekes and Lumley, 1972).

It is difficult to proceed much further without having experimental information. However, adjustment of the coefficients in (4.9) results in a relation between $f$ and $g$

$$
f(n)-\eta f^{\prime}(n)=E g^{\prime}(\eta)
$$

If a constant eddy viscosity is assumed across the wake, a Gaussian distribution for $f(\eta)$ is predicted, which agrees fairly well with observed velocity profiles in wakes for values of $\mathbf{t}$ less than 0.3. (See, e.g. Townsend (1976), Fig.6.2(a)). Knowing the velocity distributions, it is then possible to calculate the Reynolds stresses in the flow.

Energy spectra based on observations in high Reynolds number wake flows usually have a region with $-5 / 3$ slope (see, e.g. Monin and Yaglom (1975), Fig.76c), which suggests that in shear flows of sufficiently high Re, there exists an inertial subrange at high wave numbers in which the turbulence is locally isotropic.

Boundary_layer_flow:

We now consider the flow in a boundary layer over a smooth flat plate. The thickness $\delta(x)$ of the boundary layer is usually taken as the distance from the plate at which the mean velocity $0(y)$ is $99 \%$ of the velocity $U_{0}$ in the absence of the plate. Typical rates of growth (of 
thickness) of such boundary layers

downstream are $-2 \%$.

Boundary layer flows are more complicated than free shear flows in that, in addition to the thickness as a length scale, the no-slip condition on the

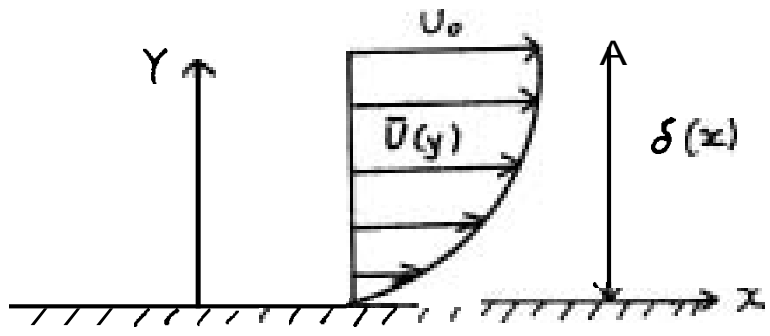
solid plate introduces a viscosity-dominated length scale. Early measurements showed that the mean velocity $\bar{U}(Y)$ could be represented in two ways:

1.) Near the wall $U$ may be taken as a function only of wall variables and independent of conditions away from the wall. The relevant variables are the wall friction velocity $u_{*}=\sqrt{\sigma_{w} / \rho}$, and the vis cous length $Y^{+}=\frac{Y}{l^{+}}=\frac{\gamma u_{x}}{\nu}$, so that

$$
\bar{U}(Y)=u_{k} f\left(Y^{+}\right)
$$

This relation is known as the law of the wall, and is found to represent experimental data very well, except in the outer portion of the boundary layer. Linear (for data very close to the wall), logarithmic, and power law forms of $f\left(Y^{+}\right)$have been used (see, e.g. Hinze (1975), Fig. $7-9)$.

2.) Away from the immediate vicinity of the wall and in most of the boundary layer, von Karman proposed a velocity-defect law (also called the law of the wake) in which the relevant variables were the wall friction velocity $u_{*}$ and the length scale $\eta=Y / \delta$

$$
v_{0}-\bar{U}=u_{*} g(\eta)
$$

This law also gives good agreement with experimental data, using a loga$r i t h m i c$ form of $g(\eta)$ away from the wall and some other function near the wall (see, e.g. Hinze (1975) Fig.7-11).

Millikan (1938) looked at the region of overlap of these representations and assumed that over some finite region the functions merge together giving

$$
\begin{gathered}
\frac{\bar{v}}{u_{*}}=f\left(\gamma^{+}\right)=f\left(\delta^{+} \eta\right)=\frac{u_{0}}{u_{*}}-g(\eta) \\
\text { where } \delta^{+} \equiv \frac{\delta u_{*}}{\nu^{*}}
\end{gathered}
$$

By elementary functional analysis, this can be shown to be true only if $f$ and $g$ are logarithmic functions.

$$
\begin{aligned}
& \frac{\bar{U}}{u_{*}}=A \ln y^{+}+B \equiv A \ln \eta+B+A \ln \delta^{+} \\
& \frac{u_{0}-\bar{U}}{u_{*}}=-A \ln \eta-B^{\prime}
\end{aligned}
$$

The logarithmic velocity profile is characteristic of the overlap region of the boundary layer in which there is no intrinsic length scale, analogous to the inertial subrange in the energy spectra of homogeneous isotropic turbulence. The same result can be obtained from matched asymptotic techniques, 
mixing length arguments, or simply from dimensional reasoning, where it is assumed that the mean velocity shear is dependent only on the wall friction velocity and the distance from the wall giving

The constant $A$ in these relations is equal to $X$ where $x \quad 0.41$ is von Karman's constant.

The log-layer is a region of nearly constant turbulent shear stress (see, e.g. Hinze (1975), Fig.7-21); addition of the viscous stresses close to the wall make that region one of constant stress as well. Outside of the log layer, the shear stress decreases with increasing distance from the wall (e.g. Hinze (1975), Fig.7-20).

The ordering of the three components of velocity fluctuations in' a wall boundary layer may be seen in Hinze (1975), Fig.7-19.

Hinze, J.O. 1975 Turbulence $\frac{\text { References }}{\text { (2nd.ed.) McGraw-Hill. }}$
Monin, A.S. and A.M.Yaglom 1975 Statistical Fluid Mechanics, Vol.2.
M.I.T.Press.

Tennekes, H. and J.L.Lumley 1972 A First Course in Turbulence. M.I.T.Press.

Townsend, A.A. 1976 The Structure of Shear Flow Turbulence (2nded.) Cambridge Univ. Press.

Notes submitted by John W. Loder

Lecture \#4. (continued)

\subsection{Boundary layer turbulence structure}

The subdivision, at high Reynolds numbers, of the boundary layer into three regions; the wake, dominated by the external conditions; the logarithmic part of the profile, characteristic of the absence of a proper length scale; and the viscous sublayer, with large mean velocity gradient; is consistent with wind tunnel and atmospheric measurements as far as the mean properties are concerned.

The most interesting region from a dynamical point of view is the one close to the wall, including the so-called wall and buffer regions, in this, production of kinetic energy is found to exceed dissipation, leading to a diffusion of fluctuating energy away from the wall. Also, the turbulence there is observed to be highly intermittent, exhibiting "bursts" of turbulence in a quasi-ordered sequence, first described in detail by Kline et al. (1967) and later extensively studied by many researchers. An extensive review of such observations has been given by Willmarth (1975). We will here only point out some of the more significant findings. For more details we refer to Willmarth's (1975) paper.

The visual observations by Kline et al., (1967), later supplemented by 
more detailed experiments from the same research group, showed the presence of a streaky sublayer structure with low-spaced streaks separated by an average spanwise distance of approximately 100 in wall units. The bursting sequence would start with a gradual lift-up of a slow-spread streak which would then suddenly begin to oscillate or go into a spiral motion. A break-up and ejection of the low-speed flow then follows shortly thereafter. The bursting sequence is completed by high-speed fluid (a "sweep") coming in from outside and replacing the low-speed fluid. Later visual observations by offen and $\mathrm{KI}$ ine (1974) show that the lift-up is associated with a sweep in the outer portion, probably due to an earlier event. They suggested that the pressure induced by such a sweep would cause locally an adverse pressure gradient leading to a separation of the flow, i.e. lift-up of the low-speed region. In support of this suggestion, Lu and Willmarth (1973) performed conditionally sampled measurements of wall pressure and Reynolds stress contributions during bursting, which clearly showed that the wall pressure is minimum at the time of bursting (see Figs.51 and 52 in Willmarth's (1975) paper).

A sizeable body of quantitative data on the bursting sequence have been generated by conditional sampling techniques. We will mention only a few of these. Wal Imarth and Lu (1971) and Wallace et al., (1972), proposed sorging of the Reynolds stress contributions to uv in each quadrant of the uv-plane, depending on the magnitude of the contribution. (As indicated in Lecture \#2 such results could also be obtained from appropriate integrals over the joint probability density of uv). The classification is explained in Fig.5.

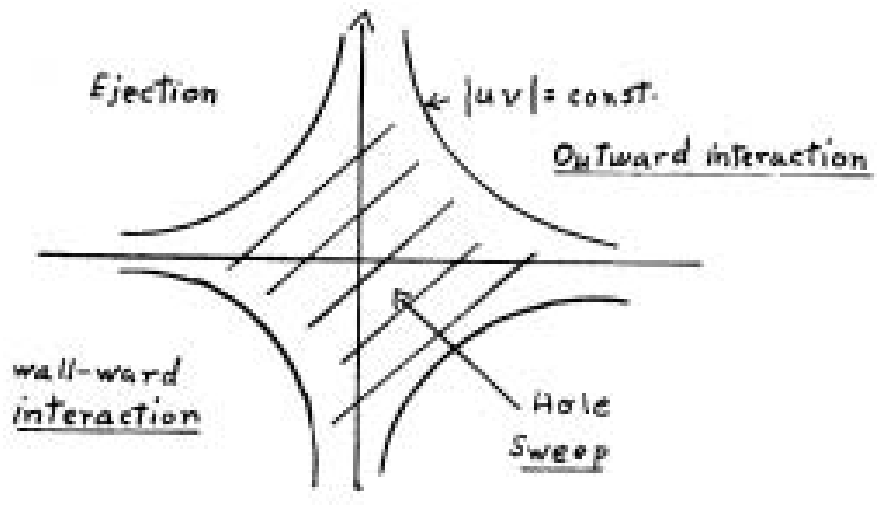

Fig. 5

The largest contribution to uv comes from ejections $(u<0, \checkmark>0)$ except for $y+<15$, see Brodkey et al., (1974). The total sum of the contributions from the ejections and the sweeps $(u>0, v<0)$ was found to be about $140 \%$ of the total, the balance being made up by negative contributions from outward and wall-ward $(u<0, v>0)$ interactions. Lu and Willmarth (1973) extended this idea by defining a hole size parameter, $H$, from $|u v|=H^{*} u^{\prime} v^{\prime}$, where $u^{\prime}$ and $v^{\prime}$ denote root-mean-square values. They measured the mean time intervals between ejections and sweeps as function of the hole size $H$. The time was found to scale with outer variables (free stream velocity and boundary layer displacement thickness) and to increase strongly with $\mathrm{H}$, as indicated conceptually in Fig.6. 


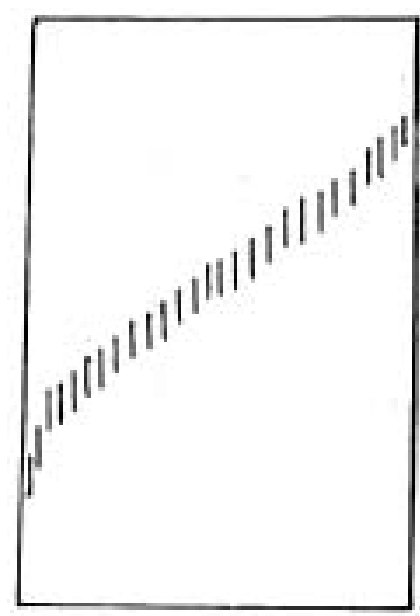

Fig. 6

Mean intervals between ejections.

One may interpret such results as indicating that a stronger burst requires more time for build-up of the energy. The time interval between sweeps exhibit a similar character, but strong sweeps occur considerably less often that strong ejection, the mean interval between sweeps of hole size $\mathrm{H}=5$ being about ten times that for ejections. The convection velocity of sampled sorted Reynolds stresses was also estimated by Lu and Willmarth (1973) by forming space-time correlations for various downstream distances. Interestingly enough, the convection velocity of the Reynolds stress maxima was found to be only about $80 \%$ of the local mean velocity at that distance from the wall. Such a value happens to be close to the phase velocity of the Tollmien-Schlichting wave for the dominating wave number for that distance from the wall, according to the dispersion characteristics for the mean velocity profile computed by Bark (1975).

Instantaneous velocity profiles, and conditionally sampled ones using their variable-internal time-averaging (VITA, see Lecture \#2) method, were obtained by Blackwelder and Kaplan (1976) employing a hot-wire rake with ten hot wires distributed within 100 viscous wall lengths from the wall. The instantaneous velocity profiles show a strong coherence in the y-direction but little or no streamwise coherence. Their conditionally sampled velocity profiles indicate the appearance of a significant repetitive event starting with a slowdown near the wall and a speed-up of velocities further out. The interface between high-speed and low-speed fluid intensifies rapidly with time giving the appearance of a strong shear layer moving toward the wall. Very rapidly, the velocity becomes in excess of the mean everywhere across the wall region, and the velocity excess then relaxes slowly back to the mean value.

The experimental data such as those described above may be interpreted kinematically on basis of vortex models. We refer to Willmarth's (1975) paper for such proposals.

\section{References}

Bark, F.G. 1975 J.Fluid Mech. 70: 229.

Blackwelder, R.F. and R.E.Kaplan 1976 J.Fluid Mech. 76. 89. 
Brodkey, R.S., J.M.Wallace and H.Eckelmann 1974 J.Fluid Mech., 63: 209.

Kline, S.J., W.C.Reynolds, G.A.Schraub and P.W.Runstadler 1963

J.Fluid Mech., 30: 741.

Lu, S.S. and W.W.Willmarth 1973 J.Fluid Mech., 60: 481.

Offen, G.R. and S.J.Kline 1974 J.Fluid Mech. 62: 223.

Wallace, J.M., H. Eckelmann and R.S.Brodkey 1972 J.Fluid Mech. 54: 39. Willmarth, W.W. and S.S.Lu 1971 J.Fluid Mech. 55: 481.

Notes submitted by Jacques Lewalle

\section{Lecture \#5}

\section{SHEAR ROW INSTABILITY}

The study of shear flow instability gives information about mechanisms which are responsible for the creation of turbulence. One such basic mechanism is the interaction between disturbances and the mean shear. Therefore, we consider wave-like disturbances on a parallel flow and assume that their amplitudes are small so linearization of the equations of motion is possible. The fluid is assumed to be incompressible. The assumption of a parallel flow is strictly correct only for flows between parallel walls (plane Poiseulle flow) but it is a rather good approximation for boundary layers.
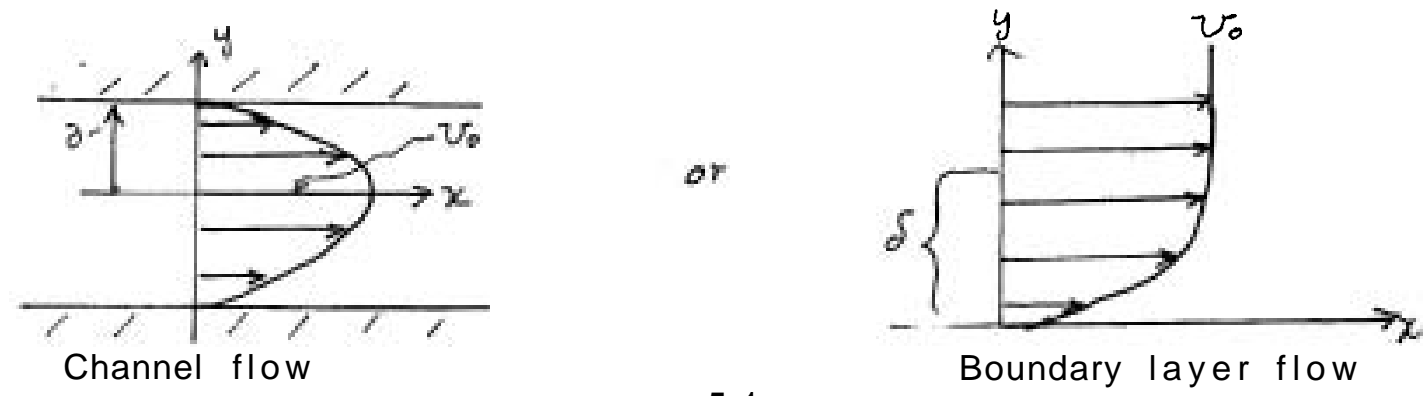

Fig. 5.1

Typical scales for velocity and length are (see Fig.5.1).

The velocity at the channel center and the half (or full) width of the channel, respectively, for channel flow, and the free stream velocity and the boundary layer thickness (e.g. displacement thickness), for a boundary layer flow.

The velocity components are

$$
\begin{aligned}
& \bar{v}_{i}=V(y) \delta_{1 i} \text { for the mean flow and } \\
& u_{i}=(\mu, v, u)=\left(\mu_{1}, u_{2}, u_{3}\right) \text { for the disturbance flow. }
\end{aligned}
$$

The equations for the fluctuating components are (see Lecture \#4) 
$\frac{\partial u_{j}}{\partial t}-\bar{v}_{j} \frac{\partial u_{i}}{\partial x_{j}}+u_{j} \cdot \frac{\partial \bar{v}_{i}}{\partial x_{j}}=-\frac{\partial p}{\partial x_{i}}+\nu \frac{\partial^{2} \mu_{i}}{\partial x_{j} \partial x_{j}}+\frac{\partial}{\partial x_{j}}\left(\overline{u_{i} u_{j}}-u_{i} u_{j}\right)$

( $p$ is the disturbance in mean pressure.)

With the given mean flow and, neglecting quadratic terms, we find that (5.1) reduces to

where

$$
\frac{D u_{i}}{D t}+v \frac{d V}{d y} \delta_{i 1}=-\frac{1}{\rho} \frac{\partial p}{\partial x_{i}}+\nu \frac{\partial^{2} u_{i}}{\partial x_{j}}
$$

$$
\frac{D}{D t}=\frac{\partial}{\partial t}+v \frac{\partial}{\partial x}
$$

From (5.2) and the incompressibility condition $\left(\frac{\partial u_{i}}{\partial x_{i}}=0\right)$ it is possible to obtain an equation for $v$ only. First take $\frac{\partial}{\partial x_{i}}$ of 45.2$)$. This gives

$$
\nabla^{2} p=-2 \rho \cdot \frac{\partial v}{\partial x} \cdot \frac{d v}{d y}
$$

The second equation in (5.2) is

$$
\frac{D v}{D t}=-\frac{1}{\rho} \frac{\partial p}{\partial y}+\nu \cdot \frac{\partial^{2} v r}{\partial x_{k} \partial x_{k}}
$$

Elimination of $\mathrm{p}$ between (5.3) and (5.4) leads finally to

$$
\begin{aligned}
\frac{D}{D t}\left(\nabla^{2} v\right)-v^{\prime \prime} \frac{\partial v}{\partial x} & =\nu \nabla^{4} v \\
v^{\prime \prime} & =\frac{d^{2} v}{d y^{2}}
\end{aligned}
$$

Equation (5.5) is of fourth order. Two of the boundary conditions are that $v=0$ on solid boundaries and at infinity. Application of the continuity equation gives a second set of boundary conditions, namely that

$$
\frac{\partial v}{\partial y}=-\frac{\partial u}{\partial x}-\frac{\partial w}{\partial z}=0 \text { at } y=0 \text { and } y=\infty \text {. }
$$

Since the $x$ and 2 coordinates are homogeneous, wave type solutions to (5.5) are sought of the form

$$
v=\hat{v}(y) \cdot e^{i(\alpha x+\beta s-\infty t)}
$$

This is the ordinary normal mode assumption. The direction of the waves is shown in Fig.5.2.

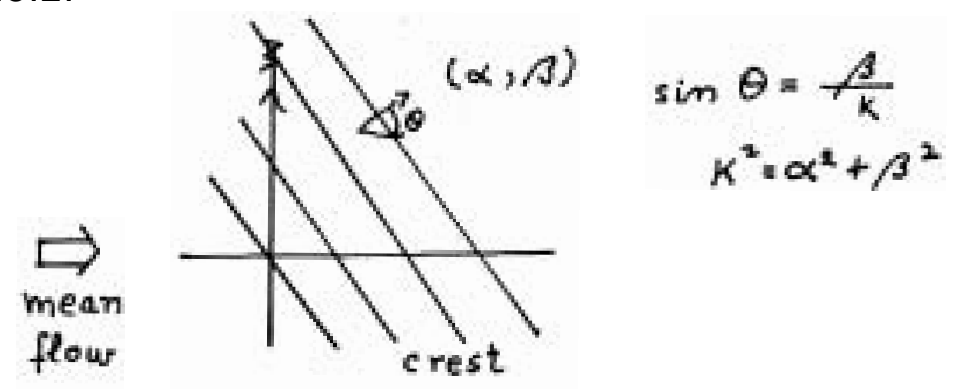

The resulting equation for $\hat{v}$ is the Orr-Sommerfeld equation,

$$
(U-c)\left(\hat{v}^{\prime \prime}-k^{2} \hat{v}\right)-U^{\prime \prime} 0-\frac{1}{i \alpha R_{e}}\left(\frac{d^{2}}{d y^{2}}-k^{2}\right)^{2} \hat{v}=0
$$


where the velocity scale $V_{a}$ and the length scale $\delta$ have been used and $\operatorname{Re}=\quad c$ is the phase velocity defined by $\omega, \propto c$. The boundary conditions for $\hat{v}$ are

$$
\hat{v}(0)=\hat{v}^{\prime}(0)=\hat{v}(\infty)=\hat{v}^{\prime}(\infty)=0 \quad \text { (Boundary layer) }
$$

Since the velocity profile is a constant far away from the boundary layer the last ot these conditions follows from the third.

Equations (5.6) with the boundary conditions constitutes an eigenvalue problem for $\alpha$ as a function of $\mathrm{d}, \beta$ and $R e$.

According to Squires' theorem (Squire, 1933) the three-dimensional problem can be reduced to a two-dimensional one for a lower Reynolds number. This is accomplished by selection of a new $x$ axis normal to the wave fronts. The mean velocity component normal to the wave front gives a smaller Reynolds number. the change is equivalent to the scaling

$$
\begin{aligned}
& \tilde{\alpha} \tilde{R}_{e}=\alpha \operatorname{Re} \tilde{c}=c \tilde{\alpha}^{2}=\alpha^{2}+\beta^{2} \\
& \quad \text { (Note: } \tilde{\alpha}>\alpha \text { and } \operatorname{Re}<\operatorname{Re}) . \\
& \text { In summary: } C=c(\alpha, \beta, \operatorname{Re})=c\left(k, O, \frac{\alpha}{k} R_{e}\right)
\end{aligned}
$$

The eigenvalue results are usually presented in two ways:

a) Temporal case. $\alpha$ real, $\omega$ complex

$$
\omega=\Omega^{(n)}\left(\alpha, R_{E}\right)=\Omega_{R}^{(n)}+i \Omega_{I}^{(n)}=\alpha\left(C_{R}^{(n)}+i C_{I}^{(n)}\right)
$$

The wave grows in time if $\Omega_{I}^{(\omega)}>0$.

b) Spatial case. $\quad \alpha$ complex, $\omega$ real

$$
\alpha=\alpha_{R}^{(n)}+i \alpha_{i}^{(n)}
$$

The wave grows in space if $\alpha_{\underline{I}}^{(n)}<0$.

Since $\Omega_{\mathbf{I}}$ and $\alpha_{\mathbf{I}}$ are very small in the unstable region, it is possible, as shown by Gaster (1962), to relate the temporal and the spatial cases to each other. To order $\left(\omega_{I} / \omega_{R}\right)^{2}$ it follows $(T=$ temporal, $S=$ spatial)

$$
\begin{gathered}
\alpha_{R}(T)=\alpha_{R}(s) \\
\Omega_{R}(T)=\Omega_{R}(s) \\
\frac{\Omega_{I}(T)}{\alpha_{I}(S)}=-\frac{\partial \Omega_{R}^{(n)}}{\partial \alpha_{R}}=- \text { group velocity }=-C_{I}^{(n)}
\end{gathered}
$$

The growth of the waves is for the two cases

a) temporal

$$
\text { growth }=e^{\Omega_{x}^{(n)} \Delta t} \quad \propto \frac{c_{T}^{(n)}}{\hat{s}_{1}^{l}+\infty} \Delta x
$$

b) spatial:

$$
\text { grotwh }=e^{-\alpha_{I}^{t}} \text {, where } \alpha_{I}=-a \frac{c_{I}^{(n)}}{c_{l}^{(n)}}
$$

Generally, for boundary layers $\frac{\alpha_{t}}{\alpha_{R}}<1$.

In experiments with Tollmien-Sclichting waves oscillating ribbons have 
often been used (Schubauer and Skramstad, 1947, the diagram from Ross et al., 1970, presented in the lecture references). This corresponds to the spatial case since the frequency is fixed. Stability data is most often presented in the following form:

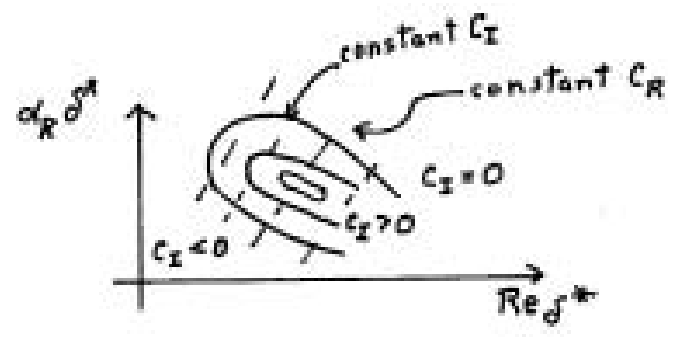

Fig. 5.3

When a wave of a given frequency travels downstream from an oscillating ribbon it will pass through regions with increasing boundary-layer thickness, and therefore the Reynolds number increases. Eventually the wave leaves the unstable region, and thus from a linear point of view a boundary layer is always stable.

For Falkner-Skan profiles (free stream velocity varies like $x^{m}$ ) which have an inflection point for $m<0$, the growth rates are increased and the critical Reynolds number is decreased. Also, for large $\operatorname{Re}, c_{R}$ becomes independent of Re. For certain Reynolds number wave number ranges increase in the viscosity, so that $\boldsymbol{R e}$ is lowered, may lead to increased instability. This is especially apparent for a velocity profile without an inflection point, for which the flow is stable in the inviscid case.

The importance of the inflection point in the mean velocity profile is seen clearly in the inviscid case $(\$=0)$ (following Reyleigh, 1890). Then Eq. (5.6) becomes

$$
(u-c)\left(\hat{v}^{\prime \prime}-k^{2} \hat{v}\right)-v^{\prime \prime} \hat{v}=0
$$

Multiplication with $\hat{v}^{*}$ and integration over the defining interval yields $-\int_{0}^{y_{1}}\left(\left|\hat{v}^{\prime}\right|^{2}+k^{2}|\hat{v}|^{2}\right) d y=\int_{0}^{y_{1}} \frac{v^{\prime \prime}}{v-c}|\hat{v}|^{2} d y=\int_{0}^{y_{1}} \frac{v^{\prime \prime}\left(U-c_{R}\right)+i v_{c i}^{\prime \prime}|\hat{v}|^{2} d y}{|v-c|^{2}}$ Identifying imaginary parts we find

$$
\begin{gathered}
\quad 0=c_{t} \int_{0}^{y_{1}} \frac{v{ }^{\prime \prime} \cdot|\hat{v}|^{2}}{|v-c|^{2}} d y \\
\text { Thus either } c_{i}=0 \text { or } \int_{0}^{y_{1}} \frac{v^{\prime \prime} \cdot \mid \hat{v} l^{v}}{|v-c|^{2} d y}=0 \text {, and if } c_{i} \neq 0, v^{\prime \prime} \text { must change sign }
\end{gathered}
$$
in the interval, which implies an inflection point in $V$. The inflection point must be of the kind' that $V^{\prime}$ has a maximum there.

A simple physical explanation for the necessity of an inflection point to give instability is given by Lin (1955).

The basic principle in Lin's argument is conservation of vorticity for a fluid element in two-dimensional flow. If the flow has a monotonically varying vorticity profile, any fluid element moved up or down experiences a restoring force. For example, if a fluid element is transferred 
from $y_{2}$ to $y_{1}$ (Fig. 5.4) it has excess vorticity. The excess vorticity induces a flow around the fluid element, pulling neighboring fluid of excess vorticity down on its left and fluid of less vorticity up on its right (Fig.5.4b) and (c)). The result is a net restoring force.

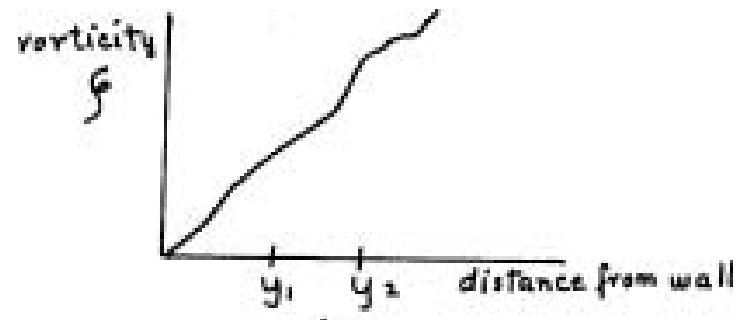

(a)

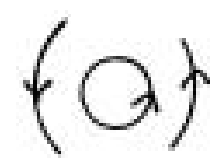

(b)

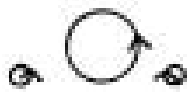

(c)

Fig. 5.4

For an element moved from $y_{1}$ to $y_{2}$, the restoring force is in the opposite direction. Thus the flow is stable. If, however, there is an extremum in the vorticity curve, this argument does not hold. Furthermore, it should be emphasized that the existence of an inflection point is not sufficient to guarantee instability, only necessary.

If there is no viscosity and no inflection point, the flow is stable, as in the Rayleigh case discussed earlier. Introduction of viscosity plays a dual role. At low Reynolds number it damps disturbances. At higher Reynolds number viscosity actually serves to divert energy from the main flow into disturbances and can cause instability - e.g. Poiseuille flow in a channel. It is possible to show that introduction of perturbations may reduce the total kinetic energy of the system. Therefore vis cosity or other means of removing kinetic energy from the system may lead to an instability. For an analogy, consider waves propagating on a flexible boundary at a speed less than the mean flow of an adjacent fluid.

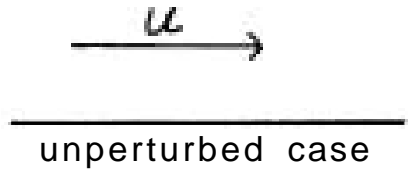

(a)

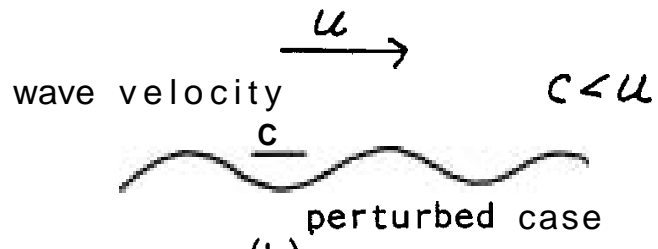

(b)

Fig. 5.5

Because there are more fluid regions with a velocity defect in the troughs than there are regions of excess velocity above the crests, the travelling wave is associated with a net kinetic energy defect. (Landahl, 1962).

Nonlinear instability

Consider a wave with wavelength $2 \pi / \propto$ and amplitude $\varepsilon$.

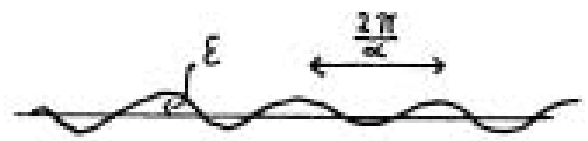

Nonlinearity has two effects: 
a) there are changes in the mean flow of order $\varepsilon^{2}$,

b) interaction between waves creates higher harmonics with wave numbers $2 \propto$ to $O\left(\varepsilon^{2}\right)$.

For the amplitude $A$ the Stuart-Landau theory gives:

$$
\begin{gathered}
\frac{d A^{2}}{d t}=2 \Omega_{i} A^{2}+a_{2} A^{*} \\
a_{2}>0, \Omega_{i}<0 \text { gives subcritical instability and } \\
a_{2}<0, \Omega_{i}>0 \text { supercritical equilibration with } \\
\left(A^{*}\right)_{2}=-2 \frac{\Omega_{i}}{a_{2}}
\end{gathered}
$$

The Stuart-Landau theory works in thermal convection but is less suited for problem in shear flow instability.

\section{References}

Gaster, N. 1962 J.Fluid Mech. 14: 222.

Landahl, M.T. 1962 J.Fluid Mech. 13: 609.

Lin, C.C. 1955 The Theory of Hydrodynamic Stability, Cambridge Univ.Press.

Rayleigh, J.W.S. 1890 Scientific Papers Vol.1: 474, Cambridge Univ.Press.

Ross, J.A., F.H.Barnes, J.G.Burns and M.A.S.Ross 1970 J.Fluid Mech. 43. 819.

Schubauer, G. B. and H. K. Skramstad 1948 NASA Report No. 909.

Squire, H.B. 1933 Proc.Roy.Soc. Al 42: 621.

Notes submitted by

L. Hakan Gustavsson and Rush D. Holt

Lecture \#6.

\section{INSTABILITY AND TRANSITION}

\subsection{Introduction}

The purpose of this lecture is to describe how a laminar boundary layer flow breaks down to turbulence and to try to explain the experimental observations on basis of theory. First, the experimental results of Klebanoff, Tidstrom and Sargent (1962) are discussed. Second, some aspects of kinematic wave theory are explained. Third, kinematic wave theory is used to explain breakdown (Landahl, 1972).

\subsection{The three-dimensional nature of transition}

Klebanoff, Tidstrom, and Sargent (henceforth referred to as KTS) carried out experiments on the transition to turbulence in the boundary layer of a flat plate. Their experiment was designed to study the growth and evolution of controlled three-dimensional disturbances. It had been 
observed that the growing waves were of a three-dimensional character; that is, the wave amplitude varied in the cross-stream direction. They therefore attached one-half inch pieces of scotch tape one-half an inch apart on the underside of a ribbon which they vibrated at a fixed frequency.

The three-dimensional nature of the growing wave is illustrated by Figs.2 and 11 of KTS. Figure 2 shows the spanwise variation in downstream fluctuating velocity $u^{\prime}$ for various downstream locations. Figure 11 gives the spanwise variation in downstream fluctuating $u^{\prime}$ and mean $\square$ velocities and cross-stream fluctuating $W^{\prime}$ and mean $\bar{W}$ velocities at a downstream location just prior to breakdown. Valleys (i.e. minimum amplitudes in the spanwise variation of velocities) are lined up with the pieces of tape on the ribbon, while peaks are lined up with the gaps between pieces of tape.

Figure 9 of KTS illustrates the harmonic content of $u^{\prime}$ as a function of downstream location. At breakdown the first harmonic has stopped growing whereas the second and third harmonics are rapidly growing.

Figure 19 of KTS shows the downstream vorticity (i.e. longitudinal eddy) structure. When the three-dimensional wave begins to grow a longitudinal eddy occupies the region from a peak to a valley. By the time breakdown occurs the eddy has almost halved its wavelength.

The suddenness of breakdown at a peak is demonstrated by Fig.22 of KTS. This suddenness indicates that some kind of critical flow condition has been reached at the breakdown.

Before breakdown an inflection point shows up in the instantaneous velocity profile. Figure 10 of KTS gives the mean velocity profile at various downstream locations. Figure 30 of KTS shows the instantaneous $u$ and Ũ velocity profile of various downstream locations. Figure 31 of KTS demonstrates that a roughness element glued to the flat plate produces a similar inflectional velocity profile.

During breakdown intense, high-frequency fluctuations develop. Figure 21 of KTS gives oscillograms of $u$ at various downstream distances around the location of breakdown. These show the sudden development of spikes of negative $u$.

The frequency of the breakdown process ( $i e$. the "hairpin" eddies) is given in Fig.29 of KTS. The Strouhal number $\frac{\delta_{5}}{U_{1}}$ is independent of Reynolds number and equal to 0.13 , where $\delta_{b}^{*}$ is the displacement thickness of the mean velocity profile at breakdown, $f_{m}$ is the frequency and $U_{1}$ is the free stream velocity.

Elder (1962) in Fig.2 shows a photograph of a growing turbulent spot approximately. The spot spreads in the downstream direction with the front facing the spot travelling at approximately $90 \%$ of the free stream velocity. The spreading angle of the spot is approximately $10^{\circ}$, which can be shown to be in good agreement with Tollmien-Schlichting theory.

The observed breakdown process is not described by the Landau equation because the instability is of much shorter wave-length than the fundamental. According to the linear theory applied to the mean velocity profile the higher wave numbers are stable. However, the breakdown process can be explained using kinematic wave theory. 


\subsection{Kinematic wave theory}

This section is devoted to kinematic wave theory, which will be applied to the problem of breakdown in the next section.

Consider a one-dimensional wave train of length scale $L$ with characteristic wave length $\lambda_{0}$. Assume that the length of the wave train is large compared with the wave length, i.e., $\in \equiv \lambda_{0} / L<<1$. (see Fig.6.1).

The dispersion relation is expressed as

$$
\omega=\Omega(k)=\Omega_{k}(k)+i \Omega_{I}(k)\left(\left|\Omega_{I} / \Omega_{k}\right|<<1\right) \text {, }
$$

where $\omega$ is the (complex) frequency and $k$ the wave number. The disturbance $v(x, t)$ may be represented by the Fourier integral

$$
v=\int_{-\infty}^{\infty} e^{i(k x-\Omega(k) t} \vec{v}_{0}(n) d k,
$$

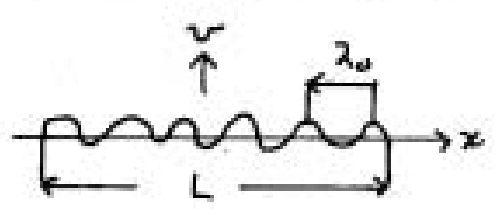

A moving wave train

Fig. 6.1

where $\hat{V}_{0}(k)$ is the Fourier transform of the initial disturbance $\left.K C^{*}\right)$ The distribution of $|\hat{v}(k)|^{2}$ has a narrow band width because $\epsilon<<1$. This fact enables us to expand the phase $(k x-\Omega(k) t)$ with respect to $\left(k-k_{0}\right)$ (or $\left.\epsilon\right)$ :

$$
k x-\Omega(k) t=\left\{k_{1} x-\Omega\left(k_{0}\right) t\right\}+\left(h-k_{0}\right)\left(k-\frac{\partial \Omega_{\alpha}\left(n_{0}\right)}{\partial k} t\right)+i\left(k-k_{0}\right) \frac{\partial \Omega_{n}\left(k_{0}\right)}{\partial k} t+\frac{1}{2}\left(k-k_{0}\right)^{2} \frac{\partial^{2} \Omega_{k}\left(n_{0}\right)}{\partial k} t+\cdots
$$

That is, we find

$$
\begin{aligned}
e^{i(k x-\Omega t)}= & e^{i\left(h_{0} x-\Omega\left(n_{0}\right) t\right)} e^{i\left(k-k_{0}\right)\left(x-\frac{a_{\Omega}\left(k_{0}\right)}{\partial t} t\right)} x \\
& x\left\{1+i\left(k-k_{0}\right) \frac{\partial \Omega_{x}\left(n_{0}\right)}{\partial \hbar} t+\frac{1}{2}\left(k-k_{0}\right) \frac{\partial^{*} \Omega_{3}\left(k_{0}\right)}{\partial k^{2}} t+\cdots\right.
\end{aligned}
$$

Therefore, as long as $(c, t / L)<\epsilon \epsilon^{-1}$ (and $\left(\Omega_{I} / \Omega_{k} \mid<<1\right)$, the disturbance is approximately given by

$$
\nu=e^{i\left(k_{0}\right)\left(-\Omega\left(k_{0}\right) t\right)} \int_{-\infty}^{\infty} e^{i\left(n-k_{0}\right)\left(x-k_{1}\left(h_{0}\right) t\right) \cdot} V(k) d k,
$$

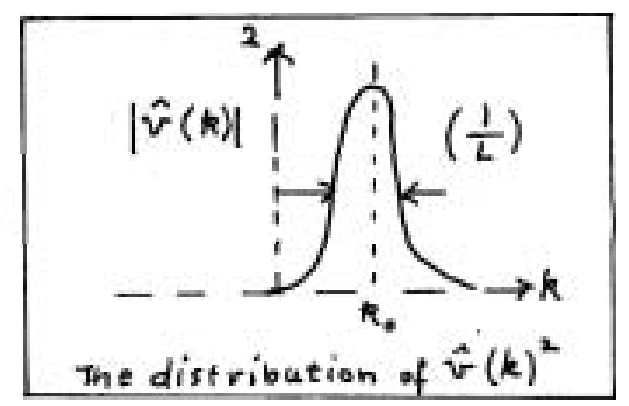

Fig. 6.2

where $\tau_{1} \equiv \frac{a \Omega_{n}\left(k_{0}\right)}{\partial k}$ is the group velocity. Alternatively, the above formula may be expressed as

$$
v=e^{l\left(k_{0} c_{1}\left(k_{0}\right)-\Omega\left(k_{0}\right)\right) t} v_{0}\left(x-c_{1}\left(k_{0}\right) t\right),
$$

which means that the wave amplitude distribution moves with the group velocity $c_{i}\left(k_{0}\right)$, with the amplitude changing exponentially with time proportional to $e^{\Omega_{0} t}$. In addition there is a phase change of $k_{0}\left(c_{1}-c_{0}\right) t$, where $\tau_{0}=\Omega\left(n_{0}\right) / m_{0} \cdot$

In the case of the initial value problem, for which the distribution of $|\hat{V}(\mathbf{k})|^{2}$ does not have a narrow band width, the method of stationary phase yields the asymptotic solution for $x$ large compared with $L$,

$$
v(x, t) \simeq \frac{\hat{v}\left(n_{0}\right)}{\sqrt{i \frac{\partial^{2} \Omega\left(n_{0}\right)}{\partial h_{2}}}} e^{i\left(k_{0} x-\Omega\left(k_{2}\right) t\right)}
$$


where $k_{0}$ is chosen so that the condition $x-\frac{a \Omega_{k}\left(k_{e}\right)}{\partial k} t=0$ is satisfied. For two-dimensional wave propagation, we find in the same way

$$
v \sim \frac{1}{t}
$$

If the growth rate $\Omega_{\boldsymbol{I}}$ is considered explicitly in this two-dimensional case, we have

$$
v \sim \frac{1}{t} e^{\Omega_{1} t}
$$

Here the factor $\left(t^{-1}\right)$ obviously originates in the wave dispersion, and may therefore be termed a "dispersion decay". Next, wave propagation through slowly varying media is considered. As is well-known, when the eikonal exists, i.e., the disturbance is expressed as proportional to $e^{i \theta}$, ( $\theta$ is the eikonal), we have $\omega=-\partial \theta / \partial t, k_{i}=\partial \theta / \partial x_{i}$, hence $-\partial x_{i}+\frac{\partial k_{i}}{\omega}=0$.

which may be regarded as an equation for the conservation of wave crests. The frequency is a function ndt only of the wave number components $k_{i}$ but also of $t$ and $x_{i}$, i.e.

$$
\omega=\Omega\left(k_{i} ; t, x_{i}\right) \text {. }
$$

Note that the group velocity is defined by the real part of $\Omega$,

$$
o_{i}=\frac{a n_{k}}{\partial k_{i}} \text {. }
$$

For simplicity we shall start out by restricting our investigation to a steady nonhomogeneous medium. Suppose a wave train is moving with the group velocity $C_{A}$ at a position $A$ and with the group velocity $C_{B}$ at another position B (see Fig.). Then a relation is easily obtained between the group velocity and the length occupied by the passing wave train. Conservation of wave crests gives

$$
\Delta t \equiv \frac{L_{A}}{C_{A}} \cdot \frac{L_{R}}{C_{a}}
$$

Therefore

$$
\frac{L_{E}}{L_{A}}=\frac{C_{B}}{C_{A}}
$$

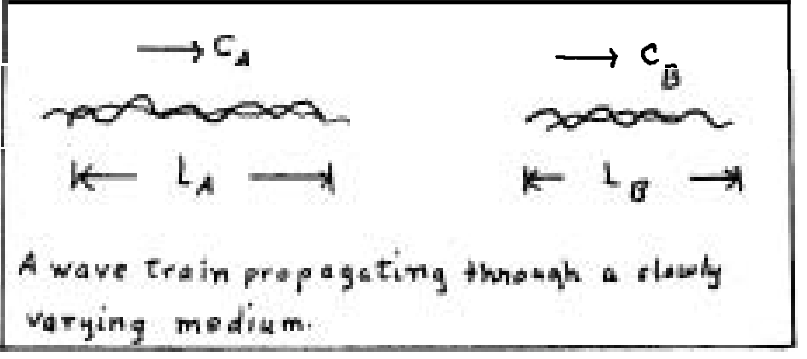

Fig. 6.3

In the conservative system a quantity called wave action with density $A$ ( $\sim$ (amplitude $)^{2}$ ) is conserved. (Whitham, 1972.) By the invariance of ( $L A$ ) we have for the wave action density

whence we find

$$
\frac{A_{A}}{A_{A}}=\frac{C_{A}}{s_{B}}
$$

$$
A \sim \frac{1}{c}
$$

These arguments show that (cA) remains constant with the variation of $x$, i.e. $\frac{\partial}{\partial x}(c, A)=0$. If generalized to an unsteady system, it reads

$$
\frac{\partial}{\partial t} A+\frac{a}{\partial x}(c, A)=0 \text {. }
$$

Moreover, if a weakly non-conservative system is considered, and extension to propagation in three dimensions is made, this conservation law becomes approximately

$$
\frac{\partial}{\partial t} A+\frac{\partial}{\partial x_{i}}\left(c_{i} \lambda\right)=2 \Omega_{I} A \text {. }
$$

For a proof of this relation, see Jimenez and Whitham, Proc.Roy.Soc. Lond. A. 349: 277 (1976). 


\subsection{Application of kinematic wave theory to breakdown}

Kinematic wave theory is now applied to boundary layer transition in order to explain the breakdown process. When there is a nonhomogeneity travel1ing with a phase velocity $c_{e}$ (i.e. $\left.\Omega=\Omega\left(x-c_{0} t\right)\right)$ in the equation for the conservation of wave action $c$ must be replaced by $c-c_{0} v$. That is, $A \approx \frac{y}{c+c}$ where $z$ is a constant. Thus, at $c=c_{0}$ there is a critical condition reached where the theory breaks down. For an arbitrary system $c=c(x, t)$, a local phase velocity may be defined as $c_{0}=-c_{t} / c_{x}$. (Landahl, 1972).

The critical condition for breakdown may be understood physically by looking at the analogous situation in a supersonic nozzle. If a flow is being accelerated from subsonic to supersonic flow through a nozzle, there is no pile up of disturbances at the location of the critical condition because disturbances (which travel at the speed of sound relative to the moving fluid) travelling downstream are swept through the nozzle by the flow. On the other hand, if a flow is being decelerated from supersonic to subsonic flow through the nozzle disturbances will pile up (i.e. a shock wave develops) at the location of the critical condition because the disturbances travelling upstream cannot pass through the nozzle. Thus a critical condition is a way of identifying a place where the flow has to change character.

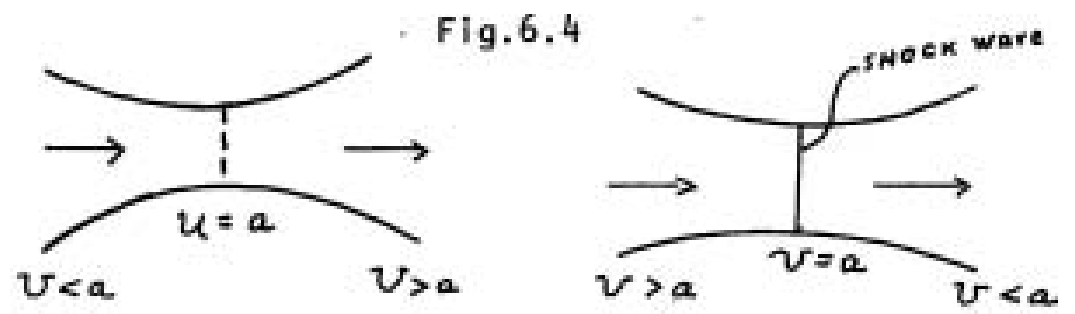

Landahl (1972) (henceforth called L) used kinematic wave theory to explain the breakdown process in this fashion. Two fundamental assumptions were made: (i) The wavelength of the secondary wave is much less than the wavelength of the primary wave which makes it possible to think of the secondary wave as riding on the primary wave, and (ii) the frequency of the secondary wave is much less than the frequency of the primary wave so that the inhomogeneity due to the primary wave is slowly varying.

Figure 6 of $L$ shows the instantaneous and mean velocity profiles before breakdown while Fig.8 of $L$ shows the velocity profile at breakdown. The Orr-Sommerfeld equations are then solved numerically in order to find the dispersion characteristics using smoothed velocity profiles from the experiments. It is then possible to determine whether the critical condition occurs somewhere in the flow, $c$ being the group velocity of the secondary wave and $c_{0}$ if the phase velocity of the primary wave. The dispersion diagram for the instantaneous velocity profile at positions upstream of breakdown is shown in Fig.7 of $L$. It can be seen that for this $c$ \# $c_{0}$. The dispersion diagram at breakdown is shown in Fig.9 of L. At breakdown $c=c_{0}$ for some $k$ and $\Omega_{\mathbf{I}} \geq 0$ where $\Omega_{\mathbf{x}}$ is the growth rate of the secondary wave. Thus, at breakdown the secondary wave focuses on the primary wave where the growth rate of the secondary wave is positive.

The phase of the primary wave is shown as a function of downstream distance in Fig.10 of L. This figure shows that the primary wave accelerates prior to breakdown. Thus, the primary wave catches up with the secondary group rather than the secondary group slowing down to meet the primary wave. 


\title{
References
}

Elder, J.W. 1962 J.Fluid Mech. 9: 235

Klebanoff, P.S., K.D.Tidstrom and L.M.Sargent 1962 J.Fluid Mech. 12: 1 Landahl, M.T. 1972 J.Fluid Mech. 56: 775.

Whitham, G. 1974 Linear and nonlinear waves, John Wiley $\&$ Sons.

\author{
Notes sdbmitted by \\ Aki r a Masuda \\ and James W. Stevenson
}

ADDENDUM (M. T. Landahl)

The Effect of Dispersion on Wave Focusing

The wave breakdown theory of Landahl (1972) was criticized by Stewartson (1973) on the grounds that the model equation (6.8) with $c$ given by (6.6) would not account properly for dispersive effects. By expansion of the linear solution around a centered wave number $k=k_{0}$ and setting $v=a \exp \left\{i\left(\bar{\theta}+k_{0} x-\Omega_{R} t\right)\right\} \equiv \psi_{\exp }\left\{k_{0} x-\Omega_{R} t\right\}$ where a is (the real) amplitude, $\tilde{\theta}$ a slowly varying phase, and $\psi$ the complex amplitude, he shows that $\psi$ satisfies approximately the differential equation

$$
i\left(\psi_{t}+c^{0} \psi_{x}\right)-b \psi_{x x}=i \Omega_{x}^{0} \psi
$$

where

$$
C=\frac{\partial \Omega_{R}}{\partial k}
$$

and

$$
b=\frac{1}{2} \frac{\partial^{2} \Omega_{B}}{\partial k^{2}}=b_{R}+i b_{I}
$$

and superscript $o$ denotes quantities evaluated at $k=k_{0}$. (For a nondissipative system with $\Omega_{t}=0$, inclusion of weakly nonlinear cubic terms leads to the well-known "nonlinear Schrödinger equation").

The solution of this equation for large times can be shown to be equivalent to the asymptotic solution (6.2). Equations (6.4) and (6.8) (when specialized to the homogeneous case), however, do not include any term corresponding to $b^{\circ} \psi_{x \times}$. Stewartson then goes on to show how the solutions based on the two model equations would differ; in particular, the imaginary part of $b$ would be important for the amplitude magnitude at large times.

The question of the importance of such dispersive effects for the evolution of the wave train has been studied by Chin (1976). Through an extension of Whitham's theory for a wave train propagating through a nonhomogeneous medium to include higher-order dispersive effects (as well as weakly nonlinear effects) he finds that (6.5) should be replaced by

$$
\omega=\Omega_{R}(k, X)+E^{2}\left[\frac{a X}{a} \frac{\partial}{\partial k}\left(\frac{\Omega_{x}}{\epsilon}\right)-b_{R} \frac{a X X}{a}\right]+a^{2} f_{2}(h)+\ldots
$$

where $X$ is a slow $x$-variable, $\chi=\epsilon x$ and $f_{2}(k)$ is a function arising from 
weak nonlinear effects (not discussed here). In this expression are left out terms involving $X$-derivatives of the wave number which are generally of less importance. Since $\Omega_{\boldsymbol{I}} / \epsilon$ must be of order unity for the concept of a slowly varying wave train to be meaningful, the correction terms for the linear case are of order $\epsilon^{2}$. This equation is to be used in the equation (6.4) for the conservation of wave crests. In a similar manner, Chin (1976) finds that the equation (6.7) for the wave action density when corrected for hiaher-order effects becomes

$$
\begin{aligned}
\frac{\partial \mathcal{A}}{\partial \bar{T}}+\frac{\partial}{\partial \bar{X}}(c \mathcal{A})= & 2 \frac{\Omega_{2}}{\epsilon} A_{3}+\epsilon^{2}\left[\frac{\partial b_{k}}{\partial k}\left(\frac{1}{3} A_{X X X}-\frac{1}{2} \frac{A_{X}^{A_{X X}}}{A^{A}}+\frac{1}{4} \frac{A_{X}}{A_{2}}\right]-\right. \\
& \left.-\frac{b_{x}}{\epsilon}\left(A_{X X}-\frac{1}{2} \frac{A_{X}^{2}}{A}\right)\right]
\end{aligned}
$$

where $T=\epsilon t$

is the slow time. Again, terms involving derivatives of $k$ have been neglected as being less important. Thus since $b_{I}=O(\epsilon)$ according to the basic assumption of slow decay or growth rate of the wave train, the effects pointed out by Stewartson (1973), which are produced by the terms within the square bracket, thus give contributions which are of order $\epsilon$ and hence negligible. However, very near a focus, terms involving higher $X$-derivatives may no longer be neglected, so that the focal structure is not properly described by (6.7) because dispersion will counteract the focussing. Through an approximate analysis, Chin (1976) finds that the thickness of the focal region is proportional to $\epsilon^{1 / 3}$ in unscaled variables. Also, the wave action density is found to remain finite, but large, at the focus.

\section{References}

Stewartson, K. 1973 Some aspects of nonlinear stability theory, paper presented at the XI Symposium on Advanced Problems and Methods in Fluid Mechanics, Kauijenny Potok, Poland 3-8 Sept. 1973.

Chin, W.C. 1976 Physics of slowly varying wavetrains in continuous systems. Ph.D.Thesis, Dept.Aeronautics $\&$ Astronautics, Mass.Inst.Technology. 
Lecture \#7.

\section{SOME THEORETICAL APPROACHES TO TURBULENCE}

\subsection{Mixing-length and eddy viscosity closures}

One of the more successful simple closure schemes for the calculation of the mean velocity distribution in a turbulent shear flow, i.e., relation between the Reynolds stress and the mean-flow properties, is Prandtl's (1925) momentum transfer theory in which a mixing length for the momentum was introduced (Taylor was the first to suggest the idea of a mixing length in his vorticity transport theory of 1915). The basic assump tion in Prandtl's (1925) theory is that each fluid element when displaced from its original position $y$ in the shear flow will retain its original streamwise momentum. Thus, if it is displaced a vertical distance $\ell$ the apparent velocity perturbation $u$ will be $k=\bar{V}(y)-\bar{v}(y+\ell)=-\ell \frac{\partial \bar{v}}{\partial y}$.

The vertical velocity component is given by $V=D l / D t$. On the basis of dimentional reasoning one could set this proportional to $\ell \mid \partial \bar{U} / \partial y l$. Thus, the Reynolds shear stress is approximated by

$$
\tau_{i 2}=-\rho \overline{u v}=\rho \overline{l^{2}} \frac{\partial \bar{v}}{\partial y} \frac{\partial \bar{v}}{\partial y}
$$

where $\ell_{m}$ is Prandtl's mixing length. He suggested that for a boundary layer the mixing length should be proportional to the distance from the wall, i.e.,

$$
l_{m}=x_{y}
$$

where $\mathcal{X}$ is a constant (Von Karman's constant). For the constant-stress region

$$
\tau_{12}+\mu \frac{\partial \bar{U}}{\partial y} \simeq \rho u_{*}^{2}=\rho l_{m}^{*}\left(\frac{\partial \vec{V}}{\partial y}\right)^{2}+\mu \frac{\partial \bar{U}}{\partial y}
$$

With $\ell_{m}=x_{y}$ and sufficiently far from the wall where the viscous stress may be neglected one finds $\partial \bar{V} / \partial y$ and, as could be expected, the logarithmic profile is recovered. Von Karman (1930) proposed a different relation between the mean velocity distribution and the mixing length, namely, he assumed it to be proportional to the local radius of curvature of the velocity profile, i.e.,

$$
\ell_{m}=-x \frac{\partial \bar{v}}{\partial y} / \frac{\partial^{2} \vec{v}}{\partial y^{2}}
$$

In Taylor's (1932) mixing length hypothesis for the vorticity he also assumed the vorticity mixing length to be proportional to the distance from the wall. All three approaches give a logarithmic region, but the velocity profiles differ in details.

The concept of a mixing length is closely related to that of the mean free path in the kinetic theory of gases. From this theory the coefficient of molecular viscosity is given by $\mathcal{\nu} \sim \ell^{\prime} a$ where $\ell^{\prime}$ is the mean free path in the gas and $a$ is the velocity of sound. Introducing a turbulent viscosity

$$
\overline{u v}=-\nu_{r} \frac{\partial \bar{v}}{\partial y}
$$

and using the mixing-length expression for $u$ we obtain $\nu_{T}=\ell_{m} v^{\prime}$

where

$$
v^{\prime}=\overline{\ell v} / \ell_{m}
$$

which could be expected to be proportional to $u^{\prime}=\sqrt{q^{2}}$. 
Many extensions of these ideas can be found in the literature; as an example, let us just sketch the modeling proposed by Jones and Launder (1972). They assume that

$$
\nu_{T}=\frac{\left(\bar{q}^{2} / 2\right)}{2 \epsilon} c_{1}
$$

The idea is to calculate $\bar{q}^{2}$ and $\bar{\epsilon}$ for the flow considered, and $C_{l}$ is adjusted to fit measured data. First, $\bar{q}^{2}$ is calculated from the energy equation

$$
\frac{1}{2} \frac{D}{D t} \bar{q}^{2}=\bar{U} \cdot \nabla \bar{q}^{2}=-\overline{u v} \frac{\partial \bar{U}}{\partial y}-\epsilon-\frac{\partial}{\partial y} \overline{u\left(p+\frac{q^{2}}{2}\right)}
$$

The dissipation $E$ is found from a separate model equation; obviously, the transport term (involving pressure correlations) has to be modeled in terms of knbwn quantities, and is assumed to be giver by

$$
\epsilon=\frac{\partial}{\partial y}\left[A_{1} \nu_{T} \frac{\partial}{\partial y}\left(\frac{\bar{q}^{2}}{2}\right)\right]^{2}-\frac{c_{2}}{\Lambda}\left(\frac{\bar{q}^{2}}{2}\right)^{3 / 2}
$$

where $\Lambda$ is same integral length scale, $A_{1}, C_{2}$ are adjustable parameters, and $\nu_{T}$ is given as above.

The model now looks comparatively simple with the two coupled equations for $q$ and $\in$ only to be solved together with the equations for the mean flow, once the parameters are fixed by comparison with experiments.

Within the range of freedom given by schemes like the above there exist many acceptable models, each with its merits and limitations; it is a matter of availability of data, specific features of a problem, (and taste), to pick the most convenient one for each case.

\subsection{Closure schemes for turbulent spectra}

We will just take a look at a different kind of closure, as applicable to the spectral equations, but only discussed here in relation to the case of an isotropic and homogeneous turbulent flow, for which the mean flow becomes uninteresting.

We have for the three-dimensional signal energy spectrum

$$
\frac{\partial E}{\partial t}=T-2 \nu K^{2} E \quad E=E(K, t)
$$

where $T$ is the non-linear spectral transfer term, of which we need not know here the explicit relation to the triple correlations.

As this transfer is a conservative process, we must have

$$
\int_{0}^{\infty} T(k) d k=0
$$

Several inertial-type models exist for $T(k)$, and attempts have been made to derive how fast the spectrum drops off in the viscous range. A well-known early attempt was made by Heisenberg (1948), leading to a spectrum for large $k$, but one could argue that the spectrum must fall off more rapidly than any inverse power of the to make spectra of derivatives of $u$ to arbitrary high order be integrable. Kovasenay (1948) proposed for hạ $\ll T$ (near equilibrium)

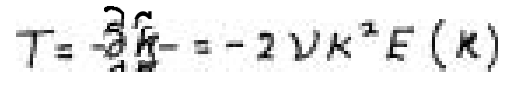

expressing that the divergence of energy transfer flux is comppletely balanced by viscous dissipation. The inertial subrange expression for $E$ is substituted, leading to 


$$
3 \frac{\partial T^{1 / 3}}{\partial k}=-2 \alpha \nu k^{1 / 3}
$$

so that, finally

$$
E=\alpha \epsilon^{2 / 3} k^{-5 / 3}\left(1-\frac{\alpha}{2} \frac{\nu k^{4 / 3}}{\epsilon^{1 / 3}}\right)^{2}
$$

An alternative version was proposed by Pao (1965), who suggested a different inertial range modeling for $\mathcal{G}$, namely $\xi(k)=f(\epsilon, k) E(K)$. Dimentional analysis yields $f-\beta \epsilon^{4 / 3} K^{5 / 3}$, with the final expression (after integration:

$$
E=\propto \epsilon^{2 / 3} k^{-5 / 3} \text { ekp }\left[-\frac{3}{2} \propto\left(\frac{k}{\kappa_{k}}\right)^{4 / 3}\right]
$$

A comparison between the two results shows that Kovasznay's formula appears as the 1st order expansion (outside the inertial range) of Pao's exponential drop off. However, as the assumption of extending the inertial scalings out of their range is a 1 st order approximation, the two formulas are equivalent, and probably of questionable validity beyond the Kolmogorov scale range.

Finally, as an example of other and more sophistocated approaches to turbulence modeling, we shall give a very short description of the first version of Kraichnan's (1959) models, the direct interaction approximation (DIA).

We start from the spectral equations for box turbulence (so as to discreticize the wave-vectors),

$$
\left(\frac{\partial}{\partial t}+\nu k^{2}\right) u_{k}(\vec{k}, t)+i K_{m} P_{k_{j}} \sum_{A} u_{m}(\vec{p}) u_{j}(\vec{q})=0+\delta(\vec{k}, t, \lambda)
$$

where - Arepresents all triangles that give $\vec{p}+\vec{q}=\vec{k}$

$-P_{k j}=\delta_{k_{j}}-\frac{k_{k} K_{j}}{k^{2}}$ is obtained through the elimination of pressure with the aid of continuity.

- $\delta(\vec{u}, \epsilon, \lambda)$ is a random forcing term, the intensity of which is measured by a small parameter $\lambda$.

Expansion of $\delta$ in powers of $\lambda$ yields:

- at order 1; direct interactions

- otherwise; higher harmonics of the perturbation.

When these equations are multiplied by $u_{k}$ and added in order to yield statistics of the field, the random forcing term appears weighted by a Green's function $\mathcal{G}$, which satisfied the equation

$$
\left(\frac{\partial}{\partial t}+\nu k^{2}\right) G+\int_{t^{\prime}}^{t} \eta\left(k, t, t^{\prime \prime}\right) G^{(0)}\left(k, t, t^{\prime \prime}\right) d t^{\prime \prime}=0
$$

with $\eta=\pi \lambda^{2} k \iint_{\Delta} k^{\prime} k^{\prime \prime} G^{(4)} b_{k k^{\prime} k^{\prime \prime}} d k^{\prime} d k^{\prime \prime}+$ higher order terms $b_{k_{i}} k_{i} k^{\prime \prime}$ being a known geometrical factor. The remaining equations read

$$
\left(\frac{\partial}{\partial t}+\nu k^{2}\right) U\left(k, t, t^{\prime}\right)=S
$$




$$
S=\pi \lambda^{2} \kappa \iint_{\Delta} k^{\prime} \kappa^{\prime \prime} d k^{\prime} d \kappa^{\prime \prime} b_{\kappa \kappa^{\prime} k^{N}}\left[\int_{0}^{b} G U^{\prime} U^{\prime \prime} d u^{\prime}-\int_{0}^{t} U^{\prime} G^{(0)} U d k^{n}\right]
$$

with $U$ defined as

$$
E(k, t)=2 \pi K^{2} U(k, t, t)
$$

Taking now $\lambda=1$ everywhere, $S^{(0)} \Xi \zeta$, yields what is known as the direct interaction approximation.

It has a serious drawback. It does not yield the expected $k^{-5 / 3}$ energy spectrum in the inertial subrange, but $k^{-3 / 2}$ instead. This has been shown to be due to the absence of Galilean invariance to random motions.

The cure was found as Lagrangian History Direct Interaction Approximation (L.H.D. I.A.) (Kraichnan, 1965).

\section{References}

Hersenberg, W. 1948 Z.Physik 124: 628

Jones, R.P. and B.E.Launder 1972 Int.J.Heat \& Mass Transfer 15: 301

Kovaznay, L.S.G. 1948 J.Aeron.Sci. 15: 745

Kraichnan, R. 1959 J.Fluid Mech. 5: 497

Kraichnan, R. 1965 Physics of Fluids B: 575

Pao, Y. 1965 Physics of Fluids 8: 1063

Prantdl, L. 1925 Z.Anzew, Math.u.Mech. 5: 136

Taylor, G.I. 1915 Phil.Trans.Roy.Soc.London A 215: 1

Taylor, G.I. 1932 Proc.Roy.Soc.London A 135: 685

Von Karman, Th. 1930 Nachrichten der Akademie der Wissenschaffen Gottengen, Math.-Phys.Klasse 58. 
Lecture \#8.

8. WAVE PROPERTIES OF WAL TURBULENCE

\subsection{Introduction}

Earlier it was shown that a spatially and temporally homogeneous system admitting disturbances in form of lightly damped propagating waves (a wave guide) which were excited by a broad-band noise source would have a wave-number frequency spectrum highly peaked around the eigenfrequency of the system. A parallel shear flow admits wave-like disturbances in form of Tollmien-Schlichting waves, and the turbulence in a boundary layer could therefore be expected to show some of the characteristics of the statistical wave-guide model. The forcing of the system is provided by the nonlinear effects, in particular by the strong mixing taking place during bursting. The observations by Kline $\boldsymbol{e} t$ al., (1967) and Kim $\boldsymbol{e} t$ al., (1971) indicate that the regions of strong mixing, i.e., of nonlinearity, are highly localized in time and space in regions very close to the wall. The strong localization implies broad-banded spectra, which therefore should satisfy well the assumption of the wave-guide model. The basic ideas of this model for boundary layer turbulence were first presented by Landahl (1967) and later extended by Bark (1975).

\subsection{Wave-auide theory}

For the fluctuating velocity field,

$$
\frac{\partial u_{i}}{\partial t}+\bar{V}_{j} \frac{\partial u_{i}}{\partial x_{j}}+u_{j} \frac{\partial \bar{U}_{i}}{\partial x_{j}}=-\frac{1}{\rho} \frac{\partial p}{\partial x_{i}}+\nu \frac{\partial^{2} u_{i}}{\partial x_{j} \partial x_{j}}+\frac{\partial}{\partial x_{j}}\left(\overline{u_{i} u_{j}}-u_{i} u_{j}\right)
$$

We assume a parallel shear flow, $\bar{V}_{i}=V(y) \delta_{i 1}$, and set

$$
\frac{D}{D t}=\frac{\partial}{\partial t}+U(y) \frac{a}{\partial x}
$$

Then (8.1) reduces to,

$$
\frac{\tilde{D} u_{i}}{D t}+u_{2} U^{\prime}(y) \delta_{i 1}=-\frac{1}{p} \frac{\partial p}{\partial x_{i}}+\nu \frac{\partial^{2} u_{i}}{\partial x_{j} \partial x_{j}}+\frac{\partial}{\partial x_{j}^{j}}\left(\overline{\left.u_{i} u_{j}-u_{1} u_{j}\right)}\right)
$$

By taking the divergence of (8.2), we obtain a relation for the pressure, writing $u_{ \pm}=v_{l}$

$$
\frac{1}{\rho} \nabla^{2} p=-2 v^{\prime} \frac{\partial v}{\partial x}+\frac{\partial^{2}}{\partial x_{i} \partial x_{j}}\left(\overline{\overline{u_{i} u_{j}}}-u_{i} u_{j}\right)
$$

At this stage either an equation for $p$ or $v$ could be obtained, but to exploit known results for the Orr-Sommerfeld equation, an equation in $v$ is used. Application of the Laplacian operator to (8.2) and use of (8.3) gives,

where,

$$
\frac{\vec{D}}{D E} \nabla^{2} v-v^{\prime \prime} \frac{\partial v}{\partial x}-\nu \nabla^{\psi} v=q
$$

$$
q=\frac{\partial}{\partial x_{j}}\left(\frac{\partial^{3} u_{i} u_{j}}{\partial x_{i} \partial x_{i}}-\frac{\partial^{2} u_{2} u_{i}}{\partial x_{k} \partial x_{k}}\right)
$$

In deriving (8.4), homogeneity in the $x$ and $z$ directions has been assumed. It would be expected that $y$-derivatives are much larger than either $x$ - or $z$-derivatives; therefore, $q$ could be approximated by

$$
q \approx \frac{\partial^{2}}{\partial y^{2}}\left[\frac{\partial}{\partial x}(u v)+\frac{\partial}{\partial z}(v w)\right]
$$

So to obtain an estimate of $q$, only the terms $u v$ and $v w$ are needed. 
Unfortunately, experimental information is only available for $u v$.

With statistical stationary turbulence and homogeneity in $\mathbf{x}$ - and $z$-directions assumed Fourier transform techniques become convenient, so we set

$$
\hat{v}\left(y_{j} \alpha_{j} \beta_{j} \omega\right)=(2 \pi)^{-3} \iiint_{-\infty}^{\infty} v(x, y, z, t) \exp [-i(\alpha x+\beta z-\omega t)] d x d z d t
$$

and similarly for $q$. The transforms are then substituted into (8.4) and the resulting equation conveniently nondimensionalized by scaling with respect to the free stream velocity $U_{0}$ and boundary layer thickness $\delta$, giving

$$
(v-c)\left(\hat{v}^{\prime \prime}-\kappa^{2} \hat{v}\right)-V^{n} \hat{v}-\frac{1}{i \alpha \operatorname{Re}}\left(\frac{d^{2}}{d y^{2}}-k^{2}\right)^{2} \hat{v}=\frac{\hat{q}}{i \alpha}
$$

where $c=\omega / \alpha$ and $K^{*}=\alpha^{*}+\beta^{2}$, with boundary conditions,

$$
\hat{v}(0)=\hat{v}^{\prime}(0)=\hat{v}(\infty)=\hat{v}^{\prime}(\infty)=0
$$

The Reynolds number is defined as $\boldsymbol{R} \boldsymbol{e}=V_{0} \delta / \nu$ and $\hat{q}$ is the transform of
the source term.

Equation (8.7) is homogeneous with respect to boundary conditions, and in general without the inhomogeneous forcing term, the solutions would be zero for a stable mean flow. The nonlinear terms would be expected to be large during bursting and therefore highly intermittent. It would therefore seem reasonable to suppose that $q$ would behave qualitatively like random shot noise, so that $\bar{q}$, and hente the energy spectrum of $q$, would be fairly flat, with a high wave number cut-off. However, one cannot exaluce the possibility that $q$ may have resonant frequencies, and its spectrum have poles and other singularities in the complexw-plane.

We look for formal solutions of (8.7). It is assumed that the mean velocity profile $U(y)$ is known for the boundary layer, and also that $q$ is known, say from experimental observation and modelled statistically as shot noise. (Bark, 1975).

The equation for $\mathrm{V}$ involves the linear Orr-Sommerfeld operator, and we can use the eigenfunctions of the homogeneous problem to represent - Denote as follows:

$$
\mathcal{L}_{\text {o.S. }}(\phi)=(V-c)\left(\phi^{\prime \prime} K^{2} \phi\right)-V^{\prime \prime} \phi-(i \alpha R e)^{-1}\left(\frac{d^{2}}{d y^{2}}-k^{2}\right)^{2} \phi
$$

and $\phi=\phi^{(n)}$, the $n$th eigenfunction, and $c=\kappa^{(n)}$ the corresponding eigenvalue satisfying

$$
\mathcal{L}_{0.5 .}\left(\phi^{(n)}\right)=0, \phi(0)=\phi^{\prime}(0)=\phi(\infty)=\phi^{\prime}(\infty)=0
$$

The adjoint operator $\tilde{\mathcal{L}}_{\text {o.s. }}$ is defined by

$$
\begin{aligned}
& \tilde{L}_{0.5 .}(\tilde{\phi})=(U-c)\left(\tilde{\phi}^{\prime \prime}-k^{2} \tilde{\phi}\right)+2 v^{\prime} \tilde{\phi}^{\prime}-\left(i \alpha R_{e}\right)^{-1}\left(\frac{\alpha^{2}}{d_{y^{2}}^{2}}-k^{2}\right)^{2} \\
& \begin{array}{r}
\left.\sim \tilde{\phi}^{(n)}\right)=0 \text { will give the adjoint eig } \\
\text { From }(8.8) \text { and }(8.9) \text { one can derive } \\
\left(\tau^{\left.(n)-c^{(m)}\right)} \int_{0}^{\infty}\left[\tilde{\phi}^{(m)} \tilde{\phi}^{(n)}+k^{2} \phi^{(n)} \tilde{\phi}^{(m)}\right] d y=\right.
\end{array} \\
& \begin{aligned}
\left(c^{(n)-c(m)}\right) \int_{0}^{\infty}\left[\tilde{\phi}^{(m)}\right) & \left.\tilde{\phi}^{(n)}+k^{2} \phi^{(n)} \tilde{\phi}^{(n)}\right] d y- \\
& =\int_{0}^{\infty}\left[\tilde{\phi}^{(m)} \mathcal{L}_{0.5 .}\left(\phi^{(n)}\right)-\phi^{(n)} \tilde{L}_{0.5}\left(\tilde{\phi}^{(m)}\right)\right] \equiv 0
\end{aligned}
\end{aligned}
$$


So, if $I^{(m, n)}=\int_{0}^{\infty}\left[\tilde{\phi}^{(m)} \phi^{(n)^{\prime}}+K^{*} \tilde{\phi}^{(m)} \phi^{(n)}\right] d y$.

then $\quad I^{[m, n]} \equiv \delta_{m n} I_{n}$

where $\quad I_{n} \equiv I^{(n, n)}$

Formally, $\hat{\boldsymbol{V}}$ may be expanded as a sum series in the eigenfunctions $\phi^{(\infty)}$, (neglecting contributions from a possible continuous part of the spectrum)

$$
\hat{v}=\sum \hat{A}_{n} \phi(n)
$$

Substituting this into (8.7), multiplying with $\widetilde{\phi}^{(m)}$ and applying the orthogonality relation $(8,10)$ we obtain

so that

$$
\int_{0}^{\infty} \mathcal{L}_{0.5}(\hat{v}) \tilde{\phi}^{(m)} d y=\frac{1}{i \alpha} \int_{0}^{\infty} \hat{q} \tilde{\phi}^{(m)} d y
$$

$$
\begin{aligned}
& \hat{A}_{n}=\left[i\left(\omega-\Omega^{(n)}\right) I_{n}\right]^{-1} \int_{0}^{0} q \Phi^{(n)} d y \\
& \Omega^{(n)}=\alpha \sigma(n)
\end{aligned}
$$

where

and thus,

$$
v=\Sigma \frac{\hat{G}_{n} \phi^{(w)}}{\omega \sim \Omega^{(n)}}, \hat{G}_{n}=\frac{1}{i I_{n}} \int^{\infty} \hat{q} \tilde{\phi}^{(n)} d y
$$

Hence, results for the wave number-frequency spectrum $\widetilde{E}_{v w}=\widetilde{E}_{\nu v}(\alpha, \beta, \omega ; y)$ can be found in terms of the spectrum for $q$. Since

$$
\tilde{E}_{v-v}=\frac{\overline{\hat{v}\left(\alpha_{j}^{\prime} \beta_{\mathrm{j}}^{\prime} \omega^{\prime}\right) \hat{\nu}^{*}\left(\alpha_{j} \beta ; \omega\right)}}{\delta\left(\alpha-\alpha^{\prime}\right) \delta\left(\alpha-\beta^{\prime}\right) \delta\left(\omega-\omega^{\prime}\right)}
$$

then,

$$
\tilde{E}_{v v}=\sum_{m} \sum_{n} \tilde{E}_{q q}^{(m)} /\left(\omega-\Omega^{(\omega)}\right)\left(\omega-\Omega^{(n)}\right)
$$

where

$$
\tilde{E}_{q q}^{(m, \nu)}=\overline{\hat{G}_{n} \phi^{(n)}\left(\hat{G}_{m} \phi^{(m)}\right)^{*}} / \delta\left(\alpha-\alpha^{\prime}\right) \delta\left(3-\beta^{\prime}\right) \delta\left(\omega-\omega^{\prime}\right)
$$

The equation (8.15) relates the spectrum of $\mathcal{V}$ to the forcing spectrum of $\$$. Included in the result is the effect of resonant forcing at $\omega=\Omega_{R}^{(n)}$ with damping $\Omega_{I}^{(n)}$, represented by the poles. But at large times other singularities, not explicitly included, such as branch points may be important. To obtain tractable result we suppose that the forcing excites the lowest mode most strongly, so that, approximately,

$$
\tilde{E}_{\nu v}=\tilde{E}_{q+}^{(0,0)} /\left|\omega-\Omega^{(\omega)}\right|^{-2}
$$

This may be inverted to give the frequency spectrum $S_{v v}$,

I.e.,

$$
\delta_{v v}=\int_{-\infty}^{\infty} \tilde{E}_{v v} e^{i(\alpha \xi+\beta \xi)} d \alpha d \beta
$$




$$
S_{V V}=\frac{1}{c_{I}^{(\infty)}} \int_{-\infty}^{\infty} e^{i\left(\alpha_{R}^{(\alpha)} f+\beta z\right)-\alpha_{I}^{(\infty)}(\xi)} \tilde{E}_{q q}\left(\alpha^{(\alpha)}, \beta\right) d \beta
$$

where $\alpha^{(d)}=\alpha_{R}^{(s)}+i \alpha_{I}^{(0)}$ is the spatial eigenvalue for the wave number corresponding to $\mathbf{n}^{\prime:}$

If we suppose that only the lowest mode is excited, we need not require too much detailed information about the mechanism of the forcing to obtain qualitative results. If the instantaneous Reynolds stresses are similar to shot noise, then the spectrum of $q$ will be substantially flat. However, q may itself be a result of resonance leading to singularity in its spectrum. Also, as the small-scale secondary-wave bursts dominate in the forcing term, they could make a significant direct contribution to the intensities and energy. But because of their noise character, their contri-

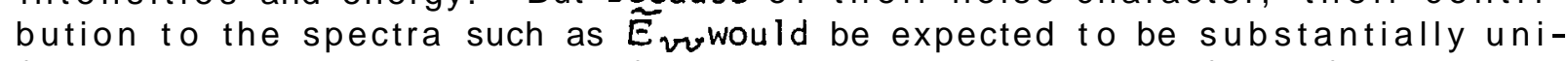
form over the wave number and frequency components. Therefore, for low and moderate frequencies and wave numbers, the structure of $\tilde{E}_{w}$ is likely to be dominated by the resonances of the primary wave motions, and there should thus be a peak of $\widetilde{E}_{v} v a t$ the resonance frequency $\omega=\Omega_{R}^{c \infty}$. From observed wave-number-frequency spectra one can deduce $\Omega_{R}$ from the location of the

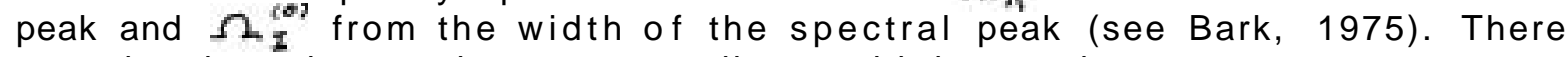
may also be other peaks corresponding to higher modes.

In deriving the result for $E_{v v}$, only propagating wave disturbances have been included. These are characterized by having a uniform phase velocity across the boundary layer. However, there may also be convected structures, such as eddies, which would be characterised by differential convection at various heights in the boundary layer. The role played by purely convected structures is here supposed to be small and therefore neglected in the spectra for $v$ and $p$, but the convected eddies may be important for the u-spectrum as will be seen later.

Calculations of pressure spectra based on the above assumptions for various Reynolds numbers, and for a mean velocity profile $U(y)$ fitted to experimental measurements were made by Landahl (1967). A plot of $C_{\mathbb{R}}$ against streamwise wave number, shows that there is only a small variation, so that dispersion is not strong for the wave modes. The eigenvalue hardly varies at all with the spanwise wave number $\beta$. A plot of $\left(c_{z} / \kappa_{R}\right)$ against $\alpha$ shows that there is a very small variation with $\alpha$, so that all scales are nearly equally damped. Wave modes will hence tend to propagate together with little dispersion and last about the same distance of travel in terms of their own wave lengths.

Details of the spectra may be compared with experiment. Willmarth and Wooldridge (1962) measured the cross-spectral density of the pressure fluctuations beneath a boundary layer. The results fitted the form

$$
S_{\rho p}(\xi, \zeta, \omega)=\Phi(\omega) A\left(\frac{\omega \xi}{v_{c}}\right) B\left(\frac{\omega s}{v_{c}}\right) \exp -\left(\frac{i \omega \xi}{v_{c}}\right)
$$

where $V_{\mathcal{L}}$ is convection velocity (= phase velocity_ and $\xi \varepsilon \xi$ are displacements in the $x, z$ directions, respectively. A plot of $U_{c} / U_{a}$ against $\omega \delta_{n} / U_{0}$ showed only a slow variation, so that the convection $V_{\text {etocity }}$ varies only weakly with frequency in qualitative agreement with the theory.

computed results showed a decrease of $U_{c}$ with a Reynolds number with values somewhat below the experimental ones. However, the decay rate as expressed 
by the function A showed excellent agreement between theory and experiment. (See also Wills, 1971.)

Bark (1975) extended the wave-guide model to the $u$-fluctuations in the viscous wall region. He used an exponential fit to the velocity profile, and the results for conditionally sampled uv-distributions during bursting obtained by Kim et al., (1971) to estimate $E_{q q}$. No information about $v w$ was available, though. The instantaneous values of $u v$ were assumed to be of the form,

$$
u v=\delta\left(t-t_{B}\right) \delta\left(x-x_{B}\right) \delta\left(z-z_{B}\right) G_{q} y^{3} \exp \left(-C_{2} y^{3}\right)
$$

for a burst occurring at $t=t_{B}, x=x_{B}$ and $z=z_{B}\left(G_{1}\right.$ and $C_{2}$ constants). This form of (uv) satisfies the conditions imposed by continuity at the boundary $y=0$. The bursting is taken to be a Poisson process both in time and in the homogeneous coordinates $x, z$. These assumptions will produce anomalous results at higher wave numbers and frequencies, but should be reasonable for lower values. Bark (1975) obtained good qualitative agreement with the experiments by Morrison, et al., (1971), particularly in estimating the wavenumber and frequencies at which the spectral peaks occurred. For example, his Fig. 3 shows close agreement for the peaks of $S_{u u}\left(\omega^{t}\right)$ and $S_{u u}\left(\beta^{+}\right)$. The agreement for $S_{u u}\left(\alpha^{+}\right)$was not as good, but the discrepancy could be due to the assumption that all bursts have the same vertical structure. The values of $c_{R}$ and $c_{I}$ for various $\alpha^{+}$were also computed for these data and agreed well with the observations. These values showed that the waves decayed on a time scale of their own period and that dispersion was weak, similar to the conclusions of Landahl (1967). Hence, a wave packet will evolve mainly through the differing decay rates of the different wave member components, and through viscous diffusion.

One of the most interesting features was the plot of $S_{\mu \omega}\left(\omega^{t}, \alpha^{+}\right)$ comparing the theory and experiment. The contours of values of $S_{u u}$ showed a similar shape, and in both the contours the maxima followed an almost straight line $\omega^{+} \sim \alpha^{+}$indicating a propagation speed which is close to but not equal to the local mean velocity.

Lu and Willmarth (1973) measured (conditionally sampled) space-time correlations of uv in the wall buffer region. They found a convection velocity of about $80 \%$ of the mean velocity, which is close to the phase velocity of the dominating wave mode at that distance from the wall. This may be interpreted either as the Reynolds stress disturbances being convected as a secondary wave motion by the primary disturbance, or that the Reynolds stress is produced by the primary waves themselves. It is the first of these that is most likely to apply.

\section{References}

1. Bark, F.H. 1975 J.Fluid Mech. 70: 229.

2. Kim, H.T., S.J.Kline and W.C.Reynolds 1971 J.Fluid Mech. 50: 133.

3. Kline, S.J., W.E.Reynolds, F.A.Schraub and P.W.Runstadler. 1967 J.Fluid Mech. 30: 741.

4. Landahl, M.T. 1967 J.Fluid Mech. 29: 441.

5. Landahl, M.T. 1972 J.Fluid Mech. 56: 775. 
6. Morrison, W.R.B., K.J.Bullock and R.E.Kronauer 1971 J.Fluid Mech. 47: 639.

7. Willmarth, W.W. and C.E.Wooldridge 1962 J.Fluid Mech. 14: 187.

8. Wills, J.A.B. 1970 J.Fluid Mech. 45: 65.

9. Lu, S.S. and W.W.Willmarth 1973 J.Fluid Mech. 60: 481.

Notes submitted by Martin R. Maxey

\section{Lecture \#9.}

\section{A TWO-SCALE MODE OF BOUNDARYLAYER TURBULENCE}

\subsection{Introduction}

In order to understand the mechanics of boundary layer turbulence, one must consider how the Reynolds stresses are maintained. In his mixinglength theory, Prandtl asserted that they arose when parcels of fluid moved across laminas of different velocities, taking their initial momentum with them for some random distance and then mixing. Although Prandtl did not attempt to relate this transfer of momentum to identifiable structures within the flow, Grant (1958) observed "mixing jets" in the water, whilst Kline et al.,* (1967) identified bursts consisting of slow fluid erupting from the sublayer. Both processes lead to strong mixing and hence the creation of turbulent stress. In particular, Kline, et al., (1967) identified a series of events in which a streak of slowly moving fluid detached from the wall, moved across the boundary layer, and then mixed with more rapidly moving fluid outside.

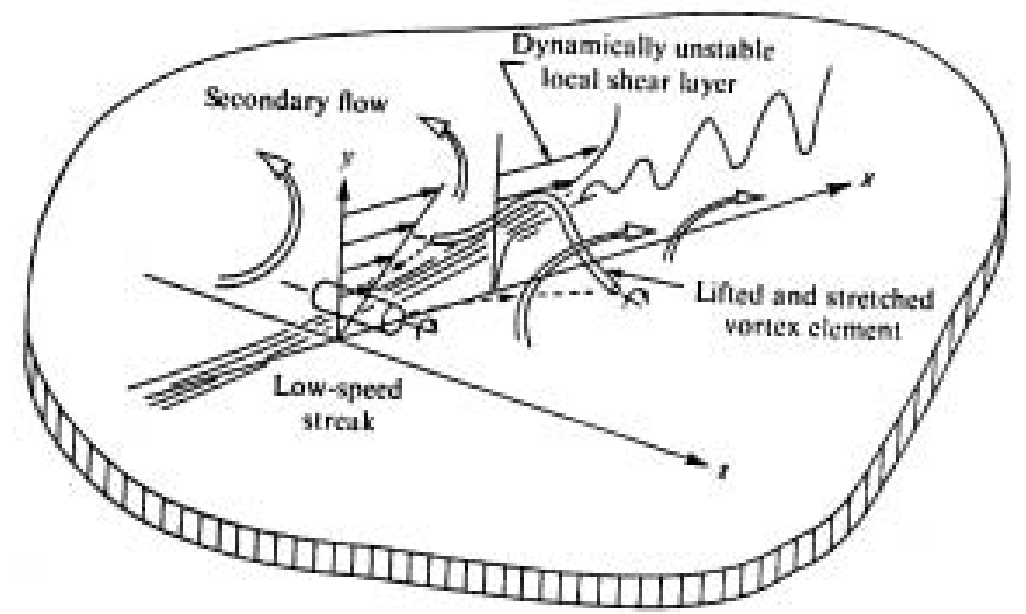

Fig.1. Structure of a Burst (from Kline et al., 1967).

Much earlier, Einstein and Li (1956) had observed the eruption of slow fluid from the sublayer, and they suggested an intermittent instability of the sublayer as its cause. 
Of course, the ejection of slowly moving fluid from the wall must for reasons of continuity also involve an injection ("sweep") of rapidly moving fluid from farther out in the boundary layer towards the wall. Using the methods of conditional sampling, Willmarth and Lu (1973) have shown that more than $100 \%$ of the mean Reynolds stress is contributed during the sweep and ejection cycles, with the (negative) remainder arising (e.g.) when slow particles move from the outer boundary layer towards the wall. The bursts are repetitive, and separated by a time characteristic of the large eddies.

That the production of turbulence is sporadic can be understood if it is noted that whenever the fluid is not well-mixed, as is compatible with the boundary conditions, there is energy in form of velocity differences available to drive mixing. The thorough mixing which results removes the energy supply for the small eddies, which consequently decay. The larger eddies will be less affected by viscosity and will last longer; during the period of decay, most of the mixing will be done by the large eddies which could thus be responsible for the bulk of the Reynolds stress during this time. As the small eddies decay, strong velocity contrasts (shear layers) can again build up eventually giving rise to new intense small-scale mixing in a manner to be described in the model below.

Both the observations and this crude argument suggest that boundary layer turbulence consists of intermittent gusts of small-scale motion which are separated by intervals of unsteady but basically laminar large-scale motion. This separation of scales is shown in idealized form in Fig.2.

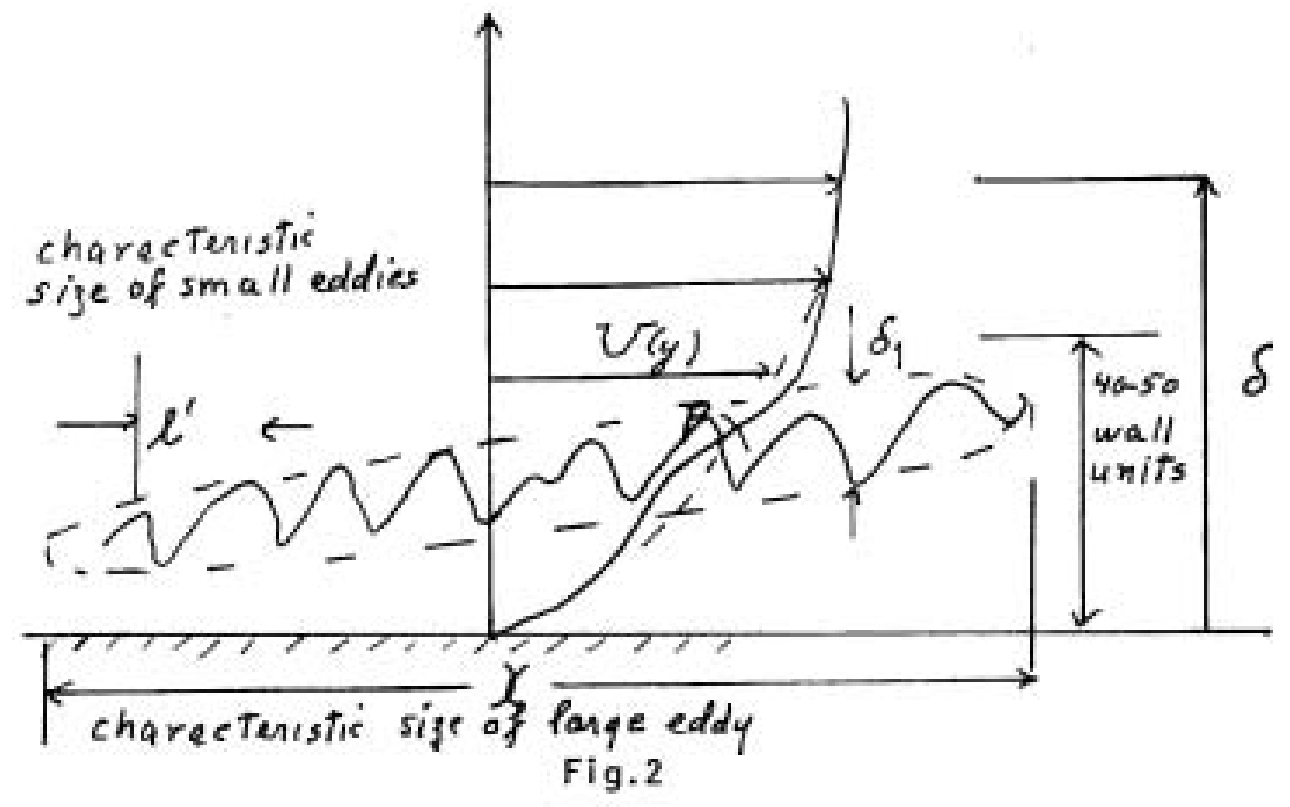

Conceptual Two-Scale Flow Model

Note - The point $P$ is a point of inflexion in the large-scale velocity field.

However, the nonlinear effects acting on the large scales will be assumed to be primarily due to the turbulent stresses produced by the small scales so that outside the burst, the flow is assumed to be governed by the equation for small perturbations in parallel shear flow.* The strong nonlinear

This ignores direct interaction of large eddies. 
effects within the burst are thought to arise when the large-scale motion breaks down through the mechanism of focusing and trapping of inflectional secondary instabilities discussed in the previous lecture. The proposed cycle which maintains the turbulent stresses is illustrated conceptually in Fig. 3.

large eddies

(primary)

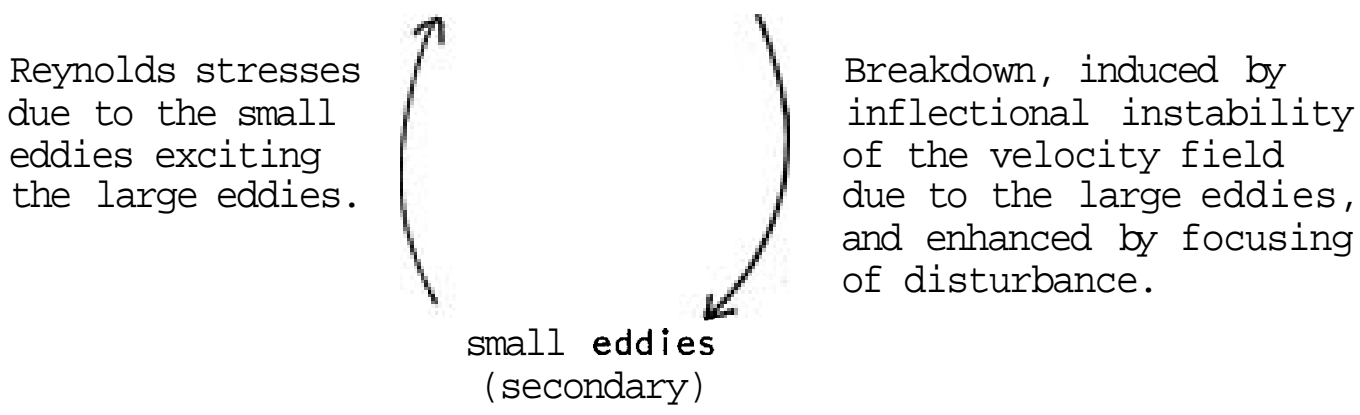

Fig.3. Conceptual Closure Model.

To verify this qualitative picture of the process by which the turbulence is maintained, the following questions must be answered: first, how strongly and in what fashion does the turbulent stress produced in the burst excite the boundary layer; secondly, what is the structure of the large-scale disturbances produced by the localized burst; thirdly, is one burst sufficient to produce the breakdown of the large-scale flow, or must several bursts interact to do so and, fourthly, how is the shape of the mean velocity profile related to the disturbance created by a burst? Whilst these questions require a full nonlinear analysis if they are to be answered completely, this lecture gives qualitative answers using the idealized two-scale model illustrated in Fig. 2.

\subsection{The two-scale model (Landahl 1976, 1977).}

In order to draw as much as possible from the theory for instability and transition developed in the earlier lectures we will assume that the mean of the boundary layer flow may be approximated by a parallel flow, i.e.

$$
U_{i}=v(y) \delta_{i}+u_{i}(\underline{x}, t)
$$

With this assumption, as shown in the previous lecture, the Navier-Stokes may be combined to give

$$
\left(\frac{\partial}{\partial t}+U \frac{\partial}{\partial x}\right) \nabla^{2} v-U^{\prime \prime}(y) v_{x}-\nu \nabla^{\prime \prime} v=q
$$

where

$$
\nabla^{2}=\frac{\partial^{2}}{\partial x_{i} \partial x_{i}}, \frac{D}{D t}=\frac{\partial}{\partial t}+U \frac{\partial}{\partial x}
$$

and where

$$
\begin{aligned}
q & =\frac{\partial}{\partial x_{j}}\left(\frac{\partial^{2} \tau_{i j}}{\partial x_{i} \partial x_{2}}-\frac{\partial^{2} \tau_{i j}}{\partial x_{k} \partial x_{k}}\right) \\
\tau_{i j} & =\overline{u_{i} u_{j}}-u_{i} u_{j} .
\end{aligned}
$$

If the quadratic terms are neglected, (9.1) reduces to the Orr-Sommer- 
feld equation which was employed in an earlier lecture to show that a shear layer can act to amplify selectively certain frequencies already present in the flow. Without additional assumptions, (9.1) is of limited usefulness since $q$ is a nonlinear function of all the fluctuating components of velocity. In the present context, (9.1) is useful if the nonlinear terms can be assumed to be strong only locally in space and time, for it can then be used to describe the flow outside such regions. For there, the equation admits large-scale, wave-like and convective solutions whose behavior is outlined below.

However, since the regions in which the nonlinear terms are important are convected with the flow, the assumption that the dynamics is linear outside these regions is not sufficient by itself. An additional assumption, that most of the convection is done by the largest eddies in the flow, is necessary. This assumption is put into the model formally by splitting the fluctuating velocity field into contributions from the-large and small eddies, viz. $u_{i}=\tilde{u}_{i}+u_{i}^{2}$ with associated length scales $l$ and $\ell$ ', respectively, where the condition $\left\langle\gg d^{\prime \prime}\right.$ implies that there are basically two radically different scales present in the flow. From (9.1), equations for $\tilde{u}_{i}$ and $u_{i}^{\prime}$ may be formed in the usual manner. They are

$$
\frac{D}{D t} \nabla^{2} \tilde{v}-v^{\prime \prime} \tilde{v}_{x}-\nu \nabla^{4} \tilde{v}=\left[\left(u^{\prime} v^{\prime}\right)_{x}+\left(v^{\prime} \omega^{\prime}\right)_{z}\right]_{y y}+O\left(l^{\prime} \hat{l}^{\prime}\right) \text {, }
$$

and

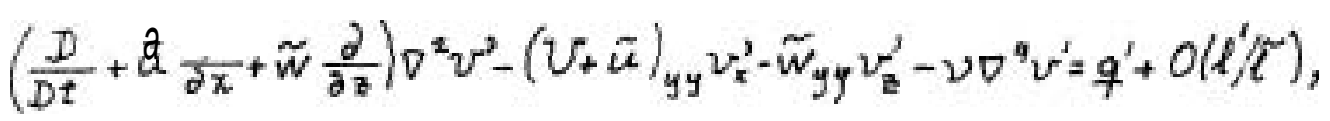

where

$$
q^{\prime}=\left(2 \frac{\partial^{\prime \prime}}{\partial y^{2}}-\nabla^{\prime}\right)\left[\left(u^{\prime} v^{\prime}\right)_{x}+\left(v^{\prime} w^{\prime}\right)_{z}\right]+\left[\left(u^{\prime 2}-v^{\prime 2}\right)_{x x}+\left(w^{\prime \prime}-v^{\prime 2}\right)_{z x}+2\left(u^{\prime} \omega^{\prime}\right)_{x z}\right]_{y},
$$

and where the tilde denotes averages over the large scales (defined in some convenient way, for example as phase averages over the small scales). As the earlier discussion suggested, the governing equation for the large scales is formally linear, with the turbulent stresses due to the small eddies in the localised shear layers driving the large eddies. In particular, note that any nonlinear interaction between the large eddies is excluded from the model. However, the evolution of the small eddies is governed by the nonlinear equation (9.3) in which the small eddies are also convected by the large ones. Because of the nonlinearity it is only possible here to discuss the solution of (9.3) for small times. However, the form of the equation will also be used to illuminate the mechanism of the instability which produces the small scale turbulence.

Thus, although nonlinearity has not been eliminated by the twoscale model, the strongest nonlinear effects have presumably been isolated so that their role in the dynamics may be understood.

\subsection{The small-scale field.}

For small times, the quadratic terms in (9.3) may be neglected. Moreover, when the viscosity is sufficiently small, its effects will be negligible until the small-scale structure is well developed. With these assumptions, (9.3) becomes 


$$
\left(\frac{\partial}{\partial t}+\tilde{V} \frac{\partial}{\partial x}+\widetilde{w} \frac{\partial}{\partial z}\right) \nabla^{1} v^{\prime}-\tilde{U}_{y y} v_{x}^{\prime}-\widetilde{w}_{y y} v_{z}^{\prime}=0 \text {, where } \tilde{U}=V+\tilde{u}
$$

If now the coordinates are totated so that the $x$ axis (Ox $\boldsymbol{x}_{*}$, say) is $10-$ cally parallel to the large-scale flow, this becomes

$$
\left(\frac{\partial}{\partial t}+\tilde{V}_{*} \frac{\partial}{\partial x_{*}}\right) \nabla_{*}^{2} v^{\prime}-\tilde{V}_{* y y} v_{x_{*}}^{\prime}=0
$$

Thus, the velocity field is unstable to the growth of small scale disturbances whenever the large-scale shear $\mathcal{U}_{* y}$ has a maximum within the flow. To determine strictly whether this instability could grow explosively, i.e. form a burst, requires a nonlinear stability analysis. However, Landahl (1975) has argued that the wave mechanism proposed for the breakdown of the laminar boundary layer (Landahl, 1972, discussed in Lecture \#7) can be extended to this case, i.e., a strong instability of the flow to smallscale turbulence occurs when the group velocity of small-scale instability wave equals the phase velocity of the large-scale disturbance in which it is imbedded. In that case, small-scale energy is focused on a given phase point in the large-scale structure, and a burst of strong small-scale disturbances would occur. Since this condition is likely to hold only locally in space and time, it seems that the explosive instability will be important only locally.

\subsection{The large-scale field}

Using the nonlinear equations discussed in the previous section to set up initial conditions for the calculation of the large-scale flow field one can show (Landahl 1977), that for vanishing $\nu$ there are two disturbance modes possible, the first a decaying wave mode. Since it has an associated pressure field, propagation of disturbances can occur across fluid elements. The second mode is a permanent eddy in $u$ and w without an associated normal velocity and pressure field and which is passively convected with the mean flow.

The short-term global effects of the localized activity can be understood most clearly by finding the angular momentum of the burst. For small times it can be assumed that both the convective and viscous terms and the pressure;? gradients in the $x$ and $z$ directions are small compared to the turbulent stresses produced by the burst. Then, large scale averaging of the Navier-Stokes equation gives

$$
\frac{\partial \vec{u}}{\partial t} \approx\left(\widetilde{u^{\prime} v^{\prime}}-\overline{u^{v}}\right)_{y} \approx\left(\widetilde{u^{\prime} v^{\prime}}\right)_{y}
$$

as

$$
\widetilde{u^{\prime} v^{\prime}} \gg \overline{u v^{\prime}},
$$

and

$$
\frac{\partial \tilde{w}}{\partial t} \simeq-(\widetilde{v})_{y}
$$

Thus,

$$
\begin{aligned}
& \tilde{u}(x, t)-\tilde{u}(x, 0)=-\tilde{f}_{y y}, \tilde{f_{1}} \equiv \int_{0}^{t}\left(\widetilde{u^{\prime} v^{\prime}}\right) d t, \\
& \tilde{w}(x, t)-\tilde{w}(\underline{x}, 0) \simeq-\tilde{f}_{\partial y^{\prime}}, \tilde{f}_{y} \equiv \int_{0}^{t}\left(\widetilde{v^{\prime} w^{\prime}}\right) d t,
\end{aligned}
$$

and since

$$
\tilde{v}_{y}=-\left(\tilde{u}_{x}+\tilde{w}_{z}\right) \text {, }
$$

"That it is consistent to neglect the pressure gradients must be verified afterwards. 


$$
\tilde{v}(\underline{x}, t)-\tilde{v}(x, 0)=\tilde{f}_{1 x}+\tilde{f}_{9 z}
$$

Note that $\tilde{f}_{7}=0=\tilde{f}_{3}$

outside the turbulent patch. The meaning of these results becomes clearer if their moments are taken over the volume of the turbulent patch;

and

$$
\begin{aligned}
& \int y\left(\tilde{u}-\tilde{u}_{0}\right) d^{3} \underline{x}=\int \tilde{f}_{1} d^{3} \underline{x}<0 \\
& \int x\left(\tilde{v}-\tilde{v}_{0}\right) d^{3} \underline{x}=-\int \tilde{f}_{1} d^{3} \underline{x}>0 \\
& \int z\left(\tilde{v}-\tilde{v}_{0}\right) d^{3} \underline{z}=-\int \tilde{f}_{2} d^{3} x,
\end{aligned}
$$

$$
\int y\left(\tilde{w}-\tilde{w}_{0}\right) d^{3} \underline{x}=\int \hat{f}_{0} d^{3} \underline{\sim} .
$$

Moreover, from (9.4) it follows that

$$
\int\left(\tilde{u}-\tilde{u}_{0}\right) d^{3} x=0
$$

and from (9.5) that

$$
\int\left(\tilde{v}-\tilde{v}_{0}\right) d^{3} \underline{x}_{x}=0
$$

Thus, the momentum before and after the burst is the same; the burst simply redistributes the initial momentum by adding an impulsive moment of momentum with components

$$
\begin{aligned}
& \tilde{M}_{(x)}=-2 \rho \int \tilde{f}_{3} \alpha^{3} \underline{x} \\
& \widehat{M}_{(y)}=0
\end{aligned}
$$

and

$$
\tilde{M}_{(z)}=-2 \rho \int \tilde{f}, d^{\prime} \underset{x}{ }<0
$$

This suggests a flow away from the wall in front of (i.e., downstream of) the burst and towards the wall behind it. Thus, when the effects of the small-scale turbulence are integrated over the burst, their net effect is to induce a large-scale flow rotation which is away from the wall at the downstream end of the eddy, and towards the wall at its upstream end.

(Fig.4). This is qualitatively compatible with the stages of ejection and sweep observed by Corino and Brodkey (1969).

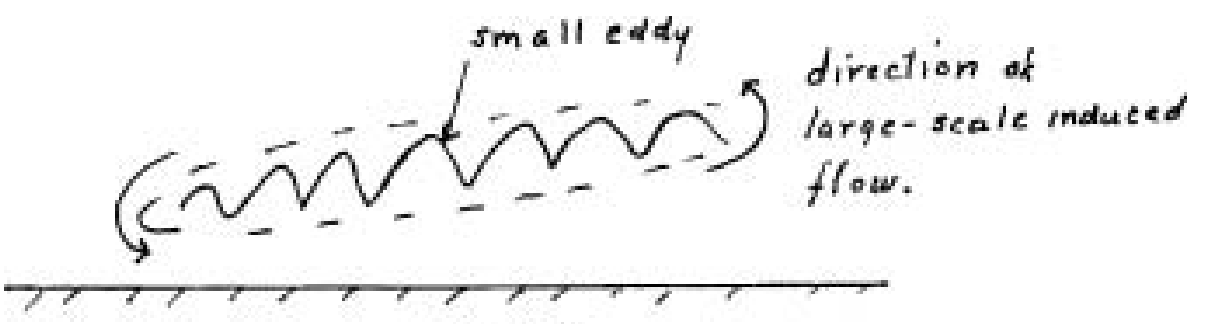

Fig. 4

The Large-Scale Flow Induced by a Burst

In the introduction it was suggested that, during the burst, most of the Reynolds stresses should be carried by the small eddies. That this is in fact the case can be verified by noting that the contribution to the 
Reynolds stresses from the small scales is (for small times) given by

$$
\left(\tilde{u}^{\prime} v^{\prime}\right)_{y}=-\frac{\tilde{v}_{y y}}{2 \alpha^{\prime}\left|\tilde{v}^{\prime}-c^{\prime}\right|^{2}}\left|\tilde{v}^{\prime}\right|^{2}
$$

where $\alpha^{\prime} \epsilon_{I}^{\prime}$ is the linear growth rate of the small-scale disturbance and $\alpha^{\prime}$ is its wavenumber. Also, the contribution of the larqe scales to the Reynolds stress is of order

where

$$
\tilde{u} \tilde{v} \sim\left(\tilde{\epsilon} U_{0}\right)^{2} \frac{\delta}{\chi_{1}}
$$

Thus

$$
\begin{aligned}
& \tilde{\epsilon}=\frac{\tilde{u}}{v_{0}} \sim\left(\frac{d_{1}}{\delta}\right)^{2} \\
& \tilde{u} \tilde{v}<<\left(\widetilde{u^{\prime} v^{\prime}}\right)
\end{aligned}
$$

\subsection{The convected eddy}

So far, it has been argued that inflectional instability of the large-scale velocity field can cause small-scale mixing which in turn induces a large-scale flow. This may be regarded as the second half of the cycle of turbulent bursting, and it remains to see how the low-speed streak, which begins the cycle, can arise. The basic idea is that the remnants of the previous burst are convected downstream whilst being simultaneously lengthened by the mean shear. If the remnants are such that they can later induce another inflectional instability the cycle will be self-sustaining.

The condition that the remnants of a burst be able to induce another inflectional instability is

$$
\frac{\partial^{2}}{\partial y^{2}}(u+v)=0
$$

Here $u$ is the contribution of the convected burst to the velocity field. The inviscid equation for $u$ is

$$
\frac{D u}{D t}=-\frac{1}{\rho} \frac{\partial p}{\partial x}-v U^{\prime} \equiv g, s \alpha y
$$

so that

$$
u=\int^{\tau} g(x(t), t) d t=\int_{0}^{t} g(x-U(t-\tau), \tau) d \tau
$$

Provided $v$ and $P$ vanish faster than $t^{-1}$, as $t \rightarrow \infty$

$$
u=\int_{0}^{\infty} g[\xi+V \tau, \tau] d \tau=u_{\infty}(\xi ; y j z) \text {, where } \xi=x-V(y) t
$$

and, using the equation of continuity with $v=0$, one finds

$$
w=w_{\infty}(\xi ; y ; z) \text { with } w_{\infty z}--u_{\infty \xi}
$$

Thus, the vorticity

$$
\begin{aligned}
& \gamma_{1}=w_{3}-v_{z}=w_{\infty y}-t V^{\prime}(y) w_{\infty \xi} \\
& \gamma_{2}=u_{z}-w_{x}=u_{\infty z}-w_{\infty \xi}
\end{aligned}
$$

and

$$
\gamma_{j}=v_{x}-u_{y}=-u_{\infty y}+t v^{\prime}(y) u_{\infty}
$$


Thus, in a frame moving with the mean flow ( $\xi=$ constant) the perturbation shear increases linearly in time, simply because the mean flow shears the eddy (Fig. 5).

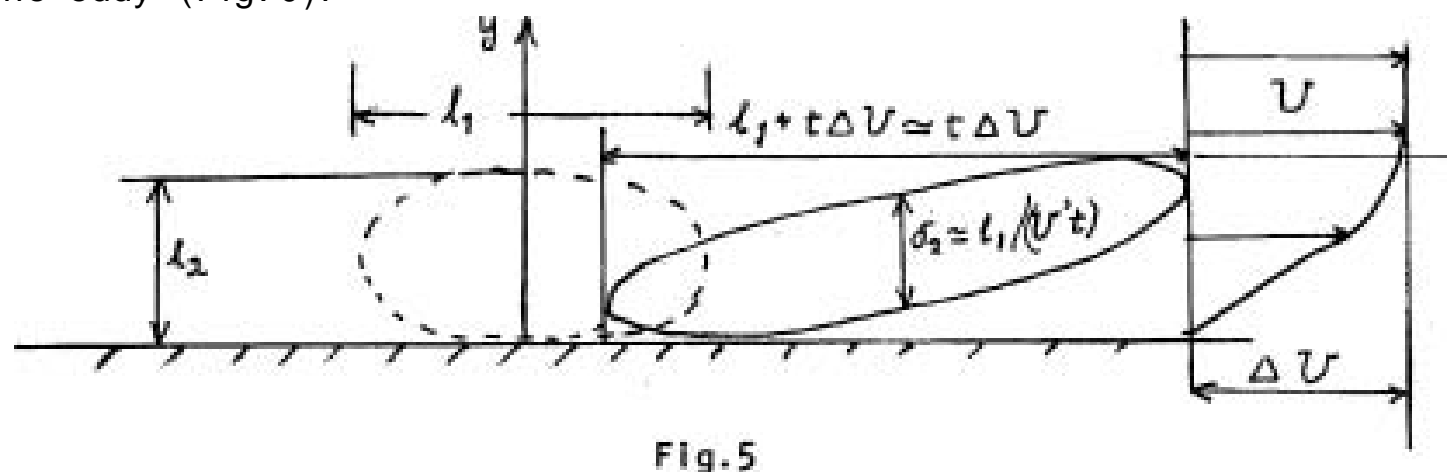

The Effect of Mean Shear on an Eddy

Shearing makes the eddy longer in the streamwise direction and narrower in the y-direction. Eventually, viscous effects enter to set a minimm thickness for the structure. The eddy lengthens at a rate given approximately by the velocity difference $\Delta U$ between its upper and lower boundary (Fig.5). Thus, after a long time $\mathbf{t}$ it is approximately

$$
l_{1}+\Delta v t=\Delta v t=l_{2} U^{\prime} t \text { long, }
$$

where $l_{2}$ is its y-direction.

The eddy will give rise to a shear layer with a thickness $\delta_{2}$ of approximately $\delta_{2} \simeq \ell_{1} / t \mathcal{V}$

Thus $\quad \frac{d \delta_{2}}{d t} \simeq-\frac{l_{1}}{v^{2} t^{2}}$

However, in time $\mathbf{t}$, the boundary of the eddy diffuses through at distance

$$
\begin{gathered}
D s(\nu t)^{1 / 2} \text { so that } \frac{d D}{d t} \approx\left(\frac{\nu}{t}\right)^{1 / 2} \text {. The equilibrium thickness occurs when } \\
\frac{d \delta_{2}}{d t}=-\frac{d D}{d t} \text {, i.e., for } t \equiv t_{\nu}=\left(\frac{l_{1}}{v^{3} \nu^{1 / 2}}\right)^{1 / 3}
\end{gathered}
$$

The equilibrium thickness of the shear layer is therefore

$$
\delta_{2 \min }=\left(\frac{\nu l_{1}}{V^{\prime}}\right)^{1 / 3}
$$

The viscous time $t_{v}$ can be compared to the lifetime $\tau_{\alpha} \sim \ell_{1} / \tau_{1}$ of the wavelike solution. One finds that near the wall the large wave-length eddies will survive longer than the convected eddies and so will dominate the statistics; away from the wall the opposite will be true.

Given this result for the minimum thickness of the convected shear layer, one can estimate the maximum initial streamwise length a shear layer can have if it is to be sustained by the process of inflectional instability. For, in order to get a local inflection point and the associated inflectional instability, the condition $\left(\partial^{2} / \partial y^{2}\right)(u+v)=0$ must hold, where $u$ is the contribution of the convected free shear layer to the large-scale field. This condition can only hold if

$$
u / \delta_{2}^{2} \sim\left|v^{n}\right|
$$




$$
\text { I.e, } \quad \delta_{2}^{2} \sim u /\left|U^{2}\right| \text { where } \delta=\ell_{1}^{2} / t\left(U^{\prime}\right)^{2}
$$

Solving for $t$ one finds that a time $t_{x}=\left(l_{1} / U^{\prime}\right)\left(U^{n /} / u\right)^{1 / 3}$ is taken for an inflection to be produced. Viscosity can prevent inflection only if viscous effects diffuse the shear layer before it can produce an inflection, i.e., $t_{v}<t_{1}$. Thus, eddies for which $\ell_{1}>\left|u / v^{n}\right|^{v} v \psi^{*}=\ell_{\max }$ cannot be sustained by the process of secondary instability. In particular, at $y^{+}=15$, the biggest eddies which could produce inflection instability can be estimated to have $f_{\max }^{*} \sim 100$ (assuming $u \sim u_{*}$ ). Moreover, any shear layer with a much smaller $l_{2}$ than this value would have such a small Reynolds number (based on the thickness of the free shear layer and $u_{*}$ ) that they could not be unstable, either. Thus, eddies of initial length of the order 100 wall units should be seen at $y^{+}=15$; this length is indeed fairly typical of low-speed streaks observed in the boundary layer.

\subsection{Conclusion}

When a layer of slow fluid detaches from the wall and moves toward the outer part of the boundary layer it meets with the more rapidly moving fluid there. Thereby, the slowly moving fluid induces an inflection point in the pre-existing (large scale) velocity field. According to the linear stability theory for parallel inviscid shear flows the flow is locally unstable. Moreover, when the group velocity of these secondary instability waves matches the phase velocity of the large scale (pre-existing) disturbance, energy could collect at some phase point on the large scale disturbance, and an explosive instability may result. The large-scale analysis (section 9.4) shows that the net effect is a flow away from the wall downstream of the burst, and towards the wall upstream of it. (Fig.4). This induced velocity field produces a large-scale flow, consisting of a decaying wave-like eddy and an eddy which is convected downstream while being sheared by the mean flow. Since only the fate of a single burst has been considered in the model, the interaction of eddies from adjacent bursts has not been treated. Moreover, the interaction between the slow fluid ejected from the sublayer and the rapidly moving fluid which replaces it has not been discussed.

In section (9.5) it was shown that only those bursts whose initial length is about 100 wall units will be self-sustaining, for only they will be able to replenish themselves by causing an inflection in the instantaneous velocity profile.

\section{References}

Corino, E.R. and R.S.Brookey 1969 J.Fluid Mech., 50: 133.

Grant, H.L. 1958 J.Fluid Mech., 41: 153.

Kline, S.J., W.C.Reynolds, F.A.Schraub and P.W.Runstadler 1967 J.Fluid Mech., 30: 741 .

Landahl, M.T. 1972 J.Fluid Mech., 56: 775.

Landahl, M.T. 1975 SIAM J.APPL.MATH., 28(4): 735.

Landahl, M.T. 1977 Phys.Fluids, 20: 555.

Willmarth, W.W. and S.S.Lu 1973 J.Fluid Mech., 60: 481. 
Grant, A. 1958 J.Fluid Mech. 4: 149.

Einstein, H.A. and H. Li 1956 Proc.Am.Soc.Civil Engrs. Paper No. 945.

Notes submitted by

Stepheh J. Morris

Lecture \#10.

10. THE DYNAMICS OF COHERENT STRUCTURES

Experiments in a turbulent wall layer show that there are localized instabilities. To emphasize this, consider the kinetic energy equation

$$
\frac{D\left(\bar{q}^{2} / 2\right)}{D t}=-\overline{u v} v^{\prime}-\varepsilon-\frac{a F_{i}}{\partial x_{i}}
$$

We will neglect the dissipation $\varepsilon$ for short times. We integrate between $t$, and $t_{2}$ over a volume surrounding the production area which makes the transport term drop out,

$$
\iiint_{(2)}\left(\frac{\bar{q}^{2}}{2}\right) d V-\iiint_{(1)}\left(\frac{\overline{q^{2}}}{2}\right) d V=-\iiint_{t_{1}^{2}}^{t_{2}} u v v^{2} \alpha V d t
$$

If there is production of Reynolds stresses, there will be an overall increase of kinetic energy of the disturbance; therefore production of Reynolds stresses and instability go together.

Suppose we have an event producing stresses in a localized region of large horizontal scale. What will be the fate of the large-scale structure?

Let us consider for that a large-scale structure in a boundary layer with the dimensions indicated in the figure below.

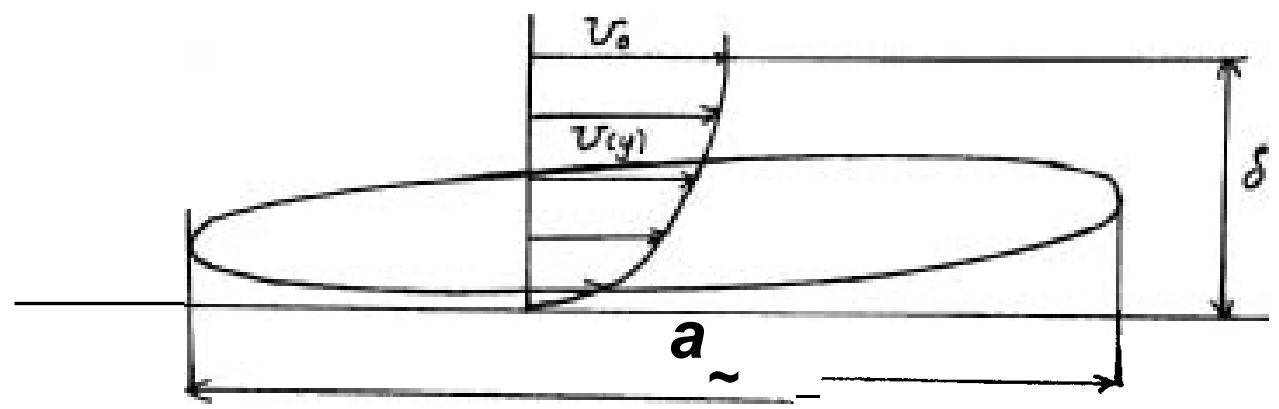

Fig.1 Large-scale Eddy

At $t=0$, we suppose that $u_{0}, v_{0}$ and $w_{0}$ are given. We assume

$$
\delta / l_{1}<l_{l} \text {, and } \delta / l_{j} \leqslant s \text {, }
$$

where $l_{3}$ is the spanwise scale.

We also take the flow to be inviscid. (Viscosity would play a role for region away from the wall only at large times.) The Euler equation of 
motion may be written,

where

$$
\begin{aligned}
& \frac{D(U+u)}{D t}=-\frac{1}{P}^{*} \cdot \partial x \\
& \frac{D W}{D t}=-\frac{1}{\rho} \frac{\partial p}{\partial z}
\end{aligned}
$$

$$
\frac{D}{D t}=\frac{\partial}{\partial t}+(U+u) \frac{\partial}{\partial x}+v \frac{\partial}{\partial y}+w \frac{\partial}{\partial z}
$$

In all of these equations we have left out the tildes $(\sim)$ introduced in the previous lecture to denote the large scales.

Because of the large horizontal scales, the pressure gradients will be small and could be neglected, at least for small times. In order to solve the equations, we introduce the material coordinates $\xi, \eta, \zeta$, (Lagrangian coordinates). The solution may then be written

$$
\begin{aligned}
v+u & =v(\eta)+u_{0}(\xi, \eta, \xi) \\
w & =w_{0}(\xi, \eta, \xi) \\
x & =\xi+\int_{0}^{t}(v+u) D t,=\xi+\left(V(\eta)+u_{0}\right) t, \\
z & =\zeta+w_{0} t
\end{aligned}
$$

The symbol $\int_{0} \approx D t$, denotes integration following the same fluid element. By property of the Euler-Lagrange transformation

$$
\alpha x d y d z=y d \xi \alpha \eta \alpha \zeta
$$

where $y$ is the Jacobian,

$$
y=\frac{\partial(x, y, z)}{\partial(\xi, \eta, \zeta)} .
$$

For an incompressible fluid

$$
y=1 \text {. }
$$

We substitute this into the equations above which gives

$$
A_{1}=\frac{\partial y}{\partial !}+A_{2} \frac{\partial y}{\partial \eta}+A_{3} \frac{\partial y}{\partial \zeta}=1 \text {, }
$$

where

$$
\begin{aligned}
& A_{1}=x_{\zeta} z_{\eta}-z_{\zeta} x_{\eta}, \\
& A_{2}=x_{\xi} z_{\zeta}-x_{\zeta} z_{\zeta}, \\
& A_{2}=x_{\eta} z_{\xi}-x_{\xi} z_{\eta} .
\end{aligned}
$$

This equation can be solved by the method of characteristics to yield

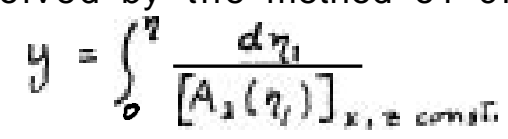

Substituting the expressions for $x$ and $z$ from the above we find

$$
A_{2}=\left(1+t w_{0 y}\right)\left(1+t u_{0 y}\right)-t^{2} u_{0 y} w_{0 \xi}
$$


$v$ is given by

$$
v=\frac{D_{y}}{D t}
$$

The pressure may be computed from the second Euler equation,

$$
\frac{D v}{D t}=-\frac{1}{P}-z_{y}^{D}
$$

It is also assumed that the disturbance is irrotational outside the boundary layer so that one can use potential-flow theory in that region. Thus,

where

$$
\frac{p}{\rho}=\frac{P_{1}}{\rho}+\int_{y}^{0} \frac{D v}{D t} d y_{1},
$$

$$
\frac{P_{\delta}}{\rho}=-\left(\frac{\partial}{\partial t}+V_{0} \frac{\partial}{\partial x}\right) \phi-\frac{1}{2}\left(\phi_{x}^{2}+\phi_{y}^{2}+\phi_{z}^{2}\right)
$$

and $v_{\delta}$ is the value of $\mathcal{V}$ at the edge of the boundary layer. The velocity Dotential $\phi$ obeys Laplace's equation and has the solution

$$
\phi_{\delta}=-\frac{1}{2 \pi} \iint \frac{v_{\delta}\left(x_{1}, z_{1}\right) d x_{1} d z_{1}}{\sqrt{\left(x-x_{1}\right)^{2}+\left(z-z_{1}\right)^{2}}} .
$$

We now iterate the solution with the full equation,

Thus

$$
\begin{aligned}
\frac{D(U+u)}{D t} & =-\frac{1}{\rho} \frac{\partial \rho}{\partial x}, \\
\frac{D W}{D t} & =-\frac{1}{\rho} \frac{\partial f}{\partial z} .
\end{aligned}
$$

which gives

$$
\begin{aligned}
U+u & =V(\eta)+u_{0}-\frac{1}{g} \int_{0}^{t} \frac{\partial p}{\partial \chi} D t_{t}, \\
w & =w_{0}-\frac{1}{p} \int_{0}^{t} \frac{\partial p}{\partial z} D t_{1},
\end{aligned}
$$

$$
\begin{aligned}
& x=f+\left(v(\eta)+u_{0}\right) t-\frac{1}{\rho} \int_{0}^{t}\left(t-t_{l}\right) \frac{\partial p}{\partial x} D t_{1}, \\
& z=3+w_{0} t-\frac{1}{\rho} \int_{0}^{t}\left(t-t_{1}\right) \frac{\partial p}{\partial z} D t_{1} .
\end{aligned}
$$

The linear approximation for $\mathbf{y}$ is found by neglecting quadratic terms in $(10.7), 10.8)$.

Thus,

$$
A_{2}^{-1} \simeq 1-\left(u_{0}+w_{0 y}\right) t=1+\nu_{0} t,
$$

which when substituted into (10.7) gives

$$
y=\eta+t \int_{0}^{\eta_{0}} v_{0,}\left(\xi_{1}, \eta_{1}\right) d \eta_{1},
$$

where

$$
\xi=x-v(\eta,) t .
$$

The linearized solution, corrected for the pressure, then becomes

$$
y-\eta \equiv l_{m}=t \int_{0}^{\eta} v_{0 \eta}\left(\xi, \eta_{1}\right) \alpha \eta_{1}+\frac{1}{\rho} \int_{0}^{\eta} d \eta_{1}\left[\int_{0}^{t}\left(t-t_{1}\right) \nabla_{H}^{2} p D t_{1}\right],
$$




$$
\text { where } \nabla_{H}^{2}=\frac{\partial^{2}}{\partial x^{2}}+\frac{\partial^{2}}{\partial z^{2}}
$$

The last term is the correction term due to the pressure. This expression gives a kind of "mixing length" and can be interpreted in that sense. We will see that, despite the factor of $t, l_{m}$ goes to a finite value as $t$ goes to infinity.

For the pressure,

$$
\frac{p}{\rho} \simeq \frac{P_{f}}{\rho}+\frac{1}{\rho} \int_{y}^{\delta} \frac{D_{v}}{D t} d y_{r}
$$

We apply the boundary-layer approximation and set

$$
\frac{\rho}{\rho} \approx \frac{\rho_{f}}{\rho}
$$

For $v_{\delta}$ we have

$$
v_{\delta}=\left(\frac{D \ell_{m}}{D t}\right)_{y=\delta}
$$

The next step is to introduce the Fourier transform of $v$,

$$
\hat{v}(y)=\frac{1}{(2 \pi)^{3}} \iiint e^{-i(\alpha x+\beta z-\omega t)} v(x, y, z, t) d x d z d t \text {. }
$$

then $\nabla_{*}^{2} p$ transforms to $-k^{2} \hat{p}$ where

$$
k^{2}=\alpha^{2}+\beta^{2} \text {. }
$$

We obtain for the pressure, using the result for potential flow

$$
\hat{P}_{\delta} \simeq \hat{p}=i \alpha \rho\left(v_{0}-\tau\right) \frac{\hat{v}_{\delta}}{k} \text { with } c=\omega / \alpha \text {. }
$$

Denoting the Fourier transform by $\mathbb{F}$, we get

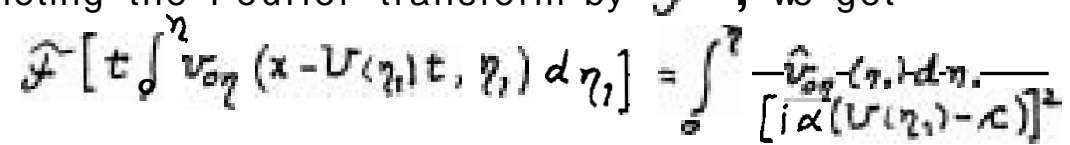

The other terms transform in a similar manner. This gives an equation for the pressure,

$$
\frac{\hat{p}}{\rho}\left[\frac{h}{i \alpha\left(v_{0}-\mu\right)}+i \alpha k^{2}\left(v_{0}-\dot{c}\right) \int_{0}^{\delta} \frac{d n_{1}}{\left[i \alpha\left(U_{1}-\tau\right)\right]^{2}}\right]=\hat{v}_{1 \delta}
$$

where

$$
v_{t \delta}=\frac{D}{D t}\left[t \int_{0}^{\delta} v_{\theta \eta}\left(\xi_{1}, \eta_{1}\right) d \eta_{1}\right] \text {. }
$$

Notes submitted by Jean-Pierre Poyet 
Lecture \#10 (second part).

Hence

$$
\frac{\hat{P}}{\rho}=\frac{i \alpha c\left(U_{0}-c\right) \hat{v}_{1 \delta}}{k\left[c+k c\left(v_{0}-c\right)^{2} \int_{0}^{\delta} \frac{\delta \eta_{1}}{\left(v_{1}-c\right)^{2}}\right]} \equiv \hat{G}_{w} \hat{v}_{1 \delta}
$$

The inversion to the physical plane gives after application of the convolution theorem

$$
P=\rho \iiint G_{w}\left(x-x_{1}, z-z_{1}, t-t_{1}\right) v_{1 \delta}\left(x_{1}, z_{1}, t_{1}\right) \alpha x_{1} d z_{1} d t_{1},
$$

where $G_{w}=\mathcal{F}^{-1}\left(\hat{G}_{w}\right)$. In, general, we may have to resort to generalized transforms; as we must have $\omega_{I}>0$ for the transforms to converge as $t \rightarrow \infty$ for the initial-value problem, we define transforms with paths of integration above the real axis in the complex $w$-plane. Now, the. integral in $\widehat{\hat{G}}_{W}$ may be evaluated to leading order in $c$ by expanding $v_{i}=U\left(\eta_{1}\right)$ about the point $\eta_{c}$ chosen such that $V_{\epsilon} \equiv U\left(\eta_{c}\right)=c_{R}$ "

$$
\int_{0}^{\delta}\left(V_{1}-c\right)^{-2} d \eta_{1} \simeq-\frac{1}{c v_{c}^{3}}+\frac{v_{c}^{\prime \prime}}{\left(v_{c}^{\prime}\right)^{3}}\left(\pi i+\ln c_{R}\right)+o(l) .
$$

From this approximation we obtain

$$
\hat{G}_{w}=\frac{i \alpha c V_{0}}{k\left(c-\epsilon_{0}\right)}
$$

where

$$
c_{0}=\frac{k v_{0}^{2}}{v_{c}^{\prime}}\left\{1+k \frac{v_{c}^{\prime \prime}}{\left(v_{c}^{3}\right)^{3}} v_{0}^{2}\left(\pi i-\ln \frac{k v_{0}^{2}}{V_{c}^{\prime}}\right)+h \cdot 0 \cdot t .\right\}
$$

This allows us to evaluate the $w$-integral in $G_{w}$ to leading order by closing the contour in the lower half plane, which for the second term leaves only the contribution from the pole at $c=c_{0}$.

Thus where

$$
G_{w}(x, z, t)=\frac{V_{0}}{2 \pi} \iint\left\{\frac{i \alpha}{k} \delta(t)+\frac{\alpha V_{t}}{V_{c}^{\prime}} \exp \left[-i\left(\frac{\alpha k V_{0}^{2}}{V_{c}^{\prime}}\right)(1+i t) t\right]\right\} e^{i\left(\alpha x+\beta z^{\prime} d x d \beta+h .0 . t .,(10.26)\right.}
$$

$$
\epsilon=\pi k v_{c}^{3} /\left(v_{c}^{3}\right)^{3}
$$

(This is valid strictly only asymptotically for $t, x$ and $z$ large.) From Gw we may construct the region of influence far downstream of a point source,

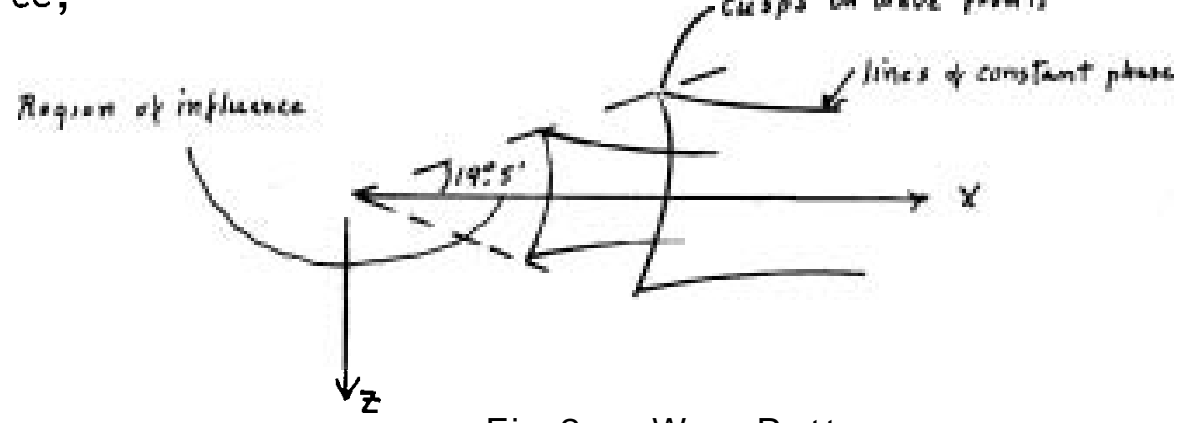

Fig.2. Wave Pattern

The spreading angle is found to be exactly the same as the Kelvin ship wave angle, $\sin ^{-1}\left(\frac{1}{3}\right)=190.5$ (probably by coincidence). 
When viscosity is included in the analysis the spreading angle is found to be approximately $10^{\circ}$. This value agrees well with the observational data for the spanwise growth of a turbulent transition spot. We expect the strongest disturbances to arise along the cusps since the disturbances decay more slowly in such regions. By introducing the expression for the pressure into the equations of motion one can demonstrate that its effect on the streamwise perturbation velocity for moderate times $\left(t<<\ell_{1}^{2} V_{c}^{\prime} / U_{0}^{2}\right)$ is small, of order $\delta / \ell_{l}$, compared to the contribution from $\ell_{m} U^{\prime}$. This will not be the case for the region very near the wall where $\ell_{m} \rightarrow \infty$. Over a longer period of time, of order $\ell_{1}^{2} U_{i}^{\prime} / V_{*}^{2}$, the pressures induced by the corrugations of the boundary layer edge will act to smooth these out, provided the boundary layer is stable. If $V_{c}^{\text {s" }}>0$ then $\boldsymbol{c}_{\varphi x}>0$, and the mean profile would be unstable. However, for a turbulent boundary layer mean profile $U^{\prime \prime}$ is always less than zero, so the disturbances are stable in the Rayleigh sense.

Ignoring the pressure effects, for a first approximation we consider the behavior at large times

$$
l_{m}=t \int_{0}^{\eta} v_{o \eta}\left(\xi_{1}, \eta_{1}\right) d \eta_{1}
$$

If we set $\xi_{1}=x-v_{1}\left(\eta_{1}\right) t$ and then consider this to define $\eta_{1}=\eta_{1}\left(\xi_{1}\right)$ and $v_{1}=v_{1}=(x-f) / t$ we have

$$
\ell_{m}=\int_{\zeta}^{x} \frac{v_{\sigma_{\eta}}\left(\xi_{1}, \eta_{1}\right) d \xi_{1}}{v_{1}^{\prime}\left(\xi_{1}\right)}
$$

For large $t, x$ will be large in the region where the disturbance is $f e l t$ $\left(x>>\ell_{1}\right)$, but $f=O\left(l_{j}\right)$ so that $v_{1}\left(f_{1}\right) \simeq x / t(x, t \rightarrow \infty)$ is independent of $\xi$ and

$$
l_{m}=\frac{1}{v_{1}^{\prime}} \int_{\xi}^{\infty} v_{o \xi}\left(\xi, \eta_{1}\right) d \xi_{1}=f(\xi, x / t) .
$$

The region of disturbance thus continuously elongates in the strearmise direction. Now

$$
u=u_{e}+V(\eta)-V(y) \simeq u_{0}-l_{m} V^{\prime}(\eta)
$$

Since $\ell_{m}$ is independent of the vertical coordinate for $t$ large (and $y$ greater than the value for which $\tau_{1}=x / t$; and $V^{\prime}(\eta)$ varies relatively slowly, $u^{\prime}=u-u_{0} \approx$ constant. Thus, we expect a marked vertical coherence above a thin shear layer of a thickness of order $\ell_{1} / U^{\prime} t$ (see Fig.3).

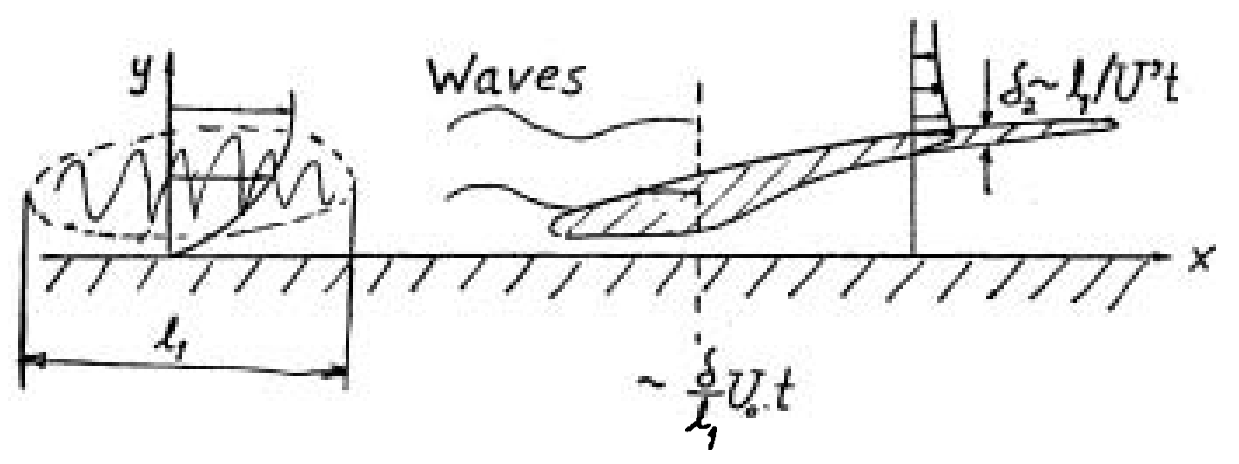

Fig.3. Nature of Solution. 
Numericil experiments were performed in which the initial velocity perturbation was

$$
v_{0} \sim \xi \eta^{2} \exp \left[-\left(\xi / l_{1}\right)^{2}-\left(\eta / l_{2}\right)^{2}\right]
$$

An exponential velocity profile approximating the mean velocity profile in the wall region of a turbulent boundary layer was used. An internal shear layer is recognizable by $t^{+}>10$. In a qualitative sense the results compare very favorably with the experimental observations of Blackwelder and Kaplan (1976).

$\underline{\text { Reference }}$

Blackwelder, R. and R.Kaplan 1976 J.Fluid Mech., 76: 89.

Notes submitted by Ross N. Hoffman 


\section{ABSTRACTS OF SEMINARS}

\section{EFFECTS OF DENSITY STRATIFICATION ON TURBULENCE}

\section{Laurence Armi}

Recent experiments have shown the importance of large scale coherent structures in most turbulent flows. The effects of stratification and density interfaces on these structures is believed to be the key to understanding stratified flow turbulence; hence much of the lecture was spent reviewing the experimental evidence for their existence.

Generally the picture one has had of turbulence is a state of chaos and disorder; but a picture is not developing of organized motion superimposed on a background of smaller scale motions. Examples of such organized motions have been known for some time, e.g., vortex shedding at high Reynolds numbers, but have always been claimed as special cases and not characteristic of "well-developed" turbulence. The simplest and most easily visualized examples of well-developed coherent structures in turbulence have been seen in recent studies of the developing free shear layer, c.f. Brown and Roshko (1974). These experiments show the development and growth of large vortex structures by pairing with adjacent vortices. The flow is wellpedigreed, i.e., it has a proper $-5 / 3$ spectrum (c.f. Champagne, Pao and Wygranski, 1976), yet the flow visualization clearly shows the existence of primarily only two scales. One sees a large outer scale and a sharp front like small scale or "braid" as described by Patnaik, Sherman and Corcos (1976) and Corcos and Sherman (1976). It is apparently the somewhat irregular modulation of these two scales which gives the developing free shear layer the appearance of having a broad spectral range of scales with a " $-5 / 3$ " spectrum. The effect of stratification on the pairing and subsequent growth by entrainment, is to suppress the process when the bulk Richardson number is greater than $0(1 / 4)$.

The importance of large scale structures in other turbulent flows is not as clear as for the mixing layer, particularly for turbulent boundary layers. From early observations it was thought that "bursting" was confined to a region near the wall, but it has now been shown by Rao, Narasimha and Narayanan (1971) and Laufer and Narayanan (1971) that the mean bursting period correlates with the overall boundary layer thickness 6 . The period is $\bar{T} \approx \delta / \mathrm{U}$. Measurements by Willmarth and Tu (1967) have shown the presence of large-scale structures convecting with a velocity $U_{c} w .8 U_{1}$. A picture is emerging of the bursting phenomenon connected with convection of characteristic 3 -dimensional structures on the scale of the boundary layer thickness. The effects of stratification on these structures is not wellunderstood. Early experiments by Townsend (1957) show a collapse of the turbulent motion when the Richardson number exceeds a limit of about 0.08. The collapse is likely due to the absence of entrainment by the large coherent structures of high momentum fluid to maintain the turbulent boundary layer.

Grid mixing experiments have been reviewed extensively by Turner (1973). There is considerable controbersy over some of these, in particular the difficulty of reconciling the difference in entrainment rates between the grid oscillation experiments of Turner (1973) and the surface stress driven experiments of Kato and Phillips (1969) and Kantha, Phillips 
and Azad (1977). We suggest that the difference can be reconciled if it is recognized that the Kato and $\mathrm{Phillips}$ style experiments are actually rotational experiments. The rotating grid introduces a smaller length scale, the turbulent Ekman layer thickness. The largest turbulent structures will scale with this smaller scale rather than the larger mixed layer depth of w $10 \mathrm{~cm}$ used by Kato and Phillips. We find with their experimental para-

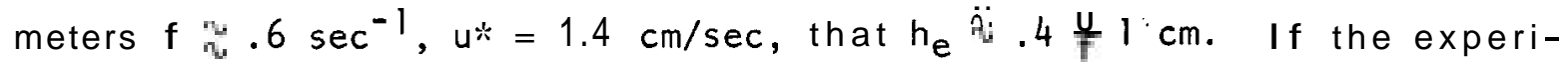
mental results are renormalized with this new shorter length they agree more closely with those of Turner (1973).

Selected References for effects of density stratification on turbulence and large scale coherent structures:

1. Developing free shear layer

2. Turbulent boundary layers

3. Stirred interfaces

4. Miscellaneous large structures

2. Arya, S.P.S., 1975 Buoyancy effects in a horizontal flat-plate boundary layer. J.Fluid Mech., 68(2):321-343.

2. Arya, S.P.S., 1972 The critical condition for the maintenance of turbulence in stratified flows. Quart.J.R.Met.Soc. 98: 264-273.

3. Baines, W.D., 1975 Entrainment by a plume or jet at a density interface. J.Fluid Mech., 68 (2) : 309-320.

1. Browand, F.K. and P.D.Weidman,1976. Large scales in the developing mixing layer. J.Fluid Mech.,L(1): 127-144.

1. Browand, F.K. and C.D.Winant, 1973. Laboratory observations of shear-layer instability in a stratified fluid, Boundary-layer Met. 5: 67-77.

1. Brown, G.L. and A. Roshko, 1974. On density effects and large structure in turbulent mixing layers. J.Fluid Mech. 64: 775-816.

2. Corcos, G.M., 1964. The structure of the turbulent pressure field in boundary-layer flows. J.Fluid Mech., 18: 353-378.

1. Corcos, G.M. and F.S.Sherman, 1976. Vorticity concentration and the dynamics of unstable free shear layers. J.Fluid Mech., 73: 241-264.

3. Crapper, P.F. and P.F.Linden, 1974. The structure of turbulent density interfaces. J.Fluid Mech., 65(1): 45-63.

1. Dimotakis, P.E. and G.L.Brown, 1976. The mixing layer at high Reynolds number: large-structure dynamics and entrainment. J.Fluid Mech. , $78(3)$ : 535-560.

4. Freymuth, P. 1966. On transition in a separated laminar boundary layer. J.Fluid Mech., 25(4): 683-704.

4. Grant, H.L., 1958. The large eddies of turbulent motion. J.Fluid Mech., 4: 149-190. 
3. Kantha, L.B., O.M.Phillips and R.S.Azad, 1977. On turbulent entrainment at a stable density interface. J.Fluid Mech., 79(4): 753-768.

3. Kato, H., and 0.M.Phillips, 1969. On the penetration of a turbulent layer into stratified fluid. J.Fluid Mech., 37(4): 643-655.

3. Keulegn, G., k949, Interfacial instability and mixing in stratified flows, U.S.Dept.Commerce, Nat.Bureau of Standards Res., J.of Research Paper RP 2040, 43: 487-500.

2. Kovasznay, L.S.G., V.Kibens, and R.F.Blackwelder, 1970. Large-scale motion in the intermittent region of a turbulent boundary layer. $\perp$ Fluid Mech., 41: 283-325.

4. Laufer, J., 1975. New trends in experimental turbulence research. Ann. Rev.of Fluid Mech., 7: 307-326.

2. Laufer, J., and M.A.B.Narayanan, 1971. Mean period of the turbulent production mechanism in a boundary layer. Phys.of Fluids, 14: 182-183.

1. Leipmann, H.W., and J.Laufer, 1947. Investigation of free turbulent mixing. TN 1257, NACA.

3. Linden, P.F. 1973. The interaction of a vortex ring with a sharp density interface: a model for turbulent entrainment. J.Fluid Mech., 60 (3): 467-480.

3. Linden, P.F. 1975. The deepening of a mixed layer on a stratified fluid. J.Fluid Mech., 71(2): 385-405.

3. Lofquist, K., 1960. Flow and stress near an interface between stratified liquids. The Physics of Fluids, $3(2)$ : 158-175.

1. Patnaik, P.C., 1976. A numerical simulation of Kelvin-Helmholtz waves of finite amplitude. J.Fluid Mech., 73: 215-240.

2. Rao, K.N., R.Narasimha, and M.A.B.Narayanan, 1971. The 'bursting' phenomenon in a turbulent boundary layer. J.Fluid Mech., 48(2): 339-352.

4. Roshko, A. 1976. Structure of turbulent shear flows: A new look, AlAA Journal, 14(10): 1349-1357.

2. Schubert, G. and G.M.Corcos 1967. The dynamics of turbulence near a wall according to a linear model. J.Fluid Mech., 22(1): 113-135.

1. Thorpe, S.A. 1971. Experiments on the instability of stratified shear flows: miscible fluids. J.Fluid Mech., 46(2): 299-319.

1. Thorpe, S.A. 1973. Experiments on instability and turbulence in a stratified shear flow. J.Fluid Mech., 61(4): 731-751.

2. Townsend, A.A., 1957. Turbulent flow in a stably stratified atmosphere. J.Fluid Mech., 3: 361-372.

3. Turner, J.S. 1973. Buoyancy Effects in Fluids, Cambridge Univ.Press.

2. Wilmarth, W.W. and B.J.Tu, 1967. Structure of turbulence in the boundary layer near the wall. Physics of Fluids (Suppl.), 10: 134-137.

1. Winant, C.D., and F.K.Browand, 1974. Vortex pairing: the mechanism of turbulent mixing-layer growth at moderate Reynolds number. J.Fluid Mech., $63(2): 237-255$. 


\title{
BOUNDARY MIXING AND LATERAL ADVECTION: THE DYNAMIC DUO RESPONSIBLE
}

\author{
FOR VERTICAL MIXING IN THE DEEP OCEAN
}

Laurence Armi

Vertical distributions of water mass properties in the deep ocean are often not inconsistent with simple models involving a constant upward vertical velocity of $w 1-4 \mathrm{~cm} /$ day and a constant vertical eddy diffusivity of $" v 1 \mathrm{~cm}^{2} / \mathrm{sec}$. A summary of these so-called "thermocline" models has been given by Veronis (1969). Recently vertical eddy diffusivities have also been calculated by Sarmiento et al., (1976), for the deep ocean using a onedimensional model which ignores horizontal transport, vertical advection and horizontal mixing relative to vertical mixing. One result of such a model is the establishment of an apparent relationship between vertical eddy diffusion and the inverse buoyancy gradient.

Vertical profiles of potential temperature, salinity, and light scattering were presented which suggest that the existence of an apparent vertical, or in the context which follows, cross isopycnal eddy diffusivity, is the result of essentially two processes: the largest contribution to the cross isopycnal mixing occurs within $\sim 50-150 \mathrm{~m}$ thick boundary layers. The layers are then advected into the interior of the basin along their respective isopycnal surfaces. The signature of these layers is found near to topographic features, whereas far in the interior of the basin the distinctive step structure is not as apparent. One is reminded of the arguments made by Iselin (1939), Montgomery (1938), and Rossby (1936) on the importance of the role of lateral turbulence and isentropic mixing for waters with surface characteristics. For the deep ocean we are suggesting that a similar combination of mechanisms is dominant. The combined effect of these two processes may be parametrically disguised as a vertical eddy diffusivity in one-dimensional models. A crude estimate shows that the two processes can account for all the vertical eddy diffusion apparent in onedimensional models. The actual mechanism is far from a simple one-dimensional process. It involves complicated dynamics both at the boundary and in the interior about which we have much to learn.

\section{References}

Iselin, C.O'D., The influence of vertical and lateral turbulence on the characteristics of the waters at mid-depths. Nat.Res.Council, Amer. Geophys. Un. , Trnas., 1939, 414-417.

Montgomery, R.B. 1938 Circulation in upper layers of southern North Atlantic. Pap.Phys.Oceanogr.Met., 6(2).

Rossby, C.-G. 1936. Dynamics of steady ocean currents in the light of experimental fluid mechanics. Pap.Phys.Oceanogr.Met., 5(1).

Sarmiento, J.L., and H.W.Feely, W.S.Moore,A.E.Bainbridge, W.S.Broecker. 1976. The relationship between vertical eddy diffusion and buoyancy gradient in the deep sea. Earth and Planetary Sci.Letters, 32: 357-370.

Veronis, G. 1969 On theoretical models of the thermocline circulation. Deep-sea Res., Suppl.to Vol.16: 301-323. 


\title{
SPIN UP OF A RAPIDLY ROTATING GAS
}

\author{
Fritz H. Bark
}

The linear spin up problem for a rapidly rotating gas is solved. The gas, which is assumed to be viscous, conductive and ideal, is contained in an axisymmetric vessel with flat top and bottom walls. The walls of the container are assumed to be perfectly conducting. By rapidIy rotating is meant that effects of the centrifugal force field strongly dominate over those of the gravitational acceleration. Furthermore, the rate of rotation is assumed to be too rapid for the Boussinesq approximation to be applicable.

Motions on the homogeneous spin up time scale is considered. It is shown that, on this time scale, a slight change of the rotation rate is equivalent to a small uniform cooling of the walls of the container. If the rotation rate is increased, and the response of the gas is looked upon on the homogeneous spin up time scale, the gas spins up to a steady state of non-uniform rotation, which is everywhere slower than the increased rotation rate of the container. This steady state slowly evolves toward the final rigid rotation on the diffusive time scale. On the diffusive time scale, the response of the gas to thermal or mechanical forcing ecomes different.

The boundary layers occurring on the homogeneous spin up time scale are on vertical boundaries, i.e., boundaries parallel to the axis of rotation, of a similar kind as for the spin up process of a homogeneous fluid. The swirl and radial velocities and the temperature field are adjusted by an unsteady Stewartson layer of thickness $E^{1 / 4}$, whereas the axial velocity is adjusted by a quasisteady Stewartson layer of thickness $E^{1 / 3}$. At the horizontal boundaries, the boundary layers resemble those occurring for the spin up of an axially stratified Boussinesq fluid, i.e., quasisteady Ekman layers and unsteady layers of thickness $E^{1 / 4}$. In contrast to the Boussinesq fluid case, though, the flow in the horizontal $E^{1 / 4}$ layers do affect the Ekman suction mechanism.

A perturbation solution for small peripheral Mach numbers and a numerical solution for finite peripheral Mach numbers is presented. It is shown that for an increased rotation rate of the container, the gas is cooled everywhere. For finite Mach numbers, it is found that the radial velocity near the centre of the container reverses, in spite of the fact gas is sucked into the Ekman layers over the whole top and bottom. 


\title{
TURBULENT INTERFACE LAYERS
}

\author{
Gabriel T. Csanady
}

Experimental data have been analyzed to determine the analogue of the law of the wall as it applies to turbulent flow along a sharp density interface. It was found possible to parameterize the waviness of such an interface in terms of an effective viscosity and a roughness length. The effective viscosity determines the interface velocity gradient and the velocity distribution in a viscous sublayer. Its value is the geometric mean of molecular viscosity and a wave-viscosity formed by wave amplitude and celerity. The properties of the effective viscosity change with the characteristics of the waves: at low speeds at the air-water interface, for example, capillarity plays an important role, because it creates a minimum in the wave celerity.

Interface roughness is affected significantly by interface stability, measured either by a Richardson number or a Keulegan parameter based on in ner variables. Higher stability reduces the roughness length. The physical reason appears to be turbulence energy loss through working against gravity.

Interface drag, shear and mass transfer coefficients may be determined by matching inner law velocity profiles to outer laws of conventional type. The parameterization scheme which results shows the drag coefficient to be a logarithmic function of a gravity to viscous force ratio and also to be affected by the Keulegan parameter based on bulk variables. Simple algebraic formulae connect shear and mass transfer coefficients to the drag coefficient.

Extrapolation of these results to turbulent Ekman layers along inclined atmospheric and oceanic fronts and just below the air-sea interface show low drag coefficients at fronts and a high wind factor at the free surface, referring to the skin velocity of the surface.

\section{THE RETURN TO ISOTROPY OF HOMOGENEOUS TURBULENCE}

\author{
John L. Lumley
}

The question of whether, and how fast, anisotropic (homogeneous, isothermal) turbulence without mean velocity gradients returns to isotropy is of fairly long standing. Rotta (1951) suggested an approximate form for the pressure gradient-velocity correlation which is responsible for the intercomponent energy interchange; this form being proportional to the energy deficit in the component, a single time-constant return to isotropy is predicted. Although there is no analytical proof that this is so, there is folk wisdom in the turbulence community that in the absence of external agencies, anisotropy should decrease to avoid a second-law violation. The first measurements of this were Uberoi (1956, 1957), followed by Milis and Corrsin (1959). These do indeed show a return to isotropy, although the downstream distance is painfully short, and the indications near the end of the ducts are ( $i f$ end effects may be excluded, which is not clear) that the return is weak. Admittedly, in Uberoi (1956) and Mills and Corrsin (1959) it was not the major purpose to investigate the return to isotropy following the contraction, but rather to examine the effect of the contraction itself. 
We intend to approach the question in a way reminiscent of that in which the school of rational mechanics approached the question of constitutive relations for non-Newtonian media. Eschewing detailed knowledge of the mechanisms responsible for the stress, rational mechanics attempted to determine the mathematical constraints to which constitutive relations were subject, and from these to delineate the possible forms of these relations. When a relation had been reduced to a small number of invariant functions, a combination of limiting cases and experiment could be used to determine these functions, determining once for all the behavior in a range of possible situations. Although we know more about the detailed mechanism of turbulence than we know about the statistical mechanics of complex media, it is evident that we do not yet know enough to make detailed predictions from first principles of such behavior as return to isotropy; there is therefore some justification for taking a similar approach to turbulence. We will finish with a rational construction which, although it may be termed semi-empirical, behaves like turbulence in a range of circumstances. The concept of viscosity, also, may be shown rationally to describe the behavior of certain media in a range of circumstances, and since the value of the viscosity in a particular medium must be determined from experiment, it may be said to be semi-empirical.

There is, in fact, considerable similarity between the return to isotropy problem, and the behavior of non-Newtonian media. In a turbulent flow at high Reynolds number, the only important stress is the Reynolds stress $-u_{i} u j$ In the experiments referred to, initially approximately isotropic turbulence is subjected to an axisymmetric contraction to produce the anisotropy, which is then allowed to decay. Measurements are taken in the principal axes of the Reynolds stress tensor. Hence, the equivalent problem in a non-Newtonian medium would be an axisymmetric distortion of material initially in a state of isotropic stress, producing unequal normal stresses, which are then allowed to decay to equality. It is, of course, dangerous to draw simplistic analogies; turbulence cannot be regarded as a non-Newtonian medium for a number of reasons. Chief among these are the fact that it does not satisfy the principles of material indifference or determine (Lumley, 1970), that there are regions near boundaries in time and space where local states are not unique functionals of flow variables (Lumley, 1970), and that the turbulent energy, which is responsible for the transport phenomena, is maintained by the external energy input. The latter makes the mechanics of turbulence evocative of (though in no way analogous to) the laminar mechanics of a gas at constant density and very low temperature in the sense that molecular momentum transport will be substantial only where the temperature is substantial due to shear or some other energy input. Nevertheless, in homogeneous turbulent flows many of the techniques and concepts of continuum mechanics can be applied to turbulence, with gratifying results.

\section{References}

Lumley, J.L. 1970 Toward a Turbulent Constitutive Relation, J.Fluid Mech. 41: $413-434$.

Mills, R.R. and S.Corrsin 1959 Effects of Contraction of Turbulence and Temperature Fluctuation Generated by a Warm Grid. NASA Memo 5-5-59W.

Rotta, J. 1951 Statistische Theorie Nichthomogener Turbulenz. ZPhy.129: 547-572. 
Uberol, M.S. 1956 Effect of Wind Tunnel Contraction on Free Stream Turbulence. J.Aeronaut.Sci., 23: 754-764.

Uberol, M.S. 1957 Equipartition of Energy and Local Isotropy in Turbulent Flows. J.Appl.Phys., 28: 1265-1170.

\section{TURBULENT VELOCITY PROFILES ROM STABILITY THEORY}

Willem V.R. Malkus

A velocity-defect law for channel flow is shown to result from a single requirement of Reynolds stress spectral "smoothness" for any mean profile maintained free of inflexions by transient instabilities. The deduced velocity is a logarithmic function of position near boundaries and parabolic in mid-regions of the flow, independent of the detailed mechanisms of momentum transport by the fluid. However, if the "smoothness" of the spectral tail is decreased, a second logarithmic layer of steeper slope emerges inside the first layer. Profile data from drag reduction experiments gathered by Virk (1975) exhibit the deduced inner log-layer and its transition region to the usual outer flow. The positive definite curvature of the mean profile is represented by $U^{\prime \prime} \approx I^{*} I$, where

$$
I=\sum_{0}^{\infty} I_{k} e^{i k \varphi}
$$

has the correct limits for general complex representation of $U^{\prime \prime}$. When

$\Delta I_{k}=O\left(I_{k} / k_{0}\right)$, the asymptotic structure of the spectrum with an exponent tail beyond $\&$, is

$$
I=I_{0} \frac{1}{1}+I_{R} \cdot\left(\frac{1-e^{-\gamma}}{1-e^{i \varphi-\gamma}}\right) \frac{\ell^{i\left(k_{0}+1\right) \varphi}}{l^{i \phi}-1}
$$

where $\gamma^{-1}$ measures the width of the tail. From the Virk data

$$
\left|I_{n_{0}}\right| /\left|I_{0}\right|=2.0 \text {, }
$$

and $\gamma$ controls the position of transition from one logarithmic region to the other.

\section{APPLICATION OF PHASE TRANSITION METHODS}

TO THE LARGE SCALE SPECTRAL PROPERTIES OF STIRRED FLUIDS

Paul C. Martin

In recent years, methods have been developed for studying the asymptotic and non-analytic behavior of the thermodynamic systems at large distances and long times. These methods, which go under the name of the renormalization group, have made it possible to deduce, for example, the singular dependence of the energy on temperature, and, at the transition temperature, the singular dependence of the correlations on distance. These singular properties result from the combined effects of a large number of degrees of freedom and can be derived by analyzing differential equations which tell 
how the properties of the system change with a small change in the number of degrees of freedom. By solving nonlinear equations with regular coefficients, one determines the nature of the singularities that appear as the number of degrees of freedom approaches infinity.

In the past year, these methods have been employed to investigate the properties of fluids, which satisfy the Navier-Stokes and similar dissipative equations, when they are subjected to random stirring forces. In this lecture an attempt has been made to describe those calculations performed by a group partly located at Harvard -- Forster, Nelson, and Stephen (PRL 36: 867 (1976) and an article to be published in Phys.Rev.A,Aug., 1977), by W.W.Loresey (J.Phys.C., Solid State Physics, 10: 1781 (1977)), and by the CNRS Turbulence group at the Observatoire de $\overline{N i c e}$ (J.D.Fournier and U.Frisch, abstract June, 1977, of a paper to be published).

What these papers especially the first two, consider is the general behavior at small wave numbers of the spectral function.

$$
\left\langle v_{i}(\underline{k} \omega) v_{j}\left(\underline{\kappa^{\prime}} \omega^{\prime}\right)\right\rangle \equiv(2 \pi)^{d+1} \delta\left(\underline{k}+\underline{k}^{\prime}\right) \delta\left(\omega+\omega^{\prime}\right) G_{i j}(\underline{k} \omega)
$$

of the Fourier components of the velocity, $v_{i}(\mathrm{r} t)$

$$
v_{i}(k \omega)=\int d t \int d r v_{i}(r t) l-i k r+i \omega t
$$

in an incompressible fluid $(\nabla \cdot v)=0$ satisfying the forced Navier-Stokes equation

$$
a_{t} \underline{v}+(\underline{v} \cdot \underline{\nabla}) \underline{v}=-\underline{\nabla} p+\nu_{0} \nabla^{2} \underline{z}+\underset{\sim}{f}
$$

in $d$-dimensions. As usual, $p$ is the pressure, $\nu_{0}$ the viscosity apart for the contribution that results from the nonlinear coupling term (i.e. the unrenormal ized viscosity), and $f$ is the random force.

The average is taken over a force which with Gaussian "white-noise" statistics specified entirely by the two-point correlation function

$$
\left\langle f_{i}(\underline{k} \omega) f_{j}\left(\underline{k}^{\prime} \omega^{\prime}\right)\right\rangle=(2 \pi)^{d+1} \delta\left(k+k^{\prime}\right) \delta\left(\omega+\omega^{\prime}\right)\left[\delta_{i j}-k_{i} k_{j} / k^{2}\right] 2 D(k)
$$

so that $D(k)$ characterizes the spectrum of the stirring force.

When $D(k)$ vanishes as $k^{2}$ or more rapidly as $k \longrightarrow 0$, the dominant behavior of $G_{\mathbf{j}}(k \omega)$ for small $k$ and $\omega$ is

$$
G(k \omega)=\operatorname{Tr} G_{i j}(k \omega) \simeq \frac{20 k^{2}}{\omega^{2}+\nu^{2} k^{4}}
$$

for $d>2$, but far more complicated for $d<2$, i.e.

$$
G(k \omega)=\omega^{-1} g\left(\omega / k^{2}\right)
$$

with $z=1+1 / 2 d$. The energy spectrum, $E(k)$, is proportional to

$$
E(k) \sim k^{d-1} \int \frac{d \omega}{2 \pi} G(k \omega) \sim k^{d-1}
$$

for all values of $d$ but for $d<2$, the corrections due to nonlinear modecoupling to the Navier-Stokes equation (which describes the short-range effects of molecular collisions) completely alter the hydrodynamic equation and dispersion relation. The manner in which these changes occur is the subject of an extensive literature which goes under the title of "longtime tails". It predicts some intriguing and unexpected results including a quite explicit correction, depending on the logariths of the wave number, to the viscosity in two dimensions. 
The results for $E(k)$ are also interesting even if they appear far from startling. The point is that $E(k)$ always has the same form at small enough wave numbers when $D(k)$ vanishes faster than $k^{2}$. It holds not only for a fluid in thermal equilibrium, but also for a quiescent fluid that is stirred randomly at large wave numbers. This prediction agrees with one made some time ago on other grounds by Saffman, and confirmed by numerical studies. It answers a question raised here last week by Malkus about how $E(k)$ varies with $k$ when $k$ is less than the'characteristic stirring wave number.

When the system is stirred more strongly at large distances, i.e., when $D(k)$ is a constant, $D_{O}$, the behavior of the system is more strongly modified at small wave numbers, and deviations from the spectrum and hydrodynamics occur whenever d 4. For such forcing, Forster, Nelson, and Stephen show that

$$
\begin{array}{ll}
E(k)=k^{d-3} & d>4 \\
E(k)-k^{2 d / 3} 5 / 3 & d \leq 4
\end{array}
$$

It is tempting to continue these arguments by considering a stirring force $D(k)-D_{0}{ }^{a}$

where a is negative.

Indeed, if one employs one of the more successful Markovian approximation techniques -- one which goes by the name of the Eddy Damped Quasi-Normal Markovian (EDQNM) approximation -- which gives

$E(k)-k^{2 / 3 d}-5 / 3$

for $a=0$, and $d \leq 4$, one finds that for a force spectrum

$D(k) \quad D_{0} k^{a}$

and $d$ sufficiently less than a number that increases as a decreases.

$E(k) \quad k-5 / 3+2 / 3(d+a)$

When $a=-d$, which is perhaps deceptively suggestive of a stirring force of the form $D(k)-\delta(k)$, one finds an energy spectrum $E(k)$

$E(k) \quad k-5 ' 3$

for all d. In contrast to the results for $a=0$, and $a=2$, which has been derived by renormalization group (RNG) techniques and can be applied to the large scale behavior of inverse "cascades", the result for $d=-\alpha$ obtained with the EDQNM has no RNG foundation. Whether the fact that it yields the Kolmogorov spectrum for all $d$ has any meaning at all remains to be seen. In general, we would expect that in problems of strong turbulence of the type discussed by Kolmogorov, the starting point is not appropriate. It seems less reasonable to go from the dissipative regime to long wavelengths or small $k$ than to begin by eliminating the effects of large eddies, and to derive the inertial Kolmogorov spectrum as a short distance or large wave number approximation. This is the picture upon which recent work of Nelkin, Bell, Siggia, and others is based.

For the moment, it is not clear that their studies using the renormalization group and interpolation methods based on it will succeed, let alone explain interrnittency. While the long wavelength results described in this lecture do not brar on this question, I think they are interesting and deserve attention. 
Gerstner's solution for finite amplitude rotational waves can be extended to treat edge waves in a rotating fluid. The wave field can be terminated by interfaces above and below, separating the wave field from a fluid moving at phase velocity in uniform motion. The addition of a constant velocity to particles in the wave field is included as a nontrivial modification in a rotating system, although the same modification in nonrotating coordinates is trivial.

\section{WAVE PACKETS \\ Erik Mollo-Christensen}

Discussion of conclusions that one can draw from recent work on theory of nonlinear waves, such as modulational instability, group formation, formations of groups of permanent envelope (group solitons), ability of groups to survive collisions. From this, one may conclude that a wave field, initially consisting of independently propagating Fourier components, will tend to concentrate an increasing fraction of the total energy in group solitons. These will survive collisions, and are basically through with nonlinear interactions that modify their structure. They should therefore be expected to dominate the statistical measures of a wave field, such as spectra, joint covariances and their Fourier transforms, the cross-spectra.

With Ramamonjiarisoa, I have constructed a model random wave field consisting of identical group solitons, and examined its statistical measures. The group solitons consist of Stokes waves, that is waves of permanent form, modified by an envelope function (Gaussian) that moves at group velocity. Stokes waves have the characteristic that all Fourier components propagate at the same phase velocity, that of the fundamental. The groups have random phase and random envelope location at time $\mathbf{t}=0$. The groups are therefore uncorrelated, and the second order statistical measures can be found by calculating them for one group and multiplying with the group population density. The resulting spectra are compared to experimental data, and show strong similarities to the celerities observed by Ramamonjiarisoa, as well as spectra observed in the ocean. The presence of internal wave group solitons is suggested as a possible explanation for the difference pointed out by Garrett and Munk between towed and buoy spectra. The representation of random fields in terms of "particles" such as group solltons, is suggested as a possible alternative to representation in terms of independently propagating Fourier components.

\section{References}

Bargsr, W.R., W.D.Garrett, E.Mollo-Christensen and K.R.Ruggles 1970 Effects of an artificlal sea sllck upon the atmosphere and the ocean. J.Appl. Meteor., 2: 396-400.

Benjamin, T.B. 1967 Instablllty of wave trains In deep water. Proc.Roy. Soc. A. $\underline{299 .}$ 
Bhartendu 1970 Comments on paper by C.R.Holmes, M.Brook, P.Krehbiel and R. McCrory, "On the power spectrum and mechanisms of thunder". J.Geophys.Res. , 76: 7441-7442.

Blanc-Lapierre, A. and R.Fortet 1953 Theorie des Fonctions Aléatoires. Masson et Compagnie, Editeurs.

Chiao, R.Y1, E.Garmire and C.H.Townes 1964 Self-trapping in optical beams. Phys. Rev. Lett. , 13: 479-482.

Chu, V.H. and C.C.Mei 1971 The nonlinear evolution of Stokes waves in deep water. J.Fluid Mech., 47: 337-351.

Courant, R. and D.Hilbert 1931 Methoden der Mathematischen Physik. (p.77) Springer, Berlin, $469 \mathrm{pp}$.

Garrett, C. and W.Munk 1972 Space-time scales of internal waves. Geophys. Fluid Dynamics, 2: 225-264.

Halpern, D., J.M.Helseth, J.R.Holbrock and P.M.Reynolds 1975 Surface wave measurements made near the Oregon Coast during August 1972 and July and August 1973. NOAA Tech. Rept. ERL 324-PMEL 22.

Haus, H.A. 1966 Higher order trapped light beam solutions. Appl.Phys.Lett., 8: $128-129$.

Houghton, D.D. 1964 An example of interaction between finite amplitude gravity waves. J.Atmos.Sci., 21: 493-499.

Landahl, M.T. 1967 A wave guide model for turbulent shear flow. J.Fluid Mech., 29: 441-459.

Leibovich, S. and A.R.Seebass 1974 Nonlinear Waves. Cornell U.Press, Ithaca and London, $331 \mathrm{pp}$.

Lighthill, J.M. 1967 Symposium on nonlinear waves. Proc.Roy.Soc. A. 299.

Medwin, H., C.S.Clay, J.M.Berkson and D.L.Jaggard 1970 Traveling Correlation Function of the Heights of Wind-blown Water Waves. J.Geophys.Res., 76: $5819-5828$.

Millard, R.C. 1971 Wind measurements from buoys. A sampling scheme. J.Geophys.Res. , 76: 5819-5828.

Ramamonjiarisoa, A. 1974 These de Doctorat d'Etat, Universite de Provence, Enregistre au C.N.R.S., No. A.O. 10.023.

and M.Coantic 1976 Loi experimentale de dispersion des vagues produites par le vent sur une faible longeur d'action. Comptes Rendus Acad.Sci., Paris, t.282: B-111-114.

-.-..-.Giovanangeli: Observations de la vitesse de propagation des vagues au large. To be published in Comptes Rendus Sci. Paris.

Ruggles, K.W. 1969 Observation of the wind field in the first ten meters above the ocean. Ph.D.Thesis, Dept.Meteorology, M.I.T.

Stokes, G.G. 1847 On the theory of oscillatory waves. Camb.Trans., 8: 441-473 (or Papers, 1: 197-229. 
Thorpe, S.A. 1968 On the shape of progressive internal waves. Phil. Trans., A, 263: 563-614.

Turner, J.S. 1973 Buoyancy Effects in Fluids. Cambridge Univ.Press, 367 - p.

von Zweck, 0. 1969 Observations of propagation characteristics of a wind-driven sea. Ph. D.Thesis, Dept.Met., M. I.T. (Rep.No.69-4).

Whitham, G.B. 1965 Nonlinear dispersion of water waves. J.Fluid Mech., 27: $399-412$.

1974 Linear and Nonlinear Waves. John Wiley and Sons, New York, $636 \mathrm{p} \overline{\mathrm{p}}$.

Yefimov, V.V.Y.U.P. Solov'yev and G.N.Kristoforov 1972 Observational determination of the phase velocities of spectral componsnts of wind waves. Isvestya, Acad. of Sci., U.S.S. R., Atmos. and Ocean. Phys., 8(4).

Yuen, H.C. and B.M.Lake 1975 Nonlinear deep water waves: theory and experiment. Phys.of Fluids, 18: 956-960.

\section{ON MODELLING THE SUPERLAYER}

\section{Stephen J. Morris}

In general, free turbulent flows at high Reynolds numbers have a sharply defined bounding surface, or superlayer, across which the vorticity falls to zero. Kelvin's circulation theorem for a homogeneous fluid implies that fluid initially outside the bounding surface can become vortical only by viscous diffusion. It is therefore natural to ask how quickly the bounding surface propagates through the fluid. The front is unlike a shock front in that both its propagation and non-zero thickness are due to the same molecular effects. Thus, it is to be expected that any model which gives the propagation speed correctly will also give a description of the internal structure of the front and, in particular, an estimate for its thickness. Moreover, the width of turbulence grows in the downstream direction at a rate which is at most weakly dependent on viscosity (e.g. Monin and Yaglom, Vol.l, equations 5.66 and 5.61). It follows that the total surface area across which the slow process of diffusion occurs must adjust itself accordingly. Similarly, if ener dissipation in homogeneous turbulence occurs primarity in vortex sheets' (say) of thickness $\lambda_{R}$ and with an associated velocity difference $\boldsymbol{V}_{k}$, the volumetric rate of energy dissipation can be independent of viscosity if the surface area of the vortex sheets increases as $v$ decreases. In both cases the surface area of a random surface is involved and it seemed to me that it would be useful to have a simple model of the bounding surface which describes how it propagates and how the adjustment of the surface area occurs.

When the turbulence is marked by a passive contaminant (heat say), the bounding surface of the turbulent velocity field and that of the con= centration field (thermal superlayer) appear to coincide well (e.g. Fiedler

(2) The actual structure of the dissipating regions is more complicated; see Kuo and Corrsin (1972). 
and Head, 1966). Since the governing equation for the passive temperature field is linear yet retains the basic effects of convection and molecular diffusion, it is better to study the structure of the thermal superlayer rather than the vorticity superlayer. Moreover the basic mechanism of propagation is most easily understood for a one-dimensional temperature field. Also, as the superlayer is a region in which convective and diffusive effects balance we wxpect that the solution of the temperature equation will be independent of the details of the initial conditions. Thus we seek a solution to the initial value oroblem

$$
\begin{aligned}
\frac{\partial \theta}{\partial t}+v(y ; t) \frac{\partial \theta}{\partial y}=x \frac{\partial^{2} \theta}{\partial y^{2}}, \quad \theta(y ; 0) & =1, y<Y_{0} \\
& =0, y>Y_{0} .
\end{aligned}
$$

Here $v(y ; t)$ is the known velocity field and $x$ the constant diffusivity. Now it is clear that for small times, the initially discontinuous temperature field will be smoothed by diffusion. Simultaneously it will be convected by the velocity field but because the front is initially very thin compared to the Kolmogorov microscale $\left(\lambda_{k}\right), v(y ; t)$ will be essentially constant within the front and equal to the velocity of the fluid particle with which the discontinuity initially coincides. As time passes, the front thickens by diffusion and successively higher order terms in the Taylor expansion of $v(y ; t)$ about that fluid'particle become important. In particular, if the (Eulerian) velocity gradient at the location of that fluid particle is negative and $\nu / x \gg 1$, only the first three terms in the Taylor expansion need be included. Now let $Y(t)$ be a function of time, shortly to be prescribed; let $\eta=y-Y(t)$ and $k(\eta ; t)=\theta(y ; t)$. Then, the dimensionless equation for $k(\eta: t)$ is

$$
\begin{aligned}
& \left(\frac{\partial k}{\partial \bar{t}}\right)_{\bar{\eta}}+\left\{\frac{v(Y(t) ; t)-\dot{Y}(t)}{\alpha \sqrt{x}}-\bar{\eta} f(t)+\varepsilon \dot{\eta}^{2} g(t)+O_{\zeta}\left(\varepsilon^{2}\right)\right\} \frac{\partial k}{\partial \eta^{2}}=\frac{\partial^{2} k}{\partial \tilde{\eta}^{2}} \text {. Here, } \alpha^{2}=\frac{v_{k}}{\lambda_{k}}, \\
& \left(\frac{\partial v}{\partial y}\right)_{y=Y(t)}=-\frac{v_{k}}{\lambda_{k}} f(t),\left(\frac{\partial^{2} v}{\partial y^{2}}\right)_{y=Y(t)}=\frac{2 V_{k}}{\lambda_{k^{2}}} g(t), \eta=\frac{\sqrt{x}}{\alpha} \bar{\eta}, t=\bar{t} / \alpha^{2} \text { and } \varepsilon=\left(\frac{\gamma}{v}\right)^{1 / 2} \text {. }
\end{aligned}
$$

Also, $f(t)$ and $g(t)$ are prescribed functions which are assumed for the time being to be of order one; $f(t)$ is also assumed positive for the moment. Letting $k=k^{(0)}+\varepsilon k^{(1)}+O\left(\varepsilon^{2}\right)$,

$$
\underline{v(Y(t) ; t)-\dot{Y}(t)}=-\varepsilon \dot{Y}_{1}(t)+O\left(\varepsilon^{2}\right) \text {, and choosing } \dot{Y}_{1}(t) \text { so that the per- }
$$

turbation expansion for $k(\eta ; t)$ is uniformly valid in time gives

$$
k(\eta ; t)=\frac{1}{2}\left(1-\operatorname{erf} \frac{\pi}{\tau}\right)+\frac{\varepsilon_{a}}{V_{\pi}} \bar{\eta}^{2} e^{-\xi^{2} / \tau^{2}}+O\left(\varepsilon^{2}\right) \text {, }
$$

and

$$
\frac{Y(t)-v(Y(t) ; t}{\alpha \sqrt{x}}=2 \varepsilon\left(a_{2} \tau\right)+O\left(\varepsilon^{*}\right) \text {. Here } a_{1} \tau \text { is a bounded function of time }
$$

which satisfies a certain ordinary differential equation, whilst the thickness $(\tau)$ of the front satisfies another.

Thus in a frame of reference which propagates through the fluid at a speed $2 \varepsilon\left(a_{2} \tau\right) \propto \sqrt{x} \simeq \varepsilon^{2} v_{k}$, the temperature profile is a slightly distorted 
error function. Physically, when $g(t)$ is positive, the velocity gradient is slightly more negative when $\eta<0$ than it is when $\eta>0$. Thus $\frac{d k}{d \eta}$ is also more negative for $\eta<0$ than for $\eta 70$ and because the heat flux is proportional to $-\frac{\partial k}{\partial \eta}$, the thermal superlayer propagates. The propagation speed is a factor of $\varepsilon^{2}$ smaller than the Kolmogorov speed $\left(v_{k}\right.$ ).

When this calculation is repeated in three dimensions the same mechinism induces a propagation velocity of order $\varepsilon^{2} v_{k}$. In addition the surface can also propagate by virtue of its curvature; it is this second term which controls the amount of surface area across which diffusion can occur.

However, Peclet number similarity is not possible using such a simple model. To see this, consider an element of area on the vortical superlayer of dimensions $\lambda_{k} \times \lambda_{k}$. Now the thermal superlayer must have more area than the vortical superlayer because its propagation speed is smaller than that of the vortical superlayer. Since the large scale structures on both the superlayers must be the same, the increased area of the thermal superlayer can only come from convolutions of wavelength much smaller than $\lambda_{s}$. Clearly, the fine structure on the thermal superlayer must be of wavelength at least $\lambda_{T}$, the thickness of the thermal superlayer. Moreover, its amplitude is unlikely to be greater than the diameter of the smallest eddies in the turbulent flow $\left(\lambda_{k}\right)$ and the area of thermal superlayer for each $\lambda_{k}^{2}$ area of vorticity superlayer is thus at most $I_{K}^{\prime} \frac{\lambda_{k}}{\lambda_{\varepsilon}}=\frac{\lambda_{k}^{2}}{\varepsilon}$. Thus the entrainment velocity for the thermal superlayer is

$$
\frac{\lambda_{k}^{k}}{\varepsilon} \cdot \varepsilon^{2} v_{n}, \rightarrow 0 \text { as } \varepsilon \rightarrow 0 \text {. Peclet number similarity is thus im- }
$$

possible according to this simple model.

The perturbation method which has been used brings out the mechanism of propagation nicely but is unable to give Peclet number similarity without being modified. This snag can be untangled by recalling that the propagation speed of the front is small because the variation in velocity gradient across its thickness is, simply because the thermal front is thin compared to $\lambda_{k}$. However if the velocity gradient at the superlayer is allowed to be random in sign rather than strictly negative, as has been assumed so far, the thermal front will be much thicker on average than the equilibrium scale $\lambda_{T}$. Hence the layer will be more strongly distorted and the propagation speed larger. I have made some very rough calculations which suggest that an entrainment velocity which varies weakly with $\varepsilon$ may be possible using this mechanism. I am about to attempt a more rigorous calculation.

\section{References}

Riedler, H. and M.R.Head 1966 Intermittency measurements in the turbulent boundary layer. J.Fluid Mech., 25: 719-735.

Kuo, A.Y.-S/ and S/ Cprrsom ;972 Experiment on the geometry of the finestructure regions in fully turbulent flow. J.Fluid Mech., 56: 447-479.

\section{Acknowl edgment}

I would like to thank my former supervisor at the University of Sydney,

Dr. R.A.Antonia for introducing me to the entrainment interface. 


\title{
PHOTON BUBBLES
}

\author{
Edward A. Spiegel
}

In a hot stellar atmosphere, radiation may produce significant dynamical as well as thermal effects. Let $F$ be the flux of radiant energy in the star's reference frame. The flux of-radiant momentum is measured by $\mathrm{F} / \mathrm{c}$, value $\mathrm{c}$ is the speed of light. Let $\mathrm{x}$ be the cross sectional area per unit mass of stellar material for scattering or absorbing light and let $\rho$ be the density of stellar material. Then $p x$ is the inverse of the mean free path of photons. The force per unit volume of the radiation or static matter is then $\rho \times F / c$. If the matter is moving with velocity $y$ with respect to the star, the radiant flux as seen in the local rest frame of the matter is, approximately, $\vec{F}=F-\frac{4}{3} E \underline{y}$, where $E$ is the density of radiant energy. (The $4 / 3$ is a correction arising from the relativistic nature of light.) Hence the radiant force density on moving matter is approximately $\rho \times E / c$.

Suppose that we think of the radiation as a fluid with velocity (w.r.t. the star) $\mathscr{Y}=F /\left(\frac{*}{3} E\right)$. The radiant force is then written $\mathrm{px}(\mathrm{v}-\mathrm{u}) / \mathrm{c}$, and is proportional to the relative velocity of the two fluids. This force can be comparable to the gravitational force on the matter. When the two forces are equal and $u=0$, astronomers say the star is at the Eddington limit. In that case the situation is in many ways like that in a fluidized bed. How good is the analogy?

There are some failures. In the fluidized bed the drag cross section per particle is rather sensitive to particle density. The analogous astrophysical quantity, $x$, does depend on density, but not so critically. Particles in a fluidized bed may feel the effects of close packing; not so the particles in a stellar atmosphere. The light may be absorbed in stellar atmospheres; no analagous phenomenon is significant in fluidization.

Despite these differences similar dynamical behavior may be encountered in the two situations. This possibility leads to the notion of photon bubbles, voids in the matter containing (almost) exclusively radiation. If these exist they must work about as follows:

Consider a spherical region in the atmosphere in which the matter density is lowered (to zero in the ideal case). Radiation flowing from below tends to move through the hole. (Picture the analogous case of flow in a porous medium with a hole.) The radiant flux lines will look like those in Fig.l. In the frame of the bubbles, out in front, the radiant force pushes the matter aside so that it goes around the hole (see Fig.2). Life is more complicated on the trailing edge, as one sees in fluidized beds.

There are some equations that go with all this, of course, but I fear that they do not help much. There is also a list of people who have, contributed to the developments such as they are - Hsieh, Lloyd, Marzek, and of course, there is a fluidization literature which has been the main guide. 

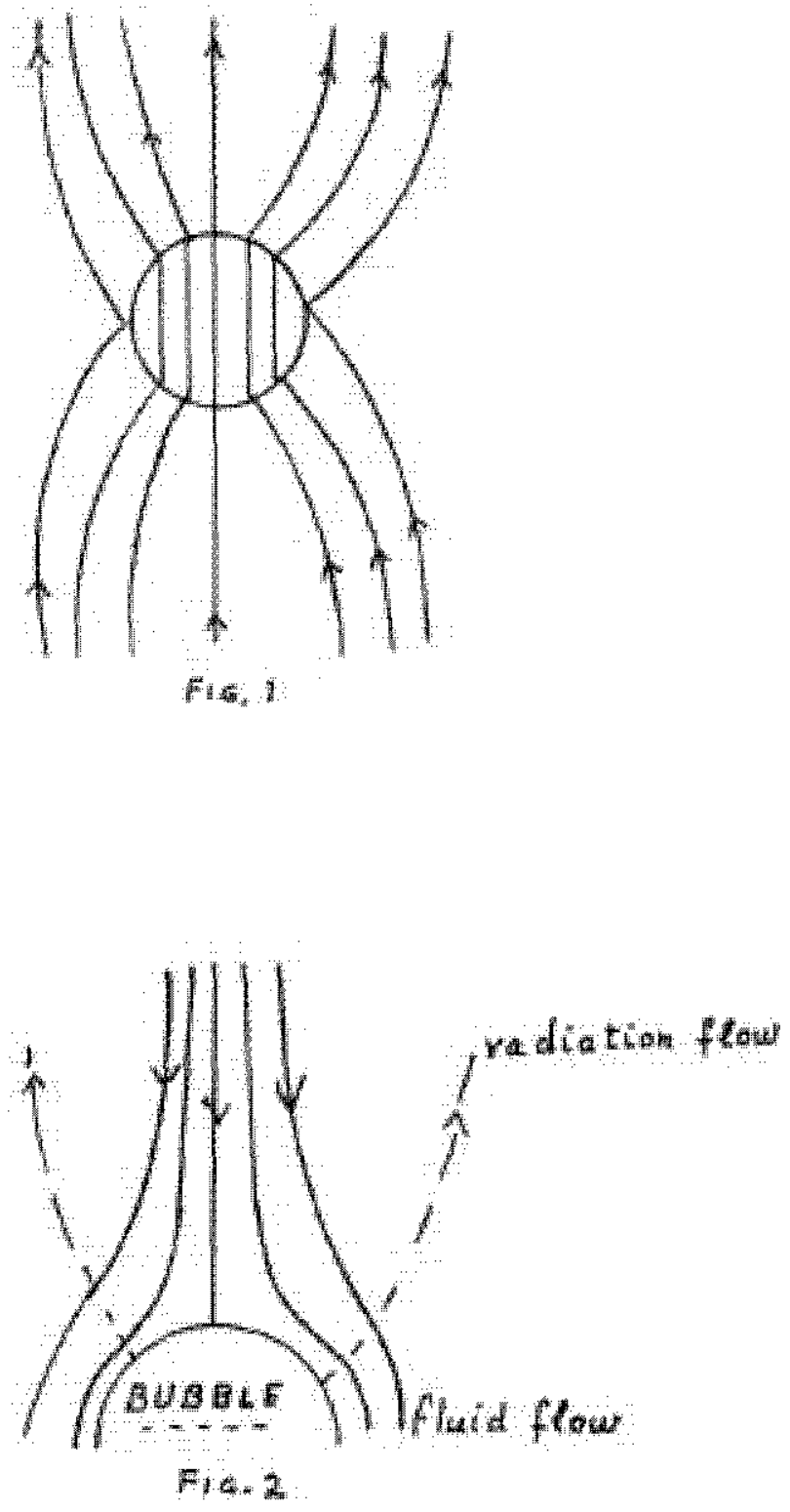


\section{EXTREME DISCHARGE IN TURBULENT AND OTHER INDETERMINATE FLOWS}

Melvin E. Stern

We seek the maximum discharge $Q=\int_{0}^{D} U(z) d z$ for a fully turbulent, mean flow $V(z)$ in a channel with given wall stress $\uparrow$ and viscosity $\nu$ subject to certain "structural constraints". One of these is the assumption that the flow is logarithmic, (or $d U / \alpha z=T^{k} / 2 k$, where $K=$ Von Karman's constant) in the far field $\mathbf{z}>\mathbf{z}_{b}$. The boundary layer depth $\mathbf{z}_{b}$ is constricted by plotting $d v / d z$ as a function of $z$ and by drawing the tangent line to that curve from the origin (i.e.z=0, $d v / d z=\uparrow / 2$ ). The intersection of that tangent with the $\mathbf{t}$ axis defines $z_{b}$, and this tangent line is a lower bound on $d v / d z$. The second important structural assumption is that there exists some "TRIAL" stream function $\psi$, with $\psi(x, 0)=0=\partial \psi(x, 0) / \alpha z$ such that

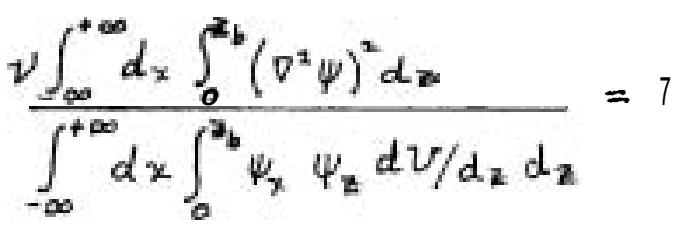

This functional inequality is shown to be correct in fact (by using the experimental $\boldsymbol{Z}_{b}$ and $d U / d$ although we do not know why. Likewise, the validity of the log law above $z_{b}$ is shown to be correct in fact, although we do not know why. Using these and other structural constraints (but using no empirically adjustable parameters) we seek a maximum for $K$, this being equivalent to maximizing $Q$. The variational formalism should also give the detailed structure of $V$ in the entire turbulent boundary layer. The results of a preliminary calculation are then presented, in which a trial form of $V$ is assumed, and (only) $K=0.32$ is computed by the theory. The correct theoretical value of $\mathrm{K}$ should be somewhat larger than this, and future work will also be directed towards the second constant in the log law. Since the constraints are known to be correct (in fact), the agreement between observed and predicted $K$ indicates the correctness of the assumption that the turbulent flow in a channel tends to minimize the discharge for a given downstream pressure gradient. This confirms one aspect of Malkus' original proposal; but our constraints are very different, in spirit as well as form.

THE BETA SPIRAL AND THE DETERMINATION OF THE ABSOLUTE VELOCITY FIELD ROM HYDROGRAPHIC STATION DATA

Henry Stommel*

Because the horizontal component of velocity rotates with depth in the open ocean, absolute velocities can be obtained from observations of . the density field alone. If in the mean, there is no flow across density surfaces, if the flow is geostrophic, and if the linear vorticity balance

Henry Stornmel and Friedrich Schott, Institut fur Meereskunde. Kiel. 
on a beta-plane obtains, then

$$
u h_{x}+v h_{y}=w ; u_{z}=-\gamma h_{y} \rho_{z} ; v_{z}=\gamma h_{x} \rho_{z} ; \gamma=g / f \rho_{0} ; f w_{z}=\beta v
$$

where $h$ represents the height of a given density surface. Differentiation of the-firstequation with respect to $\underline{z}$, and substitution from the remaining equations yields

$$
u h_{x z}+v\left(h_{y}-\beta z / f\right)_{z}=0
$$

which holds at different density surfaces at many depths. Since $h_{x z}$ and $h_{y z}$ can be determined from the observed density at any mid-ocean location, the direction of the absolute velocity, $v / u$, is known as a function of depth. If either coefficient (of $\underline{u}$ or $\underline{v}$ ) vanishes without the other vanishing at some particular depth one of the components of the absolute velocity also vanishes there, thus fixing the "depth of no motion" in that direction. Because of "eddy-noise" these coefficients can not (with present quantity of data) be very well determined, and therefore the absolute velocity can be determined better by simultaneous use of data at all depths instead of by location of the depth where the coefficients individually vanish. We will use the information contained in the main thermocline, excluding depths near the surface where unknown stresses and heating/cooling occur. Thus, introducing the notation $u=u_{0}+u^{\prime}, v=v_{0}+v^{\prime}$, where the primes represent the relative geostrophic velocity with respect to a reference level where the absolute velocities are $u_{0}$ and $v_{0}$. Equation (1) can then be rewritten in the form:

$$
u_{0} h_{x z}+v_{0}\left(h_{y z}-\beta / f\right)+u^{\prime} h_{x z}+v^{\prime}\left(h_{y z}-\beta / f\right)=R=0
$$

Writing this for several depths yields an overdetermined system for $u_{0}, v_{0}$. A "best-fit" corresponds to the solution for which the sum of the squares of the residuals, $R$, is least. Sudo (1965) used this relation in a form vertically integrated to the Ekman layer as a top boundary condition, thus introducing the very uncertain specification of the mean wind-stress; he also did not attempt to smooth out "eddy-noise".

The beta-spiral in the center of the subtropical gyre of the North Atlantic Ocean at $28^{\circ} \mathrm{N}, 36^{\circ} \mathrm{W}$ is a good example of the phenomenon. The $\mathrm{h}_{\mathrm{x}}$ was determined by smoothing over Atzantis stations 5754 - 5765 at $28^{\circ} \mathrm{N}$. The $h_{y}$ was determined from Discovery stations $3594-3607$ at $240 \mathrm{~N}$, and $3627^{-} 3641$ at $32^{\circ} \mathrm{N}$. $\rho$ was determined from the Atzantis data. The vertical derivatives of the curves shown in Fig.l are the coefficients of Eq. (1) : $h_{x z}$ approaches zero near 900 meters, suggesting that $\underline{v}$ vanished near there; near 300 meters both coefficients tend to vanish together thus making $\mathrm{y} / \mathrm{u}$ indeterminate there. The alternate curve (dashed) for $\mathrm{h}_{\mathrm{x}}$ was obtained by averaging the slopes along $24^{\circ} \mathrm{N}$ and $32^{\circ} \mathrm{N}$, and suggested a deeper level of vanishing $k$, more consistent with the findings of Leetmaa, Niiler, and Stommel (1977).

Computing the "best-fit" from Eq. (2) with reference level at 1000 meters, we find $u_{0}=+0.34 \pm .03, v_{0}-+0.06 \pm .013 \mathrm{~cm} / \mathrm{sec}-$ the errors are standard errors. Rather different values are obtained if the dashed curve for $h_{x}$ (Fig. I) is used: $u_{0}=0.55 \pm .06, v_{0}=0.25 \pm .04$, suggesting long-period, large-scale variability in the density field of the main thermocline on these sections, and pointing to the need for more hydrographic 


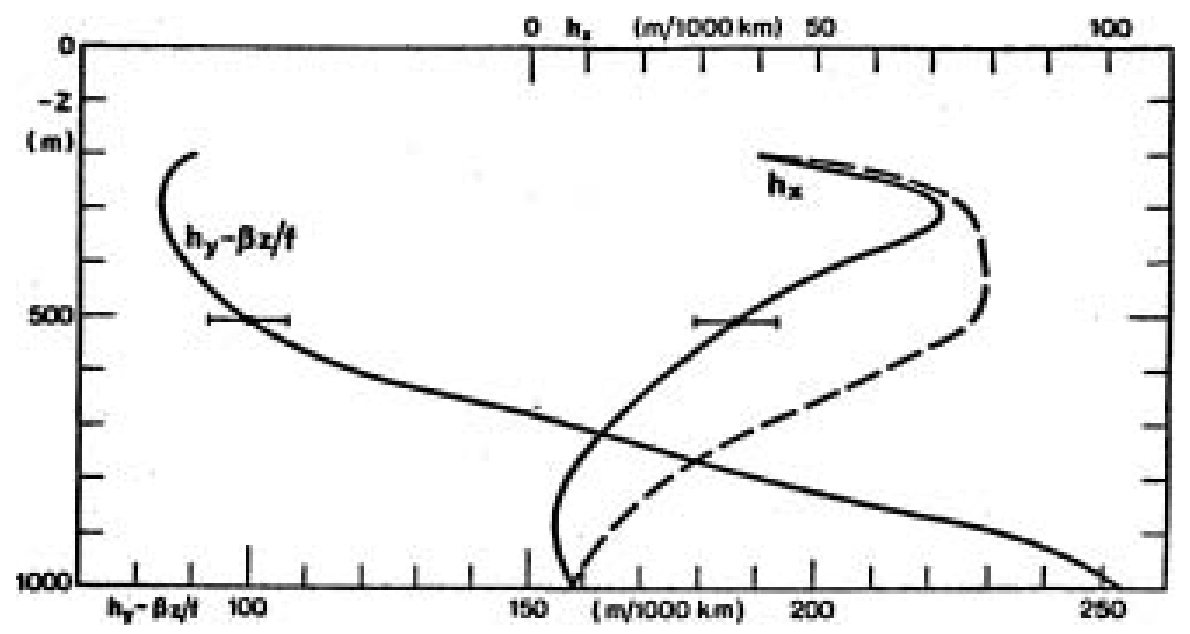

FIGURE 1. SLOPES OF THE SIGMA-T SURFACES AT $28^{\circ} \mathrm{N} 36^{\circ} \mathrm{W}$

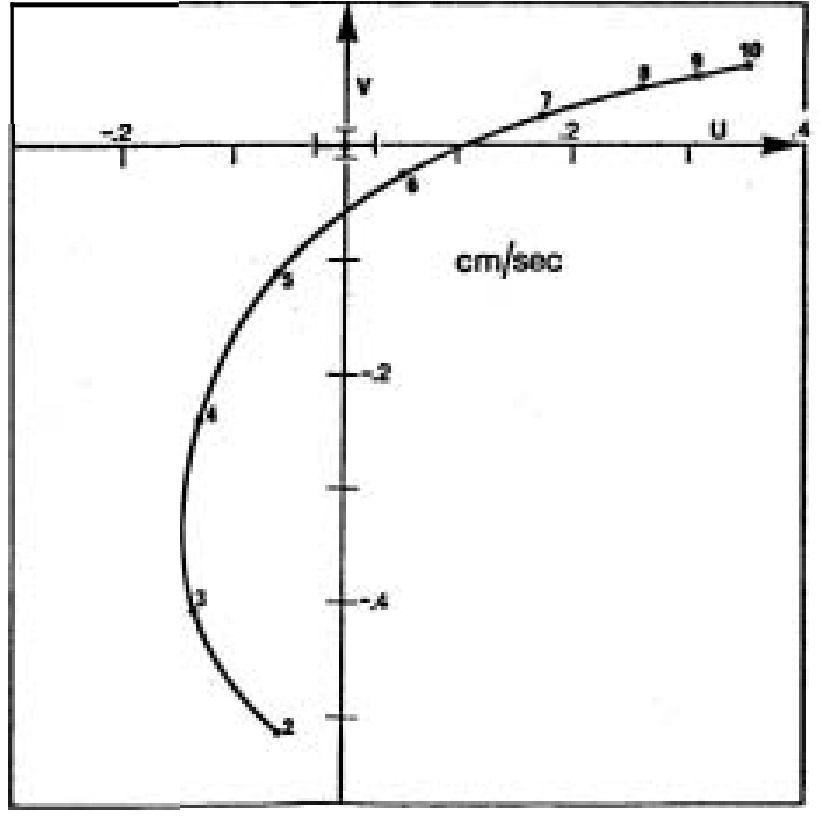

FIGURE 2. THE SPIRAL AT $28^{\circ} \mathrm{N} 36^{\circ} \mathrm{W}$.

DEPTHS IN 100 METERS

ERROR BARS AT THE ORIGIN 


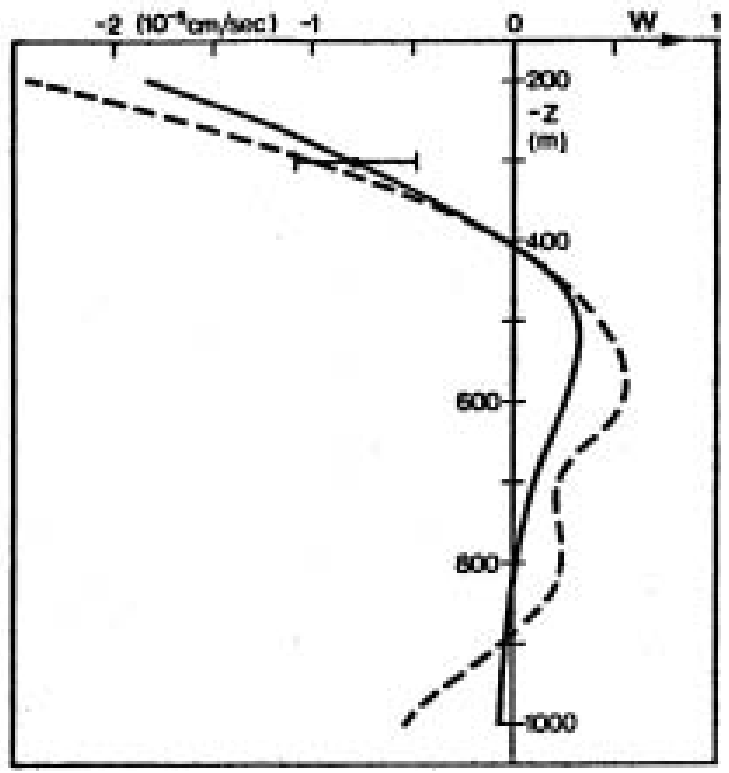

FIGURE 3. COMPUTED W AT $28^{\circ} \mathrm{N} 36^{\circ} \mathrm{W}$

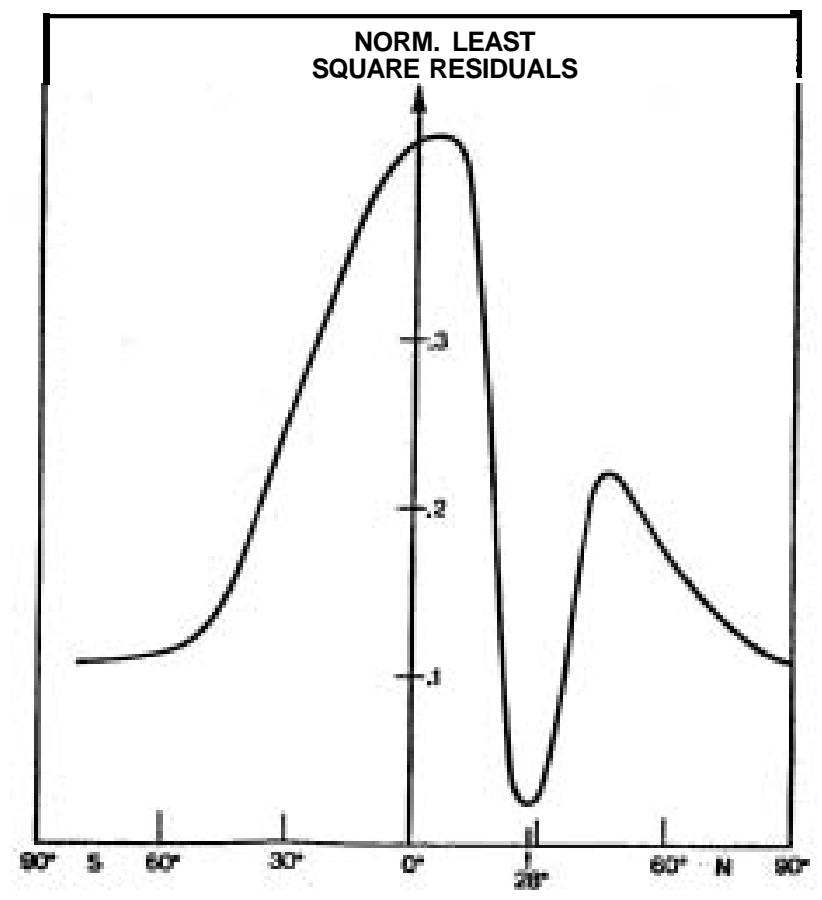

FIGURE 4. NORMALIZED LEAST SQUARE RESIDUALS FOR $28^{*} N 36^{*} W$. DENSITY DATA COMPUTED FOR VARIOUS VALUES OF LATITUDE 
station data throughout the world, in order to obtain stable results.

The spiral in absolute velocity is shown in Fig.2, with the $u_{0}$, $v_{0}$ error bars at the origin. Once $\underline{u}$ and $\underline{v}$ are known, $\underline{w}$ can be computed from the first equation and is shown in Fig.3. It approaches a negative value toward the surface as is expected from the wind-stress distribution in this location, and hence we have in principle a means of computing mean wind-stress from hydrographic station data. The least-square residuals could, with more data for better smoothing, be reduced further by addition of an eddy diffusion and flow across isopycnals, and hence we have in prin ciple a means of determining this interesting quantity. In our test location and range of depths used, $\rho_{z}$ is remarkably constant with depth, thus excluding the application of this idea.

In Fig. 4 we show the least square residual (normal ised to the nonhomogeneous term) computed for various false values of $\beta / f$ and also the real one at $28^{\circ} \mathrm{N}$. The curve shows a strong minimum at the correct latitude, thus demonstrating that the density field in the ocean "senses" its latitude.

It appears that eddy-dynamical processes are not necessary to account for the major features of the main thermocline in mid-oceanic parts of the subtropical gyres, and that with more adequate data coverage, the mean pressure field at the bottom of the ocean could be calculated. Moreover since $w$ might then be extended to the bottom, properties of the bottom frictional Tayer could be studied.

The notorious "unknown reference level" controversy, and the "law of parallel solenoids" are seen to be degenerate cases on the f-plane with wset equal to zero.

We will document the calculations of this example and for other locations more fully in a later communication. In subtropical gyres the beta-spiral rotates in the same sense as the Ekman spiral; in subpolar gyres in the opposite sense - but here we have found strong evidence of strong enhancement due to deep cooling. Some analytical examples of betaand cooling-spirals will also be given that may be useful for exposition.

\section{References}

Leetmaa, A., P.Niiler and H.Stommel 1977 Does the Sverdrup relation account for the mid-Atlantic circulation? J.Mar.Res. (Richardson memorial Volume) in press.

Sudo, H. 1965 An analysis on the deep current of the ocean with its application to the circulation of the North Atlantic Ocean. Jap.J.Geophys. 4: $1-70$. 


\title{
COHERENT TEMPERATURE STRUCTURES IN SHEAR ROW
}

\author{
Charles W. Van Atta
}

A ramp model originally developed for deducing the mean properties of certain coherent structures in atmospheric boundary layers can correctly account for the behavior of structure functions of temperature measured in a variety of laboratory and atmospheric flows. The model produces the correct sign of the odd-order structure functions, which varies with different flows, and in the inertial subrange yields the general variation with separation distance $r$ observed in the experiments. In its present state, the model can still only give the mean values for the ramp amplitude $a$ and repetition period, $(l+s)$, which are found to be not extremely sensitive to the choice of probability density for the amplitude. The contributions of the non-coherent part of the turbulent signal to the measured structure functions are found to be almost independent of the probability density a.

Including all higher terms in $\mathrm{r} / \boldsymbol{l}$, which are neglected in the earlier model, leads to two simultaneous equations for the mean values of a and ramp length $\ell$. For available data, these equations appear to have no solutions or to be at most severely ill-conditioned. Consideration of the variation of the mean length of the quiescent period $s$ with $\varepsilon$ yields useful bounds on allowable values of $\&$ and $s$.

\footnotetext{
"Effect of coherent structures on structure functions of temperature in the atmospheric boundary layer", Archives of Mechanics 22(1): 161-171.
}

\section{MODEL OF WORD OCEAN CIRCULATION}

\section{George Veronis}

A model of world ocean circulation driven by wind-stresses and differential heating is developed for a two-layer ocean with idealized boundaries and constant depth. The principal focus of the present study is the determination of the magnitudes and locations of localized sources and sinks of upper-layer water. The effect of differential heating is parameterized here by a uniform upwelling of deep water through the interface (thermocline). ,The magnitude of the upwelling, as well as the locations of the sources and sinks, is determined by specifying the latitudes of separation of the Kuroshio and the East Australian currents. The latter can be deduced if the effects of heating were known, but it seemed preferable to use the (easily identified) separation latitudes to obtain information about the complex thermal processes that exist in the ocean. The mechanism for separation emerged from an earlier study (Veronis 1973); consequently, the basic physical process is not masked by the specification of the separation latitudes. It is found that about nine Sverdrups of transport flow eastward from the Atlantic to the Indian to the Pacific to the Atlantic, coming in at the southwestern corner as a deep western boundary current and departing as the vestige of an upper-layer, separated western boundary current at the southeastern extremity of each ocean. The construction of the circulation for each ocean is not uniquely determined by the limited number of constraints 
invoked here. The actual construction is determined by the assumption that sources or sinks of upper-layer water are introduced only when they are required by the analysis. This assumption leads to a circulation in the South Pacific in which the East Australian Current separates from the coast and flows around New Zealand, part clockwise around the north and the remainder counterclockwise around the south. Where the two branches rejoin, the current separates and flows due eastward, eventually to supply the Peru and the Cape Horn Currents. Heat flux balances (determined by sinking of upper-layer water and upwelling of lower-layer water) are determined for the Atlantic and Pacific Oceans. The calculations are carried out with winter and annual mean winds, each case being treated as if it were a steady-state system. The results show, that the North Paci fic tends to absorb heat from the atmosphere and the North Atlantic tends to release heat to the atmosphere. Hence, in the overall heat balance of the earth, which requires a flux of heat from equatorial to polar regions, the principal oceanic contribution must come from the Atlantic.

\section{BUILDING BLOCKS FOR OCEAN CIRCULATION THEORY}

\section{Pierre Welander}

While the geostrophic and hydrostatic balance equations are generally accepted as the zero order dynamic equations for the interior mean ocean circulation, away from surface, bottom and side boundary layers, there is an uncertainty about the conservation equations for the density (rather, potential density). The recent $\beta$-spiral calculation by Stommel and Schott (Deep Sea Research, 1977), suggests that the ocean is nearly advective, with little cross-isopycnal mixing, at least in the central regions of the subtropical gyres (still, mixing along isopycnals may be important for the transport of heat and salt).

Thus, there are reasons for a new theoretical attack on the thermocline problem along the "advective line" proposed by the author in 1959 (see Tellus 11: 3p. 309). An outline of a new model was given in the lecture. The main point is that the density at the top is prescribed only in regions with downward Ekman vertical velocity. Solutions are worked out by an iteration scheme, starting from a guessed density field. The thermal wind equation and the Sverdrup transport equation determine the current field to the next approximation. The velocity trajectory field is then found, and going backward along these, new density values are picked up from the boundary conditions. Some general features of such solutions are:

i) The solution is statically stable only if the prescribed surface density field has no extremum away from boundaries (pointed out before by $P$. Killworth).

ii) Frequently there appears separation lines at boundaries, free discontinuity surface (fronts) between down and upwelling regions, and one may also find deep stagnant pools, which are not fed from the surface, and the density of which cannot be calculated unless some diffusion is added. 
i i i) The solutions generally differ from the similarity-type solutions studied earlier.

The side boundaries pose a problem, as there is geostrophic flow through these when there is any along-boundary density variation. Adding the simplest higher order dynamics (lateral friction of Munk's type), the condition of zero normal and tangential flow can, however, be satisfied at all side boundaries, for any prescribed surface density field. At present, this model is being tested.

The density data for the ocean actually indicate that several advective regimes exist, separated by fronts. For example, in the South Atlantic one finds different relations between potential vorticity and density next to the equator, down to $50^{\circ} \mathrm{S}$, and south of $50^{\circ} \mathrm{S}$ (see Fig.). Thus it seems unlikely that a uniform similarity type solution will work well for the oceans. If the advective model is applicable, allowance must at least be given for "cracks" at certain places. The prediction of these cracks is part of the problem to be solved.

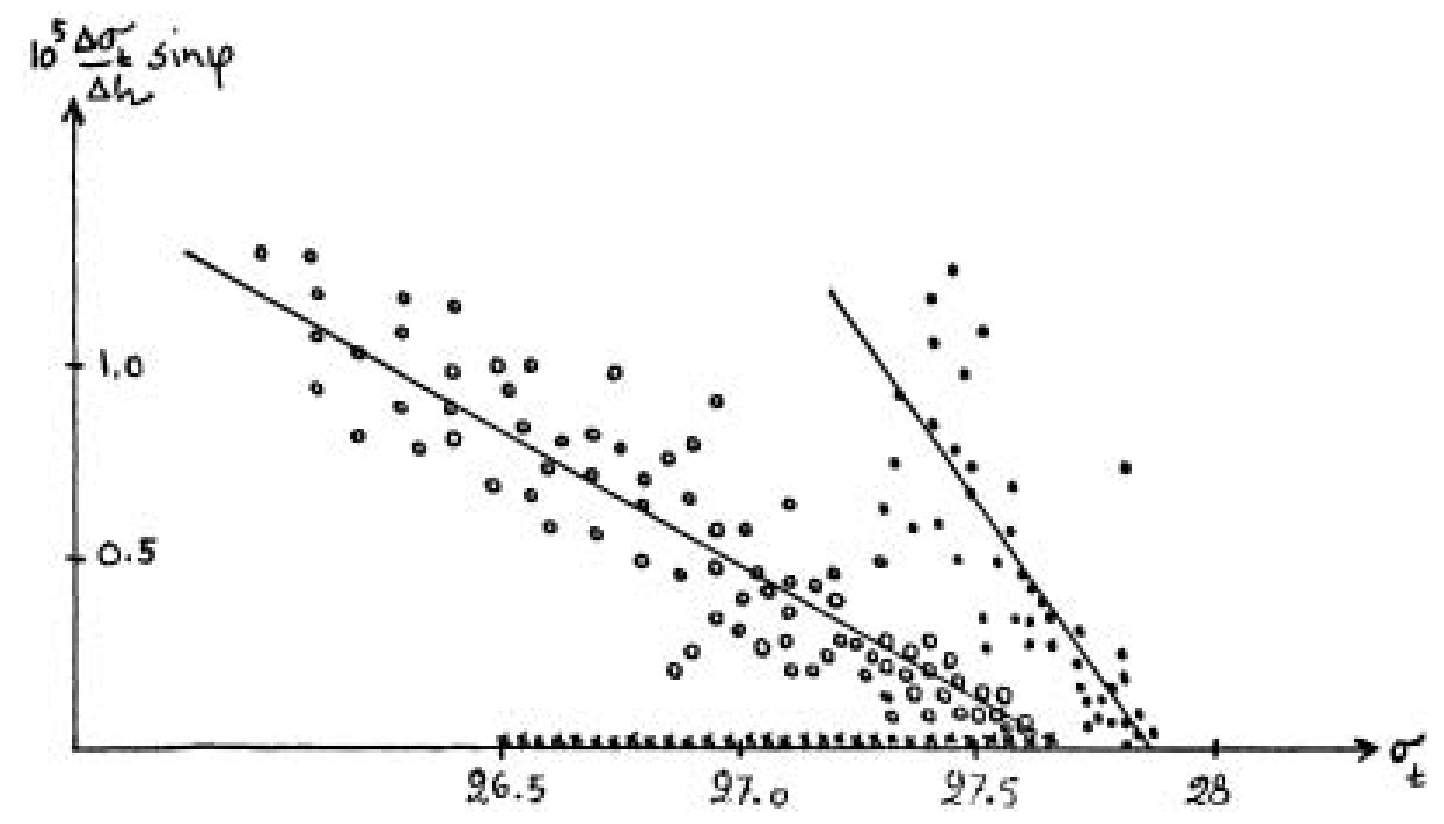

Values of geostrophic potential vorticity plotted against $\sigma_{t}$ in the South Atlantic, calculated from "Meteor" data. Crosses are near-equator values, open circles, values down to $50^{\circ} \mathrm{S}$, filled circles South of $50^{\circ} \mathrm{S}$. 


\section{RAYLEIGH-BÉNARD CONVECTION, LAMINAR AND TURBULENT}

John A. Whitehead, Jr.

A history of the experimental observations of transitions to more complicated convection states in Bénard convection was given. After reviewing the early observations of transitions in heat flux, an experiment was described where controlled perturbations prior to the onset of motion, produced two-dimensional convection cells of arbitrary width-depth ratios (Chen and Whitehead 1968). The conditions employed correspond to the finite-amplitude Rayleigh stability problem for a constant viscosity, large Prandtl number, Boussinesq fluid with rigid, conducting boundaries. The results (Busse and Whitehead (1971)) agree with the theoretical predictions by Busse and show two distinct types of instability in the form of non-oscillatory disturbances. The 'zigzag instability' corresponds to a bending of the original rolls; in the 'cross-roll instability' rolls emerge at right angles to the original rolls. Rolls within a certain bandwidth are stable at Rayleigh numbers below 23,000. At Rayleigh numbers above 23,000 rolls are unstable for all wave-numbers and are replaced by a three-dimensional form of stationary convection for which the name "bimodal convection' has since been used. At higher Rayleigh numbers (Busse and Whitehead, 1974), two mechanisms of instability for bimodal convection can be separated from the effects of random noise. The oscillatory instability of bimodal cells introduces standing waves closely resembling those occurring in low Prandtl number convection. The collective instability introduces spoke-pattern convection which is characteristic for turbulent large Prandt number convection. Both instabilities originate primarily from the momentum advection terms in the equations of motion, as is evident from the strong Prandtl number dependence of the critical Rayleigh number $R_{t}$ for the onset of oscillations. The results are discussed in relation to previous experiments and recent theoretical work. The bimodal flow is observed to be stable for given bandwidths and ranges of Rayleigh numbers when the configuration of bimodal flow is flawless -without any disruptions in the periodic matrix (whitehead and Chan (1976)). The stability range differs from earlier experiments and from our own experiments in which the bimodal planform is uncontrolled.

Dislocations can also nucleate other transition mechanisms (whitehead, 1976). When Rayleigh-Bénard convection is generated under random conditions, the finite amplitude rolls and bimodal flow are observed to possess randomly-placed dislocations where the rolls fit together poorly. The dislocations move into the small wavelength convection, and hence provide a size-adjustment mechanism. It is observed that the dimensionless speed of the movement is smaller for larger Prandtl number fluid.

Lastly, the stability range for very high Prandtl number (8600) convection admits a stable range of square convection cells, whose stability properties were discussed.

\section{References}

Busse, F.H. and J.A. Whitehead 1971 Instabilities of convection rolls in a high Prandtl number fluid, J.Fluid Mech., 47(2): 305-320. 
Busse, F.H. and J.A.Whitehead 1974 Oscillatory and collective instabilities in large Prandtl number convection. J.Fluid Mech., $66(1)$ : 67-79.

Chen, Michael M. and John A. Whitehead 1968 Evolution of two-dimensional periodic Rayleigh convection cells of arbitrary wave-numbers. J.Fluid Mech., 31(1): 1-15.

Whitehead, J.A. 1976 The propagation of dislocations in Rayleigh-Bénard rolls and bimodal flow. J.Fluid Mech., 75(4): 715-720.

Whitehead, J.A.,Jr. and Gerald L. Chan 1976 Stability of Rayleigh-Bénard convection rolls and bimodal flow at moderate Prandtl number. Dynamics of Atmospheres and Oceans, 1. 33-49.

\section{HYDROMAGNETIC TURBULENCE}

Robert H. Kraichnan

This talk reviews recent work on hydromagnetic turbulence in infinite fluids, with emphasis on the effects of helicity. As Frisch and the Nice school have shown, a lot can be learned from the relatively simple analysis of the equilibrium statistical mechanics of the dissipationless hydromagnetic system cut of $f$ at some high wavenumber. In the presence of helicity there are equilibria with strong magnetic excitation at low wavenumbers. These equilibria suggest that appropriate nonequilibrium states will transfer energy into low wavenumbers of the magnetic field. The arguments are introduced by first considering the simpler, analogous arguments that apply to two-dimensional hydrodynamic turbulence.

Even when there is no mean helicity to the turbulence, strong amplification of low wavenumbers of the magnetic field can occur provided there are persistent fluctuations of helicity in the velocity field at higher wavenumbers. This is deduced from qualitative arguments and supported also by computer simulations of the evolution of weak magnetic fields in a conducting fluid.

Hydromagnetic turbulence with helicity fluctuations makes severe demands on turbulence closure approximations. The physical and mathematical reasons why this is an especially difficult problem are discussed.

\section{AN AXISYMMETRIC OCEAN CIRCULATION MODEL}

\section{Jule G. Charney}

Over the oceans the intertropical convergence zone (ITCZ) of the atmosphere tends to parallel the equator but to be displaced several degrees from it, approximately to where the sea-surface temperature is warmest. If one wishes to explain the location of the ITCZ, it is not enough to observe that cold equatorial upwelling occurs in the presence of the 
prevailing equatorial easterlies; it is necessary to explain the generation of the easterlies as well as of the ITCZ, i.e. to explain the lowlatitude circulations of both the atmosphere and oceans as parts of a coupled mechanical system. I have conjectured that the low-level convergence of air in the ITCZ is frictionally induced l and that the ITCZ forms as a conditional instability of the second kind $(C I S K)^{2}$, whereby an initial increase of low-level cyclonic vorticity causes, in sequence, enhanced frictional convergence of moist, conditionally unstable air, enhanced release of heat of condensation by cumulus convection, and therefore enhanced increase of cyclonic vorticity by vortex stretching. If one assumes the ITCZ to be frictionally controlled by some such mechanism, then its displacement from the equator would be explained if its equilibrium configuration at the equator were shown to be unstable for lateral displacements. It seems likely that such is indeed the case, for, if we assume that the atmosphere - ocean system is approximately axisymmetric away from boundaries, the following plausability argument can be made. Let the ITCZ be displaced from the equator, say to the north. Then it will draw zonal rings of air from south of the equator to the equator, where, having lower angular momentum, they will appear with an easterly (westward) velocity component. The resulting upwelling of cold water will prevent the ITCZ from moving back to the equator. At the same time cyclonic shear across the ITCZ will be generated because rings approaching the ITCZ from the north will have lower angular momentum than rings coming from the south, since, assuming symmetry of displacements, they will have originated closer to the earth's axis. Thus the displacement will produce not only upwelling but also cyclonic shear and therefore enhanced frictional convergence.

To investigate the nature of the coupled circulations, Eugenia Kálnay-Rivas, Edwin Schneider and I have constructed simple axisymmetric Venus model for the ocean and symmetrizing the general circulation model of the Goddard Institute for Space Study for the atmosphere. To test the ocean model, preliminary numerical integrations have been performed with an axisymmetric model driven by smoothed, annually averaged, observed zonal wind stresses and heat flows 3 at the upper boundary, as shown in Fig.1. The ocean model and the circulations will now be described. The integrations for the coupled model have not yet been completed.

An unper mixed layer was incorporated in the model by the method of Rory Thompson. 4 Heat was diffused horizontally and vertically in the interior and momentum vertically. The diffusion coefficients were taken to be $x_{H}=10^{*} \mathrm{~cm}^{2} \mathrm{sec}^{-1}, x_{v}=\nu_{v}=1 \mathrm{~cm}^{2}$ sec."

The equations of motion,

$$
1.1 u_{t}+\frac{v(u \cos \phi)}{a \cos \phi} \phi+\omega u_{z}-f v=\nu_{v} u_{z z} \text {, }
$$

TCharney, J. 1971 Mathematical Problems in the Geophysical Sciences, 1 Geophys.Fl.Dyn. Lectures in applied Mathematics, Vol.13, Amer. Mathematical Society, Providence, Rhode Island, pp.355-68.

${ }^{2}$ Charney and Eliassen, 1964 J.A.S. 21: 68-75.

30ort, A.H. and T.H.VonderHaar, 1976 J.Phys.Ocean. 6: 781-800.

${ }^{4}$ Thompson, R., 1976 J.Phys. Ocean, 6. 496-503. 


$$
\begin{aligned}
& 1.2 v_{t}+\frac{v v_{u}}{a}+w v_{j}+u^{2} \frac{\tan }{a}+f u=-\frac{p_{q}}{a}+\nu_{v} v_{3}, \\
& 1.3 \quad \frac{(v \cos \phi)}{a \cos \phi} \phi+\omega_{z}=0, \\
& 1.4 \quad 0=-\frac{p_{t}}{\rho_{0}}-g \frac{\rho}{\rho_{0}} \\
& 1.5 \quad \rho_{t}+\frac{v \rho_{Q}}{a}+\omega \rho_{z}=x_{H} \frac{\left(\rho_{\theta} \cos \phi\right)}{a^{2} \cos \phi} \\
& 1.6 \rho=\rho_{0}\left(1-\frac{T-T_{0}}{T_{m}}\right)
\end{aligned}
$$

were integrated in time with the boundary conditions,

$$
\begin{array}{rlrl}
1.7 & z=0: \nu_{v} u_{z}=\frac{\tau_{0}{ }^{(x)}}{\rho_{0}}(\phi), w & =0, x_{v} T_{3}=F(\phi), \\
z & =-H: u=0, w=0, x_{v} T_{z}=0, \\
\phi & =0: v=0, \quad \tau_{\phi}=0 .
\end{array}
$$

until the solution converged to a steady state. The depth $\mathrm{H}$ was taken to be $2 \mathrm{~km}$ rather than a more realistic 4 or $5 \mathrm{~km}$ merely to save integration time. For comparison purposes, integrations were also performed for zero surface stress. For both stress conditions, advection of relative momenturn was negligible except within a degree or two of the equator, whence

1.6

$$
\begin{aligned}
& f v=\frac{-z}{\rho_{0}}=\nu_{1} u_{33}{ }^{\prime} \\
& f u=-\frac{p_{y}}{\rho_{0}}, \text { or } f u_{z}=g \frac{\rho_{y}}{\rho_{0}}
\end{aligned}
$$

from which one derives the boundary conditions:

$$
\begin{array}{ll}
1.10 & -f \int_{\mu=\infty}^{0} v d z=\frac{\tau_{0}^{(x)}}{\rho_{0}}, \\
1.11 & -f \int_{-H}^{0} v d z=\frac{\tau_{e}^{(x)}-\tau_{-H}^{(x)}}{\rho_{0}}=0
\end{array}
$$

The zonal flow consists of a barotropic component $u_{0}(\phi)$ satisfying 11. for the bottom Ekman layer, and a baroclinic component $u d \phi, z)$ satisfying 8. and 9 . Introducing the meridional stream function $\psi$ by

1.12

$$
\cos \phi=-\psi_{j}, \omega \cos \phi=\frac{\psi_{\phi}}{a},
$$

we get

$1.13 f \psi=-\frac{\tau^{(x)}}{\rho_{0}} \cos \phi+\frac{\nu u_{1} \cos \phi}{f}=-\frac{\tau^{(x)}}{\rho_{0}} \cos \phi \frac{q^{\nu} \rho_{\alpha} \cos \phi}{f^{2} \rho_{0} a}$ 
from 8 and 9 . In the zero stress case, $u_{0}(\phi)=0$ and $v$ is too small in the upper boundary layer to advect $\rho$. In the finite stress case,

$$
u_{0}(\phi)=\frac{\tau^{(\kappa)} / \rho_{0}}{v \nu_{v} \Omega \sin \phi}
$$

to satisfy $11 ., \rho$ is strongly advected in the upper boundary layer, strong upwelling occurs at the equator, and elsewhere downwelling or upwelling occurs according as the Ekman pumping is downward or upward, the vertical motion geing given by

$$
1.14 \quad \omega=\frac{\psi_{\phi}}{a}=-\frac{1}{a}\left(\frac{\tau_{0}^{(0)} \cos \phi}{f_{0}}\right)_{\phi},
$$

In the zero-stress case, temperature advection is everywhere small and the equilibrium temperature field is determined simply by diffusive equilibrium. In the non-zero stress case, it is necessary to take into account horizontal advection of temperature (density) in the upper boundary layer as well as vertical upwelling near the equator, succeeded by downwelling and upwelling poleward of the equator. The temperature, mericional stream function, and zonal velocity fields are shown in Figs. $2(a, b, c)$ and $3(a, b, c)$.

Poleward of $50^{\circ}$ to $55^{\circ}$, in the region of heat loss from the surface, vertical convection takes place and the temperature and density are nearly constant in the vertical. The high density (low temperature) water in middle and high latitudes is diffused equatorward, creating a pool of cold bottom water with weak temperature gradients. In both cases a weak thermocline structure exists equatorward of $30^{\circ}$ to $40^{\circ}$.

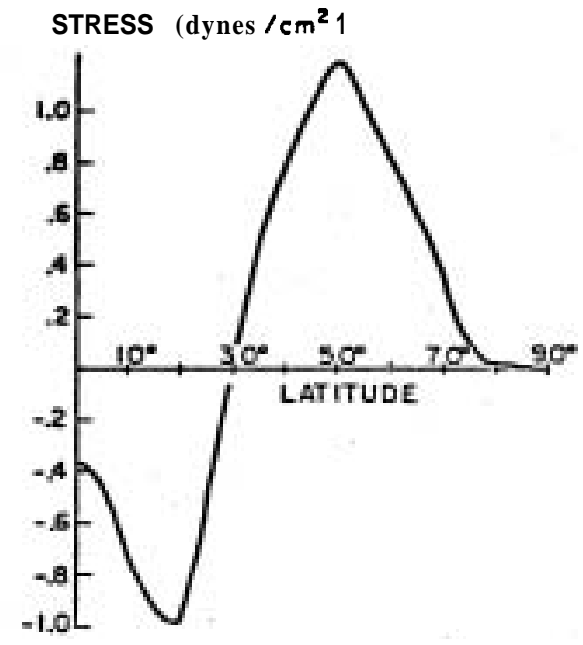

Fig. 1

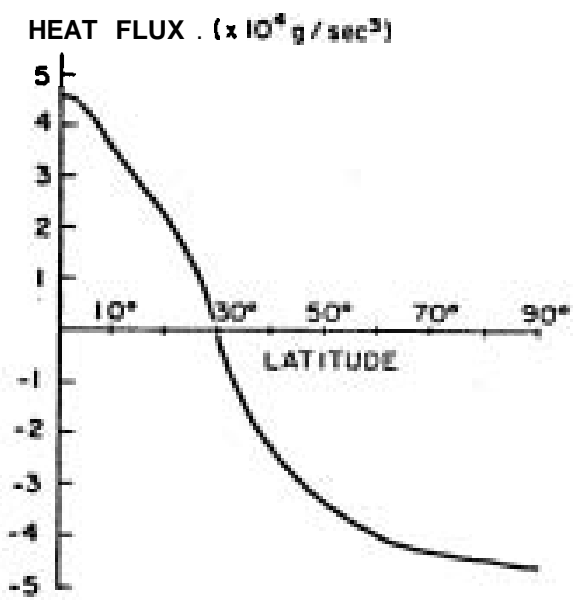

Fig. 2 
$-95$.

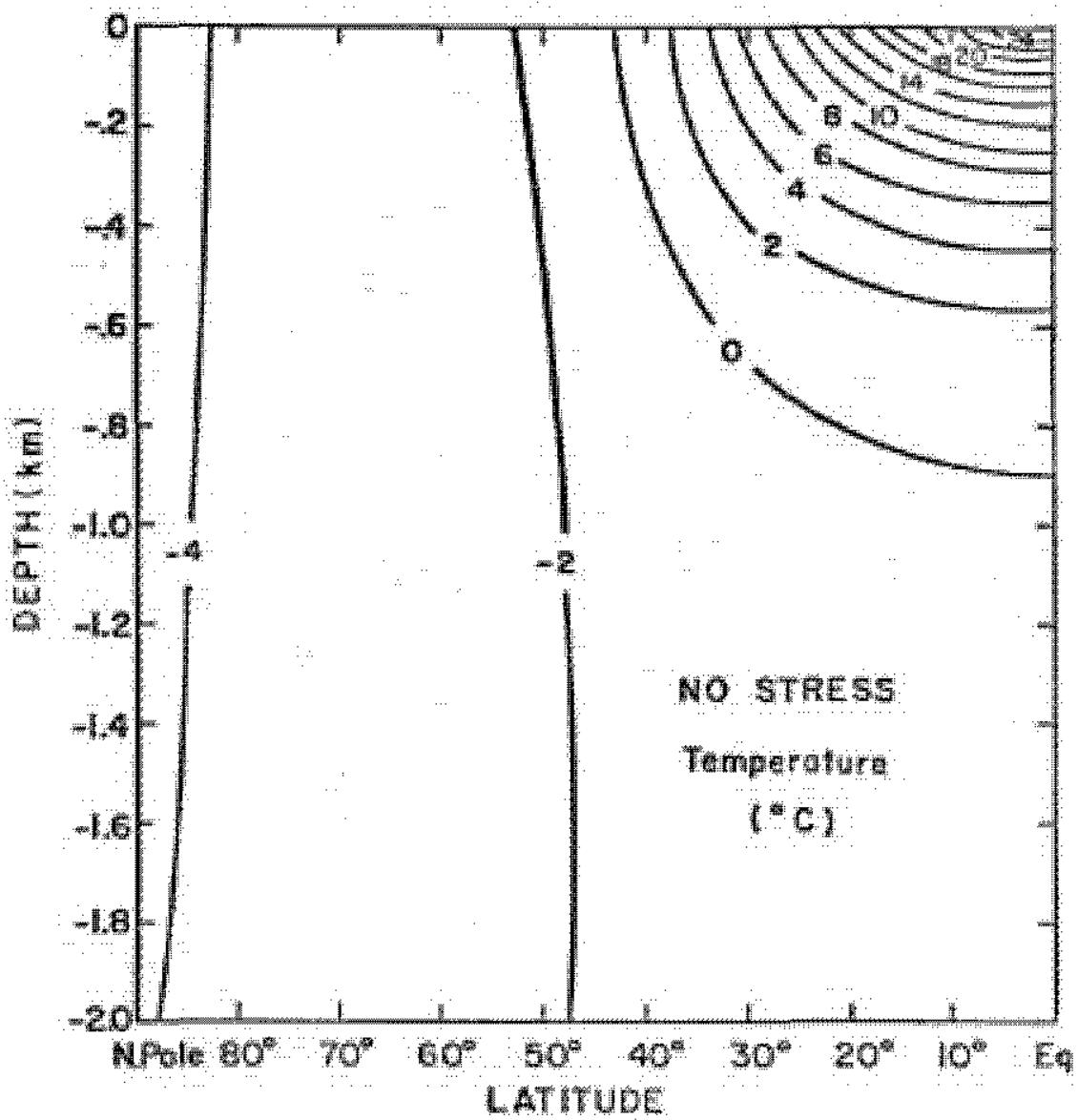

Fig.za

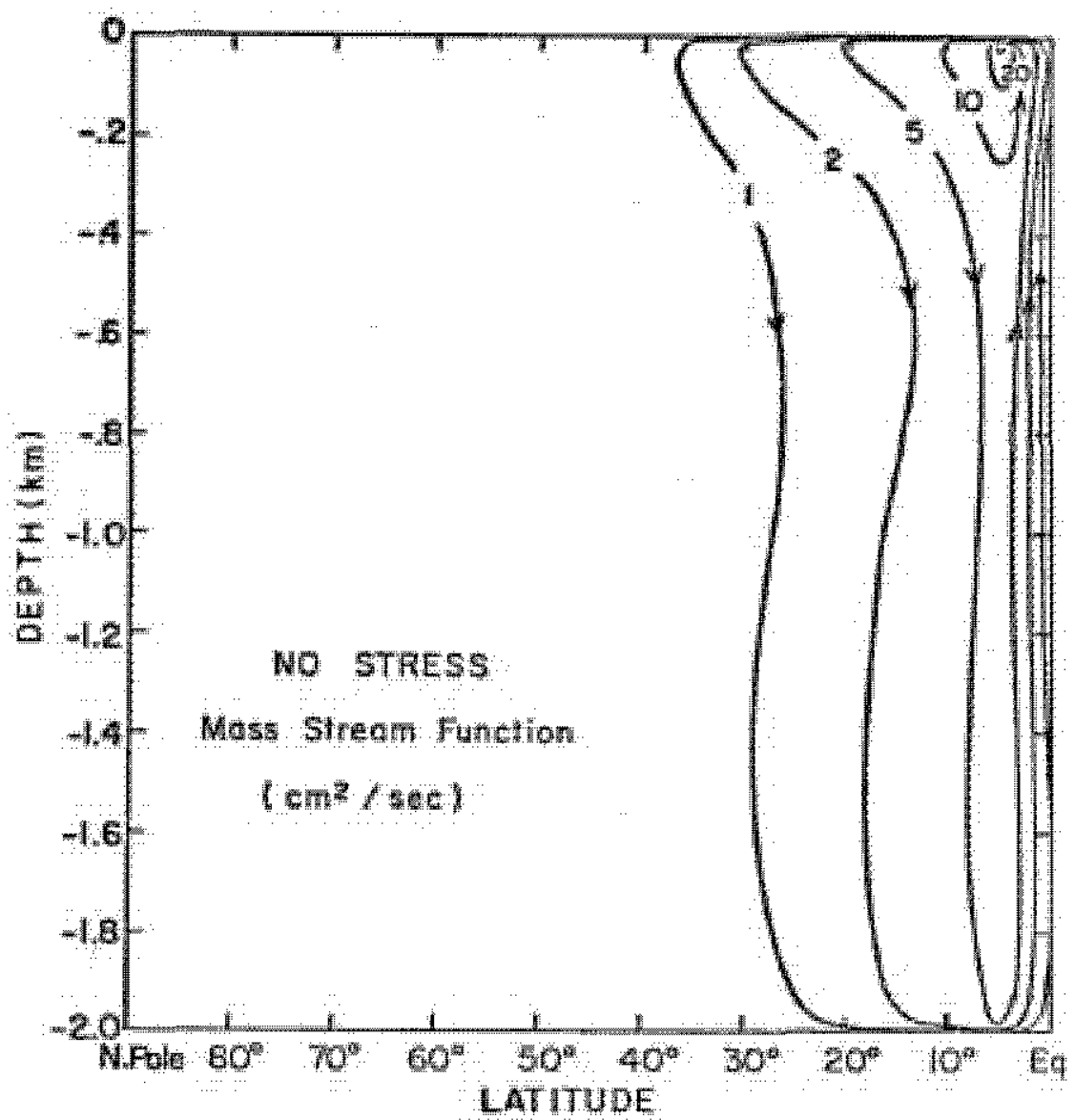

$\mathrm{Fig}, 2 \mathrm{~b}$ 


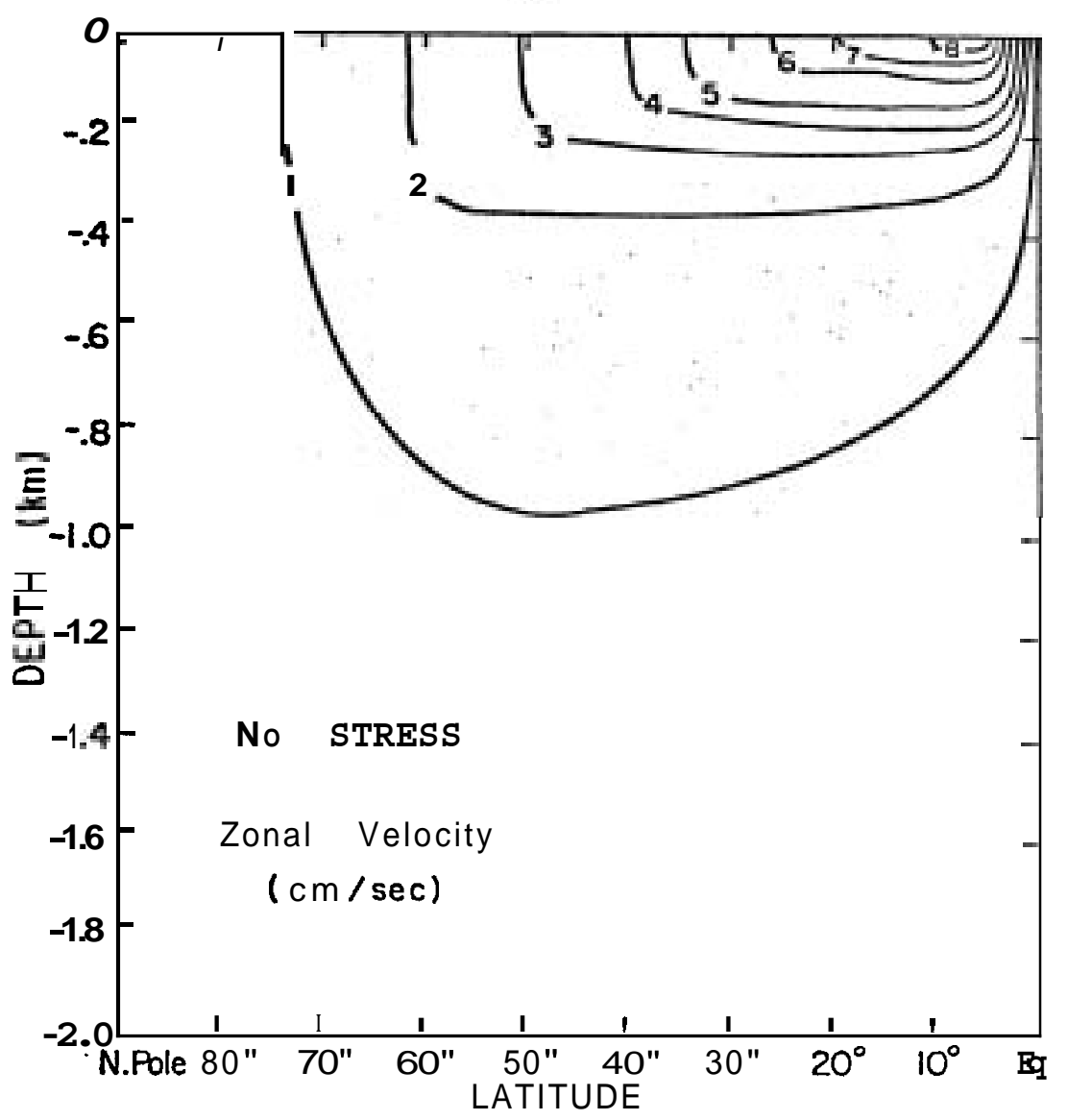

Fig. 2c

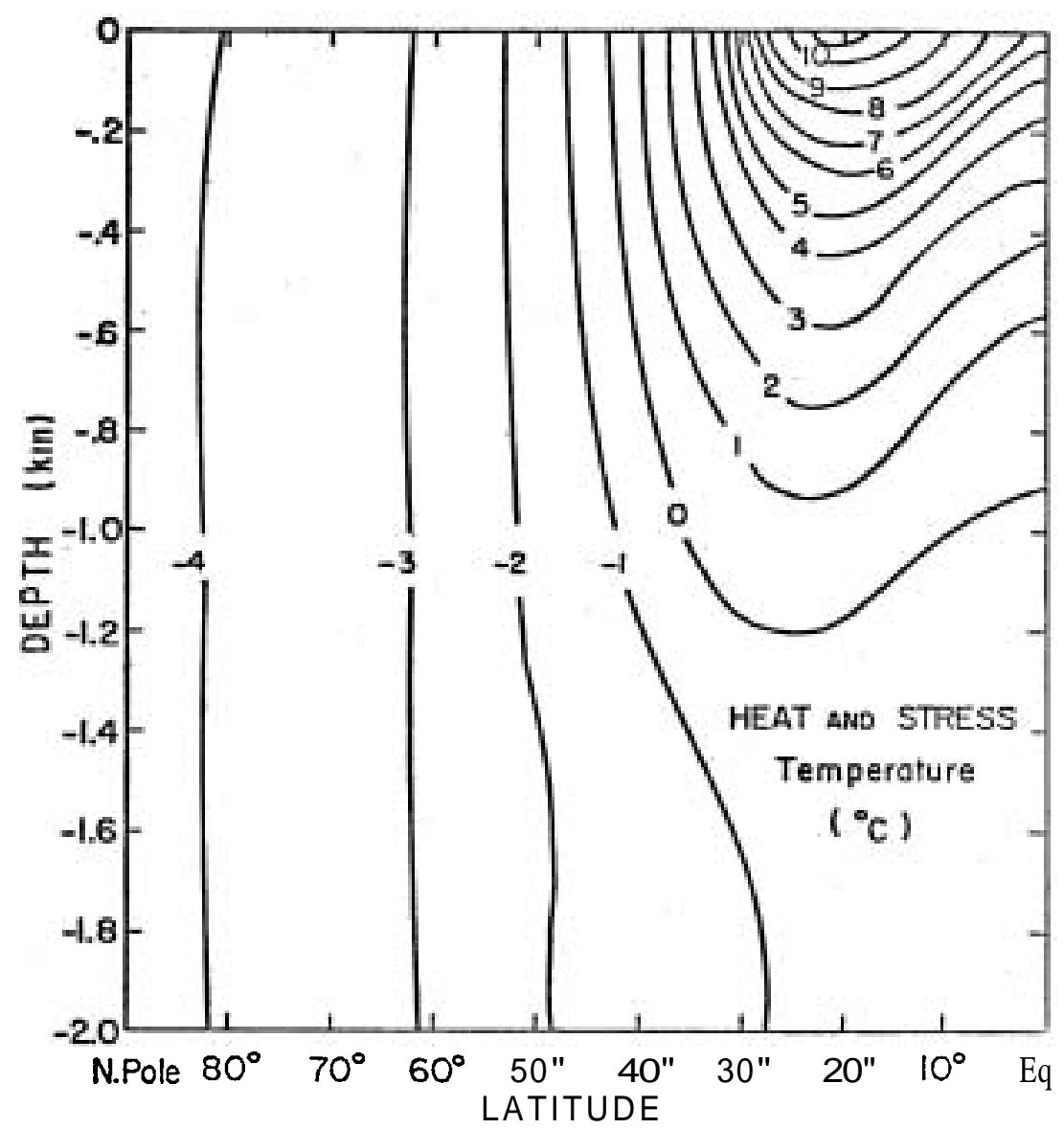

Fig. $3 a$ 
- 97 -

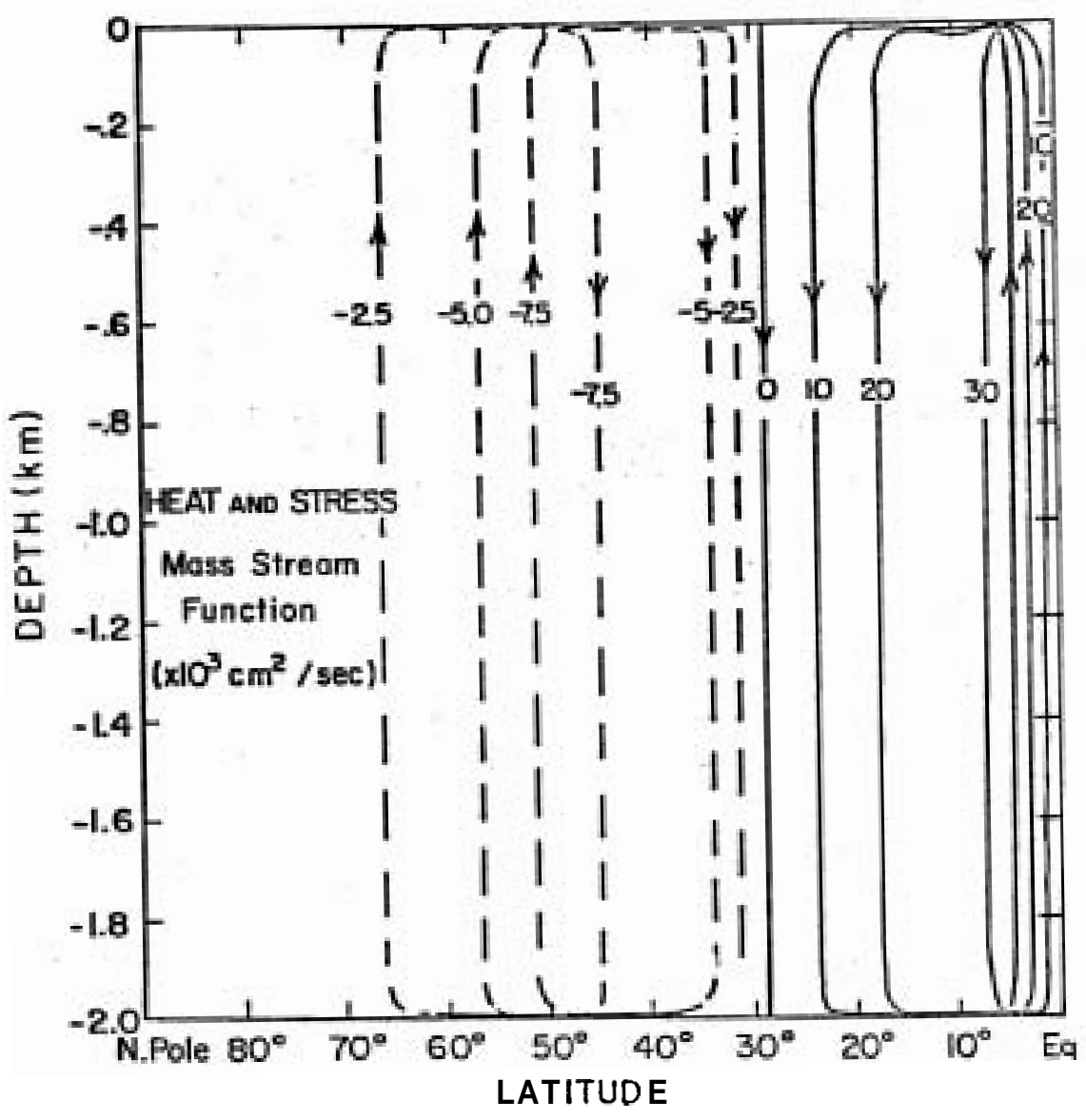

Fig. 3b

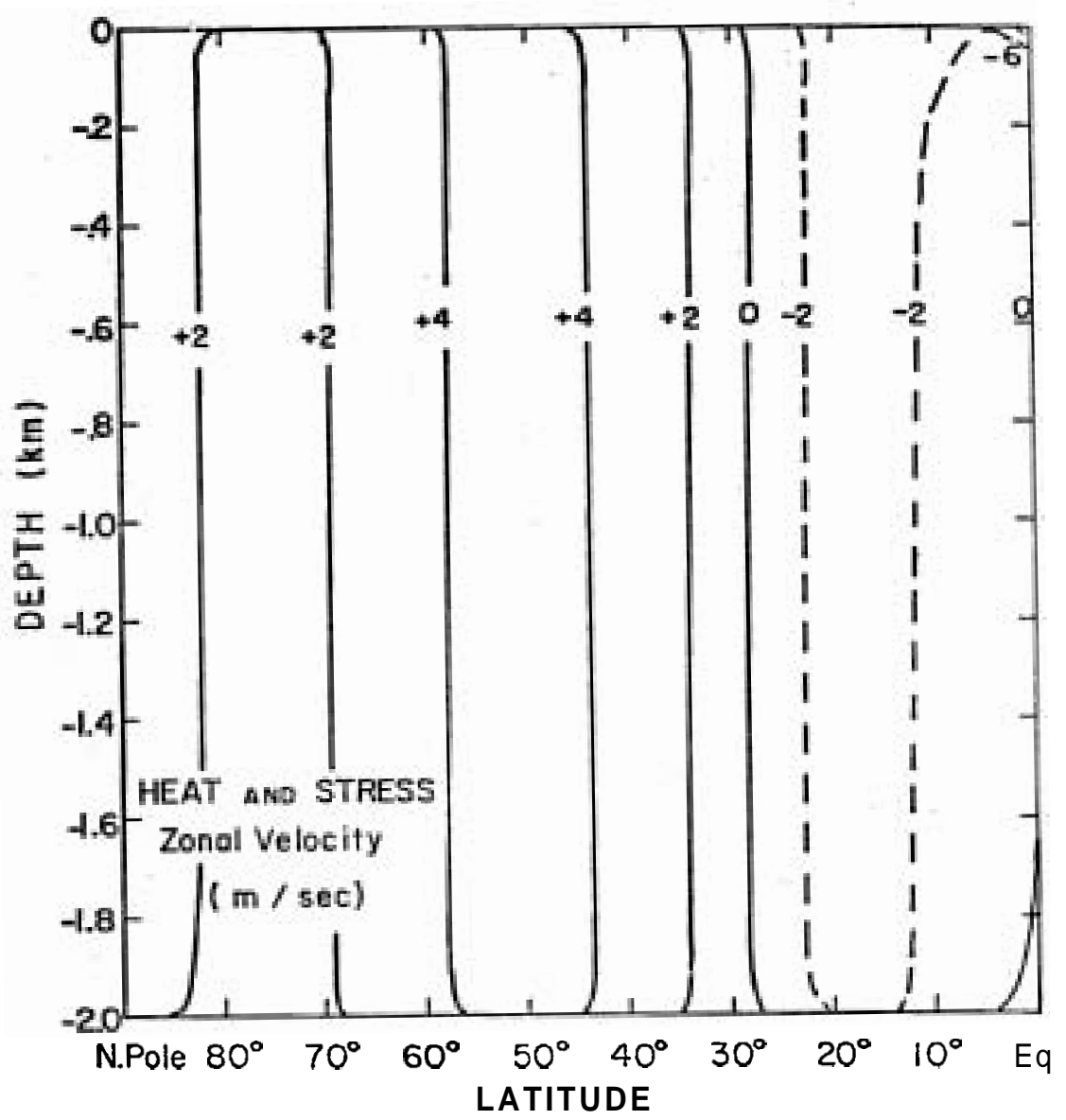

Fig. $3 \mathrm{C}$ 


\section{A TWO-DIMENSIONAL MODE OF THE THERMOHALINE CIRCULATION}

The actual circulation of the oceans is of course not axisymmetric. With a view toward constructing a simple two-dimensional model of the thermohaline circulation of the oceans for coupling with an equally simple model of the atmospheric circulation, I have adopted the following alternative to the similarity approach of Robinson, Stommel, Welander and others.

Start with the Boussinesq equations and mean density $\rho_{0}=1,{ }^{\prime \prime}$

$$
\begin{aligned}
-f v & =-p_{x} . \\
f u & =-p_{y}, \\
g \rho & =-p_{z}, \\
u_{x}+v_{y}+w_{y} & =0, \\
\rho_{t}+u \rho_{x}+v \rho_{y}+w_{\xi} & =x_{v} \rho_{z z}+x_{H} \rho_{y y},
\end{aligned}
$$

where $p$ and $\rho$ are deviations from $-\rho_{0} g z$ and $\rho_{a}$ respectively. Expand the dependent variables in powers of $x$ :

$$
\begin{aligned}
\{p, \rho, u\}(x, y, z, t) & =\sum_{n=1}^{\infty} x^{m}\left\{p_{m}, \rho_{n}, u_{n}\right\}(y, z, t), \\
\{v, w\}(x, y, z, t) & =\sum_{n=1} x^{n-1}\left\{v_{n}, \omega_{n}\right\}(y, z, t),
\end{aligned}
$$

and substitute in the above equations. Equating powers of $x$, we get

$$
\begin{aligned}
f u_{n} & =n p_{n}, \\
f u_{n} & =-p_{n y}, \\
g \rho_{n} & =-p_{n y}, \\
n u_{n}+v_{n y}+w_{n z} & =0 \\
\rho_{n t}+\sum_{r=1}^{n}\left[(n-r+1) u_{r}+v_{r}\right. & \left.\frac{\partial}{\partial y}+w_{r}^{r} \frac{\partial}{\partial z}\right] \rho_{n-r+1}=x_{v} p_{n g}+x_{w} p_{n y y}
\end{aligned}
$$

Consider now only the first power terms, corresponding to $u=1$ :

$$
\begin{aligned}
f v_{1} & =p_{1} \\
f u_{1} & =-p_{1 y} \\
g \rho_{1} & =-p_{1 z} \\
u_{1}+v_{1 y}+w_{1 z} & =0 \\
\rho_{1 t}+u_{1} \rho_{1}+Y \rho_{1 y}+w_{1} \rho_{1 z} & =x_{v} \rho_{1 z z}+x_{H} \rho_{1 y y}
\end{aligned}
$$

These may be solved subject to initial conditions and the boundary conditions, 


$$
\begin{aligned}
w_{i}(y, 0) & =w_{I E}(y, 0) \quad\left(w_{E}=x w_{I E}=\text { Ekman pumping }\right) \\
x_{v} P_{y g}(y, 0) & =-\frac{F_{1}(y)}{T_{m}}\left(F=x F_{i} ; \text { see Eqs.1.6 and } / .7\right. \text { of first section) } \\
(y,-H) & =w_{i E}(y,-H) \\
x_{v} \rho_{i g}(y,-H) & =0
\end{aligned}
$$

The zero point of $x$ must be taken at the eastern boundary to have $u$ vanish there.

All dependent variables may be expressed in terms of $w_{1}$. Thus

$$
\begin{aligned}
& p_{1}=\frac{f^{2}}{f_{y}} \omega_{i_{z}}, \\
& p_{1}=-\frac{f^{2}}{f^{2} y} \omega_{i z z}, \\
& u_{1}=-\frac{1}{f}\left(\frac{f^{2}}{f_{y}} \omega_{i z}\right)_{y}, \\
& v_{1}=\frac{f}{f_{y}} w_{i z},
\end{aligned}
$$

and substitution in the thermal equation gives

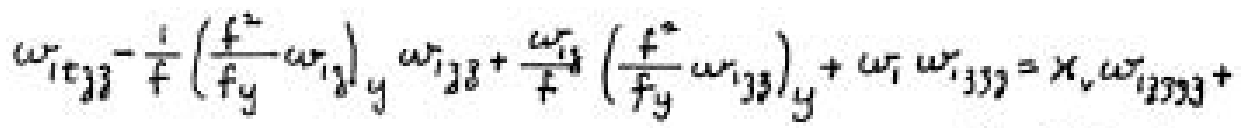

$$
\begin{aligned}
& +x_{H} \frac{f_{y}}{f^{2}}\left(\frac{f^{2}}{f_{y}} w_{z 3}\right)_{y_{y}},
\end{aligned}
$$

with the boundary conditions derived from 2:

$$
\begin{aligned}
w_{1}(y, 0) & =w_{1 e}(y, 0), \\
w_{13 s 3}(y, 0) & =\frac{g}{x_{v} T_{m}} \frac{F_{1}(y)}{f^{2} / f_{H}}, \\
w_{1}(y,-H) & =w_{1 E}(y,-H), \\
w_{133 s}(y,-H) & =0 .
\end{aligned}
$$

A particularly simple equation may be attained by ignoring lateral diffusion and assuming that $w_{1}$ is independent of $y$. Then the Ekman pumping must also be independent of $y$, and the heat flux condition requires that $F_{1}$ be proportional to $f^{2} / f$. . In this case the equation to be solved for stationary flow becomes simṕly

$$
w_{1} w_{133 z}=x_{v} w_{i j 3 z 3} \text {. }
$$

Solutions of this equation and of $2.7 \mathrm{will}$ be presented in a later publication. Those of $\mathbf{2 . 9}$ are of the boundary-layer type with a sharp thermocline. 


\title{
MICRO-SYMPOSIUM ON GEOSTROPHIC TURBULENCE
}

\author{
Abstracts of Seminars
}

\section{TWO-DIMENSIONAL TURBULENCE ABOVE TOPOGRAPHY"}

\author{
Francis P. Bretherton
}

In a turbulent two-dimemsional flow enstrophy systematically cascades to very small scales, at which it is dissipated. The kinetic energy, on the other hand, remains at large scales and the total kinetic energy is constant. Above random topography an initially turbulent flow tends to a steady state with streamlines parallel to contours of constant depth, anticyclonic around a bump. A numerical experiment verifies this prediction. In a closed basin on a beta-plane the solution with minimum enstrophy implies a westward flow in the interior, returning in narrow boundary layers to the north and south. This result is interpreted using a parameterization of the effects of the eddies on the large-scale flow. The numerical solution is in qualitative agreement, but corresponds to a minimum of a more complex measure of the total enstrophy than the usual quadratic integral.

*From a paper by Francis P. Bretherton and Dale B. Haidvogel.

\section{THE TRANSITION TO - AND THE REGION OF - GEOSTROPHIC TURBULENCE}

\section{IN A ROTATING, DIFFERENTIALLY HEATED ANNULUS*}

\section{George Buzyna}

The transition to - and the region of - geostrophic turbulence was studied experimentally in a rotating, differentially heated annulus of fluid with Prandtl number 19. The study was conducted in a region of dimensionless parameter space where the wave flow is horizontally quasi-nondivergent and quasi-geostrophic. The experimental data consisted of time sequences of fluid temperatures obtained from a network of 2016 thermistor probes distributed in the interior of the fluid. A series of 18 experiments was studied. Their location in the RoT - Ta dimensionless parameter plane is shown in Fig.l. Sixteen of these experiments were performed at the same external temperature contrast, $\Delta T$, at successively increasing rotation rates. The remaining two experiments are at the largest but at different values of $\Delta T$.

The data from these experiments reveals that the fluid motion in the wave regime is time dependent and that three different regions of time dependent motion can be identified. In particular, the time-dependent phenomena observed at successively larger values of $\Omega$ may be categorozed as amplitude vaciZlation, tilted trough vacizzation and geostrophic turbulence with a gradual transition from periodic to aperiodic time dependence. The time-averaged azimuthal temperature spectra also exhibits different characteristics in the different regions and the spectral characteristics of geostrophic turbulence are also established gradually with successively larger $\Omega$. The different characteristic regions are identified in Fig.l. The region of transition from tilted trough vacillation to geostrophic turbu- 


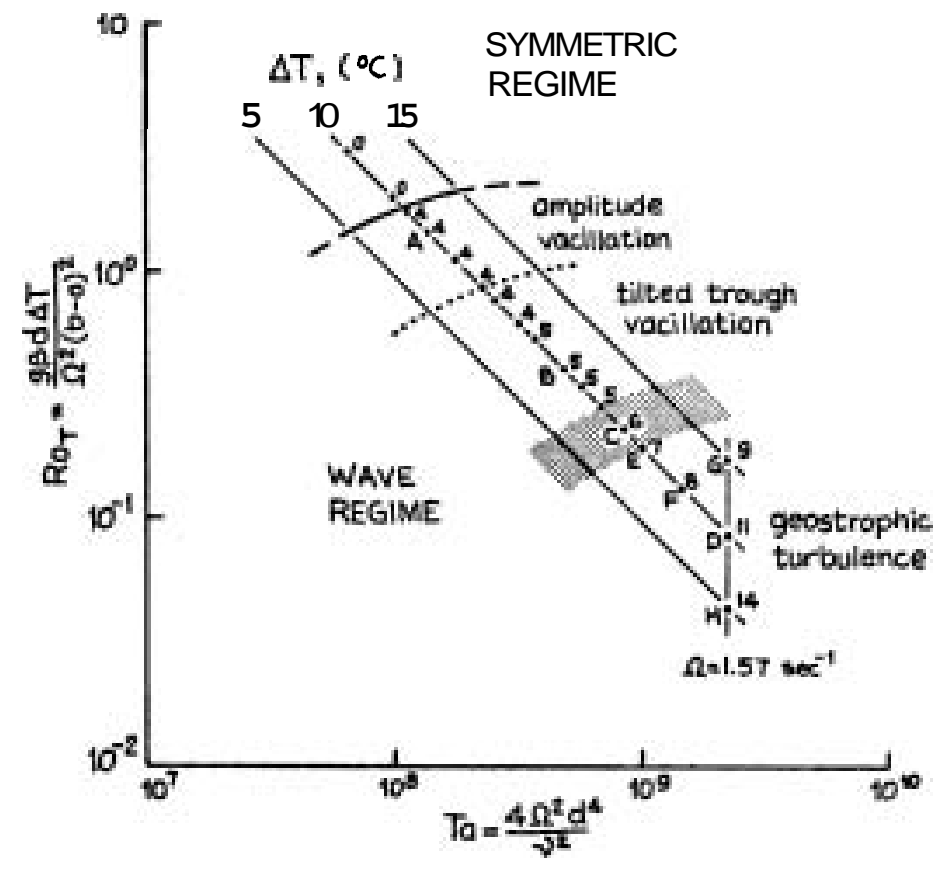

Figure 1. Regime diagram for the experiments conducted. The dots indicate experiment location, the number next to the dot indicates the dominant wave form, and the letters refer to the experiments to be presented in the following figures.

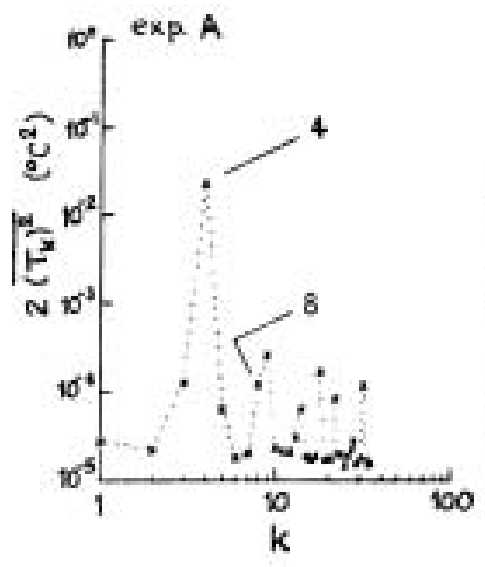

(a)

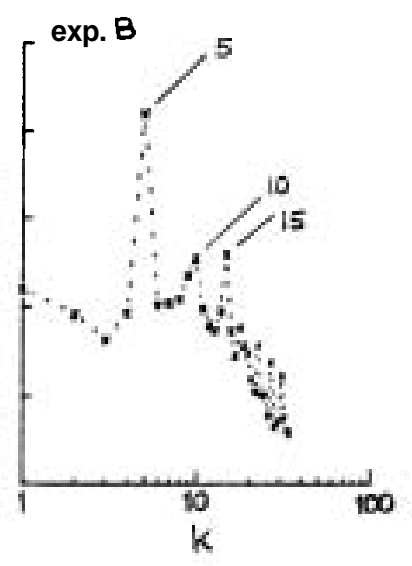

(b)

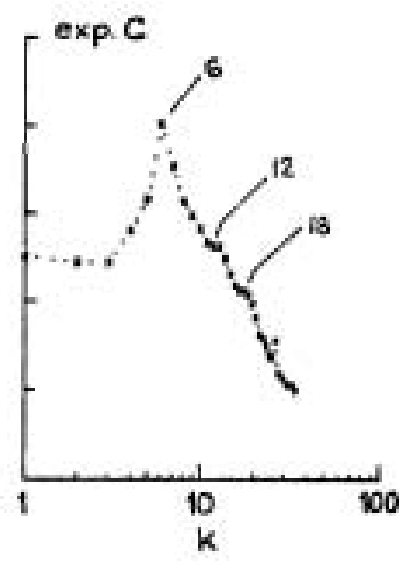

(c)

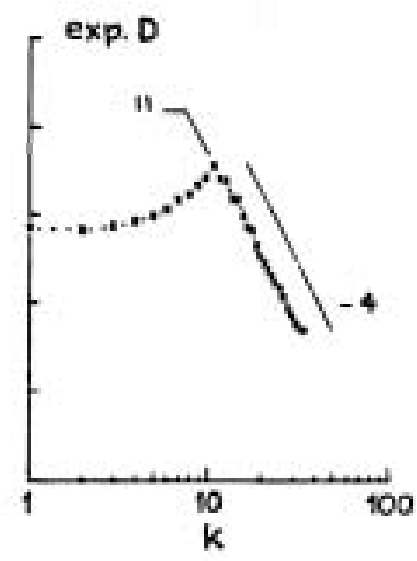

(d)

Figure 2. Time averaged azimuthal temperature variance, $\overline{\left(T_{k}\right)^{2}}$, at mid-depth and mid-gap width, as a function of wave number, $k$, at four different points in parameter space (see Fig. 1). 
lence is indicated by the stippled band. A representative time averaged azimuthal temperature variance, $\overline{\left(T_{k}\right)^{2}}$, at mid-depth and mid-gap width, as a function of azimuthal wave number, $k$, corresponding to each of the regions, including the transition region, is shown in Fig.2. The wave number spectra corresponding to the amplitude vacillation region (Fig. 2a) are characterized primarily by a single dominant mode or by a dominant mode and its side bands. The amplitude of the dominant mode and the total eddy temperature variance undergo a large cyclic variation with time (100 to 50 rotations) in this region. At higher $\Omega$, the wave number spectra corresponding to the tilted trough region (Fig. 2b) are characterized by the dominance of a single wave number and its higher harmonics. The time variation of the amplitude of the dominant mode is relatively small. The characteristic time variation here is a modulated, almost periodic fluctuation in the radial position of the main energy concentration. The time scale of this radial vacillation is between 15 to 20 rotations. The spectra in the transition region to geostrophic turbulence are characterized by a much broader spectrum of modes such that the harmonics of the dominant mode, although still distinguishable, are now less prominent (Fig. 2c). As $\Omega$ is increased in this region the time dependence acquires increasingly aperiodic characteristics.

The wave number spectra in the geostrophic turbulence region are characterized by a broad spectrum of modes. The temperature variance at the lower wave numbers is considerably larger than in the other regions, and the spectrum at high wave numbers displays a nominal -4 power slope (Fig. 2d). The time dependent behavior in this region is characterized by a considerable degree of aperiodicity in the time variation of the amplitudes of the individual modes and the total eddy temperature variance, as well as in the radial position of the amplitude concentration of the individual modes and the total eddy temperature concentration.

The time averaged azimuthal temperature variance spectra of the five experiments in the geostrophic turbulence region can be demonstrated to be similar in character by suitable normalization of the coordinates. We note that the dominant azimuthal wave number, $k$, corresponds closely to the Rossby radius of deformation scale $k_{s}=2 \pi R / \lambda_{s}$, where $\lambda_{s}$ is the Rossby radius of deformation $\left(\lambda_{\mathrm{S}}=\mathrm{Nd} /(2 \Omega), \mathrm{N}^{2}=\beta \mathrm{g}(\delta \overline{\mathrm{T}} / \delta \mathrm{z}) ; \mathrm{d}, \beta, \delta \overline{\mathrm{T}} / \delta \mathrm{z}\right.$ and $\mathrm{R}$ are the depth of the fluid, the coefficient of thermal expansion, the vertical stratification, and the radius, respectively). We also note that the time averaged temperature variance, $\overline{\left(T_{k}\right)^{2}}$, is proportional to $(A T)^{2}$. The results of the five experiments, normalized as above, with respect to the parameters in experiment $D$ (the asterisk designation), are shown in Fig. 3 . In the above representation we have assumed that $\delta \mathrm{T} / \delta \mathrm{z}$ will be proportional to $\Delta \mathrm{T}$.

The depth of the fluid was also incorporated in the temperature variance axis to account for a different fluid depth of experiment $\mathrm{H}$; the particular form was suggested by a non-dimensional formulation of the data to be given below. All experiments, with the exception of experiment $E$, show a nominal - 4 profile in the "inertial range". Experiment $E$ is represented better by a nominal -3 profile. A more extensive series of experiments in the turbulent range is currently being analized to study this 


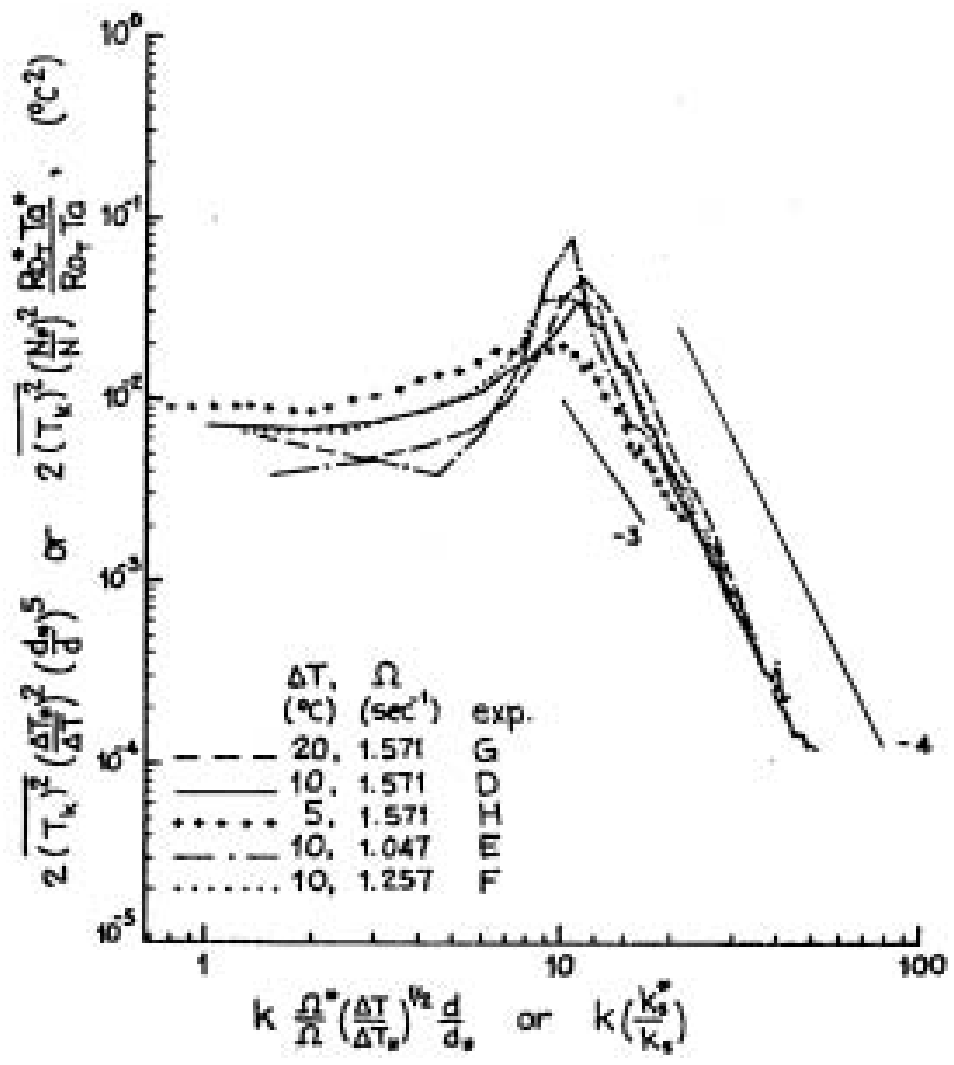

Figure 3. Time averaged azimuthal temperature variance spectra of the five experiments in the geostrophic turbulence region represented in a normalized coordinate system.

difference closer. The turbulence results presented above can also be expressed in a simple form proportional to the nondimensional parameters Ro and Ta. For such a formulation it is necessary to introduce the factor $(\overline{T N} / g)^{2},(\overline{\mathrm{T}}$ is the mean temperature and $\mathrm{g}$ is the acceleration of gravity) similar to Charney's treatment (1971, J.A.S., 28: 1087-1095) of the temperature variance spectrum. We can thus represent the data in the appropriate experiments and range by

$$
\overline{\left(T_{k}\right)^{2} /\left(\mathrm{TN}_{\mathrm{N}} / \mathrm{g}\right)^{2}=\mathrm{CRO} \mathrm{T}} \mathrm{Ta}\left(\mathrm{k} / \mathrm{k}_{\mathrm{s}}\right)^{-4} \text {, }
$$

where $C$ is a constant. We expedt that $C$ is inversely proportional to $T$ since the temperature variance spectrum should not be a function of $T$ alone with all other variables held constant.

*From a paper by George Buzyna, Richard Pfeffer and Robin Kung. 


\title{
LOW-FREQUENCY FLUCTUATIONS BENEATH THE GULF STREAM AT $55^{\circ} \mathrm{W}$ \\ Ross Hendry
}

\begin{abstract}
A kinematical and statistical description of low-frequency fluctuations of deep horizontal currents and temperature was derived from one year of moored measurements in a zonal array beneath the Gulf Stream during 1975 and 1976. Three instruments at $4000 \mathrm{~m}$ depth gave separations of 30,40 and $70 \mathrm{~km}$ between 55 and $56^{\circ} \mathrm{W}$ longitude along $40^{\circ} 30^{\prime} \mathrm{N}$ latitude. The current measurements revealed energetic fluctuations at periods greater than 20 days, with root-mean-squared currents of $12 \mathrm{~cm} / \mathrm{s}$. Energy levels in east-west and north-south fluctuations were nearly equal, but there was evidence for higher zonal energy levels at the longest time scales resolved. A rise in energy between 30- and 40-day periods was noted for both zonal and meridional currents. The correlations between horizontally separated moorings decreased noticeably over the range of scales in the array, and suggested a dominant spatial scale or eddy radius near $70 \mathrm{~km}$. Time-lagged correlation analysis showed westward propagation of features in the current variations, with apparent phase speeds in excess of $10 \mathrm{~cm} / \mathrm{s}$, while the temperature variability appeared to be dominated by large spatial scales or low frequencies and the temperature correlations between instruments was greatest at zero time lag. The Gulf Stream is a region of high energy, and although the Stream does not extend into the deep water as a coherent flow, it could contribute to the fluctuations in the deep water through instability processes, for example. Thus this experiment is located in the near-field of a large energy source and probably represents a forced regime of mesoscale oceanic variability.
\end{abstract}

\section{STATISTICAL THEORY OF STRATIFIED ROW ABOVE TOPOGRAPHY}

\author{
Jackson Herring
}

Quasi-two-dimensional rotating turbulent flow above a random topography is discussed within the framework of the direct interaction approximation (DIA), and certain ideas drawn from statistical mechanics. The DIA is presented as an expansion about randomness - in which the Eulerian acceleration is modeled as a Gaussian random stirring, and simultaneously an eddy viscosity is introduced to maintain the flows' conservation properties. The topographic component of the Eulerian acceleration is incorporated within this framework by an ensemble averaging over topographies, or equivalently, by generalizing the system studied to include an equation of motion for the topography, $h(x, t)$,

$$
\partial h(\underline{x}, t) / \partial t=0
$$

To illustrate ideas, consider a single layer whose vorticity satisfies

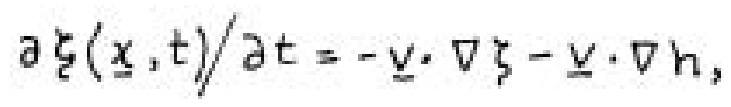

where we have chosen units such that the total layer thickness, $\mathcal{H}$, and Coriolis parameter, $f$, make no explicit appearance $(f / H=1)$. In (2), $\underline{v}(\underline{x}, t)$ is defined so that $\underline{\xi}=\bar{\nabla} \times \underline{v}(\underline{x}, t)$. Our problem is to find ensemblemean second moment information, 


$$
\begin{aligned}
\phi\left(\underline{x}, t ; \underline{x}^{\prime}, t^{\prime}\right) & \left.=\langle\hat{\xi}(\underline{x}, t)\}\left(\underline{x}^{\prime}, t^{\prime}\right)\right\rangle, \\
H\left(\underline{x}, \underline{x}^{\prime}\right) & =\left\langle h(\underline{x}) h\left(\underline{x}^{\prime}\right)\right\rangle
\end{aligned}
$$

given the initial (horizontally homogeneous) specification of $\phi$, and $H\left(x, x^{\prime}\right)$. Homogeneity makes Fourier transforms of (1) and (2) attractive and ${ }^{H}$ we denote the transformed equations as,

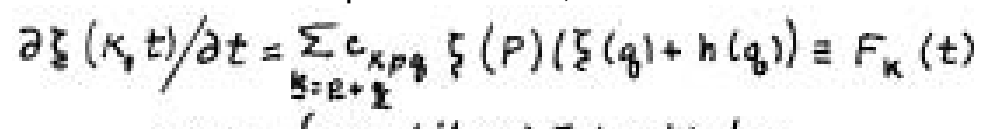

where

$$
\zeta(k)=\int \exp (i \underline{\underline{x}} \cdot \underline{\underline{x}}) \zeta(x, t) d \underline{x}, \ldots
$$

and

$$
\phi\left(\underline{k}, t, t^{\prime}\right)=\int \exp \left(-i\left(\underline{x}-\underline{x^{\prime}}\right)-\underline{k}\right) \phi\left(x, t ; x, t^{\prime}\right) d\left(\underline{x}-\underline{x}^{\prime}\right)
$$

The DIA for the system (3) reads:

$$
\begin{aligned}
\partial \phi\left(\kappa, t, t^{\prime}\right) / \partial t= & \int_{0}^{t^{\prime}} d s G\left(k, t^{\prime}, s\right)\langle F(t) F(s)\rangle_{O N} \\
& -\int_{0}^{t} d s\left(\eta(\kappa, t, s) \phi\left(k, s, t^{\prime}\right)+\hat{\eta}(k, t, s) x\left(t^{\prime}\right)\right)
\end{aligned}
$$

Here, $x(k, t)=\langle\hbar(k) \xi(k, t)\rangle$. How to obtain its DIA equation of motion will be indicated shortly.

Also $\quad \partial G\left(k, t, t^{\prime}\right) / \partial t=-\int_{t^{\prime}}^{t} d s \eta(k, t, s) G\left(k, s, t^{\prime}\right)$,

When, as in (5) $\int \eta(k, t, s) d s .$. serves the role of the relaxation rate of a small perturbation introduced into the vorticity field $\zeta(k, t)$ at time $t^{\prime}$. Similarly, $\tilde{\eta}(k, t, s)$ serves the role of the relaxation rate of the field due to a small perturbation in $h(k)$ at time $t^{i}$. We shall not record their explicit form here, but simply note that they are such as to imply for (5) energy and total entrophy conservation.

In (5), the subscript "ON ${ }^{t i}$ attached to the ensemble mean of $F(t) F(s)$ means to evaluate this term as if fields $(\xi, h)$ were multivariate Gaussian. If this were the only term on the right-hand side of (5), the equation for $\phi$ would be that for a system $\zeta$ acted on by a random Gaussian force field, $F(t)$. The last two terms in (5) $\eta$ and $\hat{\eta}$ are generalized eddy dissipation terms whose role is to restore conservation properties in the presence of a modeling of $F_{k}(t)$ as random. As is well-known (Kraichnan, 1959), (5) (without h) results from a systematic perturbation procedure applied to the statistics of $\zeta(k, t)$. It also is exact covariance equation for a model (first introduced by Phythian (1969) whose amplitude equations of motion - for the present problem - are:

$$
\partial \zeta(\underline{k}, t) / \partial t=F(\xi, h)-\int_{0}^{t} d s(k, t, s) \zeta(\underline{k}, s)-\int_{0}^{t} \hat{\eta}(k, t, s) h(\underline{k}) d s
$$

in (6) $\zeta(k, t)$ is a Gaussian random variable, whose second order statis tics are the same as $\zeta(\underline{k}, t)$. The existence of an amplitude equation of motion suffices to guarantee the realizability of the covariance equation (5). An equation for $K(K, t)$ results from multiplying (6) by $h(\underline{K})$ and ensemble averaging. 
We next briefly note certain general aspects of solutions to (5), which are amenable to study without recourse to numerical computation. First, $\emptyset\left(k, t, t^{\prime}\right)=H(k), X(k)=-H(k)$ is an exact solution to (5). Such a solution is completely static (a frozen Eulerian field), and simply corresponds to the fact that $\underline{\xi}(\underline{k}, t)=-h(\underline{k})$ satisfies $(3)$ with $\partial \underline{\zeta} / \partial t=0$. Another "non-cascading" solution to the equations of motion is the absolute equilibrium solution,

$$
\begin{aligned}
\phi\left(k, t, t^{\prime}\right) & =k^{2} G\left(k, t, t^{\prime}\right) /\left(\alpha+\beta k^{2}\right)+\left(\beta K^{2} /\left(\alpha+\beta k^{2}\right)\right)^{2}, \\
X(k) & =-\beta k^{2} /\left(\alpha+b k^{2}\right) H(k)
\end{aligned}
$$

Equation (7) may be established by the fluctuation dissipation theorem, . (Leith, 1976), which states that if $x_{i}(t)$ are a set of coupled dynamical variables possessing quadratic constant(s) of motion, then the stationary equilibrium statistics are characterized by,

$$
\left\langle x_{i}(t) x_{j}\left(t^{\prime}\right)\right\rangle=\sum_{\ell} G_{i \ell}\left(t, t^{\prime}\right)\left\langle x_{l}\left(t^{\prime}\right) x_{j}\left(t^{\prime}\right)\right\rangle
$$

Using for $x_{i}$ the set $(\zeta(k), h(k)$, and inserting the equilibrium values for $\left\langle x_{i}\left(t^{\prime}\right) x_{j}\left(t^{\prime}\right\rangle\right\rangle$ obtained by Salmon et az., (1976) yields (7). We should note that (7) is a physically realistic solution only if the a wave number range for (5) is compact, otherwise $\emptyset(k) \longrightarrow$ const. as $k \longrightarrow 0$, an unacceptable result for dissipative turbulence.

Another class of solutions to (5) are stationary, completely "frozen" in the sense of our first example above, and are most easily discussed in terms of the frequency-Fourier transform of $\emptyset\left(k, t-t^{\prime}\right), G\left(k, t-t^{\prime}\right)$. These have been known for some time (Kraichnan, 1965) for simple turbulence, but they are supposed to be unstable to non-static perturbations. Their existence corresponds to the fact that there exist a class (measure zero ?) of completely laminar solution $s$ of the Navier-Stokes equations, even for the forced dissipative case. The presence of topography may stabilize this class, indeed the numerical experiments of Bretherton and Haidvogel (1976) at one time seemed to so indicate. Numerical solutions to (5), constructed to roughly match the numerical models, indicate however that these completely static solutions are not stable; the forced, dissipative, stationary solutions have a static component (as does (7), whose magnitude increases as the topographic strength, and a dynamically active component

$\left(\emptyset^{d}\left(k, t-t^{\prime}\right) \longrightarrow 0\right.$ as $t-t^{\prime} \longrightarrow \infty$, whose lagged correlation time decreased with topography - at least for turbulence maintained by static forcing. The transition from static-dominated flow to dynamically-active flow appears to occur smoothly at

$$
|\underline{V} \cdot \nabla h| /|\underline{V} \cdot \nabla \xi| \sim \mathrm{I} \text {. }
$$

Some insight into the dynamics of the transfer mechanism implied by (5) may be obtained by wave-number Taylor series expansions for the transfer function $T(k)=\grave{\emptyset}(k, t, t)$, assuming $k$ so large that all contributions to the energy spectrum are at much smaller $k$. For example, for non-topographic turbulence, and at short times, $t,(5)$ reads,

$$
\dot{\phi}(k, t, t)=t \int^{A} d p d q\left(p^{2}=q^{3}\right)\left(q^{2}-k^{2}\right)(V(p) V(q)-U(k) V(q) \sin (p, q)
$$

$$
U(k)=k^{-3} \phi(k), \Delta \exists(k, p, q) \text { can form a triangle. }
$$


For large k, evaluate $\int^{\Delta} d p d q$. . by expanding $(q=|\underline{k}-p|)$ about $p=0$, retaining only the first non-vanishing terms. There results,

$$
\dot{\phi}=t k^{-1} \partial / \partial k\left(k^{3} \cdot \int_{0}^{k} p d p \phi(p) \partial / \partial k(k \phi(k))\right),
$$

an equation which (plausably?) states that $\dot{\emptyset}$ is proportional to the mean squared distortion of a fluid element times the length of time, $t$, the dis tortion process lasts times a homogeneous diffusion operator acting on $\emptyset(\mathrm{k})$. The latter displays a "cascade" character in that enstrophy is transferred only from neighboring $k^{\prime} s$. (Note also that $\emptyset(k)=k^{-4}$ (i.e., $E(k) \sim k^{-3}$ ) makes $\left.\dot{\emptyset}(k)=0.\right)$ At large $t, t$ in (8) must be replaced by a time scale for limiting the (strain) distortion of fluid elements. Plausibly this should be- $\left(\int_{0}^{k} \operatorname{pdp}(p)\right)^{1 / 2}$; however the DIA $(5)$ gives $\left.\sim(k) \int_{k}^{k} d p \emptyset(p)\right)^{\frac{1}{2}}$; a quantitative error whose magnitude numerical studies indicate is not serious in the energy containing region, but of serious magnitude in the enstrophy dissipation range.

Applying the above arguments to topographic turbulence (5) yields for $\underset{\alpha}{Q}(k)=\langle|\zeta(\underline{k})+h(\underline{k})|\rangle$,

$$
\begin{aligned}
\dot{D}(k)= & k^{-1} \partial / \partial k\left(k^{3} \Lambda_{1}+k^{2} \Lambda_{2}\right) \partial Q(k) / \partial k \\
& +t\left(H(k) / k^{2}\right) \int_{0}^{k} d p p^{2} Q(p),
\end{aligned}
$$

where

$$
\Lambda_{1}=t \int_{0}^{\pi} p d p \phi(p) \text {, }
$$

and

$$
\Lambda_{2}=2 t \int_{0}^{k} d p p^{3}
$$

Two effects attributable to topography are incorporated in (9). The first $\left(\sim \Lambda_{2}\right)$ is a diffusive cascade, which if acting alone would lead to an energy spectrum $k^{-1}$ instead of $k^{-3}$. It stems from the distortion of fluid elements by large-scale topography. The second represents a direct dumping of

enstrophy into scales $\mathrm{k}^{-1}$ from possibly very distant wave number regions, and hence is non-cascade in nature. Depending on the shape of $H(k)$, this term may be large, unless $Q(k) \cong 0$, or $\emptyset(k) \cong H(k) \cong-x(k)$.

We next briefly discuss two-layer turbulence above topography. First, we may note that the fluctuation dissipation theorem still implies a static component for both levels $(1=$ top and 2 =bottom). Denoting these static components $Y \phi_{i j,}^{s_{i}},(i, j=1,2)$, we find from (8),

$$
\begin{aligned}
& \phi_{11}^{3}=\bar{X}_{1}^{2} / \mathrm{H} \\
& \quad \text { st. cyc. for }(2 \rightarrow 1) .
\end{aligned}
$$

Here, $x_{i}=\left\langle\zeta_{i} n\right\rangle$. Preliminary numerical calculations indicate that for topographies typical of those used in (most?) numerical simulations, the normalized spectrum of $X_{1}$ is quite small, leading to the expectation that where topographic effects are significant, they act to significantly decorrelate levels 1 and 2 . 
In closing, we should stress that the statistical theory contains in its explicitly calculated list of ingredients auxiliary quantities which are of interest by themselves, for example, time scale information is contained in the $G_{i j}\left(k, t^{\prime}\right)$ used in (5), and its generalization to two levels. An interesting byproduct of $G$ for a barotropic layer is the time scale for the statistical diffusion of a small perturbation of very large length scale - compared to turbulence and topographic features. The reciprocal of this time scale is an effective eddy dissipation (really a topographic drag), and according to the DIA it is,

$$
\eta(k \rightarrow 0)=(\pi / 2) \int_{0}^{\infty} d p / p(x(p)+H(p)) / \eta(p)+\theta\left(\kappa^{2}\right)
$$

where we roughly may estimate $\eta(p)$ on the right-hand side of this expres-. sion by $\sqrt{\int \alpha p H[p]}$. Note that $\eta(k)$ tends to a finite limit as $k \longrightarrow 0$. At small $k, X(k) \longrightarrow 0$, so that we'expect $\eta(0)>0$, but its precise value depends on spectral details. It vanishes for the static solution, $\$(k)=-h(k)$.

Finally, we should point out that the methods of statistical closure are suspect for flows containing regions of strong trapping, a condition which will be obtained in applications. We do not know to what extent trapping effects vitiates statistical turbulence theory. However, one hopeful recent sign that trapping coes not wreck things stems from the work of Holloway (1977) ' (see also his paper in the present volume), who has applied the test field model to the topography problem. His results - which include a comparison to numerical simulations starting from random initial conditions - appear very encouraging.

A more detailed account of much of this work will soon appear in L.A.S. (Herring, 1977).

References

Bretherton, F.B. and D. Haldvogel, 1976. J.Fluid Mech. (in Press).

Herring, J.R. 1977 to appear in J.A.S.

Holloway, G. 1977 Preprint.

Kraichnan, R.H. 1959. J.Fluid Mech. 5: 497-543.

Kraichnan, R.H. 1964. Phys.Fluids, Z: 1163-1168.

Phythian, R. 1969. J.Phys. A 2: 181.

Salmon, R., G.Holloway, and N.Hendershott, 1976. J.Fluid Mech. 75: 691-703. 


\section{STATISTICAL ACCOMMODATION AMONG QUASIGEOSTROPHIC·EDDIES}

(What can you learn from a box of red and blue marbles?)

\section{Greg Hol loway}

The statistical behavior of a stratified flow in a closed basin of arbitrary shape, including the effects of self-advection, of latitudinal variation of Coriolis parameter and of irregular bottom topography, may be understood qualitatively as a tendency to increase entropy. Given very artificial restrictions whereby the motion is represented on finitely many eigenfunctions without forcing or dissipation, exact calculations of maximum entropy states ("thermal" equilibria) are possible. Generalizations of such equilibrium ideas as temperature (the inverse of the variation of entropy with respect to some parameter of the flow) may also provide a heuristic account of forced, dissipative, disequilibrium flows. A flow whose spectrum is more red than energy equipartition is characterized by a temperature defined with respect to vorticity variance, which is positive and small or "cold". If an initial condition is considered in which eddies are randomly distributed over a basin and uncorrelated among layers of motion and with underlying topography, then temperatures defined with respect to these correlations are infinite or "hot". In the ensuing evolution, the flow becomes barotropic in larger scales of motion, becomes correlated with bottom topography and develops a westward flow in the interior with eastward currents at the north and south boundaries of the basin. This evolution describes a cooling of the uncorrelated distribution with a warming of the vorticity variance spectrum. An intense western boundary current develops as a part of the "heat" transfer from hot to cold reservoirs.

Although the simple idea of a tendency toward "thermal" equilibration accounts qualitatively for many observed features of ocean circulation models, a quantitative estimate of statistical evolution depends upon finding approximations for rates of interaction. Such approximate rates can be estimated only for limited, idealized cases, here the cases of barotropic planetary waves and barotropic motion above irregular topography, both considered to be spatially statistically homogeneous in an ensemble average.

Nonlinear barotropic planetary waves are given by $D_{t}(\zeta+\beta y)=\mathscr{D}(\zeta)$ where $D_{t}$ is a substantial derivative following the motion induced by a vorticity field $\zeta=\hat{z}$. curl $\mu$ and $D(\zeta)$ is an arbitrary dissipation operator. If $\zeta$ is a Fourier coefficient of $\zeta$, evolution of the time-simultaneous variance $z_{k}=\left\langle\zeta_{k} \zeta_{-k}\right\rangle$ is sought in a form

$$
\left[\frac{d}{d t}+2 B_{k}\right] z_{k}=\sum \theta_{k p q} B_{k p q}\left(z_{p} z_{q}-z_{p} z_{k}\right)
$$

where $B_{k p q}$ is a geometric factor given by the equation of motion and $\theta_{k} \mathbf{p q}$ is a time scale estimating the coherence time among triples of vorticity coefficients $\zeta_{k}, \zeta_{p}$ and $\zeta_{s}$. An approximation for $\theta_{\kappa p q}$ is obtained by observing that the equation which purports to describe the statistical evolution of 3 in fact describes exactly the statistics of a model variable $\bar{\zeta}_{k}$ governed by 


$$
\left(\frac{d}{d t}+D_{k}+\eta_{k}+i \Omega_{k}\right) \tilde{\zeta}_{k}=f_{k}
$$

where

$$
\begin{gathered}
\eta_{K}=\frac{1}{2} \Sigma \theta_{k p q} B_{k p q} z_{p}, \\
k+p+q=0
\end{gathered}
$$

$\Omega_{k}=-B K_{x} / k^{2}$ and $f_{k}$ is a random force whose variance is

$$
\begin{gathered}
\left\langle f_{k} f_{-k}\right\rangle=\sum \theta_{k p q_{1}} B_{k p q} z_{p} z_{q} . \\
k+p+q=0
\end{gathered}
$$

Then $\theta_{k p q}$ is taken as the coherence time for triples of model variables , a d q, i.e.

$$
\boldsymbol{e}_{k p q}=\frac{\mu_{k}+\mu_{p}+\mu_{q}}{\left(\mu_{k}+\mu_{p}+\mu q\right)^{2}+\left(\Omega_{k}+\Omega_{p}+\Omega_{q}\right)^{2}}
$$

where nominally $\mu_{k}=D_{k}+\eta_{k}$. In fact, to obtain a $\theta_{k p q}$ which is not unrealistically oversensitive to motion on large scales, $\mu_{k}$ is modified to

$$
\mu_{k}=D_{k}+\frac{\lambda^{2}}{2} \Sigma \theta_{k p q} \widehat{B}_{k p q} z_{p}
$$

where $\hat{B}_{\text {rpq }}$ is another geometric factor, here suggested by the turbulent "test field model", and $\lambda$ is an empirical constant of order unity.

Nonlinear barotropic motion above irregular topography is described similarly. The equation of motion is $D_{t}(\zeta+h)=D(\zeta)$ where the depth of fluid is $H(x, y)=H_{\theta}\left(J-h(x, y) / f\right.$ with $f$ the Coriolis parameter and $h / H_{0}$ a small number of order the Rossby number. Equations for evolution of timesimultaneous variance $Z_{k}$ and correlation $R_{k}=$ Real $\left\langle I_{, n_{-k}}\right\rangle$ are sought in a form

$$
\begin{gathered}
\left(\frac{d}{d t}=2 D_{K}+2 \eta_{K}\right) Z_{K}=F_{K}+2 Y_{K} R_{K} \\
\left(\frac{d}{d t}+D_{K}+\eta_{K}\right) R_{K}=Y_{K} H_{K}
\end{gathered}
$$

where $\eta_{k}, Y_{\mu}$ and $F_{k}$ are given by sums over second moments and pairwise products of second moments $Z_{P}, R_{P}$ and $H_{P}=s h_{p} h_{-p}>$ weighted by a coherence time $\theta_{\text {mpq }}$. Again the equations for $Z_{k}$ and $R_{k}$ describe exactly a model variable governed by

$$
\left[\frac{d}{d t}+D_{k}+\eta_{k}\right] \tilde{\zeta}_{k}=f_{k}+\gamma_{k} h_{k} \text {. }
$$

Then $\theta_{k p q}=\left(\mu_{k}+\mu_{p}+\mu_{q}\right)^{-1}$ where 


$$
\begin{gathered}
\mu_{k}=D_{k}+\sum \theta_{k p q}\left(\frac{\lambda^{2}}{2} \hat{B}_{k p q} z_{p}+c_{k p q} R_{p}+D_{k p q} H_{p}\right) . \\
k+p+q=0
\end{gathered}
$$

$C_{k p q}$ and $D_{k p q}$, which appear in $\eta_{k}$, are geometric coefficients obtained from the equation of motion.

Comparison of these statistical predictions with numerical flow simulations shows good agreement. An earlier empirical estimate of from studies of two-dimensional turbulence is found to agree with statistics both of planetary waves and of flow over topography for a number of choices of topographic spectra and dissipation operators. The planetary wave closure predicts (1) the inhibition due to wave propagation of the tendency of twodimensional flows to organize energy into larger scales of motion and (2) the evolution, from an initially isotropic eddy field, of anisotropy preferring zonal motion. The closure above topography predicts (1) the effect of topography enhancing or inhibiting spectral transfer depending on the strength of correlation $R_{k}$ and (2) the development of a steady part of the vorticity field, viz. $\bar{\zeta}_{k}=\gamma_{k} h_{k}\left(D_{k}+\eta_{k}\right)^{-1}$,

Two interesting remarks are, firstly, that $\theta_{k p q}$ which appears in the planetary wave closure limits on $\theta_{\kappa p q_{i}}=\pi \delta\left(\Omega_{k}+\Omega_{p}+\Omega_{q}\right)$ when the motion is weak, i.e. $\mu_{k}{ }^{2} / \Omega_{k}{ }^{2} \rightarrow 0$, and without dissipation. In this limit the closure continuously recovers the wave resonant interaction approximation and suggests a more general formalism for approximating interactions among finite amplitude waves. Another remark is that the model variable $\widetilde{\zeta}_{k}$ corresponding to the closure above topography depends linearly on $h_{k}$ although $h_{k}$ does not appear in the equation of motion of the actual variable $\zeta_{k}$.

\section{APPROXIMATE MODES OF TURBULENCE}

\section{Robert Kraichnan}

Turbulence is a nonlinearly coupled process which exhibits welldefined structures (shear surfaces, vortices, etc.) whose precise form is plastic and which are put together in a partly random fashion to made up the whole flow. It is very difficult to adequately describe this situation in simple statistical terms. Nevertheless, considerable success has been attained by closure models which deal only with low-order moments. The direct-interaction approximation is central to such models and has a builtin consistency which explains in part why the models work. The relation of such models to perturbation theory, and the extension of the models to deal properly with the convection of small scales by large scales is discussed in this lecture. Some outstanding problems for future work, including numerical integration of the models, their use in subgrid-scale representations, and the treatment of intermittency in small scales, are outlined. 
THE GENERAL CIRCULATION IN A B-PLANE CHANNEL

\author{
James C. McWilliams
}

The problem studied can be described as follows: A stably stratified two-layer fluid is forced by a steady, zonal body force in the upper layer. The fluid is rotating with a linear meridional gradient in the Coriolis parameter (the B-plane approximation). The geometry is that of a channel with zonal periodicity. Hydrostatic and quasigeostrophic assumptions are made. There is a linear Rayleigh frictional drag force (bottom friction) in the lower layer and a (numerically weak) lateral Newtonian friction in both layers. For sufficiently intense forcing, the rectilinearly driven motion becomes unstable before a steady frictional balance can occur. Therefore, the equilibrium solution is a statistical one withthe time mean circulation balanced by transient, turbulent, geostrophic eddy fluxes. A numerical solution for this equilibrium state is described. The turbulent eddies are an essential element in the calculation, and have not been parameterized.

The solution has the character of a meandering jet, in phase in the two layers but more intense in the upper, thinner layer. The upper layer exhibits smaller horizontal scales than the lower. The transient eddies have their variance concentrated in the region of the mean jet, and have an r.m.s. amplitude in streamfunction of about $20 \%$ of the mean. The percentage is higher in velocity, vorticity, etc. The total transport of fluid down the channel is constant in time during the equilibrium period, whereas the volume integrated, total energy shows vigorous fluctuations.

The energy cycle of the equilibrium state can be summarized as follows: The mean zonal forcing does work on the fluid, generating mean upper layer kinetic energy. Partly this is communicated downwards through mean pressure work $(\bar{w} \bar{p})$ and so lost to mean bottom friction, partly this is baroclinically converted to eddy energy. - The eddyenergy is communicated vertically by eddy pressure work ( $w^{\prime} p^{\prime}$ ) either to a loss through eddy bottom friction or to a barotropic reverse conversion between eddy and mean, upper layer kinetic energies. In a mean momentum budget, downgradient transfer of momentum in the vertical can be identified with the baroclinic conversion, and an up-gradient horizontal Reynolds stress divergence with the reverse barotropic conversion. The vertical momentum transfer can also be characterized as an isopycnal form drag, a horizontal eddy mass and buoyancy flux. In a mean potential vorticity budget, expressions of these processes can also be identified.

One can make a numerical interpretation of the efficiency of the vertical momentum transfer accomplished by the geostrophic turbulence. In an equilibrium state, the upper layer upper surface stress (felt as a body force in the upper layer momentum balance) must have a magnitude equal to both the bottom frictional stress and an effective interior stress, which we might parameterize as

$$
K_{v} \frac{\partial \tilde{u}}{\partial z}
$$

For a surface stress of 1 dyne $\mathrm{cm}^{-2}$ and a numerically calculated $\partial \alpha / \partial_{z}$ of $10^{-4} 5^{-1}$ in the center of the jet, the equivalent vertical diffusivity of momentum is $k_{y}=10^{4} \mathrm{~cm}^{2} / \mathrm{s}$. This is quite a large value by traditional standards. 
An examination of eddy frequency spectra shows the presence of several moderately broad peaks superimposed upon a generally monotonically decreasing (i.e., red) structure. The spatial structure of the eddies associated with these spectral peaks is exposed by a principal component analysis; that is, the streamfunction is represented separately as

$$
\psi(x, y, z, t)=\sum_{i} a_{i}(t) f_{i}(x, y, z) \text {. }
$$

where the $f_{i}\left(\underline{x}_{\ell}\right)$ are chosen as the orthonormal eigenvectors of the spatial covariance matrix,

$$
A_{l m}=\frac{\sqrt{d V_{f} d V_{m}}}{V} \overline{\psi^{\prime}\left(\underline{x}_{l}, t\right) \psi^{\prime}\left(\underline{x}_{m}\right)^{t}} \text {. }
$$

$V$ is the fluid volume and $\mathrm{d} V_{\boldsymbol{l}}$ the volume element associated with the point $\underline{x}_{l}$. The functions thus selected are the optimally efficient descriptors of the volume integrated streamfunction variance. The first such function accounts for $34 \%$ of the total variance and represents a nearly barotropic meridional displacement of the jet without increment in transport. Other functions occur in pairs, relatively displaced by a quarter cycle in space and time: they represent zonally propagating pairs. Their character (spatial structure and phase propagation) is qualitatively similar to that of linear, neutrally stable jet modes (McWilliams, 1977, J.P.o.). Each can be identified with one of the frequency spectral peaks described above.

The first three such propagating pairs, together with the jet displacement function, account for $88 \%$ of the streamfunction variance.

There is a particular principal component pair, associated with the highest frequency spectral peak (period nine days), which can be identified with the linearly predicted maximally unstable mode for the time and zonal mean jet. lats horizontal scale is slightly larger than the internal deformation radius. This unstable component, however, is of relatively small energy. It accounts for only one percent of the total streamfunction variance. Thus, while the baroclinic energy conversion - the eddy generation rate - is associated with a baroclinically unstable wave process, the eddy enërgy does not reside in the unstable wave. Rather it is efficiently transferred to larger scale, more barotropic, energetically neutral (with respect to baroclinic conversion) eddies in a manner analogous to the reverse cascade of two-dimensional turbulence.

A fuller discussion of the preceding and related results can be found in McWilliams, Holland, and Chow (1977, Dynamics of Atmosphere and Oceans). 


\title{
SIMULATED DYNAMIC BALANCES FOR MID-OCEAN MESOSCALE EDDIES
}

\section{W. Brechner Owens}

Eulerian potential vorticity and heat balances calculated for a multi-layered quasigeostrophic model are shown to be consistent with those inferred from the MODE data. Above the thermocline the balances are the result of the turbulent cascade of relative vorticity which creates a gap between centers of mass of enstrophy and energy spectra so that relative vorticity nearly acts as a passive scalar. In the Lagrangian frame this advective effect is suppressed and one observes a coupled response by relative vorticity and vertical vortex stretching to changes in planetary vorticity. In the bottom layer there is a time-mean flow following $\mathrm{f} / \mathrm{H}$ contours which is the consequence of a down-gradient flux of vorticity. Superposed on this flow are topographic Rossby waves. The intermediate layers have a balance which is a mixture of those described above.

\section{EDDY-INDUCED ABYSSAL-OCEAN CIRCULATION}

\author{
Peter B. Rhines and W illiam R. Holland
}

The structure of eddy-induced mean circulation can be understood by invoking two properties characteristic of geostrophic turbulence: 1), the cascade of potential vorticity, q, to small scales;. 2) the transport of $q$ in space, in the presence of large-scale q-fields. The spatial vorticity transport is directly related to the stresses transferred vertically and laterally in the fluid.

The vorticity transport depends on the Taylor diffusivity tensor and the gradient of the large-scale field. In extreme cases, "memory effects" may alter the transport and cause the diffusivity to be effectively negative. This is apparent, for example, when a zonally oriented channel is driven by external forcing whose time average vanishes. Then, the net angular momentum must vanish, the area-average diffusivity vanishes, and eastward jets appear, alternating with broader westward flows.

Often, as with the ocean's abyssal flow, net forces are available to mix q. A dynamically consistent model follows from assuming that the deep flow is weak relative to the upper-level circulation, and expanding "downward". The mean thermocline shape and $\beta$-effect provide the largescale q-field, and positive diffusivity then leads to two gyres of abyssal flow beneath each surface gyre. This gives a dynamical model of Worthington's abyssal gyre, observed beneath the Gulf Stream system, and predicts a second, cyclonic cell to the south. The latter circulation is consistent with the observed energetic nature of the Western Boundary Undercurrent, which is the western boundary layer for this region. 
In the case of equal layer depths and uniform vertical energy density, the quadratic integral invariants of two-layer quasi-geostrophic flow are closely analogous to the energy and enstrophy in twodimensional turbulence. Let $U(k)$ be the barotropic kinetic energy and $E(k)$ the total baroclinic energy (i.e. the baroclinic kinetic plus available potential energy). Under adiabatic conditions, the two-layer flow conserved the total energy,

$$
\sum_{\underline{k}} U(\underline{k})+E(\underline{k})
$$

and the potential enstrophy (of either layer),

$$
\sum_{\underline{k}} k^{2} U(\underline{k})+\left(k^{2}+k_{R}^{2}\right) E(\underline{k}) \text {. }
$$

where $k_{R}^{-1}$ is the internal deformation radius.

The invariants (1) are significant because they are preserved by individual triads. Let $k, p, q$ be any three horizontal wavenumbers that sum vectorially to zero. The two-layer flow consists of UUU triads and UEE triads which are respectively constrained by

and

$$
\begin{aligned}
& \dot{U}(\underline{k})+\dot{U}(p)+\dot{U}(q)=0 \\
& k^{2} \dot{U}(k)+p^{2} \dot{U}(p)+q^{2} \dot{U}(q)=0 \\
& \dot{U}(\underline{k})+\dot{E}(p)+\dot{E}(q)=0 \\
& k^{2} \dot{U}(\underline{k})+\left(p^{2}+k_{R}^{2}\right) \dot{E}(p)+\left(q^{2}+k_{k}^{2}\right) \dot{E}(q)=0
\end{aligned}
$$

The constraints (2) are the same as in ordinary two-dimensional turbulence. As is well-known, (2b) prevents effective energy transfer between scales of motion in both extremely local and extremely nonlocal triads. For intermediate triads, the spreading of energy from middle to outer wavenumbers generally favors the lower wavenumber.

For the UEE triads, , constitutes a physically important dividing scale. If $k, p, q \gg k_{R}$, then the constraints (3) are the same as (2), but if $k, p, q$, ss $k_{R}$ then:

(i) the energy transfer is between baroclinic components only;

(ii) the triad interactions may be local or nonlocal;

(iii) there is no inhibition against energy transfer to higher horizontal wavenumbers.

The above ideas may be used to intuit the general flow of energy in a two-layer system. Let the system be driven near some minimum wavenumber $k_{0} \leftrightarrow k_{R}$, and damped principally near $k_{0}$ and $k_{D} \gg k_{R}$. The dissipation at $k_{D}$ may be construed as the scattering of enstrophy into three-dimensional flow on scales too small to feel the strong rotation. Now if $k_{D}>>k_{R}$, the net production of total energy near $k_{0}$ must be zero in a steady state. The potential enstrophy production at $k_{0}$ therefore has the sign of the baroclinic energy production (cf. lb), and it must be positive since there is only an enstrophy sink at $k_{D}$. It follows that the only possible steady state is one in which 
(i) net baroclinic energy is produced near $k_{0}$;

(i i) the baroclinic energy moves toward $k_{R}$ in UEE triads;

( $\mathrm{i} i \mathrm{i}$ ) the baroclinic energy converts to barotropic mode near $\mathrm{k}_{\mathrm{R}}$;

(iv) barotropic energy moves back toward $k_{0}$ in UUU triads. The rightward energy transfer may be extremely nonlocal as in baroclinic instability $\left(k \approx p=0\left(k_{R}\right) \gg q\right)$, or it may approach a turbulent cascade.

I have studied baroclinically-forced equilibrium flow with a simple eddy-damped-Markovian turbulence closure, which predicts the evolution of wavenumber spectra from the spectra themselves. The equilibrium spectra are surprisingly sensitive to geophysically plausible changes in the forcing and dissipation. Moderately damped flow has most of its energy in large-scale barotropic currents. However, if the damping is increased, the equilibrium barotropic decreases dramatically at the lowest wavenumbers and the total kinetic energy spectrum is actually bimodal, with a maximum in the forcing range at $k_{0}$ and another between $k_{0}$ and $k_{R}$.

\section{EDDY-ENERGY IN THE WESTERN NORTH ATLANTIC}

\section{William J. Schmitz}

Eddy kinetic energy in the western North Atlantic is observed to increase approaching the Gulf Stream System from the interior of the subtropical gyre at depths from 500 to $4000 \mathrm{~m}$ along $55^{\circ} \mathrm{W}$ longitude; by a factor of 30 in the thermocline and by over two orders of magnitude at $4000 \mathrm{~m}$, and at this latter depth tends to decrease moving up the continental rise from the Gulf Stream. The relative vertical distribution of eddy kinetic energy is found to be less depth dependent approaching the Gulf Stream System (with increasing energy level?). The shape of the kinetic energy frequency spectrum varies with depth and with geographical position; although spectral estimates in the thermocline in the MODE-I area $\left(28^{\circ} \mathrm{N}, 70^{\circ} \mathrm{W}\right)$ are dominated by longer time scales, at a site near the Gulf Stream $\left(37^{\circ} 30^{\prime} \mathrm{N}\right.$, $\left.55^{\circ} \mathrm{W}\right)$ the thermocline spectrum is more weighted toward the temporal mesoscale ( $\sim 100$ days) that dominates at $4000 \mathrm{~m}$ depth in the MODE-I area.

Spectral estimates at depth appear to be further weighted toward shorter time scales approaching the continental rise, slope, and shelf.

If the eddy kinetic energy is divided into pieces with periods less than 100 days in one band and greater than 100 days in the second, then: (1) The relative vertical distribution of eddy kinetic energy near the Gulf Stream does not differ significantly from band to band, whereas in the MODE-I region the lower frequencies are much more depth dependent than the higher. (2) The relative vertical distribution of eddy kinetic energy for periods less than 100 days is not appreciably different at the two locations. (3) The relative vertical distribution of eddy kinetic energy at the lower frequencies in the MODE-I region does not differ significantly from the total distribution at a site further into the interior of the gyre.

Time-averaged currents at all depths where data are available show a relatively narrow and comparatively depth independent return flow for the 
Gulf Stream System, along 550 with about $25 \times 10^{6} \mathrm{~m}^{3} \mathrm{~s}^{-1}$ relative to the bottom and approximately $45 \times 10^{6} \mathrm{~m}^{3} \mathrm{~s}^{-1}$ associated with the "bottom transport". 
FEUOWSHIP LECTURES

WAVE BREAKDOMN IN A SIMPLE MODEL OF A DENSITY STRATIFIED ROW

Gregory Gartrell, Jr.

\section{INTRODUCTION}

Many problems in engineering and oceanography involve the flow of density stratified fluids. Examples are the flow of sewage discharged to the ocean, the spreading of hot water discharged from power plants over cooler receiving waters, as well as the motions of oceanic currents. A good understanding of the nature of stratified shear flows is necessary in order to solve many engineering and oceanographic problems.

One problem of special interest is the sudden onset of turbulence in a stably stratified fluid. Woods $(1968$, 1969) has observed flows in the ocean which, although being initially laminar and stably stratified, suddenly experience breakdown and become turbulent. In general, he observed deep layers of laminar fluid with small density gradients separated by the layers of fluid with much higher density gradients. In addition, there were internal waves in the thin layers as well as substantial velocity gradients. Occasionally Woods observed the internal waves as they became unstable, and began to roll up. After a short time, the fluid suddenly became turbulent in the thin layer.

The writer has observed, in laboratory experiments, situations in which a thin layer of laminar fluid with moderate density and velocity gradients separates deeper regions of turbulent fluid. The fluid in the laminar region is observed to have internal waves propagating along it. In this case the mixing between the two layers is critically dependent upon the stability of the interface. In both this problem, where the interface seems to be stable to small disturbances and in the flows observed by Woods, a natural question arises as to the nonlinear stability of stratified shear flows.

The problem to be examined here is a simplified model of a stratified shear flow. The model considered consists of two infinitely deep layers of fluid of slightly different densities separated by a thin transition region. It is assumed that the density is a continuous function of the vertical coordinate, that the upper layer moves with velocity -Uo and that the transition region is stationary. Thus, there are two interfaces with a velocity discontinuity. The effects of a small wave-like perturbation on the mean flow are considered. In particular the conditions for which a secondary wave of small scale can be trapped and focused, as given by Landahl (1972) and Landahl and Criminale (1977), are examined. In section II, some of the writer's experimental work is presented as an introduction to the problem. Section 111 gives a brief review of previous work on stability in stratified flows, and a summary of some of the results of Landahl (1972) and Landahl and Criminale (1977). Sections IV and V present the problem and the breakdown conditions for the simplified flow model considered. 
11. EXPERIMENTAL WORK

Experiments in stratified shear flows are presently being conducted in the Keck Hydraulics Laboratory at California Institute of Technology. The experiments are carried out in an open-channel flume $40 \mathrm{~m}$ long, $60 \mathrm{~cm}$ deep and $110 \mathrm{~cm}$ wide. At the upstream end, there is a horizontal splitter plate along which the flow enters the channel. The water above the plate is heated to a temperature higher than that of the water below the splitter plate. In addition separate pumps are used to pump the warm and cold water. Thus, both the densities and mean velocities of the two layers are each independently controlled.

Typically, the mean velocity of the upper layer of water is between $5 \mathrm{~cm} / \mathrm{s}$ and $15 \mathrm{~cm} / \mathrm{s}$, while the velocity of the lower layer ranges between $5 \mathrm{~cm} / \mathrm{s}$ and $10 \mathrm{~cm} / \mathrm{s}$. The temperature difference can be as large as $10^{\circ} \mathrm{C}$, with a corresponding relative density difference, $\Delta \%$, of about .003 . The depth of the upper layer is typically $10 \mathrm{~cm}$ to $15 \mathrm{~cm}$, while the depth of the lower layer is $30 \mathrm{~cm}$. The overall Reynolds number of the flow is usually in the range of $8 \times 10^{4}$ to $10^{5}$.

It is observed that a two-dimensional mixing layer develops immediately following the end of the splitter plate. Then, if $\Delta \%$ is large enough and the velocity difference between the layers, $\Delta U$, is small enough, the mixing layer collapses and forms the interface between the upper and lower layers. Whether or not the mixing layer collapses, or the two layers continue to mix depends upon, among other things, the value of the Keulegan number,

$\left(\nu_{g} \Delta \%\right)^{1 / 3} \Delta U$, where $\nu$ is the kinematic viscosity and 3 is the acceleration due to gravity. (This number is obtained by combining the Reynolds number and the densimetric Froude number in such a way that the length scale is eliminated.) If the mixing layer collapses, then downstream one observes a laminar region separating a warm turbulent layer above from a colder turbulent layer below. The turbulence is generated at the boundaries of the flow.

Temperature measurements made with thermistors indicate that the interfacial region is usually thin, on the order of a few centimeters. In addition the measurements show that the interface has numerous internal waves propagating along it.

Figures 1 and 2 show typical centerline velocity and density profiles made at three locations along the flume. In this example, the mean velocity was about $12 \mathrm{~cm} / \mathrm{s}$ in the upper layer and about $6 \mathrm{~cm} / \mathrm{s}$ in the lower layer. The temperature difference $2.6^{\circ} \mathrm{C}$ corresponding to a value of $\Delta \rho / \rho$ of about $8 \times 10^{-4}$. The total depth of the flow was $40 \mathrm{~cm}$. The velocity profiles were obtained from successive photographs of a dye streak and temperature profiles were obtained from an array of thermistors. One can see that the velocity gradient gradually decreases downstream. This is typical. However, the flow is smooth, almost always laminar in the interface region. The dye (from the streaks used for photography) in the interface does not mix, but remains there in a thin streak, gradually stretching out. The dye above and below the interface mixes in a few meters.

If the density difference is large enough, and the turbulence level small enough, the interface usually changes only slightly downstream. In general, the interface sharpens a small amount, as water at the edges of the interface is mixed by the turbulence. In this case the turbulence does not penetrate the interface. 


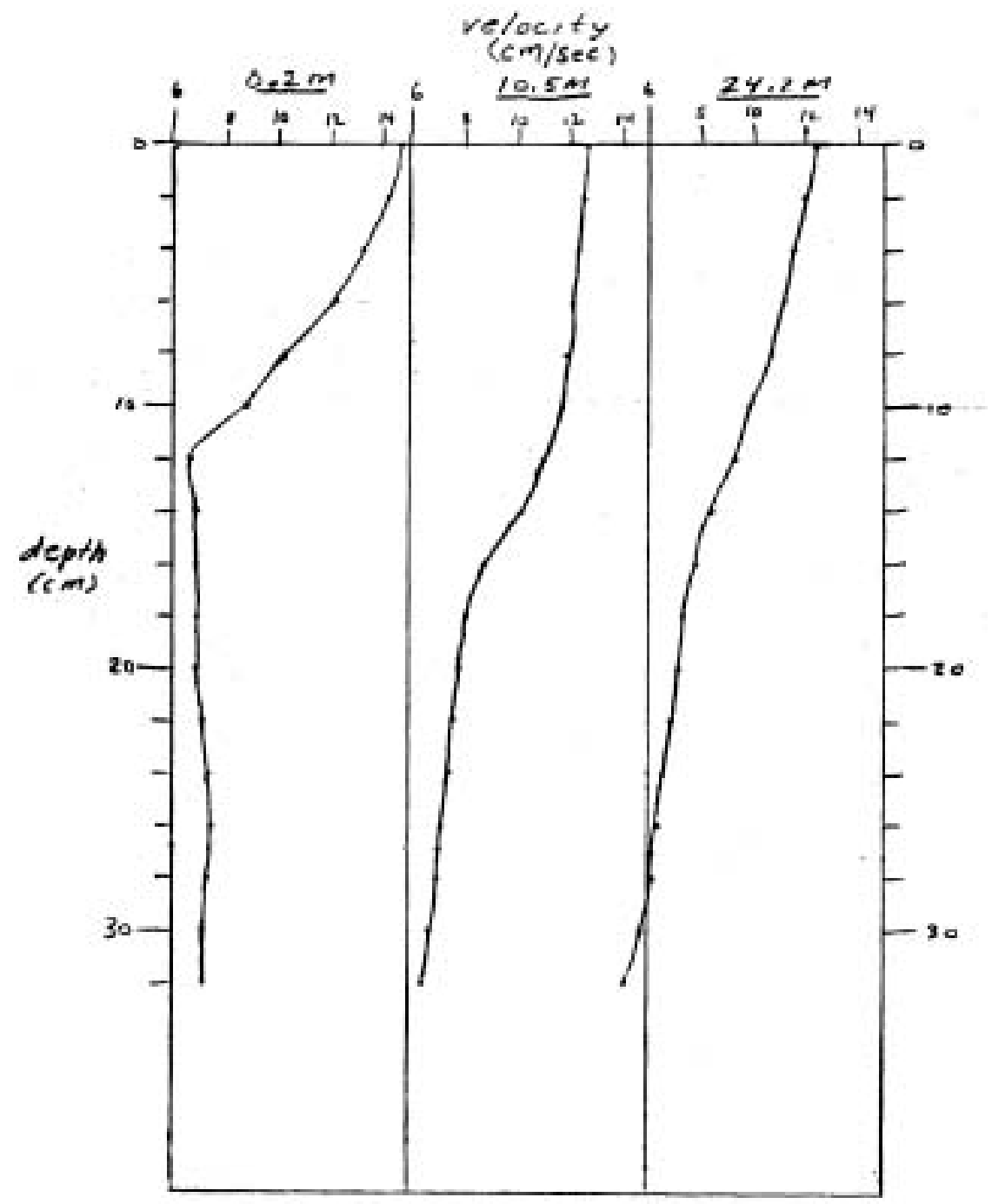

Fig.l Velocity vs. depth for three flume locations. Velocity is in $\mathrm{cm} / \mathrm{s}$, depth from surface is in $\mathrm{cm}$, locations avove graphs are in meters from the splitter plate $\Delta \rho / \rho \approx .0008$ initially, while $\Delta \bar{u} \approx 6 \mathrm{~cm} / \mathrm{s}$, initially. Splitter plate if $10 \mathrm{~cm}$ below the surface.

In the example presented, the density difference was small so that the turbulence was able to cause a very small amount of mixing in the interface. For the most part, however, the interface was laminar. There are cases, however, when the density gradient is small enough so that the water will continue to mix across the interface over the whole reach of the flume. This is in contrast to those cases in which the density gradient is high enough $\left(\sim 10^{-3} \mathrm{~cm}^{-1}\right.$, seemingly small, but still large enough to have a drastic effect) that no mixing at all occurs across the interface, after the collapse of the initial mixing layer. Turbulence seems only able to produce internal waves in this case.

The most interesting feature of Fig.l is that even far downstream of the splitter plate, there still exists a significant velocity gradient at the interface. It was found that if $\Delta \% / \rho=0$, the initial shear at the splitter 


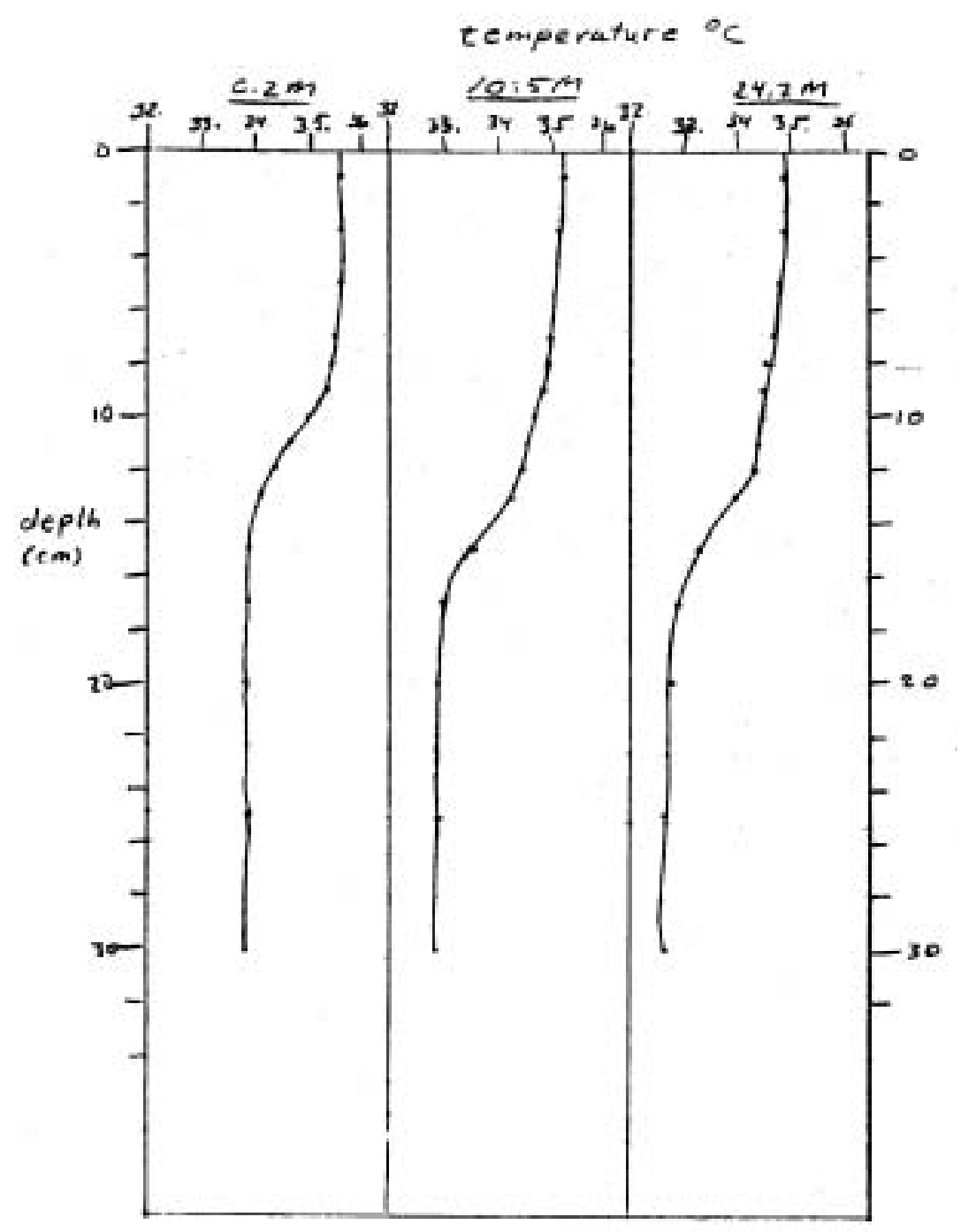

Fig.2 Temperature vs. depth for three flume locations. Temperature in ${ }^{\circ} \mathrm{C}$, other parameters as in Fig. 1.

plate is quickly destroyed by the mixing layer which develops and can no longer be detected within a few meters of the splitter plate. Thus, the downstream interface continues to exhibit significant density and velocity gradients.

The natural question arises as to what kinds of disturbances will cause the interface to become unstable and begin to mix. Certainly, one would expect that if the turbulence levels were high enough in the deep layers, mixing would occur across the interface. Other situations may cause instabilities in the flow as well. In this paper, the possibility of waves of small wavelength causing sudden mixing because of interactions with stable waves of large wavelength, will be discussed. It is hoped that with a simplified model of what is observed experimentally, a better understanding of the stratified shear layer will be obtained. 


\section{REVIEW OF SOME PREVIOUS WORK}

The stability of various types of stratified shear flows has been examined by many people in the past. In particular, Taylor (1931) and Goldstein (1931) found stability curves for several inviscid cases. Miles (1961) and Howard (1961) showed that for inviscid flow, a sufficient condition for stability is that the local Richardson number $s / \rho\left(\frac{\partial u}{\partial y}\right)^{2}$ be everywhere greater than 1/4. A review of much of the work done with stratified shear flows is given by Drazin and Howard (1966), and Thorpe (1973).

More recently, with the advent of high-speed digital computer, work has been done on more complex flows. An example is the work by Hazel (1972), in which a flow with $u \propto$ tanhy and $p \propto \exp (-\operatorname{tanhy})$ was considered.

Usually, these works have concentrated on finding neutral stability curves in the wavenumber-Richardson number plane. For free shear layers, these curves often correspond to curves where the wave speed of the disturbance, $\mathbf{C}$, vanishes. Neutral stability curves may not always coincide with the curve for $c=0$, however. A case for which $c=0$ is not the neutral stability curve was pointed out by Howard (1963), in which the flow was antisymmetric about $y=0$. In cases like this one, the principle of exchange of stabilities does not hold and one must use more complex methods for determining the curves of neutral stability.

One situation which can occur is that a finite perturbation on the mean flow can change the flow in such a way that a secondary perturbation will become unstable. Thus, one might expect a situation where internal waves in a stable stratified hsear flow modify the flow so that the local Richardson number is less than $1 / 4$, thus allowing the possibility of local instabilities. Woods (1968) felt that this phenomenon was responsible for the observed sudden onset of turbulence in the stable layers.

Landahl (1972) has given criteria for the breakdown of a flow perturbed by a wave-like disturbance of finite amplitude. The following conditions are for a two-dimensional problem. The basic idea behind the problem is that the primary wave changes the flow so that unstable secondary waves are focused where they suddenly attain large amplitudes. The first condition is that the group velocity $c_{g}$ of the secondary waves be equal to the phase speed $c$ of the neutral primary wave. If the primary wave itself is unstable, then the condition is $c_{g}=-c_{g} t / c_{g x}$, where the subscripts $t$ and $x$ indicate derivatives holding the wavenumber constant. If $c_{g}=c$, then a secondary wave can be trapped at some location along the primary wave If, for a non-dispersive system, $\mathbf{c}_{\mathrm{g}}-\boldsymbol{c}$ decreases through the critical point, where $c_{g}=c$, then the secondary waves $w$ ill be focused, since waves close to the critical point will tend to move toward the critical point. For detailed discussions, see Landahl (1972) and Landahl and Criminale (1977).

The primary requirement that allows one to use Landahl's theory for wave breakdown is that the wave number of the secondary disturbance be much larger than that of the primary wave. In this case, inhomogeneities in the fluid caused by the primary wave are small over one wavelength of the secondary wave. The other requirement is that the secondary waves be locally unstable. Landahl (1972) applied the theory to the sudden onset of turbulence in a boundary layer flow. Landahl and Criminale (1977) applied the theory to some simplified models of stratified shear flows. In their paper, they 
pointed out that "the requirement for the validity of the basic theory that the secondary wave causing breakdown must be of much smaller scale than the primary wave, is satisfied wherever the density layer's thickness is much smaller than the primary wavelength".

In the following, we consider a stratified flow where the density is continuous, but the velocity is discontinuous. It is assumed that there is a small wave-like perturbation on the mean flow. The velocity caused by the perturbation will, in general, be discontinuous at the interfaces, which are the locations where the mean velocity and density gradient are discontinuous. As pointed out by Landahl and Criminale, unstable waves induced by the discontinuity in velocity will propagate at the mean of the velocities on either side of the discontinuity. Thus, determining the condition for breakdown is much simplified.

In summary, to determine the breakdown c'ondition, it is assumed that the secondary wave disturbance has a wavelength much smaller than that of the primary wave. The group velocity of a secondary disturbance at velocity discontinuities is given by the mean of the velocities on either side of the discontinuity.

\section{THEORETICAL MODE OF A STRATIFIED SHEAR ROW}

In the following, an inviscid, incompressible fluid is assumed. The velocity in the fluid is given by $\underset{\sim}{\sim}=u_{0}(y) i+\underset{\sim}{\sim}(x, y, t)$, where $|\underline{u}|$ is small compared to $\left|u_{0}\right|$. The mean flow $u_{0}$, is given by

$$
u_{0}=\left\{\begin{array}{c}
\mid y>1 \\
0|y| \leq 1 \\
-\mid y<-1
\end{array}\right.
$$

The density of the fluid is given by $\rho=\rho_{(}(y)+\rho_{1}(x, y, t)$ where $\left|\rho_{1}\right| \leq<\left|\rho_{0}\right|$. The mean density $\rho_{0}$ is taken to be

$$
\begin{array}{ll}
\hat{\rho}_{0} e^{-\alpha} & y>1 \\
\hat{\rho}_{0} e^{-\alpha y} & |y| \leqslant 1 \\
\hat{\rho}_{0} e^{\alpha} & y<-1
\end{array}
$$

Hence the density is assumed to be continuous, but the relative density gradient is discontinuous

$$
\frac{1}{\rho_{0}} \frac{\partial A_{0}}{\partial y}=\left\{\begin{array}{c}
0|y|>1 \\
-\alpha|y| \leq 1
\end{array}\right.
$$
The overall Richardson number, $R i$, will be defined as $g \propto / / 2$. The equa-
tions of motion are:

$$
\begin{aligned}
& \text { x-momentum: } \rho \frac{\partial U}{\partial t}+\rho U \frac{\partial U}{\partial x}+\rho V \frac{\partial U}{\partial y}=-\frac{\partial p}{\partial x} \\
& \text { y-momentum: } \rho \frac{\partial V}{\partial t}+\rho U \frac{\partial V}{\partial x}+\rho V \frac{\partial V}{\partial y}=-\frac{\partial p}{\partial y}-\rho g \\
& \text { continuity: } \frac{\partial u}{\partial x}+\frac{\partial V}{\partial y}=0 \\
& \text { incompressibility: } \frac{\partial t}{t}+U \frac{\partial \rho}{a x}+V \frac{\partial \rho}{\partial y}=0
\end{aligned}
$$


The last equation requires that a fluid particle have constant density as it moves. Here, the pressure $\mathcal{P}$ is given by $-P_{0} g y+\mathcal{P}(x, y, t)$. A stream function $\Psi$ is introduced such that $u=u_{-}(y)+\frac{\partial \psi}{\partial y}$ and $V=-\frac{\partial \psi}{\partial x}$. In addition the transformations $\rho_{1}=\rho_{0}^{1 / 2} F$ and $\psi=\rho_{0}^{1 / 2} G$ are made to facilitate the reduction of the equations.

Substituting the transformations into Eqs. (4)-(7) and eliminating the pressure term, one obtains

$$
\begin{aligned}
& G_{y y} t+G_{x x} t+G_{0}\left(\frac{\rho_{0}^{\prime 2}}{4 \rho_{0}^{3}}-\frac{1}{2} \frac{\rho_{0}^{\prime \prime}}{\rho_{0}}\right)+U_{0}\left(G_{x x x}+G_{y y x}\right)+ \\
& +G_{x} U_{0}\left(\frac{\rho_{0}^{12}}{4 \rho_{0}^{1}}-\frac{1}{2} \frac{\rho_{e}^{\prime \prime}}{\rho_{0}}\right)-\frac{\left(\rho_{0} u_{e}^{\prime}\right)}{\rho_{0}} G_{x}-F_{x g}=0
\end{aligned}
$$

and

$$
F_{t}+U_{0} F_{x}=G_{x}\left(\rho_{0}^{\prime} / \rho_{0}\right)
$$

where the subscripts denote partial derivatives and only terms of first order in the perturbation quantities have been retained. It is now assumed that the perturbation quantities are wavelike in $x$ and $t$ :

$$
G=\phi(y) \exp (i k(x-c t)) \text { and } F=f(y) \exp (i k(x-c t)) \text {. }
$$

Substituting these relations as well as Eqs. (1) and (2) into (8) and (9), one finds that:

$$
\begin{aligned}
& \phi^{\prime \prime}-k^{2} \phi=0 \quad|y|>1 \\
& \phi^{\prime \prime}-\phi\left(k^{2}+\frac{y^{2}}{y}-g \alpha / c^{2}\right)=0 \quad|y| \leqslant 1 \\
& f=0, c \neq 1 \quad|y|>1 \\
& f=\frac{\alpha}{c} g \quad c \neq 0 \quad|y| \leqslant 1
\end{aligned}
$$

In addition to these equations, it is required that the fluid particle displacement, $\eta$, be continuous across the interfaces at $y= \pm 1$ and that the pressure be continuous across the interfaces as well. It is also required that the perturbation quantities vanish at $y= \pm \infty$.

Three cases may be distinguished, according to whether $\alpha^{2} \gamma^{2} \equiv k^{2}+\alpha^{2} / 4-g^{\alpha} / c^{2}$ is less than, greater than or equal to zero. If is less than zero $\phi(y)$ is wavelike in the $y$-direction; if $\alpha^{2} \gamma^{2}$ is greater than zero, $\phi(y)$ is exponential in character. Since these two cases are similar mathematically, they will be considered together.

Case 1,

Here, the solutions are:

$$
\begin{aligned}
& \phi \alpha \exp (-k(y-1)) y>1 \\
& \phi \alpha \exp (k(y+1)) y<-1 \\
& \phi=\left(A_{y}+B\right) \quad|y| \leq 1
\end{aligned}
$$


Now $\eta$, the fluid particle displacement, is given by:

$$
\eta=\psi /\left(c-u_{0}\right)
$$

For convenience, the solutions are written as:

$$
\begin{aligned}
& \psi_{I}=\delta_{I}(C-1) \exp (i \theta-k(y-i)+\alpha / 2 \\
& \eta_{I}(1)=\delta_{I} \exp (i \theta+\alpha / 2) \\
& \psi_{I I}=C\left(A_{y}+B\right) \exp (i \theta+\alpha y / 2) \\
& \eta_{\text {II }}=\left(A_{y}+B\right) \exp (i \theta+\alpha y / 2) \\
& \psi_{\text {III }}=\delta_{\text {III }}(C+1) \exp (i \theta+k(y+1)-\alpha / 2) \\
& \eta_{\text {III }(-1)=6 \pi \exp (i \theta-\alpha / 2)}
\end{aligned}
$$

where the subscripts $I, \square$ and $I I$ refer to the regions $y>1,|y|<-1$ and $y<-1$, respectively; with $\theta=k(x-c t)$ and both $\delta_{I}$ and $\delta$ III are constant. The boundary conditions require

$$
\begin{aligned}
& \eta_{x}(1)=\eta_{x}(1), \eta_{\pi}(-1)=\eta_{\mathbb{Z}}(-1) \quad \text { and } \\
& (c-1) \frac{\partial \psi_{x}(1)}{\partial y}=c \frac{\partial \psi_{x}(1)}{\partial y},(c+1) \frac{\partial \psi_{I}(-1)}{\partial y}=c \frac{\partial \psi_{m}(-1)}{\partial y}
\end{aligned}
$$

where the last two equations are obtained from the Bernoulli equation.

Applying the boundary conditions to (14) and (15) it is found that:

$$
\begin{aligned}
\delta_{I} & =A+B \text { (continuity of } \eta \text { at } y=1) \quad(16 a) \\
\delta_{m} & =-A+B \text { (continuity of } \eta \text { at } y=-1) \quad(16 b) \\
-(C=1)^{2} \delta_{I} k & \left.=\frac{\alpha}{2} C^{2}(A+B)+C^{2} A \text { (continuity of pressure at } y=1\right)(16 c) \\
(c+1)^{2} \delta_{m} k & \left.=\frac{\alpha}{2} c^{2}(B-A)+C^{2} A \text { (continuity of pressure at } y=-1\right)(16 d)
\end{aligned}
$$

In order to obtain nontrivial solutions, it is required that

$$
C^{4}\left(k^{2}+k\right)+C^{3}(2 \alpha k)+C^{2}\left(k-2 k^{2}-2 k i\right)+k^{2}=0
$$

where the facts that $2 R i=g \alpha$ and $\gamma^{*}=0$ have been used. This is the dispersion relation for $\gamma^{2} \equiv \boldsymbol{O}$.

\section{Case $2, \gamma^{2} \neq 0$}

The solutions for this case are:

$$
\begin{aligned}
& \phi_{I} \propto \exp (-k(y-1)) \\
& \phi_{I}=D_{1} \exp \left(\alpha \gamma_{y}\right)+D_{2} \exp (-\alpha \gamma y) \\
& \phi_{\square} \propto \exp (k(y+I))
\end{aligned}
$$

Following the same procedure as in the previous case, one finds 


$$
\begin{aligned}
& \psi_{I}=(c-1) \delta_{I} \exp (i \theta-k(y-i)+\alpha / 2 \\
& \eta_{I}(t)=\delta_{I} \exp (i \theta+\alpha / 2) \\
& \psi_{I I}=c\left(D_{1} e^{\alpha \gamma y}+D_{2} e^{-\alpha \gamma y}\right) \exp (i \theta+\alpha y / 2) \\
& \eta_{\bar{I}}=\left(D_{1} e^{\alpha \gamma_{y}}+D_{2} e^{-\alpha \gamma y}\right) \exp (i \theta-\alpha y / 2) \\
& \Psi_{\text {II }}=(c+1) \delta \text { III } \exp (c \theta+k(y+1)-\alpha / 2 \\
& \eta_{\text {四 }}(-1)=\text { 血 exp }(i \theta+\alpha / 2)
\end{aligned}
$$

The boundary conditions require:

$$
\begin{aligned}
& \delta_{I}=D_{1} e^{\alpha \gamma}+D_{2} e^{-\alpha \gamma} \quad \text { (continuity of } \eta \text { at } y=1 \text { ) } \\
& \left.\delta_{D^{2}}=D_{1} e^{-\alpha \gamma}+D_{2} e^{\alpha \gamma} \quad \text { (continuity of } \eta \text { at } y=-1\right) \\
& -k \delta_{I}(c-1)^{2}=c^{2} \alpha\left(D_{1} e^{\alpha \gamma}\left(y_{2}+\gamma\right)+D_{2} e^{-\alpha \gamma}\left(y_{2}-\gamma\right)\right) \text { (continuity of pressure, } y=1 \text { ) } \\
& k \delta_{\text {III }}(c+1)^{2}=c^{2} \alpha\left(D_{1} e^{-\alpha \gamma}\left(y_{2}+\gamma\right)+D_{2} e^{\alpha \gamma}\left(\frac{1}{\gamma}-\gamma\right) \text { (continuity of pressure at } y=-1\right. \text { ) }
\end{aligned}
$$

Again, for non-trivial solutions, the dispersion relation is obtained:

$$
\tan (2 \alpha \gamma)\left[C^{1}\left(2 k^{2}\right)+2 \alpha k c^{2}-c^{2}\left(2 k^{2}+2 R_{i}\right)+k^{2}\right]+2 \alpha \gamma k\left[c^{4}+c^{2}\right]=0
$$

If $|\alpha \gamma| \ll \mid$ then (22) reduces to

$$
2 \alpha \gamma\left[c^{4}\left(2 k^{2}+k\right)+(2 \alpha k) c^{2}+c^{2}\left(k-2 k^{2}-2 R i\right)+k^{2}\right]=0
$$

where the approximation $\tan t \approx \epsilon$ for $|\epsilon|<<\mid$ has been used. Equation (22), then reduces to Eq. (17) when $k r \mid \ll<1$.

For the case that $\gamma^{2}$ is real and negative, the amplitudes of the perturbation quantities are wavelike in the region of non-zero density gradient. Equation (2lb), in this case, is of the form

$$
D_{1} a=-D_{2} a^{*}
$$

where the $t$ superscript denotes the complex conjugate. This implies that , Inaddition, if $i t$ is required that at $y=0, \eta_{\pi}=b$ exp $i \theta$ where $\theta$ may include an arbitrary phase and $b$ is real, then $D_{1}+D_{2}$ is real (from Eq. (20b)) and, therefore $D_{1}=D_{2}^{*}$. This means that the amplitude of $\psi_{\text {II }}$ is wavelike in the $y$-direction, but the waves are standing waves. Hence, waves travel only in the $x$-direction.

By setting $\gamma^{2}$ to zero and using Eq. (17), which is the dispersion relation for this case, curves in the wavenumber-Richardson number plane can be found which separate the regions for the different types of solutions. Equation (17) can be rewritten as:

with

$$
\left(c^{2}-1\right)^{2} k^{2}+n c^{2}\left(c^{2}+2 a c+1\right)-\underline{c^{2} \alpha^{2}}=0
$$

$$
R_{i}=\frac{c^{2}}{2}\left(k^{2}+\alpha^{2 / 4}\right) \text {. }
$$


Then, $\boldsymbol{c}$ can be used as a parameter to find the values of $k$ and $R \boldsymbol{j}$. Two branches are found, one for $c<1$ and the other for $c>1$. These are shown in Fig.3, for the case where $\alpha=10^{-3}$. These curves correspond to one root
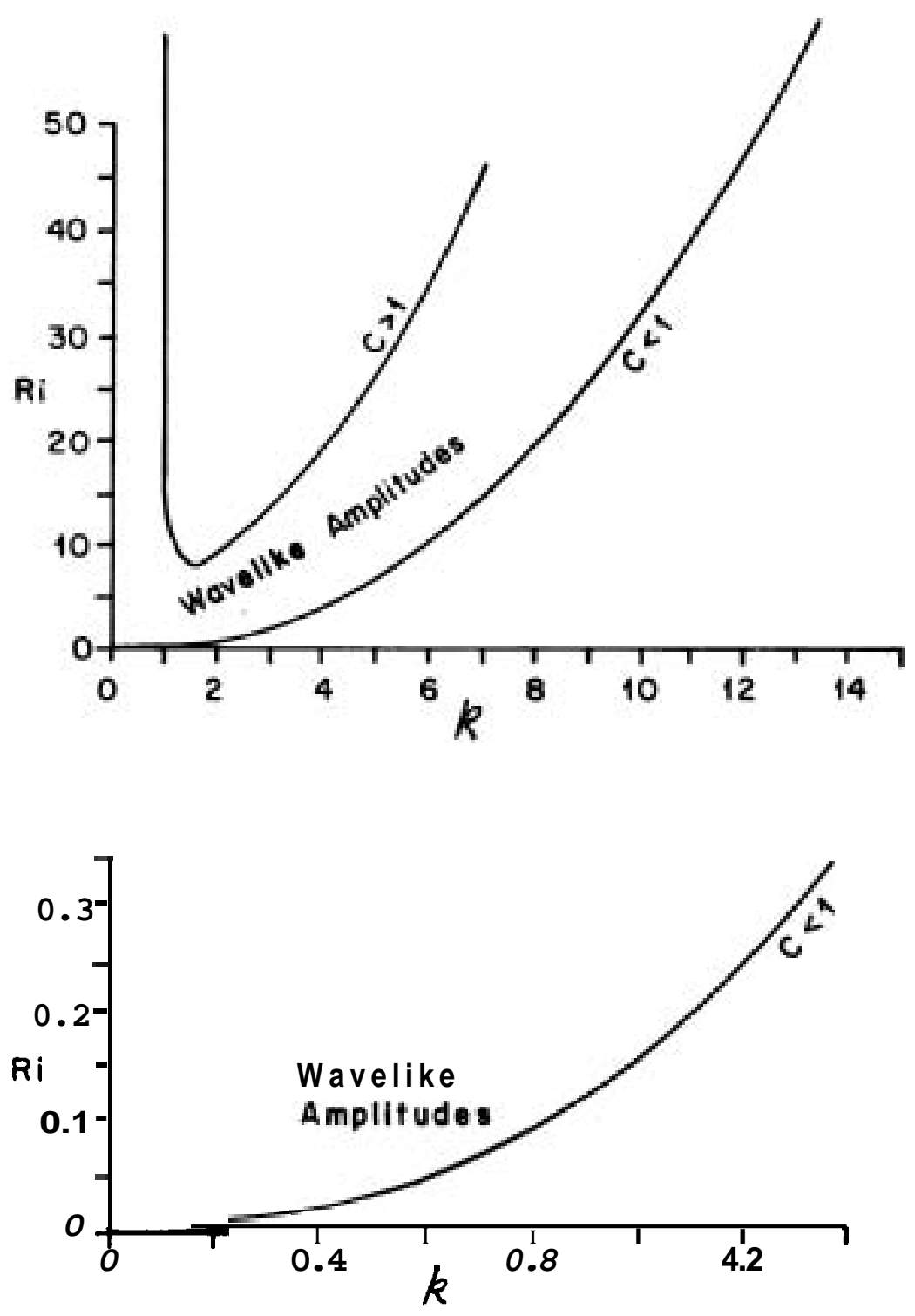

Fig.3 Boundaries for types of solutions, curves for $\gamma^{2}=0 ; \alpha=0.001$. 
of the quadratic equation for $k$. The other root corresponds to $k<10^{-6}$ for this case, and can be neglected for the purposes of this discussion. Taking Eq. (22) and assuming $\gamma^{2}=E$, where $|\epsilon|<\ll I$, the regions of wavelike amplitudes $\left(\gamma^{2}<0\right)$ and exponential amplitudes $\left(\gamma^{2}>0\right)$ are found. These are indicated on Fig. 3. The interesting features on Fig. 3 are the minimum on the branch of the curve for $c>l$ and the fact that this branch is asymptotic to the line $k=1$. Though not obvious from the figure, the two branches converge as $[c-1 \mid \rightarrow 0$.

Stability Considerations

Here, it is assumed that $\alpha<<I$. For the case where $k<<b$, one would expect the stability of the problem to be similar to the KelvinHelmholtz problem. This can be shown to be true in the following way. Since $k \ll \|$, then $\alpha^{2} \gamma^{2} \ll 1$, and $\tanh 2 \alpha \gamma \approx 2 \alpha \gamma$; the dispersion relationship reduces to Eq. (23). Since $k \ll<1, k^{2}$ is neglected in comparison to $k$ and the resulting approximation is

$$
h c^{4}+2 \alpha h c^{3}+c^{2}(k-2 R i)+h^{2}=0
$$

If $k-2 R i>0$, then by Descartes rule for polynomials, there are no real positive roots for $C$ and at most two negative real roots. (Here, we consider only positive values of $\&$.) Hence, there are two complex roots, which are conjugates, one of which corresponds to an unstable root $\left(I_{m}(c)>0\right)$. If $k-2 R_{i}<0$, then there are at most two positive real roots and two negative real roots for $C$. Hence, for small $k$, the line $R i=k / 2$ is a stability boundary, which is exactly the Kelvin-Helmholtz result.

One can also show that $c=0$ is not a stability boundary. As discussed by Howard (1963) the 'principle of exchange of stabilities' may not always be valid for antisymmetric stratified shear flows. Considering Eq. (22), let $C=i E$ where $E$ is a positive real number. Then

$$
\alpha \gamma=\left(k^{2}+\alpha^{2} / 4+2 R i / \epsilon^{2}\right)^{1 / 2} \text {, }
$$

where $h$ and $R_{i}$ are all real. Since the imaginary part of (22) is equal to zero, one finds

$$
-i \epsilon^{3} 2 \alpha k \tanh 2 \alpha \gamma=0
$$

This is satisfied for $\alpha=0, k=0$ or $\gamma=0$. Rejecting the first two cases as trivial, it is found that $\alpha=0$. Since $k, \alpha, R_{i}$ and $E$ are all real the Richardson number, $R_{i}$, must be negative, which is the case where the density in the upper layer is greater than the density in the lower layer. As $\in \rightarrow O$ the Richardson number must also approach zero, hence, $R i=0$ is the neutral stability curve associated with $C=0$. But, it has already been shown that unstable solutions exist for $R \boldsymbol{C}>0$. Therefore $C=0$ is not the stability curve for this case.

A general idea of the nature of the stability of Eq. (22) for small values of $\alpha \gamma$ can be obtained in the following way. Again, assume $\alpha \gamma<<1$ and, in addition, it is assumed that os $\leqslant<k$. Since, for a real problem, $\propto$ is often less than 0.005 , this is not a strict requirement. Then, one can neglect the term in $\mathbf{c}^{3}$ in Eq. (23) and one is left with a quadratic equation in $C^{1}$, with the solution.

$$
C^{2}=\frac{2 k^{2}+2 R i-k}{2\left(2 k^{2}+k\right)} \pm \frac{\sqrt{\xi}}{2\left(2 k^{2}+k\right)}
$$


where

$$
\xi=R i^{2}+\left(2 k^{2}-k\right) R i-k^{2}\left(k^{2}-2 k-1 / 4\right)
$$

If $\boldsymbol{\xi}$ is less than zero, $\mathbf{C}$ is complex. If $\xi$ is positive, then $\sqrt{\xi}$ is always less than $2 k^{2}+2 R i-k$, and $C$ is real or imaginary, depending upon the sign of $2 k^{2}+2 R i-k$. If $\xi>0$ and $k \ll 1$, then $R i=k / 2$ is again obtained as the stability boundary. In addition $\xi=0$ is a stability boundary. Solving (28) for $\xi=0$, one finds that

$$
R i=\frac{k}{2}-k^{2} \pm k\left(2 k^{2}+k\right)^{1 / 2}
$$

For stability, it is required that $2 k^{2}+2 R i-k>0$, hence only the root with the positive sign is accepted; the other root corresponds to the case where $c^{2}<0$. Again, from Eq. (29), $R i=k / 2$ is the stability boundary for small values of $\boldsymbol{k}$. The stability curve from Eq. (29) is shown in Fig.4, for values of $k<0.1 ;$ for larger values of $k$, the assumption that $\alpha \gamma<1$ is no longer valid. Additional stability curves have not yet been determined.

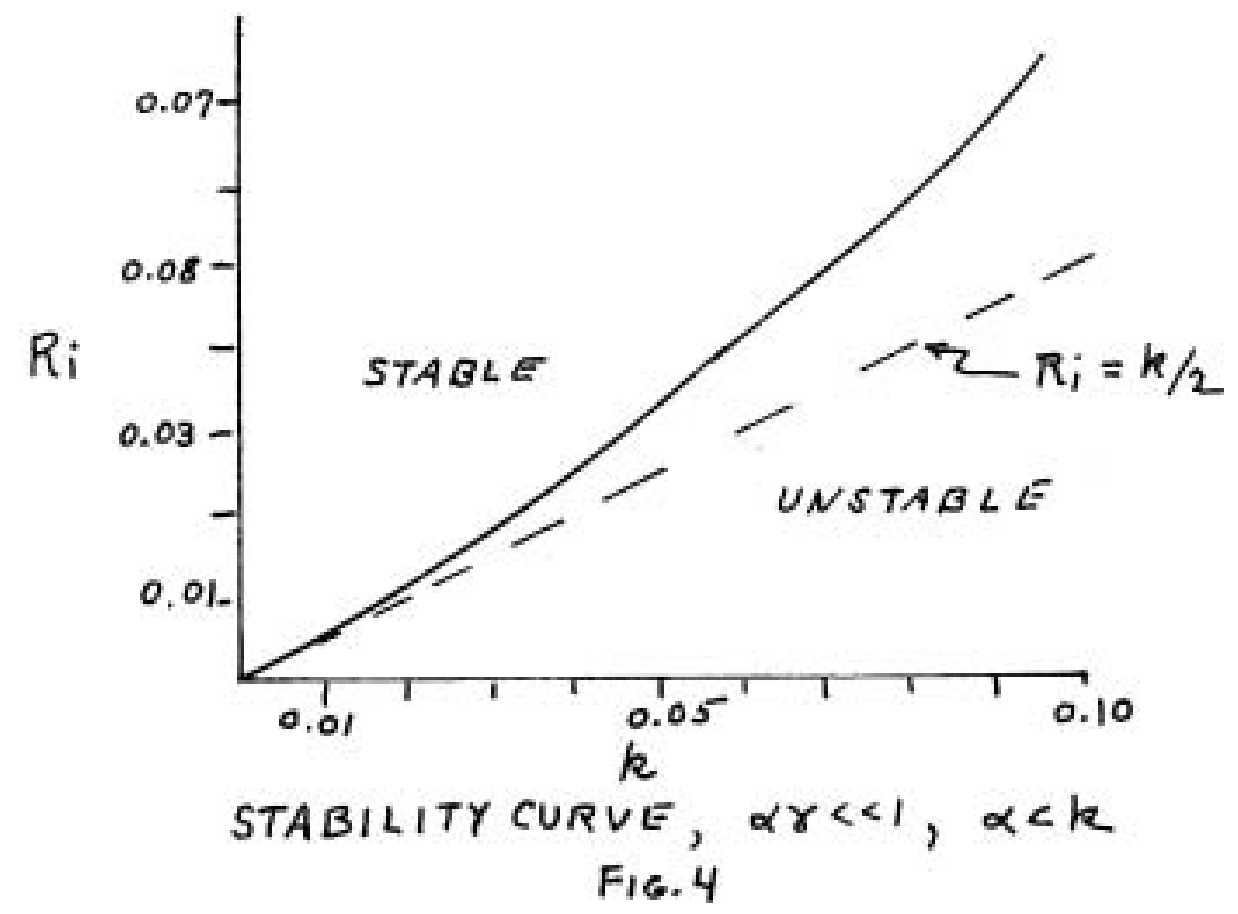

\section{BREAKDOMN CONDITIONS}

As stated previously, the conditions leading to breakdown of the flow by secondary waves are that the secondary wave be unstable and of much smaller scale than the primary wave, and that the group velocity of the secondary wave be equal to the phase velocity of the neutral primary wave. The group velocity of an unstable wave generated by the shear at the interfaces, where the velocity of the fluid is discontinuous, is given by the mean of the velocities on either side of the interface (cf. the Kelvin-Helmholtz problem). Therefore, the group velocities of the secondary waves at the interfaces are given by: 


$$
\begin{aligned}
& c_{g}(1)=\frac{1}{2}\left(1+{\frac{\partial \psi_{x}}{\partial y}}^{(1)}+\frac{\partial \psi_{\tau_{1}}(1)}{\partial y}\right)=1 / 2+u \dot{m}^{+} \\
& c_{g}(-1)=\frac{1}{2}\left(-1+\frac{\partial \psi_{\pi^{\prime}}^{(-1)}}{\partial y}+\frac{\partial \psi_{\pi}}{\partial y}{ }^{(-1)}\right)=-1 / 2 u \bar{m}
\end{aligned}
$$

Substituting the results of section IV for the stream functions, we find that

$$
\begin{aligned}
& u_{m}^{+}=-\eta_{I} k(c-1)(1-1 / 2 c) \\
& u_{\bar{m}}=\eta_{\mathbb{I}^{\prime}} k(c+1)(1+1 / 2 c)
\end{aligned}
$$

These relations hold for all values of $\gamma$. Therefore, for breakdown to occur at the upper interface, it is required that

or

$$
\begin{aligned}
& c_{g}=c=1 / 2-\eta_{x} k(c-1)(1-1 / 2 c) \\
& c\left(c-v_{2}\right)=-\eta_{x} k(c-1)(c-1 / 2)
\end{aligned}
$$

For the lower interface the requirement is

$$
c\left(c+\frac{1}{2}\right)=\eta_{\text {III }} k(c+1)(c+1 / 2)
$$

If $c \neq \pm 1 / 2$, then the requirements are

and

$$
c_{b}^{+}=\frac{\eta_{I}^{k}}{1+\eta_{x}^{k}}
$$

where $C_{b}^{*}$ and $C_{b}^{-}$are used to denote the phase speeds of the primary wave which are required for breakdown to occur on the upper and lower interfaces, respectively.

As $k$ becomes small, this condition should approach that found by Landahl and Criminale (1977) for the Kelvin-Helmholtz problem. Here, as $k \rightarrow 0$, $c_{b}^{*} \approx \eta_{t} k$, and $c_{b} \approx \eta_{\mathbb{m}}{ }^{k}$. We require that breakdown occur on both interfaces, so that $c_{b}^{+}=c_{b}^{-} \eta_{k}$. In addition the mean velocity will be given by the mean of $\boldsymbol{u m}_{\mathrm{m}}$ and $\boldsymbol{u}_{\mathrm{m}}$; this is found to be

$$
u_{m}=\frac{1}{2}\left(u_{m}^{*}+u_{m}\right) \approx \frac{1}{2}\left(\eta k-\frac{1}{2}+\eta k+\frac{1}{2}\right)=\eta k
$$

as $k \rightarrow 0$. The results of Landahl and Criminale (1977) for the Kelvin-Helmholtz case are identical to these.

The conditions that $C=1 / 2$ or $C=-1 / 2$ correspond to cases where $u_{m}^{+}=0$ or $u_{m}^{-}=0$. In either of these cases, secondary waves are trapped but not focused. Using the dispersion relation (Eq.22) one can find values of $k$ and $R i$ for the case that $c=1 / 2$. These curves are plotted in Fig.5, for $\alpha=10^{-3}$ and for a range of values of $h$ and $R i$. It is to be noted that, because of the factor involvingtanh, there are multiple values of $R_{i}$ for a given wavenumber $k$, when $\gamma^{2}$ is negative. In addition, it is noted that when $\varepsilon=1 / 2$, the condition for breakdown on the lower interface is

$$
\eta_{\text {ex }}=\frac{1}{3 k}
$$

Since it is required that the wavelength of the primary wave be smaller than the density interface thickness, (say, less than $1 / 2$ the interface thickness), 


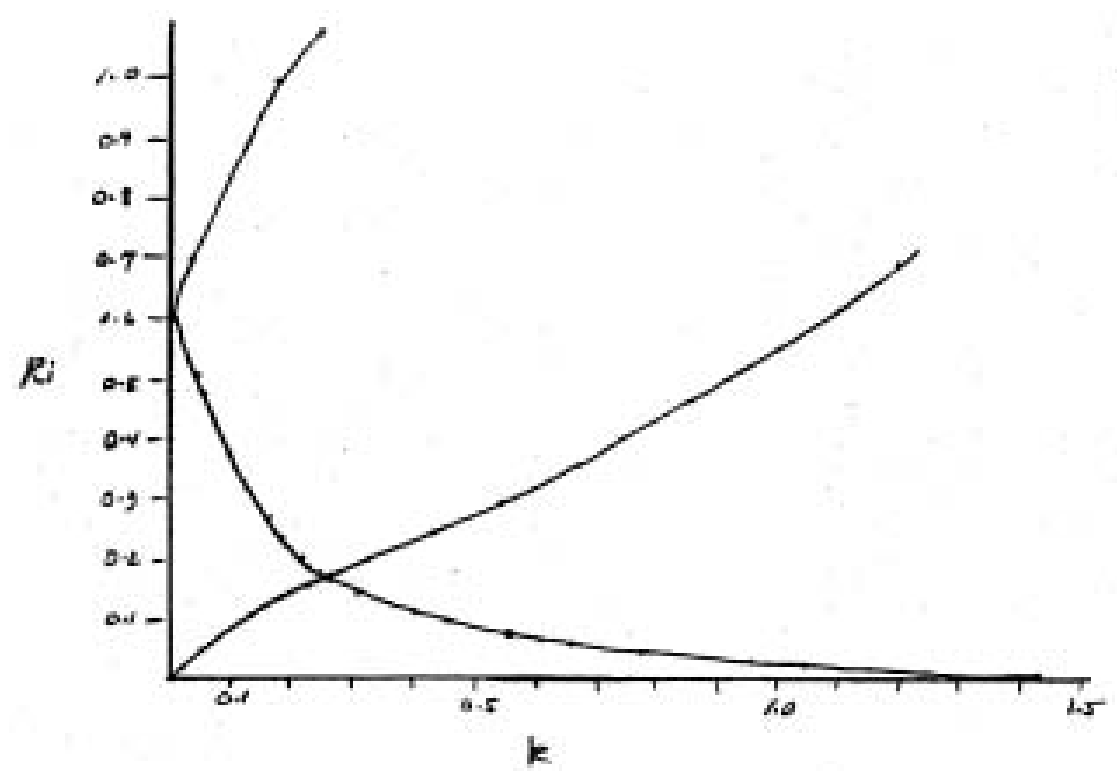

Fig.5 Breakdown conditions for $c=1 / 2, \alpha=.001$.

$k$ is at most $2 \mathrm{TL}$. (Recall that the density interface extends from +1 to -1 , therefore the unit length is $1 / 2$ the density interface thickness.) Therefore $\eta_{\mathbb{Q}}$ must be greater than 0.05 . For $\boldsymbol{t}<1 / 3, \eta$ III must be greater than 1, a deflection which is clearly outside the range permitted by the small perturbation assumption. However, it is fair to say that when $1 / 3<k<2 \pi$, and $C=1 / 2$, secondary waves can be focused, leading to breakdown, on the lower interface, while at the same time secondary waves are trapped on the upper interface. A similar result is true when $c=-1 / x$.

To find other curves in the $\boldsymbol{k}-\boldsymbol{R}$ i plane for breakdown, it is necessary to assume a value for $\eta_{\xi}$ or $\eta_{m \text { m }}$, and substitute Eq. (35a) or (35b) into Eq. (22). This results in a very complex relationship between $k$ and $R_{i}$, and values have not been computed. However, the general nature is the same as the case for $c=1 / 2$; that is, one would expect to find several values of $\boldsymbol{h}$ for a given $\boldsymbol{\eta}_{\mathbf{x}}$ and $\boldsymbol{R}_{\boldsymbol{i}}$ for which breakdown could occur. This, again, is due to the fact that the tanh factor in Eq. (22) is periodic when $\boldsymbol{r}^{2}$ is negative.

\section{CONCLUSIONS}

It has been shown that, for the simplified model of stratified shear flow considered, breakdown caused by trapping and focusing of secondary waves is possible. For the case that the phase velocity is equal to $1 / 2$, secondary waves can be trapped on the upper interface and trapped and focused on the lower interface. In addition, it has been found that, as expected, the problem becomes similar to the Kelvin-Helmholtz problem as the wavenumber approaches zero. In particular, the neutral stability curve for this case approaches the curve $R_{i}=k / 2$ as $k$ approaches zero. The breakdown conditions, in some cases, do require large amplitudes for the primary wave. These are probably not well-described by the linear theory. In 
addition, viscous effects may have important effects in the problem. Both nonlinear and viscous effects on the problem should be considered in the future.

The writer wishes to express his sincere thanks to all participants in the Geophysical Fluid Dynamics program. It has been a most interesting and productive summer. The writer would especially like to thank M. T. Landahl, who suggested the problem, and cheerfully offered advice and helpful observations throughout the summer. Special thanks are also due M. Maxey and J. Lewalle, with whom the writer had many helpful discussions.

References

1. Drazin, P.G. and L.N.Howard, (1966) Advances in Applied Mechanics, 9: 1-89.

2. Goldstein, S. (1931) Proc.Roy.Soc. A, 132: 524.

3. Hazel, P. (1972) J.Fluid Mech. 51: 39-61.

4. Howard, L.N. (1961) J.Fluid Mech. 10: 509.

5. Howard, L.N. (1963) J.Fluid Mech. 16: 333.

6. Landahl, M.T. (1972) J.Fluid Mech. 56: 775.

7. Landahl, M.T. and Criminale (1977) J.Fluid Mech. 79: 451.

8. Miles, J.W. (1961) J.Fluid Mech. 10: 496.

9. Taylor, G.I. (1931) Proc.Roy.Soc., A 132: 499.

10. Thorpe, S.A. (1973) Boundary Layer Met. 5: 95.

11. Woods, J.D. (1968) J.Fluid Mech. 37: 791.

12. Woods, J.D. (1969) Radio Science, 4: 1289. 


\section{'TWIN-EXHAUST' GALAXY MODELS AND INSTABILITIES}

IN CHANNES WITH FLEXIBLE WAUS

John L. Giuliani

\section{INTRODUCTION}

One of the current interests of galactic astronomy is the class of elliptical galaxies which are observed to have radio lobes extending far beyond the optical image along an axis through the nucleus. Such objects are commonly referred as double radio sources and more information with descriptive pictures can be found in Turland (1975). As is typical with galactic phenomena, many theories have been proposed to explain large radio lobes, but the Blandford-Rees (1974) 'Twin-Exhaust ${ }^{1}$ model has received the most attention. In this model a hot, relativistic plasma is continuously generated at the nucleus of the galaxy (see Fig.1, label A) and forms a central cavity (c). This cavity is bounded by the thermal pressure of a cold, gravitationally-confined rotating gas cloud ( $G)$. The hot plasma tends to push out along the rotation axis and form a channel. Blandford and Rees considered the one-dimensional gas flow equations and found that the channel will geometrically deform into a de Laval nozzle. Their method is equivalent to solving the nozzle flow problem when the surrounding surface is free. At the point of minimum cross section of the nozzle the hot plasma becomes transonic and the flow becomes collimated into a beam shooting out into the intergalactic medium. Interactions of the plasma with magnetic fields in the beam give rise to the emission observed in the radio lobes.

One of these radio galaxies, $M 87$, is close enough to our galaxy to allow relatively good resolution on photographic plates. Before M87 was discovered to be a double radio source, short exposures indicated an asymmetry in the optical image (see Photograph 1). What is remarkable is that this optical jet lies along the axis of the double radio lobes. This asymmetry suggests that the radio beam may be unstable and/or oscillate from side-toside, in which case we have caught the galaxy while the jet is predominantly on one side. The proposed objective during the summer was to set up a shallow water experiment modeling the double-diffuser aspects of the radio galaxy and to study possible instabilities in channel flow which could generate oscillations. The next section will show why shallow water theory is relevant and discuss the experiment. Section three will present some analysis of the experiment and the last section will consider the significance of the experiment for the galatic jef problem.

\section{SHALOW WATER THEORY AND THE EXPERIMENT}

We begin this section with a brief review of shallow water theory (Landau and Lifshitz, 1959). If one neglects any velocity in the vertical $z$-direction, the third component of Euler's equation becomes

$$
\partial_{2} E=-g \rho \Rightarrow P=P_{0}+g \rho(h-z)(\rho \text { constant) }
$$

where Po is the pressure at the free surface of height hwh $(x, y)$. Let the $x-d i-$ rection be downstream and the $y$-direction spanwise. Using the above expression for $P$ the first two components of Euler's equation become

$$
\begin{aligned}
& \partial_{t} v_{x}+v_{x} \partial_{x} v_{x}+v_{y} \partial_{y} v_{x}+g \partial x h=0, \\
& \partial_{0} v_{y}+v_{x} \partial_{x} v_{y}+v_{y} \partial_{y} v_{y}+g \partial_{y} h=0 .
\end{aligned}
$$


The corresponding continuity equation can be derived by integrating the general continuity equation over $z$ or by use of central volumes. In either case one finds

$$
\delta_{t} h+\partial_{x}\left(h v_{x}\right)+\partial_{y}\left(h v_{y}\right)=0 \text {. }
$$

The usefulness of these shallow water equations lies in their similarity to the compressible gas dynamic equations. Let

$$
\bar{\rho} \equiv \int_{0}^{h} \rho d z=g h \text { and } \overline{\mathrm{P}} \equiv \int_{0}^{h} P d z=(g h) \bar{\rho} / 2 \text {. }
$$

If one now multiplies (3) by $\rho$ and changes the last term on the left of rhe momentum equations into a pressure ( $\bar{P}$ ) gradient, one finds the identical equations for two-dimensional gas flow in terms of $\bar{g}$ and $\bar{P}$. Here the role of the sound speed is played by $\sqrt{g h}$ and the ratio of specific heats is 2 . The above correspondence allows one to experimentally investigate some types of high speed gas flow problems using shallow water flows.

The experiment went through several evolutionary phases before a workable model was developed. It is useful to discuss one of the extinct models in detail in order to provide (i) motivation for the final model and (ii) a foundation for some of the general conclusions concerning oscillations. This particular model was a double-diffuser with rigid walls following the outline of Fig.l on both sides with a water source in the central cavity. The plan was to use ping pong balls to act as buoyant flood gates in each channel. Suppose the ball on the left channel was initially depressed. This would make the flow predominantly in the opposite channel. However the water height, and equivalently the buoyancy force, would eventually increase in the channel and raise the ball. At this time the outflow from the central cavity would switch sides and the right side ball would sink and play the blocking role. The process should then repeat except for a phase change of $180^{\circ}$. Unfortunately the system was too sluggish to respond with any regularity implying that precise engineering would be required to get rid of the strong damping.

The above experiment and other similar ones seem to indicate that an oscillating mechanism does not arise from a periodic blocking process connected with the early stages of the jet formation. Rather, the most fruitful approach was first to isolate an instability mechanism in the channel and then to investigate how it might lead to oscillations in the outfield.

Guided by the 'Twin-Exhaust' model, the central channel was surrounded on both sides by channels containing ambient water which played the role of the background cold gas in the galaxy. Since the pressure of this latter gas decreases along the galactic nozzle the experimental set up must allow for a flow in the ambient water. The separation between the outer and inner flows was maintained by flexible walls. The experimental apparatus in this case is shown in Photograph 2. Since we are concentrating on the instability mechanism and because of the symmetry of the initial problem, only one side of the double diffuser needs to be considered. The central cavity has a source and is partially formed by rigid walls. Each of the outer channels has a source of the same discharge rate, and the outer walls limiting the ambient flow are fixed. The rulers in the center serve only to delineate how the central channel sits in the ambient water. (The material used for the flexible walls would have made this photograph unintelligible.) 
The main technical problem in this experiment was the construction of the channel walls. They must be extremely flexible, remain perpendicular to the bottom of the channel during any motion and succeed in separating flow in the jet from the ambient water. These requirements were found to be sufficiently satisfied by using cellophane to outline each of the three chanmels and a strip of mylar to add vertical rigidity to the walls of the central channel (Fig.2). The cellophane was layered at the bottom and attached to the mylar in such a way that the strip remained above the bottom. If the cellophane was slightly dampened on the underside the friction became negligible allowing each wall to glide smoothly and independently in the spanwise direction. At the end of the series of channels was a basin into which the water drained.

When the apparatus was set up a capillary instability immediately appeared in the central jet. This instability grew at all points along the channel and did not appear as a drift instability. Photographs 3 and 4 show an example of the oscillation at the same position in the streamwise direction but differing:in phase..by $\sim 1800$. All the photographs of the oscillation have been enhanced. The outer fixed walls are shown with full lines and the position of the flexible walls with dashed lines. Altering the parameters of the flow, such as the discharge rate or the size of the inner channel, resulted in a different character of oscillation. For example, if the flow in the outer channel was partially blocked, the oscillation was reduced in frequency and wavelength.

In some runs of the experiment it was observed that the amplitude of the oscillation was strong enough to actually shut off the flow. This is suggestive in two ways. First the capillary-type instability of the channel might be the phenomenon causing the "beady" appearance of the M87 jet shown in Photograph 1. Second, this instability might represent a possible mechanism causing the double-diffuser or twin-exhaust model to oscillate. In this theory the buoyant flood gates are replaced by flexible walls which periodically cut off the flow in one and then the other channel. If either analogy is to hold it is first of all important to analyze the physics in the shallow water experiment in order that a reasonable comparison with the galactic jet problem can be made.

\section{ANALYSIS}

The analysis of the instability in the water channel can be broken down into two modes. In the first approach a.) the outer rigid walls are taken into account giving rise to boundary conditions on the spanwise velocity at finite distances from the center line of the inner channel. The flexible walls or membrane separating the jet from the ambient flow has no properties in this part of the analysis. In the second approach however, b.) the membrane is given a tension and a mass per unit area but the outer rigid wall and the center line of the channel are removed to infinity. Fig.3.a. and b. are the respective coordinate systems for the two approaches. The displacement of the membrane from its initial position will be denoted by $\eta$. The analysis was split into these two limits so that it might be made tractable. The case a.) is clearly important in the long wavelength limit since the outer wall is within the length scale of the disturbance. Case b.) corresponds to the short wavelength limit when tension becomes important and the outer walls or center line are far out of the length scale of the disturbance. 


\section{a.) Outer Walls}

The standard linear perturbation theory can be applied to the twodimensional shallow water equations (1), (2) and (3). The unperturbed state is taken to be simple channel flow:

$$
\left.\begin{array}{rl}
u_{1} & =\text { constant } \\
u_{2} & =\text { constant }
\end{array}\right\} x \text {-direction velocity }
$$

W have ignored the change in $u$ and $h$ along the streamwise direction since it adds only complexity to the analysis and hides the important physics of the phenomena. All of the perturbations have the form

$$
\delta h_{1}=\delta h_{1}(y) e^{i k x+i \omega t}
$$

The linearized equations are

$$
\begin{aligned}
& i\left(\omega+u_{j} k\right) \delta h_{j}+h D \delta u_{j}+i k h \delta u_{j}=0 \\
& i\left(\omega+u_{j} k\right) \delta u_{j}+i k g \delta h_{j}=0 \\
& i\left(\omega+u_{j} k\right) \delta v_{j}+g D \delta h_{j}=0
\end{aligned}
$$

where $j=1,2$ and $D=d / d y$. Taking $\left(\omega+u_{j} k\right)$ times (4) and using (5) gives an equation relating $\delta h_{j}$ and $D \delta v_{j}^{j}$. This equation can be used in (6) to obtain the dispersion relation

$$
\begin{gathered}
\left(D^{2}-k^{2} m_{j}^{2}\right) \delta v_{j}(y)=0 \\
m_{j}^{2}=1-\frac{\left(\omega+u_{j} h\right)^{2}}{k^{2} g h}
\end{gathered}
$$

The general solutions are

$$
\left.\begin{array}{l}
\delta v_{j}(y)=A e^{k m_{j} y}+B e^{-k m_{j} y} \\
\delta h_{j}(y)=-i \frac{\left(\omega+u_{j} k\right)}{g k m_{j}}\left(A e^{k m_{j} y}-B e^{-k m_{j} y}\right)
\end{array}\right\} j=1,2
$$

The appropriate boundary conditions in this approach are

$$
\delta v_{1}(y=0)=0 \quad \delta v_{2}(y=L)=0
$$

The linearized matching conditions are: (i) that the interface moves with the fluid

$$
\frac{D \eta}{D t}=\partial_{t} \eta+u_{j} \partial_{x} \eta=\delta u_{j} \text { at } y=l \text {. }
$$

where $\eta=\eta_{0} e^{i \hbar x+i \omega t}$ is the equation for the interface, and (ii) that there is pressure continuity across the interface

$$
\delta h_{1}=\delta h_{2} \text { at } y=\ell \text {. }
$$

Combining these conditions with the four solutions (a) results in the 
following characteristic equation

$$
\frac{\left(\omega+u_{1} k\right)^{2}}{\left(\omega+u_{2} k\right)^{2}}=\frac{m_{1}}{m_{2}} \frac{\tanh m_{1} k \ell}{\tanh m_{2} k(l-L)} .
$$

If $g^{h} \rightarrow \infty$ (incompressible 1 imit) and the center line along with the outer wall are moved to infinity (13) becomes

$$
\frac{\left(w+u_{1} k\right)^{2}}{\left(\omega+u_{2} k\right)^{2}}=-1 \Rightarrow \omega=-\frac{k\left(u_{1}+u_{2}\right)}{2}(1 \pm i) \text {. }
$$

These are the classical Kelvin-Helmholtz waves with $g=0$ (Chandrasekhar, 1961). On the other hand if $u_{1} \rightarrow u_{2} \equiv u$ and $\ell \rightarrow L$, which represents a single large channel, (13) demands $m \ell=0$ or

$$
\omega=-\left(\frac{u}{\sqrt{g h}} \pm 1\right) k \sqrt{g h}
$$

which are shallow water waves. These limits imply that the true solution will consist of Kelvin-Helmholtz waves modified by shallow water effects and vice versa.

The following nondimensionalization will be used:

$$
\varphi=\frac{w}{\hbar g h}, \quad \Omega=-\ell, \quad F_{j}=\frac{u_{j}}{\sqrt{g h}} .
$$

In keeping with the method of approach a.) the long wavelength limit of (13) need only be considered. Let $k$ be small enough so that each of the tanh can be expanded to first order and, without lack of generality, take $F_{2}=0$ (13) then becomes a quartic

$$
\phi^{4}+2 F \varphi^{3}+\left(F^{2}-1\right) \varphi^{2}-2 F(1-\Omega) \phi-F^{2}(1-\Omega)=0
$$

which clearly indicates that the waves are non-dispersive. It should be noted that if the analysis began with the one-dimensional flow equations i.e.,

$$
v_{y}=\delta v_{y}=\underset{(3) \rightarrow}{0} \rightarrow \partial_{t}(h \ell)+\partial_{x}\left(u_{x} h l\right)=0
$$

the polynomial (15) results after a linear perturbation. This shows that the long wavelength limit is equivalent to one-dimensional flow. By using Descartes rule and theorems on the discriminant of a quartic (Dickson, 1922) the region of stability can be deduced and is shown in Fig.4. For compressible flow without walls the critical Mach number for stable flow is $81 / 2$ (Gerwin, 1968), while the presence of the outer walls acts like a stabilizing factor for long wavelengths reducing the critical Froude number to 2. Figure 5 shows some of the solutions to (15) for a fixed $\Omega$. The destabilizing part of the frequency $I_{m}(\omega / \sqrt{g h})$ is along the ordinate and the relative Froude number $F=F,-F_{2}$ along the abscissa. The slope of the curve increases as $\vec{F}$ does until $\bar{F} \cong 1.2$, after which it rapidly decreases to zero as $F$ moves toward the stability region of Fig.4. These solutions indicate that the wavenumber of maximum growth rate is infinite which is beyond the region of validity in this approach. In case b.) the analysis for larger $k$ is developed and a matching of the two solutions should pick out an optimal $k$. 


\section{b. Tension and Mass}

We now consider the coordinate system of Fig.3.b. and allow for a tension $T$ and a mass per unit area $\mu$ in the membrane. The first order perturbation theory leads to the same dispersion relation as above ( 7 ), however in this case there are different boundary and matching conditions. The former are given by

$$
\delta v_{1} \rightarrow 0 \text { as } y \rightarrow-\infty, \delta v_{2} \rightarrow 0 \text { as } y \rightarrow+\infty \text {. }
$$

The matching of the spanwise velocity $\delta v$ at the interface is still given by (11) but the difference in the pressure perturbation is now given by

$$
\mu \partial_{t}^{2} \eta=\delta P_{1}-\delta P_{2}+T \partial_{*}^{*} \eta \text {. }
$$

This means that the acceleration of a unit area of the membrane is given by the pressure jump $\left(\delta P_{1}-\delta P_{2}\right)$ across the interface and the tension due to the curvature. This equation reduces to

$$
\rho g\left(\delta h_{1}-\delta h_{2}\right)=-\mu \omega^{2} \eta+T k^{2} \eta \text {. }
$$

Using the above conditions and the solutions (9) the characteristic equation can be written in nondimensional form as

$$
\frac{(\bar{F}+\varphi)^{2}}{\sqrt{1-(\bar{F}+\varphi)^{2}}}+\frac{\varphi^{2}}{\sqrt{1-\varphi^{2}}}=-\frac{\mu k}{9} \varphi^{2}+\frac{T}{g g h}
$$

where $\bar{F}$ is the relative Froude number $F_{\mathbf{i}}-F_{\mathbf{z}}$. This equation is a twelfth order polynomial valid for all values of $\phi, \vec{F}$ and $k$. We consider the following ordering

$$
\bar{F} \sim \theta(\epsilon) \quad, \quad \sim \sim \theta\left(\epsilon^{2}\right)
$$

which holds for the case $\bar{F}<1$ and $k$ large. This parameter range is of interest since all of the flows in the experiment have a relative Froude number $<1$. Upon expanding (16) and keeping terms to order $\epsilon^{4}$ the resulting equation is a quadratic in $\varphi$ whose solution is

$$
\begin{aligned}
\varphi=\{ & -\bar{F}(1+k \tau) \pm\left[2 k \tau\left(1-F^{2}+\bar{F}^{4} / 8\right)+\tau^{2} k^{2}\left(1+\bar{F}^{2}+F^{2 / 4}\left(1+\bar{F}^{2} / 2\right)-M k F^{2}\right)+\right. \\
& \left.\left.+M \tau k^{2}\left(1-\bar{F}^{2}+\bar{F}^{4} / s\right)-\bar{F}^{2}\right]^{1 / 2}\right\} \cdot \frac{1}{2+M k+\bar{T}_{k}},
\end{aligned}
$$

where $M=\mu / \rho$ and $\tau / \rho g h$. This solution shows that the mass and relative Froude number are destabilizing while the tension is always stabilizing. The region of instability varies for different values of $k, M, T, F$ and $g h$ so no simple relation holds as in Fig.4. Some solutions of the quadratic are shown in Fig.6. The general feature of the solution is the strong destabilizing effect of the relative Froude number compared to changes by similar factors in $M$ and $T$. Analysis of the solutions in the case $\vec{F}>1$, for which (16) reduces to a complex quadratic when $\phi$ is small ( $k$ large), show the same general trend as the curves in Fig.6 - as $F$ increases so does the region of instability.

c. Comparison with Experiment

Before discussing the analysis and the experimental results some mention 
of the method of measuring the relative Froude number and the tension should be made. The mass flow rate $Q$ in each channel could be determined by measuring the amount of time needed to fill the large vessel of fixed volume $V$. If $h$ is the depth of the fluid averaged over the channel and $\boldsymbol{l}$ is a typical width, $Q / \rho h e \sqrt{g h}$ is the Froude number for the channel. The problem is that the ideal mathematical state can never be realized since the system is susceptible to infinitesimal perturbations. The measured velocity was therefore only an average in time and space during the oscillations. The tension in the flexible membrane was found by fixing one end and stretching the other end which was attached to a gram scale. A plot of the gram scale reading versus the displacement gives a straight line whose slope is the tension. Typical values ranged from 30 to 40

The crosses in Fig.7 show some typical results which are fully described in Table 1 (values in cgs units).

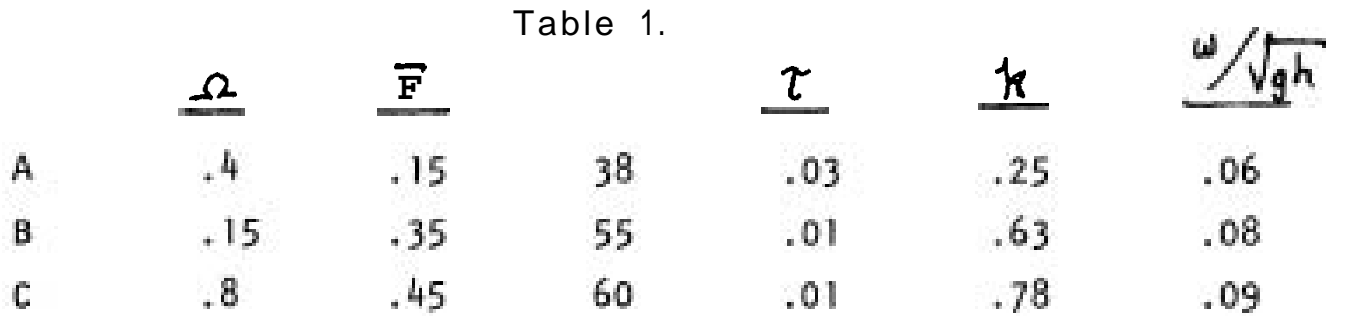

As an example of the matching process consider point B. On Fig.7 we have added the line from Fig.5 for $\Omega=.2$ and $\bar{F}=.3$ and the curve from Fig.6 for $M=.01, \tilde{I}=.01, \bar{F}=.3$. By joining the two curves we find the predicted wavenumber for maximum growth differs from the observed value by a factor $\sim 4$ and the predicted oscillation frequency is off by -2 . These are typical results. The crudeness of the theory by virtue of the approximations and the uncertainty of the experimental measurements, such as the velocity and the width $\boldsymbol{\ell}$, might be the cause of the difference between theory and observation. Also the finite length of the system should have some effect on the preferred wavelength, but not a dominant one since the system's length was not an integral multiple of the observed wavelengths.

Nonlinear effects might also play a role in altering the results of linear theory, so a nonlinear analysis was done by moving into the frame of the wave disturbance moving with speed $\omega / n$. However, because the expansion starts from one of the edges of Fig.4 it was found that many orders in the perturbation analysis would be needed to reach the region of interest which lies in the center of Fig.4. The nonlinear problem is also relevant for describing how the exterior walls enter to limit the growth of the perturbation, so another attempt with a different approach might be fruitful.

Finally consider the observation mentioned at the end of section 2 in which the frequency and wavelength of the oscillation decreased when the exterior channels were partially blocked. Besides decreasing the flow velocity in the exterior channels to almost $n$ il the blocking also raised the height of the water by $\sim 4 / 3$. This forced the level of the water in the central channel to increase and subsequently lowered the flow rate, because the discharge from all the sources remained fixed. The combined effect of slowing the flow and raising the water level reduced the relative Froude number by $\sim 1 / 2$ while altering $T=T / g h$ by only $3 / 4$. This can be translated into a shift of the 
matching curve in Fig.7 to the left and a decrease in its maximum. The wavelength with maximum growth rate and the value of this rate $(w / \sqrt{g h})$ will thereby be smaller. The analysis of the observation and others like it adds support to the correctness of the general method of analysis although some details need to be improved.

4. CONCLUSION

From the observations and analysis of the shallow water channel instability what can be said about the possibility of an oscillating 'TwinExhaust' model for double radio sources? Clearly nothing quantitative since any correspondee would have to consider the basic physical differences between the two models: a.) In the shallow water experiment the fluid density in both the jet channel and the ambient flow was the same which implies the same "sound speed" $\sqrt{g h}$. For the galaxy however the jet flow may be highly relativistic while the background gas is much denser and has a smaller speed of sound. b.) The role of the tension and mass does not carry over directly to the galactic problem, although there may be physical mechanisms in the latter case which play similar roles such as that of the tension in stretched magnetic field lines which run along the jet's surface. c.) Finally the exterior rigid walls which were seen to play an important part in the long wavelength stability problem are unrealizable in the galaxy. Again it might be possible that a corresponding mechanism such as conductive heating of the ambient gas, especially at the bulges of the unstable jet where the exposed area is large, may serve to increase the external pressure and thus act like a wall. However these analogies are totally speculative and more detailed analysis would have to be considered before any analogy could be carried over directly.

On the other hand, qualitatively one may now suspect that a type of Kelvin-Helmholtz instability could lead to the "beady" appearance of M87's jet or even to a periodic oscillation. Relativistic Kelvin-Helmholtz instabilities have been discussed in the literature (Turland and Scheuer 1976, Blandford and Pringle 1976) but not with the ramifications considered here. Due to the symmetry of the problem a rigid wall can replace the center-line of the central channel and the left half swung over to meet the right. This configuration is shown in Fig. 8 and is the same as a double-diffuser but experimentally easier since identical flows in the left and right directions for each channel are no longer demanded. Photographs 5 and 6 show strong oscillations on both sides of the center wall. Because each strip of mylar has slightly different physical properties it was impossible to get the same response on both sides. However it is possible to see that whenever the channel flow is cut off on one side due to the collapse of the membrane against the center wall the opposite channel is forced to expand. The result is an oscillating system whose frequency is dependent upon the phase speed of the pressure wave which travels back from one channel to the central cavity and out through the opposite channel.

One final comment along more general lines of interest has been suggested by Dr. W. Malkus. There are many types of walls or membranes in nature. Some of them are rigid (as were the exterior walls in the experiment) and others involve a process. In the latter category one can include the walls of the galactic jet and those of the central water channel. The former is a conduction front while the latter has been described by tension and mass. 
This research has attempted to look at possible analogies between these two types of walls in the case of a jet flow through an ambient medium. Of course the study here is incomplete and points out many more problems than solutions regarding the nature of interfaces. But the fundamental idea has been that some general principles of these types of interfaces exist and qualitative results derived from the study of one type of membrane imply phenomena for the other type.

\section{Acknowledgments}

The author wishes to thank Dr. E.A.Spiegel for suggesting the problem and for his guidance throughout the course of the research. He also wishes to thank Dr. M.Landahl and Mr. R.Frazel for informative discussions. Finally a special thanks to everyone in the Geophysical Fluid Dynamics Program for a stimulating and enjoyable summer.

\section{References}

Blandford, R.D. and J.C.Pringle 1976 Kelvin-Helmholtz Instability of Relativistic Beams. Mon.Not.Roy.Ast.Soc., 176: 443.

Blandford, R.D. and M.J.Rees 1974 A 'Twin-Exhaust' Model for Double Radio Sources. Mon.Not.Roy.Ast.Soc., 169. 395.

Chandvasekhar, S. 1961 Hydrodynamic and Hydromagnetic Stability, Oxford; Clarendon Press (\$101).

Dickson, L.E. 1922 Theory of Equations, New York; John Wiley $\varepsilon$ Sons (Chapt.VI).

Gerwin, R.A. 1968 Stability of the Interface Between Two Fluids in Relative Motion. Rev.of Mod.Pjys. 40: 652.

Landau, L.D. and E.M.Lifshitz 1959 Fluid Mechanics, Oxford; Pergamon Press (\$\$13 and 100).

Scheuer, P.A.G. and B.D.Turland 1976 Instabilities of Kelvin-Helmholtz Type for Relativistic Streaming. Mon.Not.Roy.Ast.Soc., 176: 421.

Turland, B.D. 1975 Observations of M87 at SGHz with the 5-Km Telescope. Mon.Not.Roy.Ast.Soc. 170: 281. 


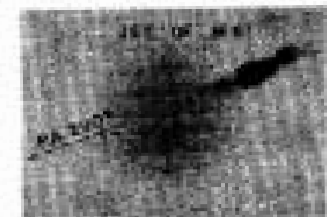

PHOTOGRAPH 1. JET QF MB7

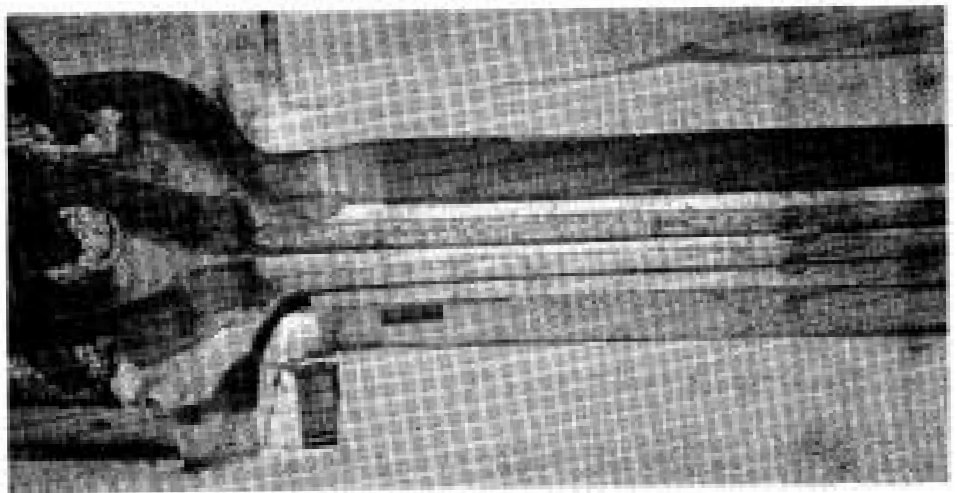

PHOTOERAPH2. OVERHEAD OF THE EXPERIMENT

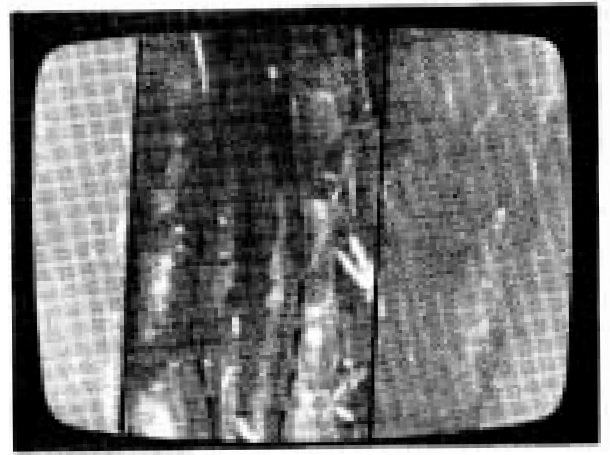

PHOTOGRAPH 3.

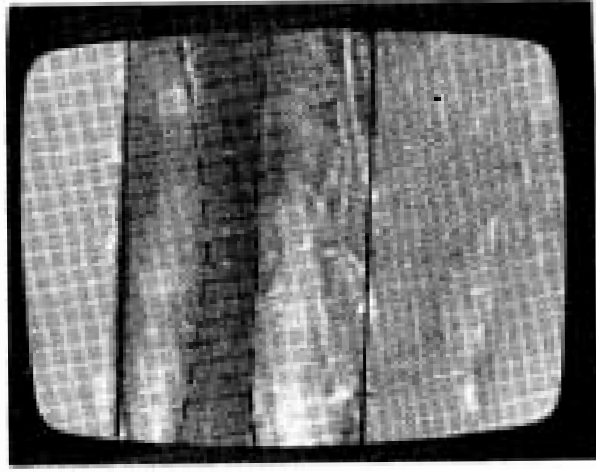

PHOTOCRAPH 4.

VIEWSOF OSCILLATING CENTRAL CHANNEL

RIGID WALLS ENHANCED BY SOLID LINES

FLEXIBLE WALLS ENHANCED BY DASHED LINES
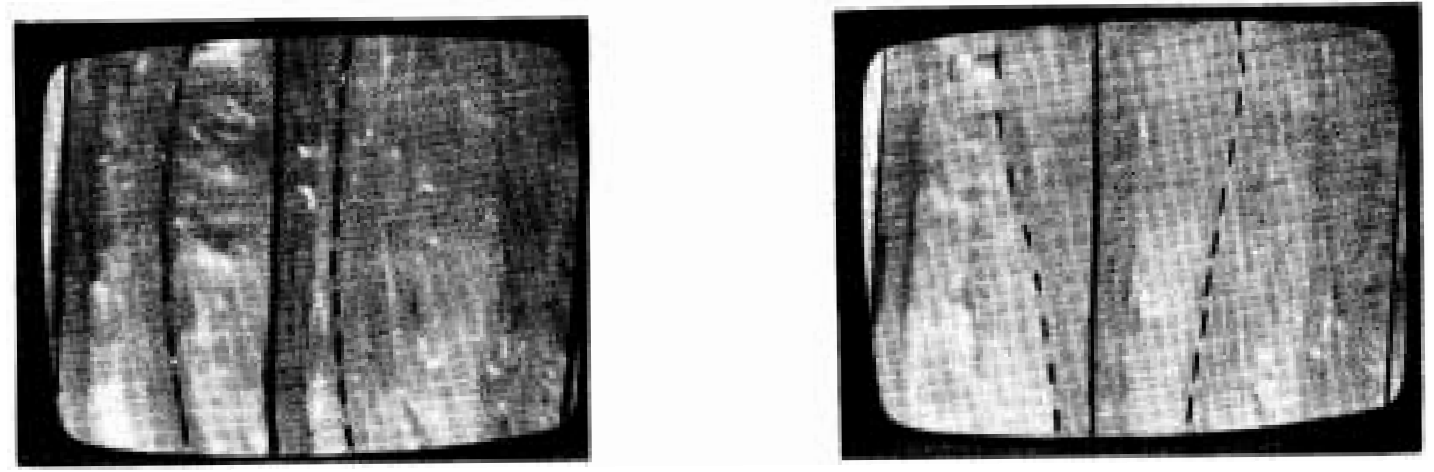

$\frac{\text { PHOTUGRAPH } 5 .}{\text { WEWS OF OSCILLATING DOUBLE-DIFFUSER }}$ 


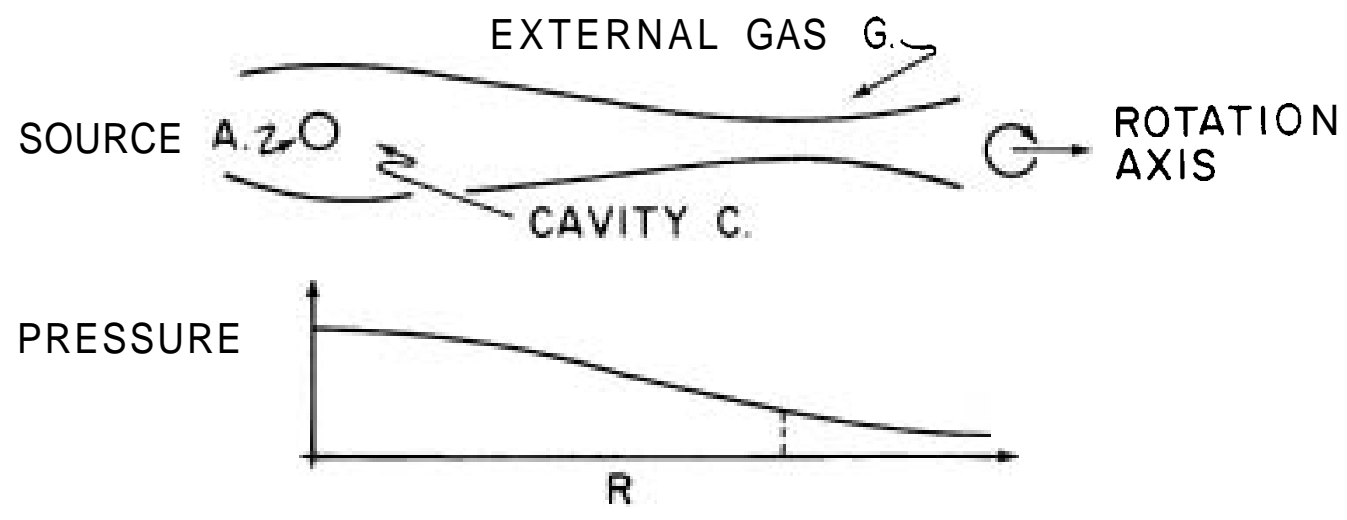

Fig. 1. Blandford-Rees 'Win-Exhaust' Model

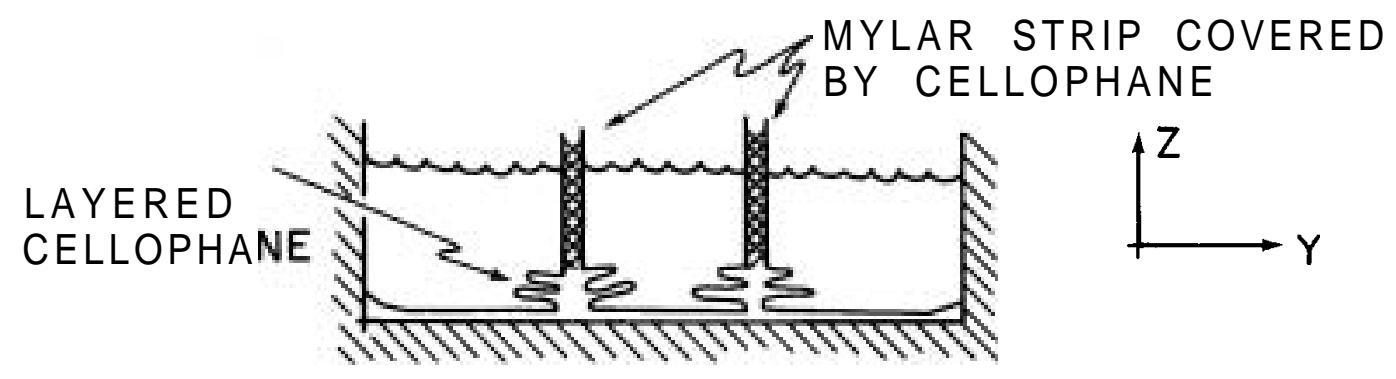

Fig. 2. Channel Construction

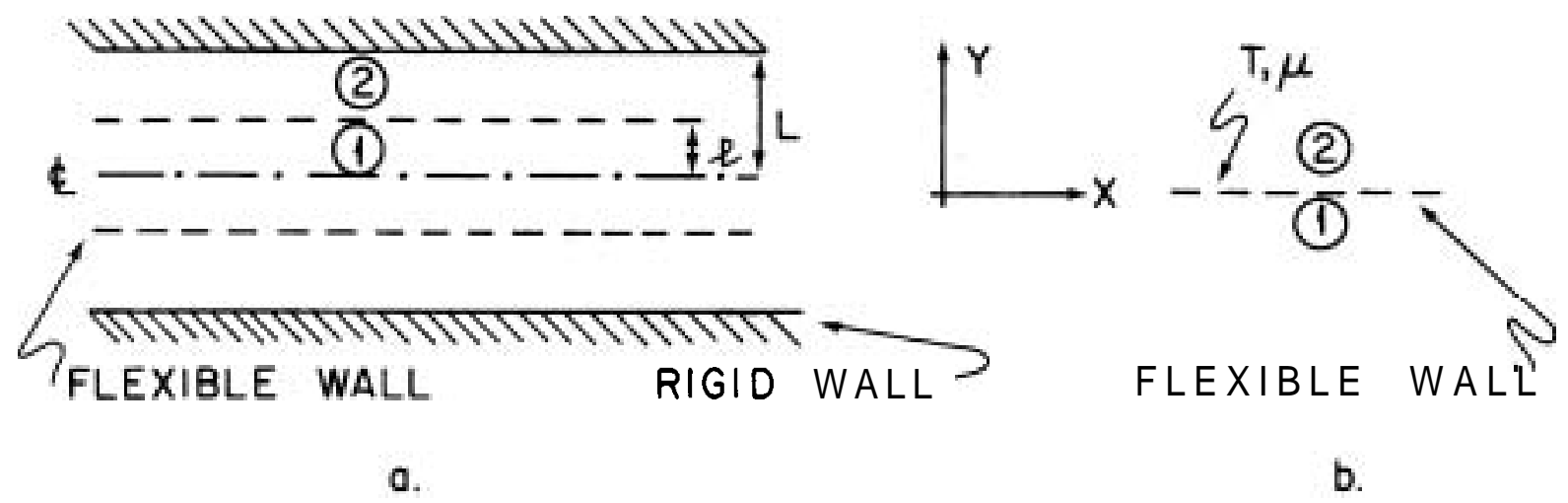

Fig. 3. Coordinate Systems Used in the Analysis

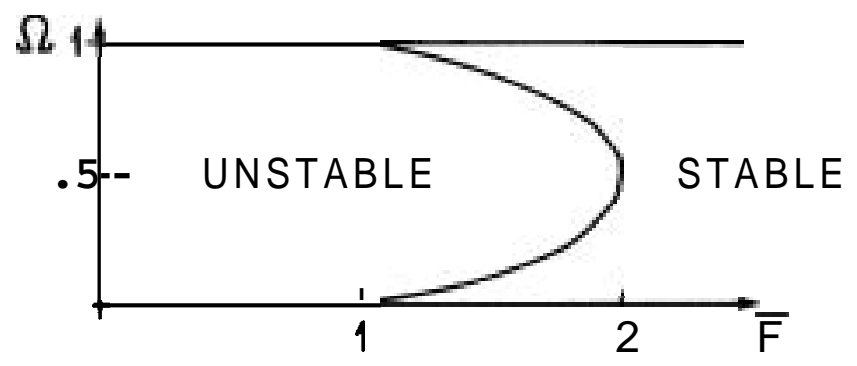

Fig. 4. Region of Stability in the Long Wavelength Limit 


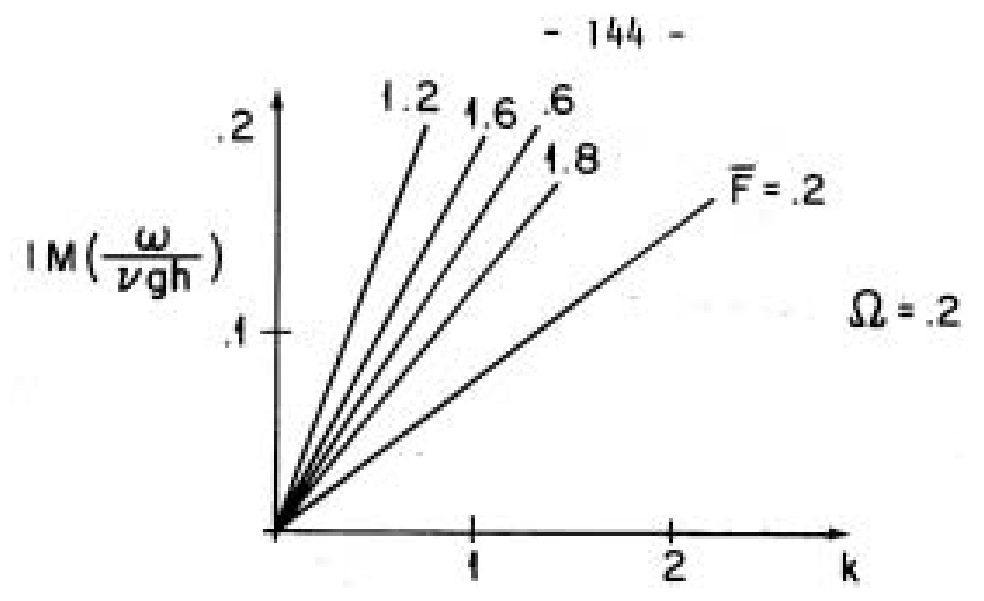

Fig. 5. Unstable Solutions for Case a.

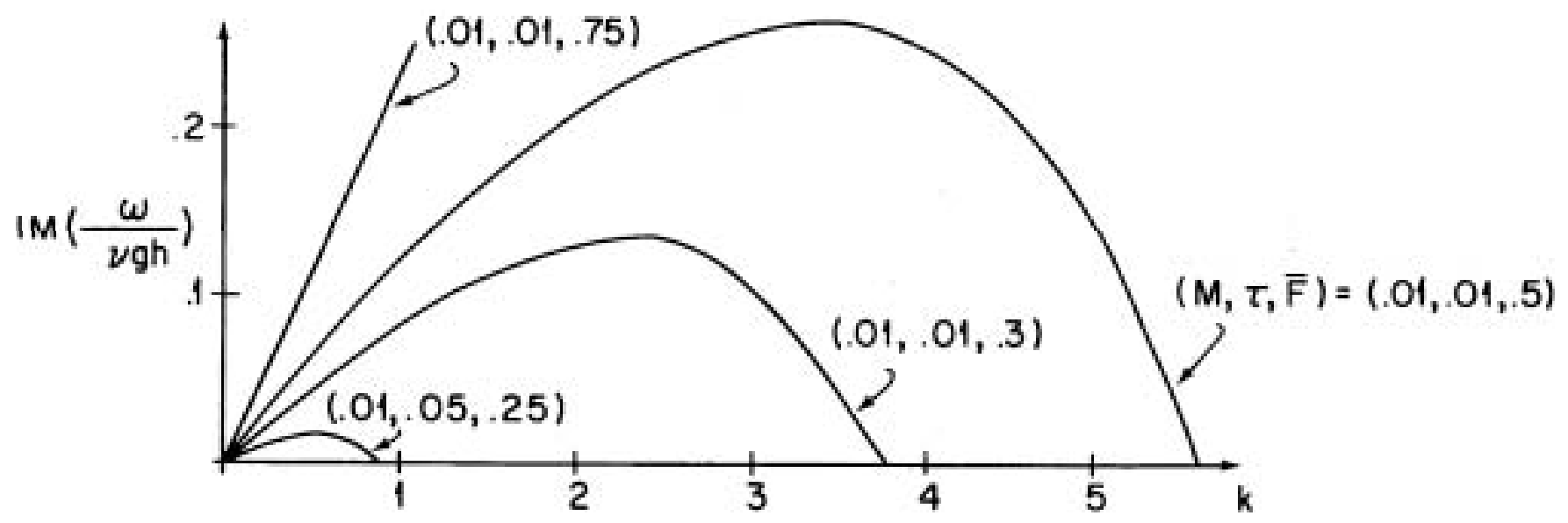

Fig. 6. Unstable Solutions for Case b.

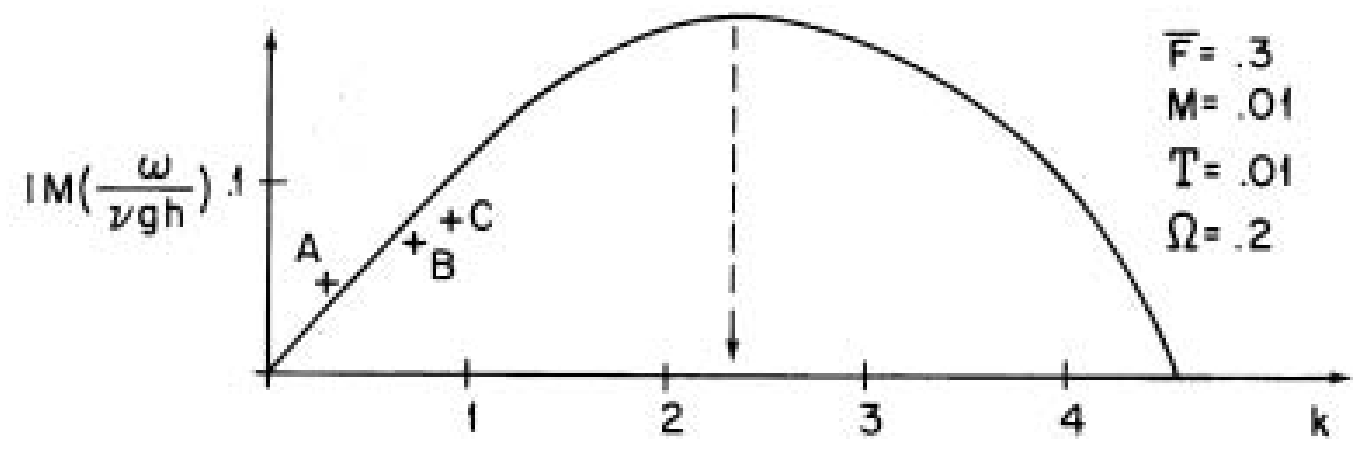

Fig. 7. Matching of Solutions

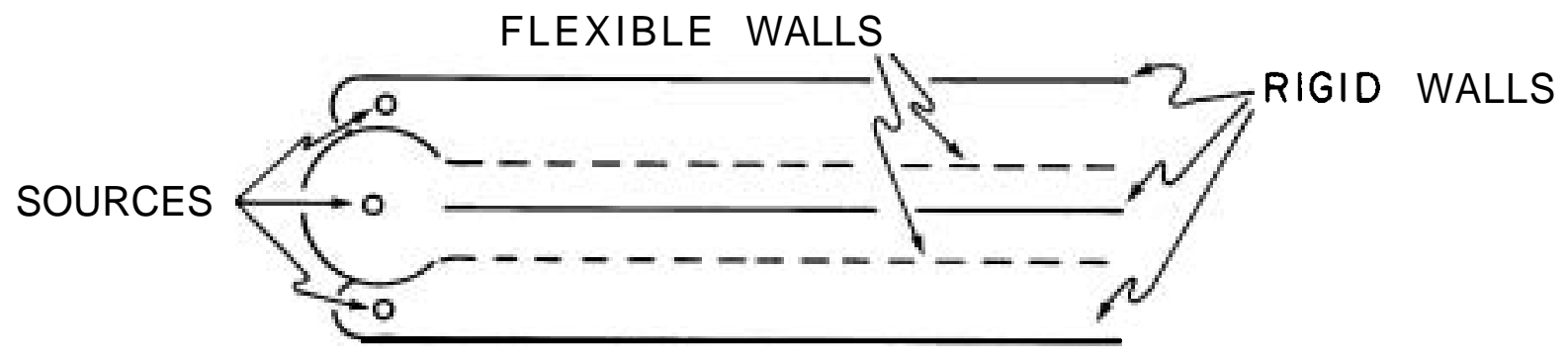

Fig. 8. Modified Construction for Double-Diffuser 


\section{THE INITIAL VALUE PROBLEM FOR AN INFINITE SHEAR LAYER WITH A DISCONTINUOUS DENSITY PROFILE \\ L. Hăkan Gustavsson}

1. Introduction

In an experiment, where there was a turbulent flow of salty water under a layer of fresh water, Lofquist (1963) notices the appearanise of wave patterns on the interface between the two layers of fluid. The waves were only present for a certain range of flow rates of the salty water: for small flow rates the interface showed no definite structure, and for very large flow rates the dye, used to detect the wave pattern, quickly dispersed. Between these two extremes, there was a range of flow rates at which waves were clearly visible on the interface. The waves were always three-dimensional and they formed a pattern of oppositely oriented arcs, one going upstream and the other going downstream. The upstream arcs were found to be slightly above the downstream arcs and Lofquist proposed that the two observed modes were associated with disturbances in respectively the upper and lower boundaries to the region of density change. Loftquist also suggested that the concentration of dye, which marked the arcs and which decreased at the ends, indicated that the vorticity was larger along the crests.

In studying the wave behavior of the flow configuration in experiments such as Lofquist's, the Kelvin-Helmholtz model is usually adopted. Without surface tension, this model shows that small enough wave disturbances are always unstable. The instability will cause typical eddies to be formed at the interface. Breakdown of the eddy structure with accompanying turbulent mixing will create finite gradients in the velocity and the density fields further downstream, a configuration more stable than the original one. It is therefore instructive to consider waves which are neutrally stable.

In the model analyzed below, the waves are considered to be generated by disturbances introduced in an infinite shear layer, with a constant shear, in which the density of the fluid is discontinuous at $z=0$.

In order to describe completely an arbitrary initial disturbance in the velocity field, it is not sufficient to consider only waves, however. In addition to the waves, there is also a convective type of solution to the initial value problem, which has quite different properties from the waves. One of these is the spreading in space; the waves spread in the cross-stream direction as well as in the streamwise direction, whereas the convected solution is always confined within the same spanwise dimensions, and is sheared as it travels downstream.

Another difference is the behavior at large times. Basically, due to their spanwise spreading, the waves, if neutrally stable, will decay faster than the convected part of the solution, which will show different time behavior for its different velocity components, however.

To bring out the effect of density discontinuity, a comparison with the initial value problem for an infinite shear layer with constant density is also made. 


\section{Initial value problem}

The flow configuration of Fig.l is considered. The perturbations of the velocity components at $t=0$ are specified and their subsequent behavior is studied. The significance of three-dimensionality, especially in connection with the treatment of the waves that are generated, will be brought out.

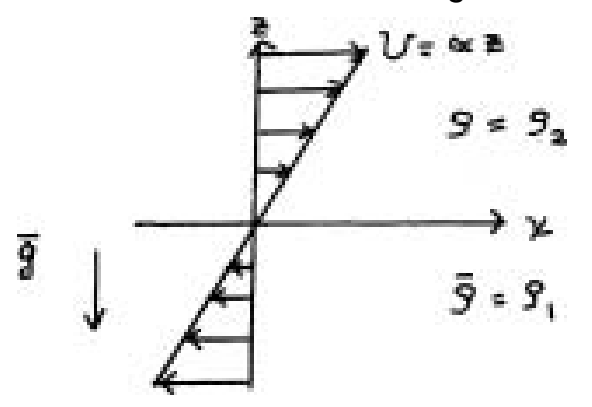

Fig. 1

The mean-flow is given by $U=\alpha \mathbf{z}$, where $z$ extends to+ $\infty$ and the mean density is $\boldsymbol{S}=$ ?, for $\bar{z}>0$ and $\bar{S}=S_{1}$, for $z<0$. y, and $S_{2}$ are constants and chosen such that $S_{1}>S_{2}$. The fluid is inviscid, incompressible and the disturbances are small compared to the mean quantities.

Equations:

$$
\begin{aligned}
\rho\left(\vec{u}_{t}+(\vec{u} \cdot \nabla) \vec{u}\right) & =-\nabla \rho+\rho \vec{g} \\
\nabla \cdot \vec{u} & =0 \\
\rho+(\vec{u} \cdot \nabla) \rho & =0
\end{aligned}
$$

Consider perturbations $(u, v, w), p, 9$ around a mean state $(U(z), 0,0), P_{0}, \overline{9}$. Linearization of Eqs. (1) - (3), with subtraction of the mean parts leads to the following set of equations:

$$
\begin{aligned}
& \bar{g}\left(u_{t}+v u_{x}+w v^{\prime}\right)=-P_{x} \\
& \bar{g}\left(v_{t}+v_{v_{x}}\right)=-P_{y} \\
& \bar{g}\left(w_{t}+v_{w_{x}}\right)=-P_{z}-g g \\
& u_{x}+v_{y}+w_{z}=0 \\
& \rho_{t}+v g_{x}+w \cdot \bar{g}^{\prime}=0 \\
& \prime^{\prime \prime \prime} \text { denotes } \frac{d}{d z}
\end{aligned}
$$

The pressure, $p$, is eliminated by first forming the divergence of the component equations (4) - (6), using continuity, and then (6) is used to give a relation between $S$ and $w$. Finally, elimination of $S$ via (8) gives the following equation for $W$ : 


$$
\frac{D}{D_{t}}\left[\frac{D}{D_{t}}\left(\nabla^{2} w\right)-U^{\prime \prime} w_{x}\right]=\frac{\bar{g}^{\prime}}{\bar{g}}\left[U^{\prime} \frac{D w_{x}}{D t}-\frac{D w_{z}}{D t^{2}}+g \nabla_{\mu}^{2} w\right]
$$

where $\quad \nabla^{2}=\frac{\partial^{\prime \prime}}{\partial x^{2}}+-\partial \partial^{2}+\frac{\partial^{2}}{\partial z^{2}}=\nabla_{H}^{*}+\frac{\partial^{2}}{a a^{2}}$ and $\frac{D}{D t}=\underset{a t}{P^{2}} \mathrm{~V} \frac{a}{a x}$

Conditions at the density interface:

$$
w \text { and } P \text { continuous }
$$

By integrating (9) over the density discontinuity, the pressure condition is found to be equivalent to

$$
\bar{\rho}\left[\frac{D^{2}}{D t^{2}} w_{z-}-\frac{D}{D t} v_{0}^{\prime} w_{x}-g \nabla_{H}^{2} w\right] \text { continuous at } z=0
$$

Conditionat $z= \pm \infty \quad W \rightarrow 0$

Equation (9) is easily solved in the two regions $z<0$ and $\boldsymbol{z}>0$, separately, and with the Fourier transform in $x$ and $t$ defined as

$$
\hat{w}\left(K_{x} K_{y}, z, t\right)=\frac{1}{2 \pi} \iint_{-\infty}^{\infty} e^{-i\left(K_{x} x+k_{y} y\right)} w(x, y, z, t) d x d y
$$

it is found that

$$
\begin{aligned}
& z>0 \quad \hat{w}_{>}=B e^{-k z}-\frac{e^{-k z}}{2 k} \int_{0}^{z} e^{k \eta} \hat{f} d \eta-e^{-k \eta} \hat{f} d \eta \\
& z<0 \quad \hat{w}_{\alpha}=C e^{k z}+\frac{e^{k z}}{2 k} \int_{0}^{z} e^{-k \eta} \hat{f} d \eta-\frac{e^{-k z}}{2 k} \int_{-\infty}^{z} e^{k \eta} \hat{f} d \eta
\end{aligned}
$$

where

$$
\hat{f}=\hat{\phi}_{0} e^{-i k_{x} V(\eta) t}=\left(\frac{d^{2} \hat{w}}{d \tau^{2}}-k^{2} \hat{w}\right)_{t=0} \cdot e^{-l k_{*} V(\eta) t}
$$

and $\quad K^{2}=K_{x}^{2}+K_{y}^{2}$

To obtain (13) and (14) the conditions at $\pm \infty$ have been used. The 'constants' of integration, $B$ and $C$, are functions of time and they are determined by application of the following conditions at $z=0$ :

$$
\begin{gathered}
\hat{w}_{<}=\hat{w}_{2} \\
\frac{D^{2}}{D t^{2}}\left(\rho_{2} \hat{w}_{z}-\rho_{1} \hat{w}_{2<}\right)=\left(\rho_{2}-\rho_{1}\right)\left[i k_{k} \alpha \cdot \frac{D \hat{w}^{2}}{D t}-g k^{2} \hat{w}\right]
\end{gathered}
$$

By eliminating $B$, the equation for $C$ becomes of second order in time and by specifying the two initial conditions:

$$
\hat{w}\left(k_{x}, k_{y}, z, 0\right)=\hat{w}_{0}\left(k_{x}, k_{y}, z\right)
$$

and

$$
\frac{\partial \hat{w}}{\partial t}\left(k_{x}, k_{y}, 0,0\right)=\hat{R}\left(k_{x}, k_{y}\right) \text {, }
$$

$C$ and thereby also $B$ are found. Inserting these values into (13) and (14) we obtain finally the following expressions for $\widehat{w}$ in the two regions $><O\left(\hat{w}_{5}\right)$ and $70\left(\hat{w}_{7}\right)$ : 


$$
\begin{aligned}
& \hat{w}_{\varepsilon}=e^{k \pi}\left\{E_{1} \cdot e^{\lambda_{1} t}+E_{2} e^{\lambda_{2} t}-\frac{1}{\lambda_{2}-\lambda_{1}}\left[\int_{0}^{\infty} \hat{f}_{1}\left(\frac{e^{\lambda_{1} t}-e^{-i k_{2} V_{(\eta) t}}}{\lambda_{1}+i k_{x} V(\eta)}-\frac{e^{\lambda_{2} t}-e^{-i k_{x} V(\eta) t}}{\lambda_{2}+i k_{x} V(\eta)}\right) d \eta^{+}\right.\right. \\
& \left.\left.+\int_{-\infty}^{0} \hat{f}\left(\frac{e^{\lambda_{1} t}-e^{-i k_{x} V(\eta) t}}{\lambda_{1}+i k_{x} U(\eta)}-\frac{e^{\lambda_{2} t}-e^{-2 k_{x} U(\eta) t}}{\lambda_{2}+i k_{x} U(\eta)}\right){ }_{d \eta}\right]+\frac{1}{2 k} \int_{0}^{\infty} e^{-k \eta} \hat{f} d \eta\right\}+\hat{w}_{n s}
\end{aligned}
$$

and

$$
\hat{W}_{>}=e^{-k_{2}}\left\{E_{1} \cdot e^{\lambda_{1} t}+E_{i} e^{\lambda_{2} t}-\lambda_{3}^{\prime}-\lambda_{i}[\cdots]+\frac{1}{2 k} \int_{0}^{\infty} e^{-k \eta \hat{f}} d \eta\right\}+w_{n s}
$$

where in Eq. (16) is the same as the term within the square bracket in Eq. (15) and where

$$
\begin{aligned}
& \hat{f}=\hat{\phi}_{0} \cdot e^{-i \kappa_{z} v(\eta) t} \\
& \hat{f}_{1}=\frac{\rho_{2}}{\rho_{m}} \frac{k_{x}^{2}}{2 k} \cdot e^{-k \eta}[U(x)-V(\eta)]^{2} \hat{\phi}_{0}(\eta) \\
& \hat{f}_{2}=\frac{\Delta \rho}{\rho_{m}} \frac{e^{k \eta}}{4 k}\left\{k_{x}^{2}\left[(U(e)-U(\eta))^{2}-\frac{\alpha U(\eta)}{k}+\frac{\alpha U(y)}{k}\right]+g^{k}\right\} \\
& \lambda_{1}=-i k_{x} U(\alpha)+i k_{x}(\mu+\nu) \\
& \lambda_{1}=-i k_{x} U(\boldsymbol{\mu})+i k_{*}(\mu-\nu) \\
& \mu=\frac{\alpha}{4 K} \cdot \frac{\Delta \rho}{\rho_{m}} \quad ; \Delta \rho_{1}-\rho_{2} \\
& \nu^{2 \prime}=\mu^{2}+\frac{\Delta \rho}{\rho_{m}} \frac{g^{k}}{2 k_{x}^{2-1}} ; \rho_{m}=\frac{\rho_{1}+\rho_{2}}{2} \\
& E_{1}=\frac{1}{\lambda_{i}-\lambda_{1}}\left[-\hat{R}+i \alpha K_{x}\left\{\int_{-\infty}^{0} e^{k \eta} \hat{w}_{0} \alpha \eta-\frac{\left.\hat{w}_{0}\right|_{x=0}}{2 k}\right\}+\frac{\lambda_{1}-2 \lambda}{2 k}\left[\frac{d \hat{w}_{0}}{d z}+k \hat{w}_{0}\right]_{z=0}\right. \\
& E_{2}=\frac{1}{\lambda_{2}-\lambda_{1}}\left[\hat{R}-i \alpha k_{x}\left\{\int_{-\infty}^{0} e^{k \eta} \hat{V}_{0} d \eta-\frac{\left.\hat{w}_{0}\right|_{2 n}}{2 k}\right\}+\frac{\lambda_{1}}{2 k}\left[\frac{d \hat{w}_{0}}{d z}+k \hat{w}_{0}\right]_{z}=0\right.
\end{aligned}
$$

$\stackrel{\mathbf{W}}{n s}_{\text {s }}$ is the solution to the initial value problem for the case of constant density throughout the fluid. It is found that

$$
\hat{w}_{n s}=-\frac{e^{-k \pi}}{2 k} \int_{-\infty}^{k \eta} \hat{f} d \eta-e^{k t}-\int_{z}^{\infty} e^{-k \eta} \hat{f} d \eta
$$

The terms in $\hat{\omega}$ are of essentially two types: wave terms represented by $\mathrm{e}^{\lambda, t}$ and $\mathrm{e}^{\lambda_{2} t}$ and convective terms represented by $\widehat{W}_{n s}$ and also by some of the integral terms, dependent on the value of $z$. Let us now consider the behavior of the different terms in $\hat{w}_{<}$and $\widehat{w}_{>}$when $t$ becomes large. The wave terms are treated in section 3 and the convective part in section 4. 


\section{Asymptotic wave behavior}

The solution for $\widehat{w}$ contains wave terms of the type $e^{\lambda_{1} t}$ and $e^{\lambda_{2} t}$. When inverting these Fourier transforms to obtain $W$, only the asymptotic form, for large times, will be considered. We are left with the following two types of integrals:

$$
\begin{aligned}
& I_{1}=\frac{1}{2 \pi} \iint_{-\infty}^{\infty} e^{i\left(k_{x} x+k_{y} y\right)} e^{\lambda_{1} t} d k_{x} d k_{y} \\
& I_{2}=\frac{1}{2 \pi} \iint_{-\infty}^{\infty} e^{i\left(k_{x} x+k_{y} y\right)} e^{\lambda_{2} t} d k_{x} d k_{y}
\end{aligned}
$$

where $\lambda_{1}$ and $\lambda_{2}$ are defined in connection with (15) and (16). For large $t$ the method of stationary phase may be used to give an estimate of $I_{1}$, and $I_{2}$. For brevity, consider just the behavior of $I$, . The result for' $I_{1}$ is obtained in the same manner and is given below.

Define the phase: $\theta=\frac{x}{t} K_{x}+\frac{y}{t} K_{y}-K_{x} v(z)+K_{x}(\mu+\nu)$ keeping $x / t$ and $y / t$ fixed, the stationary points are found as the solution to the equation

$$
\frac{\partial \theta}{\partial K_{x}}=\frac{\partial \theta}{\partial K_{y}}=0
$$

Differentiating, introducing polar coordinates $(\kappa, \varphi\rangle$ in $\left(\kappa_{x}, K_{y}\right)$ space we find the following equations:

$$
\begin{aligned}
& \xi+\left[\frac{a^{7} \sin ^{2} \phi}{k^{2}}+\frac{b}{2 K}\right]=0 \\
& \eta+\sin \phi \cdot \cos \varphi\left[-\frac{a}{k}-\frac{1}{\nu}\left(\frac{b}{2 k \operatorname{con}^{2} \phi}-\frac{a^{2}}{k^{2}}\right)\right]=0 \\
& \xi=x / t-v(a) \\
& \eta=y / t \\
& a=\frac{\alpha}{4} \cdot \frac{A \rho_{0}}{\rho m} \\
& b=\frac{g \Delta \rho_{0}}{23 m} \\
& \nu=\sqrt{\frac{a^{2}}{k^{2}}+\frac{b}{K \cos ^{2} \phi}}
\end{aligned}
$$

Equations (18) and (19) describe a mapping from $(K, \varphi)$ to $(\xi, \eta)$ and it is convenient to parameterize this mapping to obtain a relation between and $\eta$ with either $K$ or $Q$ as a parameter. It is found that eliminathon of $k$ is possible, which gives the following relationship between $\eta$ and $\varphi$ :

$$
(F+2) \xi^{2}+2 \xi \eta(F+1) \tan \varphi+F \eta^{2} \tan ^{2} \varphi+A \xi \tan ^{2} \phi-A \eta \tan \phi=0
$$

where $F=4-6 \cos ^{2} \phi$

and $A=\frac{b}{a}$.

Equation (20) is the same for both mode 1 and mode 8 and gives con-ical sections which have different form as $\varphi$ varies. From (20) it follows that a 
natural velocity scale for $\xi$ and $\eta$ is $A=\frac{b}{a}=\frac{3 g}{\alpha}$. Thus an increase in the shear gives lower group velocities as well as shorter length scales, the length scale being $b / a^{2}$, which means that the distance between wave crests becomes shorter. Instead of plotting (20) for different $\varphi$-values it is more instructive to calculate the form of the lines where the phase is a constant.

Form of wave crests

On a wave crest, $\theta$ is a constant (for a given $t$ ) and the relation between $\xi$ and $\eta$ on the crest is found in the following way: The expressions for $\xi$ and $\eta$, given by (18) and (19) are inserted into the expression for

$\theta$. In nondimensional form, where the length scale is $b / a^{2}$, it follows that

$$
\theta^{*}=\cos \varphi+\frac{\cos \varphi}{\nu_{*}}\left(\frac{1}{K^{*}}+\frac{1}{2 \cos ^{2} p}\right)
$$

From $(21), K^{*}$ can be solved and hence give $K^{*}$ and $V_{*}$ as a function of and $\varphi$.

Now (18) and $(19)^{*} \tan \varphi$ gives

$$
\xi_{*}+\eta_{*} \tan \varphi=-\frac{1}{2 k_{*} \nu_{*} \cos ^{2} \varphi}
$$

Equations (20) and (22) give two relationships between $\xi_{*}$ and $\eta_{*}$, and elimination of $\xi$ finally leads $\mathrm{to}_{3}$

$$
\eta_{*}=\frac{\tan \varphi}{2} \frac{1-\frac{3}{\kappa_{*} \nu_{*}}}{1-K_{*} \nu_{*}}
$$

For mode 2 the corresponding expressions are found to be

$$
\begin{aligned}
& \xi_{*}=\frac{1}{2 K_{*} \nu_{*} \cos ^{2} \varphi}-\eta_{*} \tan \varphi \\
& \eta_{*}=\frac{\tan \varphi \cdot \frac{\left(1+\frac{3}{K_{*} \nu_{*}}\right)}{2}}{\left(1+K_{*} \nu_{*}\right)}
\end{aligned}
$$

The wave pattern obtained from (22)-(25) is symmetric about the $\eta_{*}$-axis. From (21) it is seen that, if $\theta^{*}>0$ so is $\cos \varphi$ and it is therefore sufficient to consider only $\theta^{m} 70$. The constant phase curves are plotted for different values of $\theta^{*}$ in Figs. 2 and 3 .

In Fig.2 the upper half plane $(\eta>0)$ describes mode 1 and $(\eta<0)$ describes mode 2. For $\cos \varphi=0$, the two modes have the same phase at the same point. The locus of this point as $\theta^{*}$ varies is the dash-dot line in the upper half-plane. From Fig.2 it is also seen that mode 1 is never found for $\xi_{*}>0$. Mode 2, however, can appear for $\xi_{*}>0$, but the maximum group velocity in the $\xi_{*}^{*}$ direction is, which is reached for $\theta^{*}=2$. For $\theta^{*}<2$, the wave crests of mode 2 will be confined inside the region given by the dash-dot curves.

For $\theta^{*}>2$, Fig. 3 shows some typical curves of constant phase and it is noted that as $\theta^{*} \rightarrow \infty$ the two modes will together form a circle. This corresponds to short waves which are basically gravitational.

To follow the evolution in space of a wave crest, we start at the origin, 


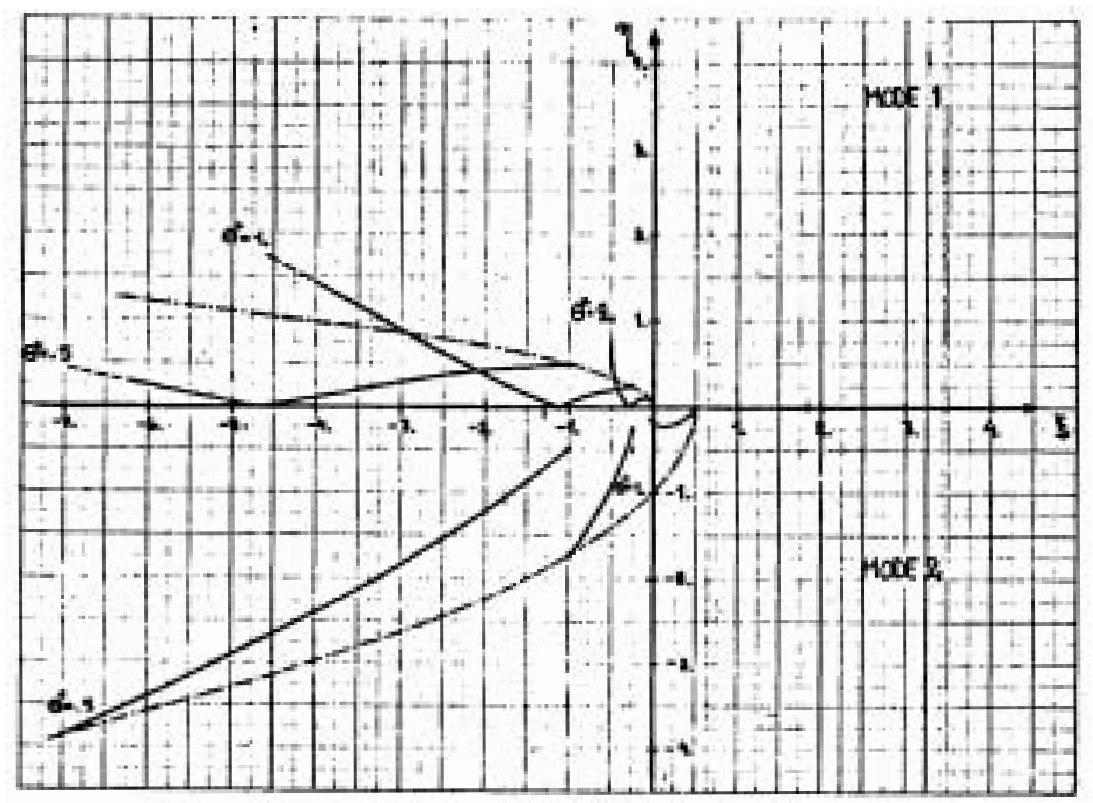

Fig.2: Curves of constant phase; $\theta^{*} \leq 2$

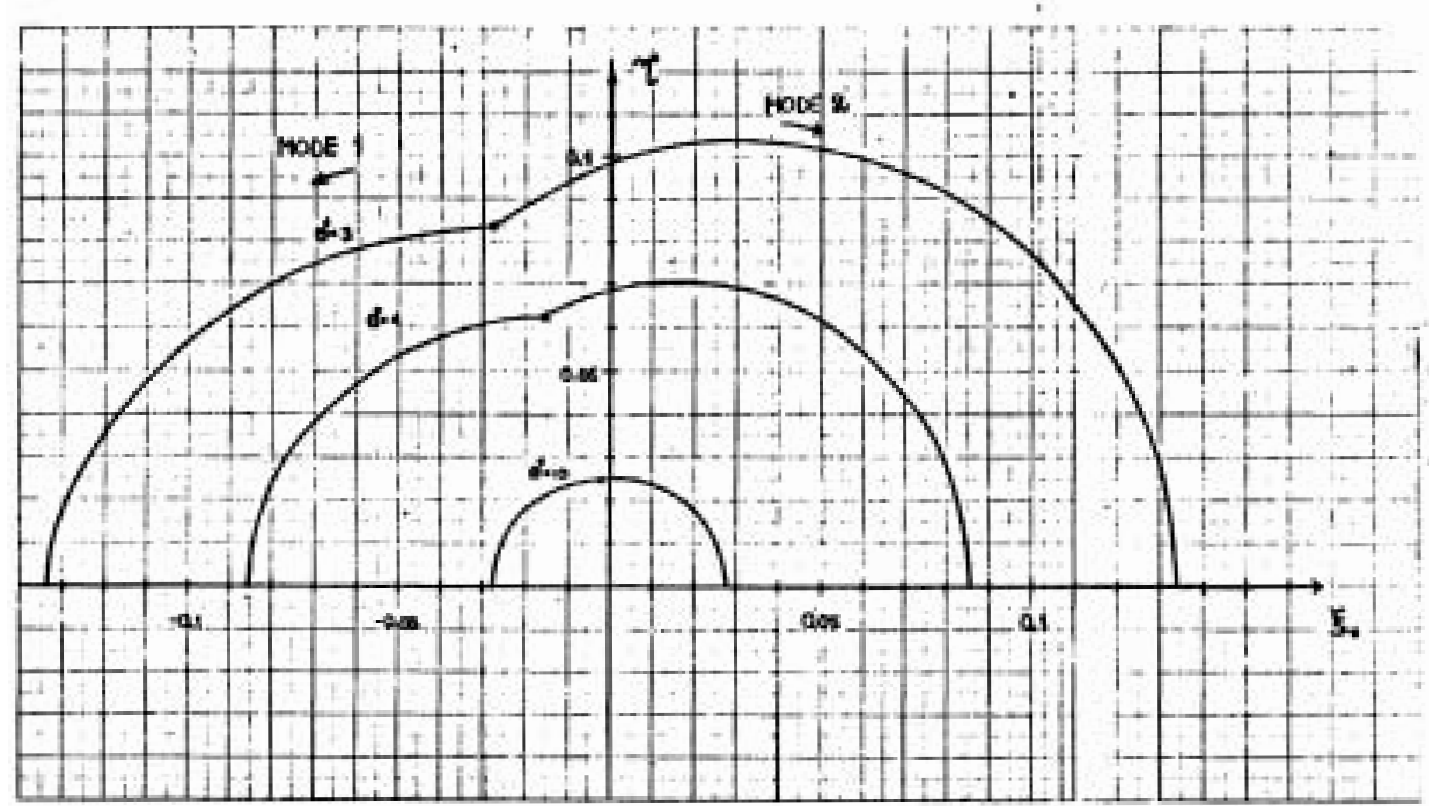

Fig.3: Curves of constant phase: $\theta^{*} \geq 2$ 
$\left(\xi_{*}=0, \eta_{*}=0\right)$, with $\theta t$ a constant as $t$ increases, $\theta^{*}$ has to decrease and we have to move out from the origin in Fig.2 or 3 . As the wave crests move out from the origin the amplitude decays and from the standard argument of stationary phase it is found that the amplitude of the waves decreases as $1 / t$, for large $\mathbf{t}$, seen from a frame of reference moving with the waves.

For an observer fixed to an outer frame of reference the decay is $1 / t^{2}$. The $1 / t$ decay may also be obtained by noticing that the decay is due to dispersivity amd therefore the total energy of the wave system is a constant. Since the area occupied by the waves grows like $t^{2}$ it follows that $\mathrm{t}^{2} \mathrm{x}$ (amplitude $)^{2}=$ constant, from which the $1 / t$ dependence follows.

4. Asymptotic behavoir of the convected solution

Starting from the end in Eq. (15) we first consider $\widehat{W}_{\text {. B }}$ Bartial integration in $\hat{\omega}_{n s}$, given by Eq. (17), it is found that the $h \xi \dot{E}$ terms cancel and $\mathbf{O}_{\text {ns }}$ goes like $1 / t^{2}$ for large times. A partial integration of (TS) shows that this term decays like $1 / t$.

of the remaining terms, $\left(T_{1}\right)-\left(T_{4}\right)$ may or may not be convective terms, depending on the location of the zeros to $\lambda_{1}+i K_{x} U(\eta)$ and $\lambda_{2}+i K_{x} V(\eta)$. Denoting the zeros by $\eta_{1}$ and $\eta_{2}$ it is found that

and

$$
\eta_{1}=z-\frac{\mu \nu}{\alpha}
$$

$$
\eta_{2}=z+\frac{\nu \mu}{\alpha}
$$

When $z<0, \eta$, becomes negative and $T_{1}$ will produce both an $e^{\lambda, t}$ term and a $\int_{0}^{-i k_{x}} U_{(\eta)} t^{\prime}(.). d \eta$ term. $\left(T_{3}\right)$ will create only an $e^{\lambda, t}$ term due to the delta function behavior of the integrand at $\eta_{1}$. Since $\eta_{2}$ can be both negative and positive, depending on the value of $k_{1}$ and $k_{x}$, no definite statement about the character of $\left(T_{2}\right)$ or $\left(T_{4}\right)$ can be made. Without making a more careful analysis we may conjecture that ( $\left.T_{2}\right)$ and $\left(T_{4}\right)$ give rise to a decay lying between that for a wave term and a purely convective term.

As $z>0$, the same argument as above may be used to show that the most important term in this case is of the type $\int^{0} e^{-i k_{x}} v(\eta) t ., d \eta$. It may also be verified that as $\Delta \rho \rightarrow 0,\left(T_{1}\right)$ and $\left(T_{2}\right)$ will cancel $\left(T_{5}\right)$.

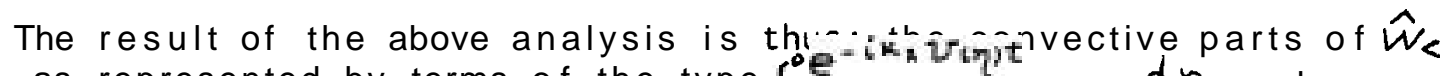
o as represented by terms of the type $\int_{-\infty}^{\infty} e^{-i k_{k} v(\eta, t)} \cdot d \eta$ and $\left\{e^{-\left[k_{x} V(\eta) t\right.} \ldots d \eta\right.$ decay as $1 / t$ for large $t$. This is a slower decay than what would be seen if there were no stratification in the system.

\section{Solution for $\stackrel{\mu}{\mu}$}

Having determined $\hat{\mathbf{W}}$, one may find the streamwise perturbation in velocity, $\hat{\mu}$, by solving

$$
\frac{\partial \hat{\mu}}{\partial t}+i k_{*} v \hat{\mu}=\frac{i k_{x}}{k^{2}}\left[\frac{\partial^{2} \hat{w}}{\partial z \partial t}+i k_{x} V \frac{\partial \hat{w}}{\partial z}-i x_{*} V^{\prime} \hat{w}\right]
$$

Even though it was found in section IV that the solution for the nonconvective case, $\hat{w}_{n s}$, behaves like $1 / t^{2}$ for large $t$, it is not obvious what kine of time behavior the corresponding $\hat{\mu}$ perturbation will have. Integration of the equation for $\hat{\mu}$ gives 


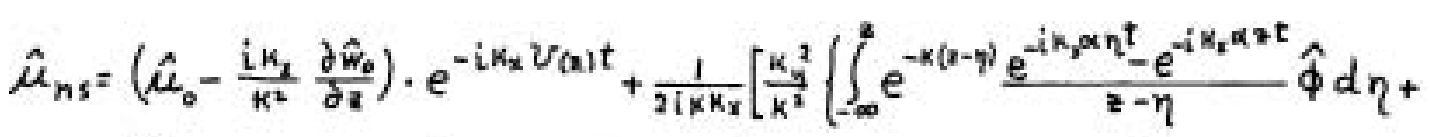

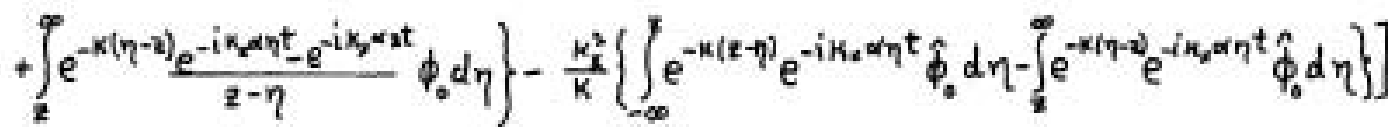

The fundamental property of $\hat{\mu}_{n s}$ is given by the appearance of the $\mathrm{e}^{i k_{x} \cup(\mathbf{a}) t}$ term, which is hidden in the integral terms but can be brought out by considering large times or making integrations by parts. This true convective term depends both on the $x$ - and $y$ - behavior of the initial disturbance as is seen from the appearance of both $k_{x}$ and $k_{y}$.

Physically, the convective term is interesting because it shows that the shear acts like a rectifier of the direction in which disturbances will occur. A given perturbation in $\hat{W}$ will eventually decay whereas it creates a non-decaying residue in $\hat{\mu}$ which is convected with the local mean velocity. Therefore, the perturbation will be first noticed where the local mean velocity is high, and the accompanying stretching of the disturbance in the streamwise direction will create intensified shear in the $z$-direction. In a real slow situation, however, the strength of the shear must be limited by the action of viscosity. For addition to the term arising from $w_{n s}$ a term of the type $\int_{0}^{\infty} \hat{h}, e^{-i k_{x} V(\eta) t} d \eta$ will also create a convective type described above. Ty integration of the equation for a term of the following form is obtained:

$$
\int_{0}^{\infty} \hat{H} \cdot \frac{e^{-i k_{x} U(x) t}-e^{-i k_{x} \alpha \eta t}}{V(\eta)-U(x)} d \eta
$$

which gives the desired term-if $z>0$, but for $z<0$ also produces a

$$
\int_{0}^{\infty} e^{-i k_{x} \infty \eta t} \ldots d \eta \text {-term proportional to } 1 / t \text { for large } t \text {. }
$$

6. Summary and discussion

When comparing the effects of an initial disturbance introduced in an infinite shear layer with and without a density discontinuity, it has been found that stratification tends to make a $w$-disturbance decay more slowly than for the case of no stratification. The density variation considered here is however extreme and for a different stratification the result would be different. Case (1960) solved the two-dimensional initial value problem for a flow with a constant shear, supported below by a rigid wall, and with an exponentially varying density. He found that the asymptotic behavior, for large times, of the non-wave part of the initial disturbance is proportional to $1 / \sqrt{t}$ for $y^{\prime}>1 / 4$ and to $t(1 / 4-j)^{1 / 2}-1 / 2$ if $j<1 / 4$, where

$$
\partial-\frac{g^{\frac{1}{s}} \frac{d j}{d z}}{\left(\frac{d y}{d z}\right)^{2}}
$$

is the Richardson number. A typical Richardson number for the present flow may be obtained as the ratio between gravity and inertial forces. With b/a as the typical velocity scale and $b / a^{2}$ as the length scale this ratio considered over the density discontinuity becomes equal to 4 . A direct comparison, in terms of Richardson number, between the present flow and that of Case, may however be hard to obtain and it is more likely that the time decay will be some functional of the density distribution. 
Whereas the $W$ component of the velocity perturbation has been found to decay more slowly with stratification, the basic action of the shear to produce convected perturbations in the streamwise direction is not changed. Besides making $\mu$ correlate over larger distances than $w$, the convection can also make nonlinearities become important. The analysis given above is linear which means that all quadratic terms that contain only perturbation quantities, have been omitted. But due to the stretching in the streamwise direction, the gradient of $\mu$ in the $z$-direction will continually increase so the nonlinear term in the equation for the $\mu$-component may be important, eventually.

The waves that are generated from an initial disturbance, will spread in the horizontal plane, forming a characteristic wave pattern which for small times is almost circular. As time increases, the wave crests of the forward-going mode are found to be confined between two curves of almost parabolic shape, one starting at the origin going in the negative $x$-direction and the other starting at a forward position going in the same direction. The group velocity in the $x$-direction is never larger than $g / \alpha$. The second mode will always be found for $x<0$ and the wave amplitudes will decay like I/t when moving with the waves object for further studies. A further point to study is also the nonlinear interaction between the convected solution and the waves.

\section{Acknowledgments}

I am indebted to Prof. Marten Landahl and to Martin Maxey for some comments on the initial value problem. I would also like to express my thanks to Mary Thayer and the other members of the F.F.D. staff for a pleasant and interesting summer.

\section{References}

Case, K.M. 1960 Stability of an Idealized Atmosphere. Physics of Fluids, Vol. 3: 149.

Loftquist, K. 1960 Stress Near an Interface Between Stratified Liquids. Physics of Fluids 3: 158. 
1. Introduction

Antarctic bottom water ( $A B W$ ) has been traced as far north as $30^{\circ} \mathrm{N}$ in the Atlantic Ocean. It is identified by chemical tracers and the variations of the thermodynamic variables. GE)SECS (1976) data from the R/V Knorr indicate that $A B N$ is present at the equator now. We present the $\mathrm{O}_{2}$ data in Fig. I; it is representative of the other variables. Within the ABN along

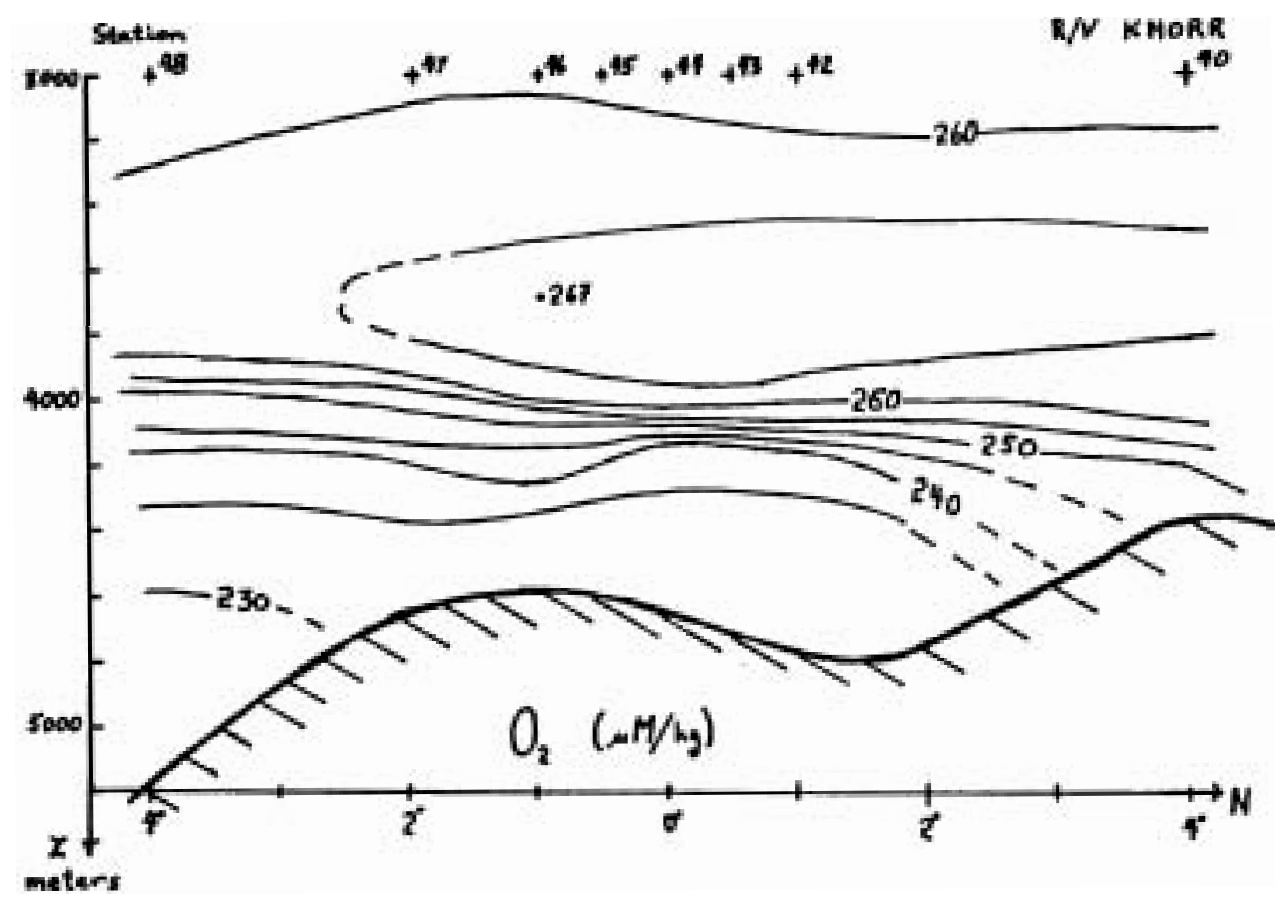

Fig. 1. Oxygen cross section drawn by the author from GEOSECS (1976) data. Horizontal axis is oriented SE-NW and lies approximately midway between South America and the Mid-Atlantic Ridge.

the coast of South America, there is a broad western boundary current. The current is most readily explained by the simple potential vorticity conserving model of Stommel and Arons (SA) (1972).

The cross sections in Fuglister's (1960) atlas show that north of the equator the $A B W$ is confined to a broad northward-flowing current on the western slope of the Mid-Atlantic Ridge.

How may we explain these phenomena? It may be that the South Atlantic basin is simply overflowing and the lowest outlet is near the western side of the ridge. Alternatively, the South Atlantic western boundary current may turn eastward near the equator and then northward upon encountering the MidAtlantic Ridge. (It may be that part of the stream is also deflected southward). 
In this paper we explore this second alternative, but do not consider the question of how or why the current turns. We suspect topography is the controlling factor. However, essentially zonal currents are observed at various depths near the equator. One may speculate that the dynamics favor zonal equatorial flows. Since in the region away from the equator the flow is geostrophic and a zonal equatorial flow is nearly geostrophic even very close to the equator, it may be 'easiest' to have a smooth transition to equatorial flow which is zonal. A good starting point for the reader who is interested in equatorial dynamics is the review paper by Moore and Philander (1977).

Since SA successfully explained the western boundary current with a potential vorticity conserving flow and we are hypothesizing a continuation of that current, we adopt a similar model here. Although we have been unsuccessful in our attempt to date we still feel that the ABN phenomena can be understood in terms of inertial effects.

2. The Stommel and Arons (SA) model

The SA model is a two-layer potential vorticity conserving model. It is assumed the constant of motion is uniform $\left(=f / H_{O}\right)$ in the current and that the flow is in the meridional direction. Thus $H_{0}=f / a$ where a is the potential vorticity of the source. Assuming geostrophy the governing equation is:

$$
\frac{d^{2}}{d x^{2}} H=\alpha^{2}\left(H-H_{0}\right)
$$

where $\mathrm{H}$ is the height of the moving layer and

$$
\alpha^{2}=f^{2} / g^{\prime} H_{0} \cdot \quad g^{\prime}=g \frac{\Delta \rho}{\rho}
$$

is the reduced gravity, $f$ is the Coriolis parameter and $\alpha^{-1}$ is the radius of deformation. The solution is

$$
H=c_{1} e^{\alpha x}+c_{2} e^{-\alpha x}+H_{0}
$$

Depending on the boundary conditions we may find solutions where $c_{1}$ and $c_{2}$ are small. In this case, for small $|x|, H \approx H_{O}$ and we may have a broad stream of uniform thickness. (A bottom slope is required for there to be motion.) As $|x|$ increases past a certain point one or the other of the exponential terms becomes important and $H$ now varies with a horizontal scale of $0\left(\alpha^{-1}\right)$.

3. A two-layer model at the equator

Near the equator we must extend this model to include more than just the vertical component of vorticity. Veronis (1963) demonstrates that it is not valid to a prioxi neglect the horizontal part of the Coriolis term.

We will still assume potential vorticity conservation describes the dynamics. We begin with Ertel's potential vorticity

$$
q=\frac{(\nabla \times \underline{\underline{v}}+2 \Omega) \cdot \nabla \eta}{\rho}
$$

$q$ is conserved follosing the flow for any scaler $\eta$ provided the following assumptions are valid.

$$
\begin{aligned}
& \text { (i) } \nabla \times E=0 \text { where is the friction force. } \\
& \text { (ii) } \nabla(I / \rho) \times \nabla P \cdot \nabla \eta=0 \\
& \text { (i i i) } \frac{D \eta}{D t}=0
\end{aligned}
$$


Thus for inviscid flow of an ideal gas any conserved variable of state is a suitable $\eta$. In the oceans $\rho$ is virtually constant and may be absorbed into $q$. If we accept the validity of a two-layer inviscid model we need only be concerned with assumption (iii). Howard (1968) shows that if the shallow water approximation is valid we may choose $\eta=\frac{z-\beta}{T-B}$ where $B=B(x, y, t)$ and $T=T(x, y, t)$ are the bottom and top of a single layer. ${ }^{T-B}(H=T-B$ as before.) Note, if $T$ and $B$ vary slowly the horizontal gradient of $\eta$ is small and we recover $\left(\frac{\partial v}{\partial x}-\frac{\partial u}{\partial y}+f\right) / H$ as the constant of motion.

Choosing this $\eta$ and hypothesizing a zonal structure (i.e. neglecting $2{ }^{2}-()$ terms $)$ the potential vorticity is

$$
q=-\frac{1}{H}\left[\left(\frac{\partial u}{\partial z}+2 \Omega \cos \phi\right)\left(\frac{\partial B}{\partial y}+\frac{\frac{-B}{H}}{\partial y}\right)+\frac{\partial H}{\partial y}-2 \Omega \sin \phi\right]
$$

We assume the pressure is hydrostatic and the zonal velocity is geostrophically balanced. (We will discuss this assumption in the next section.) Therefore $\frac{\partial u}{\partial z}=0$ and $\frac{\partial u}{\partial y}=-\frac{\partial}{\partial y}\left(g^{1} / f \frac{\partial T}{\partial y}\right)$. Now if we average the re-
resulting

$$
H_{y Y^{-}}-\left(\frac{1+\left(\beta \Omega / g^{2} y^{2}\right.}{y}\right) H_{y}-\frac{\beta C}{g^{\prime}} y H=-B_{y y}+B_{y}\left(\frac{1+\left(\frac{2 \Omega \beta}{g}\right) y^{2}}{y}\right)-\frac{\beta^{2} y^{2}}{g^{\prime}}
$$

where we have replaced $f$ by $\beta_{y}, \cos \phi$ by 1 and the average of $q$ by $C$ which we will assume is a known constant determined by the upstream conditions. For a Southern Hemisphere source $C \approx f_{g} / H_{s}<0$.

If we seek a Frobenius solution of the associated homogeneous equation we find $H=\sum_{0}^{\infty} h_{n} y_{n}$ where $h_{1}=0, h_{0}$ and $h_{2}$ are undetermined and

$$
h_{n}=\left[\frac{\beta \Omega}{g^{1}} h_{n-2}+\frac{\beta C}{g^{\prime}} h_{n-3} /(n-2)\right] / n \text { for } n=3,4, \ldots
$$

For the case of constant slope $B=b_{a}+b_{1 y}$ the particular solution is of the form $P=b_{1} y+\sum_{j} p_{n} y_{n}$. This solution though exact within the framework of our previous assumption is difficult to work with. To maintain accuracy within $5^{\circ}$ of the equator we found it necessary to use 50 terms of the series.

$$
\text { Inspecting the differential equation we see if } \mid \gg \frac{\beta \Omega}{g^{\prime}} y^{2} \text {, we may }
$$

neglect the $2 \Omega \cos \phi$ terms. For $g=10^{-1} \mathrm{~cm} \mathrm{sec}-2$ this condition holds if we are well within $7^{\circ}$ of latitude. Since we may safely ignore the $2 \Omega \cos \phi$ term relative to the $2 \Omega \sin \phi$ term for large scale flows except very close to the equator it appears we may ignore the $2 \Omega \cos \phi$ term entirely. Our equation reduces to

$$
\frac{d}{d y}\left(\frac{g^{\prime}}{A_{y}} \frac{d H}{d y}\right)-C H=-\frac{d}{d y}\left(g / \beta_{y} \frac{d B}{d y}\right)-\beta_{y}
$$

which may be solved in terms of derivatives of Airy functions. Letting $\xi=-\alpha y$ we find

$$
H(\xi)=c_{1} A^{\prime} i(\xi)+c_{2} B_{i}^{\prime}(\xi)-\pi\left(b_{1}+\beta^{2} / g \alpha^{4}\right) G_{i}^{\prime}(\xi)+\beta^{2} / g \alpha^{*} \xi
$$

where

$$
\alpha=\left(-C \beta / g^{\prime}\right)^{1 / 3} \sim O(1 / 100 \mathrm{~km}) .
$$


We experience difficulties applying this solution north at the equator $(\xi<0)$ since it predicts a wavey interface with a horizontal scale of order $\alpha-1$. If we disallow the possibility of interweaved eastward and westward flows this restricts the flow to a narrow band. The data does not seem to support such a result.

\section{Discussion}

What causes the breakdown of the two-layer model as the flow passes the equator? Our feeling is that the shallow water approximation fails and is not really conserved at the equator. The other most suspicious assumption is that of geostrophy. The neglect of friction or of longitudinal dependence may also be considered. As for geostrophy, in the meridional momentum equation the advective termsu $\frac{\partial v}{\partial x}+v \frac{\partial v}{\partial y}+w \frac{\partial v}{\partial z}$ are very small for our assumed structure so that except right at the equator the pressure gradient force must be balanced by the fu term. Besides we are using geostrophy only to estimate the velocity not to describe the dynamics so this error should be confined to the equator.

The crucial step in showing $\frac{D}{D t} \eta=0$ is to assert $w$ is linear in $Z$. Integrating the continuity equation gives

$$
w=-\int\left(\frac{\partial u}{\partial x}+\frac{\partial v}{\partial y}\right) d z
$$
so that under the hypothesis $w$ is linear in $z, \frac{\partial u}{\partial x}+\frac{\partial \varphi}{\partial y}$ must be independent
of $z$. But the umomentum equation at the equator is

$$
\frac{\partial u}{\partial t}+u \frac{\partial u}{\partial x}+v \frac{\partial u}{\partial y}+w \frac{\partial u}{\partial z}=-\frac{1}{\rho_{0}} \frac{\partial P}{\partial x}-2 \Omega w
$$

and for a two-layer system $\frac{a p}{\partial x}$ is independent of $z$ and $\frac{\partial u}{\partial z}=0$ so one of $u$ or $v$ must be a function of $z$. Only very special velocity distributions meet both these requirements (e.g. $u=\hat{u}(x, y)+a z$. We conclude the model fails since $\frac{D}{D t} \eta \neq O$ at and near the equator.

The SA model suffers, a similar breakdown; the parameter $H_{0}$ is actually $\left(\mathrm{f} / \mathrm{f}_{s}\right) \mathrm{H}_{s}$ where $\mathrm{t}, / \sim$, is the potential vorticity at the source. If we assume fluid of Southern Hemisphere origin somehow crosses the equator and flows northward all the time conserving $\eta$ then the SA model is still valid but $H_{0}<0$ and the general solution is $H=A \sin (|\alpha| x$ t $B)+H_{0}$ which implies a horizontal scale of $O\left(\alpha^{-1}\right) \sim 50 \mathrm{~km}$.

\section{Conclusion}

We have been unsuccessful in our attempt to explain the flow of ABW across the equator using potential vorticity conservation. The model predicts horizontal scales of $0(100 \mathrm{~km})$ at the equator and smaller scales northward; the data is inconclusive at the equator but to the north suggests a scale 0 $(1000 \mathrm{~km})$. Apparently the simple physics we have assumed is too far removed from reality. The direct cause of the breakdown is that $(\zeta+f) / H$ is not conserved at the equator.

In future studies we suggest that it seems more appropriate to allow for stratification and to use density (or potential density) for $\eta$. For a zonal structure the potential vorticity is then 


$$
q=\left(\frac{\partial u}{\partial z}+2 \Omega \cos \phi\right) \frac{\partial \rho}{\partial y}+\left(-\frac{\partial u}{\partial y}+f\right) \frac{\partial \rho}{\partial z}
$$

We may close the system with the thermal wind relationship. tempted to treat the system of equations. If we assume $\frac{\partial \rho}{\partial z}$ is known we obtain a single nonlinear equation in $\boldsymbol{u}$ which may be treated by the method of characteristic strips. However to proceed analytically we must take $\frac{\partial \rho}{\partial \boldsymbol{x}}$ to be constant which in turn implies $\frac{\partial u}{\partial z}$ is independent of $z$. The problem is further complicated by the presence of free boundaries.

One last alternative should be mentioned. It is conceivable that the model has not broken down; that in fact $\eta$, although not exactly conserved, is quasi-conserved and that the western boundary current does'not reach the equator but feeds the South Atlantic reservoir which in turn slowly overflows its basin. The effective source region of the North Atlantic bottom water would then be the overflow point, and the topography at this point becomes very important in formulating the problem.

\section{Acknowledgment}

I would like to thank George Veronis for suggesting this topic and supervising my work this summer.

I feel very fortunate to have been a Geophysical Fluid Dynamics summer fellow, and wish to thank the various funding agencies and the staff.

\section{References}

Fuglister, F.C. 1960 Atlantic Ocean atlas of temperature and salinity profiles and data from the International Geophysical Year of 1957-1958. Woods Hole Oceanographic Institution Atlas Ser 1. 209 pp.

GOSECS Operations Group, 1976 GEOSECS Atlantic Final Hydrographic Data Report 21 July 1972 to 31 May 1973. R/V Knorr.

Howard, L.N. 1968 'Rotating and Stratified Fluids'. Notes on the 1968 Summer Study Program in Geophysical Fluid Dynamics at Woods Hole Oceanographic Institution, Vol.L: 41.

Moore, D.W. and S.G.H.Philander 1977 'Modeling of the Tropical Oceanic Circulation'. The Sea, vol.6: 319. (Goldberg et az., editors.)

Stommel, H. and A.B.Arons 1972 Deep-sea Res., 19: 707.

Veronis, G. 1963 J.Mar.Res., 21. 199. 


\section{STEADY SOLUTIONS FOR THE LEIGHTON SOLAR DYNAMO MODEL}

Rush D. Holt

\section{Introduction}

For centuries the sun was regarded as perfectly constant. In recent centuries, after observers found blemishes on the sun, astronomers came to the conclusion that although it may not be perfectly constant, at least the imperfections (i.e., sunspots and related magnetic activities) occurred in a cycle that was regular, and so the sun was completely dependable. So strong was the belief in the regularity of the sunspot cycle that until recently most astronomers ignored the repeated claims of E.W.Maunder, a prominent British astronomer in the late 19th and early 20th centuries, that sunspot activity had come almost to a complete halt in the late 17 th century.

Recently, solar astronomer and amateur historian John Eddy (Eddy, 1976, 1977) has provided almost complete confirmation of the existence of the so-called Maunder Minimum of solar activity. Working with records of sunspot and aurora observations and carbon-14 data, Eddy has shown that not only was there a suspension of the 11-year solar cycle from 1645 to 1715 , but that there seem to have been numerous other prolonged minimums of solar activity with various durations and uneven spacing.

Eddy has also provided some suggestive evidence of the significance of the variations in solar activity. He finds a correlation between these activity minimums and cold climatic changes on earth. For example, the "Little lce Age" of unusually cold weather in Europe and North America coincided with the Maunder Minimum. Although it is difficult to find a mechanism that would make this more than just a coincidence, Eddy's evidence does point out the importance of learning about solar variability.

It is important to try to explain the irregular behavior of the sunspot cycle and the sun's magnetohydrodynamic dynamo that amplifies its magnetic fields and gives rise to sunspots. The objective of my work this summer has been to study the most commonly accepted model of the solar dynamo (Leighton, 1969), to look for steady solutions to the governing equations and to investigate their form and stability. Eventually, one should search for any mechanisms, such as coupling to a competing dynamo or competing processes within a single dynamo, that might produce the irregular cyclic behavior. This research is only a start.

Before considering the specific model, it is necessary to understand the basic nature of the convective dynamo. I will briefly consider general dynamo theory in section $\mathbf{1 1}$, and then in section III discuss the standard empirical model proposed by Babcock (1961) and modified by Leighton (1964, 1969).

\section{Dynamos}

The observed magnetic fields of the earth and the sun must be continuously generated by some dynamic mechanism -- known as a dynamo. This mechanism converts a poloidal field (a magnetic field, $\vec{B}$, whose flux line loops lie in planes that include the axis of rotation -- meridional planes) into a toroidal field (a field whose flux lines lie in planes that are perpendicular to the axis of rotation). It then converts part of that toroidal 
field back into a poloidal field. The strength of the toroidal field may be several orders of magnitude greater than the poloidal field that gave rise to it. Only the poloidal field of the sun is directly observable, though.

Because the fluids in the sun (and in the earth's core) are electrically conducting, the equations governing the dynamo mechanism are Maxwell's equations of electrodynamics and the Navier-Stokes equation of fluid motion. Faraday's law, combined with the generalized form of Ohm's law, implies that the flux $\left(\phi=\int \vec{B} \cdot d \bar{A}\right)$ through any loop moving with the fluid is constant. So, if the loop is deformed by perpendicular fluid motion so that its area increases or decreases, then the $B_{A}$ field passing through it must decrease or increase proportionally. In such a fluid, motion tends to be along field lines, and any force on the fluid perpendicular to the field lines tends to deform them rather than to cause the fluid to move across them. So the fluid particles are much like beads on a wire, or, in Cowling's words, the fields are "frozen" in the fluid (Cowling, 1957).

In the solar dynamo the toroidal field is probably produced by $\mathrm{dif}$ ferential rotation of the conducting fluid, which wraps the magnetic flux lines in a tighter and tighter spiral around the sun. (Fig.l). As they are

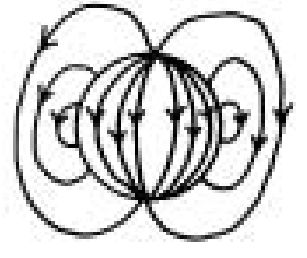

Poloidal Fields

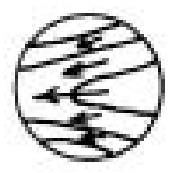

Toroidal fields formed from poloidal fields

Fig. 1

wound up, the flux lines are stretched, and the $\vec{B}$ field strength increases as the cross-sectional area of the flux lines decrease. The mechanism for producing poloidal from toroidal field may be much less efficient than the poloidal-to-toroidal mechanism. In the model we are considering this step results from convection in the rotating sun. The two parts of the cycle may take place simultaneously but at different rates, giving a cyclic variation of the net fields.

One should note that the suggested mechanisms still work if the field is reversed $(\vec{B}$ goes to $-\vec{B})$. In fact, a dynamo can reverse completely and repeatedly for certain values of the parameters if the poloidal and toroidal mechanisms are out of phase (see K.A. Robbins, 1976, for a discussion of the reversing of the simple disc dynamo).

The energy for the sun's dynamo comes from the potential energy of temperature differences, which can produce convection, and in the rotating sun, may also produce differential rotation (Busse, 1976). For the earth a possible energy source is precessional motion (Malkus, 1968).

In any dynamo, part of the cycle must be asymmetric about the axis of rotation and cannot be completely steady. The axial asymmetry of the motion is necessary to convert a toroidal field back to a poloidal field. A good example of this kind of motion is the rising of scattered convection cells in the presence of a coriolis force, which provides the necessary 
asymmetry (see Parker, 1976, for references to his earlier articles on this subject). As hot gas beneath the surface of the sun is convected upward, it bends the toroidal field lines with it. A parcel of this rising gas rotates less rapidly than the surrounding fluid, and thus not only does it form a magnetic arch at the surface, but also the arch is rotated. So a completely toroidal field can gain a poloidal component. (There are other possible mechanisms to produce the poloidal field.) There are a number of other theories about such things as the minimum radial velocity necessary to maintain amplification (Busse, 1975) and the relative sizes of large-scale and small-scale motions (Childress, 1967).

\section{The Babcock-Leighton Model}

Leighton's solar dynamo model not only requires the observed differential rotation of the sun's surface (a variation of rotation rate with latitude, the regions near the equator rotating more rapidly than the regions near the poles), but also it requires that rotation beneath the surface is different from the surface rotation. Leighton suggests that the lower regions of the convection zone rotate more rapidly than the surface. The rotation rate is written as

$$
\Omega\left(\frac{\text { radians }}{y \text { ear }}\right)=18 \sin ^{2} \theta+\left(\alpha+\beta \sin ^{n} \theta\right) \frac{R-r}{H},
$$

where $\theta$ is measured from the north pole, $H$ is the depth of the convection zone $(H \approx . \mid R), R$ is the solar radius, and $\alpha, \beta$, and $\eta$ are free parameters adjusted to describe a reasonable subsurface rotation. The first term is the standard form for the surface rotation. In the following pages $I$ assume $\alpha=0, \beta=18$, and $\eta=z$.

Leighton begins with an initial radial field (in a perfectly conducting medium) which is wound up by the differential rotation to form a subsurface toroidal field $(\beta \phi)$, amplified several orders of magnitude. This action is written as

$$
\frac{\partial B \theta}{\partial t}=\sin \theta\left(B \frac{\partial \Omega}{\partial \theta}+R B_{r} \frac{\partial \Omega}{\partial r}\right)
$$

When the growing field reaches a critical field strength $\left(B_{c} \approx 200\right.$ gauss $)$, magnetic buoyancy $\left(F_{B}=g \Delta \rho=\frac{B^{2}}{8 \pi}, \frac{M_{g}}{K T}\right)$ causes it to rise to the surface in scattered locations around the sun and appear as sunspots. The area of erupted fields has primarily poloidal fields, but the area in the direction of rotation (preceding, or $p$, spots) has the opposite sign of the following area (f spots). The rate of depletion of the toroidal flux is just the flux eruption rate, which depends on the field $B \phi$ and on the area of the eruption, which also depends on the magnitude of $B \phi$, relative to $B_{c}$. Thus

$$
\left(\frac{\partial B_{\phi}}{\partial t}\right)_{\text {depletion }}=\frac{B_{\phi}\left|B_{\phi}\right|}{100 B_{c} \tau}-\frac{B_{\phi}}{50} \text {, }
$$

where $\zeta$ is the average eruption time and 50 years is the time with which asymmetric toroidal fields diffuse evenly over the sun. We see, therefore, the depletion rate of $B_{\phi}$ is a nonlinear function of $B \phi$.

A crucial feature of the model is that the following (f) spots erupt farther from the equator than the preceding (p) spots. This is the step that generates a poloidal field. Leighton does not suggest a mechanism for 
this "tilt" of bipolar regions, but points out that it is generally observed. One may presume that it is a coriolis effect like that discussed in the section II. The equation for the growth of $B_{r}$ is

$$
\left(\frac{\partial B_{r}}{\partial t}\right)_{\text {growth }}=\frac{H a}{2 \pi R^{*} \tau} \frac{\partial(B \phi \sin \gamma)}{\partial \mu}
$$

where $a$ is the extent of the bipolar region. Leighton sets

$$
\sin \gamma=1 / 2 \cos \theta=\mu / 2
$$

empirically. Leighton assumes that this $B_{r}$ production does not take place continuously, but only when $B_{\phi}$ exceeds the critical strength $B_{c}$. He does assume, though, that there is a small, unobservable radial field which stays below the surface and which is produced continuously from the $B \phi$ field. This is the seed field that starts his cycle. It also prevents the cycle from dying out if $B_{\phi}$ should fall below $B_{c}$ everywhere.

Once erupted, each pole of the bipolar region is assumed to have some autonomy of motion. Most of the sunspots disintegrate over a period of days or weeks by peeling off flux lines which are mixed by turbulent motions and combine with flux lines of opposite polarity to neutralize each other. The rate of the diffusion of $A$, is

$$
\left(\frac{\partial B_{r}}{\partial t}\right)_{\text {depletion }}=\frac{1}{T_{D}} \frac{\partial}{\partial \mu}\left[\left(1-\mu^{2}\right) \frac{\partial B_{r}}{\partial \mu}\right],
$$

which is just the diffusion equation in spherical coordinates. Leighton believes that the dominant cause of the diffusion is the buffeting of the bipolar regions by supergranulation cells (cells about $1 / 20$ solar radius in extent and with a time scale of 10-30 hours) which sends the flux ropes on a random walk over the sun's surface. Because this kind of motion and turbulence cannot diffuse magnetic fields as well as it can scalar fields, Leighton assigns $T_{p}$ a rather large value, 20 years.

In any case, most of the flux is neutralized so that almost all of the magnetic energy in the erupting region disappears, but because there is a poloidal magnetic moment when the buoyant toroidal field erupts, after the bipolar regions are diffused over the sphere, a residual poloidal field remains. This, combined with the submerged $B_{s}$, starts the next cycle of the $B_{\phi}$ wind-up. The remaining field component $B_{\phi}$ can be calculated at each point using the fact that the divergence of the total vector $\vec{B}$ must vanish at every point.

In summary the equations describing the Leighton model are given below:

$$
\begin{aligned}
& \frac{\partial B_{s}}{\partial t}=\frac{-G H}{B 0 R \tau} \quad \frac{\partial\left(\mu B_{\phi}\right)}{\partial \mu}-\frac{B_{s}}{T_{i}} \quad G \sim \frac{B_{s}}{B_{r}}, \tau \approx .5 y r \\
& \frac{H}{R} \approx 1 / 10, T_{s} \approx 504 \mathrm{r} \text {. } \\
& \frac{\partial B_{r}}{\partial t}=-\delta \frac{H}{g \theta R \tau} \frac{\partial\left(\mu B_{\phi}\right)}{\partial \mu}+\frac{1}{T_{D}} \frac{\partial}{\partial \mu}\left[\left(1-\mu^{2}\right) \frac{\partial B_{r}}{\partial \mu}\right]
\end{aligned}
$$

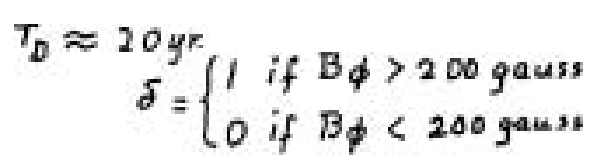




$$
\begin{aligned}
\frac{\partial B_{\phi}}{\partial t}=-18 \sin ^{3} \theta \frac{R}{H}\left(B_{r}+B_{s}\right) & +54 \cos \theta \sin ^{2} \theta B_{\theta} \\
& -\delta \frac{\left|B_{\phi}\right| B_{\phi}}{100 B_{c} \tau}-\frac{B_{2}}{T_{\phi}} \quad T_{\phi} \approx 50 \mathrm{yr}
\end{aligned}
$$

$$
\frac{R}{H}\left(B_{r}+B_{s}\right)=\frac{\partial}{\partial \mu}\left(\sin \theta B_{\theta}\right)
$$

IV. Time Independent Equations

If the time dependent part of each equation is set equal. to zero, the four equations can be reduced to a single equation:

$$
\begin{aligned}
& \frac{\partial}{\partial \mu}\left[\frac{E}{g(\mu)}\left(c \frac{1-\mu^{2}}{\mu} \frac{\partial B}{\partial \mu}+\frac{g}{\mu}\right)^{B}+\frac{F}{g(\mu)}\left(c \frac{\left(1-\mu^{2}\right)}{\mu} \frac{\partial B}{\partial \mu}+\frac{g}{\mu}\right)\right]+ \\
& +\frac{\partial}{\partial \mu}\left[\frac{f(\mu)}{g(\mu)} \frac{C}{A} \frac{\partial}{\partial \mu}\left(\left(1-\mu^{2} \frac{\partial B}{\partial \mu}\right)-\frac{f(\mu)}{g(\mu)} B\right]-\frac{D C}{A} \frac{\partial}{\partial \mu}\left(\left(1-\mu^{2}\right) \frac{\partial B}{\partial \mu}\right)+D B=0\right.
\end{aligned}
$$

with $B=B_{r}, f(\mu)=-180 \sin \theta=-180\left(1-\mu^{2}\right)^{1 / 2}, g(\mu)=54 \sin \theta \cos \theta$

$$
\begin{aligned}
C=\frac{8 O R T}{H T_{D}}, A=\frac{20 R \tau}{T_{s} Y H}, E=\frac{1}{100 B_{e} \tau}, F=1 / T_{\phi}, D=R / H
\end{aligned}
$$

I) is a constant of integration from Eq.7. For most of my calculations I chose values for the coefficients that Leighton says give solutions that agree well with observations or that at least seem reasonable $(C=20$, $A \approx 8 / 8, E \approx 10^{-4}, F \approx .02, D=10$ ).

To find approximate solutions to this equation it is necessary to make some assumptions about the form of the solutions and the boundary conditions. Radial fields are observed to have in general opposite polarity in the northern and southern hemispheres, and so the toroidal fields may be assumed to have opposite polarity also. The observed radial fields have at least some dipole character, and so I assume that they may be expanded to a good approximation in terms of a dipole and higher multipoles of odd symmetry. Although some measurements have suggested a quadrupole component of the radial field, I assume that even moments are not fundamental to the Leighton model because they would not provide significant feedback from toroidal-topoloidal fields. Thus, we have $B_{r}=0$ and $B_{s}=0$ at the equator, and it follows from Eq.7 that $\frac{\partial^{2} b_{r}}{\partial r^{2}}=0$ holds there also. I assume further that at the sun's poles, from which it follows that $B_{r}=-100 \% \frac{\partial B_{r}}{\partial \mu}$ at the poles. (There is some arbitrariness to the selection of boundary conditions. Measurement of the overall solar field is difficult because there is such a wide range of field strengths -- from less than one gauss to more than 4000 gauss -and because a large amount of flux appears to be concentrated in areas too small to resolve from earth. Furthermore, measurements of the multipole composition of the solar winds are limited by the small number of test sites.)

The most promising approach to solving Eq.10 is to assume a solution composed of odd powers of $\mu$. The simplest trial solution $B_{r}=a_{\mu}$ does not 
satisfy the boundary conditions (except for $\mathscr{H}=-\frac{T_{D}}{100}$, a condition which is too restrictive and which gives a magnitude to $B_{r}$ larger than postulated). However, any pair of odd powers of $\mu$ with a properly chosen ratio of coefficients does satisfy the boundary conditions. The expansion basis functions are not actually Legendre polynomials (zonal harmonics) but they have a close enough resemblance to them that one can identify the functions with specific multipoles. I inserted the trial solution into Eq.10 and determined the remaining coefficient by a minimization equivalent to the Ritz method without orthogonal basis functions (Galerkin method). This lengthy procedure gives the surprising result that for the steady state the octupole $\left(\mu^{s}\right)$ term dominates the solution.

Next 1 assumed a solution of the form $B_{Y}=a_{1} \mu+a_{5} \mu^{3}+a_{5} \mu^{5}$. This trial solution gave three cubic equations which when solved for $y=.003$ gave the result $B \approx 40 \mu-125 \mu^{2}+84 \mu^{5}$. So it appears that the octupole term is in fact dominant for the steady state dynamo. The other fields are accordingly

$$
\begin{aligned}
& B \phi=-6702 \mu+15900 \mu^{3}-8420 \mu^{5} \\
& B_{s}=-6.2 \mu+23.8 \mu^{3}-18.8 \mu^{5} \\
& B_{\theta}=\left(164 \mu-252 \mu^{3}+109 \mu^{5}\right) \cot \theta-(20.5) \csc \theta .
\end{aligned}
$$

The last term of the $B_{\theta}$ equation is necessary to make the field vanish at $\theta=0$. The $B_{r}, B_{\phi}$ and $B_{s}$ fields are plotted in Figs. 2, 3 and 4 .

Next I constructed two normal modes that satisfied the boundary conditions. They are $Q_{1}=\mu-.97 \mu^{3} \quad a_{2}=\mu-3,93 \mu^{3}+2.87 \mu^{5}$. The calculated steady solution shown in Fig.2 is composed mainly of $Q_{2}$; so I used $Q_{2}$ and the associated forms of $B_{\phi}, B_{s}$ and $B_{\boldsymbol{\theta}}$ in the following analysis of the time dependent equations.

V. Stability of Time Dependent Solutions

For the time dependent equations, one may assume there exist solutions of the form $\left.B_{r}(\mu, t)=B_{r_{0}}(\mu) A, I t\right)$ with analogous equations for the other components. The naught subscript indicates the time independent solution corresponding to $B_{r}=Q_{1}$. The procedure followed is shown by the following equations:

$$
\begin{aligned}
& \frac{\partial B_{r}}{\partial t}=B_{r_{0}} \frac{\partial A_{r}(t)}{\partial t}=\left(\frac{H}{\partial O R t}\right) \frac{\partial \mu B_{\phi}}{\partial \mu}-\left(\frac{1}{T_{D}}\right) \frac{\partial}{\partial \mu}\left[\left(1-\mu^{2}\right) \frac{\partial B_{r}}{\partial \mu}\right]=C_{1} \frac{\partial \mu B_{\phi}}{\partial \mu}-c_{2} \frac{\partial}{\partial \mu}\left[\left(1-\mu^{2}\right) \frac{\partial B_{r}}{\partial \mu}\right], \\
& 2 \int_{0}^{1} B_{r} \cdot B_{r} \frac{\partial A_{r} d \mu}{\partial t}=2 \int_{0}^{1} C_{1} B_{r} \frac{\partial\left(\mu B_{\phi}\right) d \mu}{\partial \mu}-2 \int_{0}^{1} C_{2} B_{r} \frac{\partial}{\partial \mu}\left[\left(1-\mu^{2}\right) \frac{\partial B_{r}}{\partial \mu}\right] d \mu \text {, } \\
& \int B_{r} \cdot B_{r} \frac{\partial A_{r}}{\partial t} \alpha \mu=\int B_{r} \cdot\left[C_{r} B_{r} \cdot \frac{\partial \mu B_{\phi}}{\partial \mu}+C_{2} \frac{\partial\left(1-\mu^{2}\right)}{\partial \mu} \frac{\partial B_{r}}{\partial \mu}\right] d \mu \text {, } \\
& \dot{A}, 2 \int_{0}^{1}\left(\mu-3.9 \mu^{3}+2.8 \mu^{5}\right)^{2} d \mu=\frac{2 A_{0}}{T_{D}} \int_{0}^{1}\left(\mu-3.9 \mu^{3}+2.8 \mu^{5}\right)\left(-25.4 \mu+100 \mu^{3}-84 \mu^{5}\right) d \mu- \\
& -\frac{2 A}{T_{0}} \int_{0}^{1}\left(\frac{\partial}{\partial \mu}\left(\left(1-\mu^{2}\right)\left(1-11 \cdot 7 \mu^{2}+14 \mu^{4}\right)\right)\right)\left(\mu-3.9 \mu^{3}+2.8 \mu^{5}\right) d u \text {, }
\end{aligned}
$$




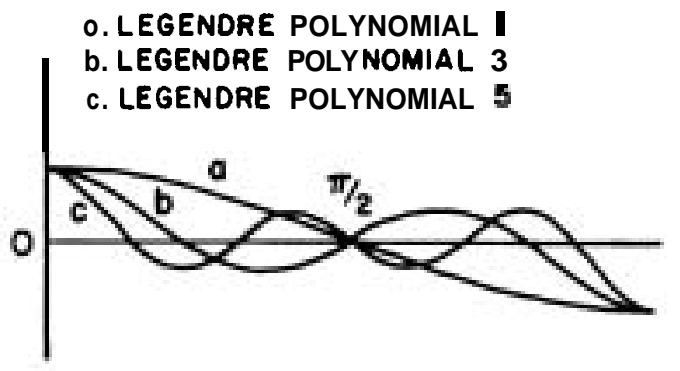

Fig. 1

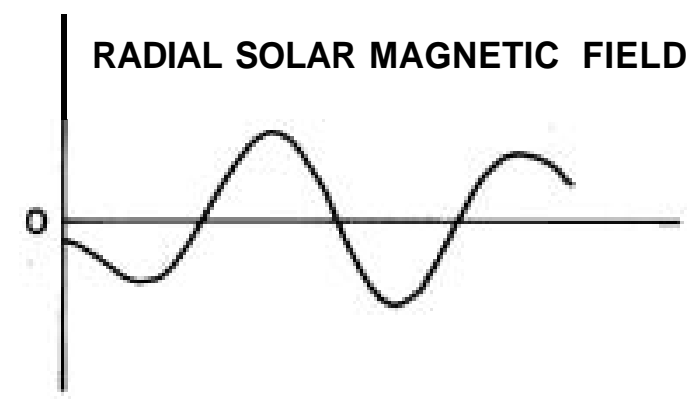

Fig. 2

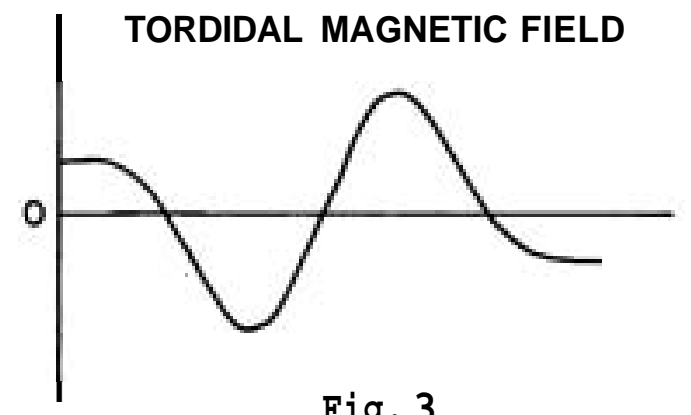

Fig. 3

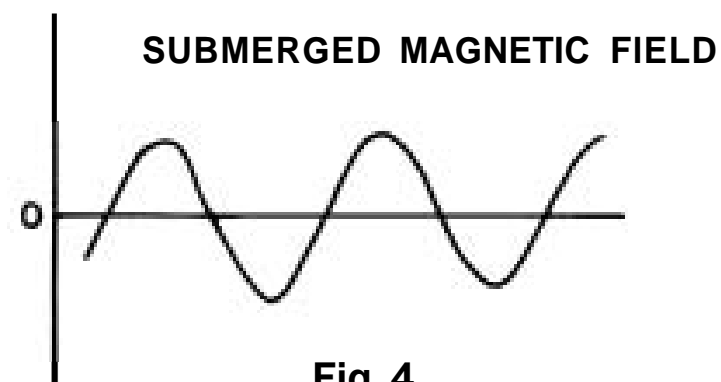

Fig. 4

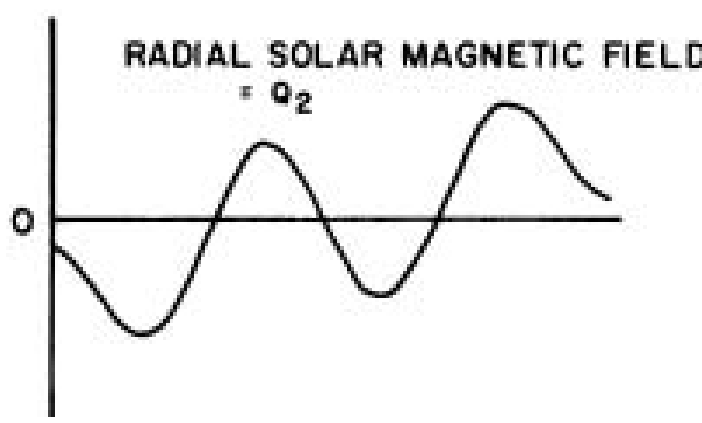

Fig. 5

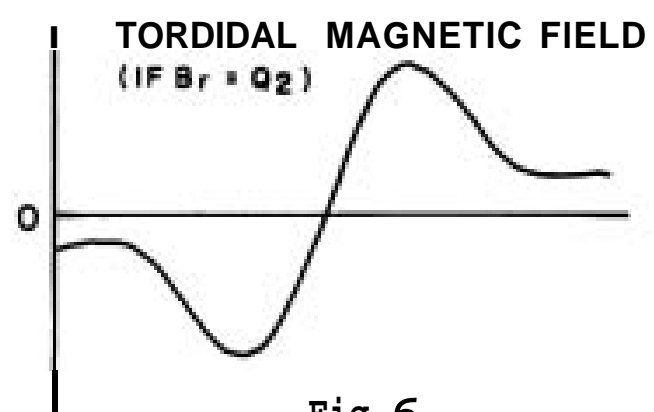

Fig. 6 
and finally

$$
\dot{A}_{r}=\frac{16}{T_{D}} A_{r}-\frac{12.3}{T_{D}} A_{\phi}=, 8 A_{r}-.6 A_{\phi} \text { (for standard coefficients). }
$$

Similar procedures yield equations for the other components:

$$
\begin{aligned}
& \dot{A}_{s}=.05 A_{\phi}=.02 A_{s} \\
& \dot{A}_{\phi}=-1.9 A_{r}+.49 A_{s}-.02 A_{\phi}-.006 A_{\phi}^{2}=.12 A_{\theta} \\
& A_{\theta}=.21 A_{r}-.03 A_{s} .
\end{aligned}
$$

The steady solutions are $A_{\phi_{0}}=0$ and $-44,3$. It is possible to perform a linear perturbation analysis, assuming time dependent solutions of the form $A_{r}(t)=\left(A_{r_{\phi}}+A_{r}^{\prime}\right) \mathrm{e}^{\eta^{t}}$ and making analogous assumptions for $A_{s}$ and $A_{\phi}$. There are two separate determinants for $n$ (one for each $A_{\phi_{0}}$ ). The solutions to the characteristic equation corresponding to the zero field case $\left(A_{\phi_{0}}=0\right)$ are $\eta=-.8,0,+1.5$, which indicate the possibility of a marginal stability $(\eta \leq 0)$. The corresponding solutions when $T_{D}$ (the surface diffusion time) is taken to be 50 years rather than 20 are similar, but the stable solutions are somewhat more stable than for the first case. These results suggest that the sun may lapse into a slightly stable mode that would suspend the solar cycle. The high field case $A_{\phi_{0}}=-44$ also has one stable solution $(\eta \approx-1.1)$ and one marginally stable solution $(\eta \approx 0)$, but it is unlikely that the sun would ever enter that mode because of the large value of $B_{\phi}$ compared to the value of $B_{r}$.

Although this study is by no means complete, it does suggest a reasonable form for some steady solutions to the Leighton dynamo equations. It is useful to see the forms of the boundary conditions and solutions to the equations, so this approach may be a good alternative to Leighton's coarsegrid numbercal integration (time-stepping) solution. I have yet to carry out the analysis for more than one mode and to investigate the range of parameters $\left(T_{D}, \&, T_{F}\right.$, etc. for which there are stable solutions. Next, it would be good to carry out a nonlinear perturbation analysis, if possible. Also, perhaps the model should be modified to give more significance to the submerged radial field, $B_{s}$, by changing its means of formation and its diffusion behavior. Leighton introduced $B_{s}$ simply to prevent the cycle from stopping completely if $B_{\phi}$ fell below $B_{c r i t i c a l}$ everywhere. Obviously, the form of $B_{\phi}$ is important in determining how long the cycle may stay in a period of minimum activity. Furthermore, because surface fields may have some difficulty penetrating the conducting fluid to begin each cycle of $B \phi$ wind-up, the form of

$B_{s}$ may be of fundamental importance to the dynamo cycle even when $B_{\phi}$ is greater than $\boldsymbol{B}_{c}$.

Acknowledgments

I want to thank Edward Spiegel, who launched me on this project and provided much helpful guidance during my fumblings, and who also provided many enjoyable moments for us all during the summer. I also extend my thanks to George Veronis, Mary Thayer, John Giuliani, and Stephen Morris, who helped me at various times and who helped make my summer in the G.F.D. program so rich. 


\section{References}

H.W.Babcock 1961 "The Topology of the Sun's Magnetic Field", Ap.J.133: 572.

F. Busse 1976 "Convection in Rotating Stars", reprint.

S.Childress 1967 "The Multiple Scale Method" GD Course Woods Hole, Lectures 1967: 165.

T.G.Cowling 1957 Magnetihydrodynamics Interscience, N.Y.

J.A.Eddy 1976 "The Maunder Minimum" Science 192. 1189.

1977 "The Case of the Missing Sunspots" Sci.Am. 5/77.

Robert B. Leighton 1964 "Transport of Magnetic Fields on the Sun" Ap. J. 140: 1547.

1969 "A Magneto-Kinematic Model of the Solar Cycle" Ap.J. 156:1-26.

W.V.R.Malkus 1968 "Precession of the Earth as the Cause of Geomagnetism" Science 160: 259.

E.N.Parker 1977 "The Generation of Magnetic Fields in Astrophysical Bodies" Ap.J. 215: 370.

K.A.Robbins 1976 "A Moment Equation Description of Magnetic Reversals in the Earth". Proc.N.A.S. 73: 4297. 


\title{
ON THE FRACTAL GEOMEIRY OF THE SMALL-SCALE STRUCTURE \\ OF HIGH REYNODS NUMBER TURBULENCE
}

\author{
Jacques Lewalle
}

$\underline{\text { Abstract }}$

An attempt is made to draw information about the geometry of turbulence using fractal objects concepts. Confirmation is found of previous suggestions that $8 / 3$ plays a special role as critical dimension in the field. In order to make some examples compatible with data, an information-theoretical entropy has to be stationary at $D=8 / 3$.

\section{Introduction}

The first part of this paper is devoted to a general introduction to the theory of fractals, developed mainly by Mandelbrot during the last few years. The purpose is not to give a complete and rigorous presentation of the topic, which is done in (10). It is felt however, that most researchers in turbulence, either applied or theoretical, still regard non-integer dimensionality as most unphysical, so that at least a quick presentation is useful. In the study, one is induced to pinpoint some of the fundamental aspects of the approach, such as self-similarity, and to discard some technical features that do not seem to be essential for the applications.

Then the present importance of fractal dimension in turbulence is briefly described. $\quad D=8 / 3$ is encountered several times, in different studies.

The next section describes some experimental results obtained by Kuo and Corrsin (5), as interpreted from the fractal viewpoint in (8). An argument based on helicity leads to prefer a representation of small scales as many-sheeted objects, but the same indecision is left as after Kuo and Corrsin's paper. The fact that the fractals would be of dimension $8 / 3$ is encouraging, when compared to $D=2.6$ obtained from the experiments. The corrections to the Kolmogorov spectrum for intermittency are mentioned.

In section 5, we introduce Mandelbrot's determining function, and Iist its properties. The fact that it connects various aspects of our problem, such as moments of the velocity derivatives distribution, the correction factor for intermittency, the width of the dissipation spectrum and dimensionality, is used in the next paragraph.

The formal relationship between dimensionality and entropy is then studied. It is shown on a collection of trial functions that general conditions on the determining function, $D=8 / 3$ and the stationarity of entropy are the conditions for the verification of the two pieces of data available.

It is suggested that the use of this entropy property could help introduce some dynamical aspects in the fractal approach.

\section{About Fractal Sets}

The concept of fractal dimension is still too often subject to misunderstanding. One hears: "Such situation occurs with fractal dimensions, but what about the real world?"

The aim of this section is to expose the problem for those readers who are not familiar with Mandelbrot's work in the field. Also, those 
elements that are necessary to the rigorous mathematical theory (e.g., the limit of zero viscosity), and those that are more fundamental for the applications (e.g., the self-similarity) are pointed out.

It seems appropriate at the beginning, to stress the fact that a fractal dimension is never to be considered as a topological property. A surface remains always of topological dimension two, and geometrical space of dimension three.

Set us take a line segment

break it into three parts, and substitute two equal segments for the central one

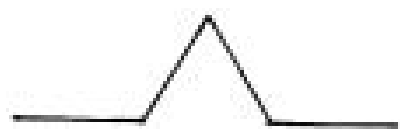

Then repeat the process ad infinitum on each subsegment. The result looks like

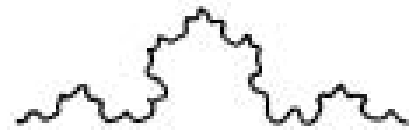

This could be a simple model of the stretching of a line by a turbulent field, as the case was studied by Cocke (3) (see also (11)). Obviously, the topological nature of the line does not change; however, its length increases without limit, if we let the process continue to arbitrarity small scales, which, in terms of turbulent motion, can be understood as the limit of 'infinite Reynolds number.

Another striking aspect of the line is that it seems to fill the paper somewhat more than the original segment. In other words, the fractal set generated by the simple process described above covers more of the plane than common one-dimensional aspects do: this is readily seen to be a metric property, and serves as a basis for the mathematical definition of fractal dimension (e.g., Kahane, in (14)).

Let us cover our fractal object with a number of balls of diameter $\delta$. As $\delta \rightarrow 0$, the number of balls increases, and their total area goes to zero in a way that depends on the fractal object A. More rigorously, let us consider a monotone function

$$
h(t)=t^{\alpha} \quad \alpha>0
$$

and denote the sequence of balls by $\left\{B_{i}\right\}$

when

$$
\begin{gathered}
\mu_{\alpha, \delta}(A)=\inf \sum_{i} h\left(\phi_{i}\right) \\
\phi_{i} \leq \delta \text { is the diameter of } B_{i}
\end{gathered}
$$

The fractal measure of the set $A$ is defined as

and depends on $\propto$.

$$
\mu_{\alpha}(A)=\lim _{\delta \rightarrow 0} \mu_{\alpha_{1} \delta}(A) \text {. }
$$


When some $\propto$ exists, such that

$$
\inf \left\{\alpha \mid \mu_{\alpha}(A)=0\right\}=\sup \left\{\alpha \mid \mu_{\alpha}(A)=\infty\right\}
$$

it is called the dimension of $A$ :

$$
\alpha=\operatorname{dim}(A)
$$

It can be proved that $\alpha \leqslant D_{T}(\mathcal{C})$, if we call $D_{T}(\mathcal{A})$ the topological dimension of the space $A$ in which $A$ is embedded, and that $\alpha \geqslant D_{T}(A)$. When $\alpha>D_{\top}(A)$, the object $A$ is called fractal.

We will need a property of the measure $\mu_{\alpha}$ :

$$
\mu_{\alpha}(\lambda A)=\lambda^{\alpha} \mu_{\alpha}(A)
$$

describing the effect of a renormalization of the space variables.

The mathematical definition of $\operatorname{dim} A$ is not easily applicable under such form. For our purposes, it is more useful to rely on the evaluation of the fractal dimension as

$$
D=\frac{\log N}{\log \frac{1}{r}}
$$

where $N$ is the number of replicas obtained at each stage of the cascade, and $r$ is the similarity ratio. In our example, and in more general cases, it can be proved (10) that

$$
D=\operatorname{dim} A \text {. }
$$

For the example: $D=\log 4 / \log 3 \approx 1.2$

where the effects of viscosity are not important, we will consider that, the viscous cutoff is beyond the resolution of the observations, so that the set behaves as a fractal for practical purposes. The dimension is given by $D$ when the breakdown is self-similar. It is well known that the inertial range in high Reynolds number turbulence fulfills these conditions.

Many examples inspired by several fields of physics, and very instructive illustrations, enlighten a more complete exposition of this material in (10).

\section{Fractals in Turbulence}

We just mentioned the papers by Cocke about the stretching of a material line by a turbulent field. The conclusion there was that the length of the segment would eventually tend to infinity: the relationship that this fact could have with a rigorous fractal formulation was not examined in more detail, but the idea does not seem to be too far from this topic.

Actually, Mandelbrot studied a very similar problem (7), namely: the dimensionality of the surfaces of equal concentration of a passive scalar contaminant in a Brownian field. When the Prandtl number is large enough, this corresponds to the description of a convected material surface. The main result of that section of the paper was to introduce the dimension $8 / 3$ as to be expected: a full proof of this fact was given shortly after by Adler (1).

Dimension $8 / 3$ creeps in also in a different context: Nelkin (12) using a 
scaling theory that will be adapted below to a different setting, shows that $8 / 3$ is likely to be a crossover dimension, i.e. a dimension at which the physical properties of the system change qualitatively. In fact, the analogy to the theory of critical phenomena (e.g. magnetism at low temperature), induces him to propose that the Kolmogorov spectrum would be strictly correct for $D<8 / 3$, and that the familiar interimittency corrections apply above that threshold.

Another study by Bell and Nelkin (2) was based on a more qualitative model of energy exchange between modes, and again on the analogy to the theory of critical points. The fundamental step there is to model the spectral transfer term so as to satisfy all energetic requirements, and superpose a forward and a backward cascade. The ratio of the intensities of these (i.e. the overall sign of the transfer term) is shown likely to depend smoothly on the dimension parameter, but no numerical estimates are made.

In these three papers, the definition of dimensionality is the same as we defined above, and so belong to the fractal approach. This cannot be said of the theory of critical points as developed by Fisher, Wilson, and others. This is the reason why we take apart the paper by Frisch, Leseur and Sulem (4), since they do not make clear their definition of D. Anyway, they find a crossover dimension at $D=2.03$ : there the direction of the energy cascade changes, which is compatible with Bell and Nelkin's conclusions.

\section{About the Small Scale Structure of Turbulence}

Several models of the dissipative patterns of a turbulent field have been proposed successively, by Townsend, Tennekes, and finally by Kuo and Corrsin (5) who tested them experimentally. They are: vortex lines, tubes, blobs, slabs, and ribbons, out of which the authors cited concluded the ribbon or rod-like structures were the most satisfactory -- without anyway reaching full agreement with the data in either case.

A review of the models and data from the fractal viewpoint was made by Mandelbrot (8): from the scaling exponent, found equal to .4, he concludes that $d=2.6$ in the case of Kuo and Corrsin; as a consequence, neither model can be satisfactory, even though Tennekes' model turns out to be compatible with fractal dimensions.

However, $d=2.6$ does not make it possible to decide between a fractal set obtained from a line, or from a surface: indeed, by a cascade similar to that described in section 1 , one can imagine to keep $\frac{1}{r}$ small and make $\mathrm{N}$ very large by convecting a line in space, and even though the induced pattern of the corresponding flow would be very complicated, it cannot be discarded, owing to our lack of feeling for what a likely structure ought to be; obtaining a fractal of proper dimension from a surface would seem easier to grasp geometrically, but this cannot be an argument at this stage.

A way to resolve this indecision is to take in'to account only the characteristics of the flow that vanish identically in two-dimensional motion. The idea is not quite to make a 2 -d section, but is comparable.

It is known in general that helicity

$$
3 \% \underline{\omega} \underline{\omega} \quad(\underline{\omega}=\nabla \times \underline{u})
$$

is identically zero for a two-dimensional $\Perp$-field; or, conversely, that 
helicity could be used to estimate how much the third dimension of geometrical space is used by the flow. Following the idea Nelkin (12) applied on another quantity, we shall set up an inertial scaling of helicity, in the line of the Kolmogorov (1941) theory. This supposes the existence of a range of wave numbers independent of external features (creating anistropy, in the simplest cases) and dissipative scales: then, local energy transfer across the wave-number scale is the only relevant parameter, and is equal to the total dissipation rate $\epsilon$ as soon as "some-sort-of-equilibrium" is achieved in the spectrum.

We consider the correlations of helicity:

$$
\langle\underline{u}(\underline{x}) \cdot \underline{\omega}(\underline{x}) \underline{u}(\underline{x}+\underline{r}) \cdot \underline{\omega}(\underline{x}+\underline{r})\rangle=H(r) \neq 0
$$

which measure somehow what is left out of the plane sections (the brackets denote an ensemble average). The magnitude $r=\| \underline{r}(\mid$ is the only scale available, and a simple dimensional argument leads to the inertial law

$$
H(r) \sim \epsilon^{4 / 3} r^{-2 / 3}
$$

with some proportionality constant.

We can also consider the spectral form of $H$, defining $h$ as the Fourier transform of $\mathrm{H}$

$$
H(0)=\int h^{2} d k
$$

Let us now work in some D-dimensional space. It seems appropriate to smooth out the small scale details by integrating over the outer shell of k-space (this is comparable to what we do without saying when we forget about the molecular structure of the fluid. We do not know in this case what the equivalent of the Knudsen number would be, but we assume its existence).

Formally:

$$
\begin{aligned}
\tilde{h}^{2}(k)=\int_{E} h^{2}\left(k^{\prime}\right) d \mu_{D}\left(k^{\prime}\right) \\
E=] \zeta, k_{k}\left[\quad \frac{\zeta}{k_{k}} \leq 1\right.
\end{aligned}
$$

We can use successively the property of $\mu_{D}$ mentioned in the introduction, and the self similarity, to obtain

$$
\begin{aligned}
& \tilde{h}^{2}(k)= \int h^{2}\left(\frac{k^{\prime \prime}}{\zeta}\right) \zeta^{+D} d \mu_{D}\left(k^{\prime \prime}\right) \quad k_{2} \zeta>1 \\
&\left.k^{*} E\right]^{\prime} \frac{k_{k}}{\zeta}[ \\
&=\zeta+D \int h^{2 *}\left(k^{\prime \prime}\right) d \mu_{D}\left(k^{\prime \prime}\right) \\
&\left.k^{\prime \prime} E\right] 1, \frac{k_{k}}{\zeta}[
\end{aligned}
$$

whence $\tilde{h}^{*^{2}}(\eta)=J^{+D} \int h^{*^{2}}\left(k^{\prime}\right) d \mu_{D}\left(k^{\prime}\right)$

So, at all scale $\xi$ in the self-similar range, we have

$$
\tilde{h}^{*^{2}}(x) \propto 3+0
$$


Integrating then $\widetilde{h}^{*^{2}}$ over the full range of its variable, we have also

$$
H(0) \propto \zeta+D \sim r-D
$$

and, since we had previously

$$
H(r) \propto \mathbf{r}-2 / 3
$$

we conclude

$$
D=2 / 3
$$

if $r$ is small enough. If we make the assumption that the flow occupies a finite portion of the 2 -d reference set, we obtain the total dimension of the flow

$$
D=2 / 3+2=8 / 3
$$

If the assumption does not hold, $D<8 / 3$. A discussion relative to plane sections of fractal objects is found in (10), p. 169, and in (8), but it seems difficult to argue whether or not it is relevant here. The above decomposition of $D$ suggests that the line vortex model should be discarded, for a many-sheeted object such as Tennekes' convected vortex tube.

Before we proceed, it may be useful to remind briefly the reader of the interest of the small scale structure for general purposes: the intermittency associated with it is responsible for small corrections to the original spectrum. Several cascade models have been built, reviewed in (11). Let it be said here that additional hypotheses need be implemented in order to obtain numerical values of the corrections (typically, the so-called lognormal assumption, not verified experimentally), and that everyone agrees, on the basis of overwhelming evidence, that the corrections must be small, say of the order of $5 \%$, and positive.

\section{Mandelbrot's Determining Function}

Let us consider that space is divided into small cells, corresponding, say, to the level of definition of our observations. As we look at smaller and smaller scales, by splitting each cell into $C$ pieces, the local dissipation rate concentrates into a few of the subcells, because of the intermittency of the involved patterns. At each level, the density of dissipation rate in each subcell is multiplied by an apparently random factor W, with the obvious property

$$
\langle W\rangle=1
$$

in the range of interest, since the dissipation rate is well defined.

Mandelbrot (6) introduces the function

$$
f(h)=\log _{c}\left\langle w^{h}\right\rangle
$$

wnere the brackets indicate again an ensemble average, $C=\gamma^{3}$ is the number of subcells in the "curdling", i.e. $\gamma$ is the inverse of the similarity ratio of the cascade.

$f$ exhibits a few remarkable properties, listed below:

$1 \%-f$ is convex (8)

$2 \%-f(0)=f(1)=0$ obviously 
$30 /$ - $f^{\prime \prime}(1)$ measures the width of the dissipation range, i.e. it is the main parameter defining the shape of $k^{2} E(k)$

$4^{\circ} /-B=-3 f(2 / 3) \quad$ (8) yields the corrected energy spectrum $E(k) \quad k^{-(5 / 3+B)}$

$5 \%$ - The Kuitosis of velocity derivations is measured by

$$
\begin{gathered}
\exp _{p}\left(\log _{\gamma} \frac{L}{r} \log \left\langle w^{2}\right\rangle\right)=\left\langle W^{2}\right\rangle \log _{\gamma} \frac{L}{r}=\left(\frac{L}{r}\right)^{3 f}(2\rangle \\
\Longrightarrow\left\langle E^{2}\right\rangle \sim r^{-3 f(2)}
\end{gathered}
$$

$6 \%$ - Similarly, the skewness is $\sim r^{-3} f(3 / 2)$.

$7^{\circ} /$ - $f^{\prime}(1)$ is such that $D=3-3 f^{\prime}(1) \quad$ (Kahane, see (8))

This is deviously the property that links $f$ to dimensionality.

$8 \%$ - One more condition can be applied though: from Wyngaard and Pao we learn that the strictly log-normal relation

$$
-S-K^{3 / g}
$$

( $S$ is the skewness, $K$ is the Kuitosis of velocity derivatives) is verified by many authors (even though the log-normal assumption itself is not).

In terms of $f$, this means

$$
f\left(\frac{3}{2}\right)=\frac{3}{8} f(2)
$$

by application of $6^{\circ}$ and $7^{\circ}$ above.

The lack of additional constraints on $f$ is due, for one part, to the current absence of dynamical arguments -- to which some remedy is brought by the present paper, it is believed --, and for the rest to the scarcity of data of higher moments of the velocity derivatives -- Klebanoff and Frenkiel made measurements at only three low Reynolds numbers.

6. A Tentative Entropy Principle. Discussion.

In (8), Mandelbrot also introduces an information-theoretical entropy function

$$
S=-f^{\prime}=\left.\frac{\partial}{\partial h} \log _{c}\left\langle w^{h}\right\rangle\right|_{h=1}=-\left\langle w \log _{C} w\right\rangle
$$

without attempting to use it.

It may be useful to notice that $S$ is not a thermodynamical entropy. Only the formal analogy with the Maxwell-Boltzmann definition of entropy in the kinetic theory of gases justifies the generalization of the denomination.

A particular feature of the above $S$ in Shannon's information theory, is that it is stationary in most time-independent processes. The immediate connection of $S$ to the dimensionality (according to the property \# 7 of $f$, as listed) was the stimulant to seek if such variational property would not be valid also in our case. More precisely, we would like to know under which conditions the stationarity of $S$ with respect to arbitrary variations in $W$ will correspond to the experimental constraints on $f$. 
The known dynamics of turbulence does not give much information about $W$ - nor about $f$, for that matter. Hence, it seems that the usual techniques of the calculus of variations do not apply in this problem. As a consequence, we tested the tentative principle

$$
\delta S=0
$$

against a collection of test functions.

Because it was felt that the dimensionality $8 / 3$ (i.e., $f^{\prime}(1)=\frac{1}{9}$ ) should play a special role, a change of functions was made, so as to take $8 / 3$ as a reference value
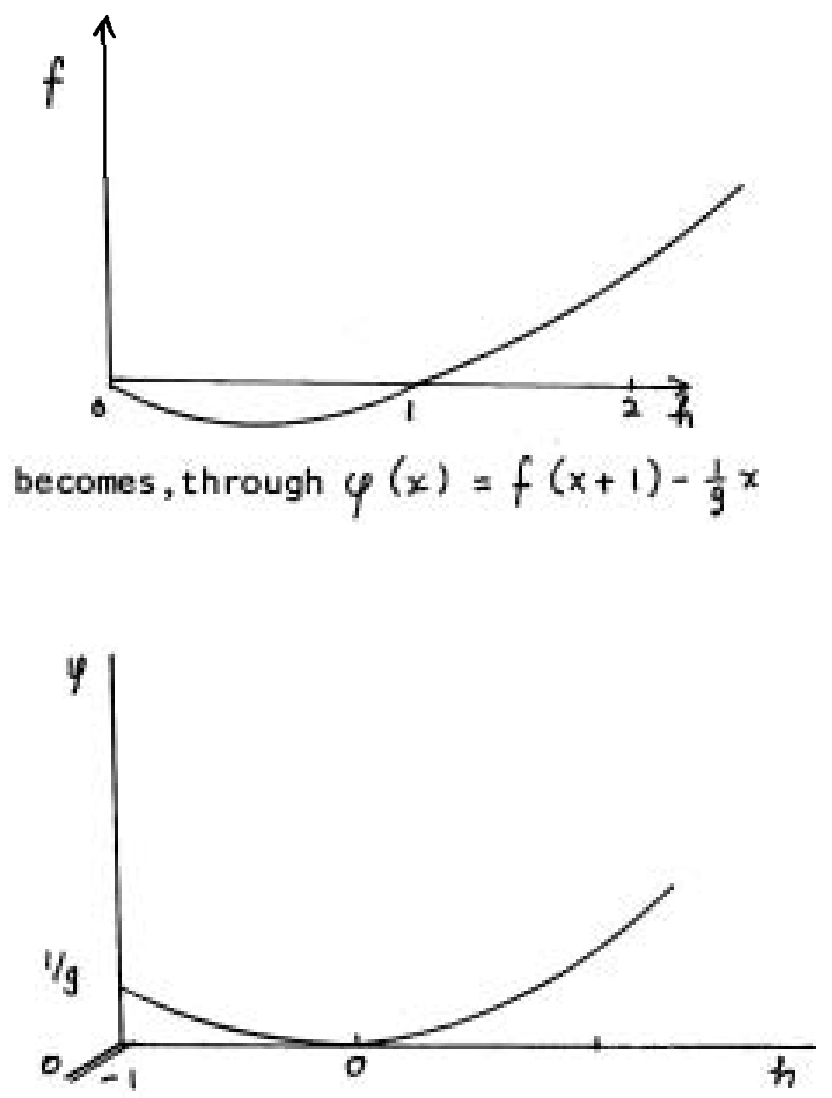

the conditions to be satisfied are $\varphi(0)=0$

$$
\varphi(-1)=\frac{1}{9}
$$

and $\varphi^{\prime}(0)=0$ is our reference slope.

The non-trivial constraints correspond to the properties $8,1,3$, and $f$ of $f$. For $\varphi$ they are written, respectively

$$
\varphi\left(\frac{1}{2}\right)=\frac{3}{8} \varphi(1)-\frac{1}{72}
$$

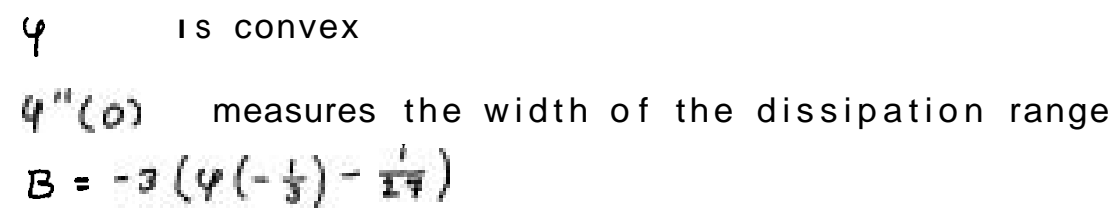

The first two are analytical, and will be satisfied rigorously. 
About the value of $\varphi^{\prime \prime}(0)$, we will use (11), Vol.2, fig.77: there one can see that the shape of the dissipation spectrum is almost universal if the Reynolds number is large enough. So our test functions should yield

$$
\varphi^{\prime \prime}(0) \cong \text { constant }
$$

in order to agree with the data.

The value of $B$ is not well known: the log-normal cascade models reviewed in (11) give it close to .05, with $50 \%$ scatter in the data. A formula derived in (8) relates $B$ and $D$; also under the log-normal assumption and for $D=8 / 3$

$$
B=\frac{3-D}{4.5}=.075
$$

Obviously, these last two constraints are not very sharp. They will be used separately as a means of screening the test functions only when the corresponding error is of an order of magnitude, and will be referred to as "data" in the following.

Finally, $\varphi^{\prime}(0)=0$ will be an interesting property if it is connected to some of the others, but is not a general requirement.

The results come out as follows, with the constraints satisfied:

$1 \% \quad \varphi(x)=n x^{4}-\frac{1}{2} n x^{2}+\left(\frac{1}{9}-\frac{3}{2} n\right) x^{2}$

$$
\frac{\partial \varphi^{\prime}(0)}{\partial n} \equiv 0
$$

The convexity condition is $0 \leqslant n<.01$

$$
\begin{array}{lll}
\text { At } n=0, \varphi^{\prime}(0)=0 & \varphi^{\prime \prime}(0)=.22 & B=.071 \\
\text { At } m=.008 \varphi^{\prime}(0)=0 & \varphi^{\prime \prime}(0) .21 & B=.077
\end{array}
$$

$$
\begin{aligned}
\varphi(x)= & -\ln \left(x+\frac{1}{n}\right)-\frac{16 x^{4}}{3} \ln \frac{2(n+1)^{3 / 8}}{(2+n)(1-n)^{1 / 8}}- \\
& -\left[\frac{16}{3} \ln \frac{2(n+1)^{3 / 8}}{(2+n)(1-n)^{1 / 8}}-\frac{1}{9}-\ln (1-n)\right] x-\ln n .
\end{aligned}
$$

if ${\frac{\partial q^{\prime}}{\partial n}}^{(0)}=0, \quad n=2, \varphi$ has a pole at $x=-\frac{1}{2}$, and must be rejected.

if $\varphi^{\prime}(0)=0, n=.078, \frac{a \varphi^{\prime}(0)}{\partial n} \neq 0, B=.94$ which is not acceptable.

$$
\begin{aligned}
& \begin{array}{l}
\varphi(x)=\exp (-x+n)+\left[\frac{8}{3} e^{n-1}+\frac{1}{3} e^{1+n}-2 e^{n}-e^{n-1}\right] x^{3}+ \\
\quad+\left(\frac{2}{3} e^{n+1}-\frac{8}{3} e^{n-\frac{1}{2}}+e^{n}+e^{n-1}-\frac{1}{g}\right) x-e^{n}
\end{array} \\
& \text { if } \varphi^{\prime}(0)=0 \quad \frac{\partial \varphi^{\prime}(0)}{\partial n} \neq 0, B=.115, \varphi^{\prime \prime}(0)=.3 \\
& \text { If } \frac{\partial \varphi^{\prime}(0)}{\partial n}=0, \varphi^{\prime}(0)=-\frac{1}{g} \text { and } \varphi \text { cannot be convec. }
\end{aligned}
$$




$$
\begin{aligned}
& 4^{\circ} \% \varphi=\sin n x^{2}+\frac{16}{3}\left(\sin \frac{n}{4}-\frac{\sin n}{4}\right) x^{4}+\left(\frac{1}{9}-\frac{16}{3} \sin \frac{n}{4}+\frac{1}{3} \sin n\right) x^{2} \\
& \varphi^{\prime}(0) \equiv 0 \\
& \text { for } n=.5 \quad B=.075 \quad \varphi^{\prime \prime}(0)=.22 \\
& n=.2 \quad B=.074 \quad \varphi^{\circ}(0)=.22 \\
& n=.8 \quad B=.077 \quad \varphi^{\prime \prime}(0)=.18 \\
& \varphi^{\prime}(0) \equiv 0 \\
& \text { for } m=.8 \quad \varphi^{\prime \prime}(0)=.23 \quad B=.075 \\
& n=.2 \quad \varphi^{\prime \prime}(0)=.21 \quad B=.07
\end{aligned}
$$

This collection is not complete of course, but represents what is left of a large trial set, of which the functions would fail to satisfy either the extremum condition $\frac{\partial \varphi^{\prime}}{\partial n}(0)=0$ or the convexity condition. The sample above is intended to give a potential variability of the curvature at the origin, and to avoid a systematic symmetry as encountered in the first case.

It is seen that acceptable values of $B$ are consistently obtained at $\varphi^{\prime \prime}(0)=.22$, with a suprising accuracy, if we impose on $\varphi$ two conditions:

$$
\left\{\begin{array}{l}
\varphi^{\prime}(0)=0 \text { (corresponding to a dimension } 8 / 3 \text { ) } \\
\varphi^{\prime}(0) \text { is stationary with respect to the free parameter. }
\end{array}\right.
$$

To summarize the results for the determing function $f$, we have shown that if $f$ satisfies the general conditions of convexity, and of fixed values at 0 and 1 ; if we impose the relationship between skewness and Kurtosis; if $D=8 / 3$ and the entropy is stationary with respect to the free parameter; then the width of the dissipation spectrum is constant and the correction coefficient $B$ has a correct value.

A more rigorous development of the idea was not possible because of the absence of any dynamics in the definition of the determining function: but an entropy property is such a dynamical feature, and it is intended to work by deductions along this line: for example, in the case of a non-Newtonian fluid, one could imagine that the width of the dissipation spectrum is different, so that the state of time independence (well-developed turbulence) would correspond to another dimensionality of the field: the $8 / 3$ has nothing magic, even if we relied heavily on it in this paper.

Also, a better understanding is gained of why $3 / 3$, (or whatever it has to be) is a critical dimension: the fact that it appeared in a derivation based on critical points theory, suggests that a connection between the critical point and the entropy of the system. Some work is suggested for both laboratory and numerical experiments, about measuring the dimensionality or establishing the extremal properties of the flow. 
Finally, a promising line seems to be opened for the introduction of functional analysis into the study of fractals in turbulence. For example, one could write $W$ as a functional of the velocity field, and its derivatives, and try to derive some consequences of the stationarity of entropy, submitted to the constraints on $f$.

\section{Acknowledgments}

The author wants to express his gratitude to Dr. W. Malkus for many useful and challenging comments; to Rush Holt for providing some of the most recent material; and in general, to all those who have supported this work in one way or another.

\section{References}

(1) Adler, R.J. 1977 Hausdorff dimension and Gaussian fields. Ann. of Proba., 5: 145

(2) Bell, T.L. and M. Nelkin 1977 Non-linear cascade model for fullydeveloped turbulence. Phys.of Fluids 20: 345.

(3) Cocke, W.Y. 1969 Turbulent hydrodynamic line stretching. Phys.of Fluids 12: 2488; 14: 1624.

(4) Frisch, U., M.Lesieur, and P.-L.Sulem 1976 Crossover dimension for fully-developed turbulence. Phys.Review Lett. 37: 395.

(5) Kuo, A.Y. and S.Corrsin 1972 Experiment on the geometry of the fine structure regions in fully turbulent fluid. J.Fluid Mech. 56: 477.

(6) Mandelbrot, B.B. 1974 Intermittent turbulence in self-similar cascades: divergence of high moments and dimension of the carried. J.Fluid Mech. 62: 331.

(7) Mandelbrot, B.B. 1975 On the geometry of homogeneous turbulence, with stress on the fractal dimension of the iso-surfaces of scalars. J.Fluid Mech. 72: 401.

(8) Mandelbrot, B.B. 1976 Intermittent turbulence and fractal dimension: Kurtosis and the spectral exponent $5 / 3+B$, in Temam (14).

(9) Mandelbrot, B.B. 1976 Fractals and turbulence: attractors and dispersion in Seminar in Turbulence, Berkeley (S.Smale, J.E.Marsden, and A.J. Chorin ed.) Lecture Notes in Mathematics, Springer, New York.

(10) Mandelbrot, B.B. 1977 Fractals: form, chance and dimension. Freeman $\varepsilon$ Co. San Francisco.

(11) Monin, A.S. and A.M.Yablom 1975 Statistical Fluid Mechanics (J.L.Lumley ed.) MIT Press.

(14) Temam, R. 1976 Turbulence and Navier-Stokes equations. Lecture Notes in Mathematics. Spinger, New York.

(15) Wyngaard, J.C. and Y.H.Pao 1972 Some measurements of the fine structure of large Reynolds number turbulence, in Statistical Models and Turbulence (Rosenblatt \& Van Atta ed.) Lecture Notes in Physics,Springer,N.Y. 


\section{A GRID-STIRRING EXPERIMENT WITH BUOYANCY INPUT}

John W. Loder

\section{Introduction}

An interesting feature of oceanographic density sections from some relatively energetic tidal regions is the existence of a vertically wellmixed water column throughout the year (e.g. in the Celtic Sea, Simpson (1976), and an Georges Bank, Bumpus (1975)). Although surface wind stress undoubtedly contributes to mixing in the upper part of the water column, the existence of shallow thermoclines in nearly less tidally energetic regions and the maintenance of the well-mixed state even in periods of low wind stress, suggests that bottom-generated turbulence is the major source of turbulent kinetic energy (TKE) for such mixing.

Simpson and Hunter (1974) used simple energetic considerations to suggest an empirical criterion for predicting the location of the transition from the well-mixed state to a stratified water column. For a well-mixed water column of depth $H$ with a rate of surface heat input $Q$, the rate of generation of potential energy (PE) required to maintain the well-mixed condition is $\frac{d P E}{\partial t}=\frac{1}{2} \frac{\alpha Q g H}{C_{p}}$ where $\alpha$ and $c_{P}$ are the coefficient of thermal expansion and the specific heat respectively. Assuming that a fraction $E$ of the rate of TKE production in the bottom boundary layer is available for the generation of $\mathrm{PE}$, they suggested that the transition from the well-mixed to the stratified regime would occur when $E \rho C_{D} \overline{\left.T_{u}\right|^{3}}=\frac{1}{2} \frac{\alpha Q g H}{C_{P}}$ where $C_{D}$ is a drag coefficient in a quadratic bottom friction law, and $|u|^{3}$ is the average over a tidal cycle of the tidal current cubed. Application of this criterion to the Irish and Celtic Seas showed contours of $\frac{H}{\text { iuls }}$ approximately parallel to the observed transition zone during summer. An interesting result is that the observations suggest a value of $\varepsilon$ of only a few tenths of a percent, indicating that very little of the TKE goes into work against gravity. Contours of $\frac{H}{\mid \overline{|u|^{3}}}$ for the Gulf of Maine (C.Garrett (personal communication) using data from Greenberg's (1975) model) and for Georges Bank (J.Fifield (personal communication) using observed tidal currents) also show rough agreement with the observed well-mixed areas during summer, and again indicate a value of $\varepsilon$ at the transition zone of only $\sim .001-.002$. This is a bit surprising since laboratory experiments by Kato and Phillips (1969) and ocean observations by Kitaigorodskii (1960) of entrainment in a surface mixed layer with mean flor suggest that-. $5 \%$ of the available kinetic energy is going into PE generation (although it is not clear that the results should be compared).

These energetic considerations suggest that, in an initially wellmixed water column with surface heating and only including contributions to vertical mixing from bottom generated turbulence, the important parameters are the rate of heat input $Q$, the depth $H$, and some velocity $U$ such as a friction velocity $U_{*}$. The nondimensional combination $\frac{Q_{H}}{U^{3}}$ (where $Q$ has units of energy/mass/time) might be expected to determine the onset of stratification. The present experiment has been motivated as an attempt to investigate the relevance of the $\frac{a n}{v^{3}}$ parameterization to a simple lab- 
oratory experiment involving a controlled rate of TKE production in the lower portion of a tank of water and controlled heat input at the surface. It might also provide a laboratory estimate of $\varepsilon$, and some indication of the dependence of the long-term evolution of the stratification in the system on $Q, H$ and $U$. The experiment $w i l l$ thus be an attempt to simulate the evolution of stratification due to buoyancy input at the ocean surface in regions such as continental shelves or shallow seas where bottom-generated turbulent velocity fluctuations are of sufficient magnitude to affect the density structure throughout the water column. Such stratification may be considered to develop during spring and summer when solar radiation and fresh water run-off provide a buoyancy input at the surface of an initially vertically well-mixed water column. The source of TKE is considered to be the kinetic energy removed in the bottom boundary layer from the oscillatory barotropic tidal flow, although the model should also be applicable to regions where some other form of bottom-generated mixing occurs.

Some obvious limitations on the applicability of such an experiment to the ocean should be noted. A variety of processes contribute to vertical mixing in the ocean; the present experiment is intended to simulate only the effects of bottom-generated turbulence (although convective motions also may have existed in the upper portion of the experimental system). The rotation of the earth has not been simulated and hence the existence of turbulent Ekman layers above the ocean floor is not represented. The applicability of the experiment might thus be limited to shallow regions where the water depth is sufficiently small that the boundary layer thickness is not limited by coriolis effects (i.e. the depth $\mathrm{H}$ is less than some characteristic Ekman layer thickness, say $\sim .1 u_{*} / f$ where $f$ is the coriolis frequency). The neglect of horizontal mixing, and horizontal and vertical advection, is also an oversimplification. This may be especially true in a discussion of bottom-generated turbulence since its intensity will vary horizontally dependent on spatial variations in the tidal current regime and bottom roughness. Such horizontal variations in vertical mixing will result in horizontal density gradients which may drive mean circulations which also may affect the density structure. A one-dimensional vertical mixing model might be useful for estimating the horizontal density variations as input to a model of the densitydriven circulation.

\section{A Discussion of Some Previous Experiments:}

A number of experimental studies involving turbulence in a stratified fluid, and relevant to the present experiment, have been reported in the literature. Most of these experiments have been attempts to model the entrainment processes associated with the growth of the ocean surface mixed layer into the underlying stratified water during storms or other periods of increased energy input from the wind. consequently they neglect the continuous buoyancy input at the ocean surface which is important in the present context for the long term evolution of oceanic stratification. A recent discussion of these experiments may be found in Price (1977). They have been mainly of two types: (1) those with mechanical energy input by an oscillating grid, thus generating turbulence without mean flow, and (2) those where a constant stress at the upper surface puts energy into a mean flow in the upper part of the fluid. They have attempted to parameterize the nondimensionalized entrainment, $E=\frac{w_{e}}{u_{*}}$ where $W_{\varepsilon}$ is the rate of growth of the mixed layer, as a function of some overall Richardson number of the system (usually 
a $R i=\frac{g \Delta p l}{\rho \mu^{\prime}}$ for the grid-stirring experiments or a $R i_{*}=\frac{g \Delta P D}{\rho u_{*}{ }^{2}}$ for the applied stress experiments, where $u^{\prime}$ is a rms horizontal turbulent velocity, $u_{*}$ is the friction velocity, $\ell$ is an integral length scale of the turbulence, $D$ is the mixed layer depth, and $\Delta \rho$ is the density difference across the mixed layer interface). There is some inconsistency in the results of these experiments, with the grid-stirring experiments showing $E \propto R t^{-3 / 2}$ or

$E \propto R_{i}^{-1}$ dependent on temperature versus salinity stratification, and on the local Péclet number. The earlier stress experiments (Kato and Phil-

lips, 1969) suggested $€ \propto R i_{*}^{-1}$ but more recent experiments (Kantha, Phillips, and Azad, 1977) suggest that this law does not hold for large Ri $i_{\text {and }}$ that $E$ is larger than in the Kato and Phillips experiments by a factor of two. These experiments raise some interesting questions. A $R i_{*}^{-1}$ law is consistent with the energetic argument that the rate of generation of PE is proportional to the availability of TKE. If this were a valid result and the proportionality factor were known, it would be quite useful for predicting entrainment and might have implications for the time dependent evolution of stratification discussed here. The Kantha, Phillips and Azad results suggest, however, that different processes control the evolution of turbulence in a stratified fluid for different ranges of $R i$. The inconsistencies in these experiments also point out the necessity of careful analysis of the importance of geometry sensitivity, sidewall friction, and other viscous effects in the laboratory experiments before their results are applied to the ocean.

Two other experimental studies are of some relevance. Kraus and Turner (1967) modeled the evolution of the seasonal thermocline for a constant rate of grid stirring in the surface layer and for a sawtooth seasonal heating function experimentally obtained by the input of buoyant fluid at the surface. The depth and temperature dependence of the upper mixed layer as functions of time and the relation to the heating and cooling cycle were in qualitative agreement with ocean observations.

C.G.H.Rooth (personal communication) has pointed out some interesting features about the dependence on stratification of the ratio of PE generation to available TKE (i.e. $£$ ) based on unpublished experimental work. Experiments were performed with both top and bottom grid stirring, and with surface heat input such that the temperature difference $\Delta T$ between the top and bottom was controllable. Estimates of the rate of generation of PE for various $\Delta T$ but constant stirring rates $(\Omega$ ) suggested a dependence of the PE generation on stratification as shown in Fig.l.

This variation of $E$ with $R i$ again implies that ranges of $R i$ exist in which the important parameters differ, thus complicating any model of the evolution of stratification in a turbulent fluid.

The present experiment can thus be considered to also have been motivated by questions raised by these past experiments, and as an attempt to obtain some feeling for the important physical processes in laboratory models and their relevance to the ocean.

\section{Experimental Apparatus}

The apparatus sketched in Fig.2, consisted of a cylindrical glass tank of diameter $29 \mathrm{~cm}$ and depth $29 \mathrm{~cm}$, with mixing provided by a vertically 


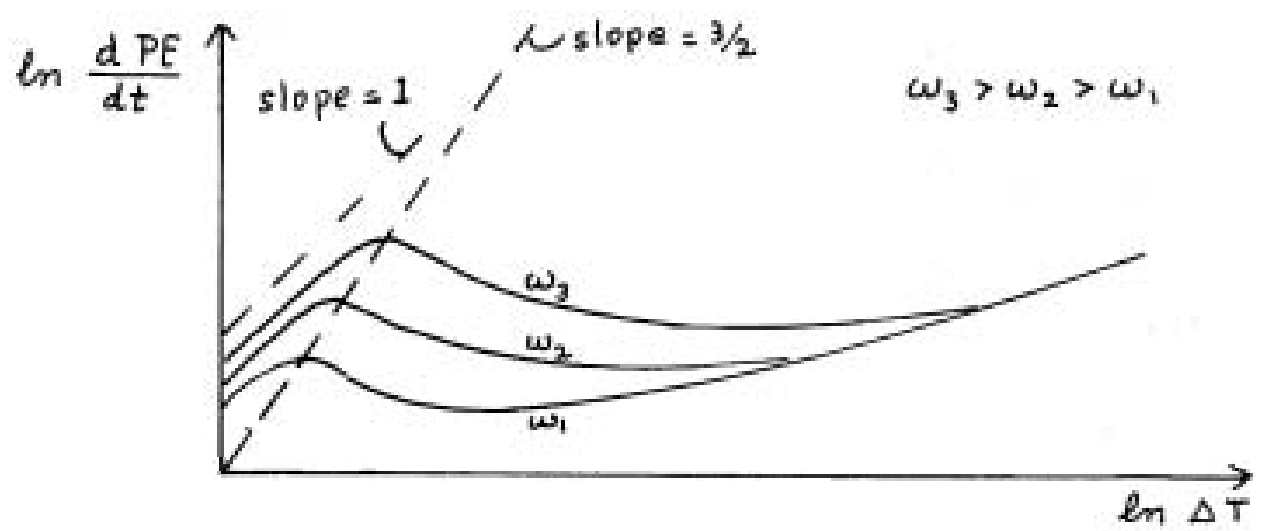

Fig.l. Rate of generation of $P E$ vs $\Delta T$ for three different stirring rates. C.G.H.Rooth (personal communication).

oscillating grid in the lower part of the tank and heating by an electric heater several centimeters below the water surface.

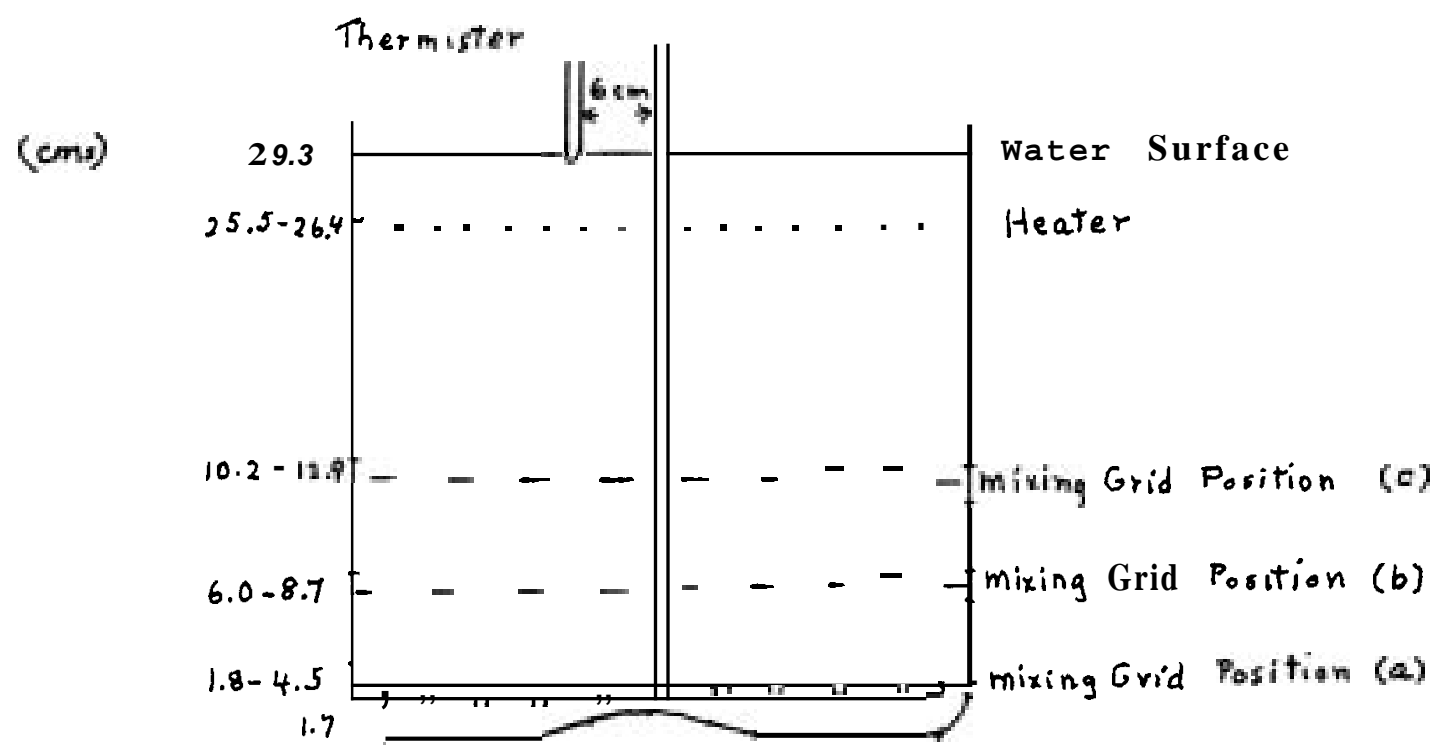

Fig.2. Experimental tank.

The aluminum mixing grid was rectangular in overall shape $(-22.2 \mathrm{~cm}$ $x 25 \mathrm{~cm})$ and quite rigid $(0.6 \mathrm{~cm}$ thick), and consisted of perpendicular rectangular crossbars $0.9 \mathrm{~cm}$ in width with $3.5 \mathrm{~cm} \times 4.0 \mathrm{~cm}$ openings between bars. It was oscillated at the desired amplitude and frequency by a variable speed gear motor attached to a scotch yoke. The heater consisted of horizontal wires of diameter $-1 \mathrm{~mm}$ and separation $\sim 1.5 \mathrm{~cm}$ with total resistance $33.5 \Omega$, and was supplied by a regulated variable output $A C$ power supply with digital read-out. The temperature was measured using a $4000 \Omega$ 
Isocurve thermistor in a linear bridge circuit and with output to a chart recorder. Vertical temperature profiles were measured $6 \mathrm{~cm}$ from the tank's center axis by mechanically lowering the thermistor at constnat speed with a total descent time of one minute.

Figure 2 also shows the positions of the heater and mixing grid relative to the water surface and tank bottom. The water surface and heater were maintained at the same position for all runs, while the mixing grid was placed at three different depths. The amplitude of the grid oscillation was fixed at $1.05 \mathrm{~cm}$ for all runs, while the frequency was varied between 92 and 137 cycles/min. The system was insulated during heating experiments.

Description of Turbulence without Buoyancy Input

Attempts were made to study the grid-induced motions in a homogeneous fluid. These involved simple and somewhat crude techniques, but they may be compared with previous experimental parameterizations (Thompson and Turner, 1975; Hopfinger and Toly, 1976) and theoretical suggestions (Long, 1977) for such flows. The Thompson and Turner, and Hopfinger and Toly experiments suggest that the integral length scale $\boldsymbol{Z}$ is proportional to distance $z$ from the grid and that the rms horizontal velocity $u^{\prime}$ is proportional to the frequency of grid oscillation, but differ in the dependence of $u^{\prime}$ on $z$ with Thompson and Turner finding a $z^{-3 / 2}$ dependence and Hopfinger and Toly a $\boldsymbol{z}^{-1}$ dependence. Long (1977) proposes a theoretical model for the statistically steady behavior of such a turbulent flow, in agreement with the Hopfinger and Toly observations.

Dye streaks left by permanganate crystals falling through the fluid were observed to qualitatively study the velocity fluctuations in the fluid and to detect any mean circulations in the tank. These observations suggested that random velocity fluctuations on a variety of scales were present throughout the tank, and showed no obvious mean circulations, although observations were at times inconclusive especially for the lowest mixing grid position.

Attempts were made to parameterize $u_{*}$ as a function of grid oscillation frequency by estimating eddy diffusivities $K$ based on diffusion times in the turbulence. For the fluid initially at rest, the rate of expansion of the bottom turbulent region as a function of time after turning on the mixing was recorded from dye streak observations. Figure 3 shows the data from three such runs for $f=108 \mathrm{cpm}$ and the mixing grid at position (b).

Such graphs were used to estimate $K$ assuming a diffusion time $T \sim L^{2} / K$ where $L$ is an outer length scale of the turbulent region. Then, assuming $K \sim X u_{*} \ell$ where $x$ is von Karman's constant and $\ell \sim x D$ where $D$ is a length scale of the tank, $u_{*}$ can be estimated from $u_{*} \sim \frac{k}{x^{2} D}$. Figure 4 shows $u_{*}$ vs. $\omega(=2 \pi f)$ using five stirring frequencies.

The deviation from the expected straight line relationship is attributed to observational uncertainties in determining $L$ s. $T / 2$ for such a small range of oscillation frequencies. Although Fig.2 shows good agreement with a diffusion time scaling, other data showed less agreement and were sometimes indistinguishable from data at a different frequency. This may also be attributable to a lack of horizontal homogeneity in the evolving turbulence. Consequently, it is felt that it cannot be concluded that a $u_{*} \propto w$ relationship does not hold for the system. 


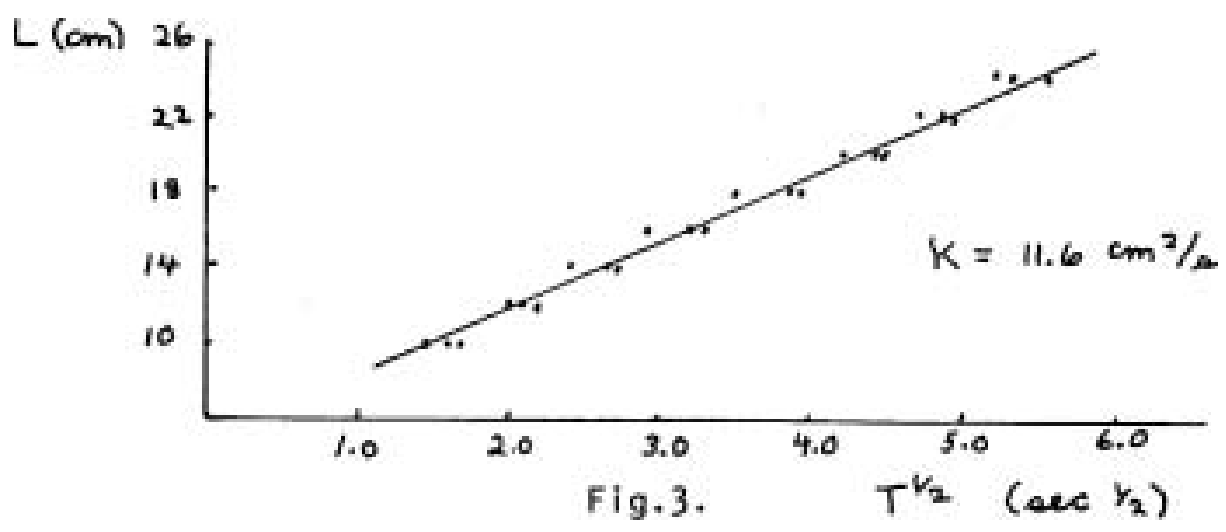

$L$ vs $T^{1 / 2}$ for three experimental runs $(x, \Delta, 0)$ with mixing grid at position (b) and $f=108 \mathrm{cpm}$.

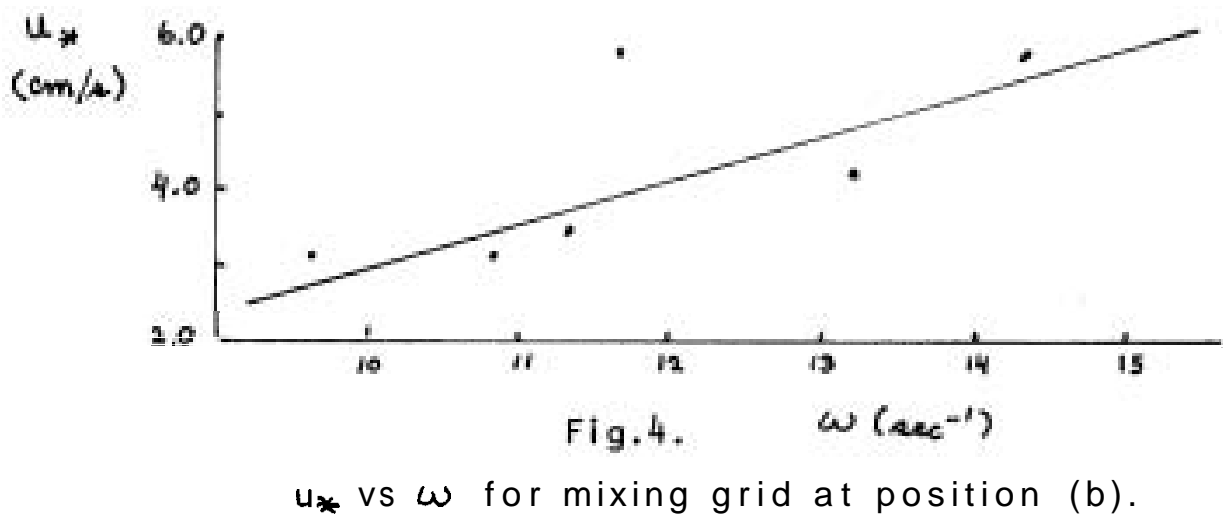

Onset of Stratification

A major aim of the experimental work was to look at the onset of stratification in an initially well-mixed system. This was done by slowly increasing the rate of heat input $Q$ for a constant rate of mixing until stratification developed. Vertical temperature profiles were measured at varying time intervals; when such profiles were not being measured, the thermistor was placed-0.2 $-0.6 \mathrm{~cm}$ below the water surface to record a time series of the near surface temperature. Figure 5 shows a typical set of such measurements.

For low heating rates, the TKE was sufficient to maintain a vertically well-mixed system, resulting in a uniform rate of temperature increase at all depths. A comparison of this with the expected rate of increase from the power dissipation in the heater showed that $\sim 15 \%$ of the heat input was being lost from the system. For increased heating rates, a regime was reached in which a thin surface layer of warmer water was observed to appear and disappear on time scales of 10 - 100 seconds. This intermittency was attributed to the intermittency of near surface turbulent fluctuations, with turbulent accelerations being insufficient to overcome buoyancy during times of stratification. The existence of such intermittent stratification 


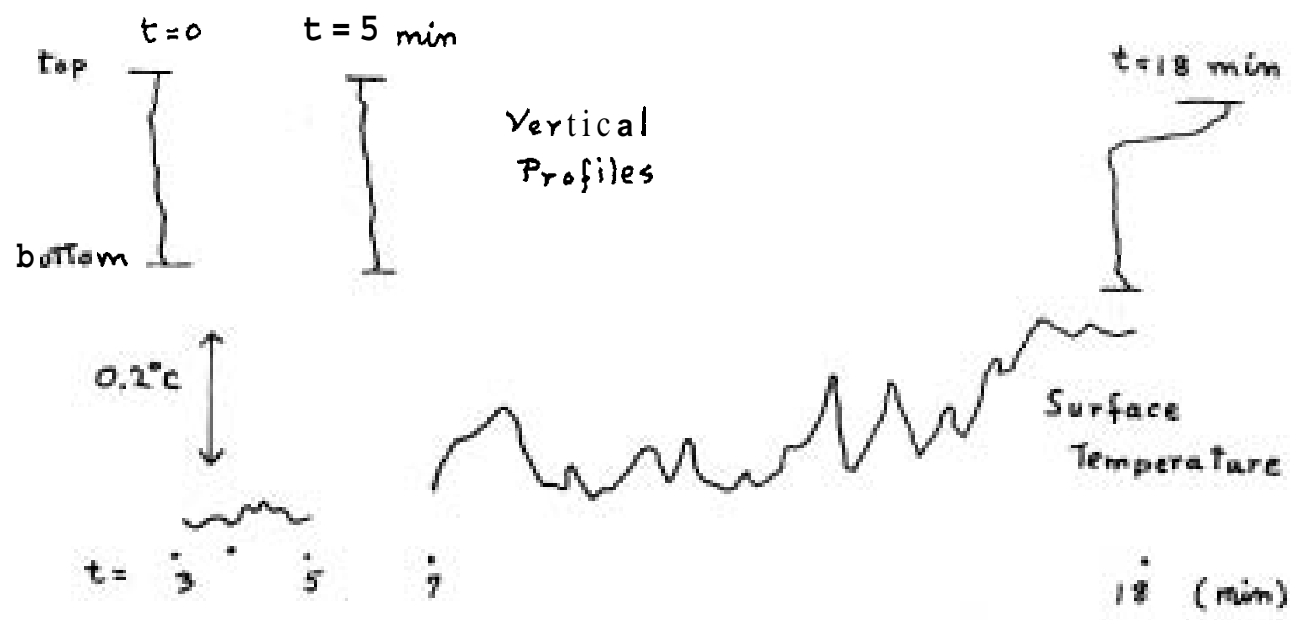

Fig. 5

Three temperature profiles and surface temperature variations for experiment with constant mixing at position

(a) and heat input

$$
\begin{array}{ll}
\mathrm{Q}=0 & \mathrm{t}<3 \mathrm{~min} \\
\mathrm{Q}=.0059 \mathrm{cal} / \mathrm{cm}^{2} / \mathrm{s} & 3<\mathrm{t}<11 \mathrm{~min} \\
Q-.0066 \mathrm{cal} / \mathrm{cm}^{2} / \mathrm{s} & \mathrm{t}>11 \mathrm{~min}
\end{array}
$$

has interesting implications for the energy budget of such a system. If the ratio of rate PE generation to rate of TKE production were constant, one might expect the onset of stratification to result in a runaway towards increasing stratification, since increasing PE generation would be required to break down such stratification. The observation of continued intermittency for a small range of $\frac{Q H}{y}$ suggests that there might be an increased rate of $P E$ generation at low stratifications, consistent with observations in the Rooth experiments.

At slightly higher heating rates, the stratification was continually maintained, and increased with time for a constant heating rate. A region of constant temperature with depth evolved above the heater with a sharp thermocline developing below the heater.

The relevance of $\frac{\mathrm{aH}}{\mathrm{U}^{3}}$ to the onset of stratification was investigated by noting the rate of buoyancy input at which the transition occurred for repeated experimental runs at each of several mixing frequencies. Figure 6 shows $(\alpha Q)$ transition, where $\alpha$ is the coefficient of thermal expansion at the transition temperature, and $Q$ is the rate of heat input/unit area, versus $u_{*}^{3}$ as estimated in the previous section for the mixing grid at position (b).

The data is not very suggestive of a linear relationship, but considering the uncertainties in the estimation of $u_{*}$, it is felt that the results are inconclusive. A plot of $\left(\alpha Q\right.$ ) transition vs $\omega^{3}$ (Fig.7) shows a much closer approach to a linear relationship. It is thus felt that the evaluation of $u_{*}$ as a function of $\omega$ was too unreliable, and that the linear relation between $\propto Q$ and $\omega^{3}$ might be interpretable as suggestive of a 


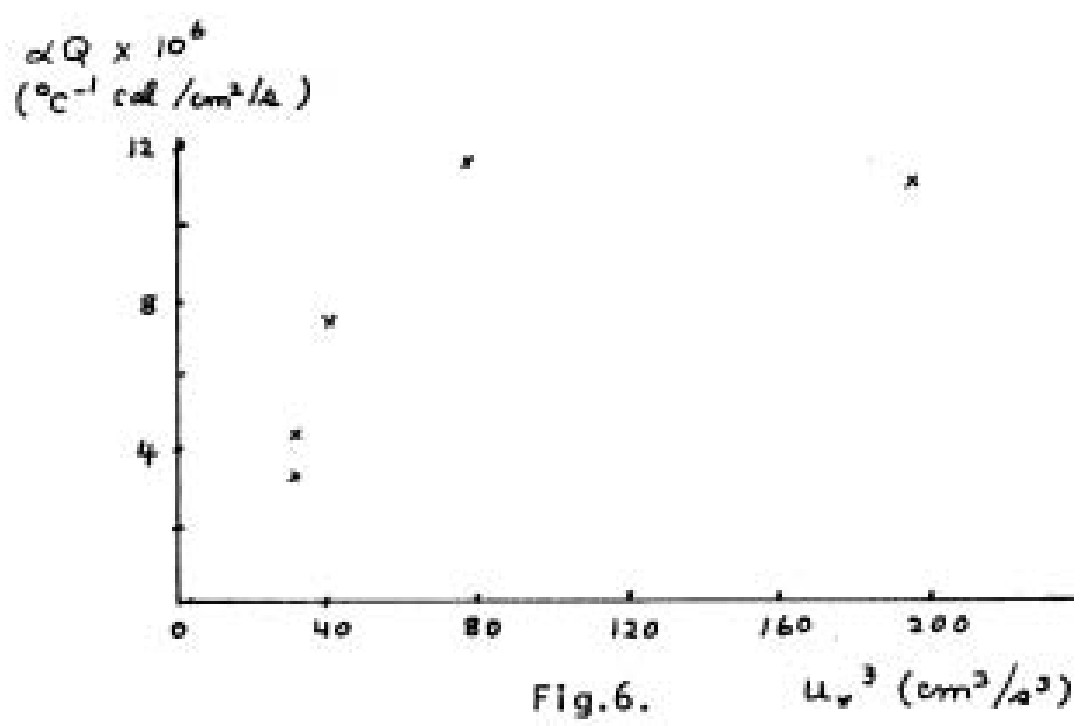

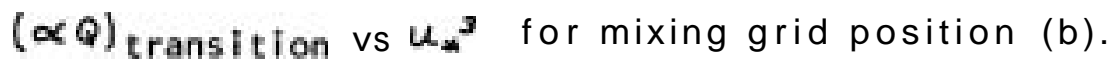

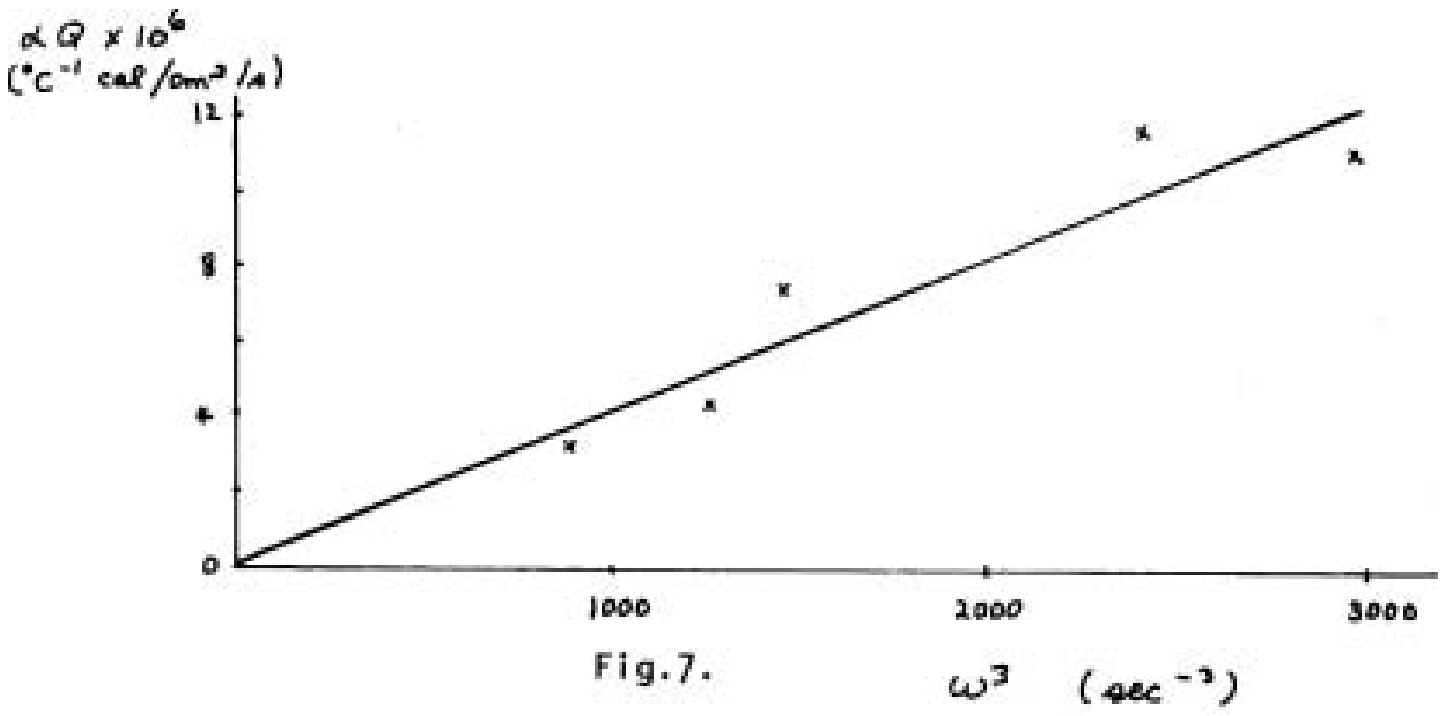

$(\propto Q)_{\text {transition }}$ vs $\omega^{\prime \prime}$ for mixing grid position (b).

linear dependence of $u_{*}$ on $w$ and of $\alpha Q$ on $u_{*}{ }^{3}$.

The rate of heating corresponding to the onset of stratification was also used to infer the dependence of the rate of TKE production/unit mass on the distance $z$ from the mixing grid. For the same mixing frequency, the value of $(\alpha Q)$ transition was observed for the three locations of the mixing grid. This was done initially to look at the sensitivity of the grid induced motions to closeness to the tank bottom. Such a sensitivity was not observed, but the results did show that considerably higher heating rates were required for stratification as the mixing grid was moved closer to the top of the tank. Figure 8 shows $(\alpha \varphi)$ transition $v s z^{-*}$ for two frequencies of grid oscillation. Interpreting the higher required heating rates as being indicative of higher turbulent energies in the near surface layer, the results suggest a $\mathbf{z}^{-\boldsymbol{f}}$ dependence for the rate of TKE production per unit mass. 


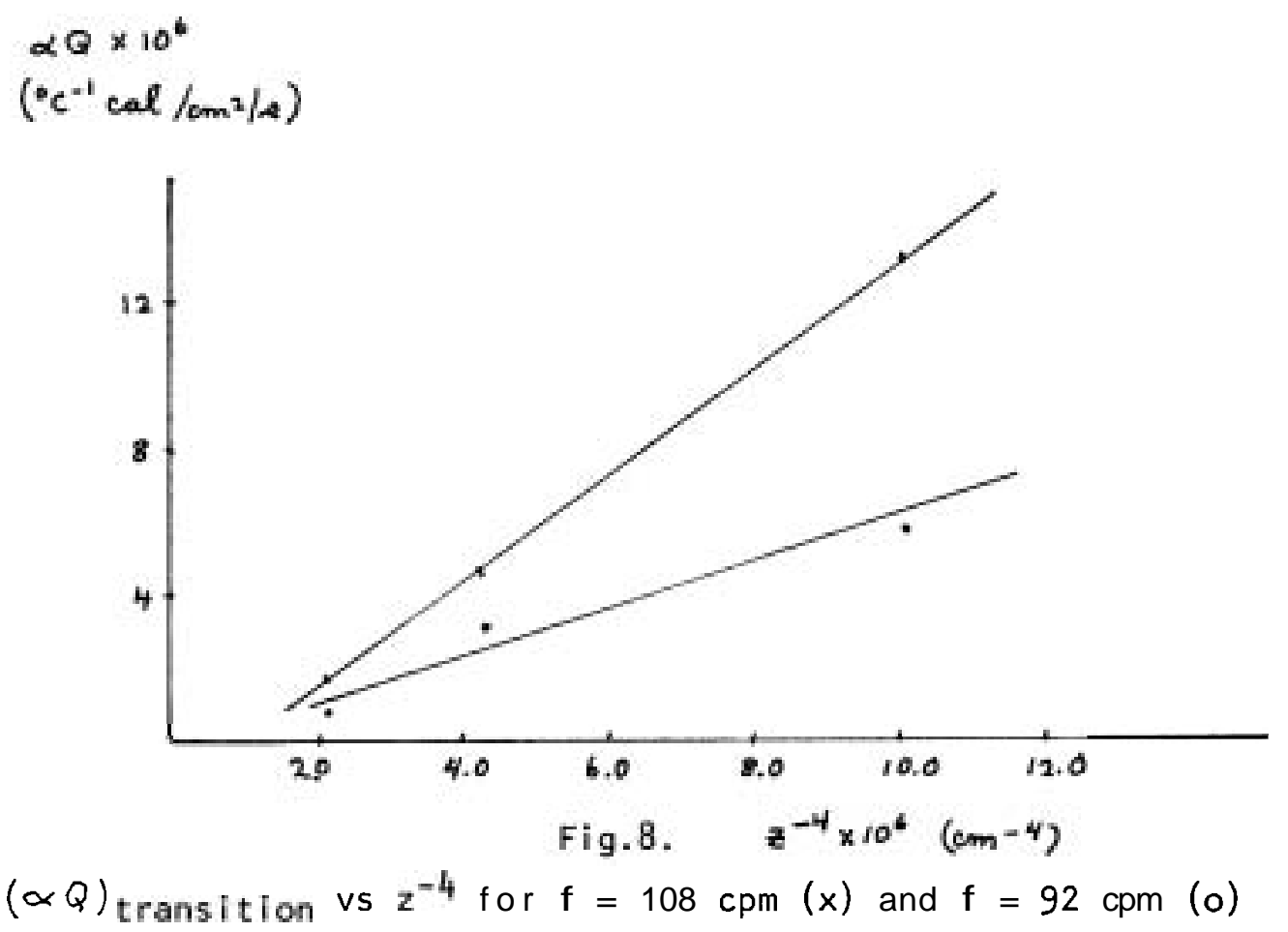

This is consistent with theoretical expectations assuming that the rate. of production of TKE per unit mass is equal to the rate of kinetic energy dissipation per unit mass, $\frac{u^{\prime 3}}{L}$, for a steady state system, and using the Hopfinger and Toly and Long dependences of $u^{\prime} \propto z^{-1}$ and $\ell \propto$ ह .

Observations of Stratified System

Several features of the behavior of the system during stratification are worthy of comment. Although time prevented more observations of the long term evolution of the stratification, the observations that were made suggested an increasing temperature difference between top and bottom for constant heating and mixing with no indication of a constant rate of heating at all depths.

An interesting behavior was the continued increase in stratification for decreasing rates of heating, and the time required for the stratification to be broken down after heating was turned off. If heating were slowly increased until stratification formed, and then slowly decreased, the system remained stratified through a range of heating rates for which well-mixed conditions were maintained during the increasing heating stage. This hysteresis makes the $\frac{a H}{v^{3}}$ criterion applicable for predicting transition only during times of increasing heating rate.

An unresolved question about these laboratory experiments is the importance of internal waves on the density interface and how realistic the presence of such waves are to the ocean thermocline. The possibility of internal modes of the wave tank being excited and containing significant energy could make less TKE available for PE generation. Rapid breaking of such waves might mean an increased cascade of TKE towards high wave numbers and viscous dissipation. Figure 9 shows temperature observations at a fixed position in the thermocline for the present experiment. 


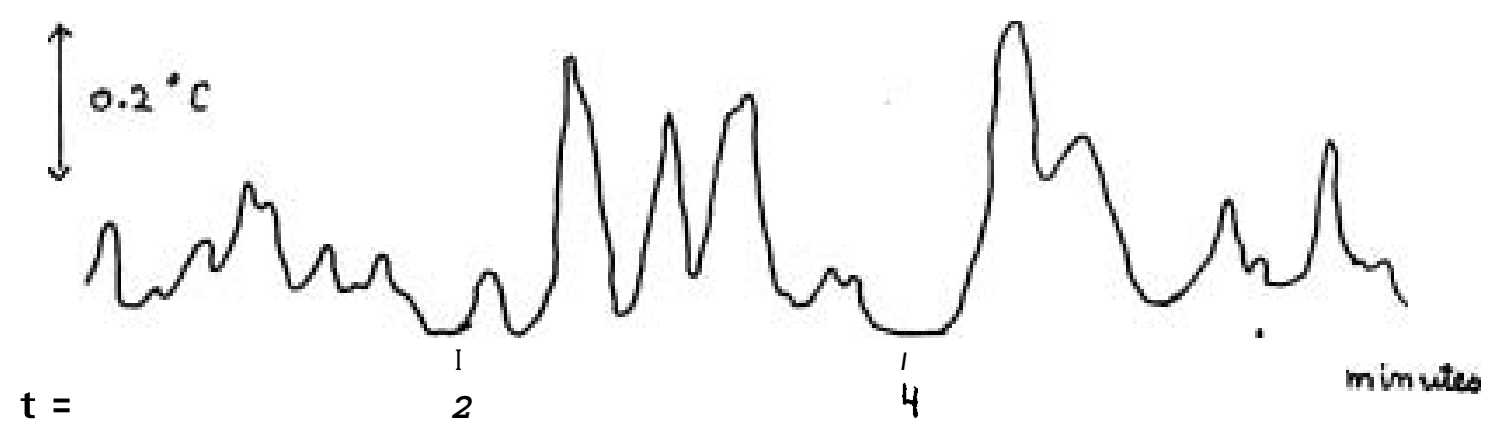

Fig.9.

Typical temperature fluctuations in the thermocline.

These temperatures have not been spectrally analyzed, and hence it is inconclusive whether the temperature fluctuations are indicative of wave-like processes on the thermocline, or whether they simply contain turbulent fluctuations associated with eddies incident upon the thermocline.

Discussion of Experiment

It should be noted that at times, unaccounted for inconsistencies were observed in the behavior of the system, which might suggest a sensitivity to some uncontrolled parameter. The limited time available for doing the experiment resulted in the use of existing, and at times less than ideal, apparatus. The effects of a curved (upwards in the center) bottom surface, a rectangular mixing grid in a cylindrical tank, sidewall friction, and external thermal gradients have not been thoroughly investigated, nor have the damping effects of the heating element on the turbulence or the effect of any law of the wall behavior in the top few centimeters of fluid on the observations. A first assessment of these factors however has not suggested that any of them had significant effects on the reliability of the results reported here.

More than anything else the present experiment has been useful as a first experiment in pointing out guidelines for similar future experiments. The occasional inconsistency in a somewhat less than simple geometry suggests that the geometry sensitivity of any experiment be carefully examined. Although it is essential that the mixing processes for simple systems be understood as a first step, there is some suggestion of a gap between the simple laboratory experiment and the more complicated ocean.

An original aim of the experiment had been to estimate a value of at the transition to a stratified system. This was not accomplished due to difficulty in quantifying the rate of working of the mixing grid; however, it might be a reasonable objective for a future experiment.

Implications for Tidal Mixing

The experiment has pointed out several qualitative features of relevance to the ocean. $\frac{a H}{U^{3}}$ appears to be the important parameter governing the onset of stratification in the present system, but the relevance of a one-dimensional model to tidal mixing is no clearer than before; a laboratory estimate of $\mathcal{E}$ would have possibly helped to clarify this. Any hori- 
zontal diffusion or advection of heat into the surface layer of a water column could be included in $Q$, in predicting the onset of stratification. The experiment also suggests that the first appearance of stratification may not immediately result in a runaway increasing stratification, but that rapid increases in stratification might be expected for slightly higher heating rates.

The hysteresis of the dependence of stratification on $\frac{9 H}{U^{3}}$ suggests that $\frac{a y}{y^{3}}$ is only a relevant parameter for predicting the stratification during the increasing heating season. It also suggests that in applying $\frac{Q H}{V ?}$ to the ocean, values of neap tidal currents may be more relevant than spring tidal cussents since stratification is more easily formed during periods of higher $\frac{Q H}{V^{3}}$ than broken down during periods of lower $\frac{g^{H}}{U^{3}}$.

\section{Acknowledaments}

The author wishes to thank Dr. Claes Rooth for his advice and many helpful discussions during the course of this work, Dr. John Whitehead for the use of his laboratory facilities, Mr. Robert Frazel for his technical assistance in assembling the apparatus, and Miss Jayne Fifield for her help with some of the experimental observations. The Geophysical Fluid Dynamics program is thanked for a very interesting summer and, together with the National Research Council of Canada, for financial support.

\section{References}

Bumpus, D.F. 1975 Review of the physical oceanography of Georges Bank. I CNAF Res. Doc. 75/107. 32 pp.

Greenberg, D.A. 1975 Mathematical studies of tidal behavior in the Bay of Fundy. Ph.D. Thesis, Univ. of Liverpool.

Hopfinger, E.J. and J.A.Toly 1976 Spatially decaying turbulence and its relation to mixing across density interfaces. J.Fluid Mech. $78(1) \div 155$.

Kantha, L.H., O.M.Phillips and R.S.Azad 1977 On turbulent entrainment at a stable density interface. J.Fluid Mech. 79(4): 753.

Kato, H. and O.M.Phillips 1969 On the penetration of a turbulent layer into a stratified fluid. J.Fluid Mech. 37: 643.

Kitaigorodskii, S.A. 1960 On the computation of the thickness of the windmixing layer in the ocean. Bull.Acad.Sci.USSR Geophys.Ser.3: 284.

Kraus, E.B. and J.S.Turner 1967 A one-dimensional model of the seasonal thermocline. I. A laboratory experiment and its interpretation. Tellus, 19. 88.

Long, R.R. 1977 A theory of turbulence in a homogeneous fluid induced by an oscillating grid. Tech.Rep.No.10 (Ser.C), Dept.Earth E Planetary Science \& Mechanics \& Materials Science, The Johns Hopkins University.

Price, J.F. 1977 Observation and simulation of storm-driven mixed-layer deepening. Tech.Rep.77-1, R.S.MAS, University of Miami.

Simpson, J.H. 1976 A boundary front in the summer regime of the Celtic Sea. Est. and Coast Mar.Sci. 4. 71.

Simpson, J.H. and J.R.Hunter 1974 Fronts in the Irish Sea. Nature 250: 404.

Thompson, S.M. and J.S.Turner 1975 Mixing across an interface due to turbulence generated by an oscillating grid. J.Fluid Mech. 67: 349. 


\title{
REFLECTION AND TRANSMISSION OF ROSSBY WAVES BY BOTTOM TOPOGRAPHY
}

\author{
Aki ra Masuda
}

\section{Introduction}

The energy transport by Rossby waves is so important that many researchers have studied its mechanism and meanings in geophysical fluid dynamics. The energy transport in the zonal direction, however, still remains as a controversial problem. Since the Coriolis parameter varies in the meridional direction the field equations have inhornogeneity in that direction. On the other hand, the vorticity equation has constant coefficients but isotropy in the zonal direction is lost (consequently Rossby waves always propagate to the west). Energy fluxes calculated by the "group-velocity concept" and the "working-pressure concept" are not the same (Longuet-Higgins (1964) and Masuda (1977)).

There is another (and more direct) motivation of this note. Recently Miura and Suginohara (1977) have carried out a numerical experiment, where they considered a two-layer ocean with a continental shelf and slope near the western boundary. As an initial condition they had a baroclinic eddy in the open ocean. The eddy propagated westward and eventually reached the slope. Then, motion in the surface layer propagated westward with almost no change, while the bottom layer motions were directed by the bottom topography to move to the south along the slope. Although this simulation was done by a nonlinear scheme, the basic mechanism may be partly explained in terms of reflection and transmission of Rossby waves.

The problem of reflection and transmission of Rossby waves in a homogèneous ocean has been discussed by Rhines (1969). The important case of non-zonal bottom topography, however, has not been investigated sufficiently. In this note we see some curious and interesting aspects of this problem. Moreover if we introduce two-layer density structure transmission and reflection coefficients are shown to become quite different from those obtained in the case of a homogeneous ocean.
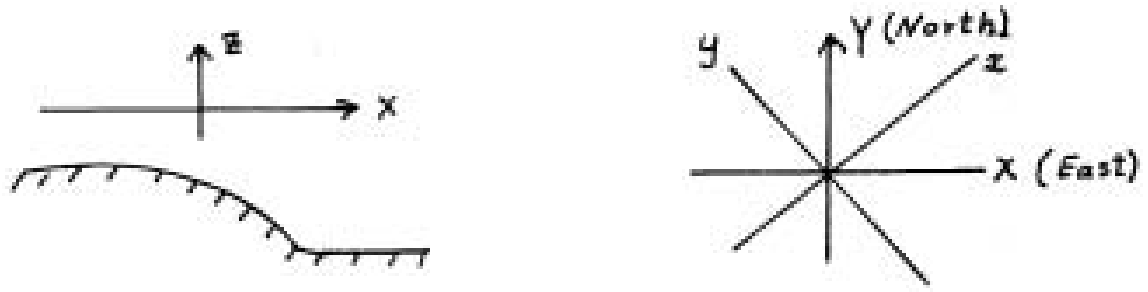

2. Formulation

Consider, first, a homogeneous ocean with one-dimensional bottom topography on a $\beta$-plane. Let us take $(x, y)$ coordinates so that the depth $H$ may be a function of $x$ only. The problem is to obtain transmission and reflection coefficients, $T / I$ and $R / I$ for the given incident wave $\sim I e^{i\left(k_{i} x+l y-\omega t\right)}$, where $I$ is the amplitude, $\left(k_{i}, l\right)$ the wavenumbers and $\omega$ is the frequency. The subscript "i" or "rru denotes "incidence" or "reflection". To the appropriate basic approximation, i.e. $\left|f_{0}\right|>>|\omega|,|\beta / \ell|,|\gamma / k|$, where $f=f_{0}+\beta y+\gamma x=f_{0}+\hat{\beta} Y$, the divergence equation for the pressure $\mathrm{p}$ is given by 


$$
\nabla^{2} p+\left(\frac{i A}{\omega}+\frac{H_{x}}{H}\right) p_{x}+\left(\frac{\partial \ell}{\omega}-\frac{f_{0} \ell H_{x}}{\omega H}\right) p=G p,
$$

where $G \quad f_{a}^{z} /(g H)$ is the inverse square of external radius of deformation. Note that $\gamma$-term appears because we use $(x, y)$ instead of $(X, Y)$. When the length scale of motion is much smaller than $G^{-1 / 2}$, the right-hand side of (2.1) can be neglected:

$$
\nabla^{2} p+\left(\frac{i \beta}{\omega}+\frac{H_{x}}{H}\right) p_{x}+\left(\frac{\gamma L}{\omega}-\frac{f_{0} H_{x}}{\omega H}\right) p=0
$$

A stream function $\psi$ is available as well in the case of length scale negligibly small compared with external radius of deformation or in the case of an artificial rigid lid on the top of the ocean. In terms of $\psi$ the velocities are expressed as $\left(H_{u}, H_{v}\right) .\left(-\psi_{y}, \psi_{x}\right)$, and the vorticity eauation becomes

$$
\nabla^{2} \psi+\left(\frac{i \beta}{\omega}-\frac{H_{x}}{H}\right) \psi_{x}+\left(\frac{\gamma l}{\omega}-\frac{f_{2} H_{x}}{\omega H}\right) \psi=0
$$

Note that (2.2) and (2.3) are the same except for the second term.

In the case of a two-layer ocean we have the equations for the surface and bottom pressure, $(p$ and $\bar{p})$ :

$$
\begin{aligned}
& \nabla^{2} p+\left(\frac{\beta}{\omega}\right) p_{x}+\left(\frac{\gamma \ell}{\omega}\right)=G p-F(\tilde{p}-p) \\
& \nabla^{2}(\tilde{p})+\left(\frac{i \beta}{\omega}+\frac{H_{x}}{H}\right) \tilde{p}_{x}+\left(\frac{\gamma \ell}{\omega}-\frac{f_{0} H_{x}}{\omega H}\right) \tilde{p}=F\left(\frac{h}{H}\right)(\tilde{p}-p) .
\end{aligned}
$$

to the same approximation as (2.1). Here $(h, H)$ are the depths of the (surface, bottom) layers,

$$
G=\frac{f_{0}{ }^{2}}{g h}, F \equiv \frac{\hat{p} f_{0}{ }^{2}}{g \Delta \rho h},
$$

$\bar{\beta}$ the density of the bottom layer and $\Delta \rho$ is the density difference between the bottom and surface layers. A tilde denotes quantities sometimes of the bottom layers and sometimes of baroclinic modes. We do not employ $\psi$-representation in the case of a two-layer ocean because it appears to become a little obscure in physical meanings.

3. Discontinuous depth profile

\subsection{Homogeneous ocean}

We try $\psi$-representation first. In both regions $I(x<0)$ and $\mathbb{L}(x>0)$, the governing equations are given by

$$
\nabla^{2} \psi+\frac{i \beta}{\omega} \psi_{x}+\frac{r L}{\omega} \psi=0 \text {. }
$$

$x=0$

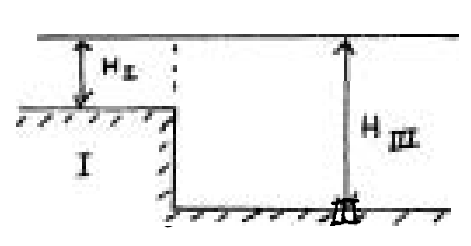

This equation yields the dispersion relation:

\footnotetext{
* Physically $G^{-1 / 2}$ and $F^{-1 / 2}$ respectively mean external and internal radia of deformation.
} 


$$
k^{2}+\ell^{2}+\frac{\beta k}{\omega}-\frac{\gamma \ell}{\omega}=0
$$

which has two roots $k_{c}$ and $k_{r}$ for given $\boldsymbol{w}$ and $\ell$. The radiation condition is used to determine which wavenumber corresponds to incident, reflected or transmitted waves. We consider that incident waves come from $x=x c_{4}$, so that they must have a group velocity to the negative $x$ direction and vice versa. Before the actual manipulation, it is necessary to check whether we can choose both incident (or transmitted) and reflected waves. Since

$$
\left\{\begin{array}{l}
\left.\frac{\partial \omega}{\partial k}\right)_{i}=-\frac{k_{i}-k_{r}}{\left(k_{i}^{2}+l^{1}\right)} \omega \\
\left.\frac{\partial \omega}{\partial k}\right)_{k_{r}}=+\frac{k_{i}-k_{r}}{\left(k_{r}^{2}+l^{2}\right)} \omega
\end{array}\right.
$$

we always have

for real $k_{i}$ and $k_{r}$.

$$
\left.\left.\frac{\partial \omega}{\partial k}\right)_{k_{i}} \cdot \frac{\partial \omega}{\partial k}\right)_{k_{r}}<0
$$

Even if $h_{i}$ and $h_{r}$ are complex, as may be possible in a two-layer ocean, they are complex conjugates of each other. So we can find such solutions that decay as $x \rightarrow \pm c$. Thus, in either case, incident, reflected and transmitted waves can be composed so as to satisfy the radiation condition.

Now, we put solutions in the form

$$
\left\{\begin{array}{l}
\psi=T e^{i\left(k_{i} x+l_{y}-\omega t\right)} \text { for } x<0 \\
\psi=I e^{i\left(n_{i} x+l_{y}-\omega t\right)}+R e^{i\left(k_{r} x+l_{y}-\omega t\right)} \text { for } x>0 .
\end{array}\right.
$$

The appropriate boundary conditions at $X=0$ are the continuity of mass flux and pressure ${ }^{*}$, i.e., the continuity of $\psi$ and $H^{-1}\left(\left(\frac{w}{2}\right) \psi_{x} t f_{0} \psi\right)$ :

$$
\left\{\begin{array}{l}
T=I+R \\
\left(\frac{H_{m}}{H_{2}}\right)\left(n_{i}+\frac{f+l}{i \omega}\right) T=\left(n_{i}+\frac{f l l}{i \omega}\right) I+\left(k_{r}+\frac{f \cdot l}{i \omega}\right) R .
\end{array}\right.
$$

From (3.4) it follows that

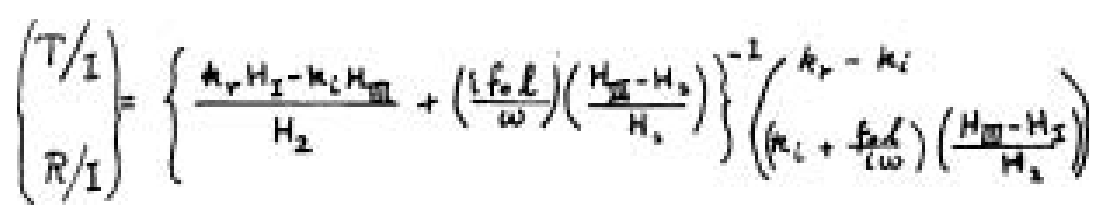

The square of the absolute transmission coefficient becomes

"In Rhines (1969) the condition for the "strength of the vortex sheets" was used, but it turns out to be the continuity of pressure combined with the mass-flux condition. 


$$
\left(\frac{T}{I}\right)^{2}=\frac{\left(k_{r}-k_{i}\right)^{2}}{\left(\frac{k_{r} H_{2}-k_{1} H_{a l}}{H_{I}}\right)^{2}+\left(\frac{f_{0} \ell}{\omega}\right)^{2}\left(\frac{H_{I I I}-H_{I}}{H_{I}}\right)^{2}}
$$

This is the same expression as was obtained by Rhines (1969) except that $k_{r}$ and $k_{i}$ are determined from the dispersion relation (3.2) and therefore $k_{r}+k_{i} \# 0$ except when topography is zonal.

Next we check $p$-representation. If we formally put $G$ as zero, we have the same equation and dispersion relation as in $\Psi$-representation. Also the boundary conditions at $x=0$ are physically the same; the continuity of $p$ and $H\left(p x-\frac{f e l}{\omega} p\right.$. Then the results are

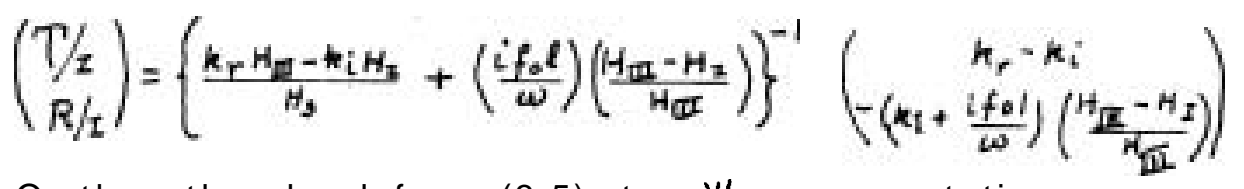

On the other hand from (3.5) to $\psi$-representation,

$$
\frac{P_{T}}{P_{I}}=\frac{k_{r}-h_{i}}{\left(\frac{h_{r} H_{I}-h_{r} H_{\text {DI }}}{H_{\text {III }}}\right)+\left(\frac{i f_{i} l}{\omega}\right)\left(\frac{H_{\text {II }}-H_{I}}{H_{\text {II }}}\right)}
$$

The ratio (3.8) cannot agree with (3.7) except when $k_{r}+k_{i}=-\beta / \omega=D$ (zonal bottom topography) or $H_{I}=H_{\text {III }}$ (trivial case). If we consider the case when $\left(f_{0} / \omega\right) \gg 1$ and $L \sim k$ both (3.7) and (3.8) give the same result:

$$
\left.\frac{P_{r}}{P_{I}} \simeq-i\left(\frac{k_{r}-k_{i}}{l}\right)\left(\frac{\omega}{f_{0}}\right)\left(\frac{H_{\text {II }}}{H_{\text {II }}-H_{I}}\right) \sim 0\left(\mid \frac{\omega}{f_{0}}\right)\right)<1 .
$$

Note, however, the difference between the two representations becomes evident for

$$
\frac{\mathrm{H}_{\mathrm{TI}}-\mathrm{H}_{\mathrm{i}}}{\mathrm{H}_{\mathrm{N}}} \sim \mathrm{O}\left(\left|\frac{\omega}{f_{0}}\right|\right) \text { and } k \sim \ell \text {. }
$$

When the horizontal divergence cannot be neglected, only $p$-representation is valid. The dispersion relation is modified into

$$
h^{2}+l^{2}+\frac{A h}{\omega}-\not L+G=0
$$

where $G=\frac{f_{0}^{2}}{g H_{I}}$ or $\frac{f_{0}^{2}}{g^{H} I}$. Now $h_{r}$ and $h_{i}$ in region I differs from those in region ㅍ. We use primes to denote the wavenumbers for region I- The transmission coefficient turns out to be

$$
\frac{T}{I_{-a}}=\frac{k_{r}-k_{i}}{\left.\frac{H_{c L} k_{i}^{\prime} H_{I}}{m}\right)+\left(\frac{i f_{0} l}{\omega}\right)\left(\frac{H_{\text {III }}-H_{I}}{H_{I I}}\right)} .
$$

immediately from (3.7), (3.8) and (3.11) it follows that the transmission coefficient is proportional to $\left(k_{r}-k_{i}\right)$, which is proportional to the group velocity (see 3.3)). Therefore we can conclude that if the group velocity of incident waves is very small, so is the transmission coefficient. In 
ordinary reflection problems, such a phenomenon cannot occur because in those cases.

3.2 Two-layer ocean

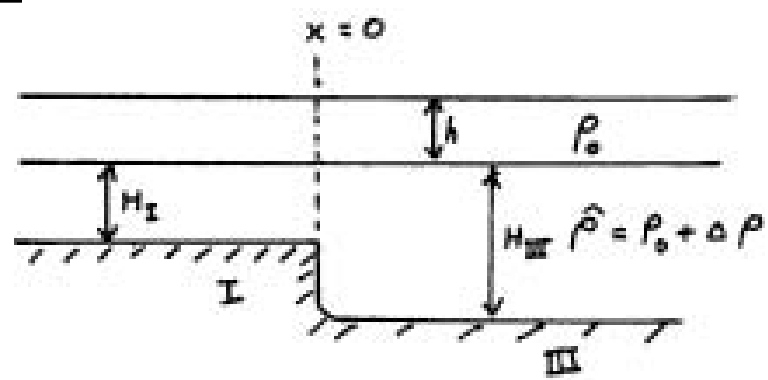

Here we treat $p$-representation only. The equations of motion in paragraph 2 yield the dispersion relation:

$$
\left\{k^{2}+l^{2}+\frac{\beta k}{\omega}-\frac{\partial l}{\omega}\right\}\left\{k^{2}+l^{2}+\frac{\beta k}{\omega}-\frac{\gamma l}{\omega}-F\left(\frac{h}{H}+1\right)-G\right\}+G F\left(\frac{h}{H}\right)=0
$$

from which derive four k's; barotropic mode $k_{\mathbf{i}}$ and $k_{r}$ and baroclinic mode $k_{i}$ and $k_{r}$. We put solutions as follows:

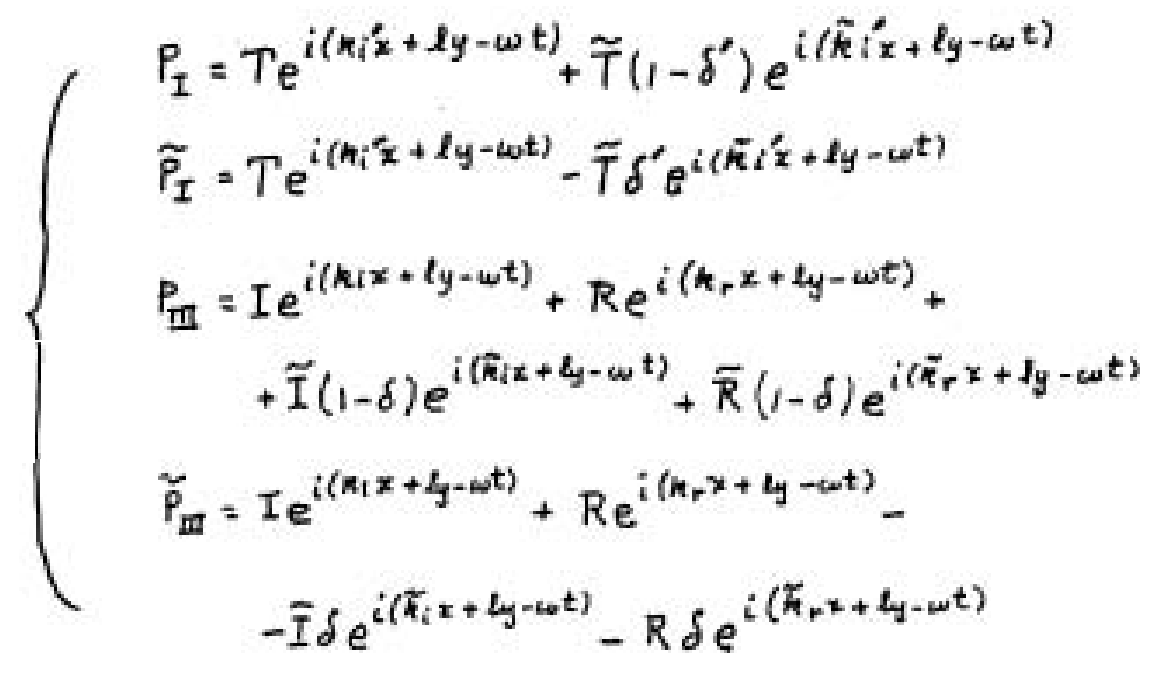

where $\delta \equiv \frac{h}{H_{\mathbb{I}^{+}}+h}$ and $\delta^{\prime}=\frac{h}{H+I}$. The continuity conditions at $x=0$ are

$$
\left\{\begin{array}{l}
P_{I}=P_{\text {III }} \\
\tilde{P}_{I}=\tilde{P}_{\text {III }} \\
P_{I_{x}}=P_{I_{x}} \\
\left(\frac{H_{I}}{H_{\text {III }}}\right) \tilde{P}_{I_{x}}+\left(\frac{f_{0} l}{\omega} \theta\right) \tilde{P}_{I}=\tilde{P}_{\text {II }},
\end{array},\right.
$$


where $\theta \equiv\left(H_{\mathbb{U}^{-}}-H_{x}\right) / H_{\text {TII }}$. From (3.13) and (3.14) we obtain for $T=I$ and $\tilde{T}=0$

$$
\left\{\begin{array}{l}
I+k=1 \\
\tilde{I}+\tilde{k}=0 \\
\left(k_{r}-n_{i}\right)_{I}=\left(k_{r}-k_{i}^{\prime}\right)+(1-\delta) \theta\left(k_{i}^{\prime}+\frac{i f_{0} l}{\omega}\right)=a_{11} \\
\left(\tilde{k}_{r}-\tilde{n}_{i}\right)_{\tilde{I}}=-\theta\left(k_{i}^{\prime}+i \frac{f}{c} l\right)=a_{21}
\end{array}\right.
$$

In the same way when $T=0$ and $\tilde{T}=1$ we have

$$
\left\{\begin{array}{l}
I+R=\delta-\delta^{\prime} \\
\tilde{I}+\widetilde{R}=0 \\
\left(k_{r}-k_{i}\right) I=\left(k_{r}-k_{i}^{\prime}\right)\left(\delta-\delta^{\prime}\right)-(1-\delta) \delta^{\prime} \theta\left(\tilde{k}_{i}^{\prime}+\frac{i f_{t} l}{\omega}\right)=a_{h} \\
\left(\tilde{k}_{r}-\tilde{k_{i}}\right) \tilde{I}=\left(\tilde{k}_{r}-\tilde{k}_{i}^{\prime}\right)+\delta^{\prime} \theta\left(\tilde{k}_{i}^{\prime}+\frac{i f_{l} l}{\omega}\right)=a_{22}
\end{array}\right.
$$

Therefore, for the incidence of barotropic modes, the following equations must hold:

$$
\left\{\begin{array}{l}
a_{11} T+a_{12} \tilde{T}=\left(k_{r}-k_{i}\right) I \\
a_{21} T+a_{32} \tilde{T}=0
\end{array}\right.
$$

Finally we obtain transmission coefficients for the incidence of barotropic modes:

$$
\left\{\left(\begin{array}{c}
T / I \\
\tilde{T} / I
\end{array}\right)=\frac{\left(k_{r}-k_{i}\right)}{\operatorname{det}}\left(\begin{array}{c}
a_{2=} \\
-a_{21}
\end{array}\right)\right.
$$

where

$$
\begin{aligned}
& \text { Let } \geq a_{11} o_{23}-a_{12} o_{21} \\
&=\left(k_{r}-k_{i}^{\prime}\right)\left(\tilde{k}_{r}-\tilde{k}_{i}^{\prime}\right)+ \\
&+\theta\left[\delta^{\prime}\left(k_{r}-k_{i}^{\prime}\right)\left(k_{i}^{\prime}+\frac{i f_{i} \ell}{\omega}\right)+\left\{(1-\delta)\left(\tilde{k}_{r}-\tilde{k}_{i}^{\prime}\right)+\left(\delta-\delta^{\prime}\right)\left(k_{r}-\tilde{k}_{i}^{\prime}\right)\right\}\left(k_{i}+\frac{i f_{i} l}{\omega}\right)\right]
\end{aligned}
$$

For the incidence of baroclinic modes, the results are

$$
\left\{\left(\begin{array}{c}
T / \tilde{I} \\
\tilde{T} / \tilde{I}
\end{array}\right)=\frac{\left(\tilde{k}_{r}-\tilde{k}_{i}\right)}{d_{e} t}\left(\begin{array}{c}
-a_{/ 2} \\
a_{u}
\end{array}\right)\right.
$$

These are complicated, so let us calculate a simple and important case when $\left|f_{0}\right| \omega|\gg\rangle \mid, h-\ell$ and $\theta \sim 1$. Then we find 


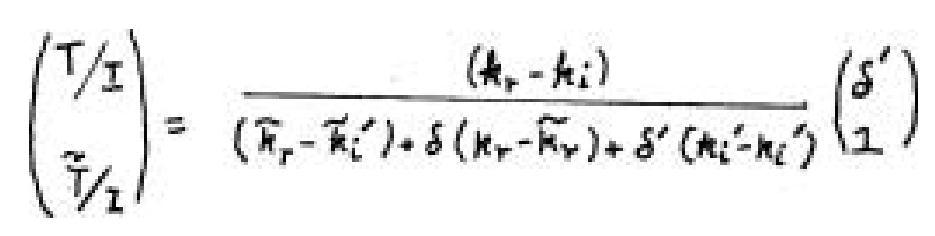

and

$$
\left(\begin{array}{c}
T / \tilde{I} \\
\tilde{T} / \tilde{I}
\end{array}\right)=\frac{\left(\tilde{k}_{r}-\tilde{k}_{i}\right)(1-\delta)}{\left(\tilde{k}_{r}-\tilde{k}_{i}^{\prime}\right)+\delta\left(k_{r}-\tilde{k}_{r}\right)+\delta^{\prime}\left(k_{i}^{\prime}-k_{i}^{\prime}\right)}\left(\begin{array}{l}
\delta^{\prime} \\
1
\end{array}\right)
$$

for the incidence of barotropic and baroclinic modes respectively. It is to be noted that in either kind of incidence $T \simeq \delta^{\prime} \bar{T}$, consequently we have

$$
\left\{\begin{array}{l}
P_{I} \simeq \tilde{T} e^{i\left(\tilde{k}_{i}^{\prime} x+l_{y}-\omega t\right)}+\delta^{-} \tilde{T}\left\{e^{i k_{i}^{\prime} x}-e^{i k^{\prime} x}\right\} e^{i\left(\ell_{y}-\omega t\right)} \\
P_{I} \simeq \delta^{\prime} \tilde{T}\left\{e^{i k^{\prime} x}-e^{i \tilde{k}^{\prime} x}\right\} e^{i\left(l_{y}-\omega^{\prime}\right)}
\end{array}\right.
$$

That is, the pressure (and $u$ ) of the surface layer behaves like a baroclinic mode while that of the bottom layer is very small within, say $|x|\left\langle\left|\widetilde{k}_{i}^{\prime}-\boldsymbol{k}_{i}^{\prime}\right|^{-1}\right.$, where $\left|\tilde{k}_{i}^{\prime}-k_{i}^{\prime}\right|$ (roughly order of $\tilde{k}_{i}^{\prime}$ ) is approximately related to internal radius deformation $\bar{F}-1 / 2$ by

$$
\left|\tilde{k}_{i}^{\prime}-\hat{k}_{i}^{\prime}\right| \simeq F\left(\frac{h}{H}\right)\left|\hat{k}_{i}^{\prime}+k_{i}^{\prime}+\frac{R}{\omega}\right|
$$

These show that the bottom topography effectively blocks the bottom layer but permits the penetration of the motion of the surface layer. Another inportant result is that transmission coefficients are order unity, which is quite different from the homogeneous case. Due to the presence of stratification Rossby waves can transport a fairly large portion of energy over the bottom topography.

\section{Continuous depth profile. \\ 4.1 Homogeneous ocean.}

Now in this section we consider such a depth profile as is shown in the figure. Regions I $(x<0)$ and III $(x>L)$ have constant depths $H_{1}$ and $H_{\text {III, }}$, respectively, while the connecting region $I(O<x \leqslant L)$ has a continuous depth profile $H(x)$. The governing equation is given by

$$
\mathcal{L}_{p}=0 \text { or } M \psi=0 \text {. }
$$

according as $p$ or $\psi$-representation, where

$$
\alpha \equiv \frac{\partial^{2}}{\partial x^{2}}+\left(\frac{i \beta}{\omega}+\frac{H_{x}}{H}\right) \frac{\partial}{\partial x}+\left(\frac{\partial \ell}{\omega}-\ell^{2}-\frac{f_{0} l H_{x}}{\omega H}\right)-G
$$

and

$$
M=\frac{\partial^{2}}{\partial \gamma^{2}}+\left(\frac{i \beta}{\omega}-\frac{H_{x}}{H}\right) \frac{\partial}{\partial x}+\left(\frac{\partial l}{\omega}-\ell^{2}-\frac{f \ell H_{z}}{\omega r}\right) \text {. }
$$

Using $\mathrm{p}$-representation we describe the general procedure of calcu- 
lating transmission coefficients. As in paragraph 3 we must put solutions as follows:

$$
\begin{aligned}
& p=T e^{i\left(k_{i}^{\prime} x+l_{\operatorname{ly}-\omega t}\right)} \quad(x<0) \\
& p=T_{q}(x) e^{i(l y-w t)} \quad(0<x<L) \\
& p=I e^{i\left(\operatorname{tei}(\mathbf{x}-L)+h_{y}-\omega t\right)}+R e^{i\left(h_{r}(x-t)+k y-\omega t\right)}(L<x),
\end{aligned}
$$

where $q(x)$ satisfies $(4.1)$ and

$$
g(0)=1, \frac{d q^{(0)}}{d x}=i k_{i} \text {. }
$$

The boundary condition at $x=L$ is that the pressure and its $x$-derivative be continuous:

$$
\begin{aligned}
& T q(L)=I+R \\
& T \frac{d q L}{d x}=i h_{i} I+i k_{i} R .
\end{aligned}
$$

Then we have

$$
\left(\begin{array}{l}
T / I \\
R / I
\end{array}\right)=\frac{1}{\operatorname{lr} q(L)+i \frac{d g(L)}{d x}}\left(\frac{k_{r}-k_{i}}{-k_{i} q_{r}(L)-i \frac{d g(L)}{d z}}\right) .
$$

Note that transmission coefficients $T / \mathcal{I}$ are always proportional to $\left(k_{\boldsymbol{r}}-k_{\boldsymbol{i}}\right)$, which causes a peculiar result as was explained in parabraph 3.

For some simple depth profiles solutions of (4.2) are expressed in terms of confluent $P$ functions. For example when $H(x)=H_{1}+\Delta x$, we easily find

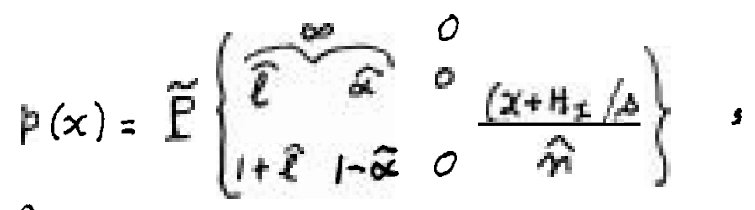

where $\hat{\alpha}, \hat{l}$ and $\hat{n}$ are determined frorn

$$
\begin{aligned}
\hat{l}(\hat{l}+1) & =(2 \hat{l}+1)\left(\frac{\omega}{i \beta}\right)\left(l^{2}-\frac{\gamma l}{\omega}\right) \\
\hat{n} & =-\left(\frac{\omega}{i \beta}\right)(2 \hat{l}+1) \\
\hat{x} & =-\left(\frac{f_{\theta}^{2}}{g^{\Delta}}+\frac{f_{0}}{i \omega}\right) \frac{(2 \hat{l}+1)}{c \beta} .
\end{aligned}
$$

When $H(x)=H_{I} \exp (\alpha x), \quad$ we again obtain

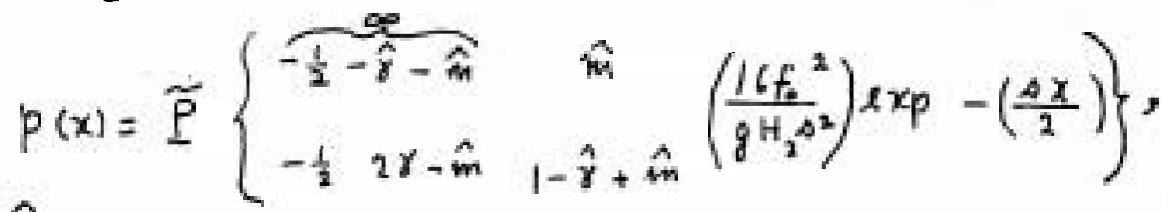

where $\hat{m}$ and $\hat{\gamma}$ are given by 


$$
\begin{aligned}
\hat{m}((\rho \omega) \hat{m}+2(\Delta \omega+i \beta)) & =\left(\frac{4 i}{s}\right)\left(t_{\partial} \Delta+\left(\ell^{2}-\frac{\gamma \ell}{\omega}\right) i \omega\right) \\
\hat{\gamma} & =-\frac{1}{2}-\frac{i \beta}{\Delta \omega}+\hat{m} .
\end{aligned}
$$

These exact solutions may be useful for investigation not only of the reflection problem but also of trapped modes. An example of the latter has been given by Longuet-Higgins (1968).

However, for the purpose of studying the effects of the smooth topography on reflection of Rossby waves, it seems the most convenient to use $\psi$-representation and exponential depth profile $H O()=H_{2} e^{\alpha x}$. In this case solutions in region 11 are expressed by exponential functions:

$$
T q(x)=A_{1} e^{i d_{1} x}+A_{2} e^{i d_{2} x},
$$

where $d_{1}$ and $d_{x}$ satisfy

$$
d^{2}+\left(i \alpha+\frac{\rho}{\omega}\right) d+\left(l^{2}-\frac{\partial \ell}{\omega}+\frac{f \alpha \ell}{H}\right)=0 .
$$

Then, along the general procedure we obtain

$$
\begin{gathered}
\left(\begin{array}{l}
T / I \\
A_{1} / I \\
A_{2} / I
\end{array}\right)=\frac{\left(k_{r}-k_{i}\right)}{\operatorname{det}}\left(\begin{array}{l}
d_{2}-d_{1} \\
d_{2}-k_{i} \\
k_{i}-d_{1}
\end{array}\right) \\
R / I=-1+e^{i d_{1} L} A_{1}+e^{i d_{2} L} A_{2} \\
\operatorname{det} \equiv-\left(k_{2}-d_{1}\right)\left(k_{1}-d_{2}\right) e^{i d_{1} L}+\left(k_{2}-d_{2}\right)\left(k_{1}-d_{1}\right) e^{i d, L} .
\end{gathered}
$$

Let us investigate transmission coefficients in detail for three special cases.

(1) $\alpha L=$ const. $\alpha \rightarrow 0, L \rightarrow \infty$.

This is a case of very gradual variation of depth. The results are

$$
\left\{\begin{array}{l}
T / I=\exp \left(\frac{k_{i}}{k_{r}-k_{i}}(\alpha L)\right) \exp \left[-i p_{1} L-\frac{i\left(f_{0} L-\gamma\right)}{k_{r}-k_{i}}\left(\frac{\alpha L}{\omega}\right)\right\} \\
A_{1} / I \simeq T / I \\
A_{1} I_{I} \simeq 0 \\
R / I \simeq 0 .
\end{array}\right.
$$

This indicates almost no reflection as is expedted. If, however, we consider the energy transport, ridiculous results are observed. That is

$$
\left(\frac{H_{I I}}{H_{I}}\right)\left|\frac{T}{I}\right|^{2} \cong \exp \left\{\left(\frac{k_{r}+h_{i}}{k_{r}-k_{i}}\right) \propto L\right\}=\left(\frac{H_{I I}}{H_{z}}\right)^{\left(\frac{k_{r}+n_{i}}{k_{r}-k_{i}}\right)}
$$


which cannot be unity except when $k_{r}+k_{i}=-\beta / w=0$ (again the zonal bottom topography!) or $H_{\underline{z}}=\mathrm{H}_{\mathrm{g}}$. If we use $p$-representation with $\mathrm{G}=0$, then the same ratio turns out to be the inverse of (4.9). This absurd result may be considered due to the inadequacy of the equation of motion in region 11 in either representation, because when $L \rightarrow \infty$ wave packets propagate so long a distance in $x$ and $y$ that the basic assumptions are violated.

(2) $\alpha L=$ const. $\propto \rightarrow \infty, L \rightarrow 0$.

In this limit opposite to (1), the bottom topography appears like a discontinuous one. Simple calculation gives

$$
T / I=\frac{k_{r}-k_{i}}{\left(\frac{\hbar_{r} H_{\Psi}-k_{i} H_{I}}{H_{I}}\right)+\left(i \frac{f_{l} l}{\omega}\right)\left(\frac{H_{T}-H_{I}}{H_{I}}\right)}
$$

which agrees with the result in paragraph 3 .

(3) $\left|f_{0} \alpha \ell / \omega\right| \gg \alpha^{2}, \ell^{2},|\gamma \ell / \omega|,|\beta \ell / \omega|, k^{2}$.

After the same procedure we obtain

$$
\left\{\begin{array}{l}
T / I \simeq-\frac{\left(k_{r}-k_{i}\right) \exp \left(-\frac{\alpha L}{2}+\frac{i \beta L}{\omega}\right)}{\sqrt{K^{2}} \sin \left(L \sqrt{k^{2}}\right)} \\
A_{1} \simeq \frac{1}{2} T \\
A_{2}=\frac{1}{3} T
\end{array}\right.
$$

where $k^{2}=-\frac{f_{0} \alpha \ell}{\omega}-\ell^{2}+\frac{\gamma \ell}{\omega}$. This is an interesting result if $\alpha \ell / \omega<0$, which conditions implies that the phase velocity in y of incident waves has the same direction as that of the phase velocity of topographic Rossby waves in region 11. As is easily seen, when $L \sqrt{k^{2}} \simeq n \pi$ ( $M$; integer), (4.11) is not valid and it must be replaced by

$$
T / I \cong \exp \left(-\frac{\alpha L}{z}+\frac{i \beta L}{\omega}\right)
$$
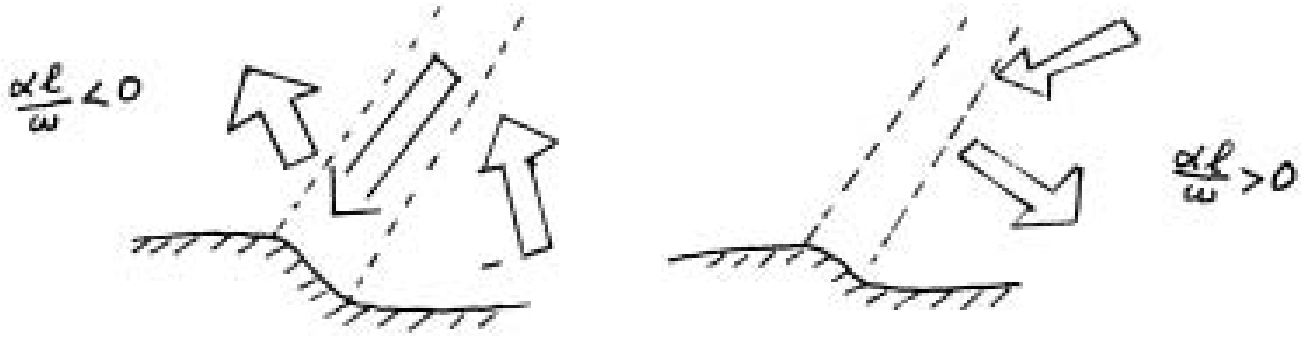

Note that (4.12) means all energy is transmitted to region 1

$\left(\because\left(\frac{H_{m}}{H_{7}}\right) \mid \frac{T}{2}\right)^{2} \quad$ Anyhow $A_{1} / I$ and $A_{x} / I$ are order unity. On the slope the motion takes a form of topographic Rossby waves. Since the wave number perpendicular to the flopw is much larger than that parallel to the slope, the "group-velocity concept" tells us that energy is transported along the slope as is schematically shown in the figure. All these phenomena occur due to the presence of wave mechanism on the slope (topographic waves). On the other hand if $\alpha \ell / \omega>0$ no wave mechanism works; the slope rather plays a role like a potential barrier. Therefore energy cannot 
penetrate into region 1. Mathematically this character is reflected on the denominator $\sinh \left(L \sqrt{-k^{2}}\right.$ of $(4.11)$. Consequently for either case $\left(\frac{f_{0} \alpha \ell}{\omega} \gtrless 0\right)$, energy seems to be transported to the negative y direction.

\subsection{Two-layer ocean}

The governing equations are expressed as

$$
\mathcal{L}(\hat{P})=0
$$

where

$$
\mathscr{L} \equiv\left(\begin{array}{ll}
\frac{\partial^{2}}{\partial x^{2}}+\frac{i \rho}{\omega} \frac{\partial}{\partial x}+\left(\frac{\partial \ell}{\omega}-\ell^{2}\right)-F-G & \frac{\partial^{2}}{\partial x^{2}}+\left(\frac{i \rho}{\omega}+\frac{H_{h}}{H}\right) \frac{\partial}{\partial x}-\left(\ell^{2}-\frac{\gamma \ell}{\omega}+\frac{f \cdot H_{x}}{\omega H}\right)-\frac{F h}{H}
\end{array}\right)
$$

This equation is too complicated to solve directly without numerical procedure. So we had better satisfy ourselves with investigation of a few special cases. First, when the width $L$ of the slope region is much smaller than any other horizontal length scales, we may expect transmission coefficients tend to those given in paragraph 3 . Next, if we take a formal limit of $G \rightarrow 0$ and $F \rightarrow 0$, the surface and bottom layers completely decouple from each other. The results of paragraph 4 apply without modification. When $G \rightarrow 0, F \sim l^{2}$ and $h / H \rightarrow 0$, the bottom layer can move independent of the surface layer. Also in this case the qualitative results pf paragraph 4.1 will be applicable to the bottom layer. The motion of the surface layer becomes a superposition of its proper motion and that excited by the bottom layer. When $E \equiv \bar{h} / \mathrm{H}$ is small enough, a formal expansion with respect to may be helpful:

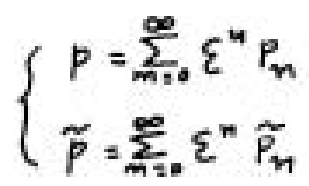

Anyway, weak coupling (strong stratification) of the surface and bottom layers is not so interesting theoretically, though it is important in the actual ocean. The opposite case, however, will require numerical computation. In carrying it out we had better determine, first, those quantities corresponding to $a_{11}, a_{2}$ and a22 in paragraph 3 . Then it is easy to obtain transmission and reflection coefficients.

\section{Summary and discussion.}

In the present note are clarified a few curious features of Rossby waves which originate in their peculiar ways of propagation in the zonal direction. First, some differences lie between $\psi$ and $p$-representation. Second, transmission coefficients are proportional to the group velocity of incident waves and therefore are usually small for waves of small group velocity.

When stratification is introduced, the aspects of energy transport change drastically. Transmission coefficients become order unity for a twolayer ocean. Consequently even barotropic Rossby waves can propagate over regions of varying depth, though, of course, some portion of energy is reflected. Propagation across the region of abrupt change of depth occurs as penetration of the motion of the surface layer and blocking of the bottom layer. 
In this note only special cases are treated for simplicity, though a lot of qualitative results are obtained. In a later work numerical calculation will be done for some typical bottom topography. Figures will be presented to illustrate transmission and reflection coefficients as functions of bottom slope, depth ratio of the surface and bottom layers, and so on. The results of Miura and Suginohara (1977), seem to demand calculation of reflection coefficients when there exists a boundary at $x=-M$. The propagation of the motion of the bottom layer observed by Miura and Suginohara has a possibility of nonlinear effects. Therefore nonlinear excitation of shelf waves by incident waves is worthy of thorough investigation from every point of view.

\section{Acknowledaments}

I wish to express my thanks to all staffs and friends for their friendship and valuable discussions, especially to Prof. G. Veronis who guided and encouraged me through this summer, Dr. J.A. Whitehead who suggested me an interesting experiment (not reported here) and Mr. R. Frazel who helped me to do experiments. I also thank Hidaka Foundation, whose financial support enabled me to attend this Geophysical Fluid Dynamics summer program.

\section{References}

Longuet-Higgins, M.A. 1964 On group velocity and energy flux in planetary wave motions. Deep Sea Res., 11: 35.

Longuet-Higgins, M.A. 1968b Double Kelvin waves with continuous depth profiles. J.Fluid Mech. 34: 49.

Masuda, A. 1977 On group velocity and energy transport by Rossby waves (preparing).

Rhines, P.B. 1969 Slow oscillations in an ocean of varying depth. Part 1. Abrupt topography. J.Fluid Mech. 37: 161. 


\title{
DEVELOPMENT OF DISTURBANCES TO AN INVISCID SHEAR LAYER
}

\author{
Martin R. Maxey
}

\section{Introduction}

The inviscid stability of a uniform shear layer was first treated by Rayleigh (1894). He analyzed the stability, to wave-like disturbances, of a plane parallel mean flow of uniform whear, bounded on either side by regions of uniform flow. The mean velocity profile is illustrated in Fig. I.

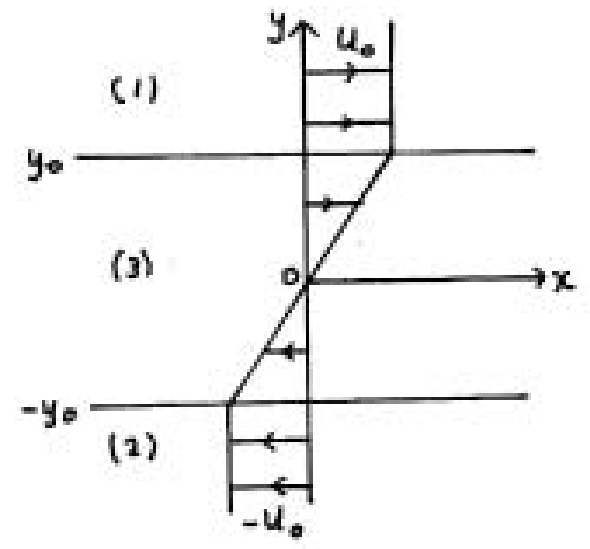

Fig. 1. Basic flow, velocity profile. $u=u_{0}, y \geq y_{0} ; u_{0}=y_{1},|y| \leqslant y_{0} ; u_{2}-u_{0}, y<-y_{0}$ Regions (1), (2) and (3) are marked.

This wss one of a series of piecewise linear velocity profiles that have since been studied for their stability characteristics. The advantage of such model profiles is that they give tractable problems for which the physical properties can be understood, as well as representing in an approximate way the behavior of continuous shear layers to longer wavelength disturbances. A comprehensive summary of these stability problems is given by Drazin and Howard (1966), where a detailed inviscid analysis is given.

The usual approach to such problems is to apply Squire's theorem, and to consider two-dimensional disturbances to the basic flow, and then to represent the disturbance by a stream function $\psi(x, y, t)$. The solution for $\psi$ is obtained in the case of infinitesimal disturbances, by taking separate Fourier modes.

$$
\psi=\operatorname{Re}\{\phi(y) \exp (i k x+\lambda t)\}
$$

where $k$ is the wave number in the direction of the mean flow, and $\lambda$ is the corresponding time constant of the disturbance. Re \{\} denotes the real part. The system of equations and boundary conditions is then solved as an eigenvalue problem for $\lambda$. Generally there are two values of $\lambda$ for every wave number $k$, these being either real or complex conjugates. So a system will have either exponentially growing and decaying solutions (unstable) or neutrally stable oscillations. In the context of the problem considered here it is found that,

$$
\lambda^{2}=-k^{2} u_{0}^{2} / 4 t^{2} y_{0}^{2}\left\{\left(2 r y_{0}^{2}-1\right)^{2}-e^{-4 r y \cdot 0}\right\}
$$

where $u_{0}$ is the free stream velocity and $2 y_{0}$ the thickness of the shear layer: 
$k=+$ in the two-dimensional case. This gives neutrally stable oscillations for large enough wave numbers, approximately $k_{y_{0}} \geq 0.64$, and instability at lower wave numbers; $\lambda$ being real or purely imaginary.

In deriving solutions to this problem, the Rayleigh equation ( $\sim r a-$ zin and Howard, 1966) is solved,

$$
(u(y)-c)\left(d^{2} / d y^{2}-k^{2}\right) \phi-d^{2} u / d y^{3} \phi=0,
$$

together with conditions of continuous normal velocity and pressure at the interfaces, and decay of the disturbance flow as $|y|$ becomes very large.

The phase speed is defined by, $c=\omega / k$ where $\lambda=i \omega$, and the mean profile is $u(y)$. For this eigenvalue problem for $c$ there are two types of solution, namely the continuous spectrum with $c=U(y)$ for some $y$ independent of wave number, or else the discrete spectrum with c taking two values for each wave number and $d \phi / d y$ continuous across any possible critical layer, i.e. $c=u(y)$. The continuous spectrum gives rise to the convective solutions, where disturbances are carried downstream by the mean flow; and the discrete spectrum corresponds to the usual 'wave propagation' of disturbances. In the problem of the shear layer all neutrally stable solutions have a corresponding critical layer, as is illustrated in Fig.2, since for this to occur

$$
\begin{aligned}
1 / 4 y_{0}^{2} k^{2}\left\{\left(2 k y_{0}-1\right)^{2}-e^{-4 y_{0} k}\right\} & \leq 1 \\
\text { i.e. } \quad 1-43 \leq c^{-43} \quad & 3=k y_{0}
\end{aligned}
$$

which is always the case here.

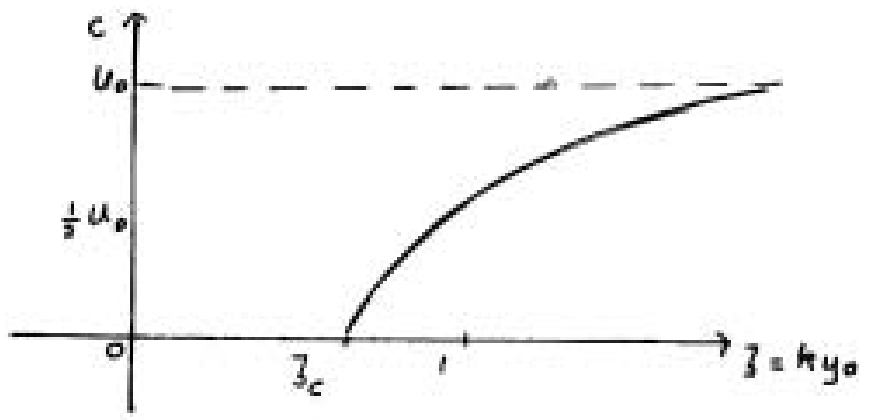

Fig.2. Sketch illustrates the variation of c with 3 . As $3 \rightarrow \infty$,

$$
\begin{aligned}
& c \rightarrow u_{0} \text {. At } 3_{c} \doteqdot 0.64, c=0 \text { and for } 3=1.0, c \div \frac{1}{2} u_{0} \text {. } \\
& c=U_{0} / 2 z\left\{(2 z-1)^{2}-e^{-43}\right\}^{1 / 2} \text {. }
\end{aligned}
$$

The purpose of the present investigation is to consider the interaction of the continuous and discrete spectra, and how the critical layers affect the development of a general disturbance. This is done in a consistent way by treating the initial value problem, with a prescribed initial disturbance and ignoring the effects of molecular viscosity. As the flow develops viscosity will naturally become important, but in the initial stages it will have small effect. The condition that the disturbance is in two dimensions only, is relaxed; and this allows the very important feature of the stretching of mean vorticity by the fluctuating velocities. It will be shown that this completely alters the dynamical response of the system. In later 
sections the nonlinear effects of such a disturbance are considered, in the context of a simple amplitude expansion. This shows up the scalar forcing and the reaction on the mean flow due to Reynolds stresses. No attempt has been made at deriving a suitable "two-timing" perturbation scheme.

Investigations of initial value problems have been made in the past by Case (1960) and Dikii (1961). Case looked at the stability of plane Couette flow, while Dikii considered in general terms the Rayleigh stability equation. Both showed the influence of the continuous spectrum, but restricted their study to two dimensions. They found that the convective motions decayed $\alpha 1 / t$. A general study of the initial value problem in two dimensions is also given by Drazin and Howard (1966). Reynolds and Poltes (1967) have examined the problem of finite amplitude stability, taking into account viscosity and the three-dimensional structure, extending the methods of Watson and Stuart (viz. Stuart 1960). However their study was restricted to considering higher order effects of a neutrally stable linear solution, and did not include any effects of convective motions.

In relation to the problem of boundary layer turbulence, Landahl (1975, 1977) used a series expansion in terms of eigenfunctions of the Orr-Sommerfeld equation to investigate the larger scale turbulent motions induced following a burst. Because of the effects of viscosity, the dominant features at large times were lightly damped waves of low streamwise wave number, for velocities normal to the boundary. For streamwise velocities the dominant behavior was convection by the mean flow, and it was suggested that this permanent 'scar' could lead to a new turbulent burst. More recently Gustavsson (1977) has examined the linear initial value problem for a simplified boundary layer profile, and considered the development of wave disturbances in relation to the growth of turbulent spots. Durbin (1977) considered a semiinfinite shear layer in the context of the rapid distortion theory and turbulence, to see how turbulence in a free stream, impinging on a region of shear could induce turbulence there.

It is in these contexts that interest in the development of flow disturbances on piecewise linear velocity profiles has recently been shown, and the coupling of wave and convection motions.

\section{Statement of the Problem}

The problem is given in terms of a formal small amplitude expansion for the disturbance flow field, up to second-order terms. Initial conditions and spatial boundary conditions are given, so that the amplitude expansion is valid at least for short time scales. Initially the flow is irrotational either side of the shear layer, and the flow is given by a velocity potential, $\phi$. Within the shear layer there is an initial mean uniform vorticity, which is convected and distorted by the disturbance, but there will be sharp interfaces between the flow regions making the edges of the shear layer, see Fig. 3 . Within the shear layer a Rayleigh problem is solved. The amplitude expansion is ordered by the parameter $\epsilon$, taken to be $u^{\prime} / U_{0}=\varepsilon$, where $u^{\prime}$ is a velocity scale of the initial disturbance.

The basic flow field is, $\nu=v_{0}$

$$
\begin{aligned}
& U=\alpha y \\
& U=-V_{0}
\end{aligned}
$$

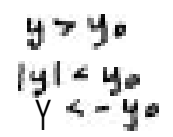

region (1)

region (2)

region (3)

where $\propto y_{0}=u_{e}$, and $\alpha$ is the basic uniform shear. 


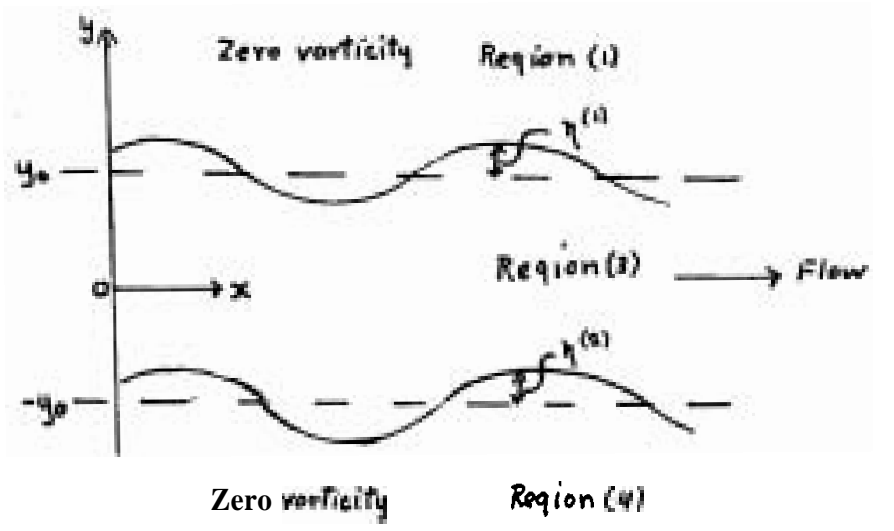

Fig.3. The interfaces $y=y_{0}+\eta^{(1)}$ and $y=-y_{0}+\eta^{(2)}$, with $\eta^{(1)} \varepsilon \eta^{(2)}$ initially zero, mark the boundaries between the flow regions. Vorticity is confined between the interfaces.

The basic flow field is,

Region (1).

$$
\begin{aligned}
& \nabla^{2} \phi=0 \\
& \phi^{(1)} \rightarrow 0 \text { as } y \rightarrow \infty \\
& \phi^{(1)}(\underline{x}, t)=\epsilon \phi_{1}^{(1)}+\sigma^{2} \phi_{2}^{(1)}+o\left(\epsilon^{3}\right)
\end{aligned}
$$

Region (2).

$$
\begin{aligned}
& \nabla^{2} \phi=0 \\
& \phi^{(2)} \rightarrow 0 \text { as } y \rightarrow \infty \\
& \phi^{(2)}(x, t)=\epsilon \phi_{1}^{(2)}+\epsilon^{2} \phi_{1}^{(2)}+o\left(6^{2}\right)
\end{aligned}
$$

Region (3).

$$
\begin{aligned}
& (\partial / \partial t+\alpha y \partial / \partial x) \nabla^{2}\left(u_{j} \frac{\partial v}{\partial x_{j}}\right)+\partial / \partial y\left(\partial u_{i} / \partial x_{j} \partial u_{j} / \partial x_{l}\right) \\
& \partial u / \partial t+\alpha y \partial u / \partial x+\alpha v+\partial p / \partial x=-u j \partial u / \partial x \\
& \partial u x / \partial t+\alpha y \partial u / \partial x+\partial p / \partial z=-u_{j} \partial \omega / \partial x \\
& \partial u / \partial x+\partial v / \partial y+\partial u / \partial z=0
\end{aligned}
$$

where the velocity field is $u^{(3)}=E y_{i}^{(3)}+\varepsilon^{2} u_{3}^{(3)}+o\left(\epsilon^{2}\right)$. unless otherwise stated the term $\&$ will refer to region (3) and the superscript will be omitted. Equation (2.2a) is essentially the homogeneous Rayleigh equation. The flow has uniform density, and kinematic pressure, $(p / \rho)$, is used.

There are also matching conditions for the various regions, and these are that pressure and the normal component of velocity at an interface should be continuous across the interface. The interfaces here are material surfaces and move with the fluid. Hence, for the surfaces $y=y_{0}+\eta^{20}$ and $y=-y_{0}+\eta^{(2)}$ 


$$
\begin{aligned}
& \partial \eta^{(1)} / \partial t+\alpha\left(y_{0}+\eta^{(i)}\right) \partial \eta^{(l)} / \partial x+u_{j} \partial \eta^{(1)} / \partial x_{j}=v\left(y_{0}+\eta^{(1)}\right) \\
& \partial \eta^{(1)} / \partial t+U_{0} \partial \eta^{(1)} / \partial x+\partial \phi^{(0)} / \partial x ; \partial \eta^{(1)} / \partial x_{j}=\partial \phi^{(1)} /\left.\partial y\right|_{y=y_{0}+\eta^{(1)}} \\
& \partial \eta^{(2)} / \partial t+\alpha\left(-y_{0}+\eta^{(2)}\right) \partial \eta(2) / \partial x+u_{j} \partial \eta^{(2)} / \partial x_{i}=v\left(-y_{0}+\eta^{(\nu)}\right) \\
& \partial \eta^{(x)} / \partial t+\left(-U_{0}\right) \partial \eta^{(2)} / \partial x+\partial \phi^{(2)} / \partial x_{j} \partial \eta^{(2)} / \partial x_{j}=\partial \phi^{(2)} /\left.\partial x_{y}\right|_{y=-y_{0}+\eta^{(x)}}
\end{aligned}
$$

The surface displacements and are zero initially, and for later times are given as an amplitude expansion,

$$
\begin{aligned}
& \eta^{(1)}=\epsilon \eta_{1}^{(1)}+\epsilon^{2} \eta_{1}^{(1)}+O\left(\epsilon^{3}\right) \\
& \eta^{(2)}=\epsilon \eta_{1}^{(2)}+\epsilon^{2} \eta_{2}^{(2)}+O\left(\epsilon^{3}\right)
\end{aligned}
$$

The pressure is matched across $y=y_{0}+\eta^{(1)}$ and $y=-y_{0}+\eta^{(\nu)}$, with the condition that pressure is zero as $|y| \rightarrow \infty$, and the uniform pressure from the basic flow is neglected.

The initial conditions on the system are that $\underline{u}_{1}^{(3)}(\underline{x}, 0), \phi_{1}^{(3)}(\underline{x}, 0)$, $\phi^{(2)}(x, 0)$ are all given, but that high order terms have zero initial values.

The equations for the first and second order components are given in Appendix A The solution for the linear first order problem is dealt with in section 3, while the second order problem is dealt with in section 4. In tackling these problems the boundary conditions are evaluated at and a Taylor expansion used to give the corrections.

3. Solution of First Order Problem

Solution and the $\hat{v}_{i}$ component

By adopting the amplitude expansion scheme, we now have a series of inhomogeneous linear equations to solve, in an unbounded $x, z$ domain. These equations can be reduced to ordinary linear differential equations, by decomposing any disturbance into Fourier modes and solving for each Fourier mode separately. The Fourier components are written as follows:

$$
\underline{u}_{1}(x, t)=\iint \hat{\underline{u}}_{1}(y, t ; k, m) \exp [i(k x+m z)] d k d m
$$

with similar expressions giving $\hat{\beta}_{1}, \hat{\phi}_{1}^{(1)}, \hat{\phi}_{1}^{(2)}$ and so on. It is also convenient to define the wavenumber + , as $\Phi^{\prime}=k^{t} \mathrm{~m}^{2}$. Referring to the equations $(A-1)-(A-8)$, a formal solution can be given in terms of a Green's function for the $\hat{v}_{1}$ component and the velocity potentials,

$$
\begin{gathered}
\hat{\phi}_{1}^{(1)}=P(t) e^{-t y} \\
\hat{\phi}_{1}^{(2)}=Q(t) e^{+y} \\
\hat{v}_{i}(\eta, t)=\int_{-y_{0}}^{y_{0}} G(\eta, y) e^{-i \hbar \alpha y t} F_{1}(y, k, m) d y-+P_{l}(t) e^{-t y_{0} \cdot G_{y}\left(\eta, y_{0}\right)+Q_{1}(t) e^{-t_{y}} G_{y}\left(\eta_{0}-y_{0}\right),}
\end{gathered}
$$


where $F_{1}(y, k, m)=\left(\partial \hat{\hat{v}}_{1} / \partial y^{2}-t^{2} \hat{v}_{a}\right)$ at $I=0$, and the Green's function $G(\eta, y)$ is given by

$$
\begin{aligned}
& y<\eta \quad G(\eta, y)=-1 / 2 r \operatorname{sh} 2 r y \cdot\left\{\cosh \left(2 r y_{0}-m+r y\right)-\cosh +(y+\eta)\right\} \\
& y>\eta \quad G(\eta, y)=-1 / 2 r \operatorname{sh} 2 r y_{0}\left\{\cosh \left(2 r y_{0}-+y+m\right)-\cosh +(y+\eta)\right\}
\end{aligned}
$$

The term $F_{1}$ is one component of the curl of the initial vorticity in the disturbance, which is convected by the flow, and would vanish if the disturbance were initially irrotational. The pressure in the shear layer is found by combining $(A-3)$ and $(A-4)$ with the continuity condition, to give,

$$
t^{2} \hat{p}_{i}^{(t)}=-(\partial / \partial t+i \propto y k) \partial \hat{v}_{i} / \partial_{y}+i h \propto \hat{v}_{i} \text {. }
$$

The details of the solution are given in Appendix $B$, the terms and $Q_{1}$ are linear combinations of the terms $x_{1}(t)$ and $Y_{1}(t)$ which are characteristic coordinates for the system.

$$
\begin{aligned}
& x_{1}(t)=x_{1}(0) e^{\lambda t}+\lambda e^{\lambda t} \int_{-y_{0}}^{y_{0}} i \hbar \alpha\left[e^{-i k \alpha y t-\lambda t}-1\right] n_{1}(y) /(\lambda+i k \alpha y) d y \\
& y_{1}(t)=y_{1}(0) e^{-\lambda t}-\lambda e^{-\lambda t-y_{0} y_{0}}\left(h \propto\left[e^{-i k \alpha y t+\lambda t}-1\right] n_{2}(y) /(-\lambda+i k \alpha y) d y\right.
\end{aligned}
$$

where as noted in the appendix, $\mathbf{h}$, and $h_{2}$ are proportional to $F$, and vanish at the endpoints. Solutions for the other velocity components are found from evaluating,

$$
\begin{aligned}
& \hat{u}_{1}(\eta, t)=\Omega_{1}(\eta) e^{-i \hbar \alpha \eta t}-m z / r=\alpha \int_{0}^{i} \hat{v}_{1}\left(\eta, t^{\prime}\right) e^{i k \alpha \eta\left(t^{\prime}-t\right)} d t^{\prime}+i k / t_{2} \partial \hat{v}_{i} / \partial \eta
\end{aligned}
$$

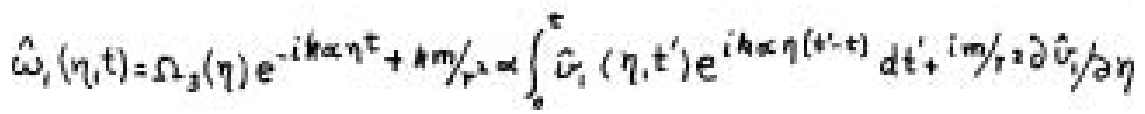

where $\Omega_{1}=\hat{u}_{i}(\eta, 0)-i k / t^{2} \partial \hat{v}_{i} /\left.\partial \eta\right|_{t=0}$ and $\Omega_{2}=\hat{\omega}_{1}(\eta, 0)-i m / r^{2} \partial \hat{G}_{i} /\left.\partial \eta\right|_{1=0}$, ane they represent the departure of the initial disturbance from a potential flow.

From the expressions for $\hat{v}_{1}$, we can see that it contains waves and convected structures. The initial vorticity distribution is both convected and interacts with the wave components to give a modified wave structure. The form of the convected terms though is not simple, but at large times does break down into simpler components. For large times, the asymptotic form of the first term of (3.4) is given by

$$
\left.\sim(1 / i k \alpha t)^{2}\left[-F_{1}\left(y_{0}\right) G_{y}\left(\eta \cdot y_{0}\right) e^{-i k U_{0} t}+F_{1}\left(-y_{0}\right) G_{y}\left(\eta_{1}-y_{0}\right) e^{i k U_{0} t}+p_{1}^{(\eta}\right) e^{-i k \alpha \eta^{t}}\right]+O\left(1 / t^{3}\right)
$$

This contains three convected components, corresponding to a velocity of the form $v(x-u t, y, z, t)$, all of which decay with time. This part of the $v$-velocity is due to the initial distribution of spanwise fluctuating vorticity, which is advected by the mean flow. Suppose this was initially periodic in $x$ and $y$, for a given position of $z$, and the vorticity distribution was locally $\tilde{\omega} \sin \left(k x_{0}+\mid y_{0}\right)$. Then with convection, $x=x_{0}+\alpha y_{0} t, y=y_{0}$ so that the vorticity distribution is now $\tilde{\omega} \sin (k x+y(l-k \alpha t))$. For large 
times the periodicity in the y-direction increases and the vortex contributions tend to cancel, increasingly with time. The only contributions that are effective are from $y=0$ locally, and from the outer edges of the shear layer since there is no further vorticity outside the layer to give cancellation. Because of the decay with time, this part of the convection solution is not significant compared to wave oscillations or to exponential growing terms. The decay rate being inversely proportional to $t^{2}$ is in contrast to both Case's (1960) and Dikii's (1961) results, but is in agreement with the results of Gustavsson (1977).

The other terms contributing to $\hat{v}_{1}$ may also be expanded for large times, and in terms of $x_{1}, Y_{1}$,

$$
\begin{aligned}
& x_{1}(t) \sim x_{1}(0) e^{\lambda t}+\lambda e^{\lambda t}\left\{-i \pi n_{1}\left(-y_{c}\right)-p \int_{-y_{0}}^{y_{0}} n_{1}(y) / i m \alpha\left(y+y_{c}\right) \cdot i k \alpha d y\right\}+o\left(\frac{1}{t^{2}} e^{\lambda t}\right) \\
& y_{1}(t) \sim y_{1}(0) e^{\lambda t}-\lambda e^{-\lambda t}\left\{-i \pi n_{2}\left(y_{e}\right)-p \int_{-y_{0}}^{y_{0}} n_{2}(y) / i k \alpha\left(y-y_{c}\right) \cdot i k \alpha d y\right)+O\left(\frac{1}{t^{2}} e^{-\lambda t}\right) \\
& \text { for the case of } \lambda \text { imaginary. } P \int_{-y_{0}}^{y_{0}} \text { denotes the Cauchy Principal Part of }
\end{aligned}
$$
the integral, and $\lambda=i k \alpha y_{c}$ so that $y=-y_{c}$ gives the position of the critical layer. A deviation of (3.12) is given in Appendix C. These results show that at large times the motion is characteristically a wave, and that the amplitude is determined in part by the initial vorticity distribution. If $\lambda$ is real, there is no problem with the critical layer,

$$
x_{1}(t) \sim x_{0}(0) e^{\lambda t}+\lambda e^{\lambda t}\left\{-\int_{-y_{0}}^{y_{0}} i k \alpha n_{2}(y) /(i k \alpha y+\lambda) d y\right\}+o\left(1 / t^{2} e^{\lambda t}\right)
$$

with a similar result for $Y_{1}(E)$. For large times then the form of $\hat{v}_{i}(\eta, t)$ may be expressed as

$$
\hat{v}_{1}(\eta, t) \sim V_{1}(\eta) e^{\lambda t}+V_{2}(\eta) e^{-\lambda t}
$$

where $V$, and $V_{2}$ both satisfy $\left(\partial^{2} / \partial m^{2}-r^{2}\right) V=0$; and in this case the convective solution only has a secondary role.

For purely wave solutions of the form (3.15), the terms $x,(t)$ and $Y_{1}(t)$ represent the normal coordinates of the oscillation. The relation of the motion at the two interfaces to the normal modes of oscillation is not simple, however at large wavenumbers, $y_{0}+\gg 1$, the mode $Y_{1}=0$ corresponds to oscillation near $y=t y_{a}$ and hardly any displacement of the other interface. The motions then tend to be in phase.

\subsection{Special Cases}

In the preceeding calculations it was assumed that both $k$ and $t$ are not-zero. If $k$ is zero, then $\lambda$ is zero as well and the disturbance is stationary in its $\hat{v}_{i}$ and $\hat{w}_{i}$ components, retaining the initial form. The $U_{1}$ component of velocity though grows linearly with time. This different

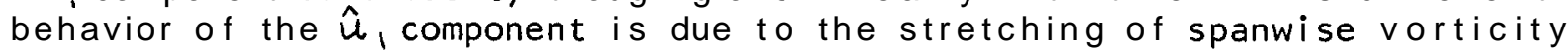
of the mean flow by the fluctuating velocity field, and would be absent in a two-dimensional flow. Eventually the stretching produces a strong component of $y$-vorticity. So although the case of $\lambda$ zero would generally be considered as neutrally stable, it in fact generates a velocity field growing linearly with time. If both $k$ and $m$ are zero, then no motion is generated. 


\section{$3.3 \hat{u}_{1}$ and $\hat{w}_{1}$ components}

The solutions for $\hat{u}_{1}$ and $\hat{\omega}_{1}$ are given by Eqs. (3.9) and (3.10). The first term of each represents the advection of an initial disturbance and the third term the simple motion forced by the $\hat{v}_{1}$ component and continuity. The second term is the more interesting and represents the motions induced by the spanwise stretching of the mean vorticity, in the disturbance flow field. This is a purely three-dimensional effect, and would be absent in two dimensions, since then $m=0$. The vortex stretching provides a mechanism of transferring energy from the mean flow to the disturbance. The integral $\int_{0}^{E} \hat{v}_{i}\left(\eta^{2}, t^{\prime}\right) e^{\left[k \times \eta\left[t^{\prime}-t\right]\right.} d t^{\prime}$ may be evaluated from $(3.4),(3.7)$ and (3.8), to give the following terms:

$$
\begin{aligned}
& \int_{-y_{0}}^{y_{0}} d y G(\eta, y) F_{1}(y, k, m) e^{-i \hbar \alpha \eta t}\left[e^{-i k \alpha \eta t(y-\eta)}-1\right](-i k \alpha[y-\eta])^{-1} \sim \\
& \sim e^{-i \hbar \alpha \eta^{t} i / / \alpha \alpha}\left\{-i \eta G(\eta, \eta) F_{1}(\eta, n, m)-P \int_{-y_{0}}^{y_{0}} G(\eta, y) F_{1}(y, k, m) /(y-\eta) d y\right\}+O\left(1 / t^{3} e^{-i h \alpha \eta^{t}}\right) \\
& \text { as } t \rightarrow \infty \text {, Similarly, }
\end{aligned}
$$

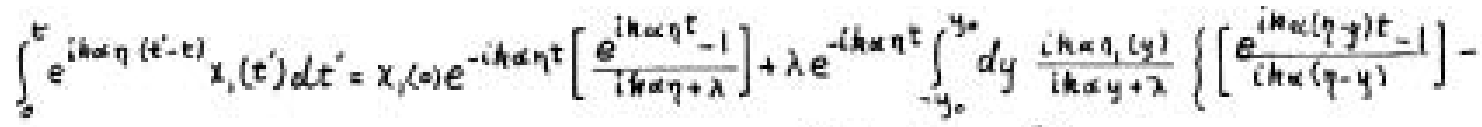

$$
\begin{aligned}
& \left.\left[\frac{e^{i h \omega \eta t+x t}-1}{i n \alpha p+\lambda}\right]\right\}
\end{aligned}
$$

This may be expanded for large times, for $\lambda$ real,

$$
\begin{aligned}
& \sim e^{-i h \alpha \eta t}\left[e^{i k \alpha \eta t+\lambda t}-1\right]\left(x_{1}(0)-\lambda \int_{-y_{0}}^{y_{0}} d y+\frac{i k \alpha \eta_{1}(y)}{i k \alpha y+\lambda}\right)+\lambda e^{i i k \alpha \eta t}\left\{i \pi \frac{n_{1}(\eta)}{i k \alpha \eta+\lambda}+\right. \\
& \left.+P \int_{-y_{0}}^{y_{0} *} \frac{n_{1}(y)}{(i k \alpha y+x)(y-\eta)} d y\right\}
\end{aligned}
$$

for $\lambda$ imaginary and $A=$ ik $\propto y_{c}$, but $\eta+y_{c} \neq 0$,

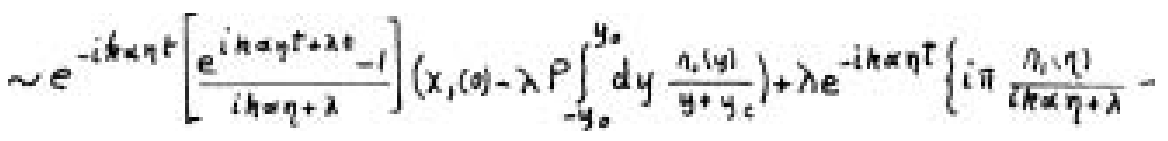

$$
\begin{aligned}
& \left.-i v \frac{n_{1}\left(-y_{c}\right)}{i h \alpha \eta+\lambda} e^{+i h \alpha \eta t+\lambda t}+\rho \int_{-y_{0}}^{y_{*}} d y \frac{n_{i}(y)}{i \hbar \eta_{\eta}+\lambda}\left[\frac{1}{y-\eta}-\frac{1}{y+y_{c}}\right]\right\}
\end{aligned}
$$

whilst for $\lambda$ imaginary and $\eta+y_{c}=0$

$$
\sim t e^{\lambda t}\left\{x_{1}(0)-\lambda P \int_{-y_{0}}^{y \cdot} \frac{n_{1}(y)}{y+y_{e}}-i \pi \lambda n_{1}(y)\right\}
$$


Similar results for the term in $Y_{1}(t)$ can be derived.

The result (3.16) shows that at large times there is a finite convective contribution due to the initial vorticity distribution, which unlike the case for $\hat{v}_{1}$, does not decay with time. Further this convective term will tend to be stronger at low wavenumbers, through the dependence on $V_{R} F_{l}$. The other contributions to the integral from $P_{1}$ and $\theta_{1}$ are found from $\psi_{1}$ and $Y_{1}$ and the equation (3.17). We can see immediately that for $\lambda$ imaginary, corresponding to a wave oscillation, the expressions in (3.17) have a positive "singular" behavior at critical layers, and that in fact the leading term will have secular growth as $x_{1}(0) t e^{\lambda t}$. This is confirmed in the result (3.20), which is the expansion for large times at the critical layer. Referring back to the forms of $\hat{u}_{1}$ and $\hat{\omega}_{1}$ in (3.9), (3.10), we see that the three-dimensional structure of the motion produces the secular growth at the critical layer, of an otherwise neutral oscillation. The

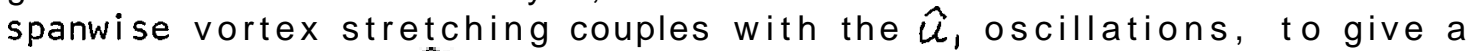

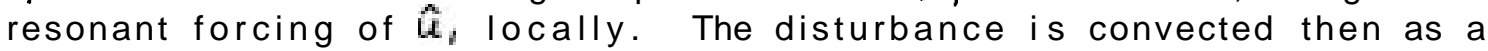
growing standing wave pattern, at the central layers. By studying only two dimensional disturbances this behavior would have been missed, since then $m=0$ and necessarily by continuity,

$$
\hat{u}_{1}=i k / t^{2} \partial \hat{v}_{1} / \partial \eta
$$

and $k=r, \hat{\omega}_{1}=0, \Omega_{1}=\Omega_{3}=0$. So both the non-zero convective motion (3.16) and the secular growth at the critical would be omitted, and the behavior of $\hat{u}_{1}$, for large times, would have been purely wave-like as in the expression (3.15).

At large times, away from critical layer, the expansions of (3.18) and (3.19) for $\int_{0}^{t} x_{1}(t) e^{i \hbar \alpha \eta\left(t^{\prime}-t\right)} d t^{\prime}$ contain both convective and exponential time dependencies, corresponding to convective motions and growing or oscillatory motions. The amplitudes of these terms are again determined by the initial vorticity distribution. The result for

$$
\begin{aligned}
& \int_{0}^{t} \hat{v}_{1}\left(\eta, t^{\prime}\right) e^{i k \alpha \eta\left(t^{\prime}-t\right)} d \quad \text { may be summarized as, }
\end{aligned}
$$

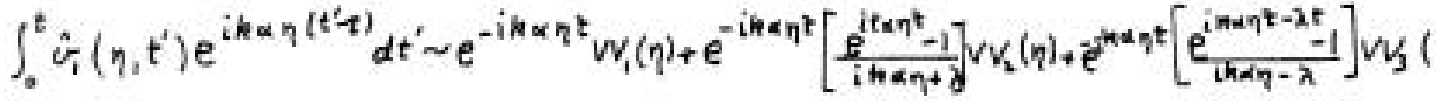

where $v v_{1}(\eta)$ is a regular function and $w_{2}(\eta), v v_{3}(\eta)$ both satisfy

$$
\left(\partial^{2} / \partial \eta^{2}-r^{2}\right) v v=0
$$

The dependence on the initial vorticity of these functions is fairly involved though, especially as $\eta$ approaches+- $y_{c}$. Combining the results* (3.22), (3.15) it is possible to determine the large time behavior of $u$, and $\hat{w}_{1}$, and

$$
\begin{aligned}
& \hat{u}_{i} \sim\left[\Omega_{1}(\eta)-v v_{1}(\eta)\left(-m^{2} / r^{2} \alpha\right)\right] e^{-i h_{\alpha} \eta^{2}}+i k / r \cdot\left[v_{1 \eta} e^{\lambda t}+v_{2 \eta} e^{-\lambda t}\right\}-
\end{aligned}
$$

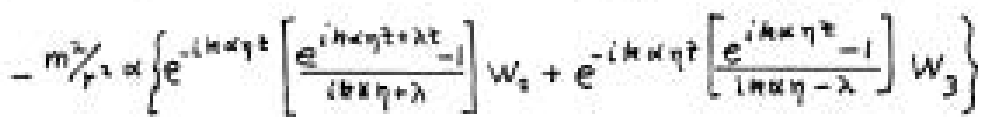

as $t \rightarrow \infty$, with a similar form for $\hat{\omega}_{1}$. 
The result (3.24) for $\hat{u}$, is valid for all $\lambda$, at large times. Even if $F_{1}=0$, provided there is some initial vorticity through the term $\Omega$, the form of $\boldsymbol{u}$, at large times is basically the same, containing convected and oscillatory motions. The main effect of $F_{1}$, in the initial data, is to alter the magnitudes of the various time dependencies.

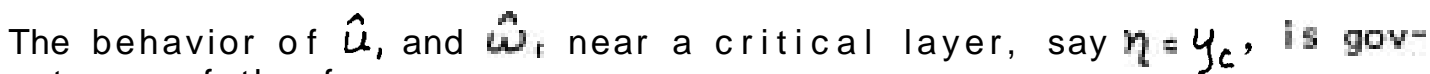
erned by terms of the form,

$$
\begin{aligned}
& e^{-i \hbar \alpha \eta \eta^{2}}\left[\frac{e^{i \alpha^{\alpha} \eta t-\lambda t}-1}{i \hbar \alpha\left(\eta-y_{c}\right)}\right] \\
& \left.=2 e^{\lambda t} \exp (-i k \alpha z t / 2)(\sin k \alpha z t) / k \alpha\right\}
\end{aligned}
$$

where $z=\eta-y_{c}$. As $t \rightarrow \infty$, this profile becomes sharper, tending eventually to a delta function, as illustrated in Fig.4. The maximum value grows linearly with time, whilst the width of the central layer region decreases with time

$$
\Delta z \sim 1 / k \alpha t \text {. }
$$

Eventually viscosity will act to limit the development of this region of increasing shear. After time from the initial disturbance, viscosity

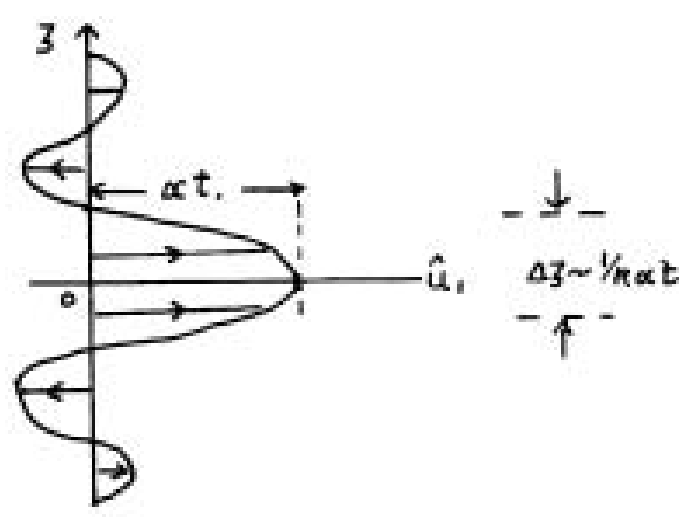

Fig.4. Sketch showing profile of $\hat{u}_{1}$ near a critical layer.

will be significant over distances of the order $(v t)^{1 / 2}, v$ is the kinematic viscosity. A balance is reached when,

$$
\begin{aligned}
& (r t)^{1 / 2} \sim 1 / k \alpha t \\
& (\alpha t) \sim\left(k y_{0}\right)^{-3 / 3} R e^{-1 / 3}
\end{aligned}
$$

where $R e=u_{0} y_{0} / v$. This $R e^{-1 / 3}$ scaling is the same as for the classical approach to critical layers, see for example Lin (1955). A similar result has been found by Landahl (1977) in relation to the time before which viscosity limits the development of shear in a convected eddy of a turbulent flow. For a Reynolds number of $10^{3}$, (3.26) implies that non-dimensional strains of $\alpha t$ equal to 10 or so are possible before account need to be taken of viscosity, in the initial value problem.

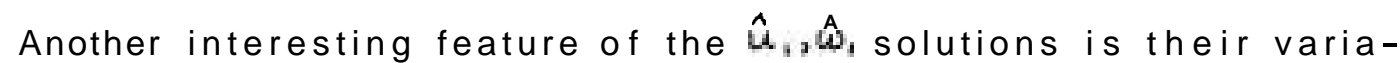
tion across the interfaces between regions (1) and (111) and (11). The 
conditions of continuity of pressure of mormal velocity have been used but these do not ensure continuity of $\hat{u}_{1}$ or $\hat{w}_{1}$. For example at $y=y_{0}$,

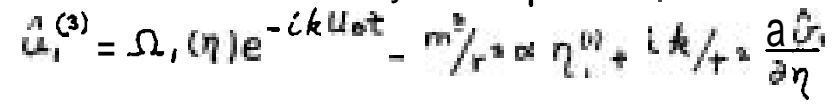

and

$$
\vec{u}_{1}^{(0)}=i k P_{1}(t) e^{-\operatorname{tg} \theta}=-i k / r v_{i}^{(n)}\left(y_{0}\right) \text {. }
$$

referring to the equation (A-9) for $\eta_{1}^{(1)}$. Using the given matching conditions and (3.6), it can be shown that

$$
\begin{aligned}
& \Delta \hat{u}_{i}\left(y_{0}, t\right)=\hat{u}_{1}^{(1)}\left(y_{0}, t\right)-\hat{u}_{1}^{(n)}\left(y_{0}, t\right)=\left[\hat{u}_{i}^{(1)}\left(y_{0}, 0\right)-u_{1}^{(s)}\left(y_{0}, 0\right)\right] e^{-i h U_{0} t}+\alpha \hat{\eta}_{1}^{(u)} \\
& \Delta \hat{\omega}_{1}\left(y_{0}, t\right)=\hat{\omega}_{1}^{(1)}\left(y_{0}, t\right)-\hat{\omega}_{1}^{(D)}\left(y_{0}, t\right)=\hat{\omega}_{1}^{(0)}\left(y_{0}, 0\right)-\omega_{1}^{(0)}\left(y_{0}, 0\right) e^{-i k u_{0} t}
\end{aligned}
$$

with similar conditions at $y=-y_{0}$. Equation (3.27) shows that in general there is a discontinuity of $\hat{u}$, at the interface due to the displacement of the interface in the mean shear flow. Such a discontinuity is to be expected in any flow with a discontinuous mean shear. The explanation is that to match normal velocity for first order it is only necessary to match at $y= \pm y_{0}$. However for $\hat{u}_{1}$, both the mean flow and $\hat{u}_{1}$ should be considered and although $u(y)$ matches at $y= \pm y_{0}$, it will not match at $y=y_{0}+\eta^{(1)}$, as illustrated in Fig.5. To the second order problem, this discontinuity will force a second harmonic motion.

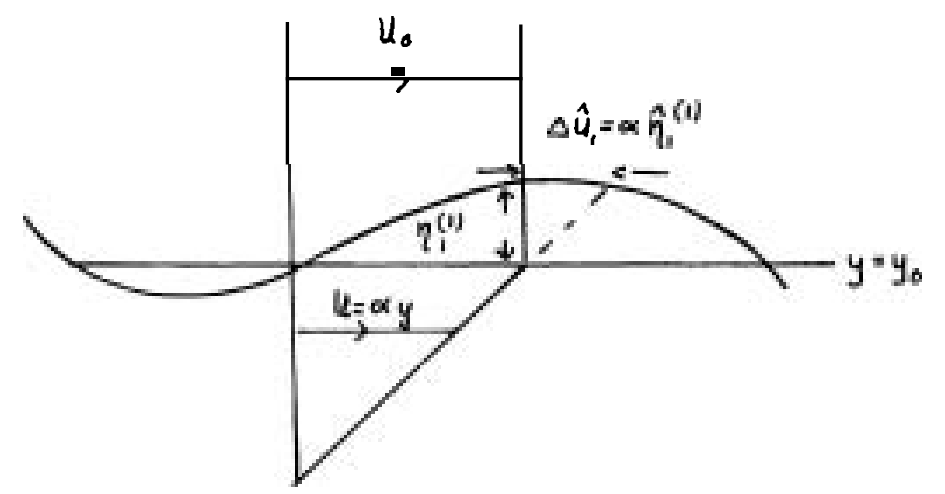

Fig.5. Sketch showing $\Delta \hat{u}_{1}\left(y_{0}, t\right)$ due to mismatch in mean flow.

\section{Second Order Problem}

The solution of the first order problem can now be used to find the second order effects of the disturbance. Equations (A-18) - (A-26) of Appendix A describe the second order motion, following the simple amplitude expansion. Formerly the solution for first order can be transformed back to real space from its Fourier modes, the source terms evaluated for the second order and then the second order problem is solved using another Fourier decomposition. If $(\theta, \psi)$ are the wave number components, then

$$
\hat{\sigma}_{1}(\eta, t)=\int_{-y_{0}}^{y_{0}} G(\eta, y) e^{-i \alpha \theta_{y} e^{2}} F_{2}(y, t, \theta, \psi) d y+G_{y}\left(\eta_{0}, y_{0}\right)\left\{\hat{h}_{1}-\sigma P_{2} e^{-\sigma_{y}}\right\}-G_{y}\left(\eta_{1}-y_{0}\right)\left\{\hat{h}_{3}+\sigma \theta_{1} e^{-\sigma y_{0}}\right\}
$$

where $\sigma^{2}=\theta^{2}+\psi^{2}$, and $F_{2}, h, h_{1}$ are found from the source terms, $q_{1}, q_{2}, q_{3}, h_{1}, h_{2}, g_{1}$ and $g_{2}$ " 


$$
F_{2}\left(y, t_{;}, \theta, \Psi\right)=\int_{0}^{t} d t^{\prime}\left\{e^{i \alpha \theta_{y} t^{\prime}} \hat{q}_{2}\left(y, t^{\prime}\right)\right\}
$$

The functions $P_{2}(t)$ and $\theta_{2}(t)$ are evaluated as before (see Appendix $B$ ), although the inhomogeneous terms $\Phi_{1}(t)$ and $\Phi_{2}(t)$ are more complicated, involving all the source terms. The solution (4.1) is very similar to (3.4) in form, however now for instance $F_{2}$ is a function of time and it is no longer clear what the long term behavior of the convective motion will be.

Rather than pursuing a general approach as above, a particular example will be considered here, to examine the character of the second order motion and to see how the first order motions will be modified. We will consider the first order motion to be that of a single Fourier of wave number $(k, m)$, so that

$$
\underline{u}_{1}(\underline{x}, t)=\operatorname{Re}\left\{\underline{\underline{u}}_{1}(y, t) \exp [i k x+i m x]\right\} \equiv \operatorname{Re}\left\{\underline{\underline{u}}_{1}\right\}
$$

This Fourier mode will then force a second order motion, which is initially zero. In this case the source terms simplify,

$$
\begin{aligned}
& q_{1}=-\frac{1}{2} \operatorname{Re}\left\{\tilde{v} \tilde{u}_{y}\right\}-\frac{1}{2} \operatorname{Re} \partial / \partial y\left\{\tilde{u}^{*} \tilde{v}\right\} \\
& q_{2}=-\frac{i}{2} \operatorname{Re}\left\{\partial_{y}\left[\tilde{v}_{y}^{2}-\tilde{v} \tilde{v}_{y y}\right]\right\} \\
& q_{3}=-\frac{1}{2} \operatorname{Re}\left\{\tilde{\omega} \tilde{\omega}_{y}-\tilde{\omega} \tilde{v}_{y}\right\}-\frac{1}{2} \operatorname{Re} \% \partial y\left\{\tilde{\omega}^{*} \tilde{v}\right\} \\
& h_{0}=\frac{1}{2} \operatorname{Re}\left\{\tilde{\eta}^{(1)}\left[2 \widetilde{\Delta}_{1} e^{-i h u_{0} t}-i k \alpha \tilde{\eta}^{(0)}\right]\right\} \\
& h_{2}=\frac{1}{2} \operatorname{Re}\left\{\tilde{\eta}^{(v)}\left[-2 \tilde{\Delta}_{2} e^{i k u, t}-i k \alpha \tilde{\eta}^{(2)}\right]\right\} \\
& g_{1}=\frac{1}{2} \operatorname{Re}\left\{t^{2} P_{1} P_{1}^{*} e^{-2 y y_{0}}\right\} \\
& g_{2}=\frac{1}{3} \operatorname{Re}\left[t^{2} \theta_{1} a_{1}^{*} \mathrm{e}^{-2 y_{2}}\right\}
\end{aligned}
$$

where $\tilde{\Delta}_{1}=\tilde{v}_{y}^{(1)}\left(y_{0}, 0\right)-\tilde{v}_{y}^{(0)}\left(y_{0}, 0\right)$ and $\tilde{\Delta}_{2}=\tilde{v}_{y}^{(3)}\left(-y_{0}, 0\right)-\tilde{v}_{y}^{(2)}\left(-y_{0}, 0\right)$. An asterisk denotes the complex conjugate. From these equations, it can be seen that two different types of flow are forced, one with an exp [i2kx+i2mz] dependence and another with no $x, z$ dependence at all. The latter motion is driven by the Reynolds stresses $\bar{u}^{*} \bar{v}$ and $\bar{w}^{*} \tilde{v}$, and will act to modify the basic mean flow.

To simplify the problem still further, it is assumed that $F_{1}=0$, but that $\Omega_{1}$ and $\Omega_{2} \neq 0$. This condition implies that $q_{2}=0$ and hence $F_{1}=0$, so there is no direct forcing of $\hat{G}_{2}$ in this instance, and motion is only produced by the second harmonic forcing at the interfaces. It can be seen from (4.4d) and (4.4e) that this interface driving motion is related to the discontinuity in $\hat{u}_{1}$, as noted in paragraph 3 . The assumption that $F_{i}=0$ is exact if $\nabla^{2} V_{1}$ is zero initially; or alternatively one can say that this is a reasonable approximation for large time behaviors, referring to (3.15) and (3.24), since the basic time dependencies of the flow are 
still retained. However care is needed, since convective motions may be important when considered in relation to terms of the form (4.2) for $F_{2}$.

Even with these assumptions, the solution for $\hat{v}_{2}(y, t ; 2 k, 2 \mathrm{~m})$ is still a complicated expression, and only the basic time dependence will be given. These are found from evaluating $X_{2}$ and $Y_{2}$, and are:-

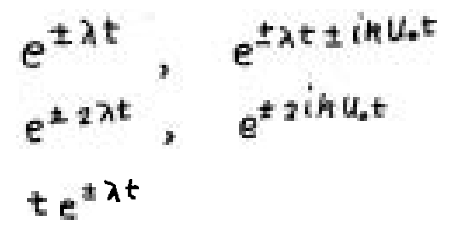

So in $\hat{v}_{2}(y, t ; 2 k, 2 m)$ there is a component representing a secularity growing oscillation, with time. If $\hat{u}_{2}$ and $\hat{\omega}_{2}$ are also evaluated, critical layer behavior will also be found with general secular motions, and dependence on $t^{2}$ at critical layers. Note that pressure forcing terms $g_{1}$ and $g_{2}$ make no contribution to $\tilde{v}_{3}(y, t ; 2 k, 2 m)$.

For the forced velocity field, with nox or $z$ dependence, and driven by the Reynolds stresses, the solution is

$$
\left.\begin{array}{ll}
\hat{v}_{2}(y, t ; 0,0)=0 \quad \phi_{2}^{(0)}=0 & \phi_{2}^{(2)}=0 \\
\hat{u}_{2}(y, t ; 0,0)=\frac{1}{2} \int_{0}^{t} d t^{\prime}\left(\hat{u}^{*} \hat{v}\right)_{y} & \dot{\omega}_{2}(y, t ; 0,0)=-\frac{1}{2} \int_{0}^{t} d t^{\prime}\left(\hat{\omega}^{*} \hat{v}\right)_{y^{*}}
\end{array}\right\}
$$

The flow field is purely in the $O x z$ planes. The possible time dependences of the $\hat{u}_{2}$ term, for $\lambda$ real or imaginary are,

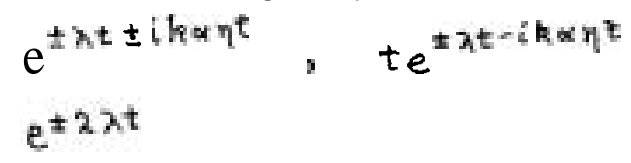

provided that $k^{2} \alpha^{2} \eta^{2}+\lambda^{2}$ is non-zero. At a critical layer, $\eta=y_{c}$ say, the form of the solution is different, and gives terms

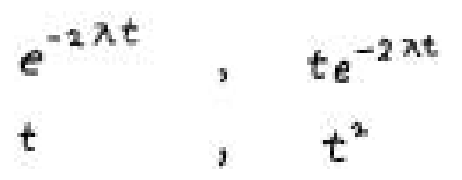

The amplitude of the term giving the $t^{2}$ behavior is found from the component of the solution,

$$
\begin{array}{r}
\frac{1}{2} m^{2} / r^{2} \alpha t \operatorname{Re}\left\{v_{1} v_{i \eta}^{*} \frac{1}{5}\left[1-\frac{e^{-3 t}-1}{-3 t}\right]-v_{1}^{*} v_{i \eta} \frac{1}{3}\left[1-\frac{e^{-3 t}-1}{-3 t}\right]-\right. \\
\left.-v_{2} v_{2 \eta}^{*} \frac{1}{3}\left[1-\frac{e^{-3 t}-1}{3 t}\right]-v_{2}^{*} v_{2 \eta} \frac{1}{3}\left[1-\frac{e^{3}-1}{3 t}\right]\right\}
\end{array}
$$

where, $\xi=\lambda+i k \alpha \eta$, and $3=\lambda-i k \alpha \eta$ and $\lambda=i k \alpha y_{e}$.

So the modification to the mean flow by the Reynolds stresses contains generally oscillatory and convective motions, as well as secularly growing oscillations. At the critical layer there are terms in $t^{2}$. 
From the form of (4.6) it would also appear that there is generally a motion growing linearly in time, but in fact the coefficient of this term is zero. It may be seen by noting that for $F_{1}=0, \hat{v}_{1}$ is given by (3. 15), and from the remarks in Appendix $B$ on the relationship of $\boldsymbol{P}_{1}, \boldsymbol{\theta}_{1}$ to $\boldsymbol{X}_{1}, Y_{1}$;

$$
V_{1}=i X_{0}\left(l_{11} e^{m}+l_{12} e^{-m}\right) \quad \frac{1}{2}=i Y_{0}\left(l_{21} e^{m}+l_{22} e^{-m}\right)
$$

where all the $\ell_{i j}$ are real. The coefficient of $(\alpha t)$ is then pure imaginary. As a critical layer is approached, $\xi \rightarrow 0$ or $\xi \rightarrow 0$, there is a strong region of shear similar to that in Fig. 4 for $\hat{u}_{1}$. The maximum value of the flow is in one direction though, and produces an indented mean profile of the type shown in Fig. 6.

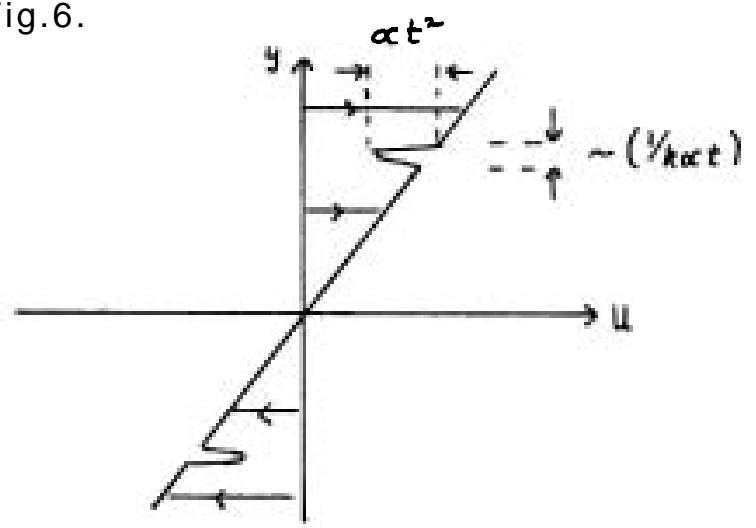

Fig.6. Modified mean velocity profile in the shear layer, showing the distortions at the critical layer.

At $\boldsymbol{\xi}=0$ the amplitude is,

$$
-\frac{1}{4} m^{2} /\left.r^{2} \alpha t^{2}\left(\left|x_{0}\right|^{3} K_{1} K_{1 \eta}+\left|Y_{0}\right|^{2} K_{2} K_{2} \eta\right)\right|_{\eta=-y_{c}}
$$

where $V_{1}=, \quad V_{2}=i Y_{0} \quad$ and $K_{1} K, \eta$ gives $\uparrow L_{11}^{2} e^{z \cdot g}-r L_{12}^{2} e^{-2 \eta}$.

The inertial stresses thus act to remove the critical layer behavior and to displace the position at which $U(y)=c(t)$, by altering $U$ and the dispersive relation. The first order disturbance flow will tend to produce a local jet in the mean flow, but eventually at times of order $1 / \epsilon$, the secondary and primary motions $w i l l$ be of the same magnitude, and the amplitude expansion scheme will cease to be valid.

An alternative view of the effect the Reynolds stress $(\tilde{u} * \tilde{v})$ would be in terms of the energy budget. The stress acts to remove energy from the mean flow and supply the disturbance. Such an interpretation has been given by Lighthill (1962), in relation to the wind generation of water waves. In that case, energy was supposed supplied to the waves from the wind at the critical wave height, where the local wind speed in the direction of wave propagation equals the phase velocity. However in the case here the extraction of energy from the mean flow will also affect the energy source.

If the problem had been treated in two dimensions alone, with $\mathrm{m}=0$ and $F,=0$, then necessarily $\Omega_{1}$ and $\Omega_{3}$ are zero and $\hat{k}_{1}$ is given by,

$$
\hat{u}_{1}(y, t ; k, m)=i k / r^{2} \frac{\partial \hat{G}_{i}}{\partial y} \text {. }
$$

Using (4.7) it can easily be shown then that all the motions with $\theta=0$, 
considered above vanish, and there is no modification to the mean flow. If $F_{1} \neq 0$, in two dimensions, then

$$
\hat{u}_{2}=-\frac{1}{2} \operatorname{Re}\left\{i k / r^{=} F_{1}(y, k, m) \int^{t} d t_{1} \hat{i}^{*}\left(y, t_{1}\right) e^{-i k \alpha y^{t} t}\right\}
$$

and this will generate a $\hat{u}_{2}$ flow similar in character to $\hat{u}_{1}(m \neq 0)$, with critical layers and growth $\alpha t$ locally. Thus again in the second order problem, the three-dimensional structure has a crucial role, and radically alters the behavior.

\section{Conclusion}

In the preceding sections it has been demonstrated that the threedimensional development of a flow disturbance is very different from that in two dimensions. In three dimensions critical layers can form by vortex stretching and give rise to local secular growth. Similar conclusions have been drawn by Wilike (1967), from his discussion of the general properties of the inviscid stability of plane parallel flows. It has also been shown that at second order inertial stresses will act to remove the first order secular growth by displacing the critical layer. The inertial stresses will become significant after times of order $\alpha t \sim u_{0} / u^{\prime}$, whilst viscous stresses will act after times of order $\alpha t=\left(R_{e}\right)^{*} / v_{3}$, so that for finite amplitude disturbances the inertial stresses may act first. At large times the simple amplitude expansion ceases to be a valid approximation. Removal of the secular growth at second order and higher can in general be achieved by a suitable "two-timing" system of fast and slow variables.

However it is not clear if it is possible to treat the first order secular growth at the critical layers, without modification to the mean flow itself.

The solutions have been given in terms of Fourier modes, for ease of calculation and simplicity; but it remains to invest these to real flow variables to examine the effect of localized disturbances both in time and space coordinates.

\section{Acknowledgments}

I am grateful to Prof. Marten Landahl for suggesting this topic for study during the summer program, and for several helpful and stimulating discussions. I am also grateful to Prof. Willem Malkus and Hakar Gustavsson for interesting discussions on this and other related topics; as well as to the staff and other participants on the program who made the summer so rewarding and enjoyable.

APPENDIX A: Equations of Motion

Equations for the linear first order problem.

$$
\begin{array}{lll}
\text { For } y \geqslant y_{0} & \nabla^{x} \phi_{1}^{(1)}=0 & \phi_{1}^{(1)} \rightarrow 0 \text { as } y \rightarrow \infty \\
\text { For } y \leqslant y_{0} & \nabla^{2} \phi^{(x)}=0 & \phi_{1}^{(x)} \rightarrow 0 \text { as } y \rightarrow-\infty \\
\text { For }|y|<y_{0} & \partial u_{1} / \partial t+\alpha y u_{1} / \partial x+\alpha v_{1}+\partial p_{1} / \partial x=0 \\
& \partial \omega_{1} / \partial t+\alpha y \partial \omega_{1} / \partial x+\partial p_{1} / \partial z=0
\end{array}
$$




$$
\begin{aligned}
& (\partial / \partial t+\alpha y \partial / \partial x) \nabla^{2} v_{t}=0 \\
& \partial u_{1} / \partial x+\partial v_{i} / \partial y+\partial w_{i} / \partial z=0 .
\end{aligned}
$$

with matching conditions, $\quad v_{1}^{(0)}\left(y_{0}\right)=\partial \phi^{(1)} /\left.\partial y\right|_{y=}$

$$
v_{1}^{(3)}\left(-y_{0}\right)=\partial \phi_{1}^{(2)} /\left.\partial y\right|_{-y_{0}}
$$

$$
\begin{aligned}
& \partial \eta_{1}^{(1)} / \partial t+u_{0} \partial \eta_{1}^{\omega} / \partial x=v_{1}^{(3)}\left(y_{0}\right) \\
& \partial \eta_{1}^{(2)} / \partial t+\left(-u_{0}\right) \partial \eta_{1}^{(2)} / \partial x=v_{1}^{(3)}\left(-y_{0}\right)
\end{aligned}
$$

and

$$
\begin{aligned}
& p_{1}^{(3)}\left(y_{0}\right)=-\partial \phi_{1}^{(1)} / \partial t-u_{0} \partial \phi_{1}^{(1)} / \partial x \\
& p_{1}^{(n)}\left(-y_{0}\right)=-\partial \phi_{1}^{\omega} / \partial t+u_{0} \partial \phi_{1}^{(\omega)} / \partial x
\end{aligned}
$$

Equations for the second order problem

$$
\begin{aligned}
& \text { For } y \geqslant y_{0}, \quad \nabla^{2} \phi_{2}^{(0)}=0 \quad \phi_{2}^{(a)} \rightarrow 0 \quad \text { as } y \rightarrow \infty \\
& \text { For } y \leqslant-y_{0} \text { : } \quad \nabla^{2} \phi_{1}^{4 \text { ul }}=0 \quad \phi_{2}^{\text {c1 }} \rightarrow 0 \text { as } y \rightarrow-\infty
\end{aligned}
$$

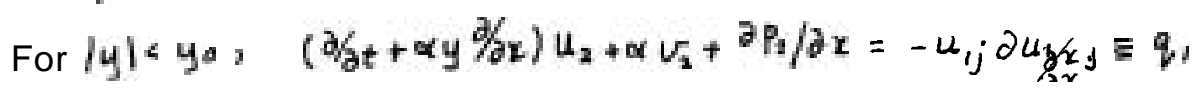

$$
\begin{aligned}
& \left(\partial / \partial t+\alpha_{y} \partial / \partial x\right) w_{2}+\partial p_{2} / \partial z=-u_{i j} \partial w_{1} / \partial x_{j}-q_{3} \\
& \left(\partial / \partial t+\alpha_{y} \partial / \partial x\right) \nabla^{2} v_{z}=-\nabla^{1}\left(u_{i j} \partial v_{i} / \partial x_{j}\right)+\partial \partial_{y}\left(\partial u_{i j} / \partial x_{j} j / \partial w_{i}\right)=q_{z} \\
& \partial w_{2} / \partial x+\partial v_{z} / \partial_{y}+\partial w_{i} / \partial z=0
\end{aligned}
$$

Matching conditions are, for normal velocity,

$$
\begin{aligned}
& v_{2}\left(y_{0}\right)-\partial \phi_{2}^{(1)} /\left.\partial y\right|_{y_{0}}=h_{1}(x, 2, t) \\
& v_{2}\left(-y_{0}\right)-\left.\partial \phi_{x}^{(x) / \partial y}\right|_{-y_{2}}=h_{2}(x, z, t]
\end{aligned}
$$

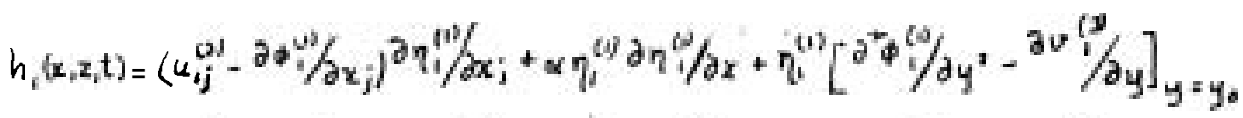

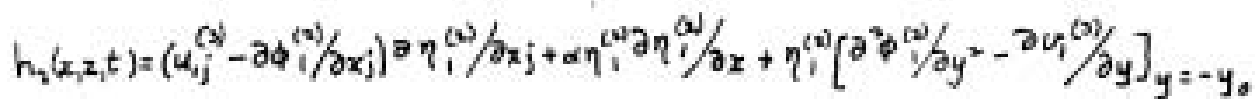
and for pressure

$$
\begin{aligned}
& {\left[-\partial \phi_{1}^{(1)} / \partial t-u_{0} \partial \phi_{2}^{(u)} / \partial x-p_{1}^{(v)}\right]_{y=y_{0}}=g_{l}(x, 2, t)} \\
& {\left[-\partial \phi_{(2)}^{(2)} / \partial t+u_{0} \partial \phi \phi_{2}^{(1)} / \partial x-p_{2}^{(2)}\right]_{y=-y_{0}}=g_{2}(x, 2, t)}
\end{aligned}
$$




$$
\begin{aligned}
& g_{1}(x, z, t)=\eta_{1}^{01}\left[-\partial p_{i}^{(1)} / \partial y+\partial p_{i}^{(3)} / \partial y\right]_{y=y_{0}}+\left[\frac{1}{2} \partial \varphi_{1}^{(1)} / \partial x_{j} \partial \phi_{i}^{(0)} / \partial x_{j}\right]_{y=y_{0}} \\
& g_{2}(x, z, t)=\eta_{1}^{(0)}\left[-\partial p_{1}^{(2)} / \partial y+\partial p_{1}^{(3)} / \partial y_{y}\right]_{y=-y_{0}}+\frac{1}{2}\left[\partial \phi_{1}^{(\nu)} / \partial x_{j} \partial \phi_{1}^{(2)} / \partial x_{j}\right]_{y=-y_{0}}
\end{aligned}
$$

For the first order problem there are non-zero initial conditions, but for the second order problem the solutions are forced by the inhomogeniety terms, such as $h$ and $g$, and the

Appendix B Pressure conditions and linear solution.

The pressure conditions (A.II) and (A.12) give two simultaneous equations,

$$
\begin{aligned}
& P_{t} e^{2 n y_{0}}+b P+\theta_{t}+Q \theta=\Phi_{1}(t) \\
& P_{t}-a P+\theta_{t} e^{2 r y_{0}}-b \theta=\Phi_{2}(t)
\end{aligned}
$$

where $a=i k u_{0}, b=i k u_{0} e^{2 n y_{0}}-(i k \alpha / r) s h 2 n y_{0}, \Phi_{1}=-\int_{-y_{0}}^{y_{0}} i k \alpha f(y) e^{-i k \alpha y t} d y$ and

$$
\begin{aligned}
& \Phi_{2}=\int_{-y_{1}}^{y_{k}} i k \alpha g(y) e^{-i k \Delta y t} d y \text {. The expressions for } f \text { and } j \text { are, } \\
& f=1 / r=e^{r y_{0}} s h r\left(y+y_{0}\right) F_{1}(y, h, m)\left(y-y_{0}\right) \\
& g=K_{r}=e^{r y} \cdot s h r\left(y-y_{0}\right) F_{1}(y, k, m)\left(y+y_{0}\right)
\end{aligned}
$$

These equations can be separated into two characteristic equations,

$$
\begin{aligned}
& x_{t}-\lambda x=-\lambda\left(\Phi_{1}+\mu_{1} \Phi_{2}\right) \\
& y_{t}+\lambda y=\lambda\left(\Phi_{1}+\mu_{2} \Phi_{2}\right)
\end{aligned}
$$

where $\quad \lambda^{2}=\left(b^{2}-a^{2}\right) /\left(e^{\left.4 n y_{0}-1\right)}=-k^{2} k_{0}^{2} / 4+2 y_{0}{ }^{2}\left[\left(2 r y_{0}-1\right)^{2}-e^{-4 n y_{0}}\right]\right.$

as given in the introduction, for the two-dimensional case. $P$ and $Q$ are related to $X$ and $Y$,

$$
\begin{gathered}
P=\left[X\left(a-\mu_{3} b\right)-Y\left(a-\mu_{1} b\right)\right] /\left[2\left(b^{2}-a^{2}\right) \sqrt{L^{2}-1}\right] \\
Q=\left[-X\left(b-a \mu_{1}\right)+Y\left(b-a-\mu_{1}\right)\right] /\left[2\left(b^{2}-a^{2}\right) \sqrt{L^{2}-1}\right] \\
\left.L=\left(a-b e^{2 n y \cdot}\right) /\left(a e^{2 n-b}-b\right) \quad \text { and } \begin{array}{c}
\mu_{1} \\
\mu_{2}
\end{array}\right]=-L \pm \sqrt{L^{2}-1} \text {, with } \\
L^{2}-1=-\lambda^{2} e^{4 y_{0} 4^{2} y_{0}^{2} / k^{2} u_{0}^{2} .}
\end{gathered}
$$

The relations (8.6) may be summarized as,

$$
\begin{aligned}
& P=d_{11} X+d_{12} Y \\
& Q=d_{21} X+d_{22} Y
\end{aligned}
$$

For $\lambda$ real corresponding to instability, $\mu_{1}$ and $\mu_{2}$ are complex conjugates, and $d_{44}^{*}=d_{12}, d_{21}^{*}=d_{22}$. For neutrally stable oscillations on the other hand $\mu_{1}$ and $\mu_{t}$ are both real, and the d's are purely imaginary. The solutions for $X$ and $Y$ are, 


$$
\begin{aligned}
& X(t)=x(0) e^{\lambda t}+e^{\lambda t} \lambda \int_{-y_{0}}^{y 0} i k \alpha\left[e^{-i k \alpha y t-\lambda t}-1\right] n_{1}(y) /(\lambda+i k \alpha y) d y \\
& Y(t)=Y(0) e^{-\lambda t}-e^{-\lambda t} \lambda \int_{-y_{0}}^{y *} i h \alpha\left[e^{i k \alpha y t+\lambda t}-1\right] n_{2}(y) /(-\lambda+i k \alpha y) d y
\end{aligned}
$$

where $\left\{\begin{array}{l}n_{1} \\ n_{2}\end{array}\right\}=\left(\mid f(y)+\left\{\begin{array}{l}\mu_{1} \\ \mu_{2}\end{array}\right\} \mathbf{j}(y)\right)$, and in particular $n_{1}\left(y_{0}\right)=n_{1}\left(-y_{0}\right)=n_{2}\left(y_{0}\right)=n_{1}\left(-y_{0}\right)=0$.

Appendix $\subset$ Expansion for large times.

Consider the form of $X_{1}(t)$ for large times, for $\lambda$ imaginary. The intergrand in (3.7) has a positive singular behavior at the critical layer, $y=-y_{c}$, where $i k \alpha y_{c}=\lambda$. However in fact the numerator gives a regular expression, $\left(-i h \alpha t n_{1}\left(-y_{c}\right)\right)$. Hence the integral may be written as two Cauchy Principal Parts, $-y$
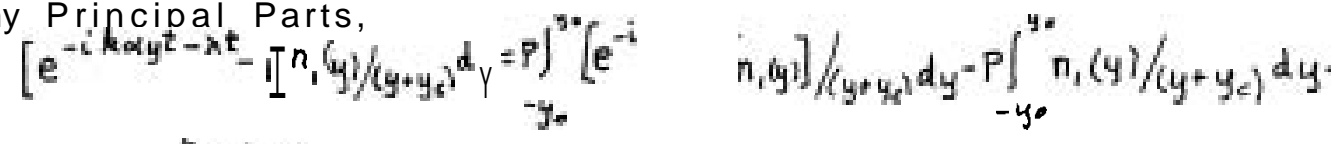

In the limit $t \rightarrow \infty$, we evaluate the first of these two integrals.

Writing $\mid \alpha \times t\left(y+y_{c}\right)=\S$

$$
\Rightarrow P \int_{-\infty}^{\infty}\left[e^{-i \xi} n_{1}\left(-y_{c}+5 / k \alpha t\right)\right] \frac{d \xi}{\xi}
$$

This may be evaluated by closing the contour in the lower complex $\mathcal{S}$ plane, as indicated in Fig.C.

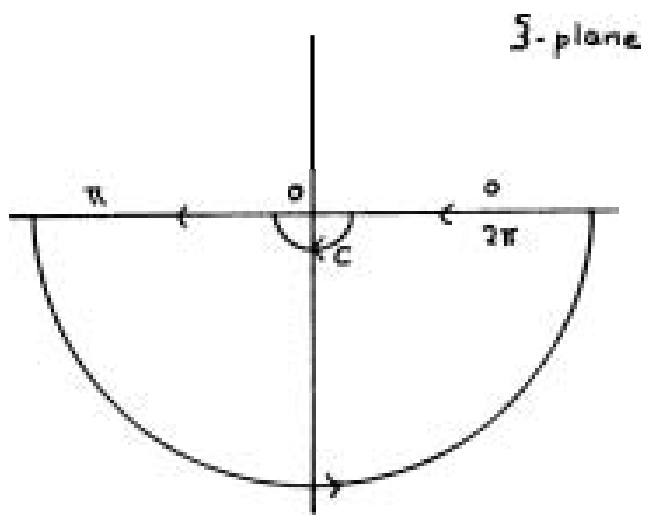

Fig.C Contour to evaluate Cauchy Principal Part.

$C$ is the semicircle; $\xi=\delta \exp (i \theta), \theta=2 \pi, \pi$ -

$$
\Rightarrow 0=-P \int_{-\infty}^{\infty} e^{-i \delta} n_{1}\left(-y_{c}+\delta / k a t\right) \frac{d \xi}{\xi}+\int_{\epsilon} e^{-i \delta} n_{1}\left(-y_{c}+\xi / k a t\right) \frac{d \delta}{\xi}
$$

where $c$ is the semicircle, $\xi=\delta e^{i \theta}, \theta=2 \pi, \pi$ and $\delta \rightarrow 0$.

$$
\Rightarrow P \int_{-\infty}^{\infty} e^{-i \xi} n_{1}\left(-y_{e}+\xi / k \alpha t\right) \frac{d \xi}{\xi}=-i n n_{1}\left(1-y_{2}\right) \text {. }
$$

So to leading order

$$
x(t) \sim x_{1}(0) e^{\lambda t}+\lambda e^{\lambda t}\left\{-i \pi n_{1}\left(-y_{2}\right)-P \int_{y_{0}}^{y_{n}} n_{1}(y) / y+y_{0} d y \cdot\right\}
$$

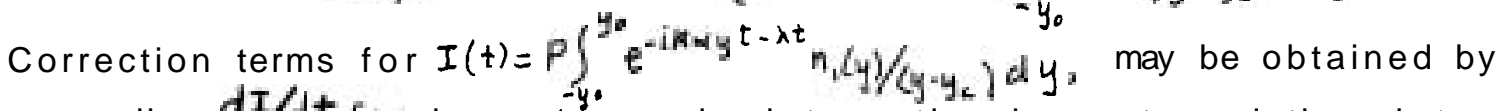
expanding $d I / d t$ for large times, by integration by parts and then integrating again so that

$$
I(t)=I(\infty)-\int_{t}^{\infty} \frac{d I}{d t}\left(t^{\prime}\right) d t^{\prime}
$$




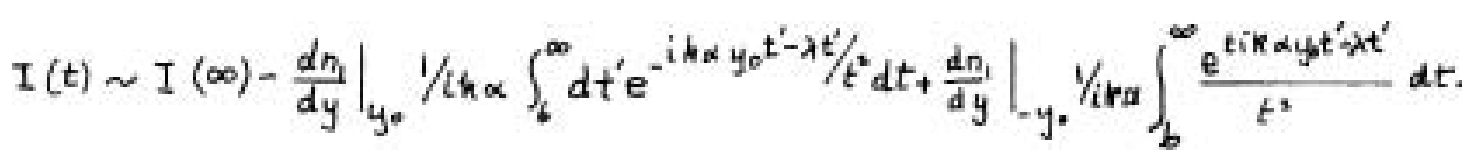

References

Case, K.M. 1960 Stability of inviscid plane Couette flows. Phys.Fluids 3 : 143.

Dikii, L.A. 1961 Stability of plane-parallel flows in an ideal fluid. Sov. Phys.Doklady 5: 1179.

Drazin, P.G. and L.N.Howard 1966 Hydrodynamic stability of parallel flows of inviscid fluid. Adv.Appl. Mech. 2: 1966.

Durbin, P. 1977 Distortion of turbulence by a constant shear layer adjacent to a wall. Smith Prize Essay, Univ.Cambridge.

Gustavsson, L.H. 1977 Private communication.

Landahl, M.T. 1975 Wave breakdown and turbulence. SIAM J.Appl.Math. 28: 735.

1977 On the dynamics of boundary layer turbulence and the mechanism of drag reduction. Phys. Fluids Supplement (in press).

Lighthill, M.J. 1962 Physical interpretation of the mathematical theory of wave generation by wind. J.Fluid Mech. 14: 385.

Lin, C.C. 1955 Theory of hydrodynamic stability. C.U.P.

Rayleigh, J.W.S. 1894 The theory of sound. Vol.II. Ch.XXI, Dover New York 1945, reprint of 2nd ed.of 1894, p.393.

Reynolds, W.C. and M.C.Potter 1967 Finite-amplitude instability of parallel shear flows. J.Fluid Mech. 27: 465.

Stuart, J.T. 1960 Non-linear mechanics of disturbances in parallel flows. J.Fluid Mech. 2: 353.

Willke, H.L. 1967 Stability in time-symmetric flows. Annals of Physics, J.Maths. E Physics 46: 151. 


\title{
A MODE OF THE UPWELLING DUE TO TOPOGRAPHICALLY GUIDED ROW
}

\author{
Stephen J. S. Morris
}

\section{Introduction}

Appreciable vertical velocities can occur in the ocean in two distinct regions. In the open ocean they can be driven either by Ekman pumping or by the change in Coriolis parameter across the equator. The vertical velocities which result in this case are characteristically of order $10^{-4} \mathrm{~cm} / \mathrm{sec}$ but vertical velocities at least an order of magnitude greater occur in coastal upwelling. These notes will only discuss mechanisms for coastal upwelling.

Any explanation of coastal upwelling must begin with the fact that whenever the wind blows over the ocean there is in the surface Ekman layer a net drift of fluid to the right of the applied stress. Near the coast, this creates a region of high pressure offshore (Fig.l) which drives a

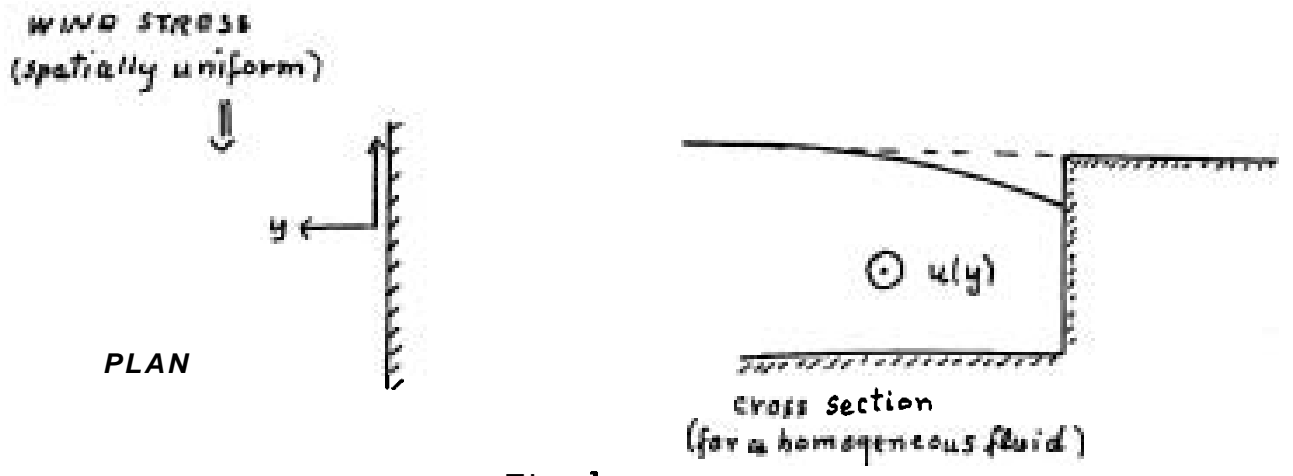

Fig. 1

southerly geostrophic flow in the interior. The simplest possible explanation of coastal upwelling points out that in the bottom Ekman layer the stress applied by the seafloor is in the opposite direction to the mean current. Thus there is a drift of fluid to the left of the mean current; similarly, the wind in the atmospheric boundary layer tends to spiral out of a high pressure region. When the current in this bottom boundary layer hits the coastline, it is converted into a vertical current which can supply the Ekman drift at the surface. Although this explanation gives a mechanism for transferring nutrients from the deep water to the surface water where they can be most efficiently used, it predicts that the onshore flow which balances the offshore drift in the surface Ekrnan layer is confined to the bottom Ekman layer. In practice the compensation flow occurs only at intermediate depths. For example, Shaffer (1976) observed that in water of depth 400 (or so) metres off the northwest coast of Africa the compensation flow occurs between 50 and $250 \mathrm{~m}$ below the surface. In addition, the upwelling is spatially intermittent along the coast with the regions of strongest upwelling seeming to be closely connected with topography. $0 \mathrm{ff}$ this coast, there are many short deep canyons (Fig.2), at the head of each of which there is a fixed centre of upwelling. Within and onshore of the canyons, the mean vertical velocities are of order $10^{-2}$ to $10^{-1} \mathrm{~cm} / \mathrm{sec}$ whilst there is little mean vertical motion between canyons (Shaffer, 1976).

A crude explanation of these observations is easily given. Using the characteristic speeds and depths given in the previous paragraph hydro- 


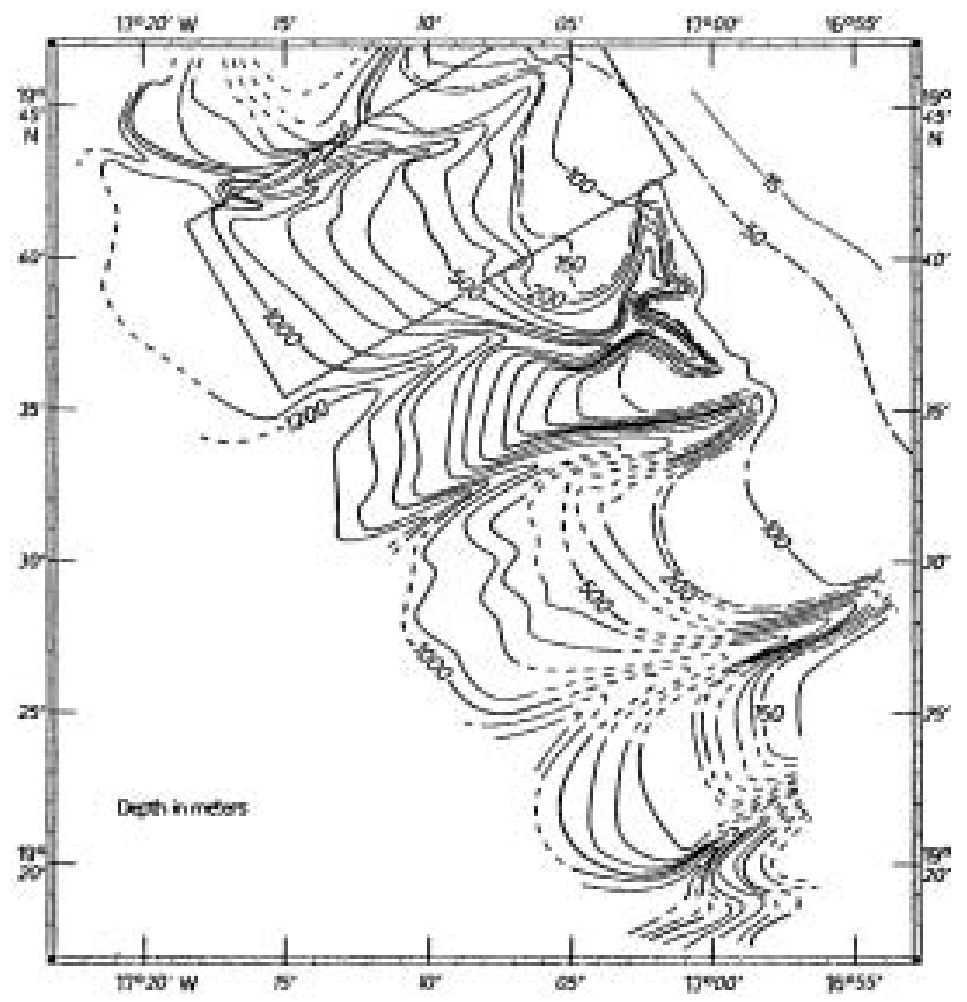

Fig.2. (Courtesy of Shaffer (1976). Canyons of $f$ the coast of Northwest Africa. NB: 1' corresponds to about $1 / 1 / 2 \mathrm{~km}$ here, so that these canyons are typically $10 \mathrm{~km}$ long, $2 \mathrm{~km}$ wide and $400 \mathrm{~m}$ (or so) deep.

statics is easily shown to be an excellent approximation. Thus if the horizontal velocities are independent of at some location, then they are everywhere independent of $z$. On letting $\zeta$ be the (vertical) component of relative vorticity, $f$ be the (constant) Coriolis parameter and $h+\eta$ be the length of a fluid column (Fig.3) it follows that $\frac{D}{D t} \frac{\text { Ltf }}{h+\eta}=0$, which is simply a special case of the Cauchy vorticity theorem. As the flow is assumed to be steady $\& . "=\eta$. Moreover, if the upstream flow is uniform it follows that $5 / f<\varepsilon_{1}$ in regions where the stream 1 ines are not too strongly curved (in a sense to be made precise shortly), so that $\mu \cdot \nabla \frac{f}{h+\eta}=0$. In particular, the flow follows the lines of constant depth. A simple energy argument shows that a fluid particle whose characteristic speed is $U$ can displace the free surface at most through a distance of order $\mathrm{U}^{3} / \mathrm{g}$. As the Froude number based on the characteristic depth $\mathrm{H}$ is much smaller than the fractional height $(a / H)$ of a typical bump on the bottom, the displacement $(\eta)$ of the free surface is thus a small fraction of the amplitude of the variations in the bottom and can be neglected for the time being. Consequently the flow follows the isobaths; because the inertia of the flow is very weak, the flow can be guided around gently curving isobaths by the topography. However, if the isobaths curve too sharply, the flow has 


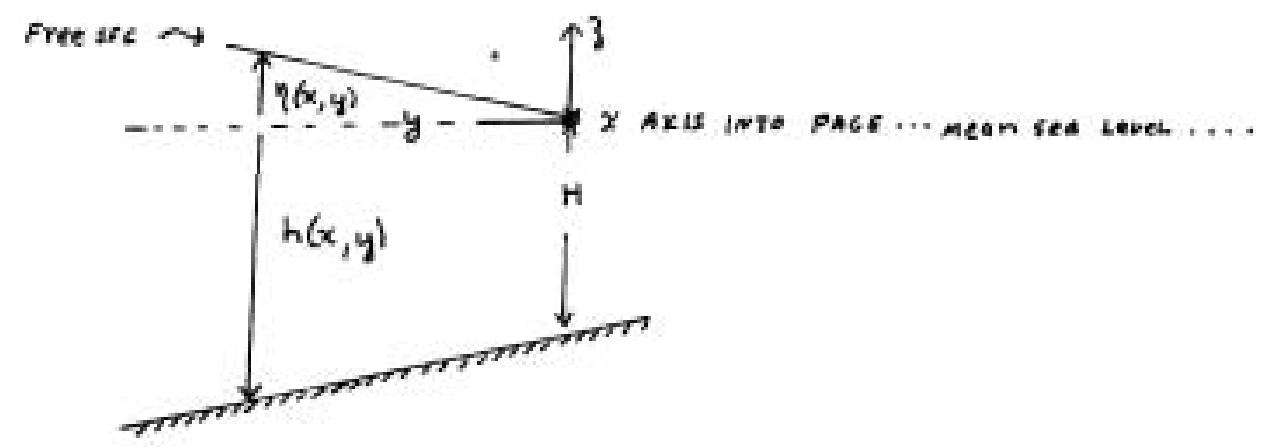

Fig.3. Definition of quantities appearing in the equations.

sufficient inertia to cut across them and in doing so, generate vertical velocities. A more detailed qualitative explanation can be given by using conservation of potential vorticity. Suppose, for example, that $u(-\infty, y)=V$ and that $h(x, y)=m\left(y+a e^{-(k x)^{2}}\right) \quad($ Fig.4) wherem《1 and $a k \sim 1$. Then the

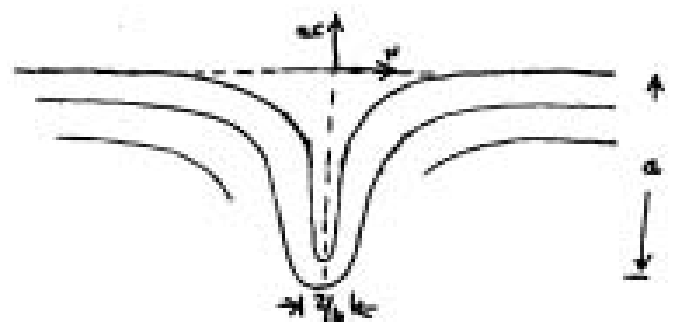

Fig.4. Contours of $h(x, y)=m\left(y-a e^{-(k x)^{2}}\right)$

mass transport stream function $\Psi=-v h^{2} / 2 m$ in the 1 imit $\zeta / f \rightarrow 0$. Thus

$$
v=-\frac{v}{m} h_{x},=-2 a k^{2} v_{x} e^{-(h x)^{2}}
$$

so that the maximum vorticity is of order $\mathrm{V} / \mathrm{a}$. This can become comparable to $f$ near $x=0$ if $a$ is not large compared to the radius $\underline{f}$ of the inertial circle. In that case the assumption that the flow follbws the contours of $h(x, y)$ is not self-consistent. The flow must therefore cut across isobaths and thus cause upwelling. However, once the flow is past the origin, the isobaths become gently curving again and the flow can once again follow the topography. How rapidly it relaxes back to following the topography determines the extent of the upwelling region. Moreover, the flow might either relax back to the contours monotonically in space or also a given streamline might oscillate in space about some isobath. For example, for southerly flow with deep water on the right, it is easily seen that the topography in Fig. 4 provides a restoring force which acts on the displaced flow so that it is possible that a standing wave can exist downstream of the canyon with an associated pattern of onshore and offshore flow. To see which of the two possibilities actually occurs, the model must be worked through in detail. In particular, it will be used to find the strength of upwelling produced and also to understand the role which the free surface plays in returning the escaped flow to the isobaths. 
Before studying this problem in more detail, it is useful to understand the part which the free surface plays in the much simpler problem of linearised rotating flow over a single obstacle (Fig.5.

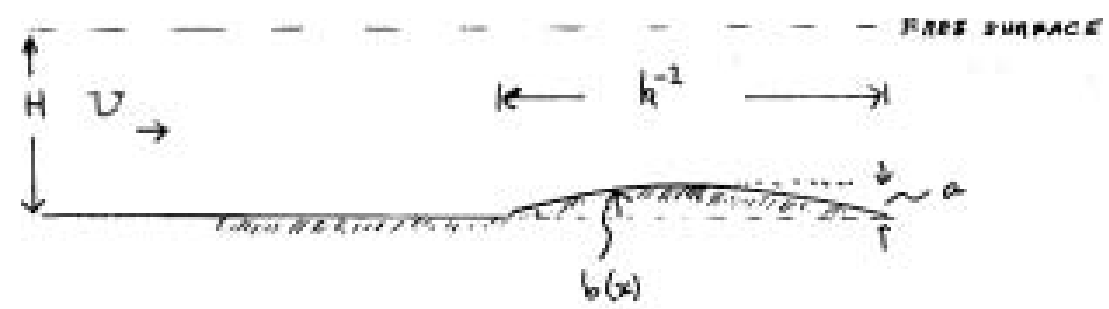

Fig.5. Flow over a small obstacle.

NB: In this section only the location of the bottom is independent of $y$.

2. The role of the free surface.

In Fig. 5 the flow is uniform of speed $U$ at $x=-\infty$ and impinges upon the obstacle shown. If $a / H \leqslant 1$, ak $\leqslant 1$ and $\frac{f}{k V}-1$, the governing equations for topography independent of $y$ are

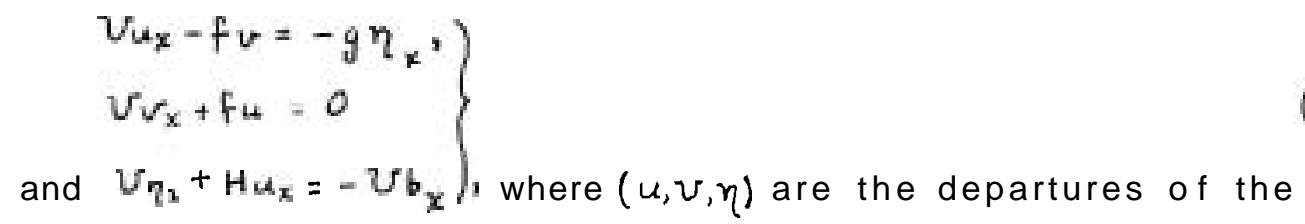
velocities and interfacial location from the basic state. Thus

where $F \equiv \frac{V^{2}}{g H}$ and $\begin{aligned} k_{L}^{2} & =\frac{f^{2}}{g H}, \\ & =F \cdot\left(\frac{f}{k V}\right)^{2} k^{2} \text { is the square of the inverse of the radius }\end{aligned}$ of deformation. First consider the case $b=a \sin k x$. Then

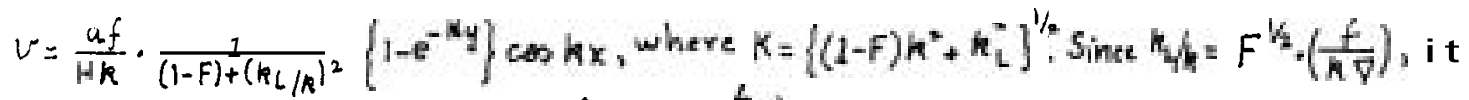
follows that as $F \rightarrow 0, v \rightarrow \frac{a f}{R H}\left(1-e^{-k y}\right) \cosh x$ so that the term $k_{L}^{2} v$ in

plays no essential role here. Neglecting this term corresponds to neglecting the term $V_{\eta_{x}}$ in the third of equations (2.1) and thus to neglecting $\eta$ altogether in a vorticity formulation of the problem. However if

$$
\begin{array}{rlrl}
b & =a \sin k x, 0 \leq k x \leq \pi \\
& =0 & & \text { otherwise, a non-zero solution which is }
\end{array}
$$

bounded at infinity will exist for $k x \leq 0$ and $k x \geqslant \pi$ only if the term $k_{L}^{2} v$ is included.

2.2. Although the displacement of the free surface is a small effect (of order $F^{1 / 2}$ here) it acts on a fluid particle for a long time (of order $F^{-1 / 3}$ ) as the fluid particle travels through a Rossby radius of deformation and can thus produce the order one effect of returning the velocity field to zero at infinity. The flow here can be fully understood by calculating 
the free surface from the potential vorticity equation, $\eta=\frac{H}{f} v_{x}+b$. In region $I$ (Fig.6), $\eta=a \frac{k_{L}}{k} e^{k_{L} x}$, i.e. $\eta$ increases with $x$ because water piles

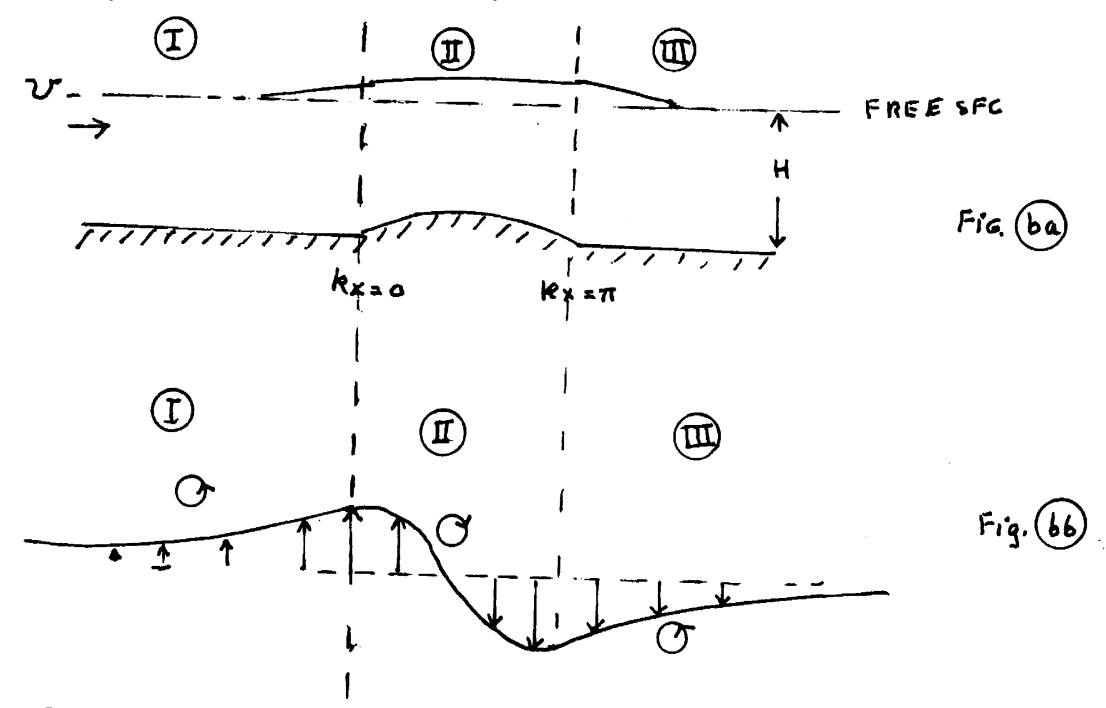

Fig.6. Flow over the obstacle

up in front of the obstacle.* Thus vortex lines in the approach flow are stretched and a flow develops to the left. Over the obstacle (region $\square$ ) variations in $\eta$ are negligible compared to those in $b$ so that vortex lines are compressed on the upstream side of the obstacle and stretched on the downstream side. Thus the vorticity over the obstacle is always less than it is at $x=0$ and the flow takes up the pattern shown in figure bb. Finally in region $\Pi, \eta=\frac{H}{f} v_{x}$, the free surface gradually subsides and the vortex columns return to their upstream height.

In the present case, the topography is complicated by the presence of a background slope $\partial h /\left.\partial y\right|_{x=}=m$. However, it seems reasonable to expect that the small but positive $m$ will serve merely to guide the approach flow into the canyon but that it will otherwise be dominated by the displacement of the free surface due to the slopes within the canyon, within which the slopes are much larger than $m$ also still small enough that hydrostatics can be assumed.

Finally, were this problem being developed simply as an exercise in perturbation theory we should need to make an expansion in two small parameters, namely $F$ and a characteristic slope. Rather than doing this we shall make an expansion in the single parameter $F$ and simply assume hydrostatics to be true. This assumption may exclude some phenomena (1) but will make it easier to understand the basic mechanics.

3. The model.

The governing equations for flow over a gently sloping bottom are

$$
\left.\begin{array}{l}
\frac{D}{D t}\left\{\frac{\zeta+f}{\eta+h}\right\}=0, \\
\frac{D}{D t}\left\{\frac{1}{2}\left(u^{2}+v^{2}\right)+g \eta\right\}=0,
\end{array}\right\}
$$

*in spite of the fact that the flow is subcritical. 


$$
\text { and }\{u(\eta+h)\}_{x}+\{v(\eta+h)\}_{y}=0 \text {. Here } \frac{D}{p t} \neq u \frac{\partial x}{\partial x}+v \frac{\partial y}{\partial y} \text { and the }
$$

other quantities are defined in Fig.3. Note that here $u$ and $v$ are the total velocities, not the fluctuations from a basic state. If $r$ is a typical radius of curvature for an isobath at the head of a canyon, then $\frac{a}{a x} \frac{1}{\frac{1}{*}} \frac{\partial}{\partial x^{\prime}}$, and $\frac{a}{\partial y}=\frac{1}{\gamma} \frac{\partial}{\partial y^{\prime}}$. Also let $(u, v)=U\left(u^{\prime}, v^{\prime}\right), h=H h^{\prime}$ and $\eta=F H \eta$ when $H$ is a typica1 depth. Then

$$
\begin{gathered}
\stackrel{u}{\prime}^{\prime} \cdot \nabla^{\prime}\left\{\frac{R y^{\prime}+1}{F \eta^{\prime}+h^{\prime}}\right\}=0, \underline{u}^{\prime} \cdot \nabla^{\prime}\left\{\frac{1}{2}\left[\left(\mu^{\prime}\right)^{2}+\left(v^{\prime}\right)^{3}\right]+n^{\prime}\right\}=0, \text { and } \\
\left(u^{\prime}\left(h^{\prime}+F \eta^{\prime}\right)\right\}_{x}+\left\{v^{\prime}\left(h^{\prime}+F \eta^{\prime}\right)\right\}_{y}=0,
\end{gathered}
$$

where the primed quantities are all of order one and $R=U / f r$. Dropping the primes on dimensionless quantities and noting that $\mu(h+F \eta)=-\psi_{y}$,

$$
\begin{gathered}
v(h+F \eta)=\psi_{x} \text { and } D \psi / D t=0 \text { gives } \\
R \nabla \cdot\left[\frac{\nabla \psi}{h+F \eta}\right]+1=P(\psi)[h+F \eta] \text { and } \frac{1}{2} \frac{(\nabla \psi)^{2}}{(h+F \eta)^{2}}+\eta=B(\psi) .
\end{gathered}
$$

The functions $P(\psi)$ and $B(\psi)$ are determined from the conditions upstream. The eastern boundary currents travel at speeds characteristically of order $10 \mathrm{~cm} / \mathrm{sec}$ in depths of (say) $100 \mathrm{~m}$ so that even if $F$ were the internal Froude number it would be small. Thus, if $P(\psi)$ were chosen to be a linear function of $\Psi$, and the upstream flow constrained accordingly, the governing equations would become linear in the limit $F \rightarrow 0$. Rather than doing this directly, choose the upstream flow to be uniform of speed $\checkmark$ with a linear bottom, $\mathrm{h}=H+m \psi_{\infty}$. Here $y_{\infty}$ satisfies $\psi\left(-\infty ; y_{\infty}\right)=\psi(x, y)$. Since uniform flows are exact solutions of the geostrophic equations,

$$
\eta\left(-\infty, y_{\infty}\right)=-\frac{U_{f}}{g} y_{\infty} \text {, Hence, } \psi\left(-\infty ; y_{\infty}\right)=-y_{\infty}+\frac{1}{2}\left(m-\frac{F U f}{g}\right) y_{\infty}^{2} \text {. }
$$

Moreover, on letting $\mu=\left(m-F \cdot \frac{U_{f}}{g}\right)$, we have

$$
\left.\begin{array}{c}
\frac{R \zeta+1}{h+F_{\eta}}=\frac{1}{1+\mu y_{\infty}}, \\
\text { wnd } \frac{1}{2} \frac{(\nabla \psi)^{2}}{\left(h+F_{\eta}\right)^{2}}+\eta=1-\frac{U_{f}}{g}
\end{array}\right\} \text {, which together with (3.2) define } P(\psi)
$$

and $B(\Psi)$. From $(3.2)$,

$$
y_{\infty}=\frac{1 \pm[1+2 \mu \psi]^{3 / 2}}{\mu} \text {. }
$$

The root which is bounded for small $\mu$ is the appropriate one; it gives

$$
\frac{R \zeta+1}{h+F_{\eta}}=\frac{1}{2-[1+2 \mu \psi]^{w_{2}}} \text { and } \frac{1}{2} \frac{(\nabla \psi)^{2}}{\left(h+F_{\eta}\right)^{2}}+\eta=1-\frac{U_{f}}{g \mu} \cdot\left\{1-(1+2 \mu \psi)^{1 / 2}\right\} \text {. }
$$

These equations are still non-linear but in the 1 imit $F \rightarrow 0, \mu \rightarrow 0$ they become linear. Following the ideas outlined at the end of the previous 
section, a perturbation expansion in powers of $F$ will be performed, using the method of multiple scales to include the slow spatial scale $k L^{\prime}$. For the time being, terms of order $\mu$ will be included in the binomial expansion of $(1+2 \mu \psi)^{1 / 2}$. Thus, $h+F_{\eta}=1+\mu \psi$ and $\frac{1}{2} \frac{(\nabla \psi)^{2}}{\left(h+F_{\eta}\right)^{2}}+\eta=1+\frac{v f f}{y} \psi$, with error of order $\mu^{2}$. Since $\left(n_{k} r\right)^{2}=F R^{-2}$, introduce the fast and slow variables $\left(x^{*}, y^{*}\right)=(x, y)$ and $(\bar{x}, \tilde{y})=F^{4} R^{-1}(x, y)$, and let $\psi(x ; F)=$ , $F$ and $\eta(x ; F)=\eta\left(x^{*}, F\right)$. The operators $\nabla$ and $\Delta(\equiv \nabla \cdot \nabla)$ become $\nabla^{*}+\frac{F^{1 / 2}}{R} \tilde{\nabla}$ and $\Delta^{*}+\frac{E}{R^{2}} \tilde{\Delta}+2 \frac{E^{*}}{R} \nabla^{*} \cdot \tilde{\nabla}$, where $\nabla_{i}^{*}=\frac{a}{\nabla x_{i}^{2}}$ and $\tilde{\nabla}_{i}=2 z_{i}-(i=1,2)$. The transformed equations are

$$
\begin{aligned}
& R \frac{\Delta^{*} \Psi+\frac{F}{R^{2}} \tilde{\Delta} \Psi+2 \frac{F^{*}}{R}\left(\nabla_{*} \cdot \tilde{\nabla}\right) \Psi}{\left(h+F_{\eta}\right)^{2}}-R\left(\nabla^{*} \Psi+\frac{F^{k}}{R} \tilde{\nabla} \Psi\right) \cdot \frac{\left\{F \nabla_{\eta}^{*}+\frac{F^{3 / 2}}{R} \tilde{\nabla}_{\eta}+\nabla^{*} h\right\}}{(F \eta+h)^{3}}+ \\
& +\frac{1}{F_{\eta+h}}-\mu \Psi=1, \\
& \text { and } \frac{\left(\nabla^{*} \Psi\right)^{2}+\frac{2 F^{1 / *}}{R} \nabla^{*} \Psi \cdot \tilde{\nabla} \Psi+\frac{F}{R^{*}}(\tilde{\nabla} \Psi)^{2}}{(F \eta+h)^{2}}-1 \text {. }
\end{aligned}
$$

On assuming expansions of the form $\Psi=\Psi_{0}+F^{\psi_{0}} \Psi_{1}+F \Psi_{2}+O\left(F^{\pi / 2}\right)$ and

$$
\begin{gathered}
\eta=\eta_{0}+F^{k_{2}} \eta_{1}+F \eta_{1}+O\left(F^{\mu^{2}}\right) \text {, we find that } 2 \eta_{0} \frac{\left(\nabla^{*} \Psi_{0}\right)^{*}}{h^{3}}-1, \\
\frac{R}{h_{1}} \Delta^{*} \Psi_{0}-\frac{R}{h^{3}} \nabla^{*} h \cdot \nabla^{*} \Psi_{0}-\mu \psi_{0}=1-\frac{1}{h}\left(x^{*}\right)
\end{gathered}
$$

and that

$$
\begin{gathered}
-h^{*}\left[\Delta^{*} \Psi_{2}+\frac{2}{R}\left(\nabla^{*} \cdot \tilde{\nabla}\right) \Psi_{2}=\frac{2 \eta_{0}}{h} \Delta^{*} \Psi_{0}+\frac{1}{R^{2}} \tilde{\Delta} \Psi_{0}\right]+\frac{R}{h^{3}}\left[\frac{\nabla_{e} h}{h} \cdot \nabla_{4} \Psi_{0}-\nabla^{*} h \cdot \nabla^{*} \Psi_{2}-\right. \\
\left.-\nabla^{*} \Psi_{1} \cdot \nabla^{*} 6-\nabla^{*} \Psi_{0} \cdot \nabla_{0} \eta_{0}\right]+\frac{\eta_{0}}{h^{2}}-\mu \Psi_{1}=0 .
\end{gathered}
$$

The dependence of $\Psi_{*}$ on $\tilde{\boldsymbol{x}}$ will be determined by the condition that there be a solution which is bounded at infinity.

Since $h$ depends both on $x$ and $y, \psi$ will also. However, if $h$ varies much more rapidly with $x$ in the canyon than it does with $y$ it seems reasonable to assume that the solution will vary much more rapidly with $x^{*}$ than with $y^{*}$ and that its variation with $y^{*}$ can therefore be neglected. At the least this approach will give some insight into how the flow works. Accordingly, let

$$
\Psi=\Phi\left(x^{*} ; \tilde{x}, \bar{y}\right)+y^{*} x\left(x^{*} ; \tilde{k}, \bar{y}\right)
$$

Equation (3.4b) will then reduce to (3.3) with $\zeta=\frac{\partial y}{\partial x^{2}}, F=0=\mu$, but the higher order equations (3-5) and (3-6), which are necessary to determine the $(\bar{x}, \bar{y})$ dependence of the flow will be more complicated. 


\section{Appendix.}

As Eqs. (3.4) to (3.6) are so complicated, I decided to study the effects of the flow's having a positive Rossby number by extending the simple argument given at the end of the first section. As the case $R \sim 1$, $F \rightarrow 0$ is appropriate, we should expect to gain some understanding of it by studying first the easier case $l \gg R \gg F$. When this is done it becomes obvious that for values of $\partial \mathrm{h} / \mathrm{\partial y}$ characteristic of the continental shelf (see, e.g., Peffley and O'Brien (1976), fig.2) the role of the free surface in steering the current is trivial in comparison to that of the topography. Hence it is not necessary to use the method of multiple scales for two-dimensional topography; the point made in paragraph 2 is irrelevant to the problem in hand. Since conservation of potential vorticity gives $\frac{R \zeta+1}{h t^{\prime} \eta}=P(\psi)$, assuming that $\psi=\Psi_{a}+R \psi_{1}+O\left(F, R^{2}\right)$

$$
\text { gives } P\left(\psi_{0}\right)=\frac{1}{h} \text {, }
$$

and $\psi_{1} \rho^{\prime}\left(\psi_{0}\right)=\frac{\xi_{0}}{h}$. If the flow upstream is uniform and $h=m y$ it follows that

$$
\begin{aligned}
P(\psi) & =H^{V_{2}}(-\psi)^{-1 / 2} \\
& =\left(\frac{H}{2 m r}\right)^{1 / 2}\left(-\psi_{0}\right)^{-V_{2}}\left(1-\frac{R}{2} \frac{\psi_{1}}{\psi_{0}}+\ldots\right)
\end{aligned}
$$

Thus, on noting that $\zeta_{p}=-m \mathrm{~m} \frac{\partial^{2} h}{\partial x^{2}}$, it is found that

$$
\psi \underset{R \rightarrow 0}{\sim} \frac{H}{m r} h^{2}\left\{-\frac{1}{2}+R \frac{H}{m r} \frac{\partial^{2} h}{\partial x^{2}}\right\}
$$

When $R=0$, isobaths and streamlines coincide, but when $R>0$ it is clear that as $x$ increases in region $I$
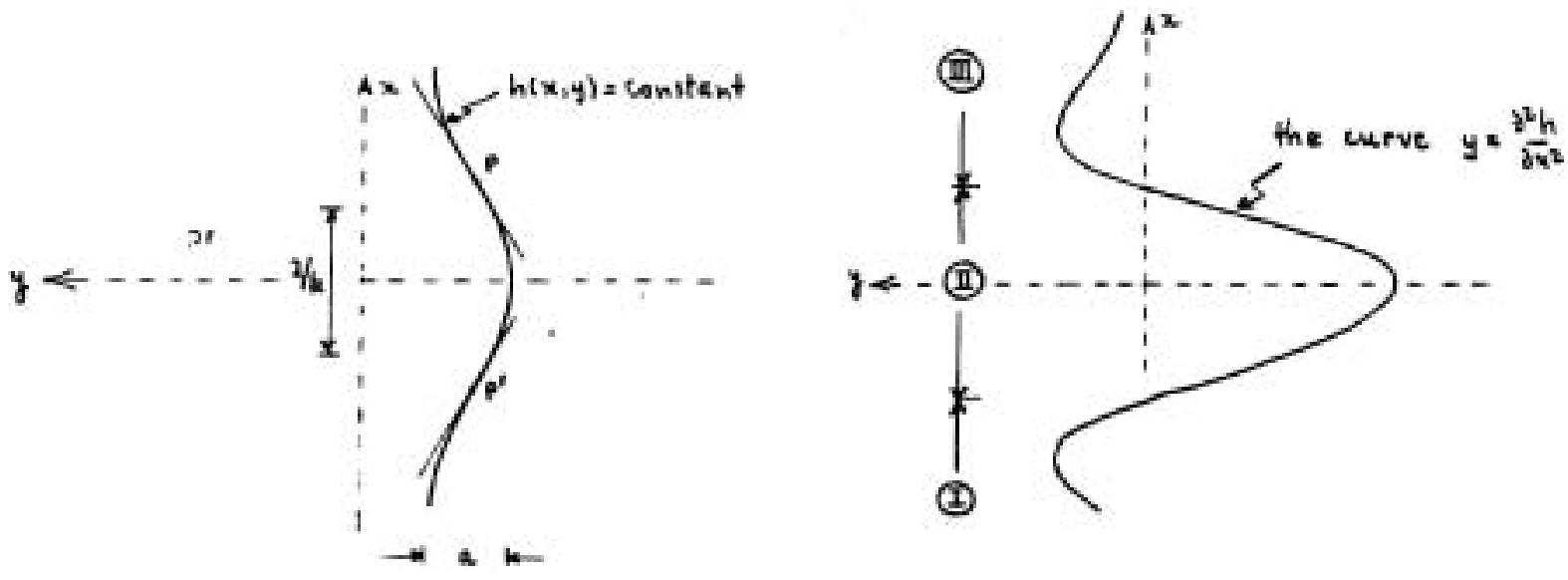

$$
P, P^{\prime} \text { are the points of inflexion on the isobath. }
$$

$\partial^{2} h / \partial x^{2}$ becomes more negative so that the term in the braces in (Al) becomes more negative and $h$ must decrease in order for $\psi$ to remain constant. Thus the streamline moves up the slope in region $I$, so that upwelling occurs there. Repeating the argument in regions II and III shows 
that the streamlines must have the form shown in the sketch.

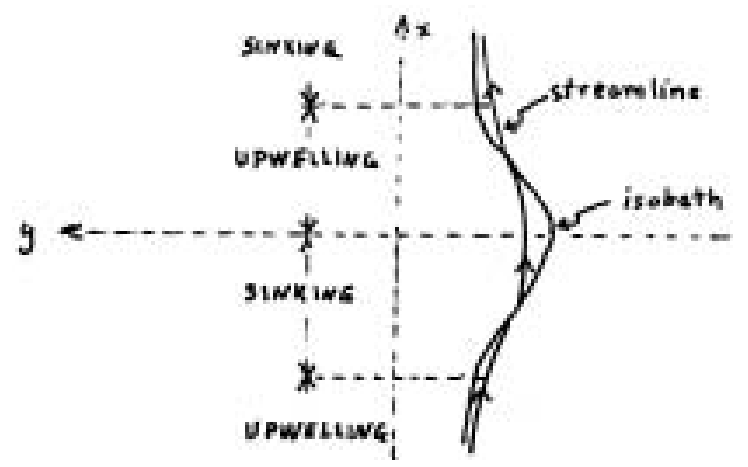

In this essentially linear case, the fluid particles do not behave as suggested in the first section; rather their trajectories adjust themselves to avoid regions where geostrophy would be violated. If we were to solve an initial value problem in which the flow was prescribed to follow the isobaths initially, we should expect the transient response of the fluid to comprise topographic Rossby waves which would act, in effect, to radiate vorticity away from areas where the streamlines were initially most sharply curved.

The initial value problem should also be solved to see whether or not upstream influence is possible. This is important for any approach to the nonlinear problem based on invariants of the flow. Finally, stratification must be included because it allows vertical structure in the velocity structure in the velocity field (q.v. section 1.).

In introducing the summer notes, Malkus once described some of the fellows' lectures as being the first pressed fruits of a novel idea. It seems more appropriate to describe mine as the first mumblings of a thesis proposal.

\section{A lledgmeis}

I am very grateful to Dr. Veronis for getting me interested in rotating, stratified fluids. I would like to thank him, Dr. Rooth and Dr. McWilliams for some helpful comments and to Mary Thayer for typing the manuscript. This summer's work was supported by the Office of Naval Research.

\section{References}

Peffley, M. and J.J.O'Brien 1976 A three-dimensional simulation of coastal upwelling off Oregon. J.Phys.Oceanogr., 6: 164.

Shaffer, G. 1976 A mesoscale study of coastal upwelling variability of $f$ N.W.Africa, in "Meteor" Forsch.-Ergebnisse, A, No. 17: 621.

(See also the sketch of a manuscript by Shaffer which appeared in the C.U.E.A. Newsletter, 5, 1, March 1976. 
COUPLING BETWEEN PULSATION AND CONVECTION IN A STAR

Jean-Pierre Poyet

\section{Introduction}

Though the title may suggest that we are going to study only pulsating cases, the method that we will present below will be applicable as well for a contracting or an expanding star, or any kind of mean behavior one can think of.

Let us mention briefly some of the interesting astronomical situations which can be studied in the frame of what will follow.

First one can investigate the Cepheid instability strip in the

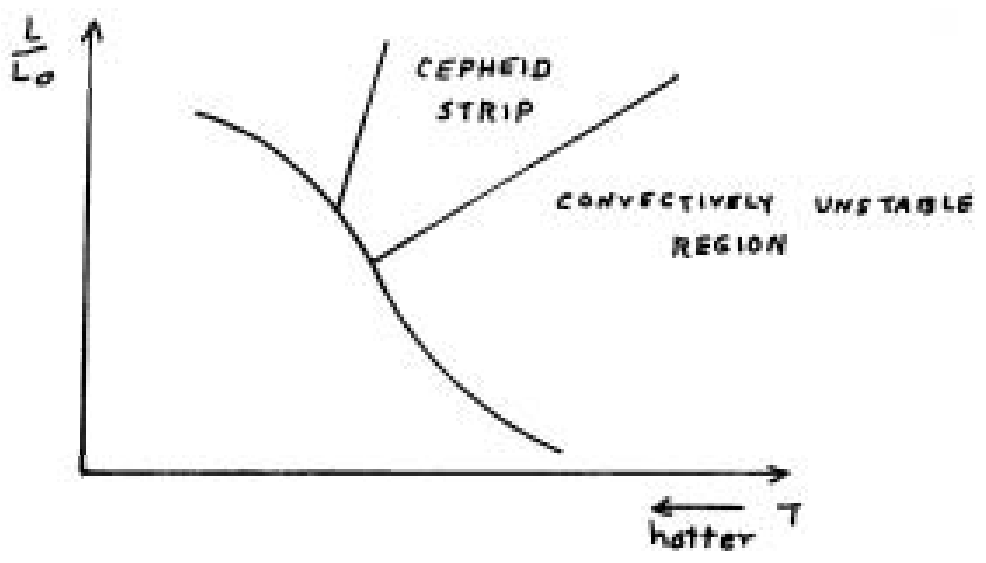

Hertsprung-Russell diagram as shown above and deduce some information about the location of the line separating the convectively unstable domain from the instability strip. Very few things are known about this line.

One may also investigate the possibility of convection after the helium flash in a star when the core expands and the outer layers contract.

Finally during the contraction of a protostar, it would be interesting to know if any convection can occur.

We will be concerned below with the direct problem; that is to say: is there any convective instability which may be caused by any time dependent mean motion? we will restrain ourselves to the linear problem.

In the frame of the nonlinear problems, another case of interest is the inverse problem: could convection itself provoke pulsations? An example of this kind of feedback could be the semi-convective $\beta$-Cephei star. (This is the reason why we will include the mean molecular weight $\mu$ in our equations for further studies of this case.)

For a very long time, the problem of the coupling between pulsation and convection has been approached by supposing that the convective motions adjusted instantaneously to the pulsation. Gough (1977) has recently proposed, in the case of the Boussinesq approximation, a two-time dependent mixing length theory. One of the drawbacks of this study is that the results depend on which type of mixing length theory is applied, that of Gough (1965) or the one of Unno (1967). 
The following study is inspired by transformations widely used in the field of cosmology and particularly cosmological turbulence. These transformations are due originally to Kurskov and Ozernoi (1976). Drury and Stewart (1976), in the case of a Robertson metric with $k=0$, show that the equations describing the matter-radiation field in the early universe, both before and after recombination can be transformed to the usual familiar hydrodynamic equations (at least as far as continuity and momentum equations are concerned, they do not give any result for the energy equation). Spiegel (1977a - b) has shown how these transformations can be applied to a newtonian cosmology and has specially emphasized the analogy with hydrodynamics when the equations are rescaled. We shall follow these ideas throughout this work.

In fluid dynamics the problem of convection in presence of pulsation has been given very little attention. Venezian (1969), Rosenblat and Tanaka (1971), Rosenblat and Herbert (1970) have treated the case of the effect of an oscillating temperature field but it is a different problem from the astrophysical problem.

\section{Equations}

We will always consider a spherical star and we will do all our scaling in this case, it is a point that we will emphasize again later on when we will consider a plane parallel assumption. For the sake of generality, we start with the full compressible equations. We will consider spherical coordinates at the center of the star and we will call $(r, \theta, \varphi)$ the coordinates.

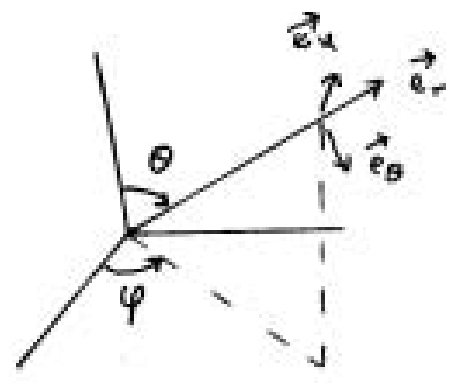

The equations are

Continuity equation

$$
\frac{\partial \rho}{a t}+\nabla \cdot(\rho \vec{v})=0
$$

Momentum equation

$$
\rho\left[\frac{\partial \vec{v}}{\partial t}+\vec{v} \cdot \nabla \vec{v}\right]=-\nabla p-\frac{6 \eta_{\eta} \rho}{n^{2}} \overrightarrow{e_{n}}+\nabla \cdot(\eta \vec{T})
$$

where, in Cartesian coordinates:

$$
\begin{aligned}
T_{i k} & =\frac{\partial v_{i}}{\partial x_{k}}+\frac{\partial v_{k}}{\partial x_{i}}+\frac{1}{3} \delta_{i k} \frac{\partial v_{k}}{\partial x_{k}} \\
\text { Let } \quad S_{i k} & =\frac{\partial v_{i}}{\partial x_{k}}+\frac{\partial v_{k}}{\partial x_{i}}
\end{aligned}
$$

In spherical coordinates: 


$$
\begin{aligned}
& S_{n \pi}=2 \frac{\partial v_{n}}{\partial \eta} \\
& S_{\eta \theta}=\frac{1}{\partial v_{\theta}}-\frac{v_{\theta}}{\eta \eta} \theta \\
& S_{\eta \varphi}=\frac{1}{n \sin \theta} \frac{\partial v_{\eta}}{\partial \varphi}-\frac{v_{\varphi}}{\eta}+\frac{\partial v_{\varphi}}{\partial \eta} \\
& S_{\theta \theta}=\frac{2}{\eta}\left[\frac{\partial v_{\theta}}{\partial \theta}+v_{\eta}\right] \\
& S_{\theta \varphi}=\frac{1}{\eta \sin \theta} \frac{\partial v_{\varphi}}{\partial \varphi}-\frac{\cot \theta}{n} v_{\varphi}+\frac{1}{n} \frac{\partial v_{\varphi}}{\partial \theta} \\
& S_{\varphi \varphi}=\frac{2}{n \sin \theta} \frac{\partial v_{\varphi}}{\partial \varphi}+\frac{2}{n} v_{\eta}+\frac{2 \cot _{g} \theta}{\eta} v_{\theta}
\end{aligned}
$$

Energy_equation

$$
\rho C_{p}\left[\frac{\partial T}{\partial t}+\vec{v} \cdot \nabla T\right]-\left[\frac{\partial p}{\partial t}+\vec{v} \cdot \nabla_{p}\right]=\sim .(A D T)+\eta \Phi
$$

We have supposed that the two coefficients of viscosity were equal to $\eta \cdot \lambda$ is the thermal conductivity.

$m_{\tau}$ is the mass contained inside the radius $\imath$. If we suppose that the star always keeps a spherical symmetry, then:

$$
\frac{d m_{n}}{d r}=4 \pi r^{2} \rho
$$

$\Phi$ is the dissipation:

$$
\Phi=\frac{1}{2}\left(s_{i \hbar}-\frac{2}{3} S_{i \hbar} \nabla \cdot 3\right)+(\nabla \cdot \vec{v})^{2}
$$

Finally we need an equation of state, we take:

$$
p=\frac{\rho k T}{4 m_{H}}
$$

where $k$ is the Boltzmann constant, $\mu$ the mean molecular weight and $m_{H}$ the mass of the hydrogen atom. The equation for $\mu$ is

$$
\frac{\partial \mu}{\partial t}+\vec{v} \cdot \nabla \mu=0
$$

when we have no nuclear reaction.

We have neglected radiation effects except for the fact that we allow for very high thermal conductivity $\lambda$ due to the radiative conductivity so that the relevant stellar case will be in the limit of small Prandtl number.

Homology

We are now going to apply a homology transformation to the previous equations analologous to the one applied to treat the problem of cosmic turbulence. Let us recall the main features of this transformation as Spiegel (1977a-b) treats it.

The coordinate $\vec{r}$ is scaled by a time factor:

$$
\vec{r}=R(t) \vec{x}
$$


$R(t)$ is in cosmology the cosine scale factor. Then:

$$
\dot{\vec{r}}=\dot{R}(t) \vec{x}+R(t) \dot{\vec{x}}=\frac{\dot{R}(t)}{R(t)} \vec{r}+R(t) \dot{\vec{x}}
$$

and the velocity can be written:

$$
\vec{v}=\vec{r}=H(t) \vec{r}+\vec{v}_{1}=\vec{v}_{0}+\overrightarrow{v_{l}}
$$

where $H(t)$ is the Hubble function and $\vec{V}_{1}$ corresponds to the velocity with respect to the expanding medium. $\overrightarrow{v_{0}}$ is the expansion velocity. $\overrightarrow{v_{i}}$ can be considered as a velocity field describing the convection process.

If we now use (13) in (1) and (2), we obtain:

$$
\begin{gathered}
\frac{\partial \rho}{\partial t}+\nabla \cdot\left(\rho \vec{v}_{1}\right)+\nabla \cdot\left(\rho \vec{v}_{0}\right)=0 \\
\rho\left[\frac{\partial \vec{v}_{1}}{\partial t}+\vec{v}_{1} \cdot \nabla_{r_{r}} \vec{v}_{1}+H \vec{r} \cdot \nabla_{R} \vec{v}_{1}\right]=-\frac{\partial \vec{v}_{e}}{\partial t}-\vec{v}_{0} \cdot \nabla_{h} \vec{v}_{0}-\nabla p-\frac{G m_{n} \rho}{\eta^{\tau}} \overrightarrow{e_{r}}+\nabla \cdot(\eta \vec{T})
\end{gathered}
$$

where the expansion velocity is alsa taken out in $\vec{T}$. We apply the same treatment to Eq. (6). This homology can also be pictured by the fact that the mean part of the radial velocity is taken to be:

$$
\overline{v_{n}}=H(t) n
$$

where the average means an average over the surface of the star. Following Spiegel (1972b) we will scale the density and the velocity by:

$$
\begin{aligned}
& \rho(\vec{r}, t)=\frac{\rho(\vec{x}, t)}{R^{2}(t)} \\
& \vec{v}_{1}(\vec{r}, t)=\frac{\vec{\mu}(\vec{r}, t)}{R(t)} \\
& \mu(\vec{r}, t)=\mu_{1}(\vec{x}, t)
\end{aligned}
$$

The scaling of the density is suggested by the fact that in cosmology the mass of the Universe is constant, so

$$
\rho R^{3}=\text { const. }
$$

Then, if a new time $\tau$ is introduced:

$$
\alpha \tau=\frac{d t}{R^{2}(t)}
$$

and if one transforms all gradients with respect to $\vec{r}$ in gradients with respect to $\vec{x}$ :

$$
\begin{aligned}
\left(\frac{\partial}{\partial t}\right)_{\vec{r}} & =\left(\frac{\partial}{\partial t}\right)_{\vec{x}}-H \vec{x} \cdot \nabla_{\vec{x}} \\
\nabla_{\vec{r}} & =\frac{1}{R} \nabla_{\vec{r}}
\end{aligned}
$$

One obtains in the cosmological case equations which look like hydrodynamic equations. Here we have also to consider a temperature and pressure scaling; the momentum equation suggests the following scaling for $p$ :

$$
P(\vec{\imath}, t)=\frac{P(\vec{x}, t)}{R^{S}(t)}
$$

The scaling for $T$ is then obtained through the equation of state (9)

$$
T(\vec{r}, t)=\frac{T,(\vec{x}, t)}{R^{2}(t)}
$$


Our scaline hypothesis would be useful only if we were able to suppose:

$$
\begin{aligned}
& \vec{T}_{1}(\vec{x}, t)=T_{1}(x) \\
& \vec{p}_{1}(\vec{x}, t)=\vec{F}_{1}(x)
\end{aligned}
$$

but further developments of the equations show that this is not possible in general (we have to consider the mean equations for showing that). Thus we lose the benefit of scaling the temperature and the pressure a priori. Given that fact, we will keep the temperature and pressure non-scaled. Moreover, the $\tau$ time dependence which conforms to the cosmological equivalent of the problem where $\tau$ can be given a physical meaning (difference between cosmic time and proper time of a particle) is not suitable here. It is not convenient because of the difficulty of getting the relation $R(\tau)$. What we impose is $R(t)$, that is to say the mean movement of the star, we have then to find $R(\tau)$ by integrating and inverting the equation $t=t(\tau)$; this is practically impossible analytically and very limiting for getting any theoretical results. So we will use everywhere the real time variable $t$.

Our hypothesis for the homology will be that:

$$
\vec{v}=H \vec{r}+\vec{v}_{1} \text { with } H=\frac{\dot{R}}{R}
$$

and

$$
\rho(\vec{r}, t)=\frac{\rho_{1}(\vec{x}, t)}{R^{3}} \text { with } \vec{\rho}_{1}(\vec{x}, t)=\bar{\rho}_{1}(x)
$$

where $\bar{S}_{1}$ means an average over the surface of the Star. (If we have a quantity $q$, its average over the surface of the star at the radius $r$ will be

$$
\bar{q}(\Omega, t)=\frac{1}{4 \pi} \int_{\varphi=0}^{2 \pi} \int_{\theta=0}^{\pi} q(\tau, \theta, \varphi, t) \sin \theta d \theta d \varphi
$$

It is the second hypothesis on $P$ which looks to us the more restrictive but it will allow an easier treatment of the coupling. We get for the momentum equation:

$$
\begin{aligned}
& \frac{\rho_{1}}{R}\left[\left(\frac{\partial \vec{v}}{\partial t}\right)_{\vec{\tau}}+\dot{H} \vec{r}+H \vec{r} \cdot \frac{\nabla_{x} \vec{v}_{i}}{R}+H^{2} \vec{r}+H \vec{v}_{i}+\frac{\vec{v}_{i} \cdot \nabla_{x} \vec{v}_{i}}{R}\right]=-\frac{\nabla_{x} p}{R}-\frac{G M_{h} \rho}{R^{2} x^{2}} \vec{e}_{r}+\eta \nabla \cdot \vec{T} \\
& \text { and } \quad\left(\frac{\partial \vec{v}_{i}}{\partial t}\right)_{\vec{r}}=\left(\frac{\partial \vec{v}_{i}}{\partial t}\right)_{\vec{x}}-H \vec{x} \cdot \nabla_{x} \vec{v}_{i}
\end{aligned}
$$

and

We can verify here that the scaling chosen for $\vec{v}_{1}$ is the only one possible. We have to make the term $H \vec{v}_{1}$ disappear in the previous equation. Let us suppose:

$$
\vec{v}_{1}=\frac{\vec{Z}}{R^{\alpha}}
$$

then: $\left.\begin{array}{rl}\left(\frac{\partial \vec{v}_{i}}{\partial t}\right)_{\vec{x}} & =\frac{1}{R^{\alpha}} \frac{\partial \vec{u}}{\partial t}-\frac{\alpha \dot{R}}{R^{\alpha+1}} \vec{u} \\ H \vec{v}_{i} & =\frac{H \vec{u}}{R^{\alpha}}=\frac{\dot{R}}{R^{\alpha+1}} \vec{u}\end{array}\right\} \Rightarrow \alpha=1$ and $\vec{v}_{i}=\frac{\vec{u}}{R}$

If we scale $\nabla \cdot \vec{T}$ with this new variable, we obtain: 


$$
\nabla \cdot T=\frac{l}{R^{3}} \frac{\partial}{\partial x_{k}}\left[\frac{\partial u_{i}}{\partial x_{k}}+\frac{\partial u_{k}}{\partial x_{i}}+\frac{1}{3} \delta_{i \hbar} \frac{\partial u_{e}}{\partial x_{e}}\right]
$$

and the momentum equation becomes:

$$
\begin{aligned}
\frac{\rho_{1}}{R^{3}}\left[\frac{1}{R} \frac{\partial \vec{u}}{\partial t}+\frac{\vec{u} \cdot \nabla_{x} \vec{u}}{R^{3}}+\left(\dot{H}+H^{2}\right) R \vec{x}\right] & =-\frac{\nabla_{x p}}{R}-\frac{G m_{n} \rho_{1}}{R^{5} x^{2}} \vec{e}+\frac{\eta}{R^{3}} \frac{\partial}{\partial x_{k}}\left[\frac{\partial u_{i}}{\partial x_{n}}+\frac{\partial u_{i n}}{\partial x_{i}}+\frac{1}{3} \delta_{i n} \frac{\partial u_{e}}{\partial x_{i}}\right] \\
\dot{H}+H^{2} & =\left(\frac{\dot{R}}{R}\right)^{*}+\left(\frac{\dot{R}}{R}\right)^{2}=\frac{\ddot{R}}{R}
\end{aligned}
$$

Let us now transform the continuity equation:

$$
\left(\frac{\partial \rho_{1}}{\partial t}\right)_{\vec{x}}+\frac{1}{R^{2}} \nabla_{x} \cdot\left(\rho_{1} \vec{u}\right)=0
$$

We transform the energy equation in the same way

$$
\begin{aligned}
& \eta \Phi=\frac{\eta}{2} \frac{1}{R^{4}}\left(\frac{\partial u_{i}}{\partial x_{k}}+\frac{\partial u_{k}}{\partial x_{i}}-\frac{2}{3} \delta_{i k} \frac{\partial u_{k}}{\partial x_{l}}\right)^{2}+9 \eta H^{2}+\frac{6 \eta H}{R^{2}} \frac{\partial u_{k}}{\partial x_{l}}+\frac{\eta}{R^{4}}\left(\frac{\partial u_{\ell}}{\partial x_{l}}\right)^{2}
\end{aligned}
$$

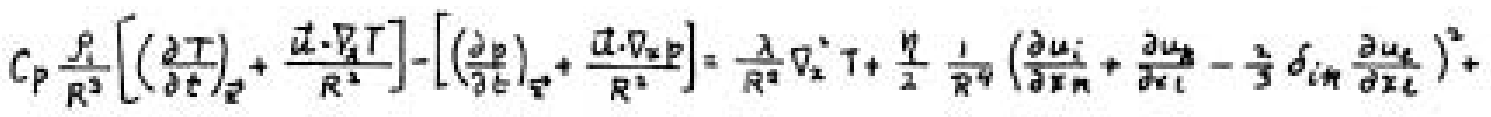

$$
\begin{aligned}
& +9 \eta H^{2}+\frac{6 \eta H}{R^{2}} \frac{\partial u_{k}}{\partial x_{t}}+\frac{\eta}{R^{4}}\left(\frac{\partial u_{t}}{\partial z_{e}}\right)^{2}
\end{aligned}
$$

We have considered all the terms with derivatives of $\eta$ and $\lambda$ negligible, but in general we take $\eta$ and $\lambda$ variable with density and temperature. We see an effective gravity term appearing in (32):

$$
-\frac{G m_{2} \rho_{1}}{R^{5} x^{2}} \hat{x}-\frac{\ddot{R}}{R^{3}} \rho_{i} x \hat{x}
$$

The remaining equations become:

$$
\begin{gathered}
\frac{d \eta_{2}}{d x}=4 \pi x^{2} \rho_{1} \\
p=\frac{k}{\mu m_{H}} \frac{\rho_{1} T}{R^{3}} \\
\left(\frac{\partial \psi}{\partial t}\right)_{\vec{x}}+\frac{\vec{u} \cdot \nabla_{x} \psi}{R^{2}}=0
\end{gathered}
$$

The equations above are far more complicated than in the cosmological case because of the presence of the temperature and pressure gradients. We will see that when we will introduce next mean and fluctuating equations, we will get still more complicated equations that do not appear in cosmology because in that case one is not interested in fluctuations of density and temperature.

Averaged and fluctuating equations

We average the equations over the sphere of radius 7 . We then subtract the averaged equations from the initial equations to get the fluctuating equations.

$$
\rho_{1}=\bar{\rho}_{1}(x)+\rho_{1}^{\prime}(\vec{x}, t)
$$




$$
\begin{aligned}
& p=\bar{p}(x, t)+p^{\prime}(\vec{x}, t) \\
& T=\bar{T}(x, t)+T^{\prime}(\vec{x}, t) \\
& \eta_{h}=\bar{\eta}_{1}(x)+m_{r}^{\prime}(\vec{x}, t) \\
& \psi=\bar{\psi}(x)+\psi^{\prime}(\vec{x}, t)
\end{aligned}
$$

We average the equations (33), (32), (35), (37), (38), (39)

$$
\nabla_{x} \cdot\left(\overline{\rho_{i}^{\prime} \vec{u}}\right)=0
$$

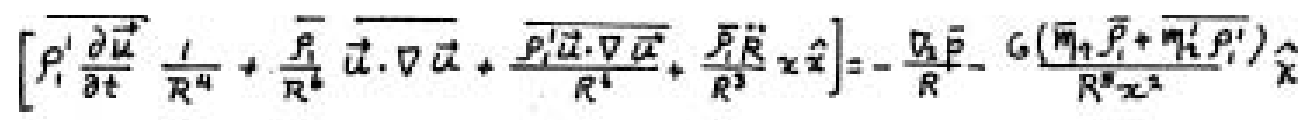

$$
\begin{gathered}
C_{p}\left\{\frac{\bar{\rho}_{1}}{R^{o}}\left(\frac{\partial \vec{T}_{1}}{\partial t}\right)_{\vec{x}}+\frac{\rho_{1}^{\prime}}{R^{3}}\left(\frac{\partial T^{\prime}}{\partial t}\right)_{\vec{x}^{2}}+\frac{1}{R^{2}}\left[\overline{\rho_{1}} \overline{\vec{u} \cdot \nabla_{x} T^{\prime}}+\overline{\rho_{r}^{\prime} \vec{u}} \cdot \nabla_{x} \bar{T}+\overline{\rho_{1}^{\prime} \vec{u} \cdot \nabla_{x} T^{\prime}}\right]\right\}- \\
-\left[\left(\frac{\partial \bar{p}}{\partial t}\right)_{\vec{x}^{\prime}}+\frac{\overline{\vec{u} \cdot \nabla_{x} p^{\prime}}}{R^{2}}\right]=\frac{\lambda}{R^{2}} \nabla_{x}^{3} \bar{T}+\eta \bar{\Phi}
\end{gathered}
$$

where

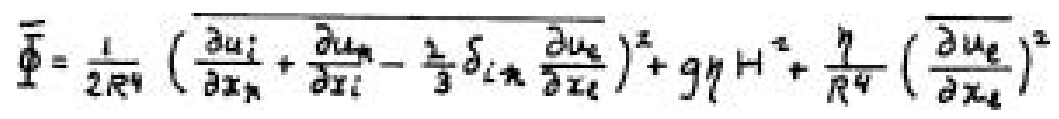

$$
\begin{aligned}
& \frac{d \bar{\eta}_{2}}{d x}=4 \pi x^{2} \bar{\rho}_{1} \\
& \bar{p} \bar{Y}+\overline{p^{\prime} Y^{\prime}}=\frac{k}{m H} \frac{1}{R^{3}}\left(\overline{\rho_{1}} \bar{T}+\overline{\rho_{1}^{\prime} T^{\prime}}\right) \\
& \overline{\vec{u} \cdot \nabla_{x} \psi^{\prime}}=0
\end{aligned}
$$

Then we deduce the fluctuation equations:

$$
\begin{aligned}
& \left(\frac{\partial \rho_{i}^{\prime}}{\partial t}\right)_{\vec{x}^{\prime}}+\frac{1}{R^{2}}\left[\nabla_{x} \cdot\left(\bar{\rho}_{1} \vec{u}\right)+\nabla_{x} \cdot\left(\rho_{1}^{\prime} \vec{u}\right)-\nabla_{x} \cdot \overline{\left(\rho_{1}^{\prime} \vec{u}\right)}\right]=0
\end{aligned}
$$

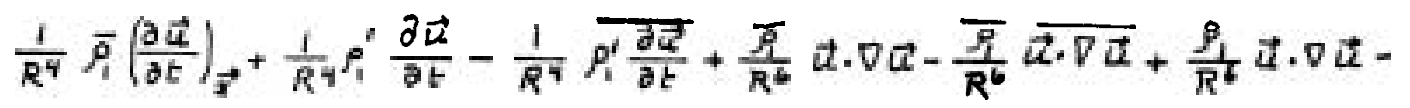

$$
\begin{aligned}
& =\frac{\overline{\rho_{1}^{\prime}} \vec{u} \cdot \nabla \vec{u}}{R}+\frac{\rho_{1}^{\prime} \ddot{R}}{R^{3}} \times \hat{x}=-\frac{\nabla_{1} p^{\prime}}{R}-\overline{R^{s} \times x^{2}}\left[\overline{\eta_{l}} \rho_{1}^{\prime}+\eta_{\tau}^{\prime} \overline{\rho_{1}}+\eta_{\tau}^{\prime} \rho_{1}^{\prime}-\overline{\overline{\eta_{\tau}^{\prime} \rho_{1}^{\prime}}}\right] \hat{x}+ \\
& +\frac{\eta}{R^{2}} \frac{\partial}{\partial x_{k}}\left[\frac{\partial u_{i}}{\partial x_{k}}+\frac{\partial u_{k}}{\partial x_{i}}+\frac{1}{3} \delta_{i k} \frac{\partial u_{k}}{\partial x_{i}}\right] \\
& \frac{d n_{1}^{\prime}}{d x}=4 \pi x^{2} \rho_{1}^{\prime} \\
& \bar{p} \psi^{\prime}+\bar{\psi} p^{\prime}+\mu p^{\prime}-\overline{\mu^{\prime} p^{\prime}}=\frac{k}{m_{H}} \frac{1}{R^{S}}\left(\rho_{1}^{\prime} \bar{T}+\bar{\rho} T_{1}^{\prime}+\rho_{1}^{\prime} T^{\prime}-\overline{\rho_{1}^{\prime} T^{\prime}}\right)
\end{aligned}
$$

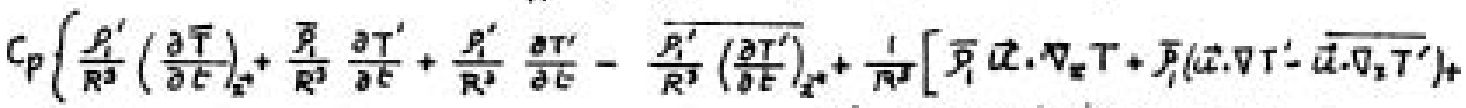

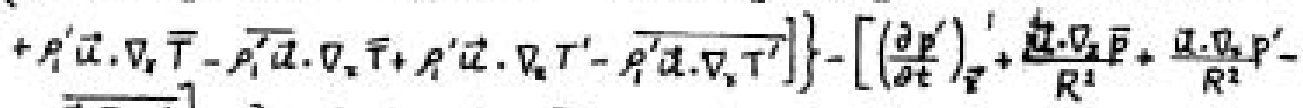

$$
\begin{aligned}
& \left.-\frac{\overline{\underline{A} \cdot \nabla_{x} p^{\prime}}}{R^{2}}\right]=\frac{\lambda}{R^{2}} \nabla_{x}^{2} T^{\prime}+\eta(\Phi-\bar{\Phi})
\end{aligned}
$$




$$
\frac{\partial \mu^{\prime}}{\partial t}+\frac{\vec{u} \cdot \nabla_{x} \bar{\mu}}{R^{2}}+\frac{\vec{u} \cdot \nabla_{x} \mu^{\prime}}{R^{2}}-\frac{\overline{\vec{u} \cdot \nabla_{x} \mu^{\prime}}}{R^{2}}=0
$$

As it has already been mentioned these equations differ much from the cosmological ones because of the introduction of fluctuating quantities.

In the kind of problem we are primarily interested in: how pulsation may provoke convection, we will assume that we know $R$, that is to say, we impose the mean movement. We look, then, at the behavior of the fluctuating quantities. This is very different from the cosmological case where $R$ is an unknown to be found.

"Pulsostatic" equilibrium

We look at a solution of the mean equations for which:

$$
\vec{u}=0 ; \quad \rho^{\prime}=0 ; \quad T^{\prime}=0 ; \quad P^{\prime}=0 ; \quad \mu^{\prime}=0
$$

We will write these mean equations for a plane parallel layer case and suppose that: $g=\frac{a m_{\eta}}{x^{2}}=$ const.

$$
\begin{aligned}
& \text { We get: } \bar{\rho}_{i} \frac{\ddot{R}}{\bar{R}^{2}} \vec{x}=-\frac{\nabla_{2} \bar{p}}{R}-\frac{G\left(\bar{\eta}_{r} \bar{\rho}_{1}\right)}{R^{5} x^{2}} \hat{x} \\
& c_{P} \frac{\bar{P}}{R^{3}}\left(\frac{\partial \vec{T}_{i}}{\partial t}\right)_{\vec{x}}-\left(\frac{\partial \beta}{\partial t}\right)_{z}=\frac{\lambda}{R^{2}} \nabla_{x}^{2} \vec{T}_{+} g \eta^{2} \\
& \bar{p}=\frac{\hbar}{\mu-m_{H}} \frac{\vec{\rho}_{1} \bar{T}}{R^{3}}
\end{aligned}
$$

and

$$
\mu=\bar{\mu}(x)
$$

We may notice here that since we have introduced in $\vec{V}=H \vec{r}+\frac{\vec{u}}{\vec{R}}$ two unknowns $R$ and $\vec{Q}$, we have one equation less than necessary for closing the system. $R$ has to be an exterior datum. We wil1 write:

or

$$
\nabla_{x} \bar{p}=-\hat{\rho}_{i}\left[\frac{\ddot{R}}{R^{2}} x+\frac{g}{R}+\hat{x}\right.
$$

where:

$$
\nabla \times \vec{p}=-\vec{\rho}_{1}(x) g_{e f f}(t) \hat{x}
$$

$$
g_{e f f}(t)=\frac{\ddot{R}}{R^{2}} x+\frac{g}{R^{4}}
$$

Linearized eauations

Even with an homology hypothesis, the problem is still quite formidable, so we will first investigate the linear stability problem. We suppose that $\rho^{\prime}, \vec{u}, T^{\prime}, p^{\prime}$ are small and we keep only the linear contributions.

$$
\begin{aligned}
& \left(\frac{\partial \rho_{1}^{\prime}}{\partial t}\right)_{\vec{x}}+\frac{1}{R^{2}} \nabla_{2} \cdot\left(\bar{\rho}_{1} \vec{u}\right)=0 \\
& \frac{1}{R^{4}} \bar{P}_{1}\left(\frac{\partial \vec{u}}{\partial t}\right)_{\vec{x}}+\frac{\rho^{\prime} \ddot{R}}{R^{3}} \times \hat{x}=-\frac{\nabla_{k} p^{\prime}}{R}-\frac{g \rho_{i}^{\prime}}{R^{3}} \hat{x}-\frac{G \eta_{i}^{\prime} \bar{P}_{i}}{R^{3} x^{2}} \hat{x}+\frac{\eta}{R^{3}} \frac{\partial}{\partial x_{k}}\left[\frac{\partial u_{i}^{\prime}}{\partial x_{k}}+\frac{\partial u_{k}}{\partial x_{i}}+\frac{1}{3} \delta_{i k} \frac{\partial u_{k}}{\partial x_{i}}\right]
\end{aligned}
$$




$$
\begin{gathered}
C_{p} \frac{\rho_{1}^{\prime}}{R^{3}}\left(\frac{\partial \bar{T}}{\partial t}\right)_{\vec{z}}+C_{p} \frac{\bar{g}}{R^{3}} \frac{\partial T^{\prime}}{\partial t}+\frac{C_{p}}{R^{3}} \overline{P_{1}} \vec{u} \cdot \nabla_{x} \vec{T}-\left(\frac{\partial p^{\prime}}{\partial t}\right)_{x}-\frac{\vec{u} \cdot \nabla_{x} \bar{p}}{R^{2}}=\frac{\lambda}{R^{2}} \nabla_{x}^{2} T^{\prime}+\frac{6 \eta H}{R^{2}} \frac{\partial u_{e}}{\partial x_{e}} \\
\rho^{\prime} \bar{\mu}+\bar{p} \mu^{\prime}=\frac{k}{m_{H}} \frac{1}{R^{3}}\left(\rho_{1}^{\prime} \bar{T}+\bar{\rho}_{1} T^{\prime}\right) \\
\frac{\partial \mu^{\prime}}{\partial t}+\frac{\vec{u} \cdot \nabla_{z} \vec{\mu}}{R^{3}}=0
\end{gathered}
$$

Boussinesq approzimation

We are going to assume that we have plane parallel layers, that is to say that we look to our fluctuating equations when:?d $<$ \& 1 where $d$ is the thickness of the layer that we study and $r$ its distance from the center of the star.

Let us notice that it is very important to make the plane parallel layer assumption after the scaling of 4 in the spherical case because in that way, we keep fundamental spherical behaviors. The density has been scaled like $\frac{P l}{R^{3}}$ and the $R^{3}$ factor stays even in the plane parallel case; if we had taken directly a plane parallel layer and made it expand, then the density would have scaled as, in that case $\rho R=$ const.) and we would have lost a fundamental feature of the problem.

For the Boussinesq approximation (Spiegel and Veronis 1961) we neglect the fluctuating mass term and set:

$$
p^{1}=0 \quad \nabla \cdot \vec{u}=0
$$

We will call ze the vertical coordinate, we call $z_{0}$ the altitude of the layer, so, inside the layer

$$
z=z_{0}+z^{\prime} \text { and } \frac{z^{\prime}}{z_{0}} \ll 1
$$

We will keep only the a, contribution in the mean quantities of the equation, so we will have:

$$
\bar{T}(z, t) \simeq \bar{T}\left(z_{0}, t\right) ; \bar{\rho}_{1}(z)=\bar{\rho}_{1}\left(z_{0}\right) \text { etc... }
$$

The equations become:

$$
\begin{aligned}
& \frac{1}{R^{4}} \bar{\rho}_{1}(z)\left(\frac{\partial \vec{u}}{\partial t}\right)_{\vec{x}}=-\frac{\nabla_{n} p^{\prime}}{R}-\frac{g f^{\prime}}{R^{3}} \hat{z}-\frac{f^{\prime}}{R^{2}} \ddot{R} z, \hat{z}+\frac{\eta}{R^{2}} \frac{\partial^{2} u_{i}}{\partial x_{k}{ }^{2}}
\end{aligned}
$$

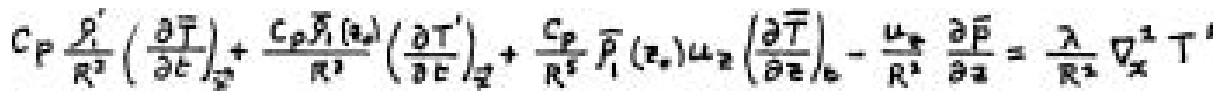

Saying that the pressure fluctuations are zero in (65) gives:

$$
\bar{P} \mu^{\prime}=\frac{k}{m_{H}} \frac{1}{R^{3}}\left(\rho_{i}^{\prime} \bar{T}+\bar{\rho}_{1}\left(z_{0}\right) T^{\prime}\right)
$$

but, neglecting the quadratic terms in the mean part of Eq. (9), we obtain

$$
\vec{p}=\frac{k}{m_{H} \bar{P}} \frac{\overline{p_{R}}}{R^{3}} \bar{T}
$$

and:

$$
\rho_{1}^{\prime} \bar{T}+\bar{\rho}_{1}\left(x_{n}\right) T^{\prime}=\frac{\mu^{\prime}}{\bar{\mu}} \bar{\rho}_{1} \bar{T}
$$


So that: $\quad \rho_{1}^{\prime}=\frac{\mu^{\prime}}{\bar{\mu}} \bar{\rho}_{1}-\bar{\rho}_{1}\left(\mu_{0}\right) \frac{T^{\prime}}{\bar{T}}$

and we can eliminate $\rho^{\prime}$ in (68) and (69). Let us go back to the "pulsostatic" equations. We have to express $\left(\frac{\partial \vec{f}}{t}\right)_{\vec{z}}$

We have: $\quad \frac{\partial \vec{p}}{\partial z}\left(z_{0}, t\right)=-\bar{\rho}_{1}\left(z_{0}\right) g_{e f f_{0}}(t)$

$$
\text { where: } \quad g_{\text {eff }}(t)=\frac{\ddot{R}}{R^{2}} z_{0}+\frac{g}{R^{*}}
$$

Using the equation of state (70):

$$
\left(\frac{\partial \bar{p}}{\partial t}\right)_{r}=\frac{k}{\bar{\mu} m_{H}} \bar{\rho}_{1}\left(z_{0}\right) \frac{\partial}{\partial t}\left(\frac{\bar{T}}{R^{2}}\right)=\frac{\hbar}{\bar{q} m_{H}} \bar{\rho}_{1}\left(z_{0}\right)\left[\frac{1}{R^{3}} \frac{\partial \bar{T}}{\partial t}-\frac{3 \dot{R}}{R^{H}} \bar{T}\right]
$$

and

$$
\begin{aligned}
& C_{p} \frac{\bar{P}_{1}}{R^{3}}\left(\frac{\partial \bar{T}}{\partial t}\right)_{\vec{x}}-\left(\frac{\partial \vec{p}}{\partial t}\right)_{\vec{x}}=\frac{\lambda}{R^{2}} \nabla_{x}^{2} \vec{T}+g \eta \frac{\dot{R}^{2}}{R^{2}} \\
& \text { Eliminating }\left(\frac{\partial \beta}{\partial t}\right)_{\vec{r}} \text { between the two equations, we get }
\end{aligned}
$$

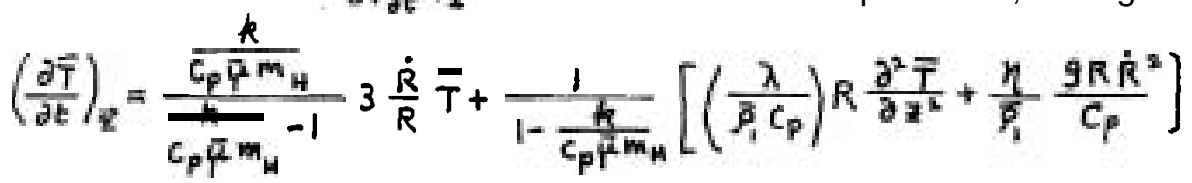

If we take all the man quantities in $\mathbf{z}_{e}$, this partial differential equation becomes an ordinary differential equation in time. We will consider

$\nu=\frac{\eta}{\mathcal{S}_{\mathrm{s}}\left(\boldsymbol{z}_{0}\right)}$ and $C_{p}$ as constants but we will allow for variations of according to:

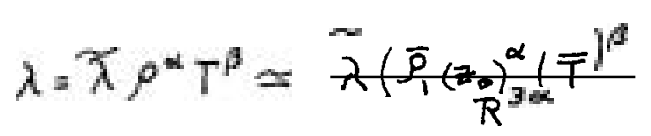

but we do not know the time dependence of $\bar{T}$.

Before restricting the study to a case where (76) can be solved easily, let us notice that the effective gravity geft. ( $t$ ) may take the value zero and negative values since $\ddot{R}$ can be of any sign. We may already predict that it is an interesting case astronomically.

Let us evaluate the parameter $\frac{k}{k^{m} \mathrm{H}}$

$$
\frac{k}{4 m_{H}} \simeq \frac{1.38 \times 10^{-16}}{10^{8} \times 2 \times 1.67 \times 10^{-24}} \simeq 0.4
$$

So since $\left(\frac{\lambda}{\overline{\bar{S}_{1}, C_{p}}}\right)$ can be very big in a star, the term in $\frac{\partial^{2} \bar{T}}{a z^{2}}$ in (76) contributes a lot.

Non-dissipative case easily.

In that case $\eta=0$ and $\lambda=0$ so that we will be able to solve (76) 
Let us call:

$$
\left(\frac{\partial \bar{T}}{\partial t}\right)_{\vec{\alpha}}=\frac{\frac{\hbar}{c_{\mu}^{m} H}}{C_{p \mu m_{\mu}}-1} 3 \frac{\dot{R}}{R} \bar{T}
$$

and:

$$
J=\frac{\frac{m}{c_{r}+m_{H}}}{\frac{m_{H}}{c^{2} m_{H}}-1}
$$

$$
\bar{T}(z, t)=\bar{T}(z, 0) \mathbb{R}^{3 J}
$$

We will suppose that and consider no effect from a fluctuating molecular weight.

The equation becomes:

$$
\begin{aligned}
& \frac{1}{R^{4}} \bar{\rho}_{1}\left(z_{0}\right)\left(\frac{\partial \vec{u}}{\partial t}\right)_{\vec{x}}=-\frac{\nabla_{x} p^{\prime}}{R}+\frac{\bar{\rho}_{1}\left(z_{0}\right)}{\left.\bar{T}_{(z,}, t\right)} T^{\prime} \frac{g_{e f f}(t)}{R} \hat{z} \\
& -3 J \frac{\dot{R}}{R^{4}} \bar{\rho}_{1}\left(z_{0}\right) T^{\prime}(z, t)+\frac{\bar{\rho}_{1}\left(z_{0}\right)}{R^{3}}\left(\frac{\partial T^{\prime}}{\partial t}\right)_{\vec{z}^{2}}+\frac{\bar{\rho}_{1}\left(t_{0}\right)}{R^{2}} u_{z}\left(\frac{1}{R}\left(\frac{\partial \bar{T}}{\partial z}\right)+\frac{2 e f f}{C_{p}}\right)=0 \\
& \text { with: } \quad g_{\text {ef }}=\frac{\ddot{R}_{z_{0}}}{R^{2}}+\frac{g}{R^{4}}
\end{aligned}
$$

Let us start to eliminate the temperature between (83) and (84). For that we first take the curl of (83) twice and keep only the $z$ component:

$$
\frac{\partial}{\partial t} \nabla_{x}^{2} u_{a}-\frac{R^{2} g_{e t t}}{\bar{T}\left(z_{0}, t\right)} \nabla_{H}^{2}\left(T^{\prime}\right)=0
$$

where $\nabla_{H}^{2}$ is the horizontal gradient: $\quad \nabla_{H}^{2}=\frac{\partial^{2}}{\partial x_{1}^{2}}+\frac{\partial^{2}}{\partial x_{z}^{2}}$ If we apply $\nabla_{H}^{2}$ to (84):

$$
\text { - } 33 \stackrel{\dot{\beta}}{\stackrel{5}{*}} \nabla_{H}^{2} T^{\prime}(z, t)+\frac{\partial}{\partial t}\left(\nabla_{H}^{2} T^{\prime}\right)+\left(\nabla_{H}^{2} u_{z}\right)\left(\frac{1}{R^{2}}\left(\frac{\partial \bar{T}}{\partial z}\right)+\frac{R g e f t}{c_{p}}\right)=0
$$

Eliminating $\nabla_{H}^{2} T^{\prime}$ between (85) and (86), we get:

$$
\begin{aligned}
& \nabla_{H}^{*} T^{\prime}=\frac{T(20, t)}{R^{3} g_{e H}(t)} \frac{a}{\partial t} \nabla_{x}^{2} \\
& -3 J \frac{\dot{R}}{R} \frac{\bar{T}\left(n_{0}, t\right)}{R^{3} g_{s H t}}\left(\frac{\partial}{\partial t} \nabla_{x}^{2} u_{s}\right)+a \bar{T}_{\left(z_{0}, t\right)} \frac{1}{R^{3} g_{e f f}} \frac{\partial}{\partial t}\left(\nabla_{x}^{2} u_{a}\right)+\bar{T}\left(z_{0}, t\right) \frac{\partial}{\partial t}\left(\frac{1}{R^{3} g_{e f t}}\right) \frac{\partial}{\partial t}\left(\nabla_{x}^{2} u_{z}\right)+ \\
& \frac{T(n}{R^{\prime} \eta_{\text {eif }}} \frac{t)}{\partial t^{2}} \nabla_{z}^{2} \cdot u_{z}+\left(\nabla_{H}^{2} u_{z}\right)\left(\frac{1}{R^{2}}\left(\frac{\partial \bar{T}}{\partial z}\right)^{*} \frac{R_{\text {gest }}}{C_{p}}\right)=0
\end{aligned}
$$

We use (81) and the first two terms of the previous equation cancel exactly. 


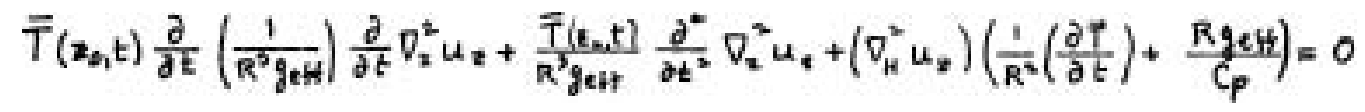

Time dependent equation

We need boundary conditions in order to solve Eq. (87). The problem itself suggests no direct physical boundary conditions. In fact this question appears rather hard and in the absence of any physical indication we will try for example free boundary conditions. For that, we assume for $u_{z}$ a solution of the form:

$$
u_{z}=f(x, y) \sin \frac{\pi}{d} z \varphi(t)
$$

where $d$ is the size of the Boussinesq layer and $f$ is such that:

$$
\nabla_{H}^{2} f=-k^{2} f
$$

(87) becomes:

$$
-\bar{T}\left(z_{0}, t\right) \frac{\partial}{\partial t}\left(\frac{1}{R^{2} g_{e s}}\right)\left(k^{2}+\frac{\pi^{2}}{d^{2}}\right) \dot{\varphi}-\frac{\bar{T}\left(c_{0} t\right)}{R^{2} g_{e t t}}\left(k^{2}+\frac{\pi^{2}}{d^{2}}\right) \ddot{\varphi}-k^{2}\left(\frac{1}{R^{2}}\left(\frac{\partial \bar{T}}{\partial z}\right)+\frac{R g_{e s t}}{C_{p}}\right) \varphi=0
$$

Let us adimensionalize this equation.

We introduce for that a nondimensional time:

$$
\widetilde{t}=\Omega t
$$

where $\Omega$ is a characteristic frequency, for instance the frequency of the pulsations of $R$, or if we do not take a pulsating $R$ a characteristic time of the evolution of $R$.

$$
g_{\text {eft }}=\frac{\ddot{R}_{z_{0}}}{R^{2}}+\frac{g}{R^{4}}=\frac{\Omega^{2} \frac{d^{2}}{d \tilde{F}^{2}} R z}{R^{2}}+\frac{\delta}{R^{4}}=\frac{g}{R^{4}}\left[1+\frac{\Omega^{2} z_{t}}{g} R^{*} \frac{d^{2} R}{d \tilde{E}^{2}}\right]
$$

If we call:

$$
\begin{aligned}
& F_{p}=\frac{3, \Omega^{2}}{g} \\
& g_{\text {eKF }}=g \frac{G}{R^{4}}
\end{aligned}
$$

where:

$$
G=1+F_{p} R^{2} \ddot{R}
$$

$F_{p}$ is a pulsational Froude number. The Eq. (90) becomes:

$$
\ddot{\varphi}+\left(\frac{\dot{R}}{R}-\frac{\dot{G}}{G}\right) \dot{\varphi}+\frac{a^{2}}{\pi^{2}+a^{2}} \frac{G}{R^{\prime}} \frac{g}{\Omega^{2} T\left(z_{,}, t\right)}\left(\left(\frac{\partial \bar{T}}{\partial z}\right)+\frac{g G}{C_{p} R}\right) \varphi=0
$$

where all the time derivatives are with respect to ' $t$ '.

$$
a=k d
$$

$G$ is the nondimensionalized effective gravity. For making the connection with cosmology, we may notice that: where $q_{0}=-\frac{\ddot{R} R}{R^{2}}$ and $H_{e}=\frac{\dot{R}}{R}$ are known respectively in cosmology as the 
adimensionalized Hubble function and the deceleration parameter.

But in (95), $\bar{T}$ is depending on time and we are obliged to introduce a time dependent "Rayleigh number" (the Rayleigh number is ordinarily defined in the dissipative case):

$$
\Lambda(\tilde{E})=-\frac{8}{\bar{T}\left(\mathbf{v}_{\theta} t\right) \Omega^{*}}\left(\left(\frac{\partial \bar{T}}{\partial z}\right)+\frac{g G}{R C_{p}}\right.
$$

Then the equation becomes:

$$
\ddot{\varphi}+\left(\frac{R}{R}-\frac{\dot{G}}{G}\right) \dot{\varphi}-\frac{a^{2}}{\pi^{2}+a^{2}} \frac{\Lambda(\tilde{E})}{R^{3}} \varphi=0
$$

and:

$$
N(\tilde{t})=-\frac{g^{G}}{\Omega^{1} \bar{T}\left(z_{n}, 0\right)}\left(\frac{d \bar{T}}{d z}\left(z_{z}, 0\right)+\frac{g^{G}}{C_{p} R^{1+13}}\right)
$$

With the numerical example taken in (78), we get:

$$
J=-\frac{2}{3}
$$

and:

$$
\hat{T}\left(z_{*}, t\right) \approx \frac{\bar{T}\left(z_{e}, 0\right)}{R^{2}}
$$

that is what we would expect from the temperature in an adiabatically expanding sphere of gas. We see that if $G$ goes to zero, we get a singularity. More specifically, let us write the equation in the case where:

$$
R=1+\varepsilon \sin \tilde{E}
$$

Then:

$$
G=1-F_{p}(1+\varepsilon \sin \tilde{E})^{2} \varepsilon \sin \tilde{E}=-F_{p} \varepsilon^{3} \sin ^{3} \tilde{E}-2 F_{p} \varepsilon^{2} \sin ^{2} E-\varepsilon F_{p} \sin \tilde{E}+1
$$

We will obviously suppose that $\varepsilon$ is always less than one. Then we may distinguish two regions in the $\left(\varepsilon, F_{p}\right)$ plane: one where $G$ is always strictly positive for any time and one where $G$ has a sign which can vary with time.

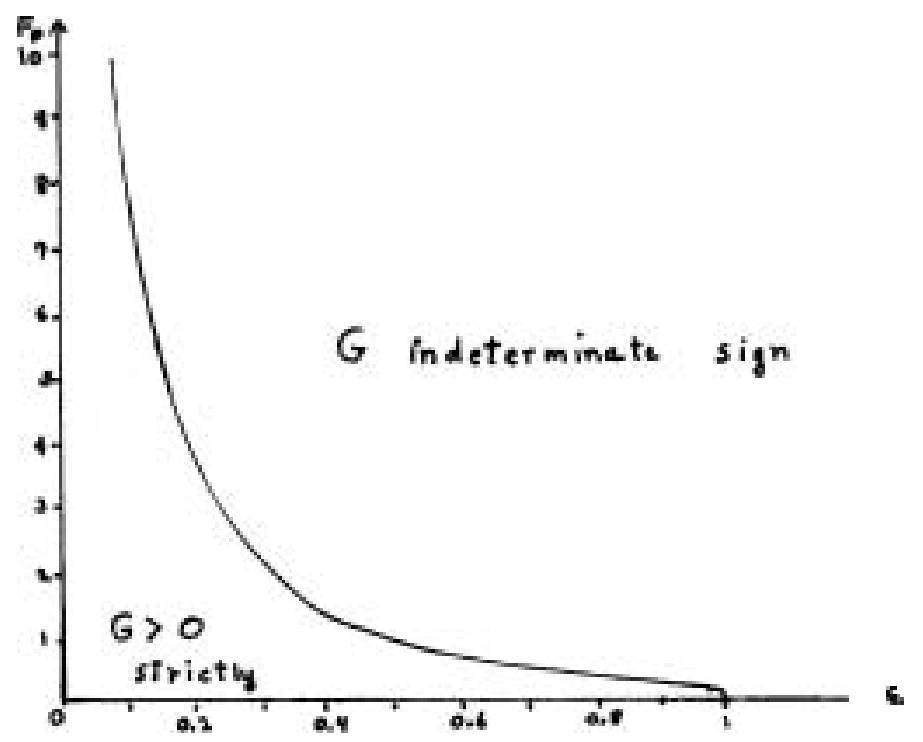

It is given by the condition that: $-F_{p \varepsilon^{3}}-2 F_{p c^{2}}-F_{p \varepsilon}+1>0$ which gives:

$$
F_{p}<\frac{1}{\varepsilon(\varepsilon+1)^{2}}
$$


We will consider only the case where stays positive. For small $\varepsilon$, the equation can be developed to any order of $\varepsilon-T_{0}$, order $\varepsilon^{2}$, we get: $\ddot{\varphi}+\left[\varepsilon\left(1+F_{p}\right) \cos \tilde{E}+\varepsilon^{2}\left(-\frac{1}{2}+\frac{F_{p}^{2}}{2}+2 F_{p}\right) \sin 2 \tilde{E}\right] \dot{\varphi}-\frac{a^{2}}{\pi^{2}+a^{2}}\left[\Lambda_{0}+\frac{\varepsilon^{2} \Lambda_{2}}{2}-\varepsilon \sin \tilde{E} \Lambda_{1}-\frac{\varepsilon^{2} \cos 2}{2} \tilde{F}_{\Lambda_{2}}\right] \varphi_{(100)}$

with: $\Lambda_{0}=-\frac{g}{\left.\Omega^{2}+z_{0}, 0\right)}\left(\frac{d \vec{T}}{d z}\left(z_{e}, 0\right)+\frac{g}{c_{p}}\right)$

$$
\begin{aligned}
& \Lambda_{1}=-\frac{g}{\Omega^{1}+\left(v_{0} 0\right)}\left[\frac{d T}{d z}\left(r_{0,0}\right)\left(F_{p}+3\right)+\frac{g}{c_{p}}\left(4+3 J+2 F_{p}\right)\right]
\end{aligned}
$$

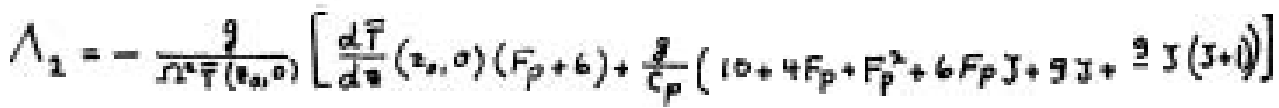

This equation, of the form: $\ddot{\varphi}+2 p \dot{\varphi}+q \varphi=0$ can be transformed to the form $\ddot{\psi}+M(\tilde{E}) \Psi \equiv 0$

$(101$

by the transformation:

$$
\begin{aligned}
& \varphi=\psi e^{-\int \frac{E}{\tilde{E}} d t} \text { with } M(E)=q-p^{2} \\
& M(\tilde{E})=\left[-\frac{a^{2}}{\pi^{2}+a^{2}}\left(\Lambda_{0}+\frac{\varepsilon^{2} A_{2}}{2}\right)-\frac{\varepsilon^{2}}{g}\left(1+F_{p}\right)^{2}\right]+\varepsilon \sin E\left[\frac{\Lambda_{1} \alpha^{2}}{\pi^{2}+\alpha^{2}}+\frac{1+F_{e}}{2}\right]+ \\
& +\varepsilon^{2} \cos 2 \tilde{E}\left[\frac{\beta_{2}}{2} \frac{a^{2}}{n^{2}+a^{2}}-\left(-\frac{1}{2}+\frac{F_{p}^{2}}{2}+2 F_{p}\right)-\frac{\left(1+F_{p}\right)^{2}}{8}\right]
\end{aligned}
$$

This is like a Hill equation. The Floquet theory tells us that there are solutions of the form $e^{\mu \widetilde{E}} \phi(\tilde{t})$ where $\phi$ is a periodic function. The problem is to determine $\mu$ which is solution of Hill's determinantal equation. In that equation, there is an indefinite determinant to evaluate which is not an easy calculation.

Conclusion

The problem is much harder than we expected at the beginning and we need some numerical investigations.

The interesting case where $G$ goes to zero periodically is extremely hard to study since we get in that case an essential periodic singularity.

The dissipative equation is much more complicated to write than the non-dissipative one because the differential equation for the time behavior of the mean temperature becomes non-trivial.

If in some aspects there was some analogy with the case of convection in presence of rotation, we would expect a stabilizing effect as shown by Chandrasekhar (1961) and Weiss (1964). Unfortunately there is no analoby so far.

As a final conclusion, I should like to emphasize that this is only a preliminary work to a much detailed study which should follow.

\section{Acknowledgments}

I would like to thank especially Dr. E.A.Spiegel for having proposed that problem and for his help and suggestions. I thank also Drs. M.Landahl and G.Veronis for very valuable discussions. Finally I am much indebted to 
the GD program for financial support during this work and to Mary Thayer and Shirlyn Baker for having typed my manuscript.

\section{References}

Baker-Moore-Spiegel 1971 Quart.J.Mech. and Appl.Math. 24: 391.

Chandrasekhar 1961 Hydrodynamic and Hydromagnetic Stability. Oxford.

Drury-Stewart 1976 Monthly Not.R.Astr.Soc. 177: 377.

Gough 1965 Geophys.Fluid Dyn. II (Woods Hole Oceanographic Institution) p. 49

Gough 1977 Astrophys.J. 214: 196.

Kurskov-Ozernoi 1974 Sov.Astr.A.J. 18: 157.

Landau-Lifschite. Mecanique des Fluides-Mir.

Ledoux-Schvarzschild-Spiegel 1961 Astrophys. J. 133: 184.

Moore-Spiegel 1966 Astrophys.J. 143: 871.

Richards 1976 SIAM J.Appl.Math. 30: 420.

Rosenblat-Herbert 1970 J.Fluis.Mech. 43: 385.

Rosenblat-Tanaka 1971 Phys.Fluids 14. 1319.

Spiegel 1977-a. preprint.

Spiegel 1977-b. private communication.

Spiegel-Veronis 1960 Astrophys.J. 131: 442.

Unno 1967 Pub.Astr.Soc.Japan. 19: 140.

Venezian 1969 J.Fluid Mech. 35: 243.

Weiss 1964 Phil.Trans. 256: 99.

Whittaker-Watson 1946 Modern Analysis, Cambridge. 
COUPLING BETWEEN A SURFACE MIXED LAYER AND

LARGE SCALE STEADY INTERIOR OCEAN MOTIONS

James W. Stevenson

\section{Introduction}

This paper considers the coupling between the surface mixed layer of the ocean and large (i.e. gyre) scale steady motions in the interior of the ocean in an anticyclonic gyre where there is downwelling at the bottom of the mixed layer. The surface mixed layer is typically the upper 10 to 100 meters of the ocean where the density is constant with depth. The wind stress and heat flux at the surface of the ocean drive motions in the interior of the ocean by acting through the mixed layer.

The derivation of the mixed layer equations will be explained in section 2. In section 3 a mixed layer model is coupled to a model of the thermocline circulation proposed by Needler (1967). There are two equations describing the coupling. First there is a heat equation where the heat is advected not only by the Ekman velocity but also by the surface interior geostrophic velocity. The second equation is the continuity equation. These two equations turn out to be highly nonlinear. Therefore in section 4 a more careful look is taken at the heat balance in the mixed layer in the southern part of a subtropical gyre where there is a net surface heating.

\section{Mixed laver model}

Several ways of parameterizing the turbulence in the surface boundary layer of the ocean have been proposed in the literature (see Niiler, (1976)). One of these is the bulk model of the mixed layer which takes advantage of the observations which show that density is constant with depth through the mixed layer. This constancy with depth allows the governing equations to be simplified by integrating them vertically through the mixed layer.

The following assumptions are made:

1) The motions are steady (i.e. $\frac{\mathrm{a}}{\partial t} \equiv 0$ )

2) The advection of momentum can be ignored.

3) The penetration of solar radiation with depth is ignored (i.e. solar radiation is assumed to be absorbed at the surface of the ocean).

4) The horizontal variation of ensemble averages of turbulent fields can be ignored compared with the vertical variations.

Total fields $\Phi$ are divided into mean $\phi$ and turbulent $\phi^{\prime}$ fields:

$$
\Phi=\phi+\phi^{\prime}
$$

The governing equations are the buoyancy, horizontal momentum, vertical momentum, continuity, and turbulent kinetic energy equations respectively:

$$
u \frac{\partial b}{\partial x}+v \frac{\partial b}{\partial y}+w \frac{\partial b}{\partial z}+\frac{\partial}{\partial z} \overline{w^{\prime} b^{\prime}}=0
$$




$$
\begin{aligned}
& f \hat{k} \times \underline{v}+\frac{\partial}{\partial z} \overline{\omega^{\prime} w^{\prime}}=-\nabla P \\
& \frac{\partial p}{\partial z}=b \\
& \frac{\partial u}{\partial x}+\frac{\partial v}{\partial y}+\frac{\partial w}{\partial z}=0 \\
& 0=-\overline{w^{\prime} w^{\prime} \cdot} \frac{\partial}{\partial z}+\overline{w^{\prime} b^{\prime}}-\frac{1}{2} \frac{\partial}{\partial t}\left[\overline{\omega^{\prime}\left(g^{2}+\frac{P^{\prime}}{P}\right)}\right]-\epsilon .
\end{aligned}
$$

where $(x, y, z)$ are the east, north, and vertical directions, $(u, v, w)$ are are the east, north, and vertical velocities, $b\left(\equiv \frac{\rho_{o}-\rho_{0}}{\rho_{o}} g\right)$ is the buoyancy, $\rho$ is density, $\rho_{0}$ is a reference density, $g$ is the acceleration of gravity, $P$ is pressure, $f$ is the Coriolis parameter, $\epsilon$ is the viscous dissipation, and $q^{2} \equiv u^{\prime 2}+w^{1^{2}}+\omega^{12}$.

The buoyancy and velocity profiles are taken to be the following:
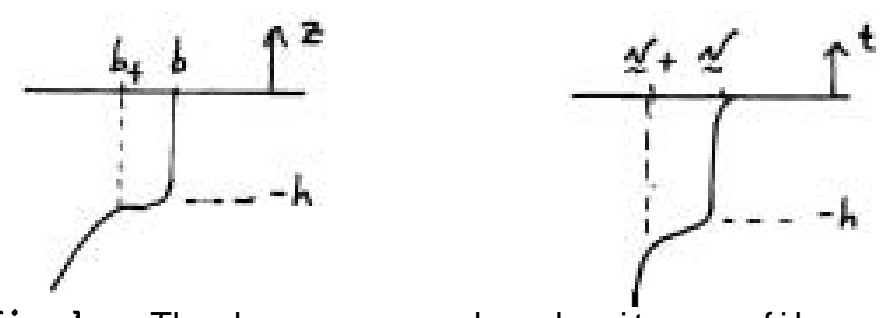

Fig.l. The buoyancy and velocity profiles.

Equations (1) are then integrated vertically from -h to 0 to obtain the following equations:

$$
\begin{aligned}
& h u \frac{\partial h}{\partial x}+h v \frac{\partial b}{\partial y}+\Lambda\left(b-b_{+}\right) e=B_{0}-B_{+} \\
& f \hat{k} \times h \sigma_{\sim}^{\prime}=\tau_{-}-h \nabla p_{+}-\Lambda\left(b-b_{+}\right) h \nabla h-\frac{1}{2} h^{2} \nabla b \\
& \frac{\partial h u}{\partial x}+\frac{\partial h v}{\partial y}=e \\
& \Lambda \frac{1}{2} h\left(b-b_{+}\right) e-\Lambda \frac{1}{2} m_{w}\left|\tau-\downarrow_{+}\right|^{2} e=m_{0} u_{*}^{3}-\varepsilon_{0} h-\frac{1}{2} B_{+} h-\frac{1}{2} B_{+} h
\end{aligned}
$$

where $\Lambda=\left\{\begin{array}{lll}\text { I } & \text { if } e>0 & \text { (entrainment) } \\ 0 & \text { if } e<0 & \text { (detrainment) }\end{array}\right.$

$$
e=w_{+}+u_{+} \frac{\partial h}{\partial x}+v_{+} \frac{\partial h}{\partial y}
$$

The symbols are as follows: $\mathfrak{W}$ is the vertically constant mixed layer velocities, $\mathbb{V}_{+}$is the velocity just below the mixed layer, $b$ is the vertically constant mixed layer buoyancy, $b_{+}$is the buoyancy just below the mixed layer, $h$ is the mixed layer depth, $e$ is the velocity of the fluid crossing the bottom interface of the mixed layer, $B_{e}$ is the surface flux of buoyancy (the sum of the solar short wave radiation, the long wave radiation, the latent heat flux, and the sensible heat flux), $\mathcal{B}_{+}$is the flux of buoyancy at the bottom of the mixed layer, $\tau_{0}$ is 
the surface wind stress, $P_{+}$is the pressure just below the mixed layer, and $u_{*} \equiv\left|\tau_{0}\right|^{\prime} f a$ is the friction velocity. The rate of generation of turbulent kinetic energy at the surface is parameterized as $m_{0} u_{*}^{3}$, the rate of generation of turbulent kinetic energy at the bottom of the mixed layer due to shear in the mean velocity is parameterized as $\Lambda \frac{i}{2} m_{w}\left(\boldsymbol{w}-\boldsymbol{\psi}_{4}\right)^{2} \boldsymbol{E}_{2}$, and the rate of dissipation of turbulent kinetic energy is parameterized as For a more detailed discussion of the mixed layer equations see Niiler and Kraus (1977) or Niiler (1976).

It is important to note the various contributions to the velocity of the mixed layer. The first term on the right-hand side of Eq. (2b) is the wind stress that drives the Ekman velocity. The last three terms are contributions to the pressure gradient in the mixed layer. The second term on the right-hand side of Eq. (2b) is the pressure gradient just below the mixed layer. The third term is the contribution to the pressure gradient due to a sloping interface with a buoyancy jump. These two contributions to the pressure gradient are constant with depth through the mixed layer. The fourth term is the contribution to the pressure gradient due to horizontal variations in buoyancy. This contribution varies linearly with depth through the mixed layer.

3. Coupling between mixed layer and thermocline models

In this section the mixed layer model is coupled to a thermocline model of an anticyclonic gyre. First, however, the thermocline model is briefly described.

Several models of the thermocline circulation have been proposed in the literature. The equations governing the flow in these models in the interior of the ocean away from horizontal and vertical boundary layers are the geostrophic, hydrostatic, continuity, and heat equations.

$$
\begin{aligned}
& f v=\frac{1}{a \cos \phi} \frac{\partial p}{\partial \lambda} \\
& f u=-\frac{1}{a} \frac{\partial p}{\partial \phi} \\
& \frac{\partial p}{\partial z}=g \alpha T \\
& \frac{1}{a \cos \phi} \frac{\partial u}{\partial \lambda}+\frac{1}{a \cos \phi} \frac{\partial}{\partial \phi}(v \cos \phi)+\frac{\partial \omega}{\partial z}=0 \\
& \frac{u}{a \cos \phi} \frac{\partial T}{\partial \lambda}+\frac{v}{a} \frac{\partial T}{\partial \phi}+\omega \frac{\partial T}{\partial z}=K \frac{\partial^{*} T}{\partial z^{2}}
\end{aligned}
$$

where $(\lambda, \phi)$ are the longitude and latitude respectively, $K$ is the vertical eddy diffusivity of heat, $\alpha$ is the coefficient of thermal expansion, $T$ is temperature, and $a$ is the radius of the Earth. The buoyancy $b$ is equal to $g \propto T$. These equations can be combined to form one nonlinear equation for $p$. Needler (1967) found a solution (though not a general solution) to this equation for $P$ of the form

$$
P=A(\lambda, \phi)+M(\lambda, \phi) e^{k z}
$$

where $k=\frac{c}{\sin \phi}$ and $c$ is a constant. Equations $(3 a, b, c, e)$ are then used to find $v, u, T$, and $w$ respectively. For a more detailed discussion of 
thermocline models see Veronis (1969) which is a review of the subject.

This solution restricts the number of boundary conditions that can be satisfied to two. In general it would be desirable to be able to specify three boundary conditions such as the surface temperature, the vertical velocity at the bottom of the mixed layer, and zero normal velocity at the bottom of the ocean.

In this work the mixed layer is forced by a given wind stress and a given surface buoyancy flux and is also influenced by the interior motions. The functions $A(\lambda, \phi)$ and $M(\lambda, \phi)$ which determine the interior motions are themselves determined from two matching conditions with the mixed layer. The matching is applied at the top of the interior thermocline solution (i.e. at $z=0$ ) and just below the mixed layer. The first condition is a matching of the buoyancies. Because there is downwelling at the bottom of the mixed layer in an anticyclonic gyre the buoyancy just below the bottom of the mixed layer, which is the buoyancy at the top of the interior, is equal to the buoyancy in the mixed layer (i.e. $\left.\mathbf{b}_{+}=\mathbf{b}\right)$. The second condition is a matching of the vertical velocity at the bottom of the mixed layer to the vertical velocity at the top of the interior.

The mixed layer equations written in polar coordinates taking into account the downwelling are the following:

$$
\begin{aligned}
& \frac{h u}{a \cos \phi} \frac{\partial b}{\partial \lambda}+\frac{h v}{a} \frac{\partial b}{\partial \phi}=B_{0}-B_{+} \\
& -f h v=\tau_{0}^{(x)}-f h v_{g}-\frac{h^{2}}{2 a \cos \phi} \frac{\partial b}{\partial \phi} \\
& f h u=f h u_{g}-\frac{h^{2}}{2 a} \frac{\partial b}{\partial \phi} \\
& \frac{h}{a \cos \phi}\left[\frac{\partial u}{\partial \lambda}+\frac{\partial}{\partial \phi}(v \cos \phi)\right]=\omega_{+}^{r} \\
& O=m_{0} u_{*}^{3}-\varepsilon_{0} h-\frac{1}{2} B_{0} h-\frac{1}{2} B_{+} h
\end{aligned}
$$

where a zonal wind stress is assumed. It should be noted that the velocities. (see Eqs. (5b,c) due to the gradient of buoyancy do not advect buoyancy since the velocity is along lines of constant buoyancy. Two equations for $A(\lambda, \phi)$ and $M(\lambda, \phi)$ are found using Eqs. (5a) (buoyancy) and (5d) (continuity). The velocities are obtained from $(5 b, c)$ and the mixed layer depth from (5e).

The buoyancy at the top of the interior is found from Eqs (4) and (3c) to be:

$$
b=g \alpha T=k M
$$

The velocity $\left(u_{g}, V_{y}\right)$ at the top of the interior is found from Eqs. (4) and $(3 a, b)$ to be:

$$
\begin{aligned}
& u_{g}=-\frac{1}{a f}\left(\frac{\partial A}{\partial \phi}+\frac{\partial M}{\partial \phi}\right) \\
& v_{g}=\frac{1}{a f \cos \phi}\left(\frac{\partial A}{\partial \lambda}+\frac{\partial M}{\partial \lambda}\right)
\end{aligned}
$$

The vertical turbulent flux of buoyancy at the top of the interior is 
found from Eqs. (4) and (3c) to be:

$$
B_{+}=\left.\alpha g K \frac{\partial T}{\partial z}\right|_{E=0}=K k^{2} M
$$

The vertical velocity at the top of the interior is found from Eqs. (4) and $(3 a, b, c, e)$ to be:

$$
\omega_{+}=k k+\frac{1}{f a^{2} k}\left(\frac{\frac{\partial A}{\partial \phi} \frac{\partial M}{\partial \lambda}-\frac{\partial A}{\partial \lambda} \frac{\partial M}{\partial \phi}}{M \cos \phi}+\frac{\frac{\partial A}{\partial \lambda}+\frac{\partial M}{\partial \lambda}}{\sin \phi}\right)
$$

One equation for $A(\lambda, \phi)$ and $M(\lambda, \phi)$ is found by substituting Eqs. $(5 b, c),(6)$, $(7 a, b)$, and $(8)$ into $(5 a)$ :

$$
-\frac{h}{f a^{2} \cos \phi}\left(\frac{\partial A}{\partial \phi}+\frac{\partial M}{\partial \phi}\right)_{k} \frac{\partial M}{\partial \lambda}+\frac{h}{f a^{2} \cos \phi}\left(\frac{\partial A}{\partial \lambda}+\frac{\partial M}{\partial \lambda}\right) \frac{\partial k M}{\partial \phi}-\frac{\tau_{0}^{(x)}}{f^{\alpha}} \frac{\partial k M}{\partial \phi}=B_{0}-K k^{2} M
$$

The second equation for $A(\lambda, \phi)$ and $M(\lambda, \phi)$ is found by substituting Eqs. $(5 b, c),(6),(7 a, b)$, and (9) into $(5 d)$ :

$$
\begin{gathered}
-\frac{h}{a \cos \phi} \frac{\partial}{\partial \phi}\left(\frac{\tau_{i}^{(x)} \cos \phi}{f h}\right)-\frac{\beta h}{f^{2} a \cos \phi}\left(\frac{\partial A}{\partial \lambda}+\frac{\partial M}{\partial \lambda}\right)+\frac{h}{2 \alpha^{2} \cos \phi}\left(k \frac{\partial M}{\partial \lambda} \frac{\partial}{\partial \phi} \frac{h}{f}-\frac{\partial k N}{\partial \phi} \frac{\partial}{\partial \lambda} \frac{h}{f}\right)= \\
=K k+\frac{1}{f a^{2} k}\left(\frac{\frac{\partial A}{\partial \phi} \frac{\partial M}{\partial \lambda}-\frac{\partial A}{\partial \lambda} \frac{\partial M}{\partial \phi}+\frac{\partial A}{\partial \lambda}+\frac{\partial A}{\partial \lambda}}{\sin \phi}\right)
\end{gathered}
$$

No solutions were found to this set of nonlinear equations.

4. Mixed layer heat balance in southern part of subtropical gyre

In this section the heat balance in the mixed layer is studied in the southern part of the subtropical gyre where there is a net surface heating. It should first be noted that the advection of heat by the Ekman transport cannot balance the positive surface heat flux. The Ekman transport is to the north between $15^{\circ} \mathrm{N}$ and $30^{\circ} \mathrm{N}$ where there is a westward wind stress. The sea surface temperature in this region decreases to the north. Therefore, the northward Ekman transport brings in warm water from the south which adds to the heating of the mixed layer by the positive surface heat flux.

The advection of heat by the interior surface velocity must balance the surface heat flux since the advection by the Ekman velocity cannot provide the balance. This is why Eq. (10) is so complicated. The interior motions that are generated by the surface wind stress and the surface heat flux acting through the mixed layer are important in advecting heat in the mixed layer.

In order to get an idea of what interior surface velocities are necessary to balance the heat (or buoyancy) equation the following simple model is solved. In the heat equation the surface heat flux and surface temperature structure are given and the interior surface velocities are solved for. The heat equation is:

$$
h u_{g} \frac{\partial b}{\partial x}+\left(h v_{g}-\frac{\tau^{(x)}}{f}\right) \frac{\partial b}{\partial y}=B_{0}
$$


where $u_{g}, V_{8}$ are geostrophic:

$$
\begin{aligned}
& f \sqrt{g}=\frac{\partial p}{\partial x} \\
& f u_{g}=\frac{\partial p}{\partial y}
\end{aligned}
$$

Using Eqs. (13a,b), Eq. (12) can be written in characteristic form):

$$
\frac{\partial P}{\partial x}=\frac{f B_{0}}{h \frac{\partial b}{\partial y}}+\frac{\tau^{(x)}}{h}
$$

where $\frac{d P}{d x}$ is the derivative with respect to $x$ along the characteristics definedby:

$$
\frac{d y}{d x}=-\frac{\partial b / \partial x}{\partial b / \partial y}
$$

The characteristics are lines of constant buoyancy. For simplicity $h$ and

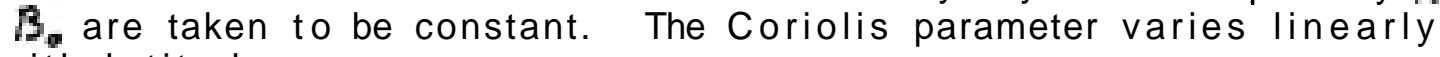
with latitude:

$$
f=f_{0}+\beta_{y}
$$

The wind stress is taken to be:

$$
\tau^{(x)}=-A \cos l y
$$

Also, for simplicity the lines of constant buoyancy are taken to be straight parallel lines:

$$
y=-c_{1}(x-L)-\frac{\dot{b}-\dot{b}_{1}}{b_{1} / \lambda}
$$

where $c_{1}$ is positive so that 1 Ines of constant buoyancy run from northwest to southeast and $b_{1} / \boldsymbol{\lambda}$ is positive so that the surface water gets colder toward the northeast.

The domain of interest is $0 \leq x \leq L$ and $04 \ell y \leq \frac{\pi}{2}$. The boundary conditions that will be speclfled are the normal velocities at the eastern and southern boundaries, The line of constant buoyancy that passes through the southeastern corner of the domain $I_{s} b=b_{1}$. The solution to (14) is:

$$
P=-(x-L)\left(f_{0}+\beta_{y}\right) \frac{B_{0}}{h b_{1} / h}-\frac{\rho c_{1} B_{0}}{2 h b_{1} / \lambda}(x-L)^{2}+\frac{A}{h c_{1} l} \sin L y+g\left(-\frac{b-b_{1}}{b_{1}}\right)
$$

For $b<b_{1} \quad g\left(-\frac{b-b_{1}}{b_{1}}\right)=P_{E}\left(\frac{y}{\lambda}+\frac{c_{1}}{\lambda}(x-L)-\frac{A}{h_{c_{1}} l} \sin \left(l_{y}+c_{1} l(x-L)\right)\right.$

For $b>b_{1} \quad g\left(-\frac{b-b_{b}}{b_{1}}\right)=P_{\xi}\left(\frac{y}{\lambda}=\frac{c^{\prime}}{\lambda}(x-L)\right)+\left(y+c_{1}(x-L) \frac{t_{1} B_{1}}{c_{1} h b_{1} / \lambda}+\left(y+c_{1}(x-L)\right)^{2} \frac{\beta B_{0}}{2 c_{3} b_{1} / \lambda}\right.$

where $P_{E}$ and $P_{S}$ are the pressures at the eastern and southern boundaries.

Two choices of boundary conditlons are considered. First let $\sqrt{9}=0$ at $y=0$ and $f u_{g}=$ constant at $x=L$. The solutions (shown in Fig.2) 


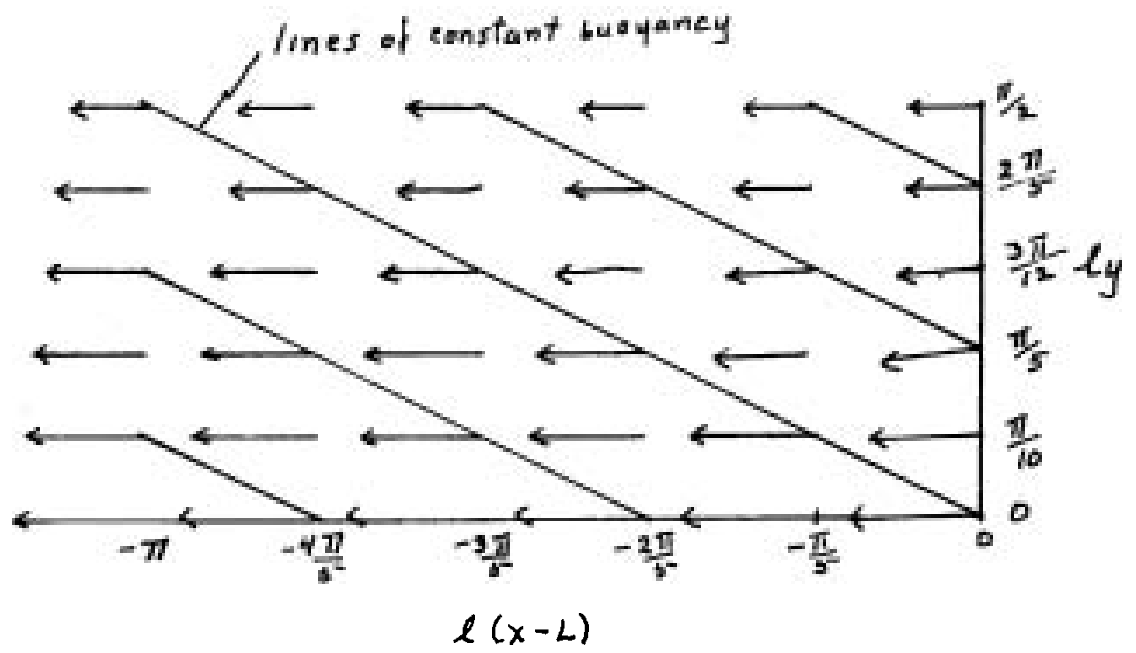

Fig.2. Interior surface velocities $\left(V_{g}=0\right.$ at $\left.y=0\right)$

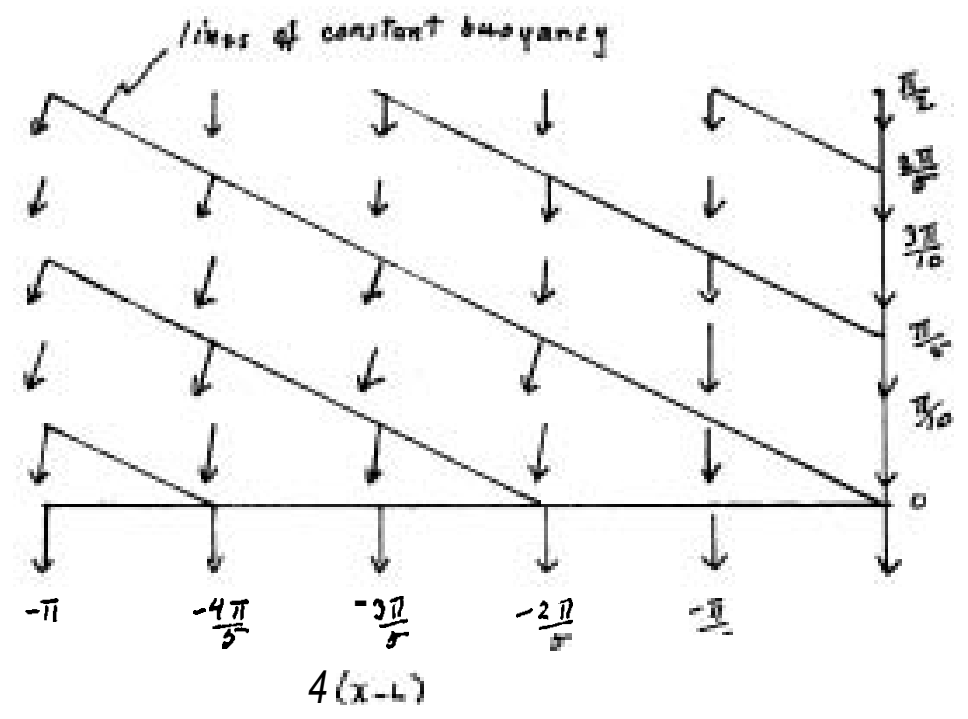

Fig.3. Interior surface velocities $\left(u_{g} 0\right.$ at $\left.x=L\right)$ 
for these boundary conditions are:

For $b<b_{1}: \frac{h u_{4}}{\beta_{0}} \frac{a b}{\partial x}=-f_{0}^{+}+\beta_{1}-\beta-\beta c_{1}+\frac{A b_{1} / \lambda}{\left(f_{0}+\beta_{u}\right) B_{0}}\left[\cos l_{y}-\cos \left(l_{y}+c_{1} l(x-1)\right)\right]+$

$$
\begin{gathered}
+\frac{A b / \lambda}{\left(f_{0}+\beta_{y}\right) B_{0}}+\frac{f_{0}}{f_{0}+\beta_{y}} \\
\frac{h \sqrt{y}}{B_{0}} \frac{\partial b}{y}=1+\frac{x-L}{f_{0}+A_{y}} \beta c_{1}+\frac{A b_{1} / \lambda}{\left(f_{0}+A_{y}\right) B_{0}} \cos \left(l y+c_{1} l(x-L)\right)-\frac{A b_{1} / \lambda}{\left(f_{0}+A_{y}\right) B_{0}}-\frac{f_{0}}{f_{0}+A_{y}}
\end{gathered}
$$

and for $b>b_{1}: \frac{h u_{0}}{B_{0}} \frac{\partial b}{\partial x}=1+\frac{A b y / \lambda}{\left(f_{0}+A_{y}\right) B_{0}} \cos \ell_{y}$

$$
\frac{h \sqrt{g}}{B_{0}} \frac{\partial b}{\partial y}=0
$$

The second choice of boundary conditions is $\sqrt{g}$ - constant at $y=0$ and $u_{g}=0$ at $x=L$. The solutions (shown in Fig.3) för these boundary conditions are:

$$
\text { For } \begin{aligned}
b<b_{1}: & \frac{h u_{0}}{B_{0}} \frac{\partial b}{\partial x}=-\frac{x-L}{f_{0}+\beta_{y}} \beta c_{1}+\frac{A b_{1} / \lambda}{\left(f_{0}+\beta_{y}\right) B_{0}}\left[\cos \ell_{y}-\cos \left(l_{y}+c_{i} \ell(x-L)\right)\right] \\
& \frac{h V_{g}}{B_{0}} \frac{\partial b}{\partial y}=1+\frac{x-d}{f_{0}+\beta_{y}} \beta c_{1}+\frac{f_{0}+\beta_{y} \beta_{B_{0}}}{f_{0}} \cos \left(l_{y}+c_{0} \ell(x-L)\right)
\end{aligned}
$$

and for $b>b_{1}$ :

$$
\begin{aligned}
& \frac{b_{1}:}{B_{0}} \frac{\partial b}{\partial x}=1+\frac{A^{b_{1} / \lambda}}{\left(f_{0}+\beta_{y}\right) B_{0}} \cos b_{y}-\frac{A^{b_{1} / \lambda}}{\left(f_{0}+\beta_{y}\right) B_{0}}-\frac{f_{0}}{f_{0}+B y} \\
& \frac{h v_{0}}{B_{0}} \frac{\partial b}{\partial y}=\frac{A^{b} / \lambda}{\left(f_{0}+\beta_{y}\right) B_{0}}+\frac{f_{0}}{f_{0}+\beta_{y}}
\end{aligned}
$$

The advection of buoyancy by the Ekman velocity is:

$$
\frac{\tau^{(x)}}{\left(f_{0}+\beta_{y}\right) B_{0}} \frac{\partial b}{\partial y}=-\frac{A^{b_{1} / \lambda}}{\left(f_{0}+\beta_{y}\right) B_{0}} \cos l y
$$

The advection of buoyancy by the velocities induced by the gradient of buoyancy in the mixed layer (i.e. the last term of (26)) is:

$$
\begin{aligned}
& \frac{h u_{i}}{B_{0}} \frac{\partial b}{\partial x}=\frac{h^{3} c_{i}\left(b / b_{a}\right)^{2}}{2\left(f_{0}+\beta_{y}\right) B_{0}} \\
& \frac{h v_{i}}{B_{0}} \frac{\partial b}{\partial y}=-\frac{h^{2} c_{1}\left(b_{b} / h_{i}\right)^{2}}{2\left(f_{a}+\beta_{y}\right) B_{0}}
\end{aligned}
$$

Figures 2 and 3 are based on the following choice of parameters:

$$
\begin{array}{rlrl}
h & =10^{4} \mathrm{~cm} & b_{1} / \lambda & =5 \times 10^{-9} \mathrm{sec}^{-2} \\
\ell & =9 \times 10^{-9} \mathrm{~cm}^{-1} & c_{1} & =\mathbf{i} / 2 \\
L & =5 \times 10^{8} \mathrm{~cm} & f_{0} & =4 \times 10^{-5} \mathrm{sec}^{-1} \\
A & =1 \mathrm{~cm}^{2} / \mathrm{sec}^{2} & \beta & =2 \times 10^{-13}(\mathrm{~cm} \mathrm{sec})-1 \\
B_{a}=2 \times 10^{-4} \mathrm{~cm}^{2} / \mathrm{sec}^{3} &
\end{array}
$$


Two nondimensional parameters of interest are:

$$
\begin{aligned}
\frac{A^{b} b_{1} / \lambda}{f_{0} B_{0}} & =0.6 \\
\frac{h_{0} c_{1}\left(b_{0} / \lambda\right)}{2 f_{0} B_{0}} & =0.08
\end{aligned}
$$

The first parameter is the ratio of the Ekman velocity and the interior surface velocity needed to balance the surface buoyancy flux. The second parameter is the ratio of the velocity induced by the gradient of buoyancy in the mixed layer and the interior surface velocity needed to balance the surface buoyancy flux. Because this second parameter is small the induced velocity if generally small compared to the Ekman velocity or the interior surface velocity.

In Fig.2 the largest velocity is $13 \mathrm{~cm} / \mathrm{sec}$ which is larger than what is observed. The largest velocity in Fig. 3 is $6.5 \mathrm{~cm} / \mathrm{sec}$. It can be seen that the solution is very sensitive to the boundary conditions.

Acknowledgments

I would like to thank George Veronis for his help during the summer. I would also like to thank Mary Thayer for typing up this manuscript.

$\underline{\text { References }}$

Needler, G.T. 1967 A model for thermohaline circulation in an ocean of finite depth. J.Mar.Res. 25: 369.

Niiler, P.P. 1976 One dimensional models of the seasonal thermocline. The Sea, vol. 6 .

Niiler, P.P. and E.B.Kraus 1977 Modelling and Prediction of the Upper Layers of the Ocean, ed.by E.B.Kraus, Pergamon Press, 325 pp.ch. 10. One-dimensional models of the upper ocean.

Veronis, G. 1969 On theoretical models of the thermocline circulation. Deep Sea Res., 16 Suppl., 301. 


\section{MANDATORY DISTRIBUTION LIST \\ FOR UNCLASSIFIED TECHNICAL REPORTS, REPRINTS, \& FINAL REPORTS PUBLISHED BY OCEANOGRAPHIC CONIRACTORS \\ OF THE OCEAN SCIENCE AND TBCHNOLOGY DIVISION \\ OF THE OFFICE OF NAVAL RESEARCH \\ (SEVISED FEBRUARY 1978)}

1 Director of Defense Research and Engineering

Office of the Secretary of .Defense Washington, D. C. 20301

ATTN: Office Assistant Director (Research)

Office of Naval Research Arlington, VA 22217

1 ATTN: (Code 460)

1 ATTN: (Code 102-OS)

6 ATTN: (Code 102IP)

1 ATTN: (Code 200)

1 CDR J. C. Harlett, (USN) ONR Representative

Woods Hole Oceanographic Inst.

Woods Hole, MA 02543

1 Office of Naval Research Branch Office

495 Summer Street

Boston, MA 02210

Director

Naval Research Laboratory

Washington, D.C. 20375

6 ATTN: Library, Code 2620
1 National Oceanographic Data

Center

National Oceanic \& Atmospheric Administration

330C Whitehaven St., N.W.

Washington, D.C. 20235

12 Defense Documentation

Center

Cameron Station

Alexandria, VA 22314

Commander

Naval Oceanographic

Office

Washington, D.C. 20373

1 ATTN: Code 1640

1 ATTN: Code 70

3 NORDA 430

NSTL Station, MS 39529

2 CO NORDA

NSTL Station, MS 39529 


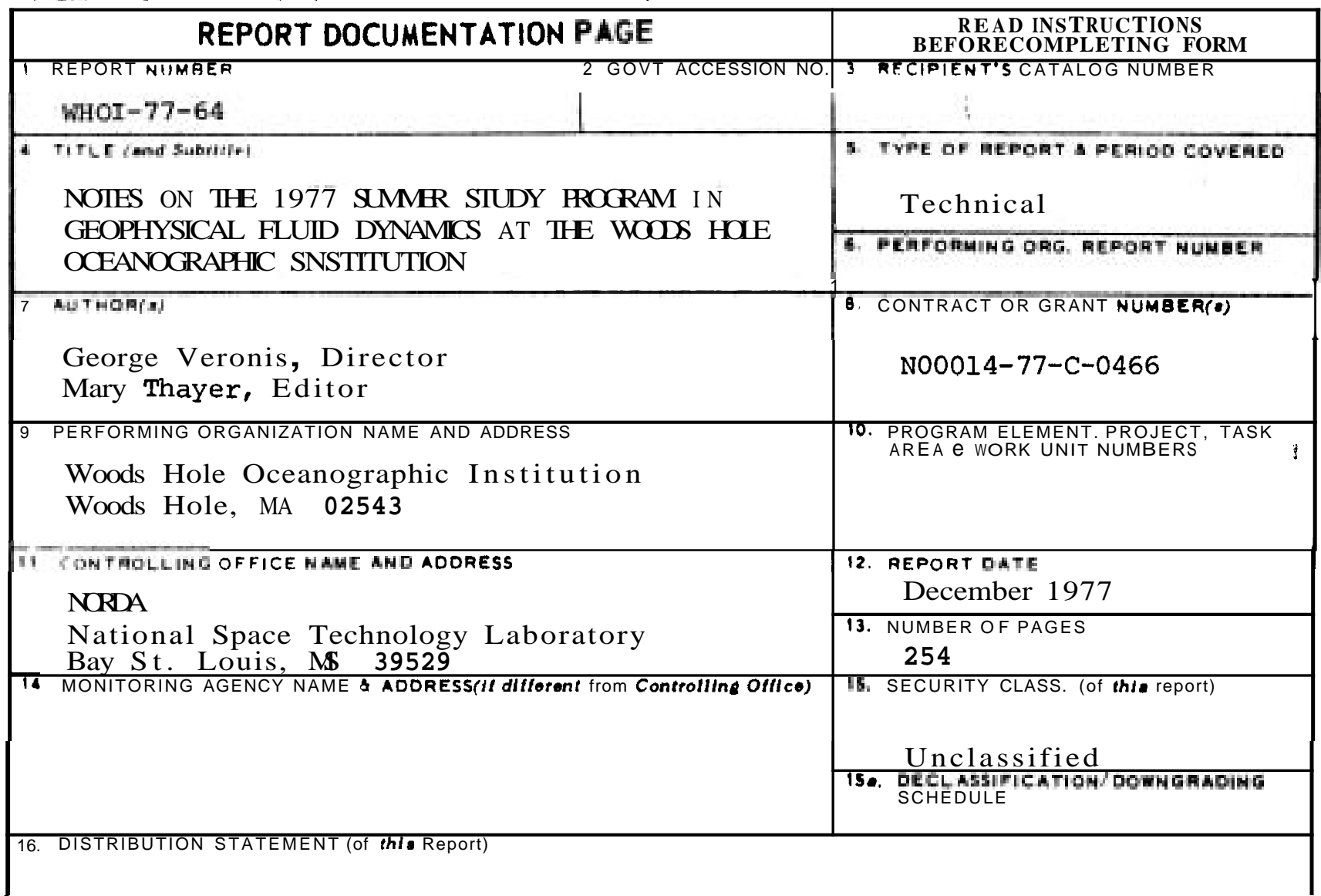

Approved for public release; distribution unlimited.

17 DISTRIBUTION STATEMENT (ol the ebstreet entered In Block 20, /1 different from Report)

18 SUPPLEMENTARY NOTES

19 KEY WORDS (Continue on reverse slde II necessary a didentlly by block number)

1. Geophysical Fluid Dynamics

2. Turbulence

3. Geostrophic turbulence

20 ABSTRACT (Contlnue on soveras elde If noceseary end ldenelfy by block number)

The lectures by Marten Landahl, recorded in the first part of this report, served as the introduction to the study of turbulence which was the principal theme of the nineteenth summer program in Geophysical Fluid Dynamics at the Woods Hole Oceanographic Institution. The reports of the lectures by the students were reworked by Professor Landahl so that they serve as a careful record of his presentation.

(Cont. on back)

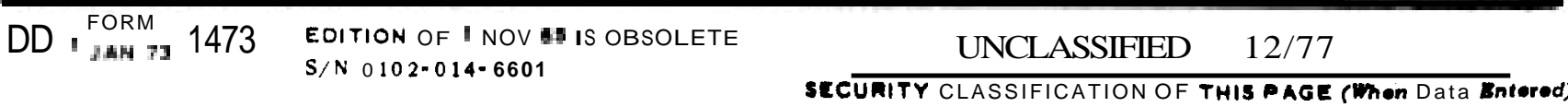


The abstracts of the seminars by invited speakers cover a broad range of topics, from astrophysics to experimental, laboratory fluid dynamics. The seminars served to introduce the student fellows to the tremendous diversity of studies that fall within the scope of geophysical fluid dynamics. Included ainong the abstracts are the seminars presented during the one-week symposium on geostrophic turbulence. Peter Rhines organized the symposium and invited many of the principal contributors to this important, developing topic.

The twelve student lectures summarize the most creative product of the summer program. These reports are necessarily preliminary because the short time available to the students does not admit a carefully worked, finished product. Some of the reports will appear later as published articles and will represent more effectively the product of the summer activity. 


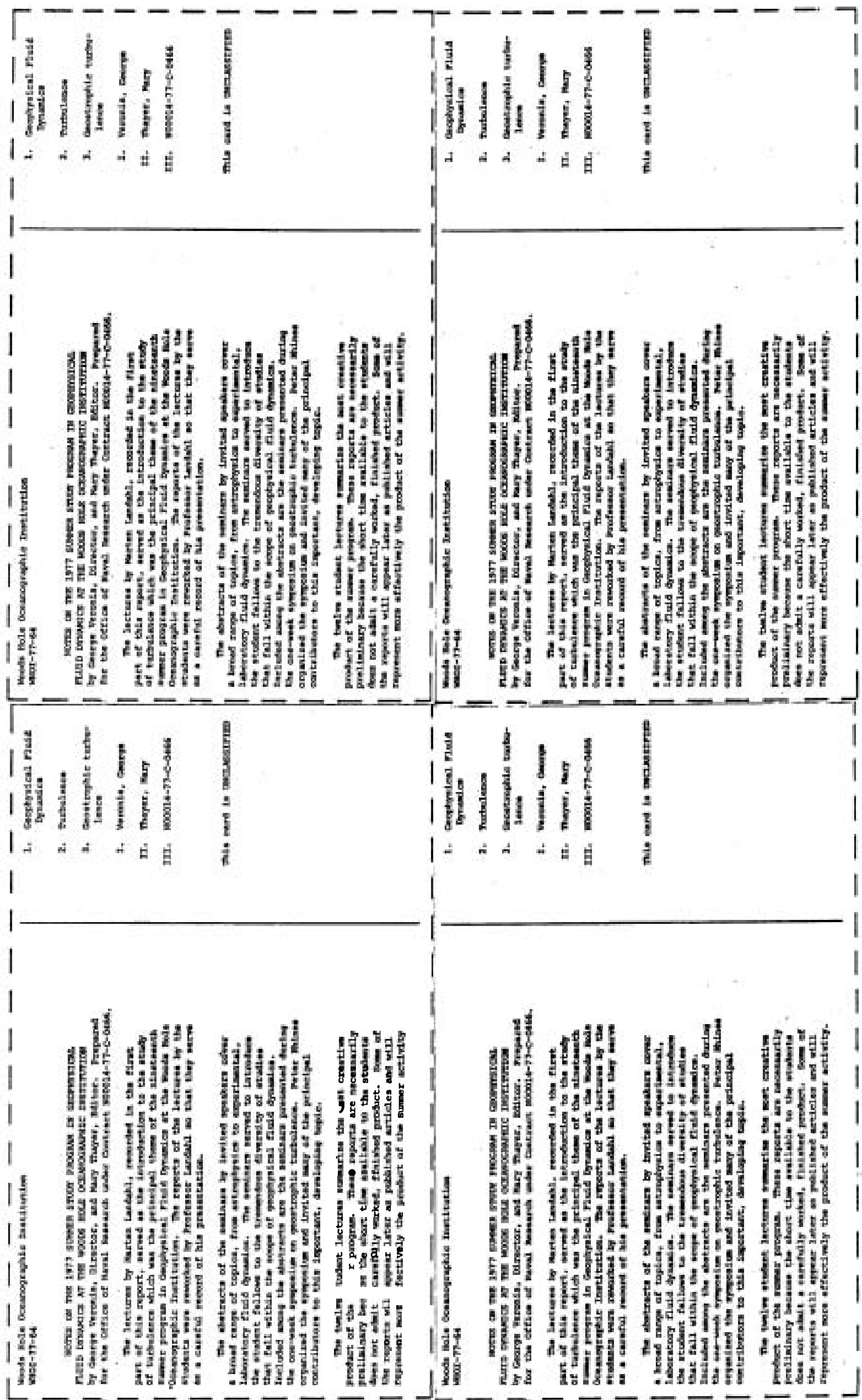

